\title{
Site-selective $\alpha-\mathrm{C}-\mathrm{H}$ Functionalization of Trialkylamines via Reversible HAT-Catalysis
}

Yangyang Shen ${ }^{1}$, Ignacio Funez-Ardoiz ${ }^{2}$, Franziska Schoenebeck, ${ }^{2,}{ }^{*}$ Tomislav Rovis ${ }^{1, *}$

Correspondence to: franziska.schoenebeck@rwth-aachen.de; tr2504@columbia.edu

${ }^{1}$ Department of Chemistry, Columbia University, New York, 10027 NY, USA.

${ }^{2}$ Institute of Organic Chemistry, RWTH Aachen University, 52074 Aachen, Germany. 


\section{$\underline{\text { Table of contents }}$}

I. General considerations $\quad$ S3

II. Optimization details and control experiments S4

III. Mechanistic experiments $\quad$ S9

IV. Computational details $\quad$ S16

V. $\quad \alpha-C\left(\mathrm{sp}^{3}\right)-\mathrm{H}$ alkylation via reversible HAT catalysis $\quad$ S64

$\begin{array}{ll}\text { VI. References } & \mathbf{S 8 3}\end{array}$

VII. ${ }^{1} \mathrm{H},{ }^{13} \mathrm{C}$ and ${ }^{19}$ F NMR spectra $\quad$ S84 


\section{General consideration}

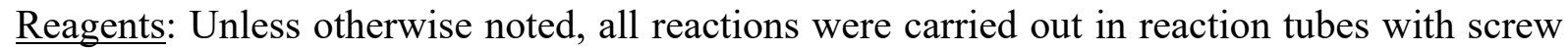
cap. Anhydrous solvents and reagents were purchased from Sigma Aldrich and used without further purification. All other reagents were purchased from commercial sources and used as received. For the pharmaceuticals received in salt form, a simple treatment with $\mathrm{K}_{2} \mathrm{CO}_{3}$ (aq.) and ethyl acetate offered pure enough sample for the reactions. Purification of the product was conducted either manually on SiliCycle ${ }^{\circledR}$ SilicaFlash ${ }^{\circledR}$ P60 (230-400 mesh) silica gel or automatically via a Teledyne Isco Lumen CombiFlash using CombiFlash gold pre-packed columns. Thin layer chromatography (TLC) was performed on Silicycle $250 \mu \mathrm{m}$ silica gel 60 Å plates. Visualization was accomplished with UV lamp (254 nm), $\mathrm{KMnO}_{4}$ or Iodine. A Kessil blue LED (34W maximum, $24 \mathrm{VDC}, 440 \mathrm{~nm}$ ) was used as the light source for the photoredox catalyzed reactions. The procedures described in this section are representative. Thus, the yields may differ slightly from those given in the tables of the manuscript.

Analytical Methods: ${ }^{1} \mathrm{H}$ NMR, ${ }^{13} \mathrm{C}$ NMR and ${ }^{19} \mathrm{~F}$ NMR spectra are included for all new compounds. ${ }^{1} \mathrm{H}$ NMR, ${ }^{13} \mathrm{C}$ NMR and ${ }^{19}$ F NMR spectra were recorded on Varian $300 / 400 \mathrm{MHz}$ or Bruker 400/500 MHz at ambient temperature. All ${ }^{1} \mathrm{H}$ NMR spectra are reported in parts per million (ppm) downfield of TMS and were measured relative to the signals for $\mathrm{CHCl}_{3}(7.26$ ppm). All ${ }^{13} \mathrm{C}$ NMR spectra were reported in ppm relative to residual $\mathrm{CHCl}_{3}(77.16 \mathrm{ppm})$ and were obtained with ${ }^{1} \mathrm{H}$ decoupling. Coupling constants, $J$, are reported in hertz $(\mathrm{Hz})$. In the case of diastereoisomeric mixtures, isolated NMR was recorded to determine the ratio. High resolution mass spectra (HRMS) were obtained from the Columbia University Chemistry Department Mass Spectrometry Facility on a Waters XEVO G2XS QToF mass spectrometer equipped with a UPC2 SFC inlet and a LockSpray source with one of the following three probes: electrospray ionization (ESI) probe, atmospheric pressure chemical ionization (APCI) probe, or atmospheric pressure solids analysis probe (ASAP). Infrared spectra were collected on a Perkin Elmer Spectrum Two FT-IR Spectrometer. Infrared spectra were recorded on a Bruker Tensor 27. Mass spectra were recorded on a Waters LCT Premier spectrometer. Stern-Volmer luminescence quenching experiments were conducted on a Fluoromax-4 fluorometer. 


\section{Optimization details and control experiments}

Table S1. Practical considerations of amine loadings

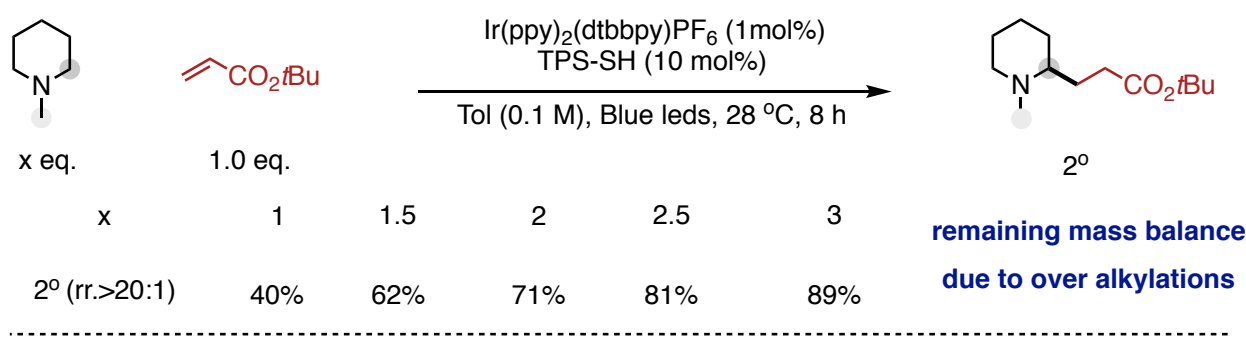

a yield and ratio are determined by GC-MS analysis with benzyl benzoate as internal standard

Table S2. Control experiments with/without TPS-SH
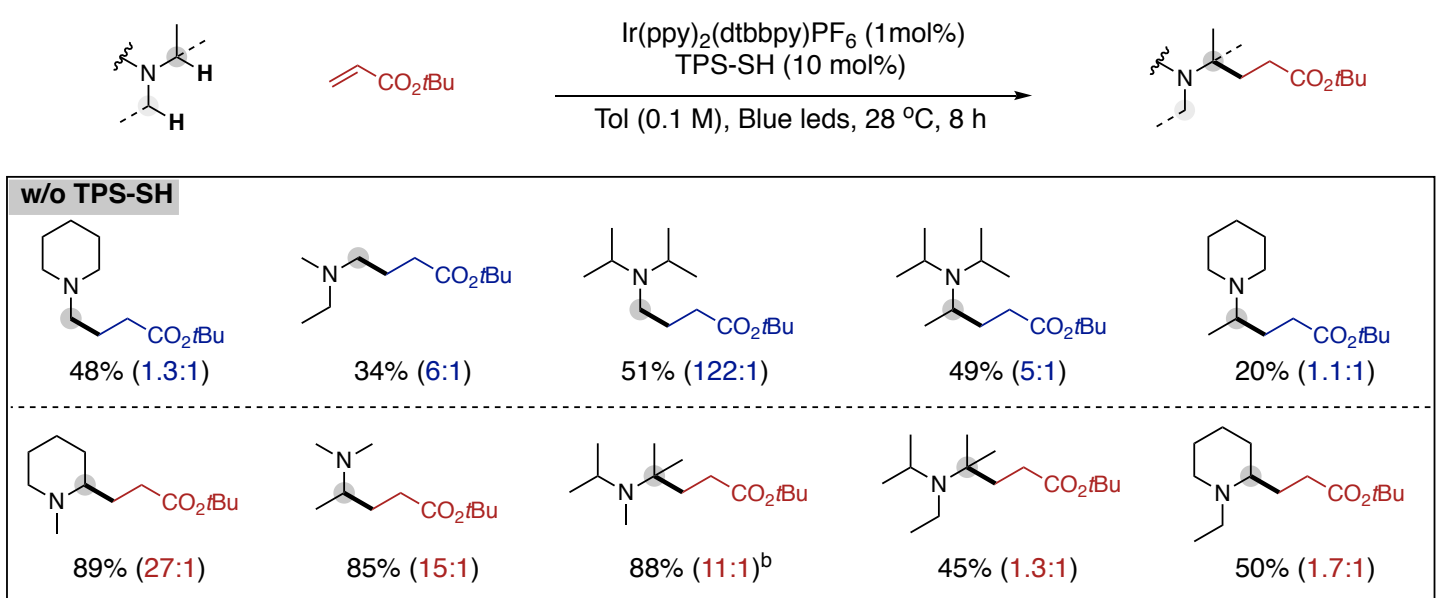

a yield and ratio are determined by GC-MS analysis with benzyl benzoate as internal standard; ${ }^{b} m$-Xylene was used
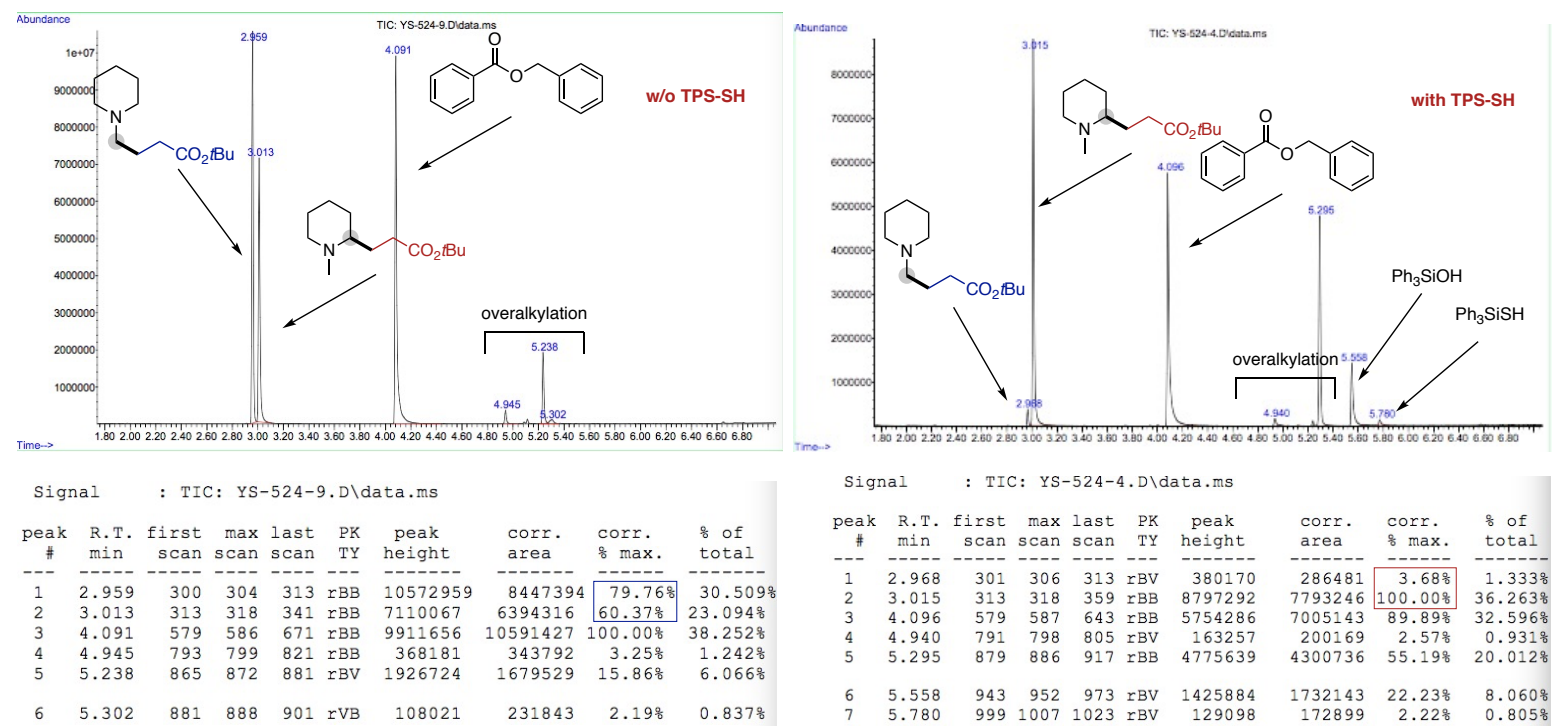


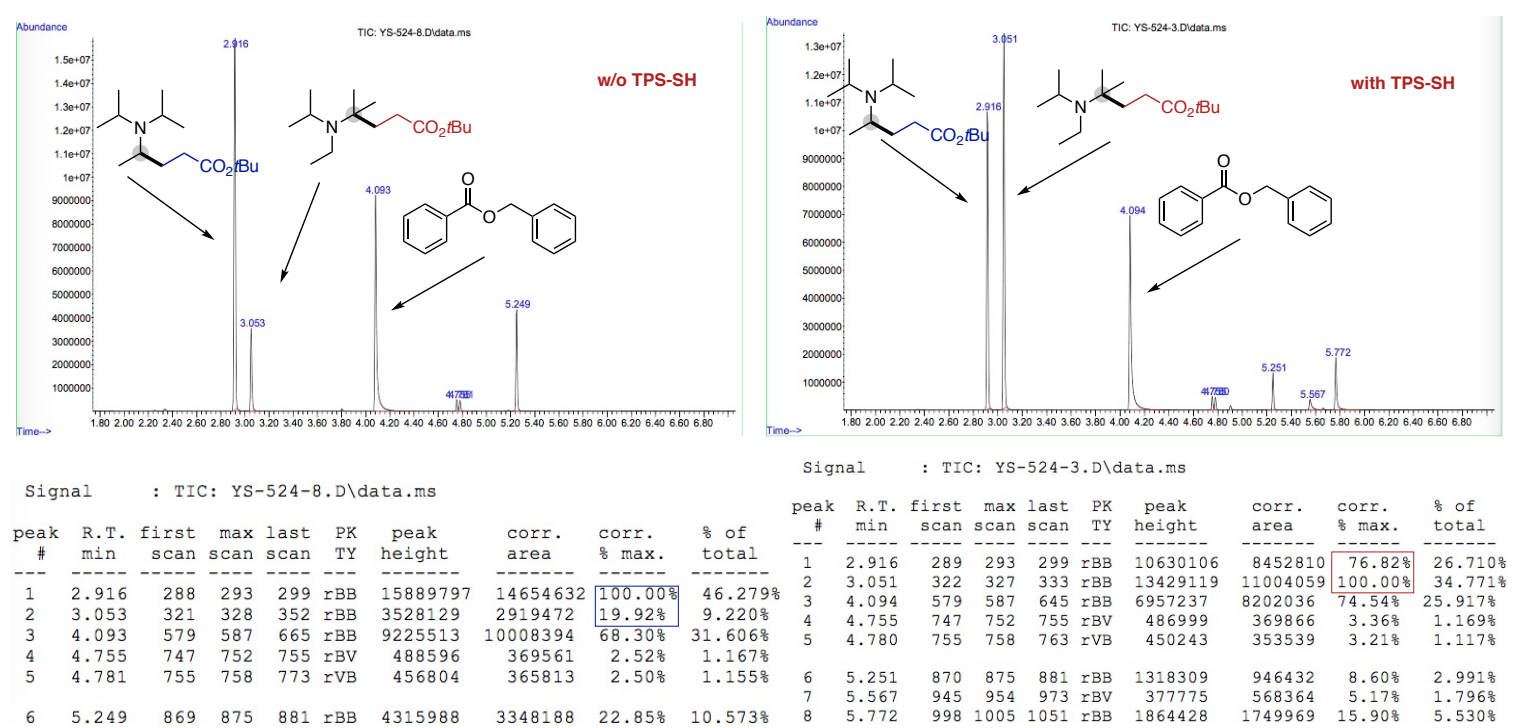

Note: Due to overlapping peaks in the spectra, ${ }^{1} H$-NMR analysis is unreliable as a method to determine the regioisomer ratios. We thus tentatively assign the ratios by GC-MS analysis, understanding that there are caveats with this method (efforts towards synthesis of minor isomer to correct the GC-MS results failed due to oligomer contamination). Notably, during the purification of the products, we only observe $<5 \%$ or trace of minor isomers (monoalkylation at the methyl position) when using more electron-deficient olefins (vinyl phenyl sulfone) as radical acceptor, unless otherwise noted.

Table S3. Optimization of thiol catalyst for $\mathrm{N}$-Me piperidine

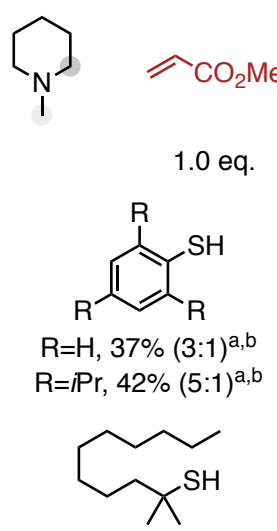

$37(2: 1)^{\mathrm{a}}$

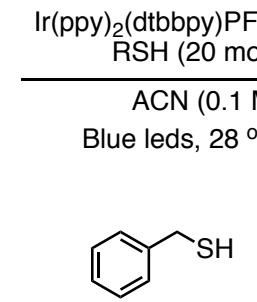

$34 \%(4: 1)^{a, b}$

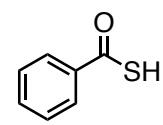

$40 \%(8: 1)^{a}$<smiles>COC(=O)CCC1CCCCN1C</smiles>

$2^{\circ}$

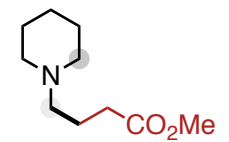

$1^{\circ}$

yield and ratio are determined by GC-MS analysis with benzyl benzoate as internal standard; a yeild of $2^{\circ}$ product, ratio of $2^{\circ}: 1^{o} ;{ }^{b}$ sulfa-Michael addition observed.

Table S4. Optimization of solvent for $N$-Me piperidine 


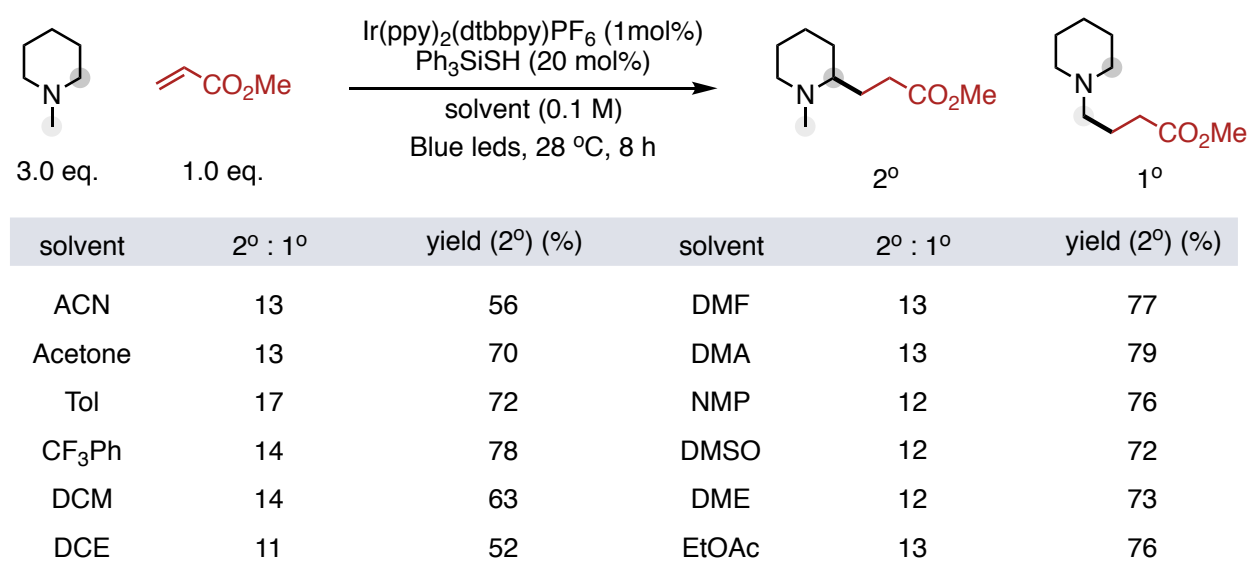

yield and ratio are determined by GC-MS analysis with benzyl benzoate as internal standard

Table S5. Optimization of solvent for $N, N$-diisopropylmethylamine

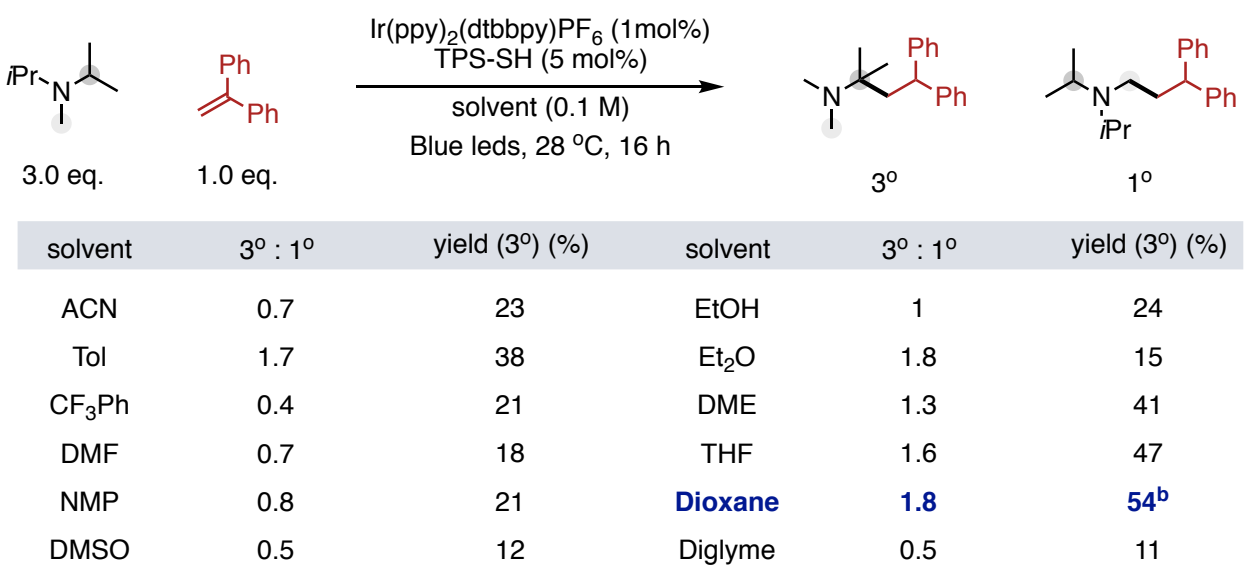

a yield and ratio are determined by GC-MS analysis with benzyl benzoate as internal standard b w/o TPS-SH, only $1^{\circ}$ product $(52 \%$ yield) observed.

Table S6. Practical consideration of alkylation of diethylcarbamazine<smiles>CCN(CC)C(=O)N1CCN(C)CC1</smiles><smiles>C=C(C(=O)OC)C(=O)OCC</smiles>

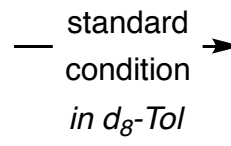
irradiation time $(\mathrm{h})$ conversion of olefin (\%)<smiles>CCN(CC)C(=O)N1CCN(C)C(C[C@H](C)C(=O)OCc2ccccc2)C1</smiles>
yield of $\mathbf{4 3}(\%)$

$\begin{array}{lcc}1 & 57 & 27 \\ 2 & 87 & 31 \\ 4 & 100 & 33\end{array}$

$0.2 \mathrm{mmol}$ scale. $0.1 \mathrm{mmol}$ of ethyl benzoate as internal standard. 


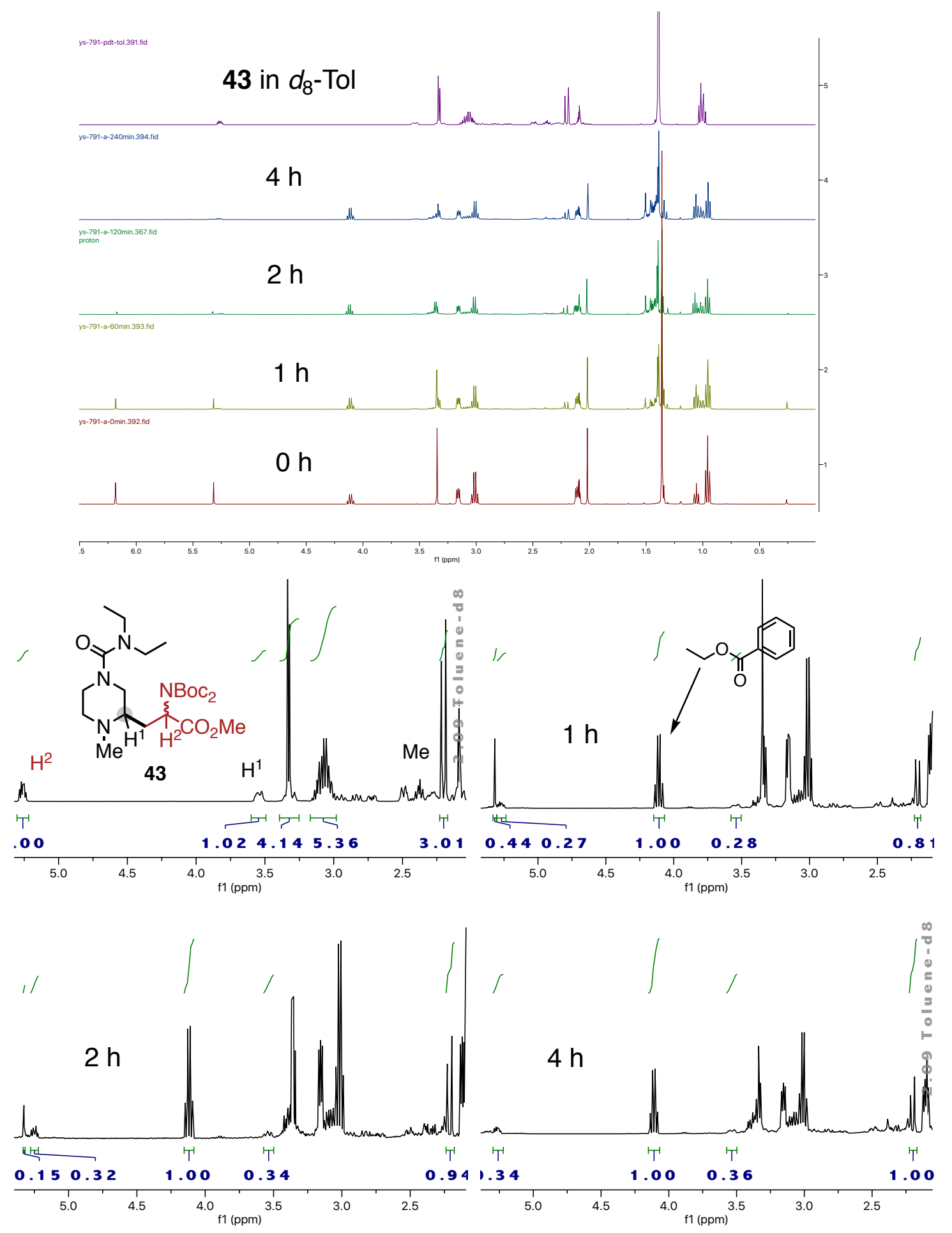


Table S7. Practical consideration of alkylation of clomipramine

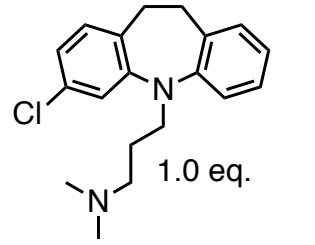

irradiation time $(\mathrm{h})$

0.5

1

2

10

$$
\begin{array}{cc}
\mathrm{SO}_{2} \mathrm{Ph} & -\begin{array}{l}
\text { standard } \\
\text { condition }
\end{array} \\
1.0 \mathrm{eq} . & \text { in } \mathrm{d}_{8} \text {-Tol }
\end{array}
$$

conversion of olefin $(0$

43

62

77

100

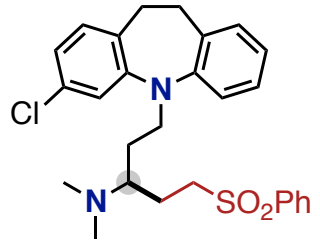

yield of 44 (\%)

17

21

23

24

$0.2 \mathrm{mmol}$ scale. $0.1 \mathrm{mmol}$ of ethyl benzoate as internal standard.
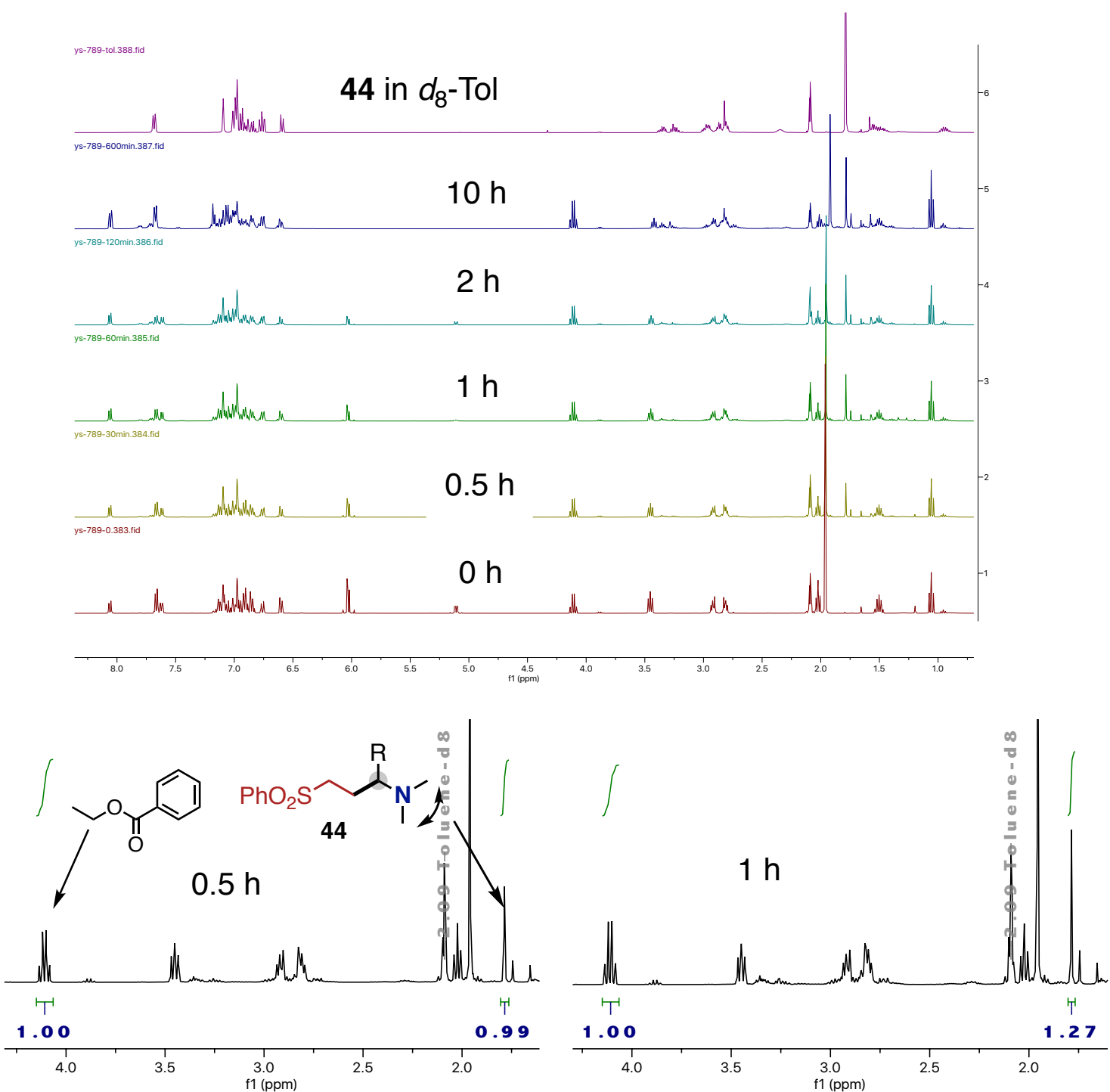


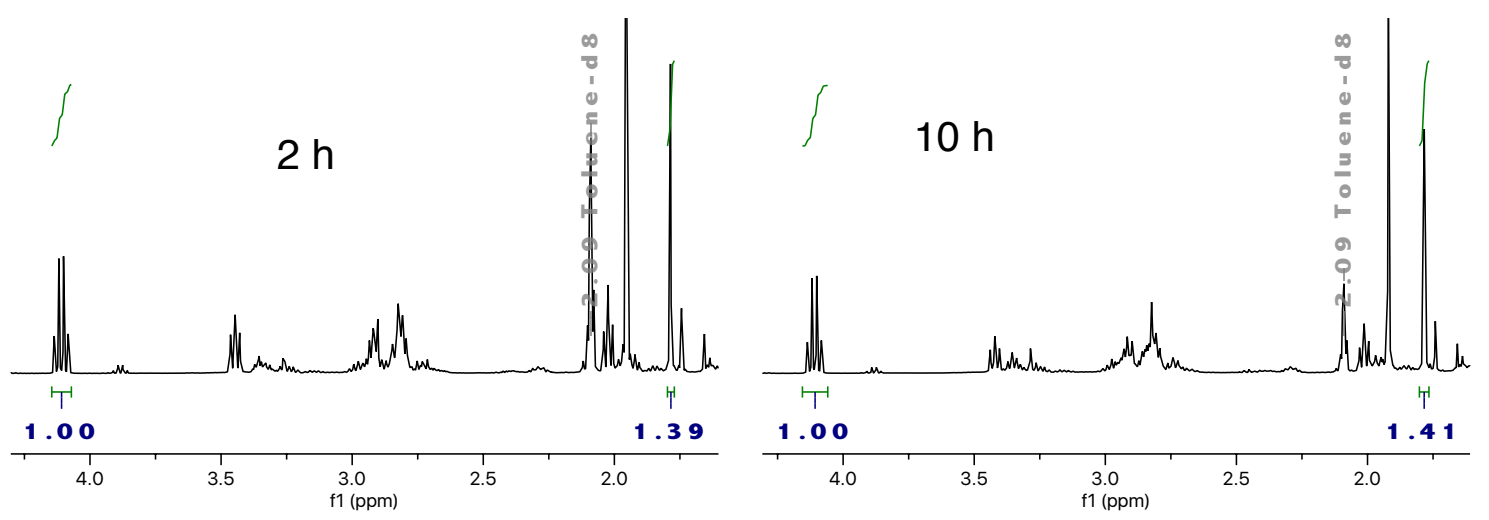

\section{Mechanistic experiments}

\section{On/Off experiments of standard reactions}
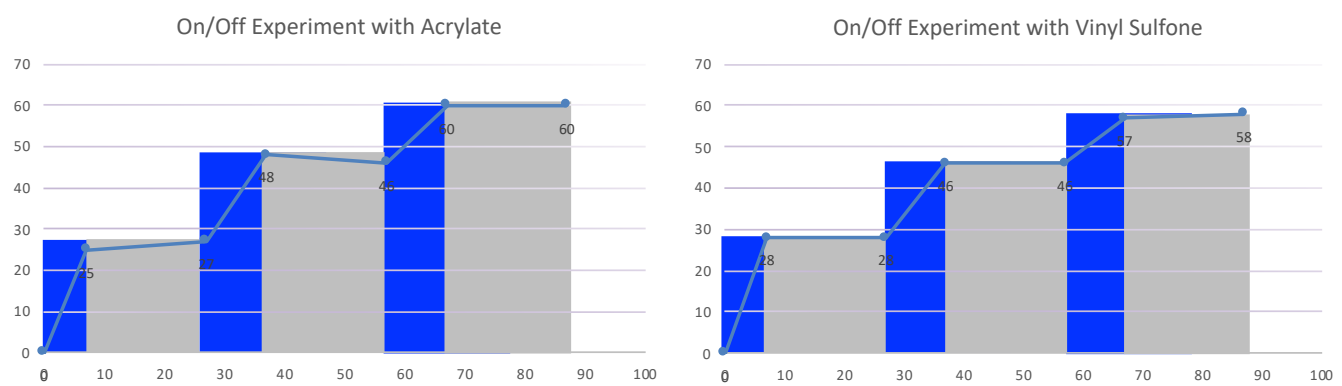

Chart 1. On/Off experiments; $Y$ axis: Yield (\%), $X$ axis: Time (min).

\section{Quenching Studies}

Standard solution of $\operatorname{Ir}(\mathrm{ppy})_{2}(\mathrm{dtbbpy}) \mathrm{PF}_{6}$ in Toluene $\left(5.0 \times 10^{-5} \mathrm{M}\right)$ was used for the analysis.

Samples were irradiated at $480 \mathrm{~nm}$ and emission was measured at $580 \mathrm{~nm}$.
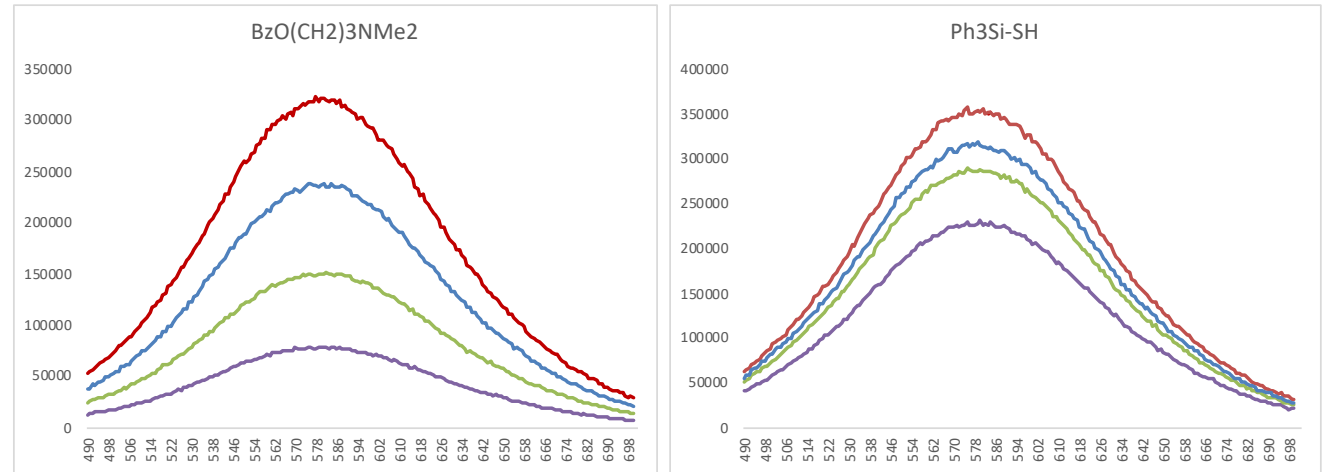

$10 / 1$

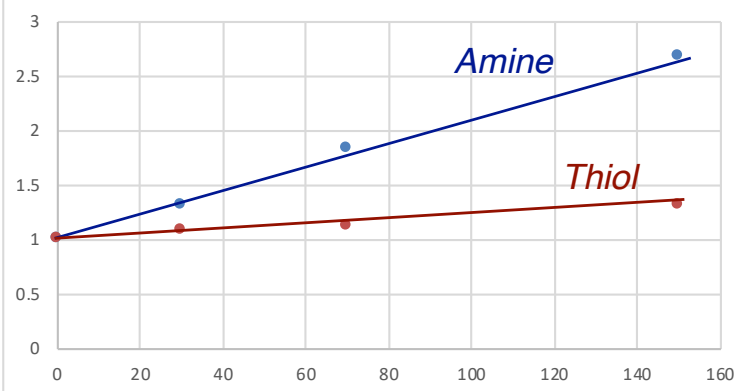

Chart 2. Quenching studies (intensity of emission at $582 \mathrm{~nm}$ was used). 


\section{Quantum yield calculation}

Quantum yield was calculated according to reported literature. ${ }^{1}$

The measurement of the photon flux (11 cm away from light source) at maximum intensity of a Kessil PR160 (34W maximum, 240 VDC, $440 \mathrm{~nm}$ ) was determined by standard ferrioxalate actinometry following already reported procedures (average of 3 measurements).

Photon flux (average of 3 times) $=4.32 \times 10^{-9}$ einstein $\cdot \mathrm{s}^{-1}$

A cuvette was charge with standard reaction solution and then placed $11 \mathrm{~cm}$ away from the light source. (Under these conditions, the fraction of light absorbed by the photocatalyst was $f$ $=0.965)$ The sample was irradiated for $420 \mathrm{~s}(7 \mathrm{~min})$. Product was detected with GC-MS in $(18+20+21) / 3 \%$ yield. The quantum yield was calculated as 23 .

$$
\Phi=\frac{\text { mol of Product }}{\text { flux } \times \mathrm{t} \times \mathrm{f}}=\frac{0.2 \times 0.2 \times 10^{-3} \mathrm{~mol}}{4.32 \times 10^{-9} \text { einstein } \cdot \mathrm{s}^{-1} \times 420 \mathrm{~s} \times 0.965} \approx 23
$$

Control studies under thermal conditions:

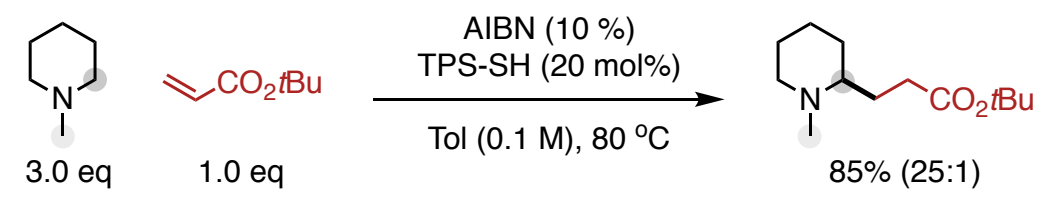

Fig. S1. Control experiment with catalytic amount of AIBN

\section{Deuteration studies under various conditions:}

Table S8. Deuteration studies with different period of irradiation time

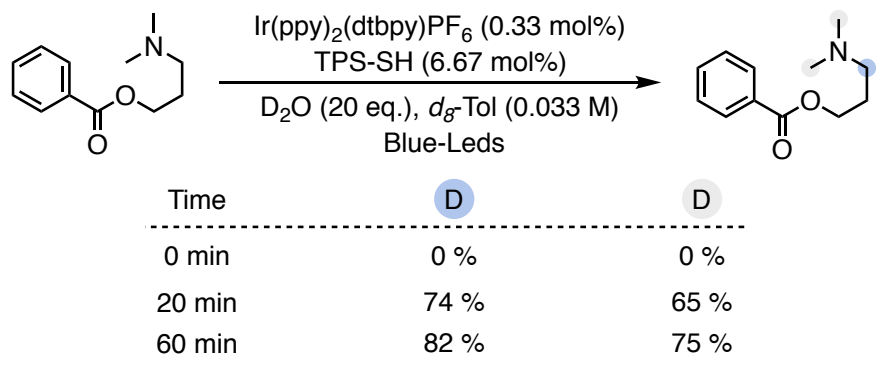

$\mathrm{D}_{2} \mathrm{O}$ (60 eq., $220 \mu \mathrm{L}, 12 \mathrm{mmol}$ ) was added to the reaction mixture. After $10 \mathrm{~min}$, the reaction was removed from the light source and TLC showed the alkene was not fully consumed. Then the mixture was directly purified by flash column chromatography in silica gel with $\mathrm{CHCl}_{3} / \mathrm{MeOH}$ and the results were analyzed by $\mathrm{NMR}$. 
Ir(III) (1 mol\%)
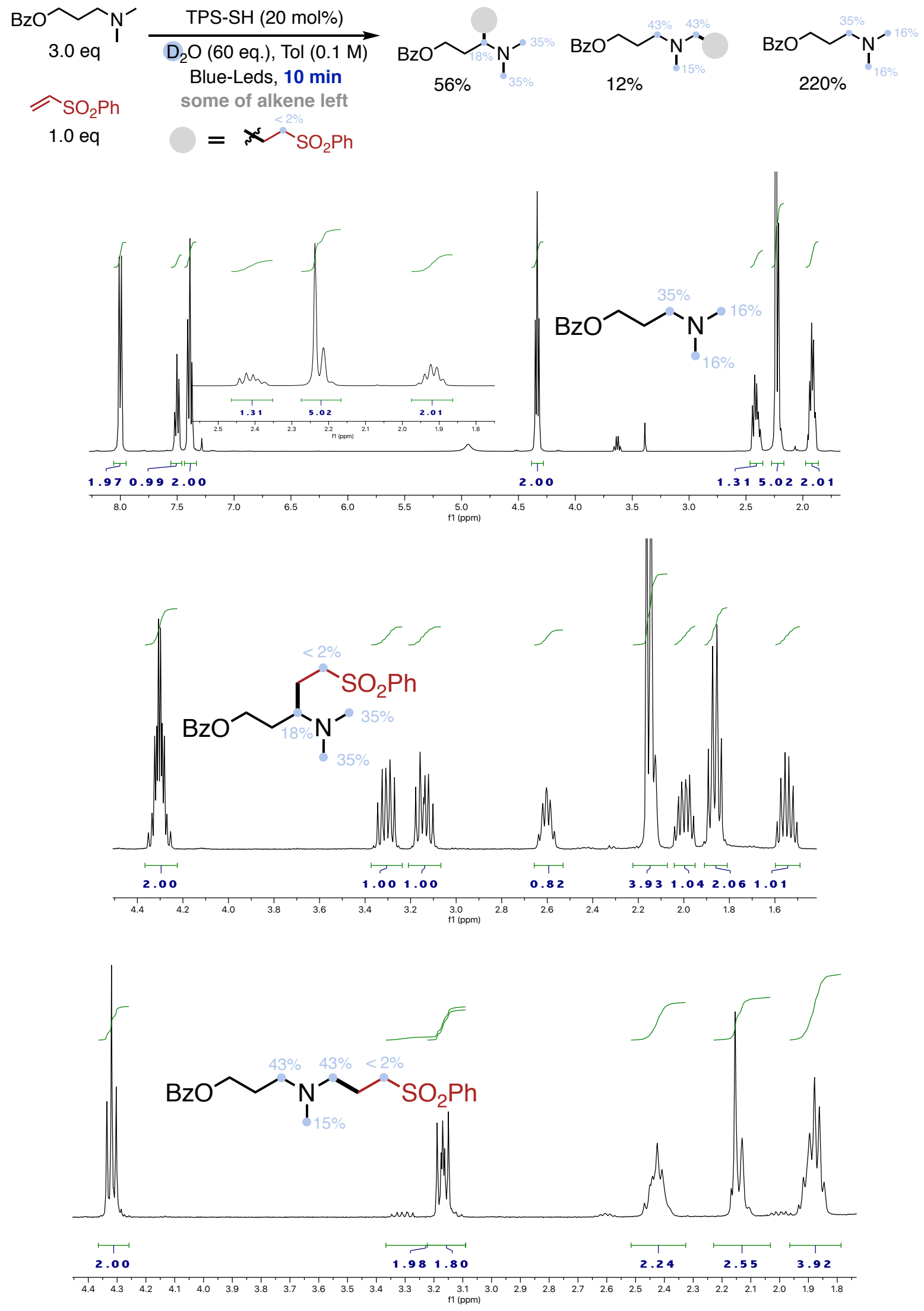

Fig. S2. Interrupted deuteration studies with vinyl sulfone under irradiation 
$\mathrm{D}_{2} \mathrm{O}$ (60 eq., $220 \mu \mathrm{L}, 12 \mathrm{mmol}$ ) was added to the standard reaction. As a control, $\mathrm{D}_{2} \mathrm{O}$ (60 eq., $220 \mu \mathrm{L}, 12 \mathrm{mmol}$ ) was added to the standard reaction without TPS-SH. After indicated time, the mixture was directly purified by flash column chromatography in silica gel with $\mathrm{CHCl}_{3} / \mathrm{MeOH}$ and the results were analyzed by NMR and shown below.
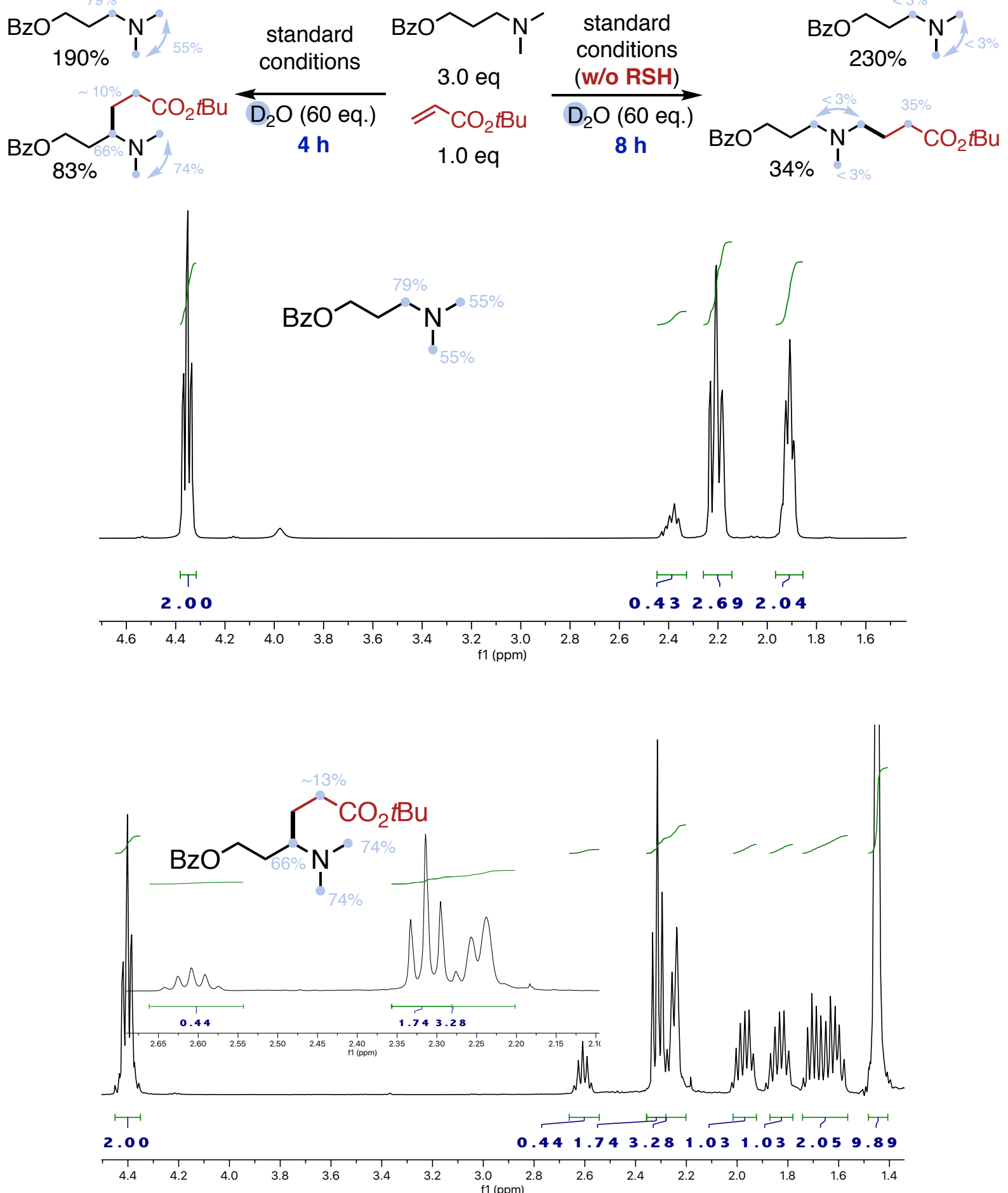

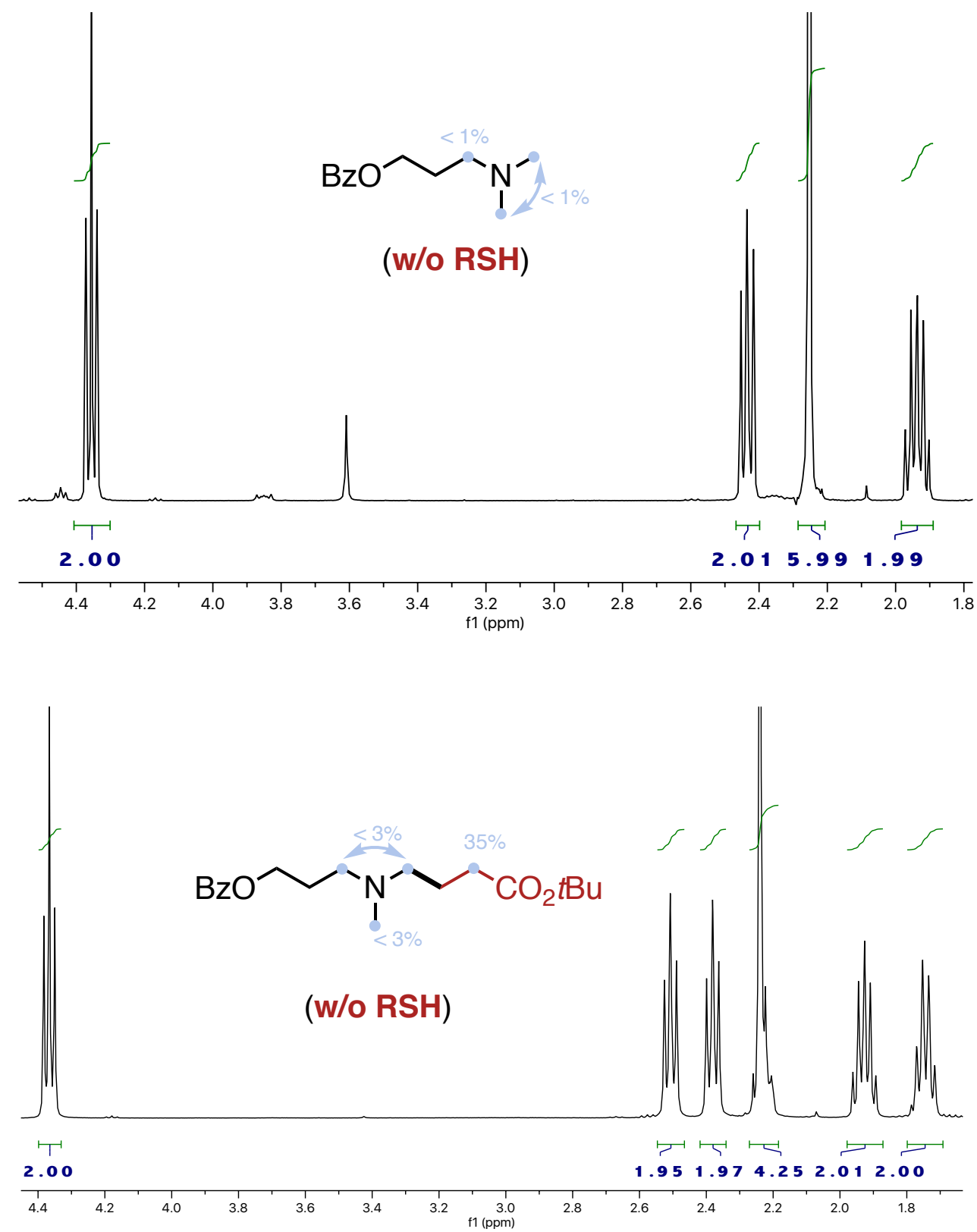

Fig. S3. Deuteration studies with acrylate with or w/o RSH under irradiation

AIBN (10 mol\%, $3.3 \mathrm{mg}, 0.02 \mathrm{mmol})$, TIPS-SH (20 mol\%, $8.6 \mu \mathrm{L}, 0.04 \mathrm{mmol})$ and $\mathrm{D}_{2} \mathrm{O}(60$ eq., $220 \mu \mathrm{L}, 12 \mathrm{mmol})$ in $m$-Xylene $(0.1 \mathrm{M})$ were used, the reaction was stirred in preheated oil-bath $\left(80^{\circ} \mathrm{C}\right)$. After $4 \mathrm{~h}$, the mixture was directly purified by flash column chromatography in silica gel with $\mathrm{CHCl}_{3} / \mathrm{MeOH}$ and the results were analyzed by NMR.

(Note: We found in toluene at $80{ }^{\circ} \mathrm{C}$, the added $\mathrm{D}_{2} \mathrm{O}$ evaporates quickly and condenses on the sides of the reaction vessel. The same issue remains in m-xylene, but with some $\mathrm{D}_{2} \mathrm{O}$ evident at the bottom.) 

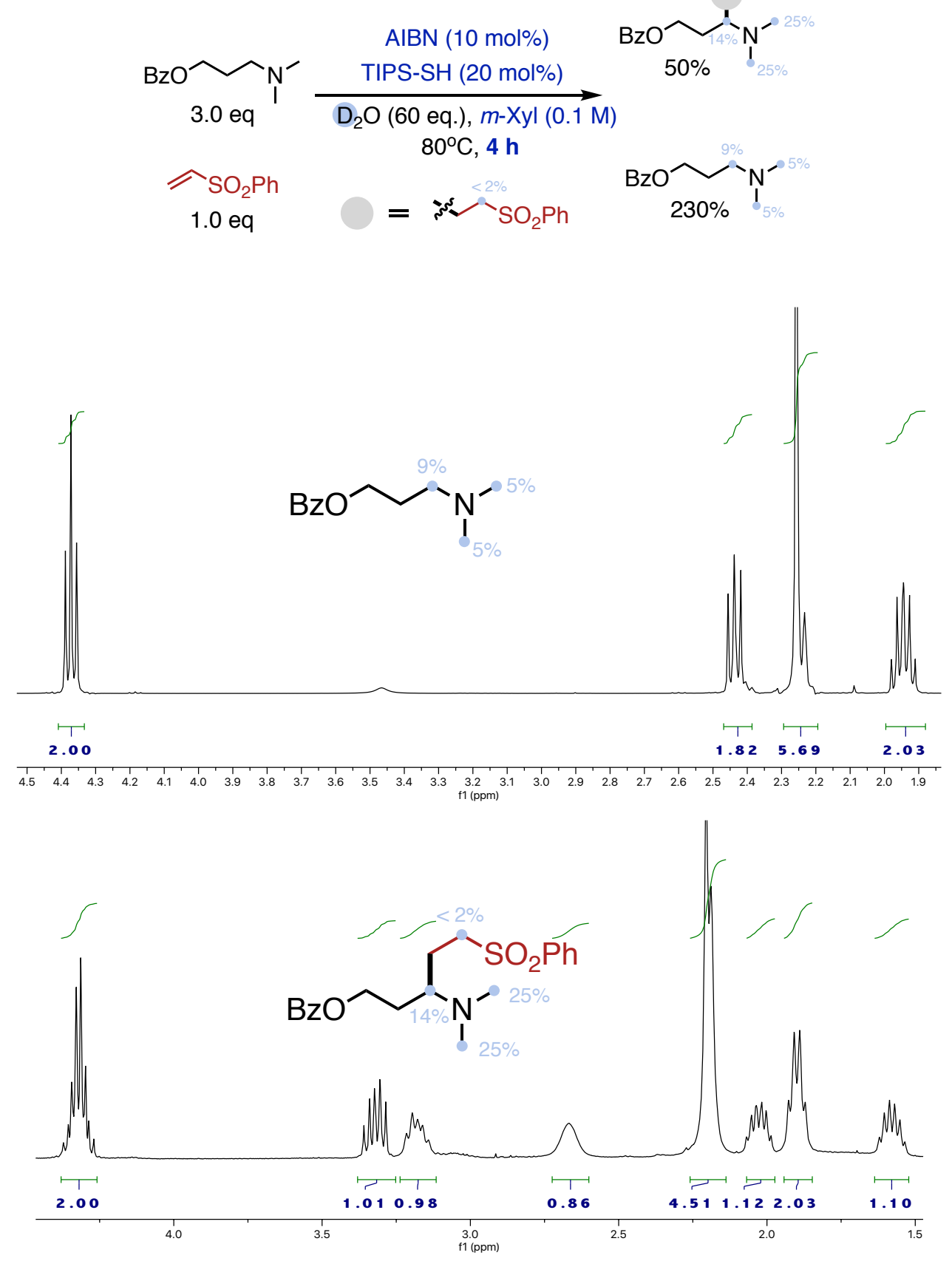

Fig. S4. Deuteration studies with vinyl sulfone under heating

AIBN (10 mol\%, $3.3 \mathrm{mg}, 0.02 \mathrm{mmol})$, TIPS-SH $(20 \mathrm{~mol} \%, 8.6 \mu \mathrm{L}, 0.04 \mathrm{mmol})$ and $\mathrm{D}_{2} \mathrm{O}(60$ eq., $220 \mu \mathrm{L}, 12 \mathrm{mmol})$ in $m$-Xylene $(0.1 \mathrm{M})$ were used, the reaction was stirred in preheated oil-bath $\left(80^{\circ} \mathrm{C}\right)$. After $4 \mathrm{~h}$, the mixture was directly purified by flash column chromatography in silica gel with $\mathrm{CHCl}_{3} / \mathrm{MeOH}$ and the results were analyzed by NMR.

(Note: We found in toluene at $80^{\circ} \mathrm{C}$, the added $\mathrm{D}_{2} \mathrm{O}$ evaporates quickly and condenses on the sides of the reaction vessel. The same issue remains in m-xylene, but with some $\mathrm{D}_{2} \mathrm{O}$ evident at the bottom.) 


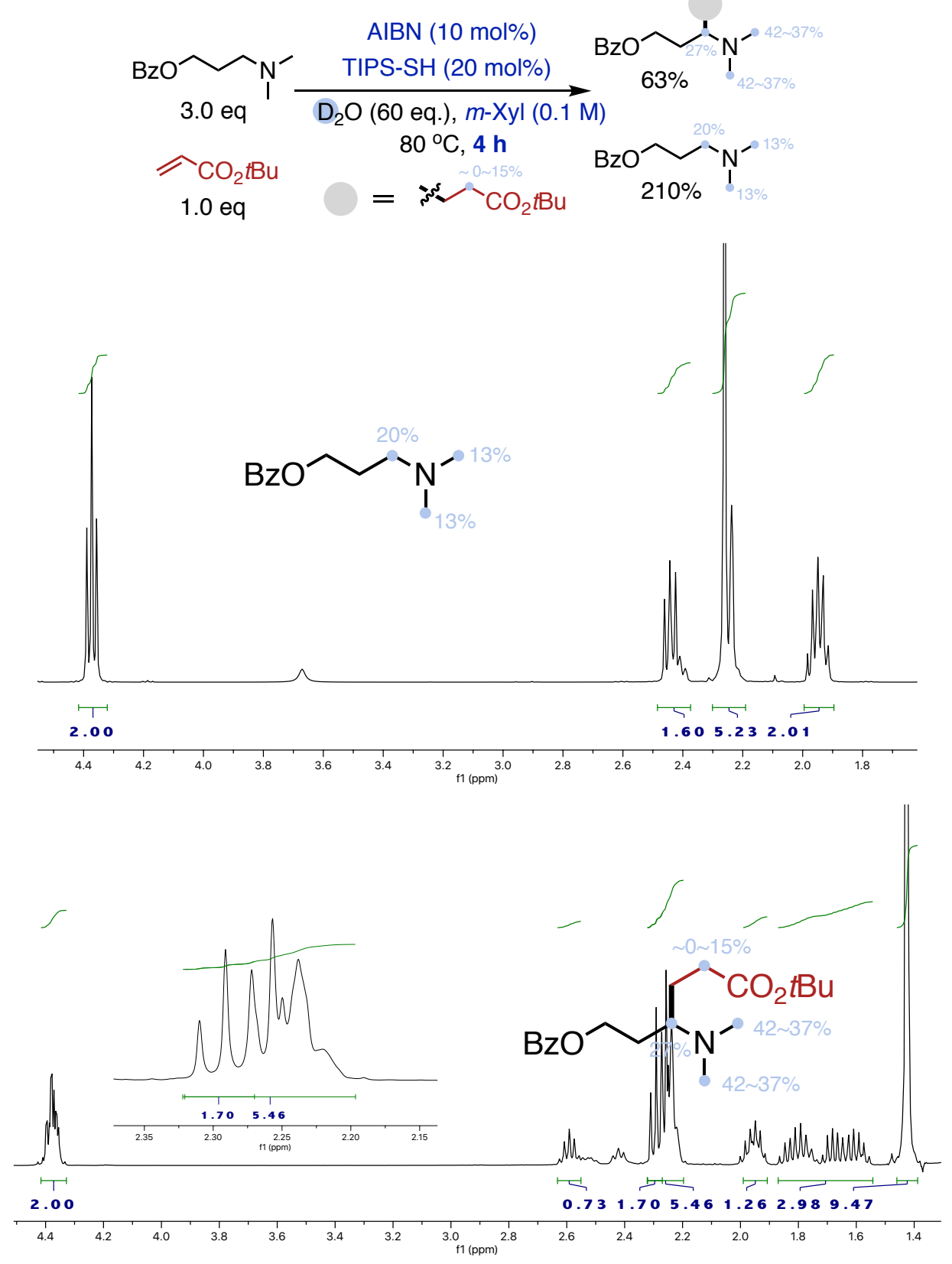

Fig. S5. Deuteration studies with acrylate under heating

\section{Cyclic Voltammetry Studies}

Cyclic voltammetry (CV) studies were performed on a $\mathrm{CH}$ instruments Model 1232B potentiostat. All experiments were run using an EDAQ 1-mm disk glassy carbon working electrode in conjunction with an $\mathrm{EDAQ} \mathrm{Ag} / \mathrm{AgCl}$ reference electrode. Platinum wire from VWR served as the counter electrode. All voltammetry measurements were run in anhydrous acetonitrile from Sigma-Aldrich at $50 \mathrm{mM}$ ( $5 \mathrm{mM}$ for photocatalyst) using tetrabutylammonium hexafluorophosphate $(0.1 \mathrm{M})$ as the electrolyte under a gentle flow of $\mathrm{N}_{2}$. The scan rate was set 
at $100 \mathrm{mV} / \mathrm{s}$ with a sensitivity of $1.0 \times 10^{-4}$. X-axis as Potential (V), Y-axis as Current (A). CV data of $i \mathrm{Pr}_{3} \mathrm{SiSH}$ is known $\left(E_{1 / 2}{ }^{\mathrm{ox}}=0.28 \mathrm{~V}\right.$ vs $\mathrm{Ag} / \mathrm{AgCl}$ in $\left.\mathrm{MeCN}\right){ }^{2}$
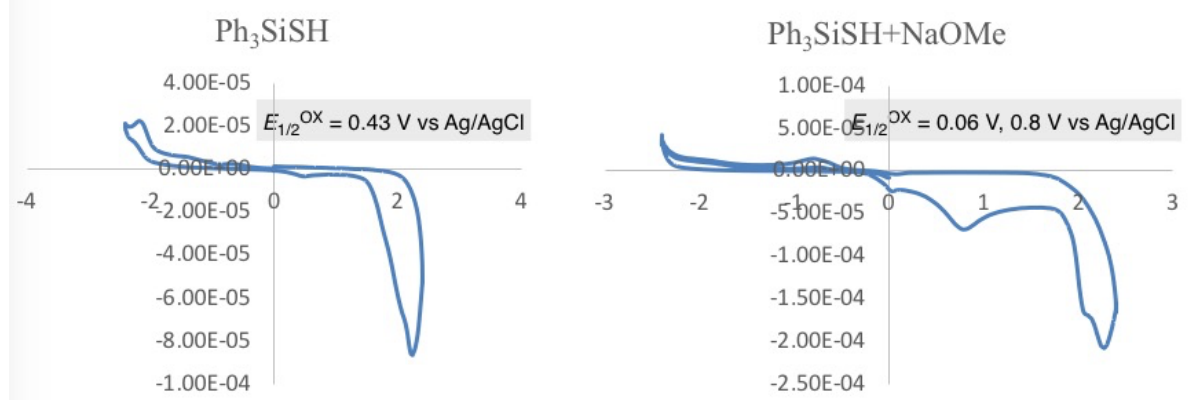

Fig. S6. $\mathrm{CV}$ of $\mathrm{Ph}_{3} \mathrm{SiSH}$ with or w/o NaOMe

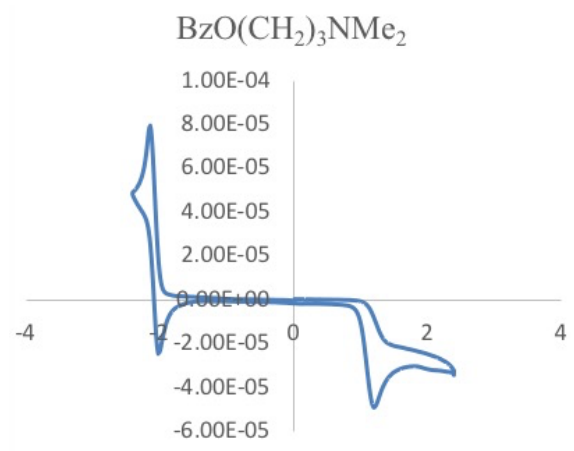

Fig. S7. $\mathrm{CV}$ of $\mathrm{BzO}\left(\mathrm{CH}_{2}\right)_{3} \mathrm{NMe}_{2}$

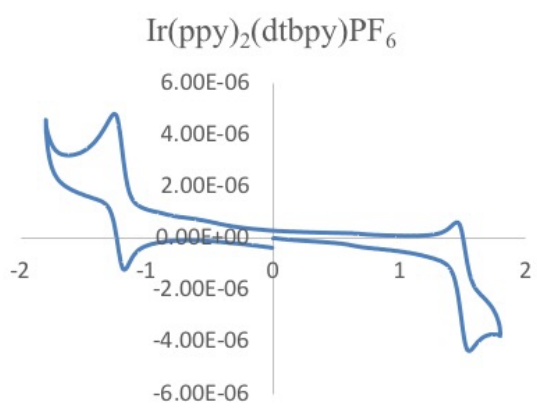

Fig. S8. $C V$ of $\operatorname{Ir}(\text { ppy })_{2}($ dtbpy $) P_{6}$

\section{Computational details}

DFT calculations were carried out with using Gaussian16 program package (v A.03). ${ }^{3}$ All structures were optimized using the M06 functional and $6-31+\mathrm{G}(\mathrm{d}, \mathrm{p})$ basis set, which is convenient for organic molecules with open-shell electronic structure, including implicit solvation (toluene) by CPCM method. Frequency calculations were conducted at the same level of theory to corroborate the nature of stationary points as minima (zero imaginary frequencies) or transition state (one imaginary frequency). Transition states were also confirmed by IRC calculations and relaxation towards reactants and products. The potential energy was further refined using the same method together with the $6-311++\mathrm{G}(3 \mathrm{~d}, 2 \mathrm{p})$ basis set. Finally, $1 \mathrm{M}$ standard state (addition of $1.89 \mathrm{kcal} / \mathrm{mol}$ ) correction was added to all species.

3D images were created by CYLview software. ${ }^{4}$

Radical stability of primary radical of $(i \mathrm{Pr})_{2}(\mathrm{Me}) \mathrm{N}$ vs $(i \mathrm{Pr})_{3} \mathrm{Si}-\mathrm{SH}$ radical was benchmarked, comparing the DFT results with CCSD(T) (+ DFT $\mathrm{G}_{\text {Corr }}+$ DFT G $\left.\mathrm{G}_{\text {Solv }}\right)$. Both methodologies yield similar results, confirming the reversibility of the process. 


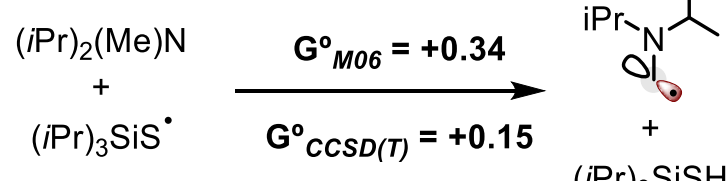

$$
\begin{aligned}
& (\mathrm{iPr})_{3} \mathrm{SiSH}
\end{aligned}
$$

For Fig. 4 in the main text, all the calculations were carried out at the same level of theory. The starting geometries of pharmaceuticals were taken from CCDC (Cambridge Crystallographic Data Centre) for all compounds except Clormipramine (not available). ${ }^{13} \mathrm{C}$ NMR shifts were calculated using the GIAO method and they are referenced to TMS (calculated at the same theory level).

Free energy profiles of complete reaction pathway with and without HAT catalyst: Initiation without $\mathrm{iPr}_{3} \mathrm{SiSH}$

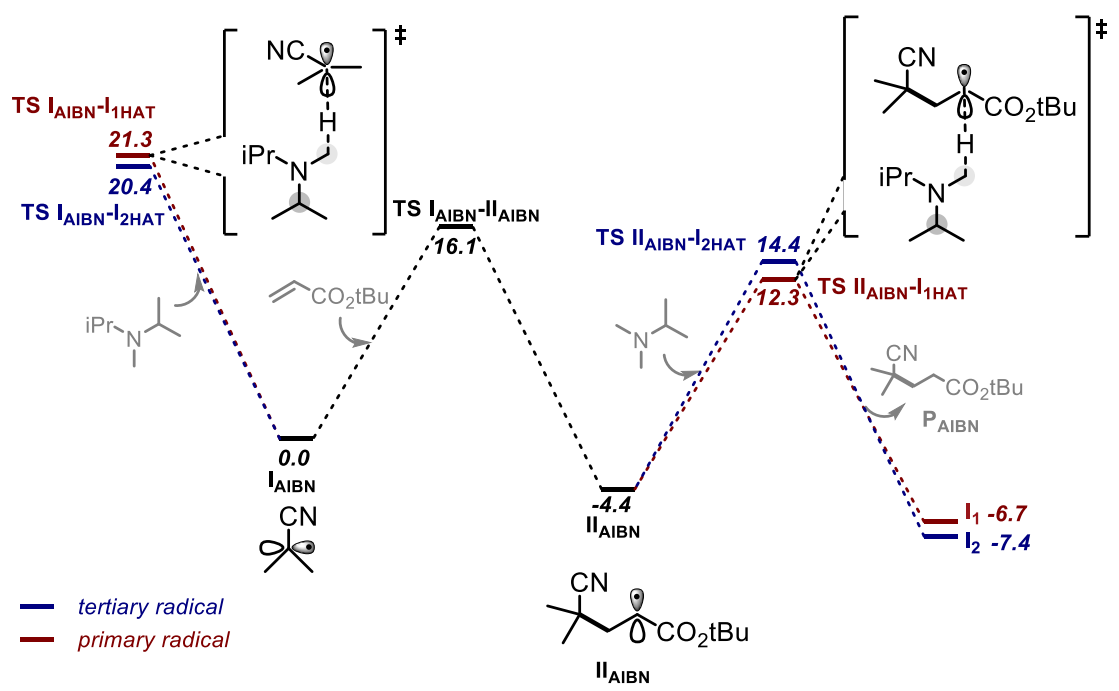

Initiation with $\mathrm{PPr}_{3} \mathrm{SiSH}$
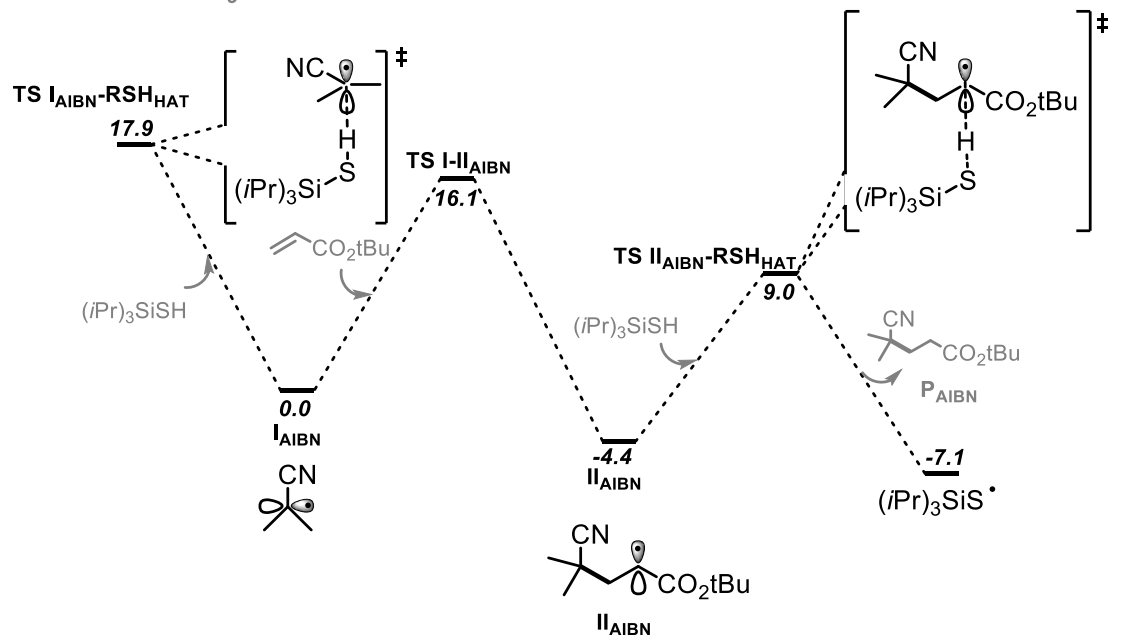

Fig. S9. Free energy (kcal/mol) profile of the initiation mechanism under HAT-catalyst free conditions (top) and with (iPr) $)_{3} \mathrm{SiSH}$ as HAT catalyst (bottom). 


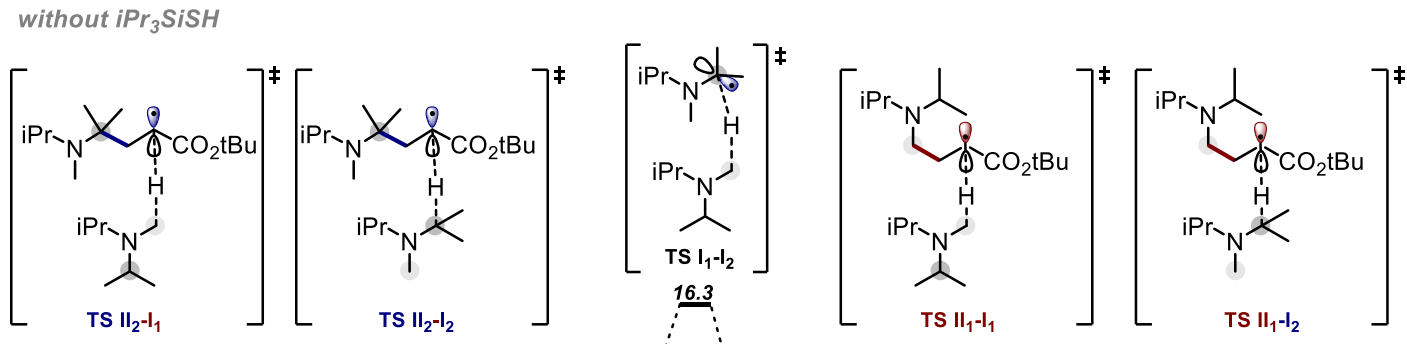

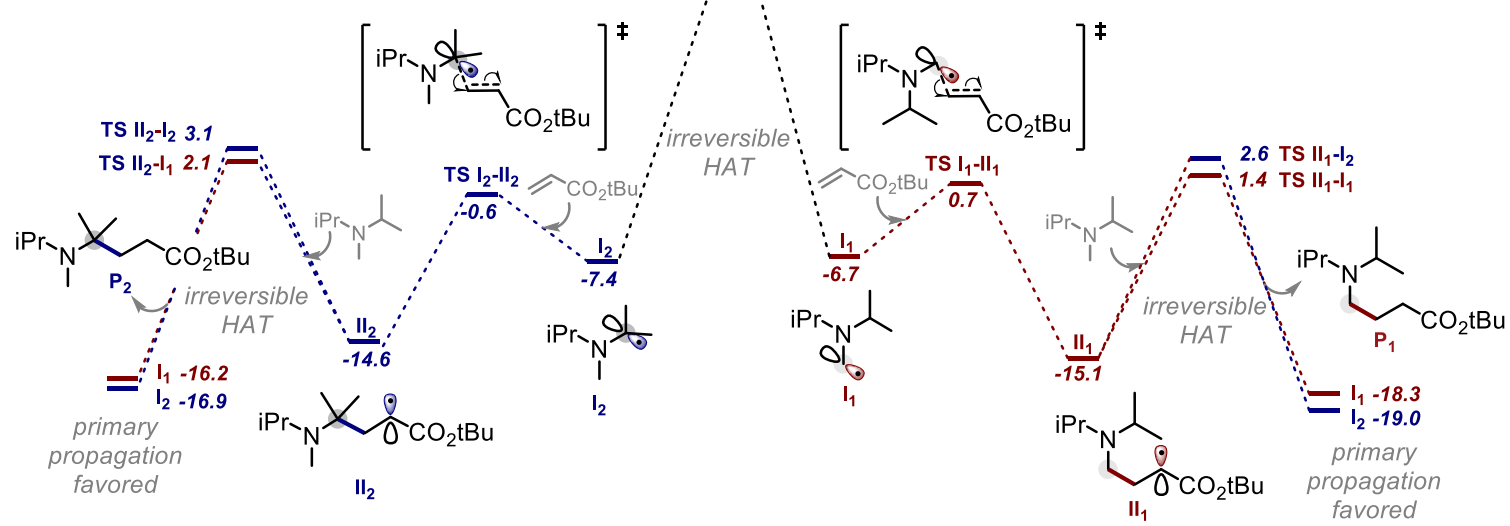

with $\mathrm{iPr}_{3} \mathrm{SiSH}$

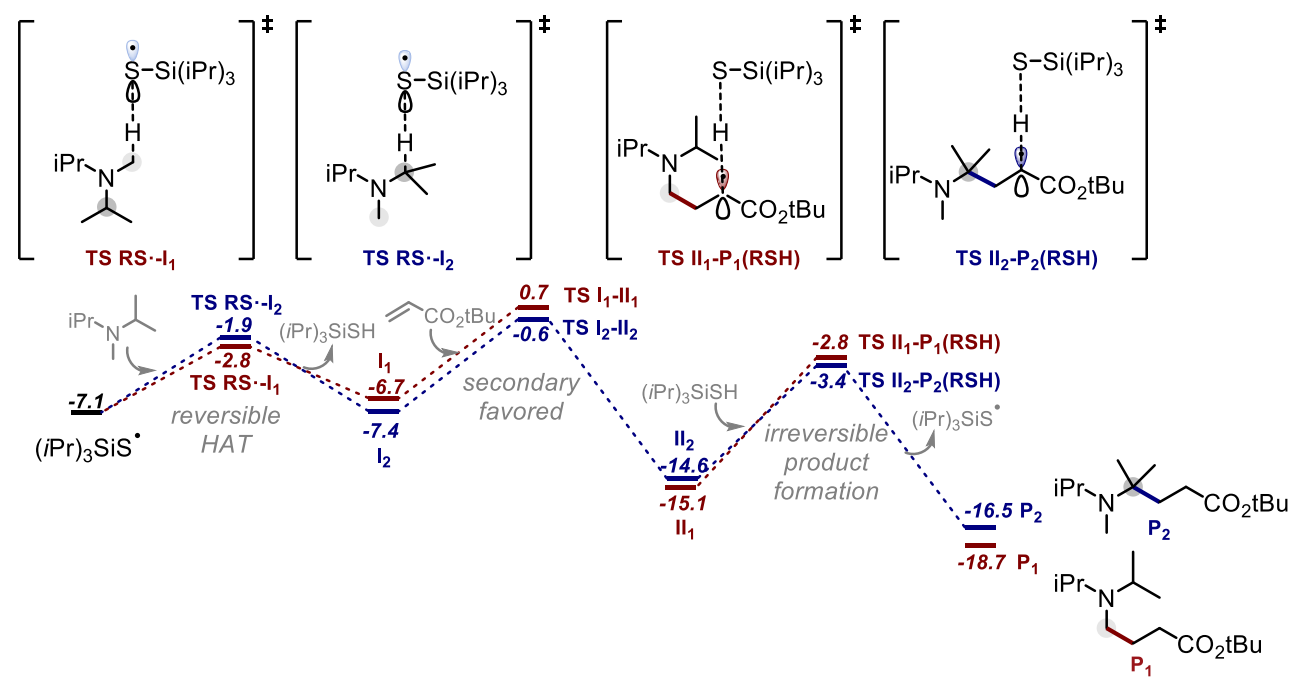

Fig. S10. Free energy profile of radical propagation without HAT catalyst and with (iPr) $)_{3} \mathrm{SiSH}$ as HAT catalyst (bottom). Free energies in $\mathrm{kcal} / \mathrm{mol}$. 

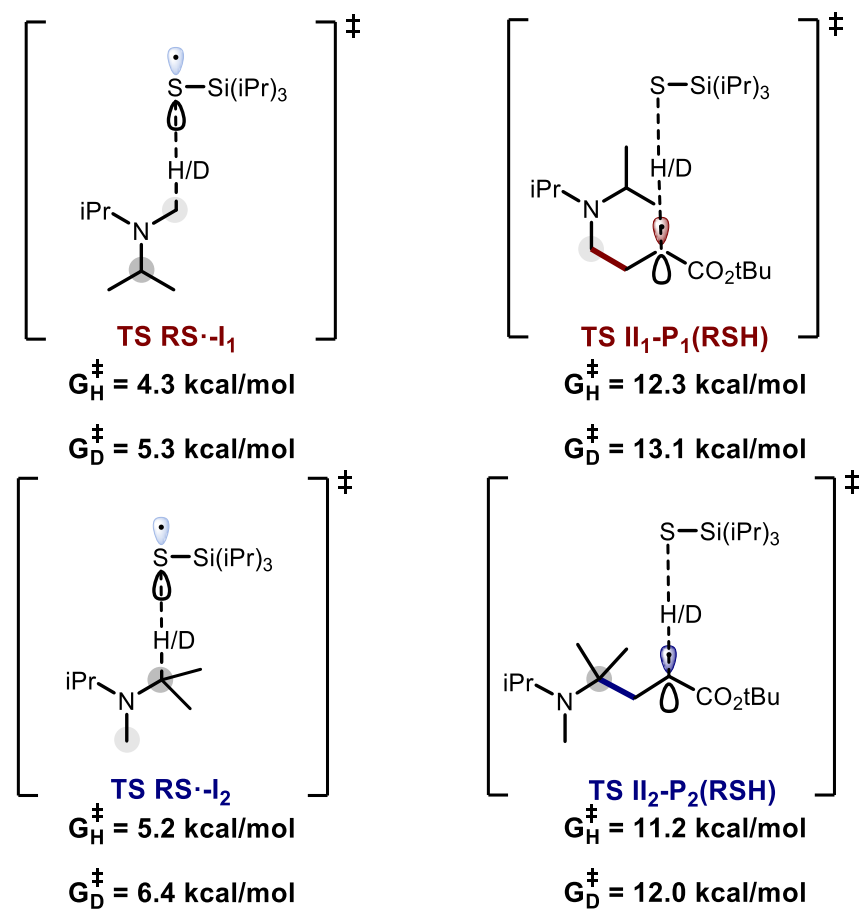

Fig. S11. Deuterium effect in HAT free energy barriers. Free energies in kcal/mol.

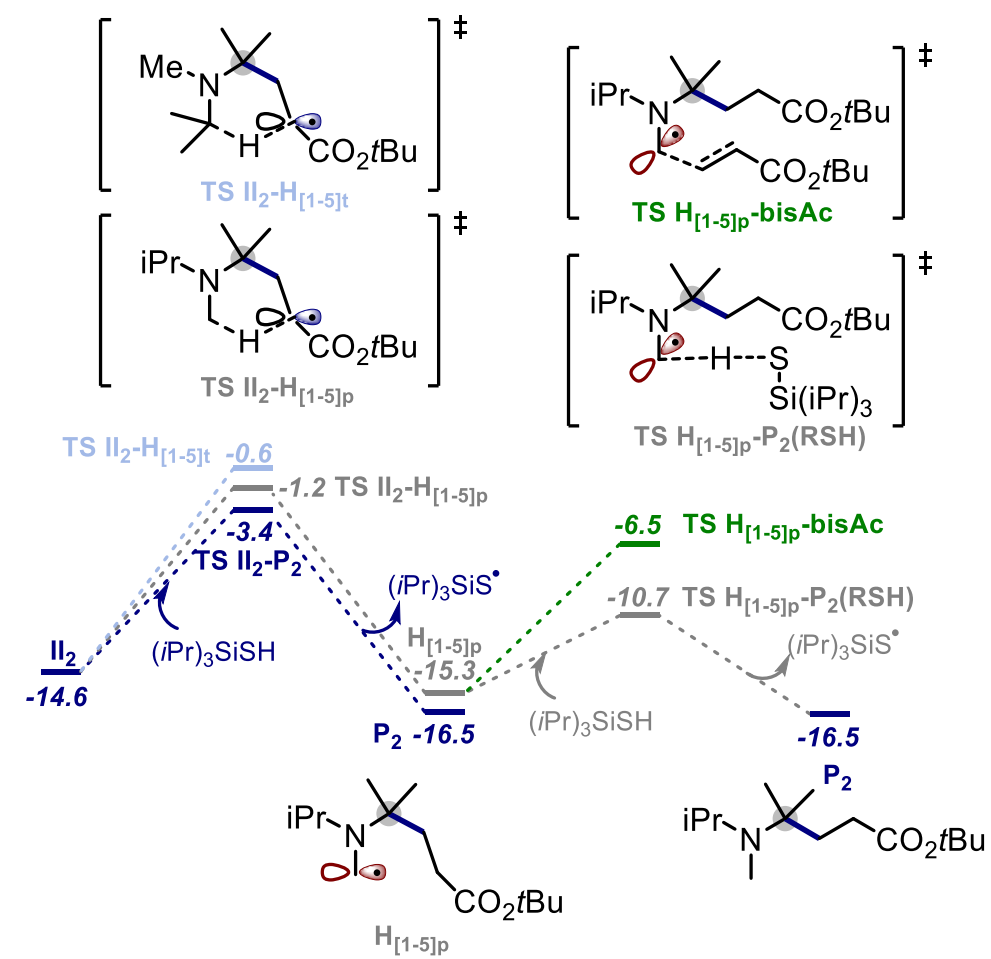

Fig. S12. Intramolecular [1,5] H-shift vs direct reaction with HAT catalyst. Free energies in $\mathrm{kcal} / \mathrm{mol}$. 


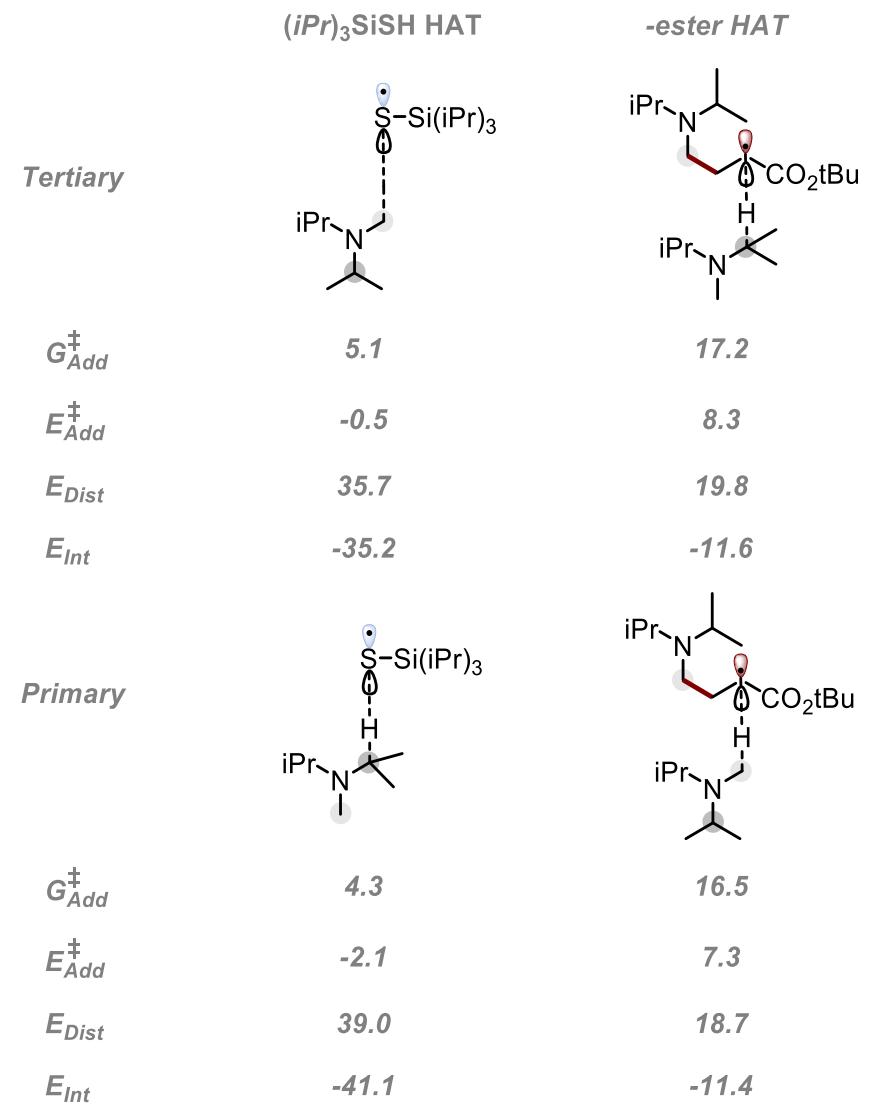

Fig. S13. Distortion interaction analysis of HAT reaction of $(i \mathrm{Pr})_{2}(\mathrm{Me}) \mathrm{N}$ and silanethiyl radical or $\alpha$-ester radical II on primary and tertiary positions. Energies in $\mathrm{kcal} / \mathrm{mol}$.

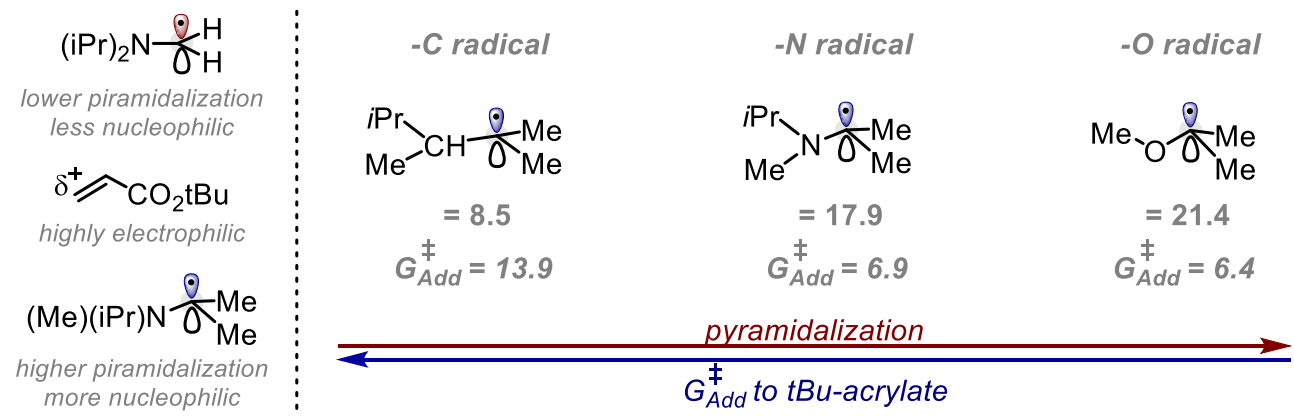

Fig. S14. Correlation between pyramidalization of radical and free energy barrier in radical addition to $t \mathrm{Bu}$-acrylate. Free energies in $\mathrm{kcal} / \mathrm{mol}$. 


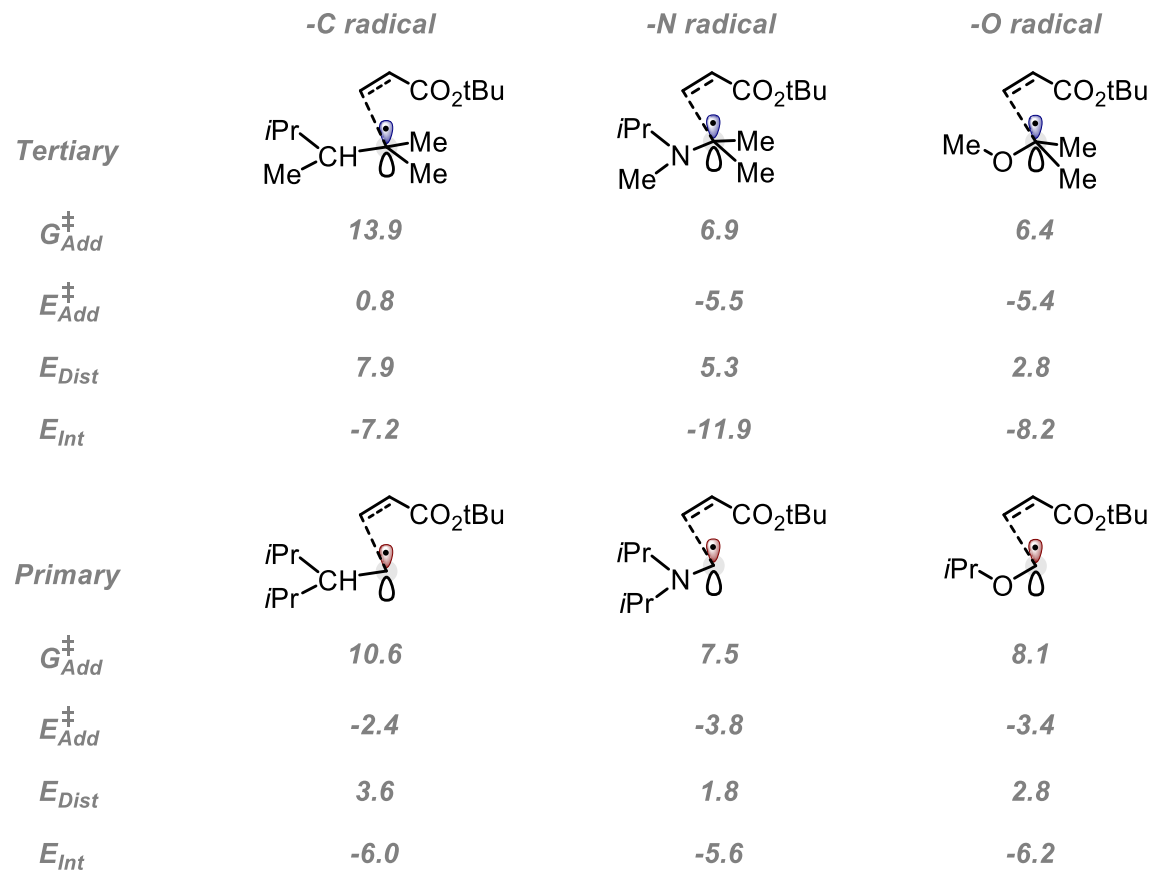

Fig. S15. Distortion interaction analysis of radical addition to $t \mathrm{Bu}$-acrylate of $\alpha-\mathrm{C}, \alpha-\mathrm{N}$ and $\alpha-O$ primary and tertiary radicals. Energies in $\mathrm{kcal} / \mathrm{mol}$.

Distortion-interaction analysis of the different radical addition transition states show that $\alpha-\mathrm{O}$ and $\alpha-\mathrm{N}$ tertiary transition states have a significantly smaller distortion energy. In addition, their pyramidalization favours the interaction of the radical fragment with the double bond, resulting in a lower activation energy barrier.

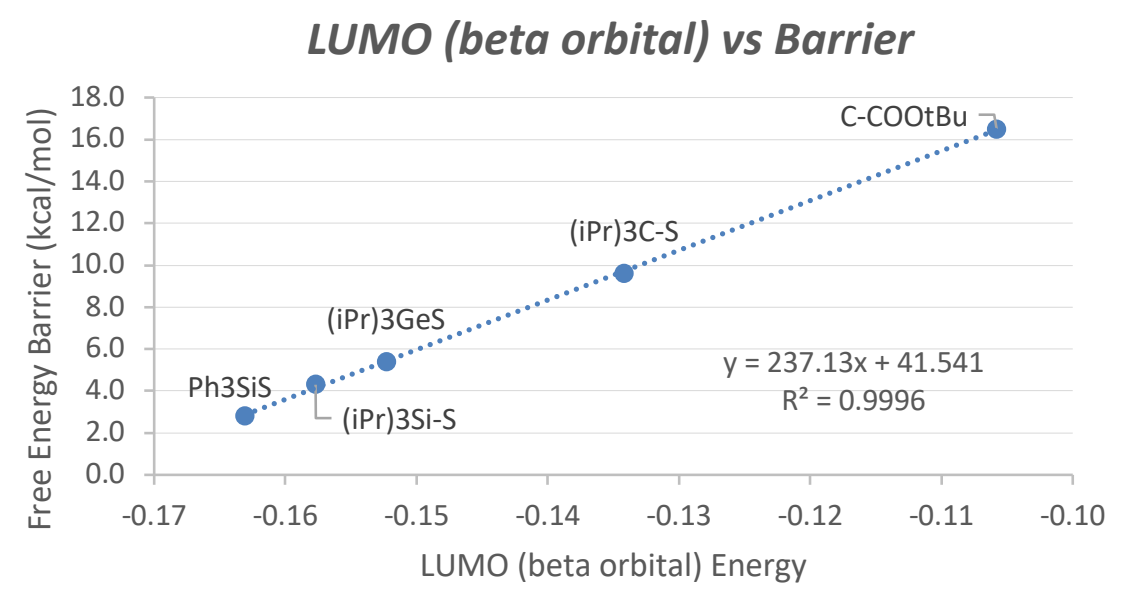

Fig. S16. Correlation between LUMO orbital of the radical and the free energy barrier of HAT from the primary site of $(i \mathrm{Pr})_{2}(\mathrm{Me}) \mathrm{N}$ to the corresponding radical.

In order to show that the HAT catalyst reacts as an electrophilic radical, we calculated the LUMO energy of the HAT catalyst radical and its correlation with the free energy barrier for 
the HAT at the primary position of substrate 3. Interestingly, there is a linear correlation of both parameters; the electrophilicity lowers the barrier considerably. The two best catalysts $\left(\mathrm{Ph}_{3} \mathrm{SiSH}\right.$ and $\left.(i \mathrm{Pr})_{3} \mathrm{SiSH}\right)$ promote the HAT with a very low free energy barrier due to the polarity match in the transition states. In contrast, nucleophilic radicals, such as the $\alpha$-ester radical $(\mathrm{C}-\mathrm{COO} t \mathrm{Bu})$, show a significant increase of the activation barrier for HAT.

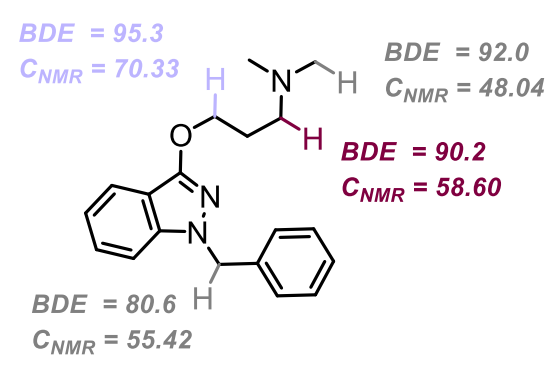

Benzydamine

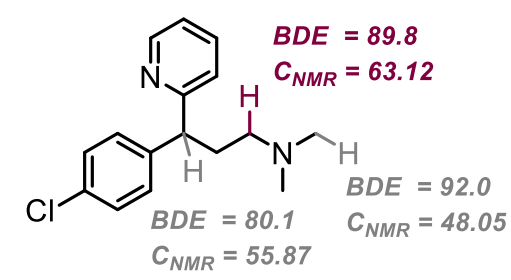

Chlorpheniramine

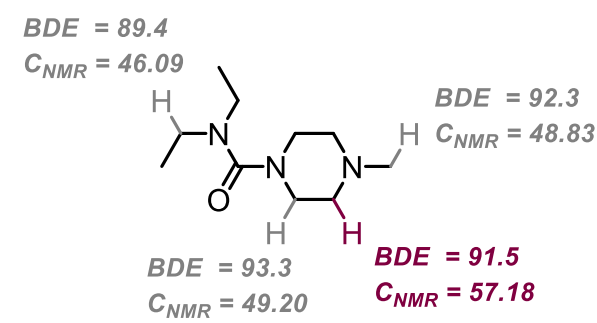

Diethylcarbamazine
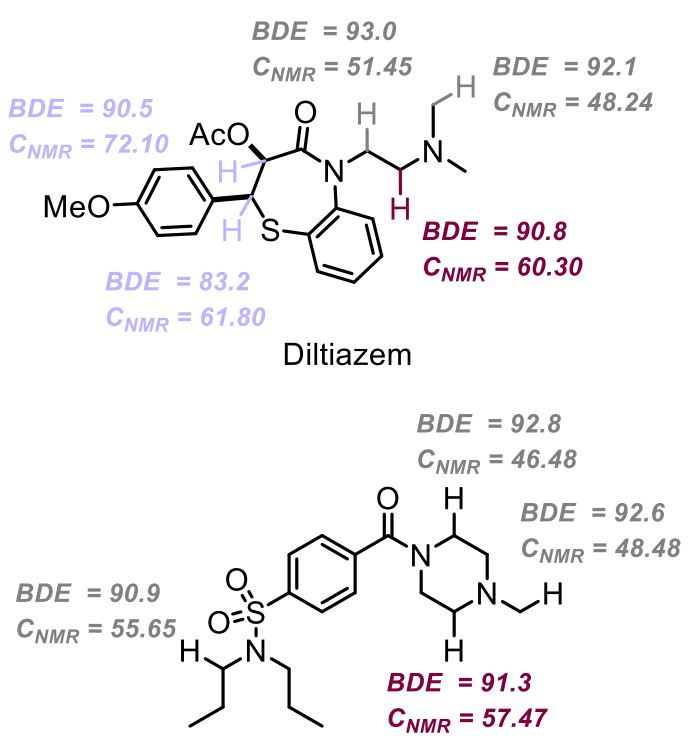

Probenecid derivative

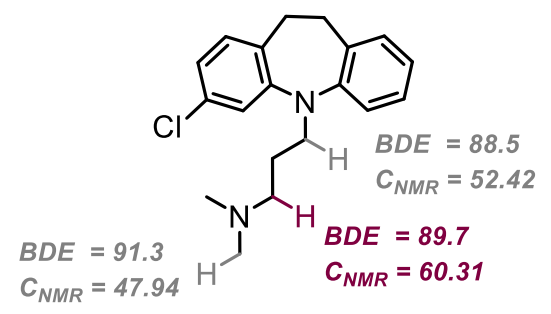

Clomipramine

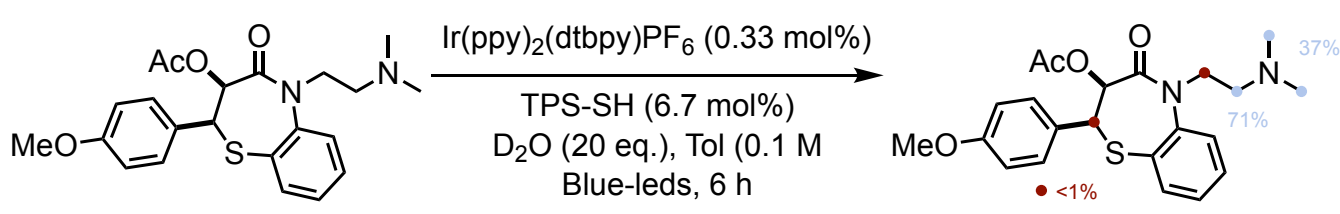

Fig. S17. Correlation between calculated $C_{N M R}$ shifts and site selectivity in late-stage drug functionalization.

Correlation is only observed for $\alpha-\mathrm{N}$ carbon centres. In the case of other heteroatoms $(\mathrm{O}, \mathrm{S})$, this correlation does not occur due to a non-reversible $\mathrm{C}-\mathrm{H}$ activation mechanism, as shown in the deuteration experiment. 

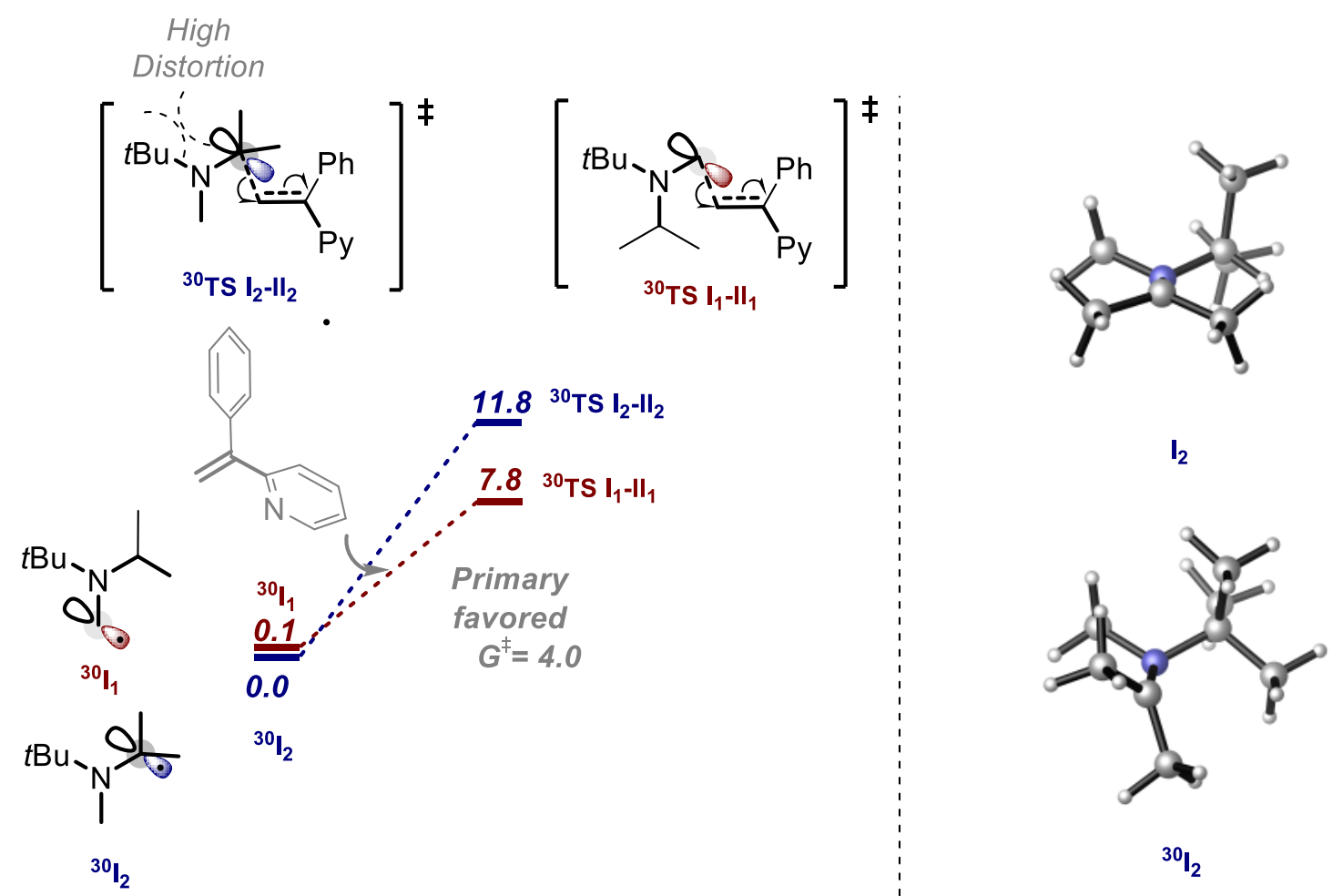

${ }^{30} \mathrm{I}_{2}$

Fig. S18. Inversion of the selectivity for radical addition to product 30 in reaction with 1phenyl-1-pyridineethane (left). Free energies in $\mathrm{kcal} / \mathrm{mol}$. 3D structures of the corresponding tertiary radical (right).

Interestingly, products $\mathbf{3 0}$ and $\mathbf{3 1}$ exhibit an opposite selectivity with respect to all the other substrates. In those cases, the least hindered position (methyl) reacts faster than the corresponding tertiary position (isopropyl). Intrigued by these exceptions, we calculated the radical addition to the alkene. We reproduced the observed selectivity inversion. The inversion is due to the $t \mathrm{Bu}$ substituent which distorts the tertiary radical structure considerably (and hence raises its energy). 

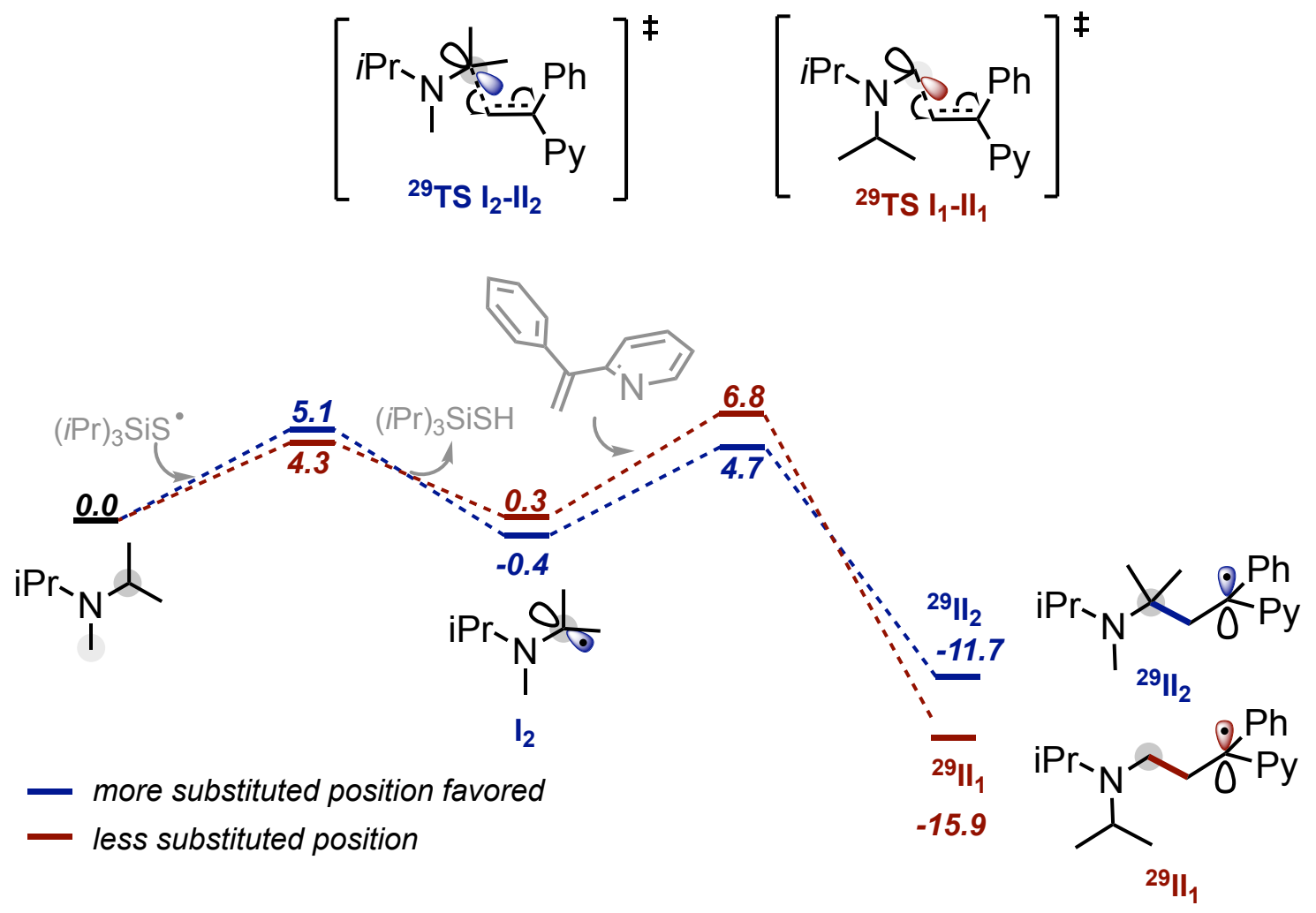

Fig. S19. Low the selectivity for radical addition to product 27 in reaction with 1-phenyl1-pyridineethane.

In the case of the reaction with less substituted tertiary amines and diarylethylenes as radical acceptor, the radical addition barrier is decreased and starts to compete with the radical generation via reversible HAT process. Thus, the selectivity of those system is reduced $(<3: 1)$ as the reversibility of HAT is less pronounced. 


\section{XYZ Coordinates of calculated species including potential energy in solvent at high basis set and free energy correction (and enthalpy correction for BDE calculation when needed):}

N(Me)(iPr)2<smiles>C[In]N(C)C(C)C</smiles>

$\mathrm{E}=-331.5660948$

$\mathrm{G}_{\text {Corr. }}=0.196476$

$\mathrm{H}_{\text {Corr. }}=0.242820$

$\begin{array}{llll}\mathrm{N} & 0.01044200 & -0.49602600 & 0.01558100\end{array}$

C $\quad 0.03259500 \quad-0.77059500 \quad 1.43709000$

$\begin{array}{llll}\mathrm{H} & -0.91443600 & -1.23353100 & 1.73789000\end{array}$

$\begin{array}{llll}\mathrm{H} & 0.82278500 & -1.49065500 & 1.67609900\end{array}$

$\mathrm{H} \quad 0.18155100 \quad 0.11968000 \quad 2.07789700$

$\begin{array}{llll}\mathrm{C} & 1.25082400 & 0.05059100 & -0.54437000\end{array}$

$\begin{array}{llll}\mathrm{C} & 2.43137200 & -0.86942000 & -0.26493600\end{array}$

C $\quad \begin{array}{llll}\text { C } & 1.59010800 & 1.48231900 & -0.12206100\end{array}$

$\mathrm{H} \quad \begin{array}{llll}\mathrm{H} & 1.09520500 & 0.05347500 & -1.63566500\end{array}$

$\mathrm{H} \quad 2.17794000 \quad-1.91250000 \quad-0.48687900$

$\mathrm{H} \quad 3.28570200 \quad-0.57982100 \quad-0.88740900$

$\mathrm{H} \quad 2.75904300 \quad-0.80733900 \quad 0.78108000$

$\mathrm{H} \quad \begin{array}{llll}\mathrm{H} & .82979600 & 2.19883200 & -0.45307300\end{array}$

$\mathrm{H} \quad \begin{array}{llll}\mathrm{H} & 1.68753200 & 1.56344400 & 0.96908800\end{array}$

$\mathrm{H} \quad 2.54736100 \quad 1.79038300 \quad-0.56111000$

$\begin{array}{lllll}\mathrm{C} & -1.22570200 & 0.13507500 & -0.46671500\end{array}$

C $\quad-1.78566100 \quad 1.25342900 \quad 0.41576700$

C $\quad-2.29717800 \quad-0.92245200 \quad-0.70705900$

$\mathrm{H} \quad-0.97096000 \quad 0.57563200 \quad-1.44375400$

$\mathrm{H} \quad-1.03407800 \quad 2.01180600 \quad 0.66090900$

$\mathrm{H} \quad-2.61563600 \quad 1.75388300 \quad-0.09758100$

$\mathrm{H} \quad-2.18181900 \quad 0.85526300 \quad 1.35916200$

$\mathrm{H} \quad-1.94029400 \quad-1.68396000 \quad-1.40854400$

$\mathrm{H} \quad-2.57037000 \quad-1.42610300 \quad 0.22999700$

$\mathrm{H} \quad-3.21056200 \quad-0.46998300 \quad-1.11346400$

\section{$t$ Bu-Acrylate}

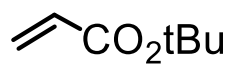

$$
\mathrm{E}=-424.2704695
$$

$$
\mathrm{G}_{\text {Corr. }}=0.142635
$$

$\begin{array}{lrrr}\mathrm{C} & -3.48113800 & 0.20164900 & -0.00007500 \\ \mathrm{C} & -2.26501700 & 0.74558800 & 0.00003600 \\ \mathrm{H} & -4.37852400 & 0.81320400 & -0.00007700 \\ \mathrm{H} & -3.60048600 & -0.87993800 & -0.00016200 \\ \mathrm{H} & -2.10963900 & 1.82205300 & 0.00013300 \\ \mathrm{C} & -1.05278100 & -0.10747400 & 0.00006600 \\ \mathrm{O} & -1.06480000 & -1.32304000 & 0.00001900 \\ \mathrm{O} & 0.04640600 & 0.65565600 & 0.00010200 \\ \mathrm{C} & 1.39544900 & 0.07385800 & -0.00001700 \\ \mathrm{C} & 1.60916800 & -0.74134900 & 1.26377700 \\ \mathrm{C} & 1.60932400 & -0.74066200 & -1.26423200 \\ \mathrm{C} & 2.28839600 & 1.30140600 & 0.00035700 \\ \mathrm{H} & 1.38072800 & -0.13702200 & 2.14974300 \\ \mathrm{H} & 0.98975700 & -1.64162600 & 1.27655900 \\ \mathrm{H} & 2.66233000 & -1.04174000 & 1.32062900 \\ \mathrm{H} & 1.38091800 & -0.13587500 & -2.14989300 \\ \mathrm{H} & 2.66251500 & -1.04094200 & -1.32115600 \\ \mathrm{H} & 0.98998900 & -1.64097800 & -1.27755700\end{array}$

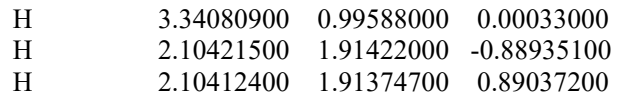

$\mathbf{I}_{\mathrm{AIBN}}$

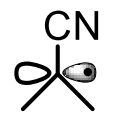

$\mathrm{E}=-210.6417195$

$\mathrm{G}_{\text {Corr. }}=0.056627$

C $\quad 0.00008000 \quad-1.06242900 \quad 1.28687500$

$\begin{array}{llll}\mathrm{H} & 0.00086800 & -0.40230800 & 2.15758200\end{array}$

$\mathrm{H} \quad-0.88071400 \quad-1.71750200 \quad 1.34654300$

$\begin{array}{llll}\mathrm{H} & 0.88051100 & -1.71813400 & 1.34544700\end{array}$

$\begin{array}{llll}\text { C } & -0.00037100 & -0.31118000 & 0.00000000\end{array}$

C $\quad 0.00008000-1.06242900 \quad-1.28687500$

$\mathrm{H} \quad 0.00086800 \quad-0.40230800 \quad-2.15758200$

$\mathrm{H} \quad 0.88051100 \quad-1.71813400 \quad-1.34544700$

$\mathrm{H} \quad-0.88071400 \quad-1.71750200 \quad-1.34654300$

$\begin{array}{llll}\mathrm{C} & -0.00010400 & 1.08171700 & 0.00000000\end{array}$

$\begin{array}{llll}\mathrm{N} & 0.00008000 & 2.25740200 & 0.00000000\end{array}$

\section{TS I $\mathbf{I}_{\text {AIBN }}-\mathbf{I I}_{\text {AIBN }}$}<smiles>CCCCOCCC(C)(C)C#N</smiles>

$\mathrm{E}=-634.9068043$

$\mathrm{G}_{\text {Corr. }}=0.222495$

$\begin{array}{lrrr}\mathrm{C} & -2.79747600 & 0.50846100 & 0.20573800 \\ \mathrm{C} & -1.64048100 & -0.85845800 & -1.09841800 \\ \mathrm{H} & -1.72476400 & -1.68516600 & -0.39380100 \\ \mathrm{H} & -2.40212400 & -0.79133100 & -1.87209600 \\ \mathrm{C} & -0.41700500 & -0.27765700 & -1.29070900 \\ \mathrm{H} & -0.24628600 & 0.45071500 & -2.08091500 \\ \mathrm{C} & 0.69893000 & -0.56003200 & -0.37854800 \\ \mathrm{O} & 0.65532400 & -1.36630300 & 0.53509500 \\ \mathrm{O} & 1.76203500 & 0.19732100 & -0.68987500 \\ \mathrm{C} & 3.00220700 & 0.14704900 & 0.09289600 \\ \mathrm{C} & 2.72653000 & 0.58665300 & 1.52117200 \\ \mathrm{C} & 3.61663500 & -1.24024300 & 0.01550600 \\ \mathrm{C} & 3.88588400 & 1.15910500 & -0.61386200 \\ \mathrm{H} & 2.23190600 & 1.56580600 & 1.52781500 \\ \mathrm{H} & 2.10074000 & -0.13361800 & 2.05463400 \\ \mathrm{H} & 3.67850300 & 0.68473300 & 2.05660900 \\ \mathrm{H} & 3.73332700 & -1.54626700 & -1.03091000 \\ \mathrm{H} & 4.61244900 & -1.21542400 & 0.47424900 \\ \mathrm{H} & 3.01091800 & -1.98387700 & 0.53882800 \\ \mathrm{H} & 4.85866900 & 1.21804700 & -0.11277500 \\ \mathrm{H} & 4.04967600 & 0.86715000 & -1.65738100 \\ \mathrm{H} & 3.42544400 & 2.15352000 & -0.59756100 \\ \mathrm{C} & -1.94925800 & 0.62474300 & 1.43533400 \\ \mathrm{H} & -1.76425800 & -0.34544000 & 1.90620800 \\ \mathrm{H} & -0.98475100 & 1.08275800 & 1.18446500 \\ \mathrm{H} & -2.44356000 & 1.27648200 & 2.17037600 \\ \mathrm{C} & -2.94280000 & 1.73284500 & -0.64786200 \\ \mathrm{H} & -3.50632800 & 2.50516800 & -0.10466000 \\ \mathrm{H} & -1.95500900 & 2.14624200 & -0.88102000\end{array}$




\section{II $_{\text {AIBN }}$

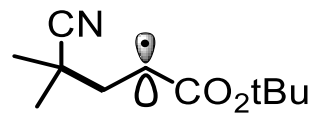

$$
\begin{aligned}
& E=-634.9429005 \\
& G_{\text {Corr. }}=0.226071
\end{aligned}
$$

$\begin{array}{llll}\text { C } & -2.74250300 & 0.26421500 & 0.13690900\end{array}$

C $\quad-1.76425300 \quad-0.22483900 \quad-0.97253400$

$\mathrm{H} \quad-1.63403600 \quad-1.30943700 \quad-0.86988000$

$\mathrm{H} \quad-2.23387500 \quad-0.02614500 \quad-1.94695700$

$\begin{array}{llll}\mathrm{C} & -0.44246400 & 0.43626400 & -0.90967900\end{array}$

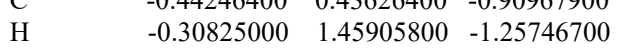

C $\quad 0.72165400 \quad-0.25936100 \quad-0.38132300$

$\begin{array}{lllll}\text { O } & 0.69405800 & -1.40550300 & 0.04343600\end{array}$

$\begin{array}{llll}\mathrm{O} & 1.80879100 & 0.52807500 & -0.42013200\end{array}$

$\begin{array}{llll}\text { C } & 3.11144000 & 0.07034600 & 0.07789200\end{array}$

$\begin{array}{llll}\mathrm{C} & 3.01988200 & -0.23612700 & 1.56326000\end{array}$

C $\quad 3.59150100-1.11980000 \quad-0.73534200$

$\begin{array}{llll}\mathrm{C} & 4.00365200 & 1.27455200 & -0.16291500\end{array}$

$\begin{array}{llll}\mathrm{H} & 2.60471900 & 0.62292200 & 2.10407200\end{array}$

$\begin{array}{llll}\mathrm{H} & 2.40104300 & -1.11513200 & 1.76004200\end{array}$

$\mathrm{H} \quad 4.02762100 \quad-0.42626600 \quad 1.95166600$

$\mathrm{H} \quad \begin{array}{llll}3.58376600 & -0.87781700 & -1.80476300\end{array}$

$\mathrm{H} \quad 4.62344500-1.35509000 \quad-0.44841300$

$\mathrm{H} \quad 2.97333600 \quad-2.00484500 \quad-0.56501300$

$\begin{array}{llll}\mathrm{H} & 5.02346500 & 1.05485500 & 0.17248500\end{array}$

$\mathrm{H} \quad 4.03504600 \quad 1.52578800 \quad-1.22915700$

$\mathrm{H} \quad 3.63585900 \quad 2.14590200 \quad 0.39075900$

$\begin{array}{llll}\mathrm{C} & -2.18731900 & -0.05168300 & 1.52867100\end{array}$

$\mathrm{H} \quad-1.95643500 \quad-1.11694500 \quad 1.63383400$

$\begin{array}{llll}\mathrm{H} & -1.26290300 & 0.51722800 & 1.68928000\end{array}$

$\begin{array}{llll}\mathrm{H} & -2.90376900 & 0.23383900 & 2.30636600\end{array}$

$\begin{array}{llll}\text { C } & -3.03536400 & 1.76147100 & 0.00092500\end{array}$

$\mathrm{H} \quad-3.78513400 \quad 2.07769500 \quad 0.73406000$

$\mathrm{H} \quad-2.11932700 \quad 2.33410700 \quad 0.18620700$

H $\quad-3.40559800 \quad 2.00729700 \quad-1.00086500$

C $\quad-4.00090800 \quad-0.47333700 \quad-0.04718000$

$\mathrm{N} \quad \begin{array}{llll}\mathrm{C} & -4.99623800 & -1.05397000 & -0.19782800\end{array}$

\section{TS I $\mathbf{I}_{\text {AIBN }}-\mathbf{I}_{1 H A T}$}

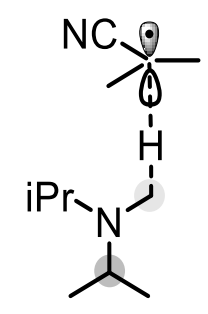

$$
\mathrm{E}=-542.189744
$$

$$
\mathrm{G}_{\text {Corr. }}=0.272013
$$

$\begin{array}{crrr}\mathrm{N} & -1.26017900 & 0.02814600 & -0.55865400 \\ \mathrm{C} & -0.14985500 & 0.34030900 & -1.33850100 \\ \mathrm{H} & -0.02749900 & -0.32738100 & -2.19976800 \\ \mathrm{H} & -0.09710700 & 1.39495500 & -1.62929700 \\ \mathrm{H} & 1.03756200 & 0.15947500 & -0.69405800 \\ \mathrm{C} & -1.78445200 & 1.04469400 & 0.36186100 \\ \mathrm{C} & -2.60685500 & 2.07664800 & -0.40018700 \\ \mathrm{C} & -0.70070100 & 1.70450500 & 1.20807700 \\ \mathrm{H} & -2.46303700 & 0.51556000 & 1.04458400 \\ \mathrm{H} & -3.41612300 & 1.59318600 & -0.95941600 \\ \mathrm{H} & -3.04626500 & 2.80618300 & 0.29029800\end{array}$

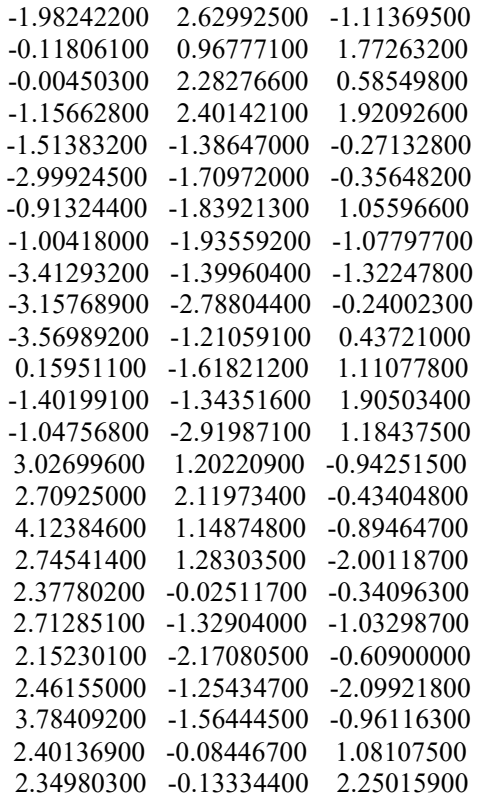

\section{TS $\mathbf{I}_{\text {AIBN- }}-\mathbf{I}_{2 H A T}$}<smiles>CC(C)N(C)C(C)(C)C(C)(C)C#N</smiles>

$\mathrm{E}=-542.1910491$

$\mathrm{G}_{\text {Corr. }}=0.271773$

$\begin{array}{lll}-1.34305400 & 0.43331300 & 0.43573600\end{array}$

$\begin{array}{lll}-1.55785400 & 0.03015100 & 1.81346500\end{array}$

$\begin{array}{lll}-2.53882700 & -0.44820600 & 1.89915300\end{array}$

$\begin{array}{lll}-0.79900800 & -0.67917100 & 2.18624600\end{array}$

$\begin{array}{lll}-1.57251600 & 0.90116700 & 2.47358300\end{array}$

$\begin{array}{lll}-0.22667900 & 1.24551100 & 0.11544100\end{array}$

$\begin{array}{lll}0.31339300 & 2.06374000 & 1.26443100\end{array}$

$\begin{array}{llll}-0.40281900 & 2.08720700 & -1.13331800\end{array}$

$\begin{array}{llll}0.82415900 & 0.44867200 & -0.15646200\end{array}$

$\begin{array}{lll}0.67523600 & 1.44251000 & 2.09255500\end{array}$

$\begin{array}{lll}1.15309000 & 2.67340000 & 0.91329900\end{array}$

$\begin{array}{lll}-0.44995500 & 2.75275300 & 1.65774300\end{array}$

$\begin{array}{llll}-0.76227300 & 1.52215500 & -1.99910100\end{array}$

$\begin{array}{lll}-1.12930600 & 2.89202300 & -0.93853800\end{array}$

$\begin{array}{llll}0.54826800 & 2.55508600 & -1.41461800\end{array}$

$\begin{array}{lll}-1.81483400 & -0.52550900 & -0.57990000\end{array}$

$\begin{array}{llll}-3.28549700 & -0.28926100 & -0.89321500\end{array}$

$\begin{array}{llll}-1.55085400 & -1.98334900 & -0.21223600\end{array}$

$-1.23559300 \quad-0.32210800 \quad-1.48994400$

$\begin{array}{llll}-3.44752000 & 0.73409900 & -1.25111700\end{array}$

$\begin{array}{lll}-3.63841300 & -0.98909400 & -1.66057800\end{array}$

$\begin{array}{lll}-3.90492800 & -0.43729200 & 0.00127600\end{array}$

$\begin{array}{llll}-0.51311500 & -2.14609100 & 0.10414200\end{array}$

$\begin{array}{lll}-2.20576500 & -2.32917400 & 0.59674400\end{array}$

$\begin{array}{llll}-1.74675000 & -2.61934000 & -1.08382300\end{array}$

$\begin{array}{llll}3.12381400 & 0.68862500 & -0.33515400\end{array}$

$\begin{array}{lll}3.24813300 & 1.10147300 & 0.67185400\end{array}$

$\begin{array}{llll}4.07875400 & 0.23056000 & -0.63186800\end{array}$

$\begin{array}{llll}2.93023700 & 1.51826900 & -1.02743200\end{array}$

$\begin{array}{llll}1.99306100 & -0.31483200 & -0.40060200\end{array}$

$\begin{array}{llll}1.74930100 & -0.90564200 & -1.77220200\end{array}$

$\begin{array}{llll}0.90563500 & -1.60571900 & -1.77479200\end{array}$

$\begin{array}{llll}1.53109500 & -0.10206000 & -2.48780400\end{array}$

$\begin{array}{llll}2.63500300 & -1.44386100 & -2.13970700\end{array}$ 


\section{TS II AIBN-I $_{1 H A T}$}<smiles>CC(C)N(CCC(=O)OCC(C)(C)C)C(C)C</smiles>

$$
\begin{aligned}
& E=-966.49896 \\
& G_{\text {Corr. }}=0.439079
\end{aligned}
$$

$\begin{array}{lllll}\text { C } & 3.37950200 & -1.31347200 & 0.24856800\end{array}$

C $\quad 1.87152700 \quad-1.50034500 \quad-0.06890400$

$\mathrm{H} \quad \begin{array}{llll}\mathrm{H} & 1.73976600 & -1.52557000 & -1.15979200\end{array}$

$\mathrm{H} \quad 1.57833400 \quad-2.49087300 \quad 0.31302500$

$\begin{array}{llll}\mathrm{C} & 0.96698700 & -0.44472600 & 0.50291300\end{array}$

$\begin{array}{llll}\mathrm{H} & 1.01938700 & -0.28858700 & 1.58385700\end{array}$

$\begin{array}{llll}\text { C } & 0.80330300 & 0.78440200 & -0.26420600\end{array}$

$\begin{array}{lllll}\mathrm{O} & 0.91685000 & 0.87179800 & -1.48161300\end{array}$

$\begin{array}{lllll}\mathrm{O} & 0.45198700 & 1.81916800 & 0.53562100\end{array}$

$\begin{array}{llll}\mathrm{C} & 0.28213400 & 3.17477100 & 0.01809500\end{array}$

$\begin{array}{lllll}\text { C } & 1.59643700 & 3.67279600 & -0.56193800\end{array}$

C $\quad-0.85368500 \quad 3.23813600 \quad-0.99081200$

$\begin{array}{llll}\mathrm{C} & -0.07888700 & 3.96710600 & 1.26315900\end{array}$

$\mathrm{H} \quad 2.39754100 \quad 3.57807200 \quad 0.18174000$

$\mathrm{H} \quad \begin{array}{llll}1.87751900 & 3.11707400 & -1.46069200\end{array}$

$\mathrm{H} \quad \begin{array}{llll}1.50013200 & 4.73371700 & -0.82313500\end{array}$

$\mathrm{H} \quad-1.77646100 \quad 2.83476000 \quad-0.55427500$

$\begin{array}{llll}-1.03839700 & 4.28655400 & -1.25582600\end{array}$

$\begin{array}{llll}-0.61879200 & 2.68171300 & -1.90183500\end{array}$

$\begin{array}{lll}-0.23094600 & 5.02172200 & 1.00598600\end{array}$

$\begin{array}{lll}-1.00252900 & 3.58207900 & 1.71210600\end{array}$

$\begin{array}{lll}0.72260200 & 3.90206600 & 2.00791800\end{array}$

$\begin{array}{lll}-2.52804100 & -1.52064900 & 0.05057300\end{array}$

$\begin{array}{llll}-2.80952700 & -1.38858200 & -1.38215800\end{array}$

$-2.11190600 \quad-2.08216900-1.87397700$

$\begin{array}{lll}-1.28662400 & -2.00702600 & 0.44001400\end{array}$

$\begin{array}{lll}-0.28944100 & -1.10652000 & 0.41497400\end{array}$

$\begin{array}{lll}-3.31341000 & -0.73419600 & 1.00924800\end{array}$

$\begin{array}{lll}-2.48655700 & 0.34090700 & 1.70525000\end{array}$

$\begin{array}{lll}-4.00505800 & -1.64743500 & 2.01398000\end{array}$

$\begin{array}{lll}-4.09184800 & -0.22769600 & 0.42309000\end{array}$

$\begin{array}{lll}-1.95785400 & 0.97679400 & 0.98546100\end{array}$

$\begin{array}{lll}-3.13727800 & 0.97629200 & 2.31753000\end{array}$

$\begin{array}{lll}-1.73372800 & -0.09994500 & 2.37210500\end{array}$

$\begin{array}{lll}-4.64537200 & -2.37606700 & 1.50415600\end{array}$

$\begin{array}{lll}-3.27012400 & -2.19992800 & 2.61360900\end{array}$

$\begin{array}{lll}-4.62377200 & -1.06223500 & 2.70459800\end{array}$

$3.65356200 \quad-1.44823600 \quad 1.75055800$

$\begin{array}{lll}3.27178800 & -2.39873000 & 2.14082100\end{array}$

$\begin{array}{lll}4.72834200 & -1.39613200 & 1.95661600\end{array}$

$3.16630300 \quad-0.62830000 \quad 2.28976100$

$\begin{array}{llll}3.91719800 & 0.02455900 & -0.26967500\end{array}$

$3.71110800 \quad 0.15320500 \quad-1.33737800$

$\begin{array}{lll}3.43693600 & 0.84704200 & 0.27519600\end{array}$

$\begin{array}{llll}4.99806700 & 0.09598400 & -0.10492200\end{array}$

$\begin{array}{lll}-0.90212600 & -2.78103800 & -0.23341800\end{array}$

$\begin{array}{lll}-1.24340500 & -2.32095000 & 1.48954400\end{array}$

$\begin{array}{llll}-2.52676600 & 0.01262700 & -1.91095800\end{array}$

$\begin{array}{llll}-1.48591200 & 0.30475100 & -1.72506200\end{array}$

$\begin{array}{llll}-2.69973200 & 0.05125800 & -2.99319000\end{array}$

$\begin{array}{llll}-3.18935700 & 0.75526300 & -1.44557900\end{array}$

$\begin{array}{llll}-4.22477100 & -1.84487000 & -1.71082900\end{array}$

$-4.40776500 \quad-2.85860000 \quad-1.33758700$

$\begin{array}{llll}-4.98035000 & -1.17698900 & -1.27743600\end{array}$

$\begin{array}{llll}-4.37518100 & -1.84256900 & -2.79638900\end{array}$

$\begin{array}{llll}4.09365800 & -2.39869300 & -0.44160500\end{array}$
TS II AIBN $-\mathbf{I}_{2 \mathrm{HAT}}$<smiles>CCOC(=O)CC(C)(C)N(C)C(C)C</smiles>

$$
\mathrm{E}=-966.4976004
$$

$\mathrm{G}_{\text {Corr. }}=0.440929$

$2.21942300 \quad-0.89045800 \quad-1.26794200$

$\begin{array}{llll}2.02728600 & -2.09555300 & 0.01402200\end{array}$

$\begin{array}{llll}0.86008700 & -0.31535600 & 0.26840400\end{array}$

$\begin{array}{llll}0.63127200 & -0.35070800 & 1.33719600\end{array}$

$\begin{array}{llll}0.51792500 & 0.95134900 & -0.37847000\end{array}$

$\begin{array}{llll}0.79351900 & 1.22942800 & -1.53793600\end{array}$

$\begin{array}{lll}-0.18872100 & 1.75549700 & 0.44555600\end{array}$

$\begin{array}{llll}-0.77666800 & 3.00609200 & -0.03510300\end{array}$

$\begin{array}{llll}0.31133300 & 3.95957500 & -0.50408300\end{array}$

$\begin{array}{lll}-1.80548300 & 2.70692400 & -1.11405000\end{array}$

$\begin{array}{lll}-1.45683300 & 3.56681700 & 1.20251200\end{array}$

$\begin{array}{lll}1.07285000 & 4.07420600 & 0.27784200\end{array}$

$\begin{array}{llll}0.79394900 & 3.60833300 & -1.41869500\end{array}$

$\begin{array}{llll}-0.13040700 & 4.94561000 & -0.69349000\end{array}$

$\begin{array}{llll}-2.52449000 & 1.96119600 & -0.74898000\end{array}$

$\begin{array}{llll}-2.35558000 & 3.62422900 & -1.35729300\end{array}$

$\begin{array}{llll}-1.33645800 & 2.32922100 & -2.02709400\end{array}$

$\begin{array}{lll}-1.91913800 & 4.53229000 & 0.96691000\end{array}$

$\begin{array}{llll}-2.23963600 & 2.88938700 & 1.56294600\end{array}$

$\begin{array}{lll}-0.72810800 & 3.71831400 & 2.00723900\end{array}$

$\begin{array}{llll}-2.31247900 & -1.24131900 & -0.28918500\end{array}$

$\begin{array}{llll}-3.47006100 & -1.04226400 & -1.13848800\end{array}$

$\begin{array}{lll}-4.22313200 & -0.45365100 & -0.60436300\end{array}$

$\begin{array}{llll}-3.20772500 & -0.48046700 & -2.04020000\end{array}$

$\begin{array}{llll}-3.94285400 & -1.99128600 & -1.45185100\end{array}$

$\begin{array}{llll}-1.14117700 & -1.83145700 & -0.82686200\end{array}$

$\begin{array}{llll}-0.92096900 & -1.56392700 & -2.29459000\end{array}$

$\begin{array}{llll}-0.84712900 & -3.27351900 & -0.45137700\end{array}$

$\begin{array}{llll}-0.16464500 & -1.14045800 & -0.25609800\end{array}$

$\begin{array}{lll}-1.00783700 & -0.49768300 & -2.53379600\end{array}$

$\begin{array}{llll}0.08895100 & -1.88310300 & -2.57666400\end{array}$

$\begin{array}{lll}-1.62898800 & -2.12691300 & -2.92189500\end{array}$

$\begin{array}{lll}-0.82506800 & -3.44516200 & 0.63048900\end{array}$

$\begin{array}{lll}-1.61686900 & -3.93483000 & -0.88238900\end{array}$

$\begin{array}{llll}0.12169400 & -3.58439800 & -0.85965300\end{array}$

$\begin{array}{lll}-2.57086900 & -1.41995000 & 1.14287700\end{array}$

$\begin{array}{lll}-3.61492300 & -2.49164300 & 1.44457400\end{array}$

$\begin{array}{lll}-2.92618200 & -0.09184100 & 1.79804200\end{array}$

$\begin{array}{lll}-1.61463900 & -1.74105800 & 1.58002400\end{array}$

$\begin{array}{lll}-3.37525100 & -3.44222000 & 0.95361600\end{array}$

$\begin{array}{lll}-3.67435300 & -2.66998000 & 2.52469700\end{array}$

$\begin{array}{llll}-4.61266200 & -2.17989800 & 1.10965900\end{array}$

$\begin{array}{llll}-2.12208600 & 0.63419700 & 1.62986200\end{array}$

$\begin{array}{lll}-3.85956600 & 0.32441400 & 1.39508600\end{array}$

$\begin{array}{lll}-3.06622100 & -0.22239300 & 2.87808000\end{array}$

$\begin{array}{lll}3.39108400 & -0.79420600 & 2.01250400\end{array}$

$\begin{array}{llll}3.19705000 & -1.85300400 & 2.21956200\end{array}$

$\begin{array}{llll}4.34762200 & -0.52131200 & 2.47157500\end{array}$

$\begin{array}{llll}2.60629400 & -0.19527200 & 2.48776300\end{array}$

$\begin{array}{llll}3.66358700 & 0.97204600 & 0.24288000\end{array}$

$\begin{array}{llll}3.67088500 & 1.19376900 & -0.82951400\end{array}$

$\begin{array}{lll}2.87113600 & 1.56798700 & 0.71313100\end{array}$

$\begin{array}{llll}4.62111300 & 1.28323900 & 0.67495500\end{array}$

$\begin{array}{llll}4.52010200 & -1.28456600 & -0.08315400\end{array}$

$\begin{array}{llll}5.38757000 & -1.90101500 & -0.55111600\end{array}$ 


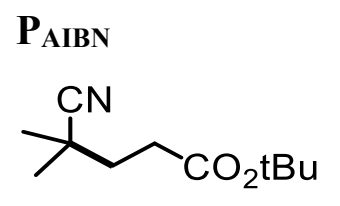

$\mathrm{E}=-635.5980074$

$$
\mathrm{G}_{\text {Corr. }}=0.240686
$$

$\begin{array}{lrrr}\mathrm{C} & -2.46022800 & 0.31618700 & -0.06927500 \\ \mathrm{C} & -1.84573200 & -0.94286000 & -0.72328500 \\ \mathrm{H} & -1.76689600 & -1.73847500 & 0.02909200 \\ \mathrm{H} & -2.54407600 & -1.29920200 & -1.49129500 \\ \mathrm{C} & 0.65766200 & -0.77559600 & -0.35066400 \\ \mathrm{O} & 0.69194200 & -1.53970200 & 0.59137200 \\ \mathrm{O} & 1.61034500 & 0.10609100 & -0.66473100 \\ \mathrm{C} & 2.83798500 & 0.24383700 & 0.13694600 \\ \mathrm{C} & 2.48272100 & 0.70866800 & 1.53839700 \\ \mathrm{C} & 3.61846300 & -1.05875900 & 0.13048700 \\ \mathrm{C} & 3.60076700 & 1.32500300 & -0.60647300 \\ \mathrm{H} & 1.88553100 & 1.62794000 & 1.49563900 \\ \mathrm{H} & 1.92784100 & -0.05328200 & 2.09223900 \\ \mathrm{H} & 3.40606500 & 0.93205000 & 2.08604800 \\ \mathrm{H} & 3.78169300 & -1.39975300 & -0.89880400 \\ \mathrm{H} & 4.59969900 & -0.88725200 & 0.58902700 \\ \mathrm{H} & 3.10639900 & -1.84509700 & 0.69006100 \\ \mathrm{H} & 4.54693000 & 1.53147200 & -0.09373600 \\ \mathrm{H} & 3.82329700 & 1.00707800 & -1.63135100 \\ \mathrm{H} & 3.01805100 & 2.25225700 & -0.64690000 \\ \mathrm{C} & -1.54624600 & 0.92281300 & 1.00238600 \\ \mathrm{H} & -1.25997900 & 0.18106600 & 1.75582000 \\ \mathrm{H} & -0.63472300 & 1.31557800 & 0.53402100 \\ \mathrm{H} & -2.04489600 & 1.75884700 & 1.50471400 \\ \mathrm{C} & -2.82404400 & 1.37772600 & -1.11526900 \\ \mathrm{H} & -3.33634000 & 2.22435800 & -0.64538600 \\ \mathrm{H} & -1.91724800 & 1.76203000 & -1.59496300 \\ \mathrm{H} & -3.47949500 & 0.96375200 & -1.88965000 \\ \mathrm{C} & -3.70810000 & -0.11359900 & 0.58226300 \\ \mathrm{~N} & -4.69658000 & -0.45141500 & 1.09160500 \\ \mathrm{C} & -0.47362900 & -0.72868100 & -1.35014000 \\ \mathrm{H} & -0.27844100 & -1.54802200 & -2.05532000 \\ \mathrm{H} & -0.41336600 & 0.19500300 & -1.93585200\end{array}$

\section{$(\text { iPr })_{3}$ SiSH}<smiles>S[SiH2][AsH2-]</smiles>

$\mathrm{E}=-1043.769941$

$$
\mathrm{G}_{\text {Corr. }}=0.2466
$$

Si $\quad 0.03299000 \quad 0.05028300 \quad 0.15902500$

S $\quad-0.75725400 \quad 0.42367700 \quad 2.17464300$

$\begin{array}{llll}\mathrm{H} & -0.14963000 & -0.64987800 & 2.72587100\end{array}$

$\begin{array}{llll}\text { C } & 1.79729200 & 0.76492700 & 0.10602700\end{array}$

$\begin{array}{llll}\text { C } & 2.30493700 & 1.08740300 & -1.30003700\end{array}$

$\begin{array}{llll}\mathrm{C} & 2.79608400 & -0.11781000 & 0.85259600\end{array}$

$\begin{array}{llll}\mathrm{H} & 1.70981600 & 1.71426600 & 0.66100800\end{array}$

$\begin{array}{llll}\mathrm{H} & 1.65201700 & 1.78900000 & -1.83373600\end{array}$

$\mathrm{H} \quad 3.30456000 \quad 1.54207500 \quad-1.25382000$

$\mathrm{H} \quad 2.39484900 \quad 0.18480800 \quad-1.91991600$

$\mathrm{H} \quad 2.46491100 \quad-0.35402900 \quad 1.87277300$

$\begin{array}{llll}\mathrm{H} & 2.95991000 & -1.06883000 & 0.32626400\end{array}$

$\begin{array}{llll}\mathrm{H} & 3.77375300 & 0.37707300 & 0.93270900\end{array}$

C $\quad 0.04154000-1.82848100 \quad-0.13150700$

$\begin{array}{lll}0.44434200 & -2.15388500 & -1.57242200\end{array}$

$\begin{array}{lll}-1.24870600 & -2.55696000 & 0.24056100\end{array}$

$\begin{array}{lll}0.83795600 & -2.20107100 & 0.53647900\end{array}$

$\begin{array}{llll}1.42481700 & -1.74170000 & -1.84148000\end{array}$

$\begin{array}{lll}0.49441800 & -3.24081600 & -1.72652600\end{array}$

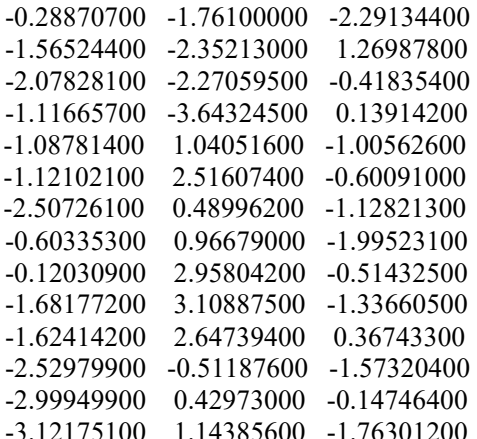

\section{$(\text { iPr })_{3} \mathrm{SiS} \cdot$}<smiles>[AsH2-][SiH2][Sn]</smiles>

$E=-1043.125448$

$\mathrm{G}_{\text {Corr. }}=0.23825$

$\begin{array}{lll}-0.55803900 & 0.12612900 & 2.25805600\end{array}$

$\begin{array}{lll}1.90691600 & 0.48126800 & 0.13983400\end{array}$

$\begin{array}{llll}2.39711700 & 0.89576400 & -1.24882600\end{array}$

$\begin{array}{llll}2.76819600 & -0.64773800 & 0.70178900\end{array}$

$\begin{array}{llll}2.00720700 & 1.35183300 & 0.80980300\end{array}$

$\begin{array}{llll}1.85359700 & 1.75794100 & -1.65336200\end{array}$

$\begin{array}{llll}3.46161400 & 1.16662900 & -1.21363600\end{array}$

$\begin{array}{llll}2.30131300 & 0.07504000 & -1.97324000\end{array}$

$\begin{array}{lll}2.42271000 & -0.98464900 & 1.68726100\end{array}$

$\begin{array}{llll}2.76703000 & -1.51809200 & 0.03019600\end{array}$

$\begin{array}{lll}3.81318100 & -0.32516000 & 0.80637500\end{array}$

$\begin{array}{lll}-0.26295200 & -1.76685400 & -0.32486800\end{array}$

$\begin{array}{llll}-0.12674000 & -1.93703000 & -1.83854100\end{array}$

$\begin{array}{lll}-1.58394100 & -2.34973800 & 0.17600900\end{array}$

$\begin{array}{llll}0.55421800 & -2.32273400 & 0.16287500\end{array}$

$\begin{array}{llll}0.84514400 & -1.59506300 & -2.21723300\end{array}$

$\begin{array}{llll}-0.23182200 & -2.99491600 & -2.11789700\end{array}$

$\begin{array}{llll}-0.90662000 & -1.38262200 & -2.37883500\end{array}$

$\begin{array}{lll}-1.65954600 & -2.32235800 & 1.27108900\end{array}$

$-2.45044400 \quad-1.80953800 \quad-0.22645200$

$\begin{array}{llll}-1.68189300 & -3.39898300 & -0.13606300\end{array}$

$\begin{array}{llll}-0.93597900 & 1.31279100 & -0.83209600\end{array}$

$\begin{array}{lll}-0.71218000 & 2.72320400 & -0.28423800\end{array}$

$\begin{array}{llll}-2.42961400 & 1.00240700 & -0.90081300\end{array}$

$\begin{array}{llll}-0.52275600 & 1.26516300 & -1.85495100\end{array}$

$\begin{array}{llll}0.34790800 & 3.00550100 & -0.25041100\end{array}$

$\begin{array}{llll}-1.23261400 & 3.46975300 & -0.89996300\end{array}$

$\begin{array}{lll}-1.11043600 & 2.80979200 & 0.73688000\end{array}$

$\begin{array}{llll}-2.63935300 & 0.05707500 & -1.41617500\end{array}$

$\begin{array}{llll}-2.86744700 & 0.93925700 & 0.10567200\end{array}$

$\begin{array}{llll}-2.96664800 & 1.79529800 & -1.44024200\end{array}$

\section{TS I AIBN-RSH ${ }_{\text {HAT }}$}<smiles>CC(C)(C#N)CS[SiH3]</smiles>

$$
\mathrm{E}=-1254.399538
$$

$$
\begin{aligned}
& \text { GCorr. }=0.322625 \\
& \begin{array}{llll}
\mathrm{C} & 3.59663300 & -1.70492800 & -0.56054200 \\
\mathrm{H} & 3.53256200 & -1.72941100 & -1.65278600 \\
\mathrm{H} & 4.64244600 & -1.87758600 & -0.27175100
\end{array}
\end{aligned}
$$




$\begin{array}{lrrr}\mathrm{H} & 2.99071800 & -2.52234900 & -0.15372600 \\ \mathrm{C} & 2.99574100 & -0.29252800 & 1.49447200 \\ \mathrm{H} & 2.62438800 & 0.68572700 & 1.81626200 \\ \mathrm{H} & 2.32748300 & -1.07567500 & 1.87164100 \\ \mathrm{H} & 3.98216400 & -0.44787500 & 1.95462900 \\ \mathrm{C} & 3.62791300 & 0.77782000 & -0.65376800 \\ \mathrm{~N} & 4.00769600 & 1.73965100 & -1.19387300 \\ \mathrm{C} & 3.11869900 & -0.38977800 & -0.00269400 \\ \mathrm{H} & 1.76928500 & -0.39025300 & -0.49958500 \\ \mathrm{Si} & -1.05858100 & 0.06509200 & 0.01578500 \\ \mathrm{~S} & 0.50252100 & -0.58491500 & -1.36796000 \\ \mathrm{C} & -0.32379000 & 1.37030400 & 1.18582800 \\ \mathrm{C} & -1.36578600 & 1.89516800 & 2.17622800 \\ \mathrm{C} & 0.34749000 & 2.53082400 & 0.45481100 \\ \mathrm{H} & 0.44623100 & 0.83377600 & 1.76476100 \\ \mathrm{H} & -1.86715500 & 1.09391100 & 2.73478800 \\ \mathrm{H} & -0.89829200 & 2.56514800 & 2.91103600 \\ \mathrm{H} & -2.14074800 & 2.47974500 & 1.66077800 \\ \mathrm{H} & 1.12202300 & 2.20025000 & -0.24850100 \\ \mathrm{H} & -0.38492100 & 3.11308500 & -0.12176100 \\ \mathrm{H} & 0.81650200 & 3.22230800 & 1.16919500 \\ \mathrm{C} & -2.43497000 & 0.80598400 & -1.07444900 \\ \mathrm{C} & -3.78715700 & 0.79855700 & -0.35594700 \\ \mathrm{C} & -2.56658200 & 0.16517200 & -2.45621100 \\ \mathrm{H} & -2.12915800 & 1.85659200 & -1.21999500 \\ \mathrm{H} & -3.74986300 & 1.25925400 & 0.63897400 \\ \mathrm{H} & -4.53727900 & 1.34607500 & -0.94330400 \\ \mathrm{H} & -4.16481900 & -0.22541100 & -0.22986800 \\ \mathrm{H} & -1.64555300 & 0.25432900 & -3.04416500 \\ \mathrm{H} & -2.81209000 & -0.90353100 & -2.38527900 \\ \mathrm{H} & -3.37336100 & 0.64542800 & -3.02756600 \\ \mathrm{C} & -1.66329500 & -1.43663700 & 1.01112100 \\ \mathrm{C} & -0.56966400 & -1.98943300 & 1.92302200 \\ \mathrm{C} & -2.21362000 & -2.54481200 & 0.11411900 \\ \mathrm{H} & -2.48621000 & -1.06303000 & 1.64511100 \\ \mathrm{H} & -0.20279600 & -1.24676300 & 2.64388200 \\ \mathrm{H} & -0.93614800 & -2.85134500 & 2.49790100 \\ \mathrm{H} & 0.28898800 & -2.34037500 & 1.32985800 \\ \mathrm{H} & -3.06783800 & -2.21361700 & -0.48969800 \\ -1.44113900 & -2.91028200 & -0.57707000 \\ \mathrm{H} & -2.54917100 & -3.40260700 & 0.71377400\end{array}$

\section{TS II AIBN-RSH $_{\text {HAT }}$}<smiles>CCOC(=O)CC(C)(C#N)CC(C)(C)C</smiles>

$$
\mathrm{E}=-1678.708917
$$$$
\mathrm{G}_{\text {Corr. }}=0.493034
$$

$\begin{array}{lccc}\mathrm{C} & 3.06590600 & -2.37696300 & 0.33015500 \\ \mathrm{C} & 1.92469100 & -1.76172800 & -0.52825400 \\ \mathrm{H} & 2.27838200 & -1.62072700 & -1.55778200 \\ \mathrm{H} & 1.09655900 & -2.48522200 & -0.56355100 \\ \mathrm{C} & 1.44310200 & -0.44803200 & -0.00579700 \\ \mathrm{H} & 1.07049500 & -0.39039800 & 1.02014400 \\ \mathrm{C} & 2.07318900 & 0.78730000 & -0.52263100 \\ \mathrm{O} & 2.68666700 & 0.83388300 & -1.57250200 \\ \mathrm{O} & 1.84607300 & 1.81535800 & 0.29974800 \\ \mathrm{C} & 2.37021900 & 3.15982900 & 0.00257400 \\ \mathrm{C} & 3.88855500 & 3.11768100 & -0.03818500 \\ \mathrm{C} & 1.76029000 & 3.67802500 & -1.28816900 \\ \mathrm{C} & 1.89477700 & 3.97632400 & 1.18980900 \\ \mathrm{H} & 4.28046900 & 2.66446800 & 0.88082500 \\ \mathrm{H} & 4.25954800 & 2.55683300 & -0.89966500 \\ \mathrm{H} & 4.27256300 & 4.14285200 & -0.09997400 \\ \mathrm{H} & 0.66568100 & 3.61664800 & -1.24602800 \\ \mathrm{H} & 2.03553000 & 4.73202700 & -1.41417300\end{array}$

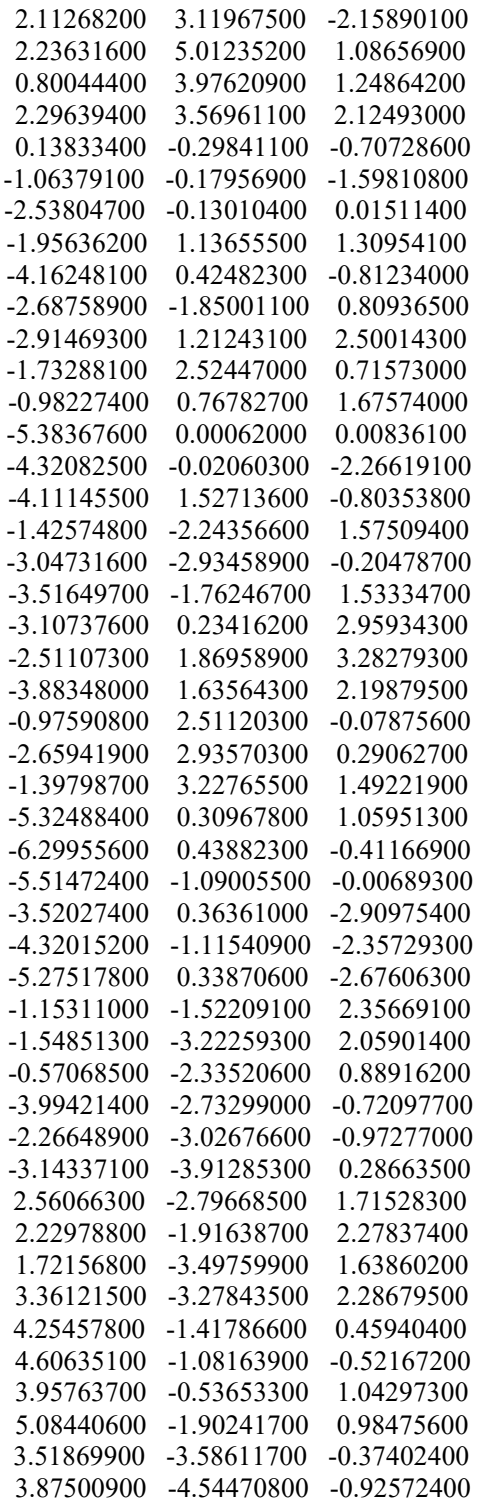

$\mathbf{I}_{1}$<smiles>CC(C)N(C)C(C)C</smiles>

$E=-330.9147445$

$\mathrm{G}_{\text {Corr. }}=0.181814$

$\mathrm{H}_{\text {Corr. }}=0.229483$

$\begin{array}{lrrr}\mathrm{N} & -0.00329100 & -0.02526600 & 0.60616200 \\ \mathrm{C} & -0.38031000 & -0.72650500 & 1.73450600 \\ \mathrm{H} & 0.34202200 & -0.77248800 & 2.54550300 \\ \mathrm{H} & -1.43760300 & -0.75363500 & 1.98198700 \\ \mathrm{C} & -0.98558000 & 0.18542300 & -0.46127700 \\ \mathrm{C} & -1.95972800 & 1.29401500 & -0.08094000 \\ \mathrm{C} & -1.72225700 & -1.09022800 & -0.86081200 \\ \mathrm{H} & -0.41779800 & 0.52678800 & -1.33757500 \\ \mathrm{H} & -1.42310200 & 2.22438600 & 0.13829400 \\ \mathrm{H} & -2.66955800 & 1.48313900 & -0.89521400 \\ \mathrm{H} & -2.53739600 & 1.01724400 & 0.81018200 \\ \mathrm{H} & -1.02073100 & -1.89731000 & -1.09993800 \\ \mathrm{H} & -2.37802200 & -1.44147200 & -0.05513500\end{array}$ 


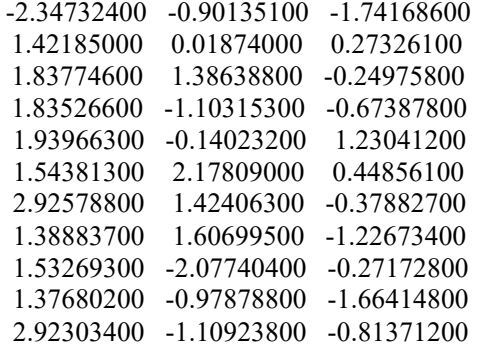

$\mathbf{I}_{2}$<smiles>CCN(C)C(C)C</smiles>

$E=-330.9165137$

$\mathrm{G}_{\text {Corr. }}=0.182447$

$\mathrm{H}_{\text {Corr }}=0.230161$

$\begin{array}{lrrr}\mathrm{N} & -0.05519800 & 0.38671900 & -0.27843000 \\ \mathrm{C} & 0.09915000 & 1.76539500 & 0.15530400 \\ \mathrm{H} & 1.06304500 & 2.15656000 & -0.17888100 \\ \mathrm{H} & -0.67438000 & 2.39655300 & -0.29083200 \\ \mathrm{H} & 0.04173200 & 1.87916600 & 1.25330900 \\ \mathrm{C} & -1.28020000 & -0.22794100 & 0.04823700 \\ \mathrm{C} & -2.49262800 & 0.64257300 & 0.09647300 \\ \mathrm{C} & -1.47000800 & -1.65597300 & -0.35064600 \\ \mathrm{H} & -2.71498800 & 1.11629900 & -0.88077700 \\ \mathrm{H} & -3.36929900 & 0.04558300 & 0.36729600 \\ \mathrm{H} & -2.41903600 & 1.45722300 & 0.82794400 \\ \mathrm{H} & -1.29544200 & -1.81994400 & -1.43149300 \\ \mathrm{H} & -0.81353500 & -2.35931100 & 0.18117300 \\ \mathrm{H} & -2.49893000 & -1.96470500 & -0.14040100 \\ \mathrm{C} & 1.15776500 & -0.43828900 & -0.13536900 \\ \mathrm{C} & 1.54563300 & -0.65889800 & 1.32374400 \\ \mathrm{C} & 2.32563600 & 0.11002000 & -0.94228000 \\ \mathrm{H} & 0.90857200 & -1.41094800 & -0.57618200 \\ \mathrm{H} & 0.69612500 & -1.04663400 & 1.90022400 \\ \mathrm{H} & 2.37377400 & -1.37357800 & 1.40103100 \\ \mathrm{H} & 1.87348100 & 0.27922800 & 1.79097700 \\ \mathrm{H} & 2.01462600 & 0.36443300 & -1.96191800 \\ \mathrm{H} & 2.77364800 & 0.99983500 & -0.48268600 \\ \mathrm{H} & 3.11490600 & -0.64811300 & -1.00255800\end{array}$

\section{TS $\mathbf{I}_{1}-\mathbf{I}_{2}$}<smiles>CC(C)N(CI)CC(C)N(C)C(C)C</smiles>

\section{$E=-662.4573924$}

$\mathrm{G}_{\text {Corr. }}=0.394573$

$\begin{array}{llll}\mathrm{N} & -2.52781100 & 0.25128700 & 0.56081200 \\ \mathrm{C} & -2.19302400 & -0.85408100 & 1.44516400 \\ \mathrm{H} & -3.06278400 & -1.49842900 & 1.59619500 \\ \mathrm{H} & -1.36423900 & -1.47853800 & 1.06102300 \\ \mathrm{H} & -1.89846800 & -0.48308700 & 2.43175400 \\ \mathrm{C} & -1.39378400 & 1.08862600 & 0.25289000 \\ \mathrm{C} & -0.70622100 & 1.61847400 & 1.49226700 \\ \mathrm{C} & -1.66937500 & 2.18718800 & -0.74610600\end{array}$

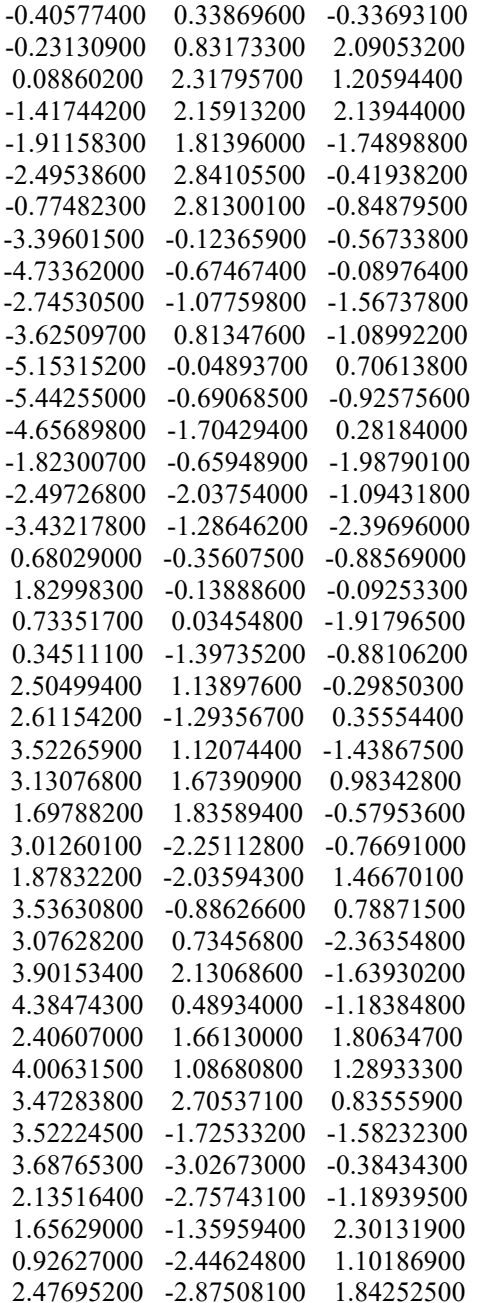

$\begin{array}{llll}2.47695200 & -2.87508100 & 1.84252500\end{array}$

TS $\mathbf{I}_{\mathbf{1}}-\mathbf{I I}_{\mathbf{1}}$

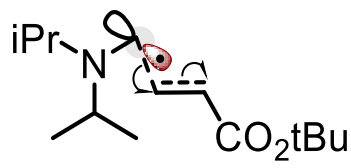

$\mathrm{E}=-755.1912092$

$\mathrm{G}_{\text {Corr. }}=0.345368$

$\begin{array}{lrrr}\mathrm{C} & -2.66806400 & 2.54434400 & -0.46621700 \\ \mathrm{C} & -2.89849500 & 1.29457400 & 0.37384800 \\ \mathrm{H} & -3.92310400 & 1.33878500 & 0.76679500 \\ \mathrm{H} & -3.38077600 & 2.59362000 & -1.29767800 \\ \mathrm{H} & -2.78535000 & 3.44476200 & 0.14776000 \\ \mathrm{H} & -1.65348300 & 2.55647900 & -0.88371200 \\ \mathrm{C} & -1.94447000 & 1.23325400 & 1.56196700 \\ \mathrm{H} & -2.14440800 & 0.35553500 & 2.18796900 \\ \mathrm{H} & -0.89629900 & 1.19047500 & 1.23843300 \\ \mathrm{H} & -2.06665400 & 2.12816600 & 2.18338400 \\ \mathrm{~N} & -2.84069700 & 0.08901600 & -0.46365700 \\ \mathrm{C} & -3.97958300 & -0.83271800 & -0.47647000 \\ \mathrm{C} & -5.23680000 & -0.16670300 & -1.01743200 \\ \mathrm{C} & -4.21200500 & -1.47203300 & 0.88762600 \\ \mathrm{H} & -3.69260600 & -1.62877500 & -1.17860100 \\ \mathrm{H} & -5.05329100 & 0.27283600 & -2.00415100 \\ \mathrm{H} & -6.04194500 & -0.90457200 & -1.11115900 \\ \mathrm{H} & -5.59885500 & 0.62579900 & -0.35017700 \\ \mathrm{H} & -3.30005000 & -1.96162600 & 1.24891300 \\ \mathrm{H} & -4.52067200 & -0.72580400 & 1.63149000 \\ \mathrm{H} & -5.00733600 & -2.22435000 & 0.82668400 \\ \mathrm{C} & -1.64575700 & -0.33045100 & -0.97012200\end{array}$ 


$\begin{array}{lrrr}\mathrm{H} & -1.67225900 & -1.10837400 & -1.72864000 \\ \mathrm{H} & -0.82636900 & 0.38225500 & -0.99832100 \\ \mathrm{C} & -0.13269700 & -1.83770900 & 0.48265000 \\ \mathrm{H} & -0.44170300 & -1.12872700 & 1.24585900 \\ \mathrm{H} & -0.78430600 & -2.68679000 & 0.29518700 \\ \mathrm{C} & 1.10890500 & -1.76963100 & -0.05245400 \\ \mathrm{H} & 1.47848700 & -2.51582200 & -0.75186500 \\ \mathrm{C} & 1.98110600 & -0.63936700 & 0.24783400 \\ \mathrm{O} & 1.66889400 & 0.32669000 & 0.93136300 \\ \mathrm{O} & 3.18681700 & -0.79353000 & -0.34119900 \\ \mathrm{C} & 4.24812800 & 0.19954600 & -0.20356300 \\ \mathrm{C} & 3.81384200 & 1.52538700 & -0.80756100 \\ \mathrm{C} & 4.66120100 & 0.33449300 & 1.25319200 \\ \mathrm{C} & 5.38105100 & -0.40236300 & -1.01748300 \\ \mathrm{H} & 3.46515400 & 1.37678700 & -1.83677100 \\ \mathrm{H} & 3.01575000 & 1.99212100 & -0.22523600 \\ \mathrm{H} & 4.67259800 & 2.20742000 & -0.83512500 \\ \mathrm{H} & 4.91054300 & -0.64926000 & 1.66887400 \\ \mathrm{H} & 5.55526300 & 0.96691100 & 1.31688700 \\ \mathrm{H} & 3.87010400 & 0.78524400 & 1.85733700 \\ \mathrm{H} & 6.25410900 & 0.25992400 & -0.99179600 \\ \mathrm{H} & 5.67177700 & -1.37790900 & -0.61111600 \\ \mathrm{H} & 5.07669500 & -0.53724400 & -2.06182200\end{array}$

\section{TS $\mathbf{I}_{2}-\mathbf{I I}_{2}$}

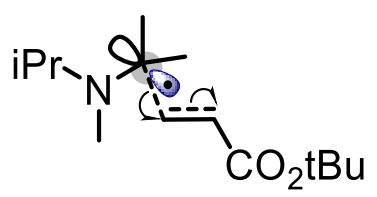

$$
\begin{aligned}
& E=-755.1957426 \\
& G_{\text {Corr. }}=0.34776
\end{aligned}
$$

$\begin{array}{lrrr}\mathrm{N} & 2.74557800 & 0.16570900 & 0.12164700 \\ \mathrm{C} & 1.98792400 & 1.43139300 & 0.16653100 \\ \mathrm{C} & 1.80465500 & 2.06234400 & -1.20800900 \\ \mathrm{C} & 2.63456700 & 2.40066500 & 1.14720200 \\ \mathrm{H} & 0.98369700 & 1.18329400 & 0.52503700 \\ \mathrm{H} & 1.35432600 & 1.35550200 & -1.91260600 \\ \mathrm{H} & 1.11715500 & 2.91135400 & -1.12026200 \\ \mathrm{H} & 2.74630000 & 2.44200200 & -1.62350300 \\ \mathrm{H} & 2.69481700 & 1.96472600 & 2.15087600 \\ \mathrm{H} & 3.65206500 & 2.66643400 & 0.83050000 \\ \mathrm{H} & 2.05413800 & 3.32924900 & 1.20667100 \\ \mathrm{C} & 2.18972800 & -1.02441500 & 0.54479400 \\ \mathrm{C} & 0.71780600 & -1.75225200 & -1.23739400 \\ \mathrm{H} & 1.06598800 & -0.86643200 & -1.76511900 \\ \mathrm{H} & 1.28954800 & -2.66615200 & -1.37573700 \\ \mathrm{C} & -0.56634500 & -1.80366600 & -0.77515500 \\ \mathrm{H} & -0.98404500 & -2.71577600 & -0.35344000 \\ \mathrm{C} & -1.37532400 & -0.60315300 & -0.69553800 \\ \mathrm{O} & -1.03598300 & 0.50710400 & -1.09319600 \\ \mathrm{O} & -2.56698700 & -0.85503700 & -0.09746000 \\ \mathrm{C} & -3.50223000 & 0.21637500 & 0.21876300 \\ \mathrm{C} & -2.85768500 & 1.20389100 & 1.18050200 \\ \mathrm{C} & -4.00451300 & 0.88624000 & -1.05074800 \\ \mathrm{C} & -4.64095800 & -0.51734400 & 0.90735000 \\ \mathrm{H} & -2.47297800 & 0.67485800 & 2.06181100 \\ \mathrm{H} & -2.03752400 & 1.75129700 & 0.70698200 \\ \mathrm{H} & -3.61075700 & 1.92559100 & 1.52032800 \\ \mathrm{H} & -4.39833600 & 0.13301200 & -1.74383100 \\ \mathrm{H} & -4.82060800 & 1.57457700 & -0.79777000 \\ \mathrm{H} & -3.21231200 & 1.44780000 & -1.55118600 \\ \mathrm{H} & -5.42112800 & 0.19325100 & 1.20425400 \\ \mathrm{H} & -5.08422200 & -1.25899300 & 0.23280500 \\ \mathrm{H} & -4.28072600 & -1.03473700 & 1.80405800 \\ \mathrm{C} & 3.92520200 & 0.15367400 & -0.72110000 \\ \mathrm{H} & 3.70555100 & -0.17286100 & -1.75163200 \\ \mathrm{H} & 4.35019800 & 1.15959600 & -0.77076500 \\ \mathrm{H} & 4.69815100 & -0.50003100 & -0.30859900 \\ \mathrm{H} & 1.19282200 & -0.97118900 & 1.65998000 \\ & 0.26747300 & -0.44040300 & 1.40172300\end{array}$

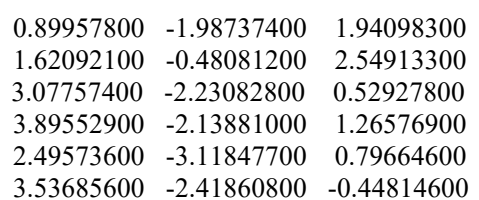

II $_{1}$

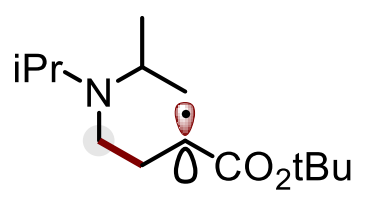

$$
E=-755.2202148
$$

$\mathrm{G}_{\text {Corr. }}=0.349125$ $\begin{array}{lll}3.62280300 & -1.12702500 & 0.09050100\end{array}$

$\begin{array}{lll}3.04012600 & -2.06197400 & 0.06526700\end{array}$

$\begin{array}{lll}3.59820500 & -1.00987400 & 2.27171700\end{array}$

$\begin{array}{lll}4.93750400 & -1.96195600 & 1.60411500\end{array}$

$\begin{array}{lll}4.98706600 & -0.19578900 & 1.51504300\end{array}$

$\begin{array}{lll}4.63639400 & -1.20948800 & -1.04330900\end{array}$

$\begin{array}{lll}4.12599000 & -1.29723600 & -2.00937800\end{array}$

$\begin{array}{lll}5.27948800 & -0.32027300 & -1.07798700\end{array}$

$\begin{array}{lll}5.29293700 & -2.07826800 & -0.91220400\end{array}$

$\begin{array}{llll}2.64582800 & -0.06400900 & -0.14279700\end{array}$

$\begin{array}{llll}3.09816600 & 1.32690900 & -0.07157500\end{array}$

$\begin{array}{lll}2.91637500 & 2.00541500 & 1.28898900\end{array}$

$\begin{array}{llll}2.43328900 & 2.14731200 & -1.17076600\end{array}$

$\begin{array}{lll}4.17715300 & 1.30310100 & -0.28417600\end{array}$

$\begin{array}{llll}3.33982800 & 1.40946500 & 2.10477300\end{array}$

$\begin{array}{lll}3.41192000 & 2.98420200 & 1.29084400\end{array}$

$\begin{array}{lll}1.85652900 & 2.18281500 & 1.51332300\end{array}$

$\begin{array}{lll}2.65582700 & 1.72122700 & -2.15603300\end{array}$

$\begin{array}{lll}1.34113100 & 2.15545800 & -1.04677400\end{array}$

$\begin{array}{llll}2.77627300 & 3.18937800 & -1.15060600\end{array}$

$\begin{array}{lll}1.31399200 & -0.34475200 & 0.33582300\end{array}$

$\begin{array}{lll}1.32272900 & -0.90624600 & 1.29004500\end{array}$

$\begin{array}{lll}0.78215900 & 0.59285200 & 0.53930900\end{array}$

$\begin{array}{lll}0.47980000 & -1.12677500 & -0.70874100\end{array}$

$\begin{array}{llll}0.44733900 & -0.50640700 & -1.61464600\end{array}$

$\begin{array}{lll}0.99861600 & -2.06328300 & -0.95199400\end{array}$

$\begin{array}{lll}-0.88792100 & -1.40108100 & -0.22590300\end{array}$

$\begin{array}{lll}-1.13257500 & -2.31969900 & 0.30443900\end{array}$

$\begin{array}{lll}-1.92113600 & -0.38921200 & -0.34043800\end{array}$

$\begin{array}{llll}-1.74632200 & 0.70887300 & -0.85419100\end{array}$

$\begin{array}{lll}-3.08397100 & -0.81410000 & 0.19120200\end{array}$

$\begin{array}{lll}-4.28150300 & 0.02832000 & 0.19936200\end{array}$

$\begin{array}{lll}-4.03108900 & 1.28848000 & 1.01146100\end{array}$

$\begin{array}{llll}-4.71753800 & 0.33161200 & -1.22456500\end{array}$

$\begin{array}{lll}-5.30727700 & -0.85228500 & 0.89086300\end{array}$

$\begin{array}{lll}-3.65974400 & 1.02908300 & 2.01017900\end{array}$

$\begin{array}{lll}-3.31002700 & 1.94901700 & 0.52337000\end{array}$

$\begin{array}{lll}-4.97667300 & 1.83107100 & 1.13145900\end{array}$

$\begin{array}{lll}-4.82691700 & -0.59876700 & -1.79459600\end{array}$

$\begin{array}{llll}-5.69304900 & 0.83241000 & -1.20180700\end{array}$

$\begin{array}{lll}-4.00494900 & 0.98218500 & -1.73756200\end{array}$

$\begin{array}{lll}-6.26590900 & -0.32555500 & 0.95946800\end{array}$

$\begin{array}{lll}-5.46016200 & -1.78107800 & 0.32948200\end{array}$

$\begin{array}{lll}-4.97719500 & -1.10759400 & 1.90430300\end{array}$

II $_{2}$

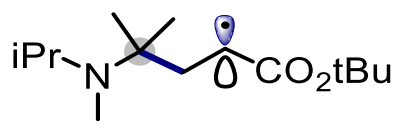

$E=-755.2202026$ 
$\mathrm{G}_{\text {Corr. }}=0.349915$

\begin{tabular}{|c|c|c|c|}
\hline $\mathrm{N}$ & -2.93918200 & -0.41215300 & 0.39310700 \\
\hline $\mathrm{C}$ & -4.08627200 & 0.38381700 & -0.07267500 \\
\hline $\mathrm{C}$ & -5.39704300 & -0.33748200 & 0.22654400 \\
\hline $\mathrm{C}$ & -4.05501500 & 0.80243100 & -1.54569700 \\
\hline $\mathrm{H}$ & -4.09830200 & 1.29650400 & 0.53484500 \\
\hline $\mathrm{H}$ & -5.40305900 & -0.73462500 & 1.24807600 \\
\hline $\mathrm{H}$ & -6.23314600 & 0.36362500 & 0.12381000 \\
\hline $\mathrm{H}$ & -5.58370500 & -1.16507600 & -0.46959100 \\
\hline $\mathrm{H}$ & -3.24443400 & 1.50570800 & -1.76661100 \\
\hline $\mathrm{H}$ & -3.94285600 & -0.06565700 & -2.20837000 \\
\hline $\mathrm{H}$ & -4.99712900 & 1.29730500 & -1.81273100 \\
\hline $\mathrm{C}$ & -1.63055800 & 0.23443400 & 0.58874500 \\
\hline $\mathrm{C}$ & -0.77171800 & 0.23124800 & -0.73431100 \\
\hline $\mathrm{H}$ & -0.68325500 & -0.80359000 & -1.08672200 \\
\hline $\mathrm{H}$ & -1.32694700 & 0.80632300 & -1.48739500 \\
\hline $\mathrm{C}$ & 0.57852500 & 0.79487400 & -0.57501200 \\
\hline $\mathrm{H}$ & 0.73615700 & 1.87097200 & -0.51724400 \\
\hline $\mathrm{C}$ & 1.74527800 & -0.05729200 & -0.45128400 \\
\hline $\mathrm{O}$ & 1.72112700 & -1.27884200 & -0.53233100 \\
\hline $\mathrm{O}$ & 2.85719700 & 0.67427400 & -0.23041900 \\
\hline $\mathrm{C}$ & 4.15820500 & 0.04338000 & -0.00647100 \\
\hline $\mathrm{C}$ & 4.10366800 & -0.83655900 & 1.23215000 \\
\hline $\mathrm{C}$ & 4.59332800 & -0.72168000 & -1.24557300 \\
\hline $\mathrm{C}$ & 5.07314900 & 1.23237000 & 0.22900000 \\
\hline $\mathrm{H}$ & 3.71646100 & -0.26583700 & 2.08534900 \\
\hline $\mathrm{H}$ & 3.47357800 & -1.71618900 & 1.07763300 \\
\hline $\mathrm{H}$ & 5.11797500 & -1.17206000 & 1.48019300 \\
\hline $\mathrm{H}$ & 4.56114600 & -0.06813800 & -2.12558300 \\
\hline $\mathrm{H}$ & 5.62773300 & -1.06146300 & -1.11268000 \\
\hline $\mathrm{H}$ & 3.96037600 & -1.59390700 & -1.42632700 \\
\hline $\mathrm{H}$ & 6.09701500 & 0.88596500 & 0.41026900 \\
\hline $\mathrm{H}$ & 5.07947600 & 1.89366300 & -0.64503800 \\
\hline $\mathrm{H}$ & 4.74140300 & 1.80896600 & 1.10007400 \\
\hline $\mathrm{C}$ & -2.89313900 & -1.74811800 & -0.17272500 \\
\hline $\mathrm{H}$ & -2.04082500 & -2.30478700 & 0.22390600 \\
\hline $\mathrm{H}$ & -3.79062500 & -2.31101400 & 0.10461900 \\
\hline $\mathrm{H}$ & -2.81340700 & -1.76934200 & -1.27537500 \\
\hline $\mathrm{C}$ & -0.86718700 & -0.52255900 & 1.68119500 \\
\hline $\mathrm{H}$ & -0.60709100 & -1.54595500 & 1.39257800 \\
\hline $\mathrm{H}$ & 0.07516400 & -0.00911300 & 1.91366300 \\
\hline $\mathrm{H}$ & -1.47524300 & -0.55994500 & 2.59255900 \\
\hline $\mathrm{C}$ & -1.80190600 & 1.67136200 & 1.07368800 \\
\hline $\mathrm{H}$ & -2.45128800 & 1.70442800 & 1.95725300 \\
\hline $\mathrm{H}$ & -0.82697500 & 2.07627400 & 1.36623800 \\
\hline $\mathrm{H}$ & -2.21642100 & 2.33723600 & 0.30739600 \\
\hline
\end{tabular}

\section{TS $\mathbf{I I}_{\mathbf{1}}-\mathbf{I}_{\mathbf{1}}$}<smiles>CC(C)N(CC(=O)OC1(C(C)C)CCN(C(C)C)C(C)C1)C(C)C</smiles>

$\mathrm{E}=-1086.774624$

$\begin{array}{llll}\text { GCorr. }= & 0.56314 & & \\ \mathrm{C} & -4.48661400 & 0.23573900 & 0.82282500 \\ \mathrm{~N} & -3.89313000 & -0.17282300 & -0.44696000 \\ \mathrm{C} & -4.31623500 & -1.43049300 & -1.04808300 \\ \mathrm{C} & -5.75741700 & -1.34993500 & -1.54573600 \\ \mathrm{C} & -4.12756100 & -2.66200700 & -0.15852600 \\ \mathrm{H} & -3.67461200 & -1.55914400 & -1.93526400 \\ \mathrm{H} & -5.88190600 & -0.50482100 & -2.23221900 \\ \mathrm{H} & -6.04502100 & -2.27146800 & -2.06745000 \\ \mathrm{H} & -6.46005600 & -1.21425200 & -0.71237300\end{array}$ $\begin{array}{lll}-3.08930800 & -2.76325400 & 0.17777800\end{array}$

$\begin{array}{lll}-4.76694700 & -2.60466200 & 0.73301000\end{array}$

$\begin{array}{llll}-4.40363800 & -3.57657000 & -0.69894300\end{array}$

$\begin{array}{llll}-2.58786500 & 0.33151500 & -0.80421400\end{array}$

$\begin{array}{llll}-1.36687800 & -0.52721600 & -0.43108000\end{array}$

$\begin{array}{lll}-1.35806500 & -0.71778900 & 0.65120900\end{array}$

$\begin{array}{llll}-1.44739700 & -1.50555900 & -0.93079200\end{array}$

$\begin{array}{llll}-0.07208900 & 0.12410800 & -0.83392700\end{array}$

$\begin{array}{llll}0.07308900 & 0.26713800 & -1.90981500\end{array}$

$\begin{array}{llll}0.36113800 & 1.26584300 & -0.03615800\end{array}$

$\begin{array}{lll}-0.01043800 & 1.50964900 & 1.10594900\end{array}$

$\begin{array}{llll}1.27581500 & 2.01418400 & -0.70440400\end{array}$

$\begin{array}{llll}1.84883000 & 3.22818100 & -0.13319500\end{array}$

$\begin{array}{llll}0.75523000 & 4.25001200 & 0.13649200\end{array}$

$\begin{array}{lll}2.65719800 & 2.91069300 & 1.11500000\end{array}$

$\begin{array}{llll}2.76650900 & 3.72290400 & -1.23916700\end{array}$

$\begin{array}{llll}0.15516600 & 4.40899900 & -0.76778400\end{array}$

$\begin{array}{llll}0.09664300 & 3.93060100 & 0.94794100\end{array}$

$\begin{array}{llll}1.21416600 & 5.20732300 & 0.41274500\end{array}$

$\begin{array}{llll}3.41523100 & 2.14788800 & 0.89422700\end{array}$

$\begin{array}{llll}3.17882500 & 3.81723200 & 1.44635500\end{array}$

$\begin{array}{llll}2.01976500 & 2.55621600 & 1.92933400\end{array}$

$\begin{array}{llll}3.26266700 & 4.64975400 & -0.92849200\end{array}$

$\begin{array}{llll}3.53708900 & 2.97652400 & -1.46702900\end{array}$

$\begin{array}{llll}2.19547100 & 3.92301600 & -2.15311800\end{array}$

$\begin{array}{llll}2.76885100 & -2.14776100 & -0.18791100\end{array}$

$\begin{array}{llll}3.10643600 & -2.03036300 & 1.23703100\end{array}$

$\begin{array}{lll}4.16939700 & -1.75684200 & 1.27846000\end{array}$

$\begin{array}{lll}1.42441000 & -2.14244700 & -0.55099800\end{array}$

$\begin{array}{llll}0.85139000 & -0.92032300 & -0.62154200\end{array}$

$3.74002300-1.69568700-1.18779900$

$\begin{array}{llll}5.02936200 & -2.50182000 & -1.11836800\end{array}$

$\begin{array}{llll}4.00283900 & -0.19467900 & -1.11838800\end{array}$

$\begin{array}{llll}3.26660900 & -1.91028300 & -2.15775100\end{array}$

$\begin{array}{llll}4.82361200 & -3.57645700 & -1.17759800\end{array}$

$\begin{array}{llll}5.68702500 & -2.22786400 & -1.95132500\end{array}$

$\begin{array}{llll}5.58425000 & -2.30878500 & -0.19141000\end{array}$

$\begin{array}{llll}3.06925200 & 0.37800000 & -1.19026600\end{array}$

$\begin{array}{llll}4.50221400 & 0.07608700 & -0.17717400\end{array}$

$\begin{array}{llll}4.66065000 & 0.11632400 & -1.93953800\end{array}$

$\begin{array}{lll}-5.35896300 & -0.41786800 & 0.97326200\end{array}$

$\begin{array}{llll}0.77076800 & -2.63976700 & 0.17438200\end{array}$

$\begin{array}{llll}1.25354600 & -2.50959600 & -1.56973200\end{array}$

$\begin{array}{llll}2.94594400 & -3.37700300 & 1.93154400\end{array}$

$\begin{array}{llll}3.56678600 & -4.14072200 & 1.44932800\end{array}$

$\begin{array}{lll}3.23625100 & -3.30523700 & 2.98648400\end{array}$

$\begin{array}{llll}1.90185200 & -3.71438100 & 1.89679200\end{array}$

$\begin{array}{lll}2.31928700 & -0.93674600 & 1.94971200\end{array}$

$\begin{array}{llll}2.42973900 & 0.03297600 & 1.45140600\end{array}$

$\begin{array}{lll}1.24732500 & -1.16866800 & 1.99101800\end{array}$

$\begin{array}{lll}2.67373200 & -0.83175400 & 2.98179700\end{array}$

$\begin{array}{llll}-3.57953300 & 0.06491300 & 2.04207600\end{array}$

$\begin{array}{lll}-2.68988300 & 0.70500700 & 1.97153200\end{array}$

$\begin{array}{lll}-4.11646100 & 0.34697000 & 2.95679800\end{array}$

$\begin{array}{lll}-3.24417900 & -0.97328400 & 2.15220100\end{array}$

$\begin{array}{lll}-5.01371500 & 1.66612100 & 0.74342700\end{array}$

$\begin{array}{llll}-5.72515200 & 1.76617700 & -0.08495400\end{array}$

$\begin{array}{lll}-5.51633600 & 1.95474700 & 1.67542400\end{array}$

$\begin{array}{lll}-4.19604400 & 2.38025500 & 0.57722400\end{array}$

$\begin{array}{llll}-2.46341100 & 1.32537300 & -0.35070800\end{array}$

$\begin{array}{llll}-2.55398400 & 0.49635900 & -1.89648400\end{array}$

\section{TS II $_{1}-\mathbf{I}_{2}$}

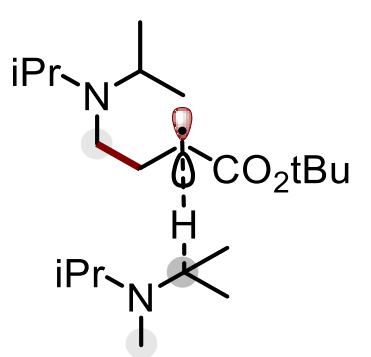


$\mathrm{E}=-1086.773119$

$$
\mathrm{G}_{\text {Corr. }}=0.563542
$$

$\begin{array}{llll}\text { C } & 4.91553700 & -2.10327000 & -0.22837900\end{array}$

$\begin{array}{llll}\mathrm{C} & 4.53119400 & -0.76743100 & 0.40208700\end{array}$

$\begin{array}{llll}\mathrm{H} & 5.46919100 & -0.23656500 & 0.62350400\end{array}$

$\mathrm{H} \quad 5.49732600 \quad-1.94127700 \quad-1.14356200$

$\mathrm{H} \quad \begin{array}{llll}5.51171600 & -2.71059100 & 0.46452100\end{array}$

$\mathrm{H} \quad 4.02345800 \quad-2.68718400 \quad-0.49174900$

$\begin{array}{llll}\text { C } & 3.81283300 & -0.98952600 & 1.73371400\end{array}$

$\mathrm{H} \quad 3.58773200 \quad-0.03978400 \quad 2.23343600$

$\mathrm{H} \quad 2.86908400-1.53342000 \quad 1.59270700$

$\mathrm{H} \quad 4.43972200 \quad-1.58722200 \quad 2.40764400$

$\begin{array}{llll}\mathrm{N} & 3.81149400 & 0.07101300 & -0.55280000\end{array}$

$\begin{array}{llll}\mathrm{N} & 3.81149400 & 0.07101300 & -0.55280000 \\ \mathrm{C} & 4.25290900 & 1.44513800 & -0.75337900\end{array}$

$\begin{array}{llll}\text { C } & 5.60523100 & 1.49417400 & -1.46023700\end{array}$

$\begin{array}{llll}\mathrm{C} & 4.28290800 & 2.30230100 & 0.51490000\end{array}$

$\mathrm{H} \quad 3.51354500 \quad 1.89061000 \quad-1.43911900$

$\mathrm{H} \quad \begin{array}{llll}5.56980200 & 0.93106800 & -2.39973800\end{array}$

H $\quad \begin{array}{llll}5.89537600 & 2.52896400 & -1.68161600\end{array}$

$\begin{array}{llll}\mathrm{H} & 6.39771500 & 1.05983300 & -0.83584000\end{array}$

$\begin{array}{llll}\mathrm{H} & 3.30857200 & 2.31910800 & 1.01733300\end{array}$

$\begin{array}{llll}\mathrm{H} & 5.02469100 & 1.92069500 & 1.22970600\end{array}$

$\begin{array}{lll}4.56274000 & 3.33644400 & 0.27645700\end{array}$

$\begin{array}{lll}2.43158400 & -0.23370800 & -0.84774800\end{array}$

$\begin{array}{lll}2.23378200 & -0.00780800 & -1.91111500\end{array}$

$\begin{array}{llll}2.28339800 & -1.31763600 & -0.74109000\end{array}$

$\begin{array}{llll}1.35297700 & 0.48708300 & -0.01664000\end{array}$

$\begin{array}{lll}1.51161100 & 0.28894900 & 1.05224000\end{array}$

$\begin{array}{lll}1.45237300 & 1.57303700 & -0.16485400\end{array}$

$\begin{array}{llll}-0.03373900 & 0.06573000 & -0.40781900\end{array}$

$\begin{array}{llll}-0.35348800 & 0.32873500 & -1.42177300\end{array}$

$\begin{array}{llll}-0.49163100 & -1.25217900 & 0.02606500\end{array}$

$\begin{array}{lll}0.03266600 & -1.92346500 & 0.90618100\end{array}$

$\begin{array}{llll}-1.62636700 & -1.60504600 & -0.62121700\end{array}$

$\begin{array}{llll}-2.37983000 & -2.80179700 & -0.26155000\end{array}$

$\begin{array}{llll}-1.55739400 & -4.04769700 & -0.54808800\end{array}$

$\begin{array}{lll}-2.83812200 & -2.72955100 & 1.18658500\end{array}$

$\begin{array}{llll}-3.58413000 & -2.73712100 & -1.18567700\end{array}$

$\begin{array}{llll}-1.21315400 & -4.04198800 & -1.58936400\end{array}$

$\begin{array}{lll}-0.68941900 & -4.11555100 & 0.11250800\end{array}$

$\begin{array}{llll}-2.18184300 & -4.93754700 & -0.40093800\end{array}$

$\begin{array}{llll}-3.38111800 & -1.79068000 & 1.36135300\end{array}$

$\begin{array}{lll}-3.52680600 & -3.55916500 & 1.38872200\end{array}$

$\begin{array}{lll}-1.99918300 & -2.79371200 & 1.88429200\end{array}$

$-4.22304700 \quad-3.61434700-1.03108600$

$-4.17523700 \quad-1.83478200 \quad-0.98362500$

$\begin{array}{lll}-3.26642400 & -2.71708400 & -2.23501400\end{array}$

$\begin{array}{lll}-2.97530900 & 1.49945000 & 0.34139900\end{array}$

$\begin{array}{lll}-4.21832700 & 1.41070700 & 1.07754500\end{array}$

$\begin{array}{lll}-5.03107400 & 1.12423700 & 0.40076200\end{array}$

$\begin{array}{lll}-4.16379100 & 0.63932300 & 1.85232700\end{array}$

$\begin{array}{lll}-4.50415000 & 2.36086300 & 1.56704800\end{array}$

$\begin{array}{lll}-1.74677300 & 1.62610200 & 1.03858800\end{array}$

$\begin{array}{lll}-1.70155200 & 0.92914600 & 2.37415700\end{array}$

$\begin{array}{lll}-1.10175500 & 2.99965800 & 1.07758700\end{array}$

$\begin{array}{lll}-0.89930600 & 0.89367500 & 0.31732000\end{array}$

$\begin{array}{lll}-2.06046000 & -0.10552500 & 2.30775100\end{array}$

$\begin{array}{lll}-0.66616700 & 0.89817800 & 2.73376900\end{array}$

$\begin{array}{lll}-2.29726900 & 1.45413400 & 3.13676800\end{array}$

$\begin{array}{lll}-0.97530000 & 3.44292000 & 0.08320800\end{array}$

$\begin{array}{lll}-1.71873600 & 3.68927200 & 1.67780800\end{array}$

$\begin{array}{lll}-0.11131400 & 2.94364200 & 1.54623400\end{array}$

$\begin{array}{lll}-3.04548500 & 2.05256700 & -1.01321500\end{array}$

$\begin{array}{llll}-3.78506500 & 3.38585200 & -1.08887800\end{array}$

$\begin{array}{llll}-3.62635100 & 1.03610300 & -1.98865700\end{array}$

$\begin{array}{llll}-2.00308500 & 2.22503600 & -1.31768600\end{array}$

$\begin{array}{llll}-3.38426100 & 4.11230900 & -0.37174700\end{array}$

$\begin{array}{llll}-3.69715300 & 3.81328400 & -2.09473200\end{array}$

$\begin{array}{llll}-4.85539600 & 3.25950400 & -0.88018300\end{array}$

$\begin{array}{llll}-3.04082300 & 0.10866900 & -1.96617700\end{array}$

$\begin{array}{llll}-4.66888800 & 0.79207700 & -1.74277500\end{array}$

$\begin{array}{llll}-3.61873200 & 1.43845800 & -3.00931000\end{array}$

\section{TS $\mathbf{I I}_{2}-\mathbf{I}_{1}$}<smiles>CC(C)OC(=O)CC(C)(C)N(C)C(C)(C)CN(CI)C(C)C</smiles>

$E=-1086.773998$

$\mathrm{G}_{\text {Corr. }}=0.563736$

C

(1)

(n)

(1)

年

(n)

,

O

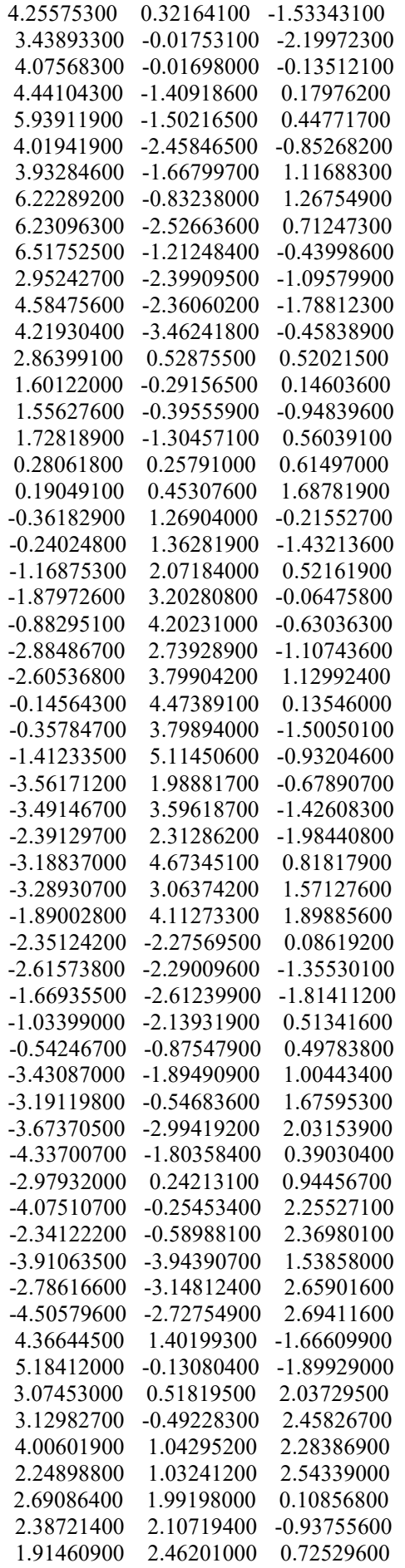




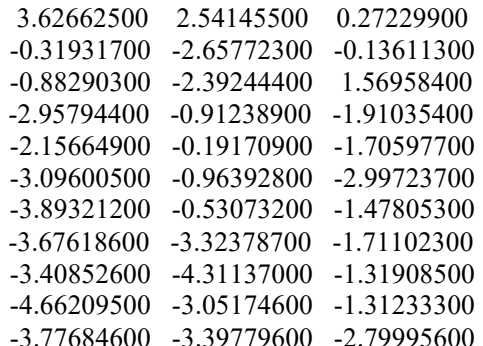

TS $\mathbf{I I}_{2}-\mathbf{I}_{2}$<smiles>CC(C)N(C)C(C)(C)CC(=O)CC(C)(C)N(C)C(C)C</smiles>

$\mathrm{E}=-1086.773233$

$$
\mathrm{G}_{\text {Corr. }}=0.564554
$$

C $\quad 4.27937600 \quad 1.12658600 \quad-1.10834700$

$\mathrm{H} \quad 3.60752700 \quad 0.85698900-1.94623300$

$\begin{array}{llll}\mathrm{N} & 3.97226700 & 0.47163400 & 0.14841200\end{array}$

$\begin{array}{llll}\mathrm{C} & 4.48002900 & -0.90970700 & 0.22537900\end{array}$

$\begin{array}{llll}\text { C } & 5.93333600 & -0.90121100 & 0.68634800\end{array}$

$\begin{array}{llll}\mathrm{C} & 4.33128700 & -1.74732900 & -1.04833800\end{array}$

$\mathrm{H} \quad 3.90143700 \quad-1.41853300 \quad 1.00635400$

$\begin{array}{llll}\mathrm{H} & 6.02360400 & -0.39747800 & 1.65556400\end{array}$

H $\quad 6.32348800 \quad-1.92259200 \quad 0.78229600$

$\mathrm{H} \quad \begin{array}{llll}6.57270400 & -0.36937200 & -0.03108000\end{array}$

$\begin{array}{llll}3.30211300 & -1.75666000 & -1.42671800\end{array}$

$\begin{array}{lll}4.98264200 & -1.38457600 & -1.85352600\end{array}$

$\begin{array}{lll}4.62184200 & -2.78455500 & -0.84081300\end{array}$

$\begin{array}{llll}2.62498700 & 0.72550000 & 0.70913300\end{array}$

$\begin{array}{lll}1.55088600 & -0.13515700 & -0.00781900\end{array}$

$\begin{array}{lll}1.65046300 & 0.01528800 & -1.09275100\end{array}$

$\begin{array}{lll}1.77979000 & -1.19241500 & 0.19657300\end{array}$

$\begin{array}{lll}0.11915900 & 0.12768600 & 0.37086500\end{array}$

$\begin{array}{lll}-0.13220900 & 0.04481500 & 1.43275900\end{array}$

$\begin{array}{llll}-0.58819200 & 1.20314400 & -0.32361600\end{array}$

$\begin{array}{lll}-0.37059900 & 1.54073000 & -1.47993100\end{array}$

$\begin{array}{lll}-1.55646700 & 1.74418800 & 0.45033300\end{array}$

$\begin{array}{lll}-2.49367500 & 2.72789100 & -0.08712900\end{array}$

$\begin{array}{llll}-1.75303800 & 3.97792100 & -0.53767700\end{array}$

$\begin{array}{llll}-3.31837700 & 2.09715600 & -1.19840000\end{array}$

$\begin{array}{lll}-3.37645000 & 3.05340400 & 1.10628900\end{array}$

$\begin{array}{lll}-1.09672000 & 4.33483300 & 0.26643000\end{array}$

$\begin{array}{lll}-1.14947800 & 3.79361600 & -1.42912700\end{array}$

$\begin{array}{llll}-2.48003300 & 4.76905000 & -0.75920500\end{array}$

$\begin{array}{llll}-3.76383600 & 1.15702500 & -0.84597500\end{array}$

$\begin{array}{lll}-4.12993700 & 2.77843600 & -1.48242800\end{array}$

$\begin{array}{llll}-2.71060300 & 1.88914100 & -2.08351300\end{array}$

$\begin{array}{lll}-4.11803900 & 3.81011600 & 0.82528600\end{array}$

$\begin{array}{lll}-3.90979300 & 2.16188900 & 1.45627400\end{array}$

$\begin{array}{llll}-2.77481900 & 3.44729600 & 1.93368800\end{array}$

$-2.52226000-1.82761000 \quad-0.30799000$

$\begin{array}{llll}-3.61600500 & -2.12892300 & -1.21019100\end{array}$

$-4.56519300 \quad-1.82799800 \quad-0.75394800$

$\begin{array}{llll}-3.52102400 & -1.56786000 & -2.14501800\end{array}$

$\begin{array}{lll}-3.68812300 & -3.20351200 & -1.46273600\end{array}$

$-1.18569700 \quad-1.98949000 \quad-0.76186300$

$-0.97947000 \quad-1.68448200 \quad-2.22346400$

$-0.43805800 \quad-3.23339900 \quad-0.31731900$

$\begin{array}{lll}-0.54241600 & -0.97805400 & -0.16105700\end{array}$

$\begin{array}{llll}-1.42418900 & -0.72277900 & -2.50537900\end{array}$

$\begin{array}{llll}0.09477900 & -1.62599000 & -2.43683000\end{array}$

$\begin{array}{llll}-1.39772600 & -2.47212600 & -2.86964600\end{array}$

$\begin{array}{llll}-0.42139300 & -3.36226000 & 0.77092200\end{array}$

$\begin{array}{lll}-0.90666600 & -4.13070500 & -0.75568700\end{array}$

$\begin{array}{llll}0.60172400 & -3.20025900 & -0.66591400\end{array}$

$\begin{array}{llll}-2.77734100 & -2.03690000 & 1.12081700\end{array}$

$\begin{array}{lll}-3.38980300 & -3.39751800 & 1.44435000\end{array}$

$\begin{array}{lll}-3.61398900 & -0.89884200 & 1.69048500\end{array}$

$\begin{array}{lll}-1.79395200 & -1.98155000 & 1.60964400\end{array}$

$\begin{array}{llll}-2.80907200 & -4.21988600 & 1.00989700\end{array}$

$\begin{array}{lll}-3.43146100 & -3.54534800 & 2.52996600\end{array}$

$\begin{array}{lll}-4.41730700 & -3.47214500 & 1.06543000\end{array}$

$\begin{array}{lll}-3.09937300 & 0.05690900 & 1.53507100\end{array}$

$\begin{array}{lll}-4.60157700 & -0.84345900 & 1.21222600\end{array}$

$\begin{array}{lll}-3.77798800 & -1.04181400 & 2.76580000\end{array}$

$\begin{array}{llll}4.25761700 & 2.21508500 & -1.00064400\end{array}$

$\begin{array}{llll}5.29953300 & 0.86690700 & -1.41196100\end{array}$

$\begin{array}{llll}2.64523000 & 0.41497100 & 2.20856700\end{array}$

$\begin{array}{lll}2.79016800 & -0.64944900 & 2.42630600\end{array}$

$\begin{array}{llll}3.45586000 & 0.97580300 & 2.69019500\end{array}$

$\begin{array}{lll}1.69943600 & 0.71324100 & 2.67619500\end{array}$

$\begin{array}{llll}2.29259500 & 2.21187800 & 0.56671500\end{array}$

$\begin{array}{llll}2.11441500 & 2.51060500 & -0.47207000\end{array}$

$\begin{array}{lll}1.38224700 & 2.44638600 & 1.13256300\end{array}$

$\begin{array}{lll}3.10727900 & 2.82135600 & 0.97793300\end{array}$

\section{TS RS·-I}<smiles>CC(C)[SiH2]SOCCN(C(C)C)C(C)C</smiles>

$\mathrm{E}=-1374.694918$

$\mathrm{G}_{\text {Corr. }}=0.447899$

$\begin{array}{lccc}\mathrm{N} & -3.05684200 & 0.15635300 & -0.33327500 \\ \mathrm{C} & -1.93609700 & 0.81464000 & -0.77744200 \\ \mathrm{H} & -1.47182900 & 0.38832100 & -1.66911900 \\ \mathrm{H} & -1.97425300 & 1.90454500 & -0.74576100 \\ \mathrm{C} & -3.95674400 & 0.81080500 & 0.62541600 \\ \mathrm{C} & -4.80137500 & 1.87090100 & -0.07034500 \\ \mathrm{C} & -3.21516500 & 1.38074300 & 1.82865500 \\ \mathrm{H} & -4.63214600 & 0.02548700 & 0.98999600 \\ \mathrm{H} & -5.36546500 & 1.43826700 & -0.90445300 \\ \mathrm{H} & -5.51089200 & 2.31862100 & 0.63517200 \\ \mathrm{H} & -4.17124500 & 2.67749500 & -0.46603900 \\ \mathrm{H} & -2.60147000 & 0.61822700 & 2.32140800 \\ \mathrm{H} & -2.55469300 & 2.20755400 & 1.53831600 \\ \mathrm{H} & -3.93501500 & 1.77119300 & 2.55713500 \\ \mathrm{C} & -3.14754500 & -1.29465500 & -0.52911600 \\ \mathrm{C} & -4.50863600 & -1.69817800 & -1.07821600 \\ \mathrm{C} & -2.78347900 & -2.07041000 & 0.73188200 \\ \mathrm{H} & -2.39449300 & -1.52388700 & -1.29774200 \\ \mathrm{H} & -4.74272900 & -1.14459800 & -1.99434300 \\ \mathrm{H} & -4.51423000 & -2.76933600 & -1.30991000 \\ \mathrm{H} & -5.31204200 & -1.51886700 & -0.35239200 \\ \mathrm{H} & -1.79641100 & -1.77167700 & 1.10754200 \\ \mathrm{H} & -3.52055400 & -1.90650400 & 1.52906500 \\ \mathrm{H} & -2.76302400 & -3.14596100 & 0.51828500 \\ \mathrm{Si} & 1.97288500 & 0.08648900 & -0.01319600 \\ \mathrm{~S} & 0.32064100 & 0.37528300 & 1.35338400 \\ \mathrm{H} & -0.71296000 & 0.57872900 & 0.29266700 \\ \mathrm{C} & 1.73462200 & 1.27026900 & -1.48904200 \\ \mathrm{C} & 2.84481700 & 1.13276500 & -2.53212200 \\ \mathrm{C} & 1.58736000 & 2.72916100 & -1.06220000 \\ \mathrm{H} & 0.78653100 & 0.95890800 & -1.95997600 \\ \mathrm{H} & 2.98071100 & 0.09943800 & -2.87776900 \\ \mathrm{H} & 2.63095500 & 1.75016600 & -3.41622500 \\ \mathrm{H} & 3.80774700 & 1.47819800 & -2.12927500 \\ \mathrm{H} & 0.76968900 & 2.87110000 & -0.34487800 \\ \mathrm{H} & 2.50735500 & 3.09912100 & -0.58706200\end{array}$




\begin{abstract}
$\begin{array}{lll}1.39406200 & 3.37469000 & -1.93112900\end{array}$
$\begin{array}{lll}3.58319700 & 0.50003400 & 0.92289600\end{array}$

$\begin{array}{lll}4.80600200 & -0.16764100 & 0.28792300\end{array}$

$\begin{array}{lll}3.53582400 & 0.19746300 & 2.42035600\end{array}$

$\begin{array}{llll}3.69365200 & 1.59264100 & 0.80785400\end{array}$

$\begin{array}{lll}4.90000300 & 0.03956800 & -0.78552100\end{array}$

$\begin{array}{llll}5.73122700 & 0.17653600 & 0.77146700\end{array}$

$\begin{array}{lll}4.76926900 & -1.25945100 & 0.40812800\end{array}$

$\begin{array}{lll}2.72331200 & 0.73332200 & 2.92466000\end{array}$

$\begin{array}{lll}3.38865700 & -0.87492500 & 2.61111200\end{array}$

$\begin{array}{lll}4.48031500 & 0.48594000 & 2.90399500\end{array}$

$\begin{array}{llll}1.98435400 & -1.71021200 & -0.64598600\end{array}$

$\begin{array}{lll}0.75391500 & -2.02119500 & -1.49585300\end{array}$

$\begin{array}{lll}2.10457900 & -2.72306700 & 0.49160800\end{array}$

$\begin{array}{lll}2.87918700 & -1.80045800 & -1.28615600\end{array}$

$\begin{array}{lll}0.66511600 & -1.36540600 & -2.37221600\end{array}$

$\begin{array}{lll}0.77805200 & -3.05792900 & -1.86112300\end{array}$

$-0.16443600 \quad-1.90995500 \quad-0.89914900$

$\begin{array}{lll}3.02157500 & -2.58979800 & 1.07992300\end{array}$

$\begin{array}{lll}1.25404800 & -2.63569700 & 1.18287700\end{array}$

$\begin{array}{lll}2.10990600 & -3.75200300 & 0.10367900\end{array}$
\end{abstract}

\section{TS RS·-I $\mathbf{I}_{2}$}<smiles>CC(C)N(C)C(C)(C)CS[SnH3]</smiles>

$$
\mathrm{E}=-1374.692377
$$

$$
\mathrm{G}_{\text {Corr. }}=0.446749
$$

$\begin{array}{llll}\mathrm{N} & -3.33876800 & 0.25074100 & 0.27887700\end{array}$

$\begin{array}{llll}\mathrm{C} & -3.33876800 & 0.25074100 & 0.27887700 \\ \mathrm{H} & -3.11074200 & -0.01073000 & 1.69166000\end{array}$

$\mathrm{H} \quad-3.23848900 \quad 0.89577700 \quad 2.29354700$

$\mathrm{H} \quad-3.82950400 \quad-0.74577700 \quad 2.05824900$

$\begin{array}{llll}\mathrm{C} & -4.14291500 & -0.74204700 & -0.45188300\end{array}$

$\begin{array}{llll}\text { C } & -5.54728300 & -0.84822000 & 0.13125900\end{array}$

C $\quad-3.47288200 \quad-2.10829800 \quad-0.54120100$

$\mathrm{H} \quad-4.25923100 \quad-0.34798800 \quad-1.46684600$

$\mathrm{H} \quad \begin{array}{llll}\mathrm{H} & -5.99963000 & 0.14198800 & 0.25363200\end{array}$

$\mathrm{H} \quad-6.17837600 \quad-1.43479200 \quad-0.54609900$

$\mathrm{H} \quad-\quad-5.56121100-1.35824000 \quad 1.10225100$

$\mathrm{H} \quad-2.50269300 \quad-2.04959900 \quad-1.04740900$

$\mathrm{H} \quad-3.30419600 \quad-2.53317400 \quad 0.45710200$

$-4.11230900-2.80542800 \quad-1.09613200$

$\begin{array}{lll}-2.39595900 & 1.05219400 & -0.37234900\end{array}$

$\begin{array}{lll}-2.00837100 & 2.31422600 & 0.35448500\end{array}$

$\begin{array}{lll}-2.54063700 & 1.22625600 & -1.85771300\end{array}$

$\begin{array}{llll}-1.05315800 & 0.20253100 & -0.33742900\end{array}$

$\begin{array}{lll}-1.69449500 & 2.14936300 & 1.38916000\end{array}$

$\begin{array}{llll}-1.17928400 & 2.80686600 & -0.16427200\end{array}$

$\begin{array}{lll}-2.85818000 & 3.01779700 & 0.37146500\end{array}$

$\begin{array}{llll}-2.47219700 & 0.28100900 & -2.40945500\end{array}$

$\begin{array}{llll}-3.49437700 & 1.71148600 & -2.12327400\end{array}$

$\begin{array}{llll}-1.73409000 & 1.87144000 & -2.22218500\end{array}$

$\begin{array}{lll}1.92717400 & -0.18453800 & 0.00243900\end{array}$

$\begin{array}{lll}-0.04225400 & -0.93264500 & -0.49895500\end{array}$

$\begin{array}{lll}-2.09875600 & -0.41114400 & 1.86838900\end{array}$

$\begin{array}{lll}3.04270700 & -1.70165500 & -0.30043700\end{array}$

$\begin{array}{lll}4.50227400 & -1.43441200 & 0.07162800\end{array}$

$\begin{array}{lll}2.95608900 & -2.23626000 & -1.72879100\end{array}$

$\begin{array}{lll}2.64870600 & -2.47928400 & 0.37578200\end{array}$

$\begin{array}{lll}4.62356500 & -1.04877500 & 1.09246400\end{array}$

$\begin{array}{llll}5.09890300 & -2.35458700 & -0.00488600\end{array}$

$\begin{array}{lll}4.95584200 & -0.70500400 & -0.61493700\end{array}$

$\begin{array}{llll}1.93046800 & -2.50181000 & -2.01086100\end{array}$

$\begin{array}{llll}3.31834800 & -1.49481400 & -2.45573100\end{array}$

$3.58153400 \quad-3.13263000 \quad-1.84828800$

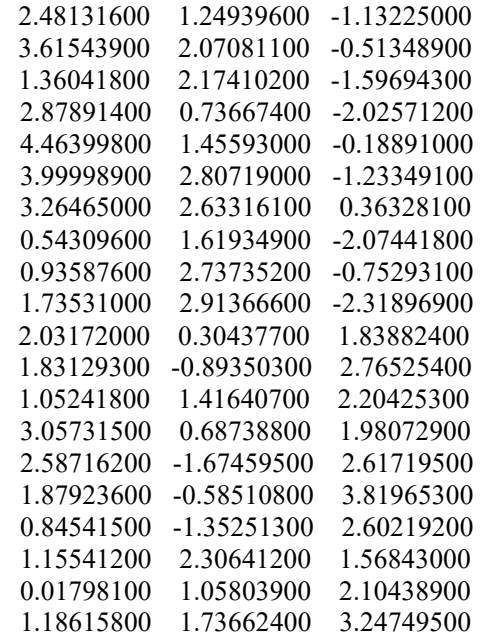

\section{TS $\mathbf{I I}_{\mathbf{1}}-\mathbf{P}_{\mathbf{1}}(\mathbf{R S H})$}

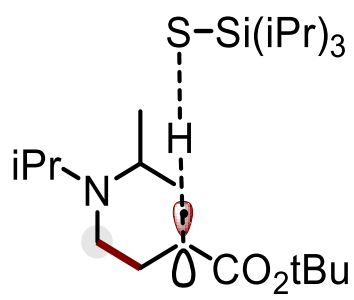

$$
\mathrm{E}=-1798.987995
$$

$$
\mathrm{G}_{\text {Corr. }}=0.61622
$$

$\begin{array}{llll}4.93797400 & -0.90043600 & -0.54300600\end{array}$

$\begin{array}{llll}5.40306100 & -1.85937100 & -0.81386300\end{array}$

$\begin{array}{llll}3.89383000 & -1.22416600 & 0.42766000\end{array}$

$\begin{array}{llll}3.51128500 & -2.61889500 & 0.62273600\end{array}$

$\begin{array}{lll}4.62857100 & -3.40641000 & 1.30084500\end{array}$

$\begin{array}{llll}3.04546300 & -3.32874300 & -0.64986100\end{array}$

$\begin{array}{llll}2.65954900 & -2.59318200 & 1.32237200\end{array}$

$\begin{array}{lll}4.92144500 & -2.92987600 & 2.24309300\end{array}$

$\begin{array}{llll}4.30768000 & -4.43382500 & 1.51205000\end{array}$

$\begin{array}{llll}5.51872100 & -3.46695200 & 0.66055500\end{array}$

$\begin{array}{llll}2.22941200 & -2.78809700 & -1.14342000\end{array}$

$3.86848200 \quad-3.42970100 \quad-1.36995400$

$\begin{array}{llll}2.68949800 & -4.34015200 & -0.41600500\end{array}$

$\begin{array}{llll}2.94887900 & -0.21620800 & 0.80711700\end{array}$

$\begin{array}{llll}1.61166200 & -0.21521500 & 0.00190900\end{array}$

$\begin{array}{lll}1.82632900 & -0.10178200 & -1.06810100\end{array}$

$\begin{array}{llll}1.12750500 & -1.18929600 & 0.15172200\end{array}$

$\begin{array}{llll}0.70750800 & 0.86673100 & 0.45285300\end{array}$

$\begin{array}{lll}0.29448300 & 0.81061000 & 1.46468100\end{array}$

$\begin{array}{llll}0.93711500 & 2.23654800 & -0.04286900\end{array}$

$\begin{array}{lll}1.73318500 & 2.51544800 & -0.92329300\end{array}$

$\begin{array}{llll}0.11389200 & 3.09634100 & 0.56718700\end{array}$

$\begin{array}{lll}-0.05390900 & 4.47853100 & 0.10361900\end{array}$

$\begin{array}{lll}1.22484500 & 5.26234500 & 0.34040500\end{array}$

$\begin{array}{llll}-0.48644800 & 4.48729700 & -1.35293200\end{array}$

$\begin{array}{llll}-1.17420600 & 4.98810400 & 0.99208700\end{array}$

$\begin{array}{llll}1.52919200 & 5.18702600 & 1.39113000\end{array}$

$\begin{array}{llll}2.03953800 & 4.90325400 & -0.29391700\end{array}$

$\begin{array}{llll}1.04607800 & 6.32030300 & 0.11308700\end{array}$

$\begin{array}{llll}-1.36046600 & 3.83889500 & -1.49284200\end{array}$

$\begin{array}{llll}-0.76960800 & 5.50877200 & -1.63414300\end{array}$

$\begin{array}{llll}0.31316300 & 4.15315700 & -2.01911800\end{array}$

$\begin{array}{llll}-1.40313700 & 6.03091000 & 0.74485500\end{array}$

$\begin{array}{llll}-2.08011900 & 4.38700600 & 0.84701800\end{array}$

$\begin{array}{llll}-0.88508100 & 4.93546700 & 2.04802500\end{array}$

$\begin{array}{llll}-0.55934600 & 0.62275200 & -0.29508600\end{array}$

$\begin{array}{llll}2.68732900 & -0.33683300 & 1.87151800\end{array}$

$\begin{array}{lll}3.41484300 & 0.77334100 & 0.70925600\end{array}$

$\begin{array}{lll}4.42931500 & -0.26151900 & -1.83445600\end{array}$ 


\begin{tabular}{lrrr}
$\mathrm{H}$ & 5.26481900 & -0.09331900 & -2.52505000 \\
$\mathrm{H}$ & 3.69596300 & -0.90020400 & -2.34055900 \\
$\mathrm{H}$ & 3.96050400 & 0.71304200 & -1.64306600 \\
$\mathrm{C}$ & 6.02348300 & -0.03970100 & 0.09642600 \\
$\mathrm{H}$ & 6.84340000 & 0.14450200 & -0.60877600 \\
$\mathrm{H}$ & 5.62686900 & 0.93816000 & 0.40012000 \\
$\mathrm{H}$ & 6.43045500 & -0.53331200 & 0.98669600 \\
$\mathrm{~S}$ & -1.81782800 & 0.53819700 & -1.13801900 \\
$\mathrm{Si}$ & -2.78127200 & -1.05195500 & 0.00151500 \\
$\mathrm{C}$ & -2.10717800 & -0.96953500 & 1.78104200 \\
$\mathrm{C}$ & -4.65251300 & -0.69681200 & -0.04430900 \\
$\mathrm{C}$ & -2.36010800 & -2.73452100 & -0.77781700 \\
$\mathrm{C}$ & -2.59933200 & -2.12972300 & 2.64812100 \\
$\mathrm{C}$ & -2.40426000 & 0.36369600 & 2.46628600 \\
$\mathrm{H}$ & -1.01220900 & -1.06916000 & 1.67796600 \\
$\mathrm{C}$ & -5.47701600 & -1.94535600 & 0.28011100 \\
$\mathrm{C}$ & -5.14297200 & -0.05945600 & -1.34438700 \\
$\mathrm{H}$ & -4.80787100 & 0.03720100 & 0.76522200 \\
$\mathrm{C}$ & -0.86472000 & -3.03816900 & -0.70874200 \\
$\mathrm{C}$ & -2.85036800 & -2.84318300 & -2.22128700 \\
$\mathrm{H}$ & -2.89632300 & -3.48580800 & -0.17194600 \\
$\mathrm{H}$ & -2.39689200 & -3.11232100 & 2.20318100 \\
$\mathrm{H}$ & -2.11629100 & -2.10906000 & 3.63511600 \\
$\mathrm{H}$ & -3.68199700 & -2.05939400 & 2.82374000 \\
$\mathrm{H}$ & -2.07943500 & 1.22893700 & 1.87364500 \\
$\mathrm{H}$ & -3.48176400 & 0.47889300 & 2.64978000 \\
$\mathrm{H}$ & -1.90588800 & 0.41947300 & 3.44479800 \\
$\mathrm{H}$ & -5.17369600 & -2.43010800 & 1.21665600 \\
$\mathrm{H}$ & -6.54244000 & -1.69207200 & 0.37200600 \\
$\mathrm{H}$ & -5.39436200 & -2.69553600 & -0.51849800 \\
$\mathrm{H}$ & -4.63990300 & 0.89185400 & -1.55391100 \\
$\mathrm{H}$ & -4.97564600 & -0.71841200 & -2.20748600 \\
$\mathrm{H}$ & -6.22303500 & 0.13855900 & -1.29328300 \\
$\mathrm{H}$ & -0.47228700 & -3.03155800 & 0.31825300 \\
$\mathrm{H}$ & -0.64290900 & -4.02643800 & -1.13646000 \\
$\mathrm{H}$ & -0.29457100 & -2.29876200 & -1.29173000 \\
$\mathrm{H}$ & -3.93993200 & -2.74522000 & -2.30473300 \\
$\mathrm{H}$ & -2.39741000 & -2.06318400 & -2.84959300 \\
& -2.57449900 & -3.81434500 & -2.65600900 \\
\hline
\end{tabular}

\section{TS II $-\mathbf{P}_{2}$ (RSH)}<smiles>CCOC(=O)CC(C)(C)N(C)C(C)C</smiles>

$$
\mathrm{E}=-1798.98948
$$

$$
\mathrm{G}_{\text {Corr. }}=0.616775
$$

$\begin{array}{lccc}\mathrm{C} & 4.92057300 & -0.91682800 & -1.05108100 \\ \mathrm{H} & 4.34834200 & -0.64760300 & -1.95862700 \\ \mathrm{~N} & 4.10024800 & -1.29549000 & 0.08319500 \\ \mathrm{C} & 3.61967900 & -2.68842400 & 0.03245800 \\ \mathrm{C} & 4.67928800 & -3.62367300 & 0.60303600 \\ \mathrm{C} & 3.16172700 & -3.17156400 & -1.34550900 \\ \mathrm{H} & 2.74719600 & -2.74939400 & 0.69507600 \\ \mathrm{H} & 4.92827800 & -3.34091700 & 1.63212700 \\ \mathrm{H} & 4.32767300 & -4.66296100 & 0.59870300 \\ \mathrm{H} & 5.60176600 & -3.58374900 & 0.00862700 \\ \mathrm{H} & 2.43501600 & -2.49376400 & -1.80832900 \\ \mathrm{H} & 4.00436000 & -3.28742900 & -2.03831600 \\ \mathrm{H} & 2.68447600 & -4.15421400 & -1.24661800 \\ \mathrm{C} & 3.18144900 & -0.28168700 & 0.61388200 \\ \mathrm{C} & 1.89144400 & -0.20176600 & -0.27494400 \\ \mathrm{H} & 2.18436300 & -0.02211300 & -1.31878200 \\ \mathrm{H} & 1.40877500 & -1.18749700 & -0.22250500 \\ \mathrm{C} & 0.91179800 & 0.83283700 & 0.13730000 \\ \mathrm{H} & 0.51592800 & 0.79812800 & 1.15538800 \\ \mathrm{C} & 0.97563600 & 2.18882700 & -0.44365500\end{array}$

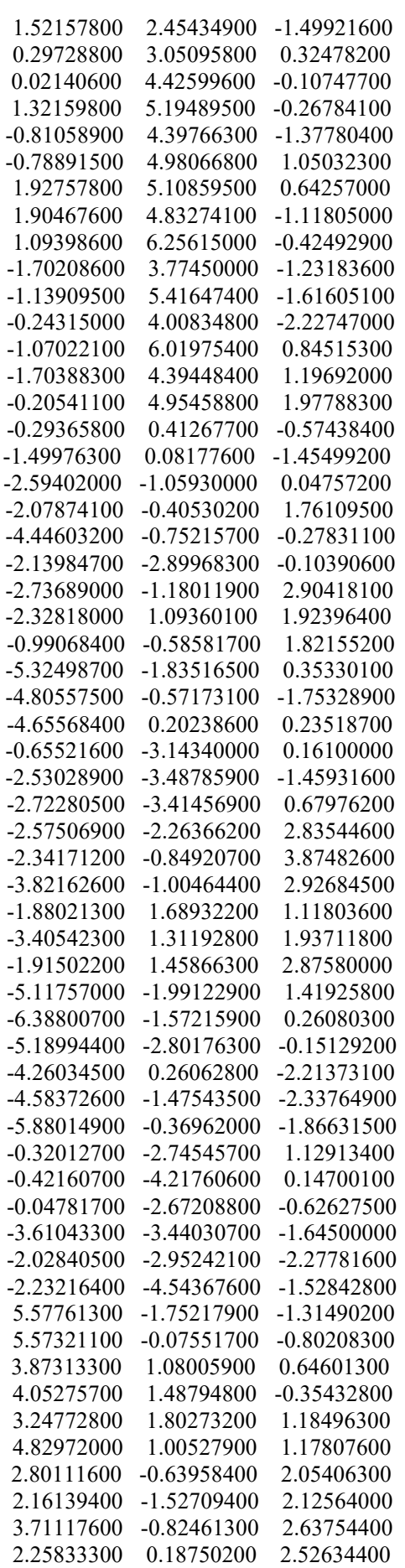

$\mathbf{P}_{1}$

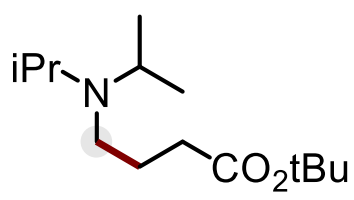

$\mathrm{E}=-755.8761941$

$\mathrm{G}_{\text {Corr. }}=0.363292$

$\begin{array}{llll}\text { C } & 4.16591500 & -0.91489000 & 1.60857500\end{array}$

C $\quad 3.58062900 \quad-1.06806100 \quad 0.20384300$

$\begin{array}{llll}\mathrm{H} & 3.02840400 & -2.02122400 & 0.18571800\end{array}$

$\mathrm{H} \quad 3.37768700 \quad-0.83661300 \quad 2.36789000$ 


$\begin{array}{lrrr}\mathrm{H} & 4.78930100 & -1.78189900 & 1.86053800 \\ \mathrm{H} & 4.80164200 & -0.02227100 & 1.67991000 \\ \mathrm{C} & 4.68662000 & -1.18120600 & -0.83747900 \\ \mathrm{H} & 4.25996900 & -1.34477700 & -1.83383600 \\ \mathrm{H} & 5.30131300 & -0.27216600 & -0.87655100 \\ \mathrm{H} & 5.35805200 & -2.01581300 & -0.60073000 \\ \mathrm{~N} & 2.59804100 & -0.05132200 & -0.17198700 \\ \mathrm{C} & 3.00938500 & 1.35287900 & -0.11383600 \\ \mathrm{C} & 2.69089500 & 2.08252700 & 1.19475900 \\ \mathrm{C} & 2.42487400 & 2.11073600 & -1.30044600 \\ \mathrm{H} & 4.10341200 & 1.35382300 & -0.22980900 \\ \mathrm{H} & 3.05025200 & 1.53303400 & 2.07157200 \\ \mathrm{H} & 3.16429000 & 3.07232900 & 1.19777200 \\ \mathrm{H} & 1.61158300 & 2.24310200 & 1.31446000 \\ \mathrm{H} & 2.75045000 & 1.65592500 & -2.24325500 \\ \mathrm{H} & 1.32618000 & 2.08442400 & -1.27661600 \\ \mathrm{H} & 2.73218200 & 3.16424600 & -1.29106700 \\ \mathrm{C} & 1.23687500 & -0.34774800 & 0.23051300 \\ \mathrm{H} & 1.19761800 & -0.81459600 & 1.23760900 \\ \mathrm{H} & 0.67075800 & 0.58805700 & 0.31410900 \\ \mathrm{C} & 0.51990300 & -1.24524200 & -0.77221700 \\ \mathrm{H} & 0.49565200 & -0.72947800 & -1.74195600 \\ \mathrm{H} & 1.09790400 & -2.16812700 & -0.92034100 \\ \mathrm{C} & -1.86580700 & -0.46469400 & -0.39534800 \\ \mathrm{O} & -1.64733100 & 0.60767500 & -0.92047300 \\ \mathrm{O} & -3.01715500 & -0.80918700 & 0.19400200 \\ \mathrm{C} & -4.17210100 & 0.09732200 & 0.24203900 \\ \mathrm{C} & -3.82420400 & 1.34373100 & 1.03740800 \\ \mathrm{C} & -4.64415300 & 0.41656200 & -1.16606400 \\ \mathrm{C} & -5.21355900 & -0.72755900 & 0.97681200 \\ \mathrm{H} & -3.42664600 & 1.06875600 & 2.02165200 \\ \mathrm{H} & -3.09169200 & 1.96615800 & 0.51789000 \\ \mathrm{H} & -4.73499000 & 1.93451800 & 1.19295900 \\ \mathrm{H} & -4.82350600 & -0.50880400 & -1.72644100 \\ \mathrm{H} & -5.59111200 & 0.96683100 & -1.10948900 \\ \mathrm{H} & -3.91944900 & 1.02789100 & -1.70948200 \\ \mathrm{H} & -6.14043900 & -0.15179400 & 1.07913800 \\ \mathrm{H} & -5.43553100 & -1.64919800 & 0.42694300 \\ \mathrm{C} & -4.85846200 & -0.99580700 & 1.97832500 \\ \mathrm{H} & -0.88837400 & -1.61224000 & -0.33837000 \\ -1.30497100 & -2.40582800 & -0.97385500 \\ \mathrm{H} & -0.90162500 & -2.02204500 & 0.68149800\end{array}$

$\mathbf{P}_{2}$<smiles>CCCOC(=O)CCC(C)(C)N(C)C(C)C</smiles>

$$
E=-755.8729926
$$

$\mathrm{G}_{\text {Corr. }}=0.363508$

$\begin{array}{lrrr}\mathrm{C} & 4.88302500 & 0.38055000 & 0.68295400 \\ \mathrm{C} & 3.75984200 & -0.52063300 & 0.16372000 \\ \mathrm{H} & 3.31958300 & -1.00630000 & 1.04326300 \\ \mathrm{H} & 4.50259400 & 1.21475500 & 1.28294700 \\ \mathrm{H} & 5.56163200 & -0.20559300 & 1.31456600 \\ \mathrm{H} & 5.48368700 & 0.79814000 & -0.13490100 \\ \mathrm{C} & 4.32834200 & -1.62741300 & -0.71667500 \\ \mathrm{H} & 3.54204400 & -2.33905400 & -0.99319100 \\ \mathrm{H} & 4.75103000 & -1.21613800 & -1.64320900 \\ \mathrm{H} & 5.13015100 & -2.17074900 & -0.20027000 \\ \mathrm{~N} & 2.66460400 & 0.16889700 & -0.54273000 \\ \mathrm{C} & 3.13005400 & 1.23079400 & -1.41204200 \\ \mathrm{H} & 4.03626100 & 0.89907200 & -1.93073000 \\ \mathrm{C} & 1.43058500 & 0.43502500 & 0.23754000 \\ \mathrm{C} & 0.80934400 & -0.93575000 & 0.58888000 \\ \mathrm{H} & 0.77163400 & -1.53407900 & -0.33162000 \\ \mathrm{H} & 1.47392100 & -1.46748900 & 1.28042600 \\ \mathrm{C} & -1.70550500 & -0.82089900 & 0.21627300 \\ \mathrm{O} & -1.70782500 & -1.39285500 & -0.85410400 \\ \mathrm{O} & -2.70531300 & -0.06229100 & 0.68216200 \\ \mathrm{C} & -3.92696200 & 0.17544700 & -0.09958800 \\ \mathrm{C} & -3.57941300 & 0.92244700 & -1.37578100\end{array}$

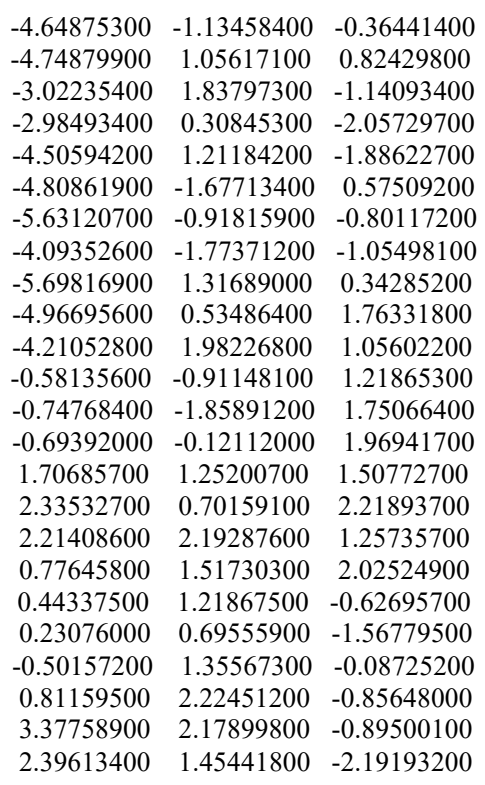

TS II $2-H_{[1-5] p}$

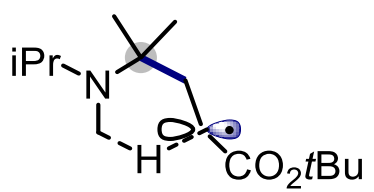

$E=-755.1973975$

$\mathrm{G}_{\text {Corr. }}=0.348372$ 


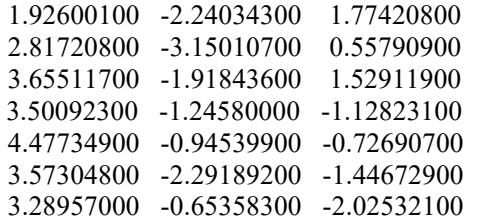

TS II $\mathbf{I}_{2}-\mathbf{H}_{[1-5] \mathbf{t}}$<smiles>CCOC(=O)[C@H]1CC(C)(C)N(C)C(C)(C)[C@H]1C(C)(C)C</smiles>

$\mathrm{E}=-755.1974886$

$\mathrm{G}_{\text {Corr. }}=0.349522$

$\mathrm{N} \quad-2.50541600 \quad 0.54131900 \quad 0.41002700$

C $\quad-2.62741600 \quad-0.78735300 \quad-0.25786800$

C $\quad-1.28465900 \quad-1.55667000 \quad-0.13221500$

$\begin{array}{llll}\mathrm{H} & -1.08584700 & -1.75982200 & 0.92868900\end{array}$

$\mathrm{H} \quad-1.41875800 \quad-2.53191500 \quad-0.62622500$

C $\quad-0.11715900-0.81416800 \quad-0.71662700$

$\begin{array}{llll}\mathrm{H} & 0.05692900 & -0.91503000 & -1.79011400\end{array}$

C $\quad 1.10904100 \quad-0.73124800 \quad 0.07509000$

$\begin{array}{llll}\mathrm{O} & 1.18145900 & -0.92984800 & 1.28146700\end{array}$

O $\quad 2.15079100 \quad-0.32964300 \quad-0.68823300$

$\begin{array}{lllll}\text { C } & 3.46370400 & -0.05012100 & -0.11245600\end{array}$

$\begin{array}{llll}\mathrm{C} & 3.36820300 & 1.09702800 & 0.88067300\end{array}$

C $\quad 4.05232100-1.30709000 \quad 0.50811200$

$\begin{array}{llll}\mathrm{C} & 4.28085600 & 0.36910200 & -1.32262400\end{array}$

$\begin{array}{llll}\mathrm{H} & 2.89448000 & 1.96731000 & 0.40900800\end{array}$

$\begin{array}{llll}\mathrm{H} & 2.79462500 & 0.81683300 & 1.76814100\end{array}$

$\begin{array}{llll}\mathrm{H} & 4.37784400 & 1.38943400 & 1.19433000\end{array}$

$\mathrm{H} \quad 4.04357800 \quad-2.12771600 \quad-0.21945300$

$\begin{array}{llll}\mathrm{H} & 5.09482800 & -1.11536800 & 0.79067400\end{array}$

$\mathrm{H} \quad 3.49891000 \quad-1.61587300 \quad 1.39812400$

$\mathrm{H} \quad \begin{array}{llll}5.30494800 & 0.61289200 & -1.01721000\end{array}$

$\mathrm{H} \quad 4.32112900 \quad-0.44091900 \quad-2.05994800$

$\mathrm{H} \quad 3.84003000 \quad 1.25217500 \quad-1.79950600$

C $\quad-1.47613900 \quad 1.38809900 \quad-0.13030900$

$\mathrm{H} \quad-0.62126500 \quad 0.44540100 \quad-0.61900000$

C $\quad-2.98700300-0.63450800 \quad-1.73594500$

$\mathrm{H} \quad-2.20914900-0.13930200 \quad-2.32412300$

$\mathrm{H} \quad-3.13268800 \quad-1.63100100 \quad-2.17054000$

$\mathrm{H} \quad \begin{array}{llll}-3.92160900 & -0.07171100 & -1.85081700\end{array}$

C $\quad-3.73352100 \quad-1.61361800 \quad 0.38987100$

H $\quad-4.67974300 \quad-1.05789600 \quad 0.40280600$

$\begin{array}{lll}-3.88734700 & -2.52694900 & -0.19599000\end{array}$

$-3.49187000 \quad-1.92290300 \quad 1.41216000$

$\begin{array}{lll}-1.89450100 & 2.30831300 & -1.25707100\end{array}$

$\begin{array}{llll}-2.34953500 & 3.21703900 & -0.83075400\end{array}$

$\begin{array}{lll}-1.02389200 & 2.62516000 & -1.84559600\end{array}$

$\begin{array}{lll}-2.62886500 & 1.86668700 & -1.93208200\end{array}$

$\begin{array}{lll}-0.56062100 & 2.09389000 & 0.84078400\end{array}$

$\begin{array}{lll}0.27227700 & 2.53351600 & 0.27629400\end{array}$

$\begin{array}{lll}-1.06238900 & 2.92340000 & 1.36257700\end{array}$

$\begin{array}{lll}-0.13392100 & 1.41735300 & 1.58994800\end{array}$

$\begin{array}{lll}-2.60050600 & 0.49925000 & 1.86336000\end{array}$

$\begin{array}{lll}-1.82724100 & -0.12268300 & 2.34850200\end{array}$

$\begin{array}{lll}-2.51996600 & 1.51178900 & 2.26410000\end{array}$

$\begin{array}{lll}-3.58117500 & 0.12135900 & 2.16329800\end{array}$

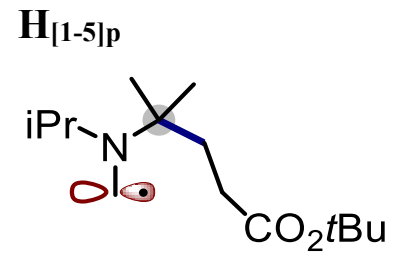

$\mathrm{E}=-755.2215814$

$\mathrm{G}_{\text {Corr. }}=0.350148$

N $\quad 3.17494100 \quad-0.24531600 \quad 0.33065600$

$\begin{array}{llrr}\text { C } & 3.51943500 & 0.96516600 & -0.43357700\end{array}$

$\begin{array}{llll}\text { C } & 4.94209200 & 0.86520200 & -0.97116900\end{array}$

$\begin{array}{llll}\mathrm{C} & 3.31236200 & 2.26510800 & 0.34083600\end{array}$

$\begin{array}{llll}\mathrm{H} & 2.84197200 & 0.99807900 & -1.29447500\end{array}$

$\begin{array}{llll}5.06045800 & -0.02368900 & -1.60142100\end{array}$

$\begin{array}{llll}5.19407500 & 1.75132100 & -1.56639600\end{array}$

$\begin{array}{llll}5.66493200 & 0.79627300 & -0.14823100\end{array}$

$\begin{array}{llll}2.31329200 & 2.31342500 & 0.78766400\end{array}$

$\begin{array}{lll}4.04864300 & 2.38371100 & 1.14434500\end{array}$

$\begin{array}{llll}3.42758300 & 3.11784600 & -0.33874100\end{array}$

$\begin{array}{lll}1.97113500 & -1.01511500 & -0.07408600\end{array}$

$\begin{array}{lll}0.72680500 & -0.13854700 & 0.16646400\end{array}$

$\begin{array}{llll}0.75253900 & 0.19371000 & 1.21501100\end{array}$

$\begin{array}{llll}0.80113400 & 0.77134100 & -0.44516400\end{array}$

$\begin{array}{llll}-0.61056800 & -0.79927500 & -0.11885500\end{array}$

$\begin{array}{llll}-0.66937700 & -1.18286900 & -1.14743200\end{array}$

$\begin{array}{lll}-1.77754500 & 0.13683000 & 0.06742000\end{array}$

$\begin{array}{llll}-1.68656200 & 1.30686200 & 0.37445500\end{array}$

$\begin{array}{llll}-2.92656200 & -0.51150100 & -0.15810400\end{array}$

$\begin{array}{llll}-4.23154200 & 0.15553100 & -0.05124800\end{array}$

$\begin{array}{lll}-4.45387400 & 0.63819500 & 1.37173700\end{array}$

$\begin{array}{llll}-4.32878600 & 1.27797500 & -1.07014700\end{array}$

$\begin{array}{llll}-5.20495000 & -0.95806500 & -0.39308700\end{array}$

$\begin{array}{lll}-4.31225900 & -0.18679300 & 2.08008600\end{array}$

$\begin{array}{lll}-3.77653700 & 1.45442900 & 1.63489700\end{array}$

$\begin{array}{lll}-5.48566500 & 0.99635000 & 1.47094500\end{array}$

$\begin{array}{llll}-4.09939100 & 0.90155100 & -2.07423900\end{array}$

$\begin{array}{llll}-5.35520400 & 1.66407000 & -1.07973000\end{array}$

$\begin{array}{lll}-3.65025200 & 2.10146000 & -0.83395200\end{array}$

$\begin{array}{llll}-6.23297800 & -0.58111700 & -0.34701700\end{array}$

$\begin{array}{llll}-5.01893000 & -1.33792300 & -1.40414200\end{array}$

$\begin{array}{lll}-5.10710300 & -1.78858900 & 0.31522300\end{array}$

$\begin{array}{llll}3.64999100 & -0.31430400 & 1.62832200\end{array}$

$\begin{array}{lll}3.59427300 & -1.26120800 & 2.15225100\end{array}$

$\begin{array}{lll}4.47902000 & 0.34120900 & 1.87592500\end{array}$

$\begin{array}{llll}-0.78783100 & -1.66896300 & 0.52812200\end{array}$

$\begin{array}{llll}1.88092200 & -2.30116700 & 0.74441900\end{array}$

$\begin{array}{llll}1.63488300 & -2.10997600 & 1.79528700\end{array}$

$\begin{array}{lll}1.09890200 & -2.94735800 & 0.33199000\end{array}$

$\begin{array}{llll}2.82478000 & -2.85727900 & 0.69756500\end{array}$

$\begin{array}{llll}2.10692900 & -1.42138000 & -1.54519100\end{array}$

$\begin{array}{llll}3.04900100 & -1.96267800 & -1.69558100\end{array}$

$\begin{array}{llll}1.28888700 & -2.09007600 & -1.83621400\end{array}$

$\begin{array}{llll}2.08710900 & -0.56885700 & -2.23301300\end{array}$

TS $\mathrm{H}_{[1-5] p-b i s A c}$<smiles>CCOCCCC(C)(C)N(C(=O)C=CC(=O)OCC(C)(C)C)C(C)C</smiles>

$\mathrm{E}=-1179.497962$

$\mathrm{G}_{\text {Corr. }}=0.51575$

$\begin{array}{lrrl}\mathrm{N} & -0.57295600 & 1.71454800 & 0.34724200 \\ \mathrm{C} & -0.84655500 & 1.92700100 & -1.08764300 \\ \mathrm{C} & -1.92455000 & 2.98702300 & -1.27726900 \\ \mathrm{C} & -1.19555200 & 0.63771000 & -1.82387000 \\ \mathrm{H} & 0.07884100 & 2.31601500 & -1.52666600 \\ \mathrm{H} & -1.64420100 & 3.92469300 & -0.78374800 \\ \mathrm{H} & -2.07725700 & 3.18689900 & -2.34429800 \\ \mathrm{H} & -2.88548300 & 2.65830000 & -0.86054300 \\ \mathrm{H} & -0.39682600 & -0.10742200 & -1.73443100 \\ \mathrm{H} & -2.12209800 & 0.19470100 & -1.43863900 \\ \mathrm{H} & -1.34681800 & 0.84928900 & -2.88936600 \\ \mathrm{C} & 0.82171500 & 1.86998200 & 0.84062900 \\ \mathrm{C} & 1.68885500 & 0.79331400 & 0.16084800\end{array}$ 


\begin{tabular}{|c|c|c|c|}
\hline $\mathrm{H}$ & 1.22909200 & -0.18611400 & 0.36002200 \\
\hline $\mathrm{H}$ & 1.65189100 & 0.93044700 & -0.92822900 \\
\hline $\mathrm{C}$ & 3.14699000 & 0.75760400 & 0.58457200 \\
\hline $\mathrm{H}$ & 3.64055200 & 1.72925400 & 0.44102600 \\
\hline $\mathrm{C}$ & 3.94886200 & -0.26141900 & -0.18584500 \\
\hline $\mathrm{O}$ & 3.51185600 & -0.94880300 & -1.08479800 \\
\hline $\mathrm{O}$ & 5.20686300 & -0.29153800 & 0.26587300 \\
\hline $\mathrm{C}$ & 6.21909900 & -1.19427200 & -0.30271000 \\
\hline $\mathrm{C}$ & 5.80486300 & -2.63954500 & -0.08792300 \\
\hline $\mathrm{C}$ & 6.44827700 & -0.86377200 & -1.76729400 \\
\hline $\mathrm{C}$ & 7.45471400 & -0.86722800 & 0.51602900 \\
\hline $\mathrm{H}$ & 5.58688300 & -2.82000500 & 0.97145900 \\
\hline $\mathrm{H}$ & 4.92749300 & -2.90428500 & -0.68317400 \\
\hline $\mathrm{H}$ & 6.63369400 & -3.29575000 & -0.37938900 \\
\hline $\mathrm{H}$ & 6.67698500 & 0.20207000 & -1.88618900 \\
\hline $\mathrm{H}$ & 7.30898100 & -1.43712300 & -2.13204000 \\
\hline $\mathrm{H}$ & 5.57987300 & -1.11365900 & -2.38177800 \\
\hline $\mathrm{H}$ & 8.29563300 & -1.48637600 & 0.18377900 \\
\hline $\mathrm{H}$ & 7.73056000 & 0.18666600 & 0.39650500 \\
\hline $\mathrm{H}$ & 7.27760600 & -1.06343300 & 1.57956700 \\
\hline $\mathrm{H}$ & 3.26400400 & 0.52304500 & 1.65080500 \\
\hline $\mathrm{C}$ & 0.86442800 & 1.70467600 & 2.35669100 \\
\hline $\mathrm{H}$ & 0.62632800 & 0.68207200 & 2.67331600 \\
\hline $\mathrm{H}$ & 1.86972200 & 1.94007300 & 2.72035200 \\
\hline $\mathrm{H}$ & 0.17247600 & 2.39811400 & 2.84865600 \\
\hline $\mathrm{C}$ & 1.30560000 & 3.28691900 & 0.51596900 \\
\hline $\mathrm{H}$ & 0.60727400 & 4.02227000 & 0.93339600 \\
\hline $\mathrm{H}$ & 2.28870000 & 3.47053300 & 0.96378500 \\
\hline $\mathrm{H}$ & 1.39884800 & 3.47311300 & -0.55966900 \\
\hline $\mathrm{C}$ & -1.53308300 & 1.07519300 & 1.07812000 \\
\hline $\mathrm{H}$ & -1.47203600 & 1.10084200 & 2.15932800 \\
\hline $\mathrm{H}$ & -2.53393500 & 1.05942300 & 0.65370500 \\
\hline $\mathrm{C}$ & -1.70612200 & -1.39436400 & 1.15557900 \\
\hline $\mathrm{H}$ & -0.75210400 & -1.53590400 & 1.65816900 \\
\hline $\mathrm{H}$ & -1.67863600 & -1.30903800 & 0.07410800 \\
\hline $\mathrm{C}$ & -2.87251900 & -1.61332600 & 1.82043900 \\
\hline $\mathrm{H}$ & -2.88285700 & -1.83459100 & 2.88564200 \\
\hline $\mathrm{C}$ & -4.19678200 & -1.48668800 & 1.23250000 \\
\hline $\mathrm{O}$ & -5.23462900 & -1.71240800 & 1.84194600 \\
\hline $\mathrm{O}$ & -4.15877800 & -1.07074700 & -0.05669000 \\
\hline $\mathrm{C}$ & -5.38205500 & -0.81322700 & -0.81081800 \\
\hline $\mathrm{C}$ & -6.18326700 & -2.09441400 & -0.97785400 \\
\hline $\mathrm{C}$ & -6.18466200 & 0.29511800 & -0.14735300 \\
\hline $\mathrm{C}$ & -4.86477500 & -0.34136400 & -2.15970600 \\
\hline $\mathrm{H}$ & -5.55630800 & -2.87741600 & -1.42135400 \\
\hline $\mathrm{H}$ & -6.57475700 & -2.45138500 & -0.02238100 \\
\hline $\mathrm{H}$ & -7.02556100 & -1.90834300 & -1.65568600 \\
\hline $\mathrm{H}$ & -5.54283900 & 1.16781100 & 0.03229500 \\
\hline $\mathrm{H}$ & -6.99736100 & 0.60386200 & -0.81639200 \\
\hline $\mathrm{H}$ & -6.61579300 & -0.02872700 & 0.80282800 \\
\hline $\mathrm{H}$ & -5.70567600 & -0.15687100 & -2.83824900 \\
\hline $\mathrm{H}$ & -4.29578600 & 0.59166200 & -2.05523400 \\
\hline $\mathrm{H}$ & -4.21179100 & -1.09810000 & -2.61116600 \\
\hline
\end{tabular}

\section{TS H[1-5]p-P $\mathbf{P}_{2}(\mathbf{R S H})$}

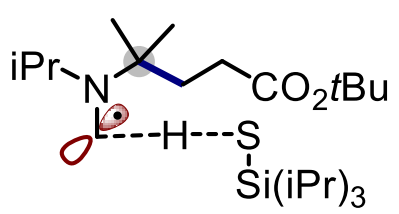

$E=-1799.001666$

$$
\begin{array}{lrll}
\text { GCorr. }= & 0.614254 & & \\
\mathrm{~N} & 0.20793200 & 2.71299200 & 0.28602000 \\
\mathrm{C} & 0.32732000 & 3.30335700 & -1.05956900 \\
\mathrm{C} & -0.23189600 & 4.72077200 & -1.07496500 \\
\mathrm{C} & -0.30799900 & 2.43864100 & -2.14300300 \\
\mathrm{H} & 1.39928400 & 3.36648000 & -1.27819300 \\
\mathrm{H} & 0.26921800 & 5.35090800 & -0.33150700 \\
\mathrm{H} & -0.09364900 & 5.17309800 & -2.06397200 \\
\mathrm{H} & -1.30697000 & 4.72344700 & -0.85522100
\end{array}
$$

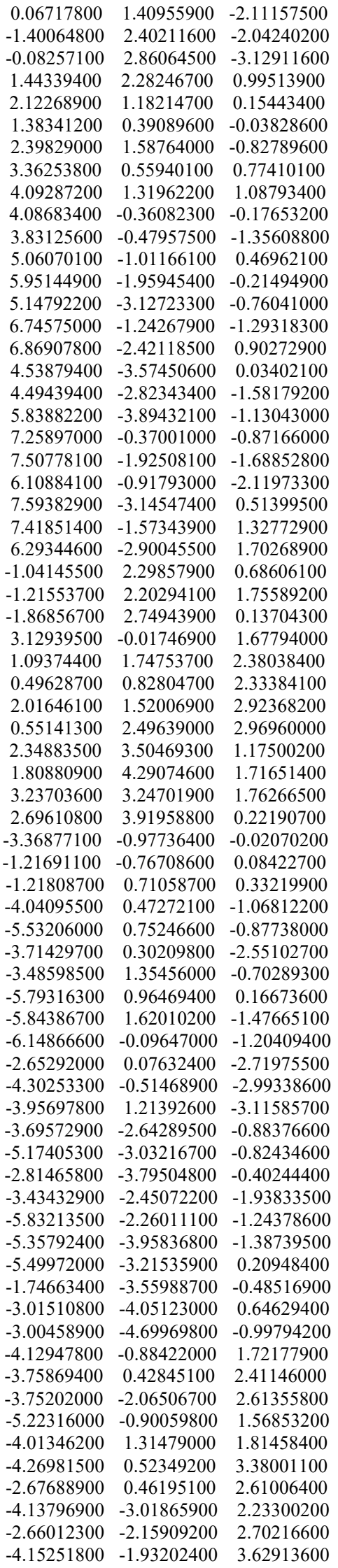

$\alpha-C I_{1}$

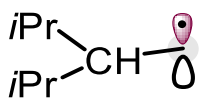




$\begin{array}{lrrr}\mathrm{E}=-314.8686079 & & \\ \mathrm{G} & & \\ \mathrm{G}_{\text {Corr. }}= & 0.191976 & & \\ \mathrm{C} & -1.82762000 & -1.02602400 & -0.67600300 \\ \mathrm{C} & -1.46339400 & 0.07139800 & 0.31696000 \\ \mathrm{H} & -2.01514400 & -0.13563500 & 1.25030800 \\ \mathrm{H} & -1.45863300 & -2.00830000 & -0.35267600 \\ \mathrm{H} & -2.91680600 & -1.09480400 & -0.78875300 \\ \mathrm{H} & -1.41281600 & -0.81739600 & -1.67156700 \\ \mathrm{C} & -1.93896700 & 1.42723700 & -0.19130400 \\ \mathrm{H} & -1.69825400 & 2.23181000 & 0.51552700 \\ \mathrm{H} & -1.48251300 & 1.68194700 & -1.15731900 \\ \mathrm{H} & -3.02573900 & 1.42542500 & -0.34101500 \\ \mathrm{C} & 1.04010900 & 0.33812600 & -0.41330500 \\ \mathrm{C} & 1.48465900 & -0.92475900 & -1.14923400 \\ \mathrm{C} & 2.26730200 & 1.07739100 & 0.11198900 \\ \mathrm{H} & 0.54842500 & 0.99916300 & -1.14518900 \\ \mathrm{H} & 0.64408300 & -1.53642500 & -1.49265000 \\ \mathrm{H} & 2.08760600 & -0.65881000 & -2.02674100 \\ \mathrm{H} & 2.10970200 & -1.55749600 & -0.50528400 \\ \mathrm{H} & 1.99908900 & 2.04934100 & 0.54452300 \\ \mathrm{H} & 2.77287700 & 0.49263100 & 0.89386800 \\ \mathrm{H} & 2.99736700 & 1.25291200 & -0.68815000 \\ \mathrm{C} & 0.36840900 & -1.10515900 & 1.54974300 \\ \mathrm{H} & -0.39859200 & -1.60334800 & 2.14122300 \\ \mathrm{H} & 1.40195400 & -1.38120700 & 1.74911400 \\ \mathrm{C} & 0.02571900 & 0.08855800 & 0.73162800 \\ \mathrm{H} & 0.11009200 & 0.97958200 & 1.39193000 \\ & & & \end{array}$

\section{$\alpha-O \mathbf{I}_{1}$}

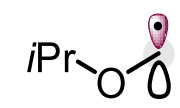

$\mathrm{E}=-232.908669$

$$
\mathrm{G}_{\text {Corr. }}=0.091096
$$

$\begin{array}{lrrr}\mathrm{C} & 0.60416500 & 1.43032800 & -0.13316000 \\ \mathrm{C} & 0.37709100 & 0.00383000 & 0.32353100 \\ \mathrm{H} & 0.17169600 & -0.01477200 & 1.40859900 \\ \mathrm{H} & -0.27164900 & 2.05589900 & 0.07215600 \\ \mathrm{H} & 1.46792500 & 1.86382500 & 0.38326000 \\ \mathrm{H} & 0.79900000 & 1.45125800 & -1.21268500 \\ \mathrm{C} & 1.54186200 & -0.90195000 & 0.00289900 \\ \mathrm{H} & 1.34714300 & -1.92694500 & 0.33392900 \\ \mathrm{H} & 1.72054800 & -0.91431100 & -1.07928700 \\ \mathrm{H} & 2.45059200 & -0.54179300 & 0.49703300 \\ \mathrm{C} & -1.96781200 & -0.08492100 & 0.07927800 \\ \mathrm{H} & -2.04448100 & 0.28309900 & 1.10356200 \\ \mathrm{H} & -2.81695200 & -0.53125800 & -0.42577200 \\ \mathrm{O} & -0.76945700 & -0.55109100 & -0.33951100\end{array}$

\section{$\alpha-C I_{2}$}

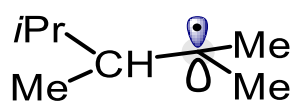

$$
\mathrm{E}=-314.8826651
$$

$$
\mathrm{G}_{\text {Corr. }}=0.192038
$$

$\begin{array}{lccc}\mathrm{C} & -1.11029600 & -0.59827500 & -0.03423300 \\ \mathrm{C} & -2.53698900 & -0.27038600 & -0.45766300 \\ \mathrm{C} & -1.00668000 & -0.71048300 & 1.48196200 \\ \mathrm{H} & -0.86156000 & -1.58679700 & -0.45518100 \\ \mathrm{H} & -2.61023700 & -0.11065000 & -1.54167700 \\ \mathrm{H} & -3.21935300 & -1.08714200 & -0.19134300 \\ \mathrm{H} & -2.90567000 & 0.63611300 & 0.04090700 \\ \mathrm{H} & -0.01592500 & -1.06165300 & 1.79756000 \\ \mathrm{H} & -1.19148500 & 0.25605100 & 1.97115300\end{array}$

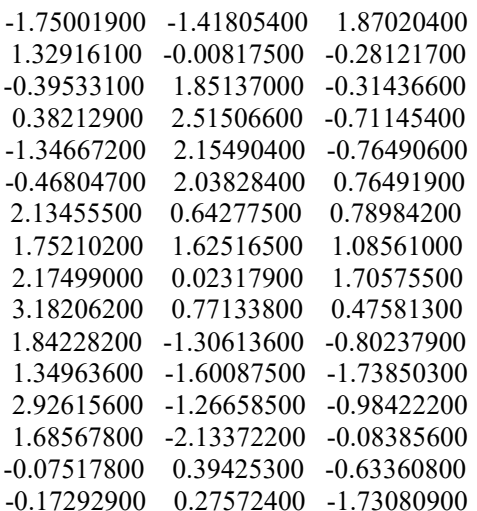

\section{$\alpha-O I_{2}$}

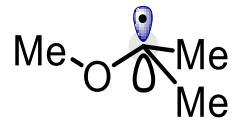

$E=-232.912363$

$\mathrm{G}_{\text {Corr. }}=0.090838$

$\begin{array}{lrrc}\mathrm{C} & -0.46253900 & 0.00902200 & 0.20110500 \\ \mathrm{C} & 1.90398200 & -0.18234000 & 0.05741600 \\ \mathrm{H} & 2.01744100 & 0.14488900 & 1.10082800 \\ \mathrm{H} & 2.63617800 & -0.96453600 & -0.15438600 \\ \mathrm{H} & 2.09354700 & 0.66985800 & -0.60897200 \\ \mathrm{C} & -0.42802000 & 1.47753300 & -0.04166100 \\ \mathrm{H} & 0.40360900 & 1.98489300 & 0.46208600 \\ \mathrm{H} & -0.34503200 & 1.71365200 & -1.12009200 \\ \mathrm{H} & -1.35718000 & 1.93056700 & 0.31870600 \\ \mathrm{C} & -1.72636300 & -0.74623800 & 0.01627500 \\ \mathrm{H} & -1.64012000 & -1.76146000 & 0.41974000 \\ \mathrm{H} & -2.55979000 & -0.24119900 & 0.51643300 \\ \mathrm{H} & -1.99509400 & -0.84274500 & -1.05220000 \\ \mathrm{O} & 0.62801000 & -0.74772300 & -0.16011900\end{array}$

\section{$\alpha-C$ TS I-II}<smiles>CC(C)C(C(=O)C=CC(=O)OC(C)(C)C)C(C)C</smiles>

$\mathrm{E}=-739.1428412$

$\mathrm{G}_{\text {Corr. }}=0.358198$

$\begin{array}{lrrr}\mathrm{C} & -3.72050500 & -0.55775500 & 1.57727800 \\ \mathrm{C} & -2.22555100 & -0.61730900 & 1.28411000 \\ \mathrm{H} & -1.93406900 & -1.68202000 & 1.26259800 \\ \mathrm{H} & -4.32445700 & -0.99547100 & 0.77195300 \\ \mathrm{H} & -3.94801000 & -1.10872300 & 2.49815900 \\ \mathrm{H} & -4.05660100 & 0.47710800 & 1.72879000 \\ \mathrm{C} & -1.43353400 & 0.03247600 & 2.41245100 \\ \mathrm{H} & -0.35540400 & -0.10989300 & 2.26528300 \\ \mathrm{H} & -1.63116400 & 1.11185200 & 2.47780100 \\ \mathrm{H} & -1.70328200 & -0.40496500 & 3.38169400 \\ \mathrm{C} & -2.24404300 & 1.40593500 & -0.39264200 \\ \mathrm{C} & -3.64010800 & 1.55045600 & -0.99482800 \\ \mathrm{C} & -1.22094100 & 2.07371300 & -1.30758700 \\ \mathrm{H} & -2.23079600 & 1.94673900 & 0.56803300 \\ \mathrm{H} & -4.41314000 & 1.04949700 & -0.40330900 \\ \mathrm{H} & -3.91229000 & 2.61069800 & -1.07116800 \\ \mathrm{H} & -3.67748500 & 1.13278500 & -2.01017700 \\ \mathrm{H} & -0.22188800 & 2.09556100 & -0.85115800 \\ \mathrm{H} & -1.13259400 & 1.53314700 & -2.26164900\end{array}$ 


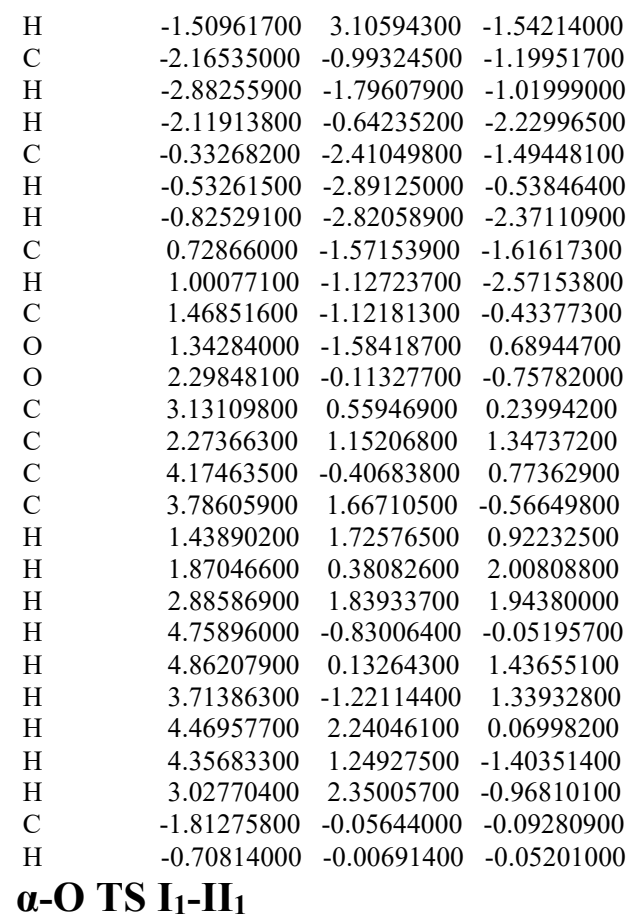

Pro

$$
\mathrm{E}=-657.1845584
$$$$
\mathrm{G}_{\text {Corr. }}=0.255107
$$

C $\quad-2.67060400 \quad-2.04283500 \quad-0.99068400$

$\begin{array}{llll}\text { C } & -1.95499300 & -1.08987600 & -0.05701700\end{array}$

$\mathrm{H} \quad-1.17917600 \quad-0.53700300 \quad-0.61196200$

$\mathrm{H} \quad-3.15446700 \quad-1.50687200 \quad-1.81496500$

$\begin{array}{llll}\mathrm{H} & -1.95635000 & -2.75455100 & -1.42122300\end{array}$

$\mathrm{H} \quad-3.43683400 \quad-2.60622500 \quad-0.44413200$

$\begin{array}{llll}\mathrm{C} & -1.35024700 & -1.78394900 & 1.13998100\end{array}$

$\mathrm{H} \quad-0.78823100-1.07922700 \quad 1.76290700$

$\mathrm{H} \quad-2.14143300 \quad-2.24185800 \quad 1.74618900$

$\mathrm{H} \quad-0.66818800 \quad-2.57857900 \quad 0.81485100$

C $\quad-3.10114100 \quad 0.96376200 \quad-0.30479900$

$\mathrm{H} \quad-2.81194400 \quad 0.91620300 \quad-1.35627800$

$\mathrm{H} \quad-3.97024500 \quad 1.54711300 \quad-0.01435100$

$\begin{array}{llll}\mathrm{C} & -1.40567300 & 2.56470100 & 0.32403100\end{array}$

$\mathrm{H} \quad-1.38431600 \quad 2.86955900 \quad-0.71960300$

$\mathrm{H} \quad-2.11771300 \quad 3.06138200 \quad 0.97610200$

$\begin{array}{llll}\mathrm{C} & -0.38876200 & 1.82753600 & 0.83714000\end{array}$

$\begin{array}{llll}\mathrm{H} & -0.33268400 & 1.58529800 & 1.89597100\end{array}$

$\begin{array}{llll}\mathrm{C} & 0.60206200 & 1.21801100 & -0.04790500\end{array}$

$\begin{array}{llll}\mathrm{O} & 0.65138200 & 1.37817900 & -1.25878000\end{array}$

$\begin{array}{lll}1.43315900 & 0.41661100 & 0.64665800\end{array}$

$\begin{array}{lll}2.48072100 & -0.36600000 & -0.00854300\end{array}$

$\begin{array}{lll}1.87711500 & -1.33141700 & -1.01765900\end{array}$

$\begin{array}{lll}3.50579800 & 0.56185400 & -0.63901100\end{array}$

$3.09731100 \quad-1.13399600 \quad 1.14758100$

$\begin{array}{llll}1.10276700 & -1.94887100 & -0.54261800\end{array}$

$\begin{array}{lll}1.44103400 & -0.80773200 & -1.87213000\end{array}$

$\begin{array}{lll}2.66344100 & -2.00331800 & -1.38277200\end{array}$

$\begin{array}{lll}3.88006300 & 1.27409400 & 0.10593800\end{array}$

$\begin{array}{llll}4.35546400 & -0.03007100 & -1.00061200\end{array}$

$\begin{array}{llll}3.08338300 & 1.11647100 & -1.48072900\end{array}$

$\begin{array}{lll}3.91463700 & -1.76706100 & 0.78361500\end{array}$

$3.49852800 \quad-0.44431200 \quad 1.89884800$

$\begin{array}{lll}2.34811600 & -1.77472200 & 1.62837100\end{array}$

$\begin{array}{lll}-2.89646300 & -0.12184900 & 0.45585700\end{array}$
$\alpha-C$ TS $\mathbf{I}_{2}-\mathbf{I I}_{2}$<smiles>CCOC(=O)C(C)(C)C(C)C(C)C</smiles>

$\mathrm{E}=-739.1518953$

$\mathrm{G}_{\text {Corr. }}=0.358605$

$\begin{array}{lll}3.72925800 & -0.93368400 & 0.41912200\end{array}$ $\begin{array}{llll}5.25488400 & -0.97962500 & 0.44942900\end{array}$ $\begin{array}{llll}3.21035700 & -1.88458600 & -0.65532400\end{array}$ $\begin{array}{llll}3.38493700 & -1.31321800 & 1.39362500\end{array}$ $\begin{array}{llll}5.66868000 & -0.26792600 & 1.17567300\end{array}$ $\begin{array}{llll}5.60325200 & -1.98211500 & 0.72598300\end{array}$ $\begin{array}{llll}5.68694400 & -0.74727800 & -0.53283900\end{array}$ $\begin{array}{llll}2.11606200 & -1.94784900 & -0.67317600\end{array}$ $\begin{array}{llll}3.54706000 & -1.58300400 & -1.65661400\end{array}$ $\begin{array}{llll}3.59580200 & -2.89676000 & -0.48074600\end{array}$ $\begin{array}{lll}1.72797800 & 0.70653600 & 0.56614400\end{array}$ $\begin{array}{llll}0.57686800 & 0.32101600 & -1.41532200\end{array}$ $\begin{array}{llll}0.48683200 & 1.39919900 & -1.52697500\end{array}$ $\begin{array}{lll}1.43377100 & -0.15378500 & -1.88317000\end{array}$ $\begin{array}{llll}-0.53115300 & -0.41963700 & -1.12353700\end{array}$ $\begin{array}{lll}-0.51460100 & -1.50767600 & -1.15427400\end{array}$ $\begin{array}{llll}-1.75621800 & 0.21381400 & -0.64340200\end{array}$ $\begin{array}{llll}-1.90521800 & 1.41630700 & -0.48100300\end{array}$ $\begin{array}{lll}-2.70112600 & -0.71227200 & -0.38675000\end{array}$ $\begin{array}{lll}-4.00424700 & -0.35199200 & 0.17081300\end{array}$ $\begin{array}{lll}-3.82508200 & 0.27914000 & 1.54216000\end{array}$ $\begin{array}{llll}-4.76333800 & 0.54819000 & -0.79007200\end{array}$ $\begin{array}{llll}-4.70054700 & -1.69609000 & 0.29517600\end{array}$ $\begin{array}{lll}-3.22014400 & -0.37427500 & 2.18316200\end{array}$ $\begin{array}{lll}-3.34497000 & 1.25887300 & 1.47755500\end{array}$ $\begin{array}{lll}-4.80753700 & 0.40208100 & 2.01403200\end{array}$ $\begin{array}{llll}-4.81202000 & 0.08656300 & -1.78359300\end{array}$ $\begin{array}{llll}-5.78970300 & 0.67743900 & -0.42525600\end{array}$ $\begin{array}{llll}-4.29688500 & 1.53223200 & -0.87773100\end{array}$ $\begin{array}{llll}-5.70491900 & -1.55978600 & 0.71218400\end{array}$ $\begin{array}{lll}-4.79337900 & -2.17466400 & -0.68646800\end{array}$ $\begin{array}{lll}-4.13653700 & -2.36392800 & 0.95638200\end{array}$ $\begin{array}{llll}3.71660800 & 1.21558400 & -0.96254000\end{array}$ $\begin{array}{llll}3.31292400 & 2.22820300 & -1.06005100\end{array}$ $\begin{array}{llll}4.80774200 & 1.30827400 & -0.94909500\end{array}$ $\begin{array}{llll}3.44609500 & 0.66772900 & -1.87297100\end{array}$ $\begin{array}{lll}1.29474700 & 2.12782700 & 0.78040700\end{array}$ $\begin{array}{lll}1.69303600 & 2.82560900 & 0.03595600\end{array}$ $\begin{array}{lll}0.20192500 & 2.21615600 & 0.77477300\end{array}$ $\begin{array}{lll}1.65154100 & 2.47025500 & 1.76843000\end{array}$ $\begin{array}{lll}1.06838400 & -0.23893900 & 1.52207400\end{array}$ $\begin{array}{lll}1.52387400 & -0.13253200 & 2.52244700\end{array}$ $\begin{array}{llll}0.00167400 & -0.00507200 & 1.62991400\end{array}$ $\begin{array}{lll}1.15502600 & -1.29230200 & 1.23508300\end{array}$ $\begin{array}{lll}3.21281400 & 0.51648200 & 0.30228900\end{array}$ $\begin{array}{lll}3.68640400 & 1.05306500 & 1.15528300\end{array}$

\section{$\alpha-O$ TS I $\mathbf{I}_{2}-\mathrm{II}_{2}$}<smiles>COC(C)(C)OCCOC(C)(C)C</smiles>

$\mathrm{E}=-657.191497$

$\mathrm{G}_{\text {Corr. }}=0.255301$

$\begin{array}{llll}\text { C } & -2.87608000 & 0.17799800 & 0.42237900\end{array}$

C $\quad-1.49402700 \quad 1.16514300 \quad-1.29426000$

$\mathrm{H} \quad-1.67718400 \quad 0.23320500 \quad-1.82437900$ 


$\begin{array}{lrrr}\mathrm{H} & -2.22549400 & 1.95808200 & -1.42305900 \\ \mathrm{C} & -0.26197400 & 1.43136300 & -0.78804600 \\ \mathrm{H} & -0.01577000 & 2.39762900 & -0.35309000 \\ \mathrm{C} & 0.74434200 & 0.37914700 & -0.69493000 \\ \mathrm{O} & 0.60401700 & -0.76463700 & -1.10887000 \\ \mathrm{O} & 1.85027000 & 0.82725700 & -0.06666500 \\ \mathrm{C} & 2.97029200 & -0.05792600 & 0.24638000 \\ \mathrm{C} & 2.50802100 & -1.17082600 & 1.17376200 \\ \mathrm{C} & 3.60296500 & -0.58730800 & -1.03006100 \\ \mathrm{C} & 3.93647400 & 0.86332800 & 0.97094700 \\ \mathrm{H} & 1.99358500 & -0.74639900 & 2.04524300 \\ \mathrm{H} & 1.83480100 & -1.86731000 & 0.66710600 \\ \mathrm{H} & 3.38148200 & -1.72828100 & 1.53332500 \\ \mathrm{H} & 3.86783900 & 0.24409600 & -1.69425800 \\ \mathrm{H} & 4.52399500 & -1.12708300 & -0.77779600 \\ \mathrm{H} & 2.93285400 & -1.26700500 & -1.56176900 \\ \mathrm{H} & 4.83576800 & 0.30846900 & 1.26197300 \\ \mathrm{H} & 4.23532400 & 1.69515900 & 0.32292300 \\ \mathrm{H} & 3.47346100 & 1.27518300 & 1.87504800 \\ \mathrm{C} & -2.53407000 & -2.07005200 & -0.29802100 \\ \mathrm{H} & -1.55530300 & -1.81164900 & -0.72548600 \\ \mathrm{H} & -2.39080800 & -2.53756900 & 0.68432700 \\ \mathrm{H} & -3.04990500 & -2.77301900 & -0.95452600 \\ \mathrm{C} & -1.89828600 & 0.00519300 & 1.53174300 \\ \mathrm{H} & -1.01494700 & -0.57678500 & 1.24249900 \\ \mathrm{H} & -1.55265200 & 0.98826200 & 1.86834900 \\ \mathrm{H} & -2.36771900 & -0.50046100 & 2.39263000 \\ \mathrm{C} & -3.91656300 & 1.23360500 & 0.54429100 \\ \mathrm{H} & -4.64845700 & 0.97362400 & 1.32727500 \\ \mathrm{H} & -3.46507700 & 2.19425400 & 0.81563400 \\ \mathrm{H} & -4.46710300 & 1.35121000 & -0.39605900 \\ \mathrm{O} & -3.37119400 & -0.92532000 & -0.20334100\end{array}$

\section{$\mathrm{Ph}_{3} \mathrm{SiS} \cdot$}

$$
\begin{aligned}
& \mathrm{Ph}_{3} \mathrm{Si}^{-\mathrm{S}^{\bullet}} \\
& \mathrm{E}=-1382.281865
\end{aligned}
$$

$$
\mathrm{G}_{\text {Corr. }}=0.222907
$$

$\begin{array}{cccc}\mathrm{Si} & 0.00385900 & -0.00721600 & 0.62072100 \\ \mathrm{~S} & 0.15155100 & 0.34503300 & 2.73199200 \\ \mathrm{C} & -1.73835900 & -0.43953200 & 0.08444200 \\ \mathrm{C} & -2.66316400 & -1.02304600 & 0.95910000 \\ \mathrm{C} & -2.13097700 & -0.21613100 & -1.24402400 \\ \mathrm{C} & -3.93791900 & -1.37787100 & 0.52290300 \\ \mathrm{H} & -2.38905800 & -1.19460200 & 2.00039400 \\ \mathrm{C} & -3.40369000 & -0.56787500 & -1.68549000 \\ \mathrm{H} & -1.43383600 & 0.24504100 & -1.94584300 \\ \mathrm{C} & -4.30852100 & -1.15120900 & -0.80056300 \\ \mathrm{H} & -4.64371000 & -1.82771300 & 1.21753300 \\ \mathrm{H} & -3.69092000 & -0.38421800 & -2.71836600 \\ \mathrm{H} & -5.30438800 & -1.42471600 & -1.14181900 \\ \mathrm{C} & 1.21566500 & -1.33130300 & 0.07942100 \\ \mathrm{C} & 2.52127000 & -1.36395600 & 0.59262400 \\ \mathrm{C} & 0.85157100 & -2.29657900 & -0.86939500 \\ \mathrm{C} & 3.43730300 & -2.31876800 & 0.16156200 \\ \mathrm{H} & 2.82290200 & -0.63600300 & 1.34653900 \\ \mathrm{C} & 1.76531900 & -3.25541300 & -1.30285000 \\ \mathrm{H} & -0.15985100 & -2.30464600 & -1.27566400 \\ \mathrm{C} & 3.05911900 & -3.26616200 & -0.78868300 \\ \mathrm{H} & 4.44512100 & -2.32801400 & 0.57049900 \\ \mathrm{H} & 1.46437700 & -3.99721800 & -2.03917900 \\ \mathrm{H} & 3.77223800 & -4.01597200 & -1.12375500 \\ \mathrm{C} & 0.48679800 & 1.68559800 & -0.03844000 \\ \mathrm{C} & -0.39857500 & 2.76598000 & 0.11213100 \\ \mathrm{C} & 1.72487400 & 1.91610000 & -0.65432800 \\ \mathrm{C} & -0.06308600 & 4.03350100 & -0.35278100 \\ \mathrm{H} & -1.36520900 & 2.60998300 & 0.59241000 \\ \mathrm{C} & 2.06115400 & 3.18277400 & -1.12410000 \\ \mathrm{H} & 2.43031900 & 1.09485600 & -0.77929900 \\ \mathrm{C} & 1.16806400 & 4.24200900 & -0.97251100 \\ \mathrm{H} & -0.76082300 & 4.85888400 & -0.23227000 \\ & & & \\ & & & \\ \mathrm{H} & & & \end{array}$

$\begin{array}{llll}\mathrm{H} & 3.02055100 & 3.34407200 & -1.61027300 \\ \mathrm{H} & 1.43236400 & 5.23205600 & -1.33697800\end{array}$

\section{TS Ph $\mathbf{P h}_{3} \mathrm{SiS} \cdot-\mathbf{I}_{1}$}<smiles>CC(C)N(CC=[SH][SnH][AsH2])C(C)F</smiles>

$\mathrm{G}_{\text {Corr. }}=0.433982$

$\begin{array}{llll}\mathrm{C} & -2.39002000 & -0.01008100 & 0.34269600\end{array}$

$\begin{array}{llll}\mathrm{H} & -2.03037000 & 0.84002800 & 0.92562400\end{array}$

H $\quad-2.14658000 \quad-0.99519500 \quad 0.74191100$

$\begin{array}{lllll}\text { C } & -4.31791500 & -1.04725900 & -0.75923300\end{array}$

$\begin{array}{lll}-4.86259000 & -1.90771700 & 0.37405900\end{array}$

$\begin{array}{lll}-3.43668300 & -1.85848900 & -1.70258500\end{array}$

$-5.17033200 \quad-0.66748700 \quad-1.33804600$

$\begin{array}{llll}-5.52510000 & -1.32608700 & 1.02520500\end{array}$

$\begin{array}{lll}-5.42621100 & -2.75776000 & -0.02763700\end{array}$

$\begin{array}{lll}-4.04565400 & -2.30937000 & 0.98814800\end{array}$

$\begin{array}{lll}-3.03542500 & -1.23999000 & -2.51329300\end{array}$

$-2.58824800 \quad-2.30776000 \quad-1.17009000$

$\begin{array}{lll}-4.02042200 & -2.67317700 & -2.14645200\end{array}$

$\begin{array}{llll}-4.05843800 & 1.47501800 & -0.63952200\end{array}$

$\begin{array}{llll}-5.50632100 & 1.74688800 & -0.25832500\end{array}$

$\begin{array}{lll}-3.79757100 & 1.72356100 & -2.12066700\end{array}$

$\begin{array}{lll}-3.42441000 & 2.16481800 & -0.06299500\end{array}$

$\begin{array}{llll}-5.67247600 & 1.56366600 & 0.80906100\end{array}$

$\begin{array}{llll}-5.75735400 & 2.79176600 & -0.47315100\end{array}$

$\begin{array}{llll}-6.20544900 & 1.12214900 & -0.82849700\end{array}$

$\begin{array}{lll}-2.74049500 & 1.56282100 & -2.36613900\end{array}$

$\begin{array}{llll}-4.40449100 & 1.05875900 & -2.74942100\end{array}$

$\begin{array}{llll}-4.05811200 & 2.75558600 & -2.38388100\end{array}$

$\begin{array}{llll}1.30788200 & 0.00628100 & -0.23093100\end{array}$ $\begin{array}{llll}-0.08067700 & 0.03479800 & -1.86685100\end{array}$

$\begin{array}{llll}-1.19059900 & 0.02717000 & -0.90485100\end{array}$

$\begin{array}{llll}2.99376900 & -0.49825300 & -0.88293100\end{array}$

$\begin{array}{llll}3.59853800 & 0.25293500 & -1.90218500\end{array}$

$\begin{array}{llll}3.69526800 & -1.59420100 & -0.36529000\end{array}$

$\begin{array}{lll}4.85460200 & -0.08510700 & -2.39598700\end{array}$

$\begin{array}{llll}3.07705300 & 1.11648100 & -2.31859500\end{array}$

$\begin{array}{llll}4.95535800 & -1.93669700 & -0.85436200\end{array}$

$\begin{array}{llll}3.25303500 & -2.19262700 & 0.43150800\end{array}$

$\begin{array}{llll}5.53520500 & -1.18344000 & -1.87124500\end{array}$

$\begin{array}{llll}5.30481400 & 0.50777200 & -3.18916000\end{array}$

$\begin{array}{llll}5.48399100 & -2.79246100 & -0.43996900\end{array}$

$\begin{array}{llll}6.51720100 & -1.45021800 & -2.25562400\end{array}$

$\begin{array}{lll}1.43616400 & 1.70550100 & 0.56851300\end{array}$

$\begin{array}{llll}2.49995700 & 1.98266400 & 1.44061200\end{array}$

$\begin{array}{lll}0.48335000 & 2.70887700 & 0.35300200\end{array}$

$\begin{array}{lll}2.60488500 & 3.21464600 & 2.08093900\end{array}$

$\begin{array}{lll}3.26270500 & 1.22273400 & 1.62166000\end{array}$

$\begin{array}{llll}0.58197300 & 3.94448400 & 0.99024100\end{array}$

$\begin{array}{llll}-0.34200400 & 2.52483700 & -0.33733900\end{array}$

$\begin{array}{lll}1.64253000 & 4.19805300 & 1.85653600\end{array}$

$\begin{array}{lll}3.43857100 & 3.40971600 & 2.75206400\end{array}$

$\begin{array}{lll}-0.16656500 & 4.71222000 & 0.80594200\end{array}$

$\begin{array}{lll}1.72342500 & 5.16276400 & 2.35257600\end{array}$

$\begin{array}{lll}0.68658500 & -1.23338300 & 1.04321700\end{array}$

$\begin{array}{lll}0.36623900 & -2.54192200 & 0.64786000\end{array}$

$\begin{array}{lll}0.46403500 & -0.87839700 & 2.37971900\end{array}$

$\begin{array}{lll}-0.16150100 & -3.45997300 & 1.55061600\end{array}$

$\begin{array}{llll}0.51526100 & -2.84151800 & -0.39079800\end{array}$

$\begin{array}{lll}-0.06495700 & -1.79314000 & 3.29027600\end{array}$

$\begin{array}{lll}0.69226100 & 0.13339800 & 2.71628800\end{array}$ 


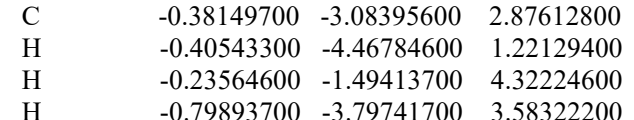

\section{$(i \mathrm{Pr})_{3} \mathrm{GeS} \cdot$}

$$
(\text { iPr })_{3} \mathrm{Ge}^{-\mathrm{S}^{\bullet}}
$$

$\mathrm{E}=$

$\mathrm{G}_{\text {Corr. }}=$

$\begin{array}{llll}\text { S } & 0.58522100 & -0.16499600 & 2.29279200\end{array}$

$\begin{array}{llll}\mathrm{C} & -1.95855000 & -0.50696900 & 0.11290500\end{array}$

$\begin{array}{llll}\text { C } & -2.43603600 & -0.88688200 & -1.28367100\end{array}$

$\begin{array}{llll}\text { C } & -2.79231500 & 0.62312200 & 0.69734300\end{array}$

$\mathrm{H} \quad-2.03602900 \quad-1.38622700 \quad 0.77054000$

$\mathrm{H} \quad-1.86684900 \quad-1.72071900 \quad-1.71291700$

$\mathrm{H} \quad-3.49440200 \quad-1.18405900 \quad-1.26893700$

$\mathrm{H} \quad-2.35296900 \quad-0.03891700 \quad-1.97855300$

$\begin{array}{llll}\mathrm{H} & -2.41989500 & 0.94481400 & 1.67836100\end{array}$

$\begin{array}{llll}\mathrm{H} & -2.78607400 & 1.49947600 & 0.03320200\end{array}$

$\begin{array}{llll}\mathrm{H} & -3.84182200 & 0.32025800 & 0.81901300\end{array}$

$\begin{array}{lllr}\mathrm{H} & -3.84182200 & 0.32025800 & 0.81901300 \\ \mathrm{C} & 0.25522900 & 1.83854300 & -0.35533200\end{array}$

$\begin{array}{llll}\text { C } & 0.18862300 & 1.98938000 & -1.87074500\end{array}$

$\begin{array}{llll}\text { C } & 1.54551200 & 2.42068600 & 0.20671600\end{array}$

$\mathrm{H} \quad-0.59353600 \quad 2.37414200 \quad 0.09590400$

$\mathrm{H} \quad-\quad-0.75177000 \quad 1.60820100 \quad-2.29106000$

$\mathrm{H} \quad 0.27465200 \quad 3.04522900 \quad-2.16539900$

$\mathrm{H} \quad 1.01115000 \quad 1.45050900 \quad-2.36159300$

$\begin{array}{llll}\mathrm{H} & 1.58146000 & 2.36133700 & 1.30236300\end{array}$

$\mathrm{H} \quad 2.43065900 \quad 1.89779600 \quad-0.17939000$

$\mathrm{H} \quad \begin{array}{llll}\mathrm{H} & 1.64606900 & 3.47926100 & -0.07293900\end{array}$

C $\quad 0.99516000 \quad-1.32597200 \quad-0.90608000$

C $\quad 0.76004700 \quad-2.73063700 \quad-0.36376700$

C $\quad 2.47994200 \quad-0.99168600 \quad-0.90408200$

$\mathrm{H} \quad 0.61213300 \quad-1.26689200 \quad-1.93850400$

$\mathrm{H} \quad-0.29986300 \quad-3.01653400 \quad-0.37001200$

$\mathrm{H} \quad 1.30710400 \quad-3.47972500 \quad-0.95366100$

$\mathrm{H} \quad \begin{array}{llll}1.11706300 & -2.80837500 & 0.67322300\end{array}$

$\begin{array}{llll}\mathrm{H} & 2.69115300 & -0.02030600 & -1.36910000\end{array}$

$\mathrm{H} \quad 2.87329700 \quad-0.96156700 \quad 0.12208900$

$\mathrm{H} \quad 3.05572200 \quad-1.75051400 \quad-1.45329900$

Ge $\quad-0.05457700 \quad-0.04014900 \quad 0.12300900$

\section{TS (iPr) $)_{3} \mathrm{GeS} \cdot-\mathbf{I}_{1}$}

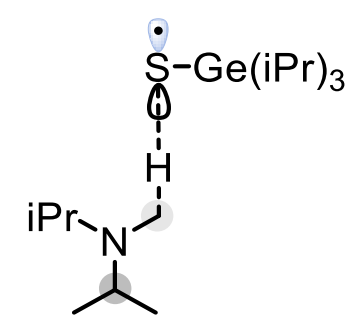

$$
\mathrm{E}=
$$

$\mathrm{G}_{\text {Corr. }}=$

$\begin{array}{cccc}\mathrm{C} & -3.86468972 & -2.29018737 & -0.90627693 \\ \mathrm{~N} & -3.22358100 & -0.17995600 & 0.33423900 \\ \mathrm{C} & -2.13298100 & -0.90991800 & 0.74330000 \\ \mathrm{H} & -1.64136600 & -0.53939400 & 1.64605400 \\ \mathrm{H} & -2.23640800 & -1.99447800 & 0.68354700 \\ \mathrm{C} & -4.17281300 & -0.76132400 & -0.62356600 \\ \mathrm{C} & -5.05971800 & -1.79363000 & 0.06135300 \\ \mathrm{C} & -3.48437700 & -1.33734200 & -1.85542600 \\ \mathrm{H} & -4.81298500 & 0.06727800 & -0.95463600 \\ \mathrm{H} & -5.58583500 & -1.35316900 & 0.91601100\end{array}$

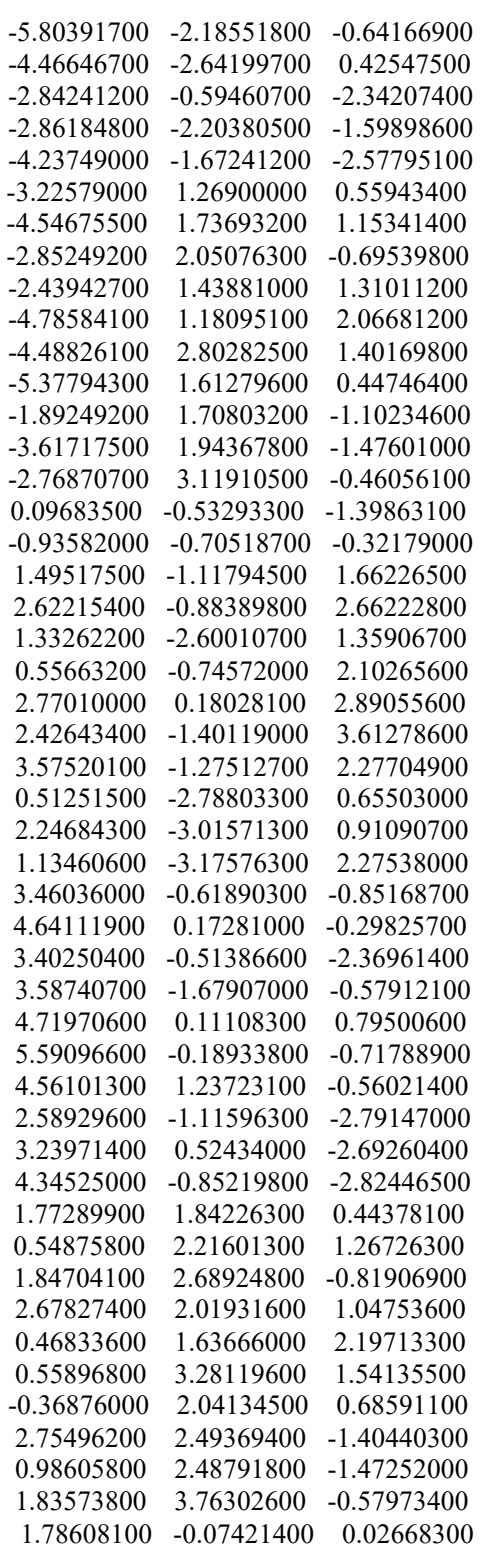

(iPr) ${ }_{3} \mathrm{CS}$.<smiles>[SH2+][AsH2]</smiles>

$E=-791.6594977$

$\mathrm{G}_{\text {Corr. }}=0.250675$

$\begin{array}{lrrr}\mathrm{S} & -0.24571600 & 0.35152300 & 1.92541600 \\ \mathrm{C} & 1.56535100 & 0.40237900 & -0.06206400 \\ \mathrm{C} & 2.05846200 & 0.26334300 & -1.50130300 \\ \mathrm{C} & 2.51735900 & -0.35790400 & 0.86247100 \\ \mathrm{H} & 1.65610800 & 1.46098300 & 0.21681400 \\ \mathrm{H} & 1.42945300 & 0.79085700 & -2.22746100 \\ \mathrm{H} & 3.06870600 & 0.68387200 & -1.57677600 \\ \mathrm{H} & 2.12459800 & -0.78877000 & -1.80643400 \\ \mathrm{H} & 2.29189700 & -0.19844000 & 1.92482400 \\ \mathrm{H} & 2.51037200 & -1.43964300 & 0.67911600 \\ \mathrm{H} & 3.54344400 & -0.00915000 & 0.69391000 \\ \mathrm{C} & -0.21044700 & -1.46339400 & -0.26023100 \\ \mathrm{C} & -0.60951900 & -1.68803100 & -1.72405500 \\ \mathrm{C} & -1.18929700 & -2.23431800 & 0.62747400 \\ \mathrm{H} & 0.75711900 & -1.97049200 & -0.12920000 \\ \mathrm{H} & 0.03842900 & -1.17480200 & -2.44079200 \\ \mathrm{H} & -0.55309200 & -2.76052000 & -1.94651100\end{array}$ 


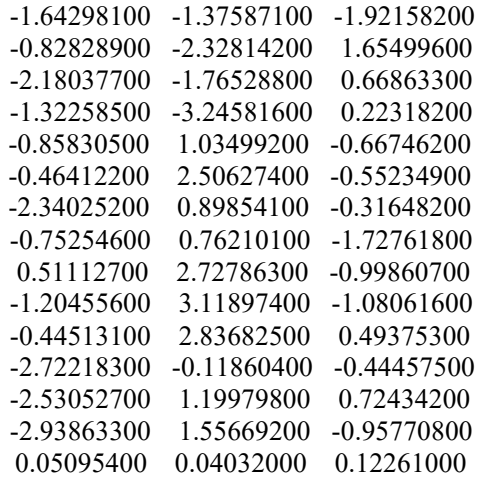

TS $(i \mathrm{Pr})_{3} \mathrm{CS} \cdot \mathbf{I}_{1}$<smiles>CC(C)N(CC=CC(C)(C)C(C)(C)C)C(C)C</smiles>

$\mathrm{E}=-1123.220647$

$\mathrm{G}_{\text {Corr. }}=0.460532$

N $\quad-2.90944600 \quad-0.09012600 \quad 0.37560500$

$\begin{array}{llll}\mathrm{C} & -1.74352000 & -0.55660200 & 0.94369500\end{array}$

$\mathrm{H} \quad-1.41714900 \quad-0.00268100 \quad 1.82747600$

$\mathrm{H} \quad-1.66754600-1.64267500 \quad 1.03511600$

$\begin{array}{llll}\text { C } & -3.64764500 & -0.93812700 & -0.56857500\end{array}$

$\begin{array}{llll}\text { C } & -4.40271300 & -2.03389400 & 0.17382000\end{array}$

$\begin{array}{llll}\text { C } & -2.75707400 & -1.51234300 & -1.66418200\end{array}$

$\mathrm{H} \quad-\quad-4.38778000 \quad-0.28396400 \quad-1.04851400$

$\mathrm{H} \quad \begin{array}{rrrr}-5.07426400 & -1.60565600 & 0.92670000\end{array}$

$\begin{array}{llll}\mathrm{H} & -4.99902400 & -2.63080800 & -0.52606700\end{array}$

$\mathrm{H} \quad-3.70810800 \quad-2.71402800 \quad 0.68314700$

$\mathrm{H} \quad-2.22242200 \quad-0.72277500 \quad-2.20446800$

$\mathrm{H} \quad-2.00927700 \quad-2.20262200 \quad-1.25330200$

$\mathrm{H} \quad-3.36662300 \quad-2.07145900 \quad-2.38356900$

$\begin{array}{llll}\text { C } & -3.18036100 & 1.35091400 & 0.41023300\end{array}$

$\begin{array}{llll}\text { C } & -4.62085500 & 1.63632100 & 0.81196200\end{array}$

$\begin{array}{llll}\mathrm{C} & -2.80221700 & 2.04331700 & -0.89462200\end{array}$

$\begin{array}{llll}\mathrm{H} & -2.52710500 & 1.74298900 & 1.20355100\end{array}$

$\begin{array}{llll}\mathrm{H} & -4.86921000 & 1.14625000 & 1.75999400\end{array}$

$\begin{array}{llll}\mathrm{H} & -4.76801500 & 2.71580300 & 0.93116900\end{array}$

$\mathrm{H} \quad \begin{array}{llll}\mathrm{H} & -5.33332300 & 1.29413900 & 0.05049700\end{array}$

$\mathrm{H} \quad-\quad-1.75084200 \quad 1.85678100 \quad-1.14820900$

$\mathrm{H} \quad \begin{array}{llll}-3.42751600 & 1.69379600 & -1.72692500\end{array}$

$\mathrm{H} \quad-2.95055700 \quad 3.12603800 \quad-0.80369300$

S $\quad 0.52367600 \quad-0.02304500 \quad-1.07003500$

$\mathrm{H} \quad-0.54657700 \quad-0.28283300 \quad-0.01087100$

$\begin{array}{llll}\mathrm{C} & 1.77395900 & -1.10557300 & 1.15492800\end{array}$

$\begin{array}{lll}2.83824500 & -1.19166200 & 2.25279200\end{array}$

$\begin{array}{lll}1.49934300 & -2.52750000 & 0.66346400\end{array}$

$\begin{array}{lll}0.85072700 & -0.77484400 & 1.65007900\end{array}$

$\begin{array}{lll}3.11059700 & -0.22046100 & 2.68048300\end{array}$

$\begin{array}{lll}2.45666000 & -1.81370600 & 3.07251000\end{array}$

$\begin{array}{lll}3.75433000 & -1.67493400 & 1.89072100\end{array}$

$\begin{array}{lll}0.77271300 & -2.55345200 & -0.15534300\end{array}$

$\begin{array}{lll}2.41384800 & -3.02962700 & 0.32132500\end{array}$

$\begin{array}{lll}1.10245600 & -3.12611000 & 1.49382400\end{array}$

$\begin{array}{lll}3.27208400 & -0.48090900 & -0.84903500\end{array}$

$\begin{array}{llll}4.63717700 & -0.05432000 & -0.29333800\end{array}$

$\begin{array}{lll}3.23273500 & -0.10856200 & -2.33457600\end{array}$

$3.25943400-1.58076000-0.83338700$

$\begin{array}{llll}4.76900000 & -0.26732400 & 0.77088900\end{array}$

$\begin{array}{lll}5.42598500 & -0.59256700 & -0.83355200\end{array}$

$\begin{array}{llll}4.82328500 & 1.01667300 & -0.44333200\end{array}$

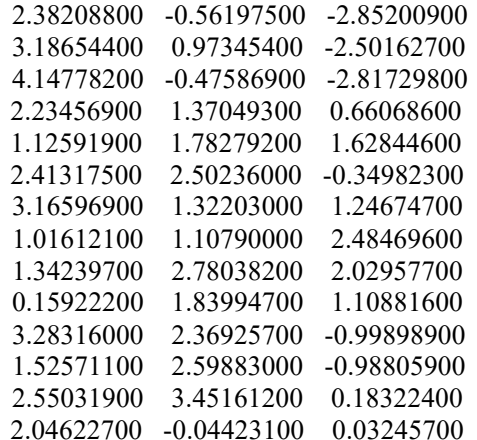

NEtMe $_{2}$

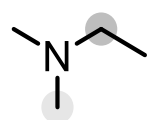

$E=-213.686664$

$\mathrm{H}_{\text {Corr. }}=0.118215$

$\begin{array}{llll}\mathrm{N} & 0.45665100 & -0.00296100 & -0.34575800\end{array}$

$\begin{array}{llll}\text { C } & -0.69783600 & -0.65112700 & 0.26487300\end{array}$

$\mathrm{H} \quad-0.68238200 \quad-1.70399600 \quad-0.04908500$

$\begin{array}{llll}\mathrm{H} & -0.59580600 & -0.65256800 & 1.37375100\end{array}$

$\begin{array}{llll}\mathrm{C} & 1.66283000 & -0.75065900 & -0.04890100\end{array}$

$\mathrm{H} \quad \begin{array}{llll}\mathrm{H} & 1.56835000 & -1.77965100 & -0.41430100\end{array}$

$\mathrm{H} \quad 2.52410600-0.28890300 \quad-0.54480200$

$\begin{array}{llll}\mathrm{H} & 1.87550400 & -0.79074300 & 1.04029900\end{array}$

$\begin{array}{llll}\mathrm{C} & 0.60387600 & 1.37117900 & 0.09483400\end{array}$

$\mathrm{H} \quad 1.50206900 \quad 1.80813100 \quad-0.35531700$

$\mathrm{H} \quad-\quad-0.25227600 \quad 1.97797400 \quad-0.21606100$

$\begin{array}{llll}\mathrm{H} & 0.70144900 & 1.44674800 & 1.19895400\end{array}$

C $\quad-2.02318500 \quad-0.03029100 \quad-0.13001900$

$\begin{array}{llll}\mathrm{H} & -2.85262200 & -0.64844900 & 0.22965500\end{array}$

$\mathrm{H} \quad-2.15778400 \quad 0.97117500 \quad 0.29353900$

$\mathrm{H} \quad-2.10127300 \quad 0.04640000 \quad-1.22104500$

${ }^{N E t M e} \mathbf{I}_{1}$

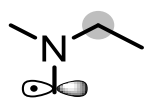

$\mathrm{E}=-213.029550$

$\mathrm{H}_{\text {Corr. }}=0.103418$

$\mathrm{N} \quad 0.52330700 \quad-0.06449000 \quad-0.17053700$

C $\quad-0.71818700 \quad-0.61427400 \quad 0.35924300$

$\mathrm{H} \quad-0.71714300 \quad-1.68703700 \quad 0.12978700$

$\mathrm{H} \quad-0.72385100 \quad-0.52521800 \quad 1.46304200$

C $\quad \begin{array}{llll}\text { C } & 1.64059200 & -0.87525200 & -0.08259300\end{array}$

$\mathrm{H} \quad \begin{array}{llll}1.48389500 & -1.94454100 & -0.19520700\end{array}$

H $\quad 2.59027600 \quad-0.44166400 \quad-0.38306100$

$\begin{array}{llll}\mathrm{C} & 0.73331500 & 1.34528500 & 0.08890100\end{array}$

$\begin{array}{llll}\mathrm{H} & 1.67446300 & 1.66323200 & -0.36938800\end{array}$

$\mathrm{H} \quad-0.07244400 \quad 1.94529400 \quad-0.34482500$

$\begin{array}{llll}\mathrm{H} & 0.78493500 & 1.55606600 & 1.17239400\end{array}$

$\begin{array}{llll}\mathrm{C} & -1.95967100 & 0.03037500 & -0.22507800\end{array}$

$\mathrm{H} \quad-2.85264900 \quad-0.50553700 \quad 0.11316300$

$\begin{array}{llll}\mathrm{H} & -2.07174500 & 1.07500900 & 0.08567200\end{array}$

$\mathrm{H} \quad-1.93517600 \quad-0.00098600 \quad-1.32066000$

${ }_{\text {NEtMe2 }} \mathbf{I}_{2}$ 
<smiles>CC(=O)N(C)C</smiles>

$\mathrm{E}=-213.031943$

$\mathrm{H}_{\text {Corr. }}=0.103496$

N $\quad 0.45556700 \quad-0.03126800 \quad-0.24330700$

$\begin{array}{llll}\mathrm{C} & -0.69506500 & -0.72549700 & 0.12599600\end{array}$

$\begin{array}{llll}\mathrm{H} & -0.60772400 & -1.80665000 & 0.02566500\end{array}$

$\begin{array}{llll}\mathrm{C} & 0.50869100 & 1.38723700 & 0.04834700\end{array}$

$\mathrm{H} \quad \begin{array}{llll}\mathrm{H} & 1.39087700 & 1.82341500 & -0.43184100\end{array}$

$\mathrm{H} \quad-0.37587500 \quad 1.89750500 \quad-0.34314400$

$\begin{array}{llll}\mathrm{H} & 0.57210300 & 1.58288200 & 1.13558100\end{array}$

C $\quad-2.03115100 \quad-0.08967300 \quad-0.00914200$

$\mathrm{H} \quad-2.81365300 \quad-0.81605900 \quad 0.23023100$

$\begin{array}{llll}\mathrm{H} & -2.17090400 & 0.76893800 & 0.66365900\end{array}$

$\begin{array}{llll}\mathrm{H} & -2.22874000 & 0.27844700 & -1.03341700\end{array}$

$\begin{array}{llll}\mathrm{C} & 1.70563400 & -0.71034800 & 0.02950300\end{array}$

$\mathrm{H} \quad \begin{array}{llll}1.63900900 & -1.75401400 & -0.29483100\end{array}$

$\mathrm{H} \quad 2.52023800 \quad-0.22908100 \quad-0.52275800$

$\mathrm{H} \quad 1.95704600 \quad-0.69682100 \quad 1.10577700$

\section{Me-pyperidine}<smiles>CN1CCCCC1</smiles>

$\mathrm{E}=-291.077792$

$\mathrm{H}_{\text {Corr. }}=0.154896$

C $\quad-1.15067600 \quad-1.24747900 \quad-0.22619600$

$\begin{array}{llll}\text { C } & 0.31474000 & -1.20075300 & 0.17220100\end{array}$

$\begin{array}{llll}\text { C } & 0.31474300 & 1.20075000 & 0.17221200\end{array}$

$\begin{array}{llll}\text { C } & -1.15066600 & 1.24748100 & -0.22620500\end{array}$

$\begin{array}{llll}\mathrm{C} & -1.87776100 & 0.00000600 & 0.25965200\end{array}$

$\mathrm{H} \quad 0.39717900 \quad-1.24288000 \quad 1.28375700$

$\mathrm{H} \quad \begin{array}{llll}0.85164900 & -2.07529200 & -0.21951100\end{array}$

$\mathrm{H} \quad-1.21834700 \quad-1.31125400 \quad-1.32252800$

$\begin{array}{llll}\mathrm{H} & -1.61303600 & -2.15597300 & 0.18079000\end{array}$

$\mathrm{H} \quad \begin{array}{llll}0.39717500 & 1.24285900 & 1.28376800\end{array}$

$\begin{array}{llll}\mathrm{H} & 0.85166300 & 2.07529000 & -0.21948300\end{array}$

$\mathrm{H} \quad-1.61303100 \quad 2.15598500 \quad 0.18075100$

$\mathrm{H} \quad-1.21831700 \quad 1.31123400 \quad-1.32254100$

$\begin{array}{llll}\mathrm{H} & -1.89987300 & 0.00000900 & 1.36169900\end{array}$

$\begin{array}{llll}\mathrm{H} & -2.92219200 & 0.00000700 & -0.07712600\end{array}$

$\mathrm{N} \quad 0.96701400 \quad 0.00000000 \quad-0.33383200$

$\begin{array}{llll}\mathrm{C} & 2.37452600 & -0.00000100 & 0.00393900\end{array}$

$\mathrm{H} \quad 2.86262900 \quad-0.88826300 \quad-0.41395400$

$\mathrm{H} \quad 2.86262800 \quad 0.88827000 \quad-0.41393500$

$\begin{array}{llll}\mathrm{H} & 2.54334500 & -0.00001400 & 1.10151900\end{array}$

\section{Me-pyperidine $\mathbf{I}_{1}$}<smiles>CC(=O)N1CCCCC1</smiles>

$$
\mathrm{E}=-290.420603
$$

$$
\mathrm{H}_{\text {Corr. }}=0.140606
$$

$\begin{array}{llll}\text { C } & 1.08387200 & 1.24878100 & -0.25846600\end{array}$

$\begin{array}{llll}\mathrm{C} & -0.34508400 & 1.22105900 & 0.25641400\end{array}$

C $\quad-0.34508400-1.22105900 \quad 0.25641400$

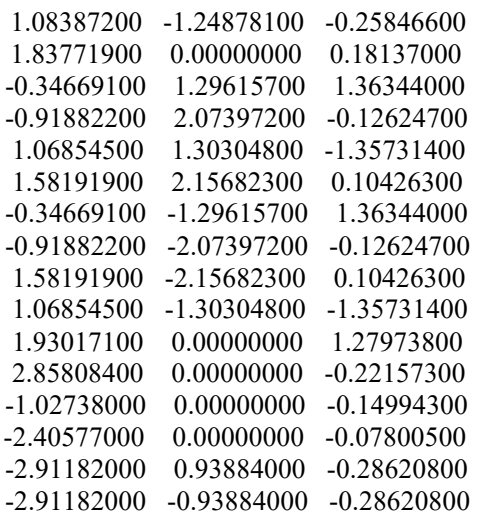

Me-pyperidine $\mathbf{I}_{2}$<smiles>CC1CCCCN1C</smiles>

$\mathrm{E}=-290.422432$

$\mathrm{H}_{\text {Corr. }}=0.140366$

C $\quad-1.13357800 \quad-1.21542300 \quad-0.27099400$

C $\quad 0.32935700 \quad-1.20467700 \quad 0.13303200$

$\begin{array}{llll}\mathrm{C} & 0.29510200 & 1.21887300 & 0.09454700\end{array}$

C $\quad-1.17419600 \quad 1.26801500 \quad-0.14952400$

$\begin{array}{llll}\mathrm{C} & -1.85787900 & -0.01347300 & 0.31918700\end{array}$

$\mathrm{H} \quad 0.41131600 \quad-1.35887700 \quad 1.22991000$

$\mathrm{H} \quad 0.87726600 \quad-2.02318300 \quad-0.35203300$

$\begin{array}{lll}-1.20544400 & -1.18587400 & -1.36920000\end{array}$

$\begin{array}{lll}-1.59010700 & -2.15734400 & 0.05774200\end{array}$

$\begin{array}{lll}0.88893500 & 2.12911600 & 0.01657600\end{array}$

$\begin{array}{lll}-1.60094100 & 2.14923100 & 0.34544900\end{array}$

$\begin{array}{lll}-1.38491500 & 1.39296600 & -1.23196100\end{array}$

$\begin{array}{lll}-1.81793000 & -0.06933700 & 1.41832200\end{array}$

$\begin{array}{lll}-2.91674200 & -0.01534900 & 0.03151200\end{array}$

$\begin{array}{llll}0.97995800 & 0.05037200 & -0.22891400\end{array}$

$\begin{array}{llll}2.39872100 & 0.05164600 & 0.05431700\end{array}$

$\begin{array}{llll}2.87845400 & -0.79812700 & -0.44392400\end{array}$

$\begin{array}{lll}2.85301100 & 0.97409800 & -0.32195900\end{array}$

$\begin{array}{lll}2.60222700 & -0.01968800 & 1.13857400\end{array}$

\section{Et-pyperidine}<smiles>CCN1CCCCC1</smiles>

$\mathrm{E}=-330.371275$

$\mathrm{H}_{\text {Corr. }}=0.180962$

C $\quad-1.78811400 \quad-1.04123400 \quad-0.35270100$

$\begin{array}{llll}\text { C } & -0.38644800 & -1.30431100 & 0.16988200\end{array}$

$\begin{array}{llll}\mathrm{C} & 0.08200800 & 1.04119900 & 0.32449900\end{array}$

C $\quad-1.30514000 \quad 1.39457900 \quad-0.18678800$

$\begin{array}{llll}\mathrm{C} & -2.30493200 & 0.29842600 & 0.15369200\end{array}$

$\begin{array}{llll}\mathrm{H} & -0.42284900 & -1.41297200 & 1.27911200\end{array}$

$\mathrm{H} \quad \begin{array}{llll}0.00089200 & -2.25144600 & -0.22923800\end{array}$

$\mathrm{H} \quad-1.76208900-1.03556100 \quad-1.45272400$

$\mathrm{H} \quad-2.45157900 \quad-1.86100100 \quad-0.04824500$

$\begin{array}{llll}\mathrm{H} & 0.07040100 & 1.01240500 & 1.43963800\end{array}$

$\begin{array}{lll}0.79425300 & 1.82183500 & 0.03273800\end{array}$

$\begin{array}{lll}-1.61498200 & 2.35754000 & 0.23936200\end{array}$

$\begin{array}{llll}-1.25712400 & 1.52386000 & -1.27847500\end{array}$ 


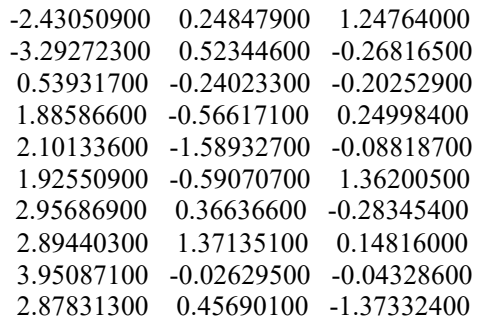

Et-pyperidine $\mathbf{I}_{1}$<smiles>CC(C)N1CCCCC1</smiles>

$\mathrm{E}=-329.716513$

\section{$\mathrm{H}_{\text {Corr. }}=0.166446$}

$\begin{array}{lrrr}\mathrm{C} & 1.78219300 & -1.02230300 & 0.32185500 \\ \mathrm{C} & 0.40809100 & -1.32585400 & -0.24882300 \\ \mathrm{C} & -0.14589000 & 1.03902900 & -0.32965300 \\ \mathrm{C} & 1.21918000 & 1.40366300 & 0.23095700 \\ \mathrm{C} & 2.25748800 & 0.35122400 & -0.13266300 \\ \mathrm{H} & 0.47506800 & -1.41577900 & -1.35333600 \\ \mathrm{H} & 0.03183800 & -2.28427200 & 0.12994900 \\ \mathrm{H} & 1.72981100 & -1.05088300 & 1.42061100 \\ \mathrm{H} & 2.48492800 & -1.80549000 & 0.00988800 \\ \mathrm{H} & -0.11774600 & 1.07903200 & -1.43991000 \\ \mathrm{H} & -0.89533200 & 1.76631100 & -0.00027300 \\ \mathrm{H} & 1.51048400 & 2.39178400 & -0.14763700 \\ \mathrm{H} & 1.14447800 & 1.48466200 & 1.32578700 \\ \mathrm{H} & 2.39889800 & 0.34271500 & -1.22578900 \\ \mathrm{H} & 3.23120900 & 0.59490000 & 0.31039900 \\ \mathrm{~N} & -0.55807200 & -0.29168700 & 0.10241500 \\ \mathrm{C} & -1.89345700 & -0.64748300 & -0.07174900 \\ \mathrm{H} & -2.09334100 & -1.70466500 & 0.09870500 \\ \mathrm{C} & -2.98165000 & 0.34000200 & 0.14951400 \\ \mathrm{H} & -2.99857800 & 1.14596100 & -0.59887000 \\ \mathrm{H} & -3.95185300 & -0.16348100 & 0.09863400 \\ \mathrm{H} & -2.91908600 & 0.83135400 & 1.13830600\end{array}$

\section{Et-pyperidine $\mathbf{I}_{2}$}<smiles>CCN1CCCCC1</smiles>

$\mathrm{E}=-329.715910$

$$
\mathrm{H}_{\text {Corr. }}=0.16626
$$

$\begin{array}{lrrr}\mathrm{C} & 1.77749100 & -1.00613200 & 0.39009200 \\ \mathrm{C} & 0.38707000 & -1.31629400 & -0.13295200 \\ \mathrm{C} & -0.07432000 & 1.05136400 & -0.25335600 \\ \mathrm{C} & 1.32458100 & 1.41817500 & 0.10761300 \\ \mathrm{C} & 2.29880300 & 0.28932200 & -0.21466300 \\ \mathrm{H} & 0.44258500 & -1.53116200 & -1.22107100 \\ \mathrm{H} & -0.02092800 & -2.21075200 & 0.35539300 \\ \mathrm{H} & 1.73756900 & -0.91080100 & 1.48622500 \\ \mathrm{H} & 2.44189200 & -1.84928700 & 0.16275000 \\ \mathrm{H} & -0.82938200 & 1.83163400 & -0.31255800 \\ \mathrm{H} & 1.60534200 & 2.34489200 & -0.40798900 \\ \mathrm{H} & 1.39976200 & 1.63688300 & 1.19307700 \\ \mathrm{H} & 2.37823300 & 0.17428300 & -1.30700400 \\ \mathrm{H} & 3.30270500 & 0.52212000 & 0.16233100\end{array}$

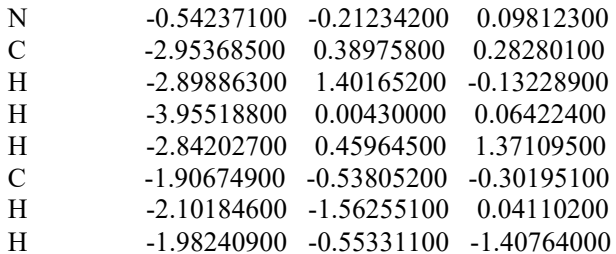

\section{$\operatorname{NEt}(i \operatorname{Pr})_{2}$}<smiles>CCN(C(C)C)C(C)C</smiles>

$$
E=-370.859482
$$

$\mathrm{H}_{\text {Corr. }}=0.222521$

$\begin{array}{lrrr}\mathrm{N} & -0.01030600 & 0.26664800 & 0.15228900 \\ \mathrm{C} & -0.39931500 & 1.40802600 & -0.66088400 \\ \mathrm{H} & -1.48929700 & 1.39264200 & -0.78522300 \\ \mathrm{H} & 0.01771800 & 1.34627700 & -1.68682600 \\ \mathrm{C} & 1.40635500 & -0.09178200 & 0.04873900 \\ \mathrm{C} & 1.76526300 & -0.99972400 & -1.12901100 \\ \mathrm{C} & 1.92363500 & -0.67100400 & 1.35913300 \\ \mathrm{H} & 1.93450600 & 0.86157500 & -0.10722400 \\ \mathrm{H} & 1.40279200 & -0.59240400 & -2.08101100 \\ \mathrm{H} & 2.85410900 & -1.10945000 & -1.20669600 \\ \mathrm{H} & 1.34365100 & -2.00587500 & -1.00271600 \\ \mathrm{H} & 1.71133000 & 0.01112000 & 2.19028500 \\ \mathrm{H} & 1.45789500 & -1.63902100 & 1.58719300 \\ \mathrm{H} & 3.00675700 & -0.83730700 & 1.30836200 \\ \mathrm{C} & -0.97321700 & -0.83722200 & 0.22124600 \\ \mathrm{C} & -1.58547500 & -1.27053200 & -1.11401500 \\ \mathrm{C} & -2.06952400 & -0.50537800 & 1.22711800 \\ \mathrm{H} & -0.41924600 & -1.70041600 & 0.61864500 \\ \mathrm{H} & -0.82191500 & -1.47124700 & -1.87319600 \\ \mathrm{H} & -2.17372200 & -2.18629800 & -0.97625700 \\ \mathrm{H} & -2.26702900 & -0.50838800 & -1.51345200 \\ \mathrm{H} & -1.63686700 & -0.31496400 & 2.21600500 \\ \mathrm{H} & -2.62045000 & 0.39529400 & 0.92209100 \\ \mathrm{H} & -2.79530800 & -1.32439100 & 1.31068000 \\ \mathrm{C} & -0.01445200 & 2.73310800 & -0.02281400 \\ \mathrm{H} & 1.06646300 & 2.80666400 & 0.14821400 \\ \mathrm{H} & -0.30583300 & 3.57655900 & -0.66038000 \\ \mathrm{H} & -0.51302800 & 2.84014300 & 0.94841500\end{array}$

$\operatorname{NEt}(i \operatorname{Pr}) \mathbf{I}_{1}$<smiles>CC(C)N(C(C)C)C(C)C</smiles>

$\mathrm{E}=-370.209413$

$\mathrm{H}_{\text {Corr. }}=0.2081$

$\begin{array}{llll}\mathrm{N} & 0.01600000 & 0.23155900 & 0.22770900\end{array}$

C $\quad 0.08725300 \quad 1.59706500 \quad-0.03512000$

$\begin{array}{llll}\mathrm{H} & -0.87255600 & 2.10913800 & -0.01960800\end{array}$

$\begin{array}{llll}\mathrm{C} & 1.23744100 & -0.57201000 & 0.11632500\end{array}$

C $\quad \begin{array}{llll}\text { C } & 1.52966400 & -1.00560400 & -1.31777700\end{array}$

$\begin{array}{llll}\mathrm{C} & 1.23337800 & -1.75568000 & 1.07436000\end{array}$

$\begin{array}{llll}\mathrm{H} & 2.05343700 & 0.08679000 & 0.43996200\end{array}$

$\mathrm{H} \quad 1.54020800 \quad-0.13778500 \quad-1.98928400$

$\mathrm{H} \quad 2.50448200-1.50472600 \quad-1.38153000$

$\mathrm{H} \quad \begin{array}{llll}0.77155800 & -1.71237300 & -1.68150100\end{array}$

$\mathrm{H} \quad 0.99552600 \quad-1.43162500 \quad 2.09403700$

$\mathrm{H} \quad 0.51224600 \quad-2.52957700 \quad 0.78264800$ 


$\begin{array}{lrrr}\mathrm{H} & 2.22298400 & -2.22751700 & 1.08342100 \\ \mathrm{C} & -1.26693200 & -0.43571200 & -0.03503700 \\ \mathrm{C} & -1.89471100 & -0.06988900 & -1.37878400 \\ \mathrm{C} & -2.24001000 & -0.19141100 & 1.11215700 \\ \mathrm{H} & -1.05268300 & -1.51216900 & -0.06190000 \\ \mathrm{H} & -1.17782800 & -0.18115800 & -2.19994200 \\ \mathrm{H} & -2.75254000 & -0.72382100 & -1.57724100 \\ \mathrm{H} & -2.25841100 & 0.96448300 & -1.38810200 \\ \mathrm{H} & -1.81523700 & -0.53770800 & 2.06147400 \\ \mathrm{H} & -2.46603100 & 0.87806000 & 1.21199800 \\ \mathrm{H} & -3.18620700 & -0.71869600 & 0.93910300 \\ \mathrm{C} & 1.30630600 & 2.38507100 & 0.28994500 \\ \mathrm{H} & 2.17417400 & 2.13423000 & -0.34007700 \\ \mathrm{H} & 1.10296600 & 3.44973200 & 0.13727000 \\ \mathrm{H} & 1.63757300 & 2.26282900 & 1.33890500\end{array}$

\section{$\operatorname{NEt}(i \operatorname{Pr}){ }^{2} \mathbf{I}_{2}$<smiles>CCN(CC)N(C)C</smiles>

$\mathrm{E}=-370.205607$

$$
\mathrm{H}_{\text {Corr. }}=0.206858
$$

$\mathrm{N} \quad \begin{array}{llll}-0.29638700 & 0.22939400 & -0.20477600\end{array}$

$\begin{array}{llll}\text { C } & -0.44269900 & -1.14369100 & 0.33826700\end{array}$

C $\quad 0.42466200 \quad-1.36434800 \quad 1.57230600$

C $\quad-0.21860300 \quad-2.22094200 \quad-0.71251400$

$\begin{array}{llll}\mathrm{H} & -1.49415000 & -1.22123500 & 0.65315800\end{array}$

$\mathrm{H} \quad 0.23634400 \quad-0.59489500 \quad 2.33191200$

$\begin{array}{llll}\mathrm{H} & 0.21982900 & -2.34363400 & 2.02184100\end{array}$

$\mathrm{H} \quad \begin{array}{llll}\mathrm{H} & 1.49179800 & -1.33130700 & 1.31449000\end{array}$

$\mathrm{H} \quad-0.83174500 \quad-2.02518200 \quad-1.60034300$

$\mathrm{H} \quad 0.83049300 \quad-2.27328100 \quad-1.02582800$

$\mathrm{H} \quad-0.49238200 \quad-3.20430200 \quad-0.31012000$

$\begin{array}{llll}\mathrm{C} & 0.99979300 & 0.76339900 & -0.32161700\end{array}$

$\begin{array}{llll}\mathrm{C} & 1.55563500 & 1.73900100 & 0.66667000\end{array}$

$\begin{array}{llll}\text { C } & 1.96586600 & 0.13427500 & -1.26412700\end{array}$

$\begin{array}{llll}\mathrm{H} & 0.79554800 & 2.14843300 & 1.34064000\end{array}$

$\mathrm{H} \quad 2.32457100 \quad 1.26127300 \quad 1.30425500$

$\begin{array}{llll}\mathrm{H} & 2.05847300 & 2.58385900 & 0.17224800\end{array}$

$\mathrm{H} \quad \begin{array}{llll}\mathrm{H} & 1.45905400 & -0.29896700 & -2.13433300\end{array}$

$\mathrm{H} \quad 2.68539500 \quad 0.88444500 \quad-1.62373600$

$\mathrm{H} \quad 2.57587200 \quad-0.66668000 \quad-0.80271000$

$\begin{array}{llll}\text { C } & -2.65901000 & 0.90203200 & -0.46722400\end{array}$

$\mathrm{H} \quad-3.03554800 \quad-0.11560300 \quad-0.30695000$

$\mathrm{H} \quad-3.42724300 \quad 1.59780900 \quad-0.10943600$

$\begin{array}{lllll}\mathrm{H} & -2.53498900 & 1.04539000 & -1.54680000\end{array}$

$\begin{array}{llll}\mathrm{C} & -1.34058600 & 1.14119800 & 0.25002000\end{array}$

$\begin{array}{llll}\mathrm{H} & -1.00735500 & 2.16421500 & 0.03799400\end{array}$

$\mathrm{H} \quad-1.48961100 \quad 1.07837000 \quad 1.34647800$

\section{Benzydamine}<smiles></smiles>

$\mathrm{E}=-976.895668$

$\mathrm{H}_{\text {Corr. }}=0.410364$

$\mathrm{O}$ $\begin{array}{lll}-1.60801300 & 1.06745000 & 0.01901800\end{array}$ $\begin{array}{llll}1.55479400 & 0.31126900 & -1.10705400\end{array}$

$\begin{array}{llll}0.23037600 & -0.01654400 & -0.94976000\end{array}$

$\begin{array}{llll}-0.31144600 & 0.99058400 & -0.30141200\end{array}$

$\begin{array}{lll}0.64060500 & 2.00490100 & 0.01824100\end{array}$

$\begin{array}{llll}1.84667300 & 1.50415000 & -0.51129500\end{array}$

$3.19255200-1.45721000-0.51216400$

$\begin{array}{llll}-2.42170700 & -0.03823800 & -0.37723600\end{array}$

$\begin{array}{lll}-2.02611800 & -0.95884400 & 0.07848700\end{array}$

$\begin{array}{lll}-2.35911300 & -0.15904800 & -1.46887300\end{array}$

$\begin{array}{llll}2.73057800 & -1.47942500 & 0.80364900\end{array}$

$\begin{array}{lll}1.84561100 & -0.90645500 & 1.07612900\end{array}$

$\begin{array}{llll}2.48147200 & -0.67856100 & -1.59443600\end{array}$

$\begin{array}{llll}3.21613900 & -0.18145500 & -2.24343800\end{array}$

$\begin{array}{llll}1.90262300 & -1.35381000 & -2.23648400\end{array}$

$\begin{array}{llll}1.79395200 & 3.94114500 & 0.79879300\end{array}$

$\begin{array}{lll}1.80600200 & 4.90268100 & 1.30547800\end{array}$

$\begin{array}{llll}3.39640400 & -2.23003200 & 1.77061000\end{array}$

$\begin{array}{lll}3.02750900 & -2.23726400 & 2.79369700\end{array}$

$\begin{array}{llll}4.33240900 & -2.19296700 & -0.84433300\end{array}$

$\begin{array}{llll}4.70319100 & -2.17286400 & -1.86927300\end{array}$

$\begin{array}{lll}-3.83442900 & 0.24184800 & 0.07283900\end{array}$

$\begin{array}{lll}-3.82027700 & 0.45603500 & 1.14982600\end{array}$

$\begin{array}{llll}-4.21624100 & 1.13850100 & -0.43458300\end{array}$

$\begin{array}{llll}-4.73761000 & -0.93989700 & -0.23869200\end{array}$

$\begin{array}{llll}-4.57998700 & -1.24717200 & -1.28378800\end{array}$

$\begin{array}{llll}-4.44228600 & -1.80982200 & 0.39228000\end{array}$

$\begin{array}{llll}3.05420000 & 2.20459700 & -0.37734000\end{array}$

$\begin{array}{llll}3.99077800 & 1.80971600 & -0.76417600\end{array}$

$\begin{array}{llll}4.52859600 & -2.96615800 & 1.43226800\end{array}$

$\begin{array}{llll}5.04755700 & -3.55030000 & 2.18848900\end{array}$

$\begin{array}{lll}0.60735500 & 3.24119500 & 0.67522900\end{array}$

$\begin{array}{lll}-0.32526900 & 3.63055600 & 1.07674100\end{array}$

$\begin{array}{lll}3.00045200 & 3.42111700 & 0.27985000\end{array}$

$\begin{array}{lll}3.91640600 & 3.99418500 & 0.40476100\end{array}$

$\begin{array}{llll}4.99614300 & -2.94586300 & 0.11929700\end{array}$

$\begin{array}{llll}5.88323100 & -3.51301000 & -0.15343000\end{array}$

$\begin{array}{lll}-6.15426400 & -0.65031300 & -0.07984300\end{array}$

$\begin{array}{lll}-6.94622200 & -1.77448400 & -0.54191700\end{array}$

$\begin{array}{llll}-6.75489200 & -2.69457300 & 0.04891500\end{array}$

$\begin{array}{llll}-6.71779200 & -1.99031200 & -1.59183800\end{array}$

$\begin{array}{lll}-8.01333000 & -1.53804800 & -0.46534400\end{array}$

$\begin{array}{lll}-6.49289300 & -0.33453700 & 1.29567700\end{array}$

$\begin{array}{lll}-7.57230300 & -0.16736100 & 1.37860100\end{array}$

$\begin{array}{lll}-5.98932100 & 0.57991100 & 1.62482500\end{array}$

$\begin{array}{lll}-6.21685700 & -1.15472700 & 1.99169300\end{array}$

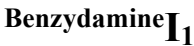<smiles>CN(C)CCCOc1nn(Cc2ccccc2)c2ccccc12</smiles>

$\mathrm{E}=-976.238166$

$\mathrm{H}_{\text {Corr. }}=0.397017$

$\begin{array}{llll}\mathrm{O} & -1.66536000 & 1.00675100 & 0.02336600\end{array}$

$\begin{array}{llll}1.50969700 & 0.31028600 & -1.10634800\end{array}$

$\begin{array}{llll}0.19232300 & -0.04209900 & -0.94640100\end{array}$

$\begin{array}{llll}-0.36730800 & 0.95559700 & -0.29892500\end{array}$

$\begin{array}{llll}0.56523600 & 1.98851900 & 0.01746100\end{array}$

$\begin{array}{llll}1.77975300 & 1.50965800 & -0.51329300\end{array}$

$\begin{array}{llll}3.18161900 & -1.42669000 & -0.51366600\end{array}$

$-2.45454800 \quad-0.11725500 \quad-0.36760300$

$\begin{array}{llll}-2.03894100 & -1.02788500 & 0.09008300\end{array}$

$\begin{array}{llll}-2.39269100 & -0.24074900 & -1.45899800\end{array}$

$\begin{array}{llll}2.72722500 & -1.45063300 & 0.80471700\end{array}$

$\begin{array}{lll}1.83548500 & -0.88959400 & 1.08003900\end{array}$

$\begin{array}{llll}2.45396800 & -0.66224500 & -1.59504600\end{array}$ 


$\begin{array}{lrrr}\mathrm{H} & 3.17774300 & -0.15152400 & -2.24568200 \\ \mathrm{H} & 1.88682200 & -1.34857200 & -2.23574900 \\ \mathrm{C} & 1.68365300 & 3.94685800 & 0.79368500 \\ \mathrm{H} & 1.67866000 & 4.90907000 & 1.29917700 \\ \mathrm{C} & 3.40931900 & -2.18766800 & 1.77081200 \\ \mathrm{H} & 3.04622200 & -2.19641100 & 2.79594800 \\ \mathrm{C} & 4.33019900 & -2.14708700 & -0.84947800 \\ \mathrm{H} & 4.69491600 & -2.12564600 & -1.87655800 \\ \mathrm{C} & -3.87156300 & 0.13559900 & 0.08605700 \\ \mathrm{H} & -3.85883800 & 0.34522100 & 1.16392700 \\ \mathrm{H} & -4.26963300 & 1.02781500 & -0.41653200 \\ \mathrm{C} & -4.75065700 & -1.06388500 & -0.22695200 \\ \mathrm{H} & -4.62806000 & -1.34005500 & -1.28340900 \\ \mathrm{H} & -4.42237400 & -1.93835600 & 0.37006600 \\ \mathrm{C} & 2.97429400 & 2.23253900 & -0.38202400 \\ \mathrm{H} & 3.91749800 & 1.85442600 & -0.76949500 \\ \mathrm{C} & 4.55028700 & -2.90838700 & 1.42893900 \\ \mathrm{H} & 5.08194400 & -3.48191800 & 2.18444800 \\ \mathrm{C} & 0.51007800 & 3.22501900 & 0.67291800 \\ \mathrm{H} & -0.42883100 & 3.59768500 & 1.07560600 \\ \mathrm{C} & 2.89890000 & 3.44864300 & 0.27363700 \\ \mathrm{H} & 3.80420500 & 4.03875700 & 0.39659300 \\ \mathrm{C} & 5.01018900 & -2.88638800 & 0.11328100 \\ \mathrm{H} & 5.90388500 & -3.44164900 & -0.16226800 \\ \mathrm{~N} & -6.16627500 & -0.81464900 & -0.00848400 \\ \mathrm{C} & -7.04451800 & -1.63456800 & -0.69569200 \\ \mathrm{H} & -6.74445100 & -1.95510000 & -1.68949900 \\ \mathrm{H} & -8.10073100 & -1.52539300 & -0.46699800 \\ \mathrm{C} & -6.53017500 & -0.48398800 & 1.35517900 \\ \mathrm{H} & -7.60857100 & -0.30947400 & 1.40980300 \\ \mathrm{H} & -6.02418900 & 0.42907400 & 1.68357900 \\ \mathrm{H} & -6.27310000 & -1.30033100 & 2.05358200\end{array}$

Benzydamine $\mathbf{I}_{2}$<smiles></smiles>

$E=-976.240946$

$\mathrm{H}_{\text {Corr. }}=0.397041$

$\begin{array}{llll}\mathrm{O} & -1.64271500 & 1.05973600 & 0.01280400\end{array}$

$\mathrm{N} \quad \begin{array}{llll}1.52121900 & 0.30317600 & -1.10936600\end{array}$

$\begin{array}{llll}\mathrm{N} & 0.19668800 & -0.02519700 & -0.95277800\end{array}$

$\begin{array}{llll}\text { C } & -0.34627400 & 0.98244000 & -0.30608200\end{array}$

$\begin{array}{llll}\text { C } & 0.60527900 & 1.99754700 & 0.01326700\end{array}$

C $\quad 1.81207700 \quad 1.49668200 \quad-0.51446300$

C $\quad 3.16382500-1.45739000 \quad-0.50578600$

C $\quad-2.45515400 \quad-0.04942000 \quad-0.37562200$

$\mathrm{H} \quad-2.06524300-0.96763900 \quad 0.08702600$

H $\quad-2.39582400 \quad-0.17649900 \quad-1.46655900$

$\begin{array}{llll}\mathrm{C} & 2.69274400 & -1.48623500 & 0.80675800\end{array}$

$\begin{array}{llll}\mathrm{H} & 1.79854700 & -0.92474800 & 1.07296800\end{array}$

C $\quad 2.44930100-0.68770600-1.59215200$

$\mathrm{H} \quad 3.18186500 \quad-0.19363400 \quad-2.24585000$

$\mathrm{H} \quad 1.87023600 \quad-1.36827700 \quad-2.22834800$

$\begin{array}{llll}\mathrm{C} & 1.75727400 & 3.93498400 & 0.79315200\end{array}$

$\begin{array}{lll}1.76845900 & 4.89696600 & 1.29904000\end{array}$

$3.36104500-2.22890800 \quad 1.77800100$

$2.98490500-2.24157100 \quad 2.79839000$

$\begin{array}{llll}4.31531300 & -2.17821300 & -0.83015900\end{array}$

$\begin{array}{lll}4.69334000 & -2.15247800 & -1.85231500\end{array}$

$\begin{array}{lll}-3.86862500 & 0.23591100 & 0.07336900\end{array}$

$\begin{array}{lll}-3.85658200 & 0.41616400 & 1.16025400\end{array}$

$-4.19957000 \quad 1.18778100-0.38691200$

$\begin{array}{llll}-4.76359800 & -0.89689500 & -0.29077600\end{array}$
$-4.49043100 \quad-1.52489200 \quad-1.14052700$

$\begin{array}{llll}3.01922600 & 2.19767000 & -0.37992600\end{array}$

$\begin{array}{lll}3.95632700 & 1.80263000 & -0.76536000\end{array}$

$\begin{array}{lll}4.50486800 & -2.95052600 & 1.44731200\end{array}$

$\begin{array}{llll}5.02569900 & -3.52857100 & 2.20693700\end{array}$

$\begin{array}{lll}0.57102000 & 3.23442500 & 0.66896000\end{array}$

$\begin{array}{llll}-0.36212800 & 3.62375900 & 1.06930500\end{array}$

$\begin{array}{llll}2.96446600 & 3.41487200 & 0.27599700\end{array}$

$3.88013700 \quad 3.98831200 \quad 0.40135600$

$\begin{array}{lll}4.98157700 & -2.92341800 & 0.13782800\end{array}$

$\begin{array}{llll}5.87781000 & -3.47898600 & -0.12879500\end{array}$

$\begin{array}{llll}-6.13292000 & -0.81568700 & -0.06368400\end{array}$

$\begin{array}{lll}-6.58181300 & -0.14782300 & 1.14185900\end{array}$

$\begin{array}{lll}-7.66944900 & -0.02846600 & 1.10472000\end{array}$

$\begin{array}{lll}-6.13630300 & 0.84767300 & 1.22341300\end{array}$

$\begin{array}{lll}-6.32529700 & -0.72222100 & 2.05142300\end{array}$

$\begin{array}{llll}-6.88659700 & -2.01643700 & -0.36379000\end{array}$

$\begin{array}{llll}-7.95460000 & -1.78047600 & -0.42271900\end{array}$

$\begin{array}{lll}-6.56905300 & -2.42125300 & -1.33015900\end{array}$

$\begin{array}{lll}-6.74175300 & -2.79687300 & 0.40461300\end{array}$

\section{Benzydamine $\mathbf{I}_{3}$}<smiles>CN(C)CCOc1nn(Cc2ccccc2)c2ccccc12</smiles>

$E=-976.232079$

$\mathrm{H}_{\text {Corr. }}=0.396224$

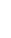

$\begin{array}{llll}-1.62281500 & 1.14168800 & -0.01936100\end{array}$

$\begin{array}{llll}1.53555900 & 0.31341500 & -1.11029800\end{array}$

$\begin{array}{llll}0.20448600 & 0.01771400 & -0.97679700\end{array}$

$\begin{array}{llll}-0.31723600 & 1.03353500 & -0.32765500\end{array}$

$\begin{array}{lll}0.64808300 & 2.02344700 & 0.01282600\end{array}$

$\begin{array}{llll}1.84898500 & 1.49625500 & -0.50388200\end{array}$

$\begin{array}{llll}3.14347500 & -1.48160800 & -0.52268500\end{array}$

$\begin{array}{lll}-2.47902700 & 0.13262500 & -0.37301200\end{array}$

$\begin{array}{llll}-2.03004100 & -0.83022300 & -0.60801800\end{array}$

$\begin{array}{llll}2.65205800 & -1.53532200 & 0.78169800\end{array}$

$\begin{array}{lll}1.75544700 & -0.97654000 & 1.04590500\end{array}$

$\begin{array}{lll}2.44566600 & -0.69011100 & -1.60390200\end{array}$

$\begin{array}{llll}3.18732900 & -0.20281400 & -2.25214200\end{array}$

$\begin{array}{llll}1.85320800 & -1.35262500 & -2.24655300\end{array}$

$\begin{array}{lll}1.83957500 & 3.92368100 & 0.82219400\end{array}$

$\begin{array}{llll}1.86912900 & 4.88101200 & 1.33599500\end{array}$

$\begin{array}{llll}3.30325900 & -2.29939500 & 1.74778400\end{array}$

$\begin{array}{lll}2.91150600 & -2.33158800 & 2.76181600\end{array}$

$\begin{array}{llll}4.29819700 & -2.19838400 & -0.84397500\end{array}$

$\begin{array}{lll}4.69231200 & -2.15274500 & -1.85926300\end{array}$

$\begin{array}{lll}-3.86431800 & 0.31374700 & 0.10798100\end{array}$

$\begin{array}{lll}-3.86167200 & 0.45228300 & 1.20438900\end{array}$

$\begin{array}{llll}-4.30209200 & 1.23560600 & -0.30973000\end{array}$

$\begin{array}{llll}3.07180100 & 2.16490400 & -0.34997800\end{array}$

$\begin{array}{lll}4.00281600 & 1.74906400 & -0.72795600\end{array}$

$\begin{array}{lll}4.45019000 & -3.01746400 & 1.41996500\end{array}$

$\begin{array}{lll}4.95777300 & -3.61222400 & 2.17559800\end{array}$

$\begin{array}{lll}0.63726900 & 3.25560800 & 0.67920100\end{array}$

$\begin{array}{lll}-0.28994700 & 3.66592500 & 1.07197800\end{array}$

$\begin{array}{lll}3.03972800 & 3.37783300 & 0.31480300\end{array}$

$\begin{array}{llll}3.96763800 & 3.92732200 & 0.45553800\end{array}$

$\begin{array}{llll}4.94733100 & -2.96506400 & 0.11897000\end{array}$

$\begin{array}{llll}5.84633700 & -3.51738700 & -0.14489000\end{array}$

$\begin{array}{llll}-6.15099700 & -0.65456800 & -0.06445000\end{array}$

$\begin{array}{lll}-6.46685000 & -0.48281800 & 1.34169100\end{array}$

$\begin{array}{lll}-7.54930200 & -0.36597300 & 1.46277000\end{array}$

$\begin{array}{lll}-5.98939100 & 0.41561600 & 1.74570700\end{array}$ 


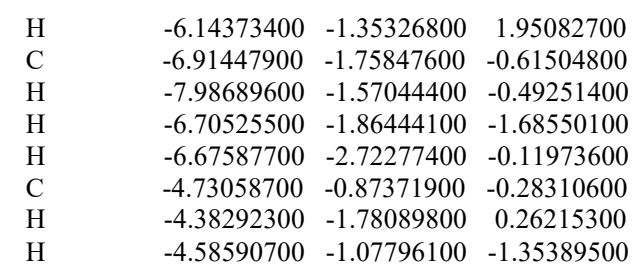

Benzydamine $\mathbf{I}_{4}$<smiles>CN(C)CCCOc1nn(Cc2ccccc2)c2ccccc12</smiles>

$\mathrm{E}=-976.256496$

$\mathrm{H}_{\text {Corr. }}=0.397174$

$\begin{array}{llll}\mathrm{O} & -1.34665400 & 1.37601600 & -0.07045300\end{array}$

$\begin{array}{llll}\mathrm{N} & 1.99142100 & 0.55964200 & 0.00194300\end{array}$

$\begin{array}{llll}\mathrm{N} & 0.66628100 & 0.19544100 & -0.02216300\end{array}$

$\begin{array}{lllll}\mathrm{C} & -0.01670500 & 1.31879000 & -0.04354700\end{array}$

$\begin{array}{llll}\text { C } & 0.82353400 & 2.47288500 & -0.03450200\end{array}$

$\begin{array}{llll}\mathrm{C} & 2.12333500 & 1.93856500 & -0.00463400\end{array}$

$\begin{array}{llll}\text { C } & 2.97986100 & -1.75570700 & 0.03080100\end{array}$

$\begin{array}{llll}\text { C } & 1.81001800 & -2.56198000 & 0.00208700\end{array}$

$\begin{array}{llll}\mathrm{H} & 0.83659300 & -2.08509800 & -0.02315800\end{array}$

$\begin{array}{llll}\mathrm{C} & 3.02706600 & -0.34238000 & 0.02931900\end{array}$

$\mathrm{H} \quad 4.00040600 \quad 0.13984400 \quad 0.05123500$

$\begin{array}{llll}\text { C } & 1.72966100 & 4.67633200 & -0.03219400\end{array}$

$\begin{array}{llll}\mathrm{H} & 1.60772100 & 5.75618800 & -0.04263300\end{array}$

C $\quad 1.91009000 \quad-3.94527700 \quad 0.00579300$

$\mathrm{H} \quad 0.99874700 \quad-4.53951600 \quad-0.01692000$

$\begin{array}{llll}\text { C } & 4.23412400 & -2.42786800 & 0.06368800\end{array}$

$\mathrm{H} \quad \begin{array}{llll}\mathrm{H} & 5.14648100 & -1.83293700 & 0.08635000\end{array}$

$\begin{array}{llll}\text { C } & -3.51822500 & 0.41427900 & -0.10934200\end{array}$

$\begin{array}{lllr}\mathrm{H} & -3.75583200 & 1.08857700 & 0.72412300\end{array}$

$\begin{array}{llll}\mathrm{H} & -3.77509400 & 0.93923300 & -1.03968000\end{array}$

$\begin{array}{llll}\mathrm{C} & 3.25319300 & 2.76304400 & 0.01206000\end{array}$

$\begin{array}{llll}\mathrm{H} & 4.26363700 & 2.36404000 & 0.03490300\end{array}$

$\begin{array}{llll}\text { C } & 3.15157800 & -4.58431800 & 0.03795600\end{array}$

$\mathrm{H} \quad 3.21335800 \quad-5.66970300 \quad 0.04043200$

C $\quad 0.61551600 \quad 3.85521600 \quad-0.04871200$

$\mathrm{H} \quad-0.39183100 \quad 4.26347300 \quad-0.07239700$

$\begin{array}{lllll}\mathrm{C} & 3.02987500 & 4.13016800 & -0.00208400\end{array}$

$\begin{array}{lll}3.88417300 & 4.80276600 & 0.01018000\end{array}$

$\begin{array}{lll}4.31529500 & -3.80735000 & 0.06703600\end{array}$

$\begin{array}{lll}5.29114100 & -4.28742600 & 0.09243300\end{array}$

$\begin{array}{lll}-5.73452100 & -0.70101700 & -0.30430900\end{array}$

$\begin{array}{lll}-6.38464600 & 0.15891300 & 0.66766200\end{array}$

$\begin{array}{lll}-7.45435300 & 0.22476900 & 0.44137600\end{array}$

$\begin{array}{llll}-5.97528300 & 1.17347800 & 0.63160000\end{array}$

$\begin{array}{lll}-6.27462700 & -0.22529900 & 1.70372000\end{array}$

$\begin{array}{llll}-6.39436900 & -1.99238100 & -0.34719300\end{array}$

$\begin{array}{llll}-7.45052400 & -1.86493100 & -0.60923500\end{array}$

$-5.92746900-2.62915600-1.10715900$

$\begin{array}{lll}-6.34345800 & -2.52301300 & 0.62641800\end{array}$

$\begin{array}{llll}-4.31927800 & -0.87389900 & -0.01717600\end{array}$

$\begin{array}{llll}-4.17575700 & -1.32943900 & 0.98975400\end{array}$

$\begin{array}{lll}-3.92178200 & -1.59766700 & -0.74489600\end{array}$

$\begin{array}{llll}-2.03871500 & 0.12178300 & -0.07798300\end{array}$

$\begin{array}{lll}-1.75519300 & -0.44846800 & 0.81951000\end{array}$

$\begin{array}{lll}-1.71732900 & -0.45918900 & -0.95483200\end{array}$

\section{Diethylcarbamazine}<smiles>CCN(C(=O)N1CC=CN(C)CC1)C(C)C</smiles>

$\mathrm{E}=-632.953165$

$\mathrm{H}_{\text {Corr. }}=0.32961$

O $\quad 0.48507200 \quad-1.93854400 \quad-0.65798400$

$\begin{array}{lll}1.82739400 & -0.14260500 & -0.23323500\end{array}$

$\begin{array}{llll}-0.37430300 & 0.15596400 & -1.01889500\end{array}$

$\begin{array}{lll}-2.92100400 & 0.41863600 & 0.27907500\end{array}$

$\begin{array}{lll}2.88810600 & 2.06346300 & 0.20912100\end{array}$

$\begin{array}{llll}2.10277100 & 2.37446700 & 0.90887100\end{array}$

$\begin{array}{llll}3.28462600 & 2.95924000 & -0.28107800\end{array}$

$\begin{array}{llll}3.69999800 & 1.62428900 & 0.80106500\end{array}$

$\begin{array}{llll}2.36312600 & 1.08289500 & -0.82453300\end{array}$

$\begin{array}{llll}3.17813800 & 0.80635500 & -1.51369800\end{array}$

$\begin{array}{llll}1.58661300 & 1.54165400 & -1.44318200\end{array}$

$\begin{array}{llll}0.63771300 & -0.71719000 & -0.63417100\end{array}$

$\begin{array}{lll}-0.70117500 & 1.33317300 & -0.21385700\end{array}$

$\begin{array}{lll}-1.11508700 & 2.10427400 & -0.87978900\end{array}$

$\begin{array}{lll}0.19715500 & 1.73787100 & 0.25515600\end{array}$

$\begin{array}{lll}-1.71393000 & 0.98123900 & 0.86162400\end{array}$

$\begin{array}{lll}-1.24351500 & 0.26393900 & 1.57474400\end{array}$

$\begin{array}{lll}-1.97971000 & 1.88262300 & 1.43029300\end{array}$

$\begin{array}{lll}-3.90014300 & 0.12162800 & 1.30451500\end{array}$

$\begin{array}{lll}-3.52939000 & -0.62725800 & 2.03390400\end{array}$

$\begin{array}{lll}-4.81373400 & -0.27338500 & 0.84569100\end{array}$

$\begin{array}{lll}-4.15814400 & 1.03485400 & 1.85327300\end{array}$

$-2.59585000 \quad-0.77633600 \quad-0.49137000$

$\begin{array}{lll}-3.51742200 & -1.16151600 & -0.94779500\end{array}$

$\begin{array}{lll}-2.18836400 & -1.57199400 & 0.16757600\end{array}$

$\begin{array}{lll}-1.58111600 & -0.45322600 & -1.57250100\end{array}$

$\begin{array}{llll}-1.30270100 & -1.35694100 & -2.11881200\end{array}$

$\begin{array}{llll}-2.01777800 & 0.26640800 & -2.27975100\end{array}$

$\begin{array}{llll}2.80687200 & -1.05211300 & 0.36512400\end{array}$

$\begin{array}{lll}3.79356500 & -0.58739100 & 0.25328900\end{array}$

$\begin{array}{llll}2.82374500 & -1.99182000 & -0.20005200\end{array}$

$\begin{array}{llll}2.51198800 & -1.31544900 & 1.82996100\end{array}$

$\begin{array}{llll}2.52028000 & -0.37915600 & 2.40210100\end{array}$

$\begin{array}{llll}3.25537900 & -1.99302800 & 2.26484700\end{array}$

$\begin{array}{lll}1.52485000 & -1.77760300 & 1.94512100\end{array}$

Diethylcarbamazine $\mathbf{I}_{1}$<smiles>CCN(C(=O)N1CC=CC2CC1CN2)C(C)C</smiles>

$E=-632.294933$

$\mathrm{H}_{\text {Corr. }}=0.316117$

O $\quad 0.44178100 \quad-1.97532300 \quad-0.51259400$

$\begin{array}{ccc}1.79174100 & -0.15533100 & -0.24637300\end{array}$

$\begin{array}{lll}-0.42974800 & 0.08970700 & -0.99513000\end{array}$

$\begin{array}{lll}-2.90445100 & 0.34080800 & 0.39551100\end{array}$

$\begin{array}{lll}2.84292200 & 2.08052500 & 0.04600800\end{array}$

$\begin{array}{llll}2.07232000 & 2.42368300 & 0.74711400\end{array}$

$\begin{array}{lll}3.21802800 & 2.95053300 & -0.50366500\end{array}$

$\begin{array}{llll}3.67359100 & 1.68238100 & 0.64076600\end{array}$

$\begin{array}{llll}2.30303900 & 1.03897800 & -0.91789400\end{array}$

$\begin{array}{lll}3.10479800 & 0.72927000 & -1.60828200\end{array}$ 


$\begin{array}{lrrr}\mathrm{H} & 1.50912000 & 1.45556300 & -1.54424000 \\ \mathrm{C} & 0.59535400 & -0.75590600 & -0.57578300 \\ \mathrm{C} & -0.75092000 & 1.30594900 & -0.24833800 \\ \mathrm{H} & -1.20906300 & 2.02550200 & -0.94220100 \\ \mathrm{H} & 0.15522000 & 1.75898500 & 0.15733100 \\ \mathrm{C} & -1.70789700 & 0.99900600 & 0.89104400 \\ \mathrm{H} & -1.19409400 & 0.35546700 & 1.63561200 \\ \mathrm{H} & -2.00136900 & 1.92647400 & 1.39798400 \\ \mathrm{C} & -3.99849300 & 0.30860800 & 1.23843000 \\ \mathrm{H} & -4.85192500 & -0.28114500 & 0.91600900 \\ \mathrm{H} & -4.15121300 & 1.16982500 & 1.88314500 \\ \mathrm{C} & -2.61573600 & -0.86851100 & -0.36530000 \\ \mathrm{H} & -3.55741500 & -1.25294200 & -0.77559600 \\ \mathrm{H} & -2.18861800 & -1.64406000 & 0.29628900 \\ \mathrm{C} & -1.64063900 & -0.56193400 & -1.48777200 \\ \mathrm{H} & -1.36256800 & -1.48084600 & -2.00838800 \\ \mathrm{H} & -2.11080100 & 0.12236900 & -2.20829000 \\ \mathrm{C} & 2.79063900 & -1.01901900 & 0.38745200 \\ \mathrm{H} & 3.77299000 & -0.56610000 & 0.20854400 \\ \mathrm{H} & 2.78894700 & -1.99806100 & -0.10638300 \\ \mathrm{C} & 2.54273900 & -1.17190300 & 1.87648500 \\ \mathrm{H} & 2.56685800 & -0.19575400 & 2.37695800 \\ \mathrm{H} & 3.30109800 & -1.81511500 & 2.33676100 \\ \mathrm{H} & 1.56100700 & -1.62448400 & 2.05722500\end{array}$

Diethylcarbamazine $\mathbf{I}_{2}$<smiles>CCN(C(=O)N1CCN(C)CC1)C(C)C</smiles>

$\mathrm{E}=-632.296589$

$$
\mathrm{H}_{\text {Corr. }}=0.31644
$$

$\begin{array}{lrrr}\mathrm{O} & 0.40651200 & -1.88631600 & -0.72302100 \\ \mathrm{~N} & 1.80048700 & -0.14680300 & -0.24110300 \\ \mathrm{~N} & -0.38711100 & 0.24282900 & -1.02641300 \\ \mathrm{~N} & -2.86338700 & 0.34347700 & 0.39851900 \\ \mathrm{C} & 2.88276700 & 2.01314600 & 0.34887400 \\ \mathrm{H} & 2.09799000 & 2.28089900 & 1.06687200 \\ \mathrm{H} & 3.28629500 & 2.93710000 & -0.07940600 \\ \mathrm{H} & 3.69030800 & 1.52907000 & 0.91099200 \\ \mathrm{C} & 2.35308800 & 1.10752100 & -0.74896800 \\ \mathrm{H} & 3.16978500 & 0.86576200 & -1.44906600 \\ \mathrm{H} & 1.58682300 & 1.61408200 & -1.34243500 \\ \mathrm{C} & 0.59564500 & -0.67110600 & -0.65755000 \\ \mathrm{C} & -0.70702300 & 1.39605100 & -0.17523600 \\ \mathrm{H} & -1.03109300 & 2.21219200 & -0.85278100 \\ \mathrm{H} & 0.18471300 & 1.75325000 & 0.34326000 \\ \mathrm{C} & -1.75528100 & 1.05955800 & 0.82981300 \\ \mathrm{H} & -1.93251300 & 1.74767100 & 1.65497000 \\ \mathrm{C} & -2.58664700 & -0.75505100 & -0.52275100 \\ \mathrm{H} & -3.53293600 & -1.06984300 & -0.98155300 \\ \mathrm{H} & -2.16957400 & -1.61620100 & 0.03050200 \\ \mathrm{C} & -1.60499500 & -0.32237600 & -1.59572900 \\ \mathrm{H} & -1.34559700 & -1.17235000 & -2.23070400 \\ \mathrm{H} & -2.06177600 & 0.45627700 & -2.22383100 \\ \mathrm{C} & 2.76524500 & -1.10429300 & 0.30358100 \\ \mathrm{H} & 3.76194700 & -0.66111600 & 0.19070500 \\ \mathrm{H} & 2.74589800 & -2.01962300 & -0.30009600 \\ \mathrm{C} & 2.49139600 & -1.42231500 & 1.76162500 \\ \mathrm{H} & 2.53933900 & -0.51348700 & 2.37412800 \\ \mathrm{H} & 3.22277000 & -2.14014600 & 2.14990800 \\ \mathrm{H} & 1.49302700 & -1.85886000 & 1.87701900 \\ \mathrm{C} & -3.84648700 & 0.01263900 & 1.40821600 \\ \mathrm{H} & -4.07582000 & 0.89972700 & 2.00749600 \\ \mathrm{H} & -4.76919900 & -0.32873100 & 0.92656200 \\ \mathrm{H} & -3.48865900 & -0.78430300 & 2.08335000\end{array}$

Diethylcarbamazine $\mathbf{I}_{3}$<smiles>CCN(C(=O)N1CCN(C)CC1)C(C)C</smiles>

$E=-632.293428$

$\mathrm{H}_{\text {Corr. }}=0.316191$

O $\quad 1.11996000 \quad-2.10614000 \quad-0.29199900$

$\mathrm{N} \quad \begin{array}{llll}1.91923200 & 0.03906300 & -0.22496000\end{array}$

$\mathrm{N} \quad-0.40771700 \quad-0.41336900 \quad-0.21681600$

$\mathrm{N} \quad-3.14114000 \quad 0.33657600 \quad-0.11312600$

$\begin{array}{llll}\text { C } & 2.37171000 & 2.48162200 & -0.26061600\end{array}$

$\begin{array}{llll}\mathrm{H} & 1.73253800 & 2.68707700 & 0.60634600\end{array}$

$\mathrm{H} \quad 2.36820200 \quad 3.36742500 \quad-0.90496600$

$\begin{array}{llll}\mathrm{H} & 3.39523100 & 2.34414700 & 0.10825900\end{array}$

$\begin{array}{llll}1.88651900 & 1.26708600 & -1.02875800\end{array}$

$\begin{array}{llll}2.51482300 & 1.11007900 & -1.92015900\end{array}$

$\begin{array}{llll}0.86651400 & 1.42994600 & -1.38957400\end{array}$

$\begin{array}{llll}0.90332300 & -0.89494200 & -0.26488600\end{array}$

$\begin{array}{lll}-0.76744700 & 0.84648600 & 0.24801800\end{array}$

$\begin{array}{lll}0.01151400 & 1.38281900 & 0.78126100\end{array}$

$\begin{array}{lll}-2.77269900 & -1.06835200 & -0.07594900\end{array}$

$-3.54228400 \quad-1.65713900 \quad-0.59032700$

$\begin{array}{lll}-2.71802200 & -1.43461500 & 0.97307800\end{array}$

$\begin{array}{llll}-1.45076800 & -1.27985300 & -0.77368000\end{array}$

$-1.11374800-2.31347200 \quad-0.67226000$

$\begin{array}{llll}-1.56802500 & -1.05194100 & -1.84369700\end{array}$

$\begin{array}{lll}3.25843500 & -0.51013200 & 0.01788600\end{array}$

$\begin{array}{llll}3.98110900 & 0.22421100 & -0.35527700\end{array}$

$\begin{array}{llll}3.39071900 & -1.42580700 & -0.57219900\end{array}$

$\begin{array}{lll}3.50358900 & -0.77898300 & 1.48955700\end{array}$

$\begin{array}{lll}3.40158200 & 0.14464600 & 2.07279300\end{array}$

$\begin{array}{llll}4.51110100 & -1.17913600 & 1.64897900\end{array}$

$\begin{array}{llll}2.78524400 & -1.51027000 & 1.87575500\end{array}$

$\begin{array}{lll}-4.48203700 & 0.54638800 & 0.39366200\end{array}$

$\begin{array}{llll}-4.73512800 & 1.61177300 & 0.35035300\end{array}$

$\begin{array}{llll}-5.20368900 & -0.00322800 & -0.22063700\end{array}$

$\begin{array}{lll}-4.59126500 & 0.20876800 & 1.44465500\end{array}$

$\begin{array}{lll}-2.17502700 & 1.08895300 & 0.66840100\end{array}$

$\begin{array}{lll}-2.29135300 & 0.82646700 & 1.75282200\end{array}$

$\begin{array}{lll}-2.40095600 & 2.16185100 & 0.59329200\end{array}$

Diethylcarbamazine $\mathbf{I}_{4}$<smiles>CCN(CC)C(=O)N1CCNCC1C</smiles>

$\mathrm{E}=-632.299170$

$\mathrm{H}_{\text {Corr. }}=0.315758$

$\begin{array}{llll}0 & 0.47556600 & -2.04882500 & -0.50486800\end{array}$

$\mathrm{N} \quad \begin{array}{llll}1.85513400 & -0.23406400 & -0.29600300\end{array}$

$\begin{array}{rrr}-0.35988100 & 0.02140400 & -1.04678400\end{array}$

$\begin{array}{lll}-2.87696300 & 0.49951200 & 0.24354400\end{array}$

$\begin{array}{llll}3.16636100 & 1.90110900 & -0.19468500\end{array}$

$\begin{array}{lll}2.65019100 & 2.36988800 & 0.66456300\end{array}$

$\begin{array}{lll}3.47018000 & 2.70512900 & -0.87101400\end{array}$

$\begin{array}{llll}4.08315700 & 1.44987900 & 0.20988700\end{array}$

$\begin{array}{llll}2.29595700 & 0.93559700 & -0.90925100\end{array}$

$\begin{array}{llll}1.75085000 & 1.22756500 & -1.80185500\end{array}$

$\begin{array}{lll}0.63456000 & -0.83227200 & -0.59711600\end{array}$ 


$\begin{array}{lrrr}\mathrm{C} & -2.59648600 & -0.78040100 & -0.39609200 \\ \mathrm{H} & -3.53475700 & -1.18799400 & -0.79498900 \\ \mathrm{H} & -2.19822300 & -1.51071400 & 0.34005600 \\ \mathrm{C} & -1.59162100 & -0.60559400 & -1.52011100 \\ \mathrm{H} & -1.34658000 & -1.56964800 & -1.97087000 \\ \mathrm{H} & -2.01733100 & 0.04931400 & -2.29343700 \\ \mathrm{C} & 2.76192400 & -1.00682200 & 0.56239100 \\ \mathrm{H} & 3.78194500 & -0.67211900 & 0.35141100 \\ \mathrm{H} & 2.69342300 & -2.05583300 & 0.25802400 \\ \mathrm{C} & 2.41797900 & -0.84801500 & 2.03091300 \\ \mathrm{H} & 2.47065200 & 0.20418300 & 2.33756800 \\ \mathrm{H} & 3.10786300 & -1.42337600 & 2.65799200 \\ \mathrm{H} & 1.40155400 & -1.21014900 & 2.22779000 \\ \mathrm{C} & -3.85014500 & 0.34362100 & 1.30533400 \\ \mathrm{H} & -4.07461200 & 1.31770000 & 1.75505200 \\ \mathrm{H} & -4.78059200 & -0.07133400 & 0.90142400 \\ \mathrm{H} & -3.49068100 & -0.33319000 & 2.10722400 \\ \mathrm{C} & -1.64642900 & 1.08533800 & 0.74878600 \\ \mathrm{H} & -1.18892700 & 0.43590800 & 1.53169600 \\ \mathrm{H} & -1.87839100 & 2.05064400 & 1.21860900 \\ \mathrm{C} & -0.63819300 & 1.28988600 & -0.36743600 \\ \mathrm{H} & -1.03822200 & 1.99516000 & -1.10984500 \\ \mathrm{H} & 0.28250000 & 1.71693700 & 0.03795800\end{array}$

\section{Probenecid derivative (38)}<smiles>CCCN(C(C)CC)S(=O)(=O)c1ccc(C(=O)N2CC3CCN(C)CC3C2)cc1</smiles>

$$
\mathrm{E}=-1491.069206
$$

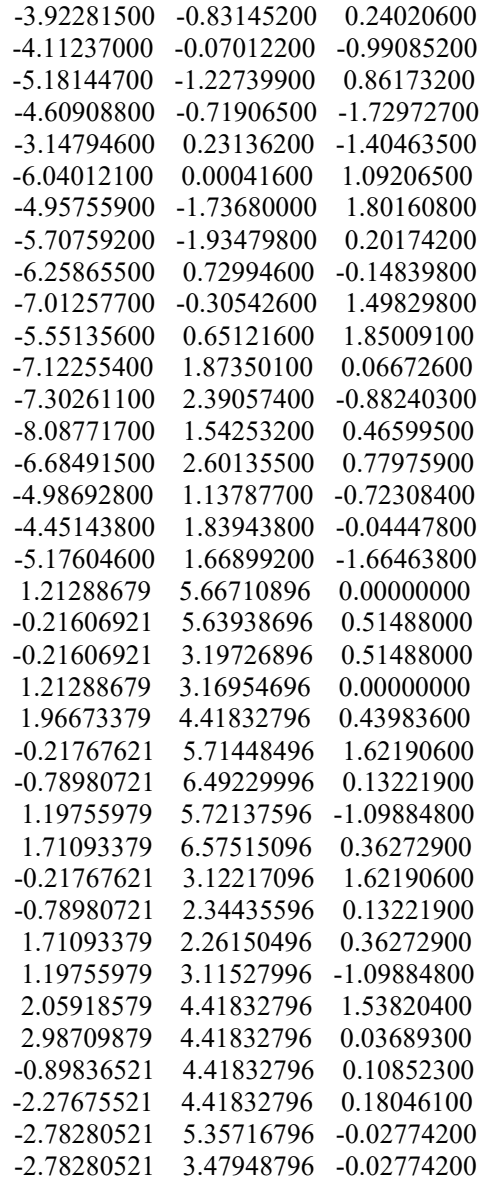

Probenecid I $_{1}$<smiles>CCPN(C(C)CC)S(=O)(=O)c1ccc(C(=O)N2CCN(C=O)CC2)cc1</smiles>

$E=-1490.410538$

$\begin{array}{lccc}\mathrm{H}_{\text {Corr. }}= & 0.474103 & & \\ \mathrm{~S} & 2.56963000 & 0.51566800 & -1.38444200 \\ \mathrm{O} & -2.77944000 & -2.30867700 & 1.45573400 \\ \mathrm{O} & 2.95514100 & -0.33168000 & -2.50743700 \\ \mathrm{O} & 2.51400500 & 1.96663500 & -1.52400400 \\ \mathrm{~N} & 3.62797300 & 0.19803200 & -0.14349300 \\ \mathrm{C} & -2.80712500 & -1.39335000 & 0.63629600 \\ \mathrm{C} & -1.51880400 & -0.87794700 & 0.06886400 \\ \mathrm{C} & -1.16900700 & 0.47081500 & 0.15794200 \\ \mathrm{H} & -1.86756000 & 1.18608900 & 0.58835400 \\ \mathrm{C} & 0.07421100 & 0.90389600 & -0.28907700 \\ \mathrm{H} & 0.35270300 & 1.95305300 & -0.23547000 \\ \mathrm{C} & 0.95522100 & -0.02286700 & -0.84079200 \\ \mathrm{C} & 0.61946700 & -1.37269600 & -0.93912700 \\ \mathrm{H} & 1.31037000 & -2.07791300 & -1.39410000 \\ \mathrm{C} & -0.61482300 & -1.79787900 & -0.46605100 \\ \mathrm{H} & -0.88965000 & -2.84864500 & -0.51481400 \\ \mathrm{C} & 3.53084600 & 1.06992600 & 1.03948500 \\ \mathrm{H} & 3.57950200 & 0.42369900 & 1.92866800 \\ \mathrm{H} & 2.54500100 & 1.55651800 & 1.07638400 \\ \mathrm{C} & 4.62082700 & 2.12650900 & 1.08447700 \\ \mathrm{H} & 4.53088300 & 2.75587200 & 0.18981800 \\ \mathrm{H} & 5.60153600 & 1.63431400 & 1.02367300 \\ \mathrm{C} & 4.52264900 & 2.96629000 & 2.34721100\end{array}$




$\begin{array}{lrrr}\mathrm{H} & 5.30318200 & 3.73294200 & 2.38125800 \\ \mathrm{H} & 4.62664400 & 2.34606100 & 3.24711100 \\ \mathrm{H} & 3.55323300 & 3.47726900 & 2.40879700 \\ \mathrm{H} & 5.69754800 & -3.37121900 & -0.13049700 \\ \mathrm{C} & 3.99354900 & -1.20252900 & 0.10083600 \\ \mathrm{H} & 3.87864600 & -1.75173300 & -0.84051100 \\ \mathrm{H} & 3.30355200 & -1.65004000 & 0.83995100 \\ \mathrm{C} & 5.42912300 & -1.33679200 & 0.58067800 \\ \mathrm{H} & 5.56709000 & -0.77579000 & 1.51625800 \\ \mathrm{H} & 6.09202600 & -0.87480400 & -0.16342900 \\ \mathrm{C} & 5.79510700 & -2.79597200 & 0.79860100 \\ \mathrm{H} & 6.82697800 & -2.90111600 & 1.14817900 \\ \mathrm{H} & 5.14162100 & -3.26182300 & 1.54698300 \\ \mathrm{~N} & -3.96351900 & -0.78420700 & 0.24766500 \\ \mathrm{C} & -4.14538400 & -0.00140300 & -0.97061600 \\ \mathrm{C} & -5.22586500 & -1.18740200 & 0.85624500 \\ \mathrm{H} & -4.66317400 & -0.62818800 & -1.71426200 \\ \mathrm{H} & -3.17811700 & 0.28402600 & -1.38909800 \\ \mathrm{C} & -6.06589900 & 0.04206000 & 1.14243500 \\ \mathrm{H} & -5.00783900 & -1.73641200 & 1.77492000 \\ \mathrm{H} & -5.76265900 & -1.86097500 & 0.16985700 \\ \mathrm{~N} & -6.24257100 & 0.84186900 & -0.06034900 \\ \mathrm{H} & -7.05377900 & -0.26053700 & 1.50835300 \\ \mathrm{H} & -5.58239800 & 0.64633300 & 1.93385300 \\ \mathrm{C} & -7.24572600 & 1.79280400 & -0.03925500 \\ \mathrm{H} & -7.30622600 & 2.46643800 & -0.88919200 \\ \mathrm{H} & -8.14299100 & 1.55115800 & 0.52303700 \\ \mathrm{C} & -4.98436300 & 1.22636900 & -0.67799100 \\ \mathrm{H} & -4.42521700 & 1.91533600 & -0.01437200 \\ \mathrm{H} & -5.19985200 & 1.76031000 & -1.61071300\end{array}$

Probenecid $\mathbf{I}_{2}$<smiles>CCCN(C(CC)CC)S(=O)(=O)c1ccc(C(=O)N2CCN3CCCC2C3)cc1</smiles>

$E=-1490.412828$

$\mathrm{H}_{\text {Corr. }}=0.474404$

S $\quad 2.57482500 \quad 0.25865800 \quad-1.46563600$

$\begin{array}{llll}\mathrm{O} & -2.82680100 & -2.28596200 & 1.49136500\end{array}$

$\begin{array}{llll}\mathrm{O} & 3.07248400 & -0.75614400 & -2.38788100\end{array}$

$\begin{array}{llll}\mathrm{O} & 2.49110600 & 1.65878200 & -1.86668000\end{array}$

$\begin{array}{llll}\mathrm{N} & 3.54302300 & 0.20927000 & -0.11373200\end{array}$

$\begin{array}{llll}\text { C } & -2.85298100 & -1.50176900 & 0.54277800\end{array}$

$\begin{array}{llll}\text { C } & -1.55791200 & -1.03700600 & -0.05244800\end{array}$

$\begin{array}{lllll}\mathrm{C} & -1.25153300 & 0.32162100 & -0.15684300\end{array}$

$\begin{array}{llll}\mathrm{H} & -1.99976400 & 1.06838900 & 0.11168900\end{array}$

$\begin{array}{llll}\mathrm{C} & 0.00400600 & 0.72414000 & -0.59835900\end{array}$

$\begin{array}{llll}\mathrm{H} & 0.24878000 & 1.77846300 & -0.69668100\end{array}$

$\begin{array}{llll}\text { C } & 0.94148400 & -0.24484200 & -0.94813800\end{array}$

$\begin{array}{llll}\text { C } & 0.64820700 & -1.60502600 & -0.85426500\end{array}$

$\mathrm{H} \quad \begin{array}{llll}\mathrm{C} & 1.38470700 & -2.34608100 & -1.15422900\end{array}$

$\begin{array}{llll}\mathrm{C} & -0.60014500 & -1.99563400 & -0.38890600\end{array}$

$\mathrm{H} \quad \begin{array}{llll}\mathrm{H} & -0.84297300 & -3.05027500 & -0.28512900\end{array}$

$\begin{array}{llll}\mathrm{C} & 3.33840300 & 1.28067100 & 0.87595900\end{array}$

$\begin{array}{llll}\mathrm{H} & 3.31139400 & 0.81064800 & 1.87051800\end{array}$

$\begin{array}{llll}\mathrm{H} & 2.35190900 & 1.74652400 & 0.73772700\end{array}$

$\begin{array}{llll}\mathrm{C} & 4.41601700 & 2.34934000 & 0.81658600\end{array}$

$\begin{array}{llll}\mathrm{H} & 4.40528700 & 2.79668400 & -0.18561600\end{array}$

$\begin{array}{llll}\mathrm{H} & 5.40028600 & 1.87554600 & 0.93684900\end{array}$

$\begin{array}{llll}\mathrm{C} & 4.20047300 & 3.41008100 & 1.88306300\end{array}$

$\begin{array}{llll}\mathrm{H} & 4.97263700 & 4.18480400 & 1.84058300\end{array}$

$\begin{array}{llll}\mathrm{H} & 4.22332600 & 2.97302400 & 2.88993100\end{array}$

$\begin{array}{llll}\mathrm{H} & 3.22814300 & 3.90397000 & 1.75954000\end{array}$

$\begin{array}{llll}\mathrm{H} & 5.61823700 & -3.24964900 & 0.75708000\end{array}$

$\begin{array}{lll}3.89180200 & -1.11389400 & 0.41829800\end{array}$

$\begin{array}{llll}3.87259100 & -1.82858200 & -0.41231300\end{array}$

$\begin{array}{llll}3.13548400 & -1.43556700 & 1.15831000\end{array}$ $\begin{array}{lll}5.27437800 & -1.12501100 & 1.04862300\end{array}$

$\begin{array}{lll}5.31842300 & -0.39859400 & 1.87285400\end{array}$

$\begin{array}{lll}6.00501800 & -0.79364700 & 0.29832300\end{array}$

$\begin{array}{lll}5.62319100 & -2.51052600 & 1.56780500\end{array}$

$\begin{array}{lll}6.61667400 & -2.52676200 & 2.02693200\end{array}$

$\begin{array}{lll}4.90185800 & -2.84396600 & 2.32454700\end{array}$

$\begin{array}{lll}-4.01140100 & -1.01826200 & 0.02217300\end{array}$

$\begin{array}{lll}-4.17175700 & -0.17797400 & -1.16206200\end{array}$

$\begin{array}{lll}-5.26566200 & -1.19066700 & 0.73850900\end{array}$

$\begin{array}{lll}-4.82910400 & -0.73722200 & -1.85784000\end{array}$

$\begin{array}{lll}-3.21738900 & -0.04486500 & -1.67602200\end{array}$

$\begin{array}{lll}-4.76885000 & 1.13407200 & -0.78448200\end{array}$

$\begin{array}{lll}-5.75028100 & 0.16909400 & 1.20725600\end{array}$

$\begin{array}{lll}-5.10176400 & -1.86138000 & 1.58442200\end{array}$

$\begin{array}{lll}-6.00246300 & -1.64757400 & 0.06202100\end{array}$

$\begin{array}{lll}-5.84195800 & 1.10978400 & 0.09608400\end{array}$

$\begin{array}{lll}-4.77958300 & 1.93995500 & -1.51616600\end{array}$

$\begin{array}{lll}-6.74097000 & 0.08011100 & 1.66999500\end{array}$

$\begin{array}{lll}-5.05111300 & 0.55792800 & 1.97370500\end{array}$

$\begin{array}{lll}-6.40025000 & 2.39302400 & 0.46975000\end{array}$

$\begin{array}{llll}-6.53063400 & 3.01328200 & -0.42246000\end{array}$

$\begin{array}{lll}-7.37997700 & 2.24719600 & 0.93688200\end{array}$

$\begin{array}{lll}-5.74970700 & 2.93198500 & 1.17986900\end{array}$

${ }_{\text {Probenecid }} \mathbf{I}_{3}$<smiles>CCCN(C(CC)CC)S(=O)(=O)c1ccc(C(=O)N2CC3CC(CN(C)C3)C2)cc1</smiles>

$\mathrm{E}=-1490.409741$

$\mathrm{H}_{\text {Corr. }}=0.473694$

$2.61091300 \quad 0.61601100 \quad-1.35918700$

$\begin{array}{lll}-2.71567500 & -2.31563000 & 1.40415100\end{array}$

$\begin{array}{llll}2.95353100 & -0.16064800 & -2.54540600\end{array}$

$\begin{array}{llll}3.68893000 & 0.19111300 & -0.16671900\end{array}$

$\begin{array}{llll}-2.74397600 & -1.32973600 & 0.67020400\end{array}$

$\begin{array}{lll}-1.47428500 & -0.79237900 & 0.09644900\end{array}$

$\begin{array}{llll}-1.08450500 & 0.53719400 & 0.28210300\end{array}$

$\begin{array}{llll}-1.74802100 & 1.23175300 & 0.79510100\end{array}$

$\begin{array}{llll}0.15492700 & 0.97570100 & -0.16863800\end{array}$

$\begin{array}{llll}0.46067300 & 2.01066300 & -0.04006500\end{array}$

$\begin{array}{llll}0.99763300 & 0.07547400 & -0.81650400\end{array}$

$\begin{array}{llll}0.62573800 & -1.25497300 & -1.00730700\end{array}$

$\begin{array}{llll}1.28655900 & -1.93870600 & -1.53410400\end{array}$

$\begin{array}{lll}1.0 .60523000 & -1.68733800 & -0.53324100\end{array}$

$\begin{array}{llll}-0.90690500 & -2.72473900 & -0.65453000\end{array}$

$\begin{array}{lll}3.64279400 & 0.98721200 & 1.07153200\end{array}$

$\begin{array}{llll}3.67058600 & 0.28387800 & 1.91740900\end{array}$

$\begin{array}{llll}2.68106400 & 1.51461600 & 1.15117500\end{array}$

$\begin{array}{lll}4.77944800 & 1.98980000 & 1.17000700\end{array}$

$\begin{array}{lll}4.71078200 & 2.67677000 & 0.31684600\end{array}$

$\begin{array}{llll}5.73716100 & 1.46046700 & 1.06870200\end{array}$

$\begin{array}{lll}4.72886200 & 2.75343800 & 2.48287800\end{array}$

$\begin{array}{llll}5.54383000 & 3.48053000 & 2.55608000\end{array}$

$\begin{array}{lll}4.81104300 & 2.07462800 & 3.34179600\end{array}$

$\begin{array}{lll}3.78422000 & 3.30278700 & 2.58553300\end{array}$

$\begin{array}{llll}5.63225700 & -3.43884000 & -0.41736100\end{array}$

$\begin{array}{llll}4.00839000 & -1.23407400 & -0.01790100\end{array}$

$\begin{array}{llll}3.86500000 & -1.71646300 & -0.99140500\end{array}$

$\begin{array}{llll}3.31071100 & -1.70518500 & 0.69918900\end{array}$

$\begin{array}{llll}5.44308100 & -1.44980400 & 0.43400600\end{array}$

$\begin{array}{llll}5.61025600 & -0.96012500 & 1.40410300\end{array}$

$\begin{array}{llll}6.11462400 & -0.96121100 & -0.28497000\end{array}$

$\begin{array}{lll}5.75914000 & -2.93237800 & 0.54751800\end{array}$

$\begin{array}{llll}6.78991000 & -3.09700900 & 0.87680300\end{array}$

$\begin{array}{llll}5.09667000 & -3.42529800 & 1.27023000\end{array}$

$\begin{array}{llll}-3.92898200 & -0.67154500 & 0.38843000\end{array}$ 


$\begin{array}{lrrr}\mathrm{C} & -4.09536300 & 0.20211500 & -0.78696900 \\ \mathrm{C} & -5.08932600 & -1.10654300 & 0.99909200 \\ \mathrm{H} & -4.34875600 & -0.42688200 & -1.65150800 \\ \mathrm{H} & -3.15836100 & 0.71630000 & -1.00175500 \\ \mathrm{C} & -6.30538300 & -0.25661300 & 0.96773500 \\ \mathrm{H} & -4.96446900 & -1.88085700 & 1.74669800 \\ \mathrm{~N} & -6.44656800 & 0.49919700 & -0.26706900 \\ \mathrm{H} & -7.19521200 & -0.88257700 & 1.11616700 \\ \mathrm{H} & -6.27785200 & 0.44920400 & 1.83797600 \\ \mathrm{C} & -5.20675800 & 1.19728200 & -0.54972500 \\ \mathrm{H} & -4.92956200 & 1.88421400 & 0.28151600 \\ \mathrm{H} & -5.33471700 & 1.80743100 & -1.45217800 \\ \mathrm{C} & -7.56728400 & 1.41385600 & -0.18375800 \\ \mathrm{H} & -7.68713800 & 1.94481100 & -1.13433200 \\ \mathrm{H} & -8.48863200 & 0.85556600 & 0.01596600 \\ \mathrm{H} & -7.43788200 & 2.16471700 & 0.62189200\end{array}$

Probenecid $\mathbf{I}_{4}$<smiles>CCCN(CCC)S(=O)(=O)c1ccc(C(=O)N2CCN(C)CC2)cc1</smiles>

$\mathrm{E}=-1490.412331$

$\mathrm{H}_{\text {Corr. }}=0.473264$

S $\quad 2.80697500 \quad-1.67462700 \quad 0.33071900$

$\begin{array}{llll}\mathrm{O} & -2.34083100 & 2.41995700 & 1.21761100\end{array}$

$\begin{array}{llll}\mathrm{O} & 3.41197800 & -1.93827400 & 1.63084800\end{array}$

O $\quad 2.68928700 \quad-2.73125800 \quad-0.66527400$

$\mathrm{N} \quad 3.70338200 \quad-0.42469300 \quad-0.40766400$

$\begin{array}{llll}\mathrm{C} & -2.44582900 & 1.22540400 & 0.94512200\end{array}$

$\begin{array}{llll}\mathrm{C} & -1.20381400 & 0.39447700 & 0.81837900\end{array}$

C $\quad-0.92627300 \quad-0.32781300 \quad-0.34485100$

$\mathrm{H} \quad-1.65275700 \quad-0.35150000 \quad-1.15526400$

C $\quad 0.28068100 \quad-1.00371500 \quad-0.47978800$

$\mathrm{H} \quad 0.50928800 \quad-1.56871100 \quad-1.38018200$

$\begin{array}{llll}\text { C } & 1.19743000 & -0.95765900 & 0.56897600\end{array}$

$\begin{array}{llll}\mathrm{C} & 0.93982400 & -0.23902600 & 1.73500000\end{array}$

$\begin{array}{llll}\mathrm{H} & 1.67237400 & -0.21993300 & 2.53830400\end{array}$

$\begin{array}{llll}\mathrm{C} & -0.26341700 & 0.44479800 & 1.84992900\end{array}$

$\begin{array}{llll}\mathrm{H} & -0.48174500 & 1.02717300 & 2.74161500\end{array}$

$\begin{array}{llll}\mathrm{C} & 3.28215000 & -0.07187800 & -1.69234800\end{array}$

$\mathrm{H} \quad 3.16805700 \quad-0.89629400 \quad-2.39329300$

$\begin{array}{llll}\mathrm{C} & 2.79156400 & 1.29401700 & -2.02912500\end{array}$

$\mathrm{H} \quad 3.59751300 \quad 2.04575800 \quad-1.96291100$

$\begin{array}{llll}\mathrm{H} & 2.04171700 & 1.61249800 & -1.28083500\end{array}$

C $\quad 2.17421400 \quad 1.32792900 \quad-3.41912900$

$\mathrm{H} \quad 1.82080000 \quad 2.33163000 \quad-3.67423000$

$\mathrm{H} \quad 1.31958100 \quad 0.64234000 \quad-3.48205100$

$\mathrm{H} \quad 2.90200800 \quad 1.02290000 \quad-4.18033800$

$\begin{array}{llll}\mathrm{H} & 6.19656800 & 1.90793100 & 1.93921500\end{array}$

$\begin{array}{lllll}\mathrm{C} & 4.24257000 & 0.62389000 & 0.47031200\end{array}$

$\begin{array}{llll}\mathrm{H} & 4.27346700 & 0.22702500 & 1.49056000\end{array}$

$\begin{array}{llll}\mathrm{H} & 3.57476200 & 1.50139200 & 0.47903400\end{array}$

$\begin{array}{llll}\mathrm{C} & 5.64404900 & 1.02099400 & 0.03474900\end{array}$

$\begin{array}{llll}\mathrm{H} & 5.63455500 & 1.29892000 & -1.02864700\end{array}$

$\begin{array}{llll}\mathrm{H} & 6.29706300 & 0.14242200 & 0.11883600\end{array}$

$\begin{array}{llll}\text { C } & 6.17205300 & 2.17182000 & 0.87442900\end{array}$

$\begin{array}{llll}\mathrm{H} & 7.18826200 & 2.44997600 & 0.57775100\end{array}$

$\begin{array}{llll}\mathrm{H} & 5.53875600 & 3.06197900 & 0.76824600\end{array}$

$\begin{array}{llll}\mathrm{N} & -3.64284200 & 0.62475100 & 0.70017500\end{array}$

$\begin{array}{llll}\mathrm{C} & -3.90738600 & -0.80962300 & 0.76230700\end{array}$

$\mathrm{H} \quad-4.40609000 \quad-1.03130300 \quad 1.71934000$

$\begin{array}{llll}\mathrm{H} & -2.97132100 & -1.37117400 & 0.73469400\end{array}$

C $\quad \begin{array}{llll}-5.75679100 & 0.97465900 & -0.44355900\end{array}$

$\mathrm{N} \quad-6.04990700 \quad-0.44794300 \quad-0.34926800$

$\mathrm{H} \quad-6.70070800 \quad 1.53321000 \quad-0.40848600$

$\mathrm{H} \quad \begin{array}{llll}\mathrm{H} & -5.26452600 & 1.21136600 & -1.41244700\end{array}$

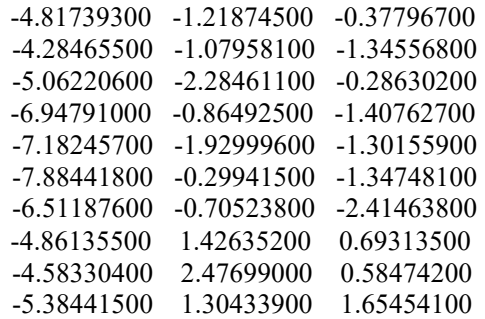

\section{Clomipramine}

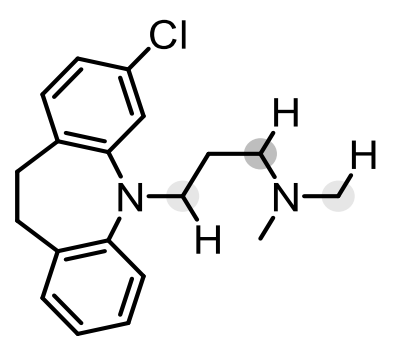

$$
\begin{aligned}
& E=-847.555701 \\
& H_{\text {Corr. }}=0.41038
\end{aligned}
$$

\begin{tabular}{|c|c|c|c|}
\hline $\mathrm{C}$ & 0.30578900 & 3.15410200 & -1.45399400 \\
\hline $\mathrm{C}$ & 0.34096500 & 1.78125800 & -1.25421300 \\
\hline $\mathrm{C}$ & -0.50978300 & 1.13778800 & -0.33742600 \\
\hline $\mathrm{C}$ & -1.41645700 & 1.92658700 & 0.40578500 \\
\hline $\mathrm{C}$ & -1.43963800 & 3.30583700 & 0.16906700 \\
\hline $\mathrm{C}$ & -0.60037400 & 3.93255100 & -0.74159600 \\
\hline $\mathrm{H}$ & 0.98337300 & 3.60808500 & -2.17360600 \\
\hline $\mathrm{H}$ & 1.04738800 & 1.19240700 & -1.83337100 \\
\hline $\mathrm{H}$ & -2.14338400 & 3.90593200 & 0.74694900 \\
\hline $\mathrm{H}$ & -0.64691500 & 5.00923200 & -0.88408400 \\
\hline $\mathrm{C}$ & -2.37748200 & 1.43484400 & 1.46735000 \\
\hline $\mathrm{H}$ & -3.40697300 & 1.57463700 & 1.10046200 \\
\hline $\mathrm{H}$ & -2.28138300 & 2.10278000 & 2.33509900 \\
\hline $\mathrm{C}$ & -2.23381200 & -0.00988400 & 1.93042500 \\
\hline $\mathrm{H}$ & -2.92832500 & -0.18767800 & 2.76081500 \\
\hline $\mathrm{H}$ & -1.21581900 & -0.17808300 & 2.31046400 \\
\hline $\mathrm{N}$ & -0.41620700 & -0.27071200 & -0.18647900 \\
\hline $\mathrm{C}$ & -1.60595100 & -1.04745600 & -0.24361300 \\
\hline $\mathrm{C}$ & -1.85202400 & -1.91768400 & -1.30884500 \\
\hline $\mathrm{C}$ & -2.52129400 & -0.96023600 & 0.81378500 \\
\hline $\mathrm{C}$ & -3.00636100 & -2.69572800 & -1.32975600 \\
\hline $\mathrm{H}$ & -1.13985900 & -1.97791000 & -2.12977100 \\
\hline $\mathrm{C}$ & -3.68199200 & -1.73195200 & 0.76924800 \\
\hline $\mathrm{C}$ & -3.92849400 & -2.60096000 & -0.29076800 \\
\hline $\mathrm{H}$ & -3.18816100 & -3.36796900 & -2.16518500 \\
\hline $\mathrm{H}$ & -4.39294600 & -1.66033700 & 1.59201500 \\
\hline $\mathrm{H}$ & -4.83354700 & -3.20354100 & -0.30352500 \\
\hline $\mathrm{C}$ & 0.81945100 & -0.94299100 & -0.57071600 \\
\hline $\mathrm{H}$ & 0.64699600 & -2.01789000 & -0.43890400 \\
\hline $\mathrm{H}$ & 1.06413700 & -0.80850500 & -1.64148800 \\
\hline $\mathrm{C}$ & 1.99995500 & -0.52990700 & 0.29713900 \\
\hline $\mathrm{H}$ & 1.84452300 & -0.90062400 & 1.32045300 \\
\hline $\mathrm{H}$ & 2.04933200 & 0.56516800 & 0.35895300 \\
\hline $\mathrm{C}$ & 3.29609000 & -1.08925200 & -0.26222000 \\
\hline $\mathrm{H}$ & 3.15662600 & -2.15553400 & -0.49961600 \\
\hline $\mathrm{H}$ & 3.52634900 & -0.58099800 & -1.22902900 \\
\hline $\mathrm{N}$ & 4.42539700 & -0.98905500 & 0.64836200 \\
\hline $\mathrm{C}$ & 5.57758900 & -1.67238900 & 0.09245500 \\
\hline $\mathrm{H}$ & 5.91672100 & -1.21656500 & -0.86137700 \\
\hline $\mathrm{H}$ & 6.41281600 & -1.63732000 & 0.80086000 \\
\hline $\mathrm{H}$ & 5.33570300 & -2.72391100 & -0.09992500 \\
\hline $\mathrm{C}$ & 4.76027100 & 0.39096400 & 0.94771500 \\
\hline $\mathrm{H}$ & 5.64095400 & 0.42223600 & 1.59846100 \\
\hline $\mathrm{H}$ & 4.98936100 & 0.97236400 & 0.02949100 \\
\hline $\mathrm{H}$ & 3.94002000 & 0.88943100 & 1.47375500 \\
\hline
\end{tabular}

Clomipramine $\mathbf{I}_{1}$ 
<smiles>CN(C)CCCN1c2ccccc2CCc2ccc(Cl)cc21</smiles>

$\mathrm{E}=-846.899095$

$$
\mathrm{H}_{\text {Corr. }}=0.396916
$$

$\begin{array}{llll}\text { C } & 2.93761500 & -2.91313100 & -0.96105100\end{array}$ C $\quad 1.76580500-2.16815600 \quad-0.94514300$

C $\quad 1.63080200 \quad-1.01406700 \quad-0.15514900$

$\begin{array}{llll}\text { C } & 2.72590100 & -0.59919700 & 0.63448300\end{array}$

$\begin{array}{llll}\text { C } & 3.89787300 & -1.36134400 & 0.58391700\end{array}$

C $\quad 4.02255600 \quad-2.50685300 \quad-0.19167200$

$\mathrm{H} \quad 3.00127000 \quad-3.80094900 \quad-1.58610700$

$\mathrm{H} \quad 0.93840200 \quad-2.47975900 \quad-1.57692000$

$\begin{array}{llll}\mathrm{H} & 4.73828800 & -1.04009600 & 1.20025700\end{array}$

$\begin{array}{llll}\mathrm{H} & 4.95046000 & -3.07321200 & -0.19097000\end{array}$

$\begin{array}{llll}\text { C } & 2.76223600 & 0.59860200 & 1.56050600\end{array}$

$\begin{array}{llll}\mathrm{H} & 3.48656200 & 1.32549800 & 1.15922100\end{array}$

$\begin{array}{llll}\mathrm{H} & 3.18266500 & 0.26462500 & 2.51972200\end{array}$

$\begin{array}{llll}\mathrm{C} & 1.45264900 & 1.33618900 & 1.82088700\end{array}$

$\mathrm{H} \quad \begin{array}{llll}\mathrm{H} & 1.63237100 & 2.12215200 & 2.56493900\end{array}$

$\begin{array}{llll}\mathrm{H} & 0.71342600 & 0.64256700 & 2.24708500\end{array}$

$\mathrm{N} \quad 0.39607000 \quad-0.31774300 \quad-0.16551400$

$\begin{array}{llll}\mathrm{C} & 0.41183100 & 1.08195000 & -0.42417000\end{array}$

$\begin{array}{lllll}\text { C } & -0.08281300 & 1.60726100 & -1.61986300\end{array}$

$\begin{array}{llll}\mathrm{C} & 0.91263600 & 1.93893200 & 0.56403400\end{array}$

C $\quad-0.07627800 \quad 2.98155200 \quad-1.83945100$

$\mathrm{H} \quad-0.47388500 \quad 0.93646300 \quad-2.38281800$

$\begin{array}{llll}\mathrm{C} & 0.93082400 & 3.31268400 & 0.32208800\end{array}$

$\begin{array}{lllll}\mathrm{C} & 0.43554500 & 3.83891900 & -0.86830800\end{array}$

$\mathrm{H} \quad-0.46442400 \quad 3.38054900 \quad-2.77377200$

$\begin{array}{llll}\mathrm{H} & 1.32581200 & 3.97761700 & 1.08974600\end{array}$

$\begin{array}{lll}0.44562700 & 4.91324800 & -1.03589000\end{array}$

$\begin{array}{lll}-2.05865100 & -0.43120600 & 0.09298200\end{array}$

$\begin{array}{llll}-2.28491100 & 0.54460300 & -0.35873900\end{array}$

$\begin{array}{lll}-1.85943000 & -0.25423000 & 1.16003600\end{array}$

$\begin{array}{lll}-4.52429700 & -0.73816700 & 0.23244100\end{array}$

$\begin{array}{llll}-5.64920600 & -1.31821700 & -0.32655800\end{array}$

$\begin{array}{lll}-6.61276800 & -0.92790900 & -0.01165600\end{array}$

$-5.54359500-1.73729000-1.32345100$

$\begin{array}{lll}-4.64918800 & -0.28070500 & 1.60201000\end{array}$

$\begin{array}{lll}-5.63338700 & 0.17520700 & 1.74353100\end{array}$

$\begin{array}{lll}-4.54425900 & -1.11398800 & 2.31975300\end{array}$

$\begin{array}{lll}-3.89078400 & 0.47446300 & 1.83033600\end{array}$

$-0.81192000 \quad-1.04528200 \quad-0.52664300$

$\begin{array}{lll}-0.69901900 & -2.06729900 & -0.14046100\end{array}$

$\begin{array}{lll}-0.94833000 & -1.14198300 & -1.62146300\end{array}$

$\begin{array}{llll}-3.24989300 & -1.35519200 & -0.09777300\end{array}$

$\begin{array}{lll}-3.10964700 & -2.27908600 & 0.49927400\end{array}$

$-3.30279000 \quad-1.66625900 \quad-1.15089500$

Clomipramine $\mathbf{I}_{2}$

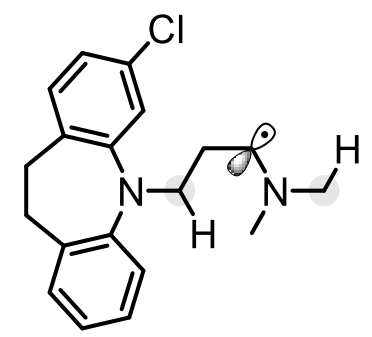

$E=-846.901736$

\begin{tabular}{|c|c|c|c|}
\hline $\mathrm{C}$ & 2.83391500 & -3.00592700 & -0.94795500 \\
\hline $\mathrm{C}$ & 1.69399200 & -2.21313300 & -0.94102300 \\
\hline $\mathrm{C}$ & 1.59969600 & -1.05531900 & -0.15014300 \\
\hline $\mathrm{C}$ & 2.70433200 & -0.68762700 & 0.64971000 \\
\hline $\mathrm{C}$ & 3.84409300 & -1.49759700 & 0.60812400 \\
\hline $\mathrm{C}$ & 3.92811200 & -2.64626500 & -0.16829500 \\
\hline $\mathrm{H}$ & 2.86610600 & -3.89479900 & -1.57401500 \\
\hline $\mathrm{H}$ & 0.85935100 & -2.48886000 & -1.57993500 \\
\hline $\mathrm{H}$ & 4.69156000 & -1.21243500 & 1.23256100 \\
\hline $\mathrm{H}$ & 4.83170300 & -3.25068800 & -0.16054900 \\
\hline $\mathrm{C}$ & 2.78236600 & 0.50528400 & 1.57955900 \\
\hline $\mathrm{H}$ & 3.54090200 & 1.20183300 & 1.18765200 \\
\hline $\mathrm{H}$ & 3.17871500 & 0.15117800 & 2.54187200 \\
\hline $\mathrm{C}$ & 1.50263300 & 1.29685600 & 1.82915800 \\
\hline $\mathrm{H}$ & 1.70796400 & 2.07324600 & 2.57667800 \\
\hline $\mathrm{H}$ & 0.73084200 & 0.63400700 & 2.24638100 \\
\hline $\mathrm{N}$ & 0.39608700 & -0.30793300 & -0.17176800 \\
\hline $\mathrm{C}$ & 0.47460400 & 1.08992800 & -0.42648900 \\
\hline $\mathrm{C}$ & 0.01516900 & 1.63764800 & -1.62648100 \\
\hline $\mathrm{C}$ & 1.00121100 & 1.92374700 & 0.56809300 \\
\hline $\mathrm{C}$ & 0.08337800 & 3.01043100 & -1.84413300 \\
\hline $\mathrm{H}$ & -0.39696000 & 0.98471500 & -2.39377000 \\
\hline $\mathrm{C}$ & 1.08021300 & 3.29594500 & 0.32869800 \\
\hline $\mathrm{C}$ & 0.62075200 & 3.84427000 & -0.86606300 \\
\hline $\mathrm{H}$ & -0.27716500 & 3.42672600 & -2.78199400 \\
\hline $\mathrm{H}$ & 1.49504600 & 3.94248700 & 1.10164800 \\
\hline $\mathrm{H}$ & 0.67872000 & 4.91742100 & -1.03181000 \\
\hline $\mathrm{C}$ & -2.06146300 & -0.32827900 & 0.08942300 \\
\hline $\mathrm{H}$ & -2.18530000 & 0.69884800 & -0.30456100 \\
\hline $\mathrm{H}$ & -1.86228100 & -0.20779800 & 1.16872900 \\
\hline $\mathrm{C}$ & -3.27746600 & -1.14755000 & -0.16842900 \\
\hline $\mathrm{H}$ & -3.27229900 & -1.83496100 & -1.01644000 \\
\hline $\mathrm{N}$ & -4.53902200 & -0.66321500 & 0.16216000 \\
\hline $\mathrm{C}$ & -5.63708900 & -1.58323700 & -0.05436900 \\
\hline $\mathrm{H}$ & -5.66683800 & -2.37831300 & 0.71237500 \\
\hline $\mathrm{H}$ & -6.58814100 & -1.04031900 & -0.02841300 \\
\hline $\mathrm{H}$ & -5.53657800 & -2.05557000 & -1.03697300 \\
\hline $\mathrm{C}$ & -4.66719200 & 0.09379500 & 1.39192600 \\
\hline $\mathrm{H}$ & -5.66513400 & 0.54181100 & 1.43923300 \\
\hline $\mathrm{H}$ & -4.52899900 & -0.54493100 & 2.28429000 \\
\hline $\mathrm{H}$ & -3.93171800 & 0.90238600 & 1.42824800 \\
\hline $\mathrm{C}$ & -0.84003000 & -0.98267600 & -0.54125100 \\
\hline $\mathrm{H}$ & -0.77425800 & -2.01233400 & -0.16750200 \\
\hline $\mathrm{H}$ & -0.98205700 & -1.05736900 & -1.63682200 \\
\hline
\end{tabular}

Clomipramine $\mathbf{I}_{3}$

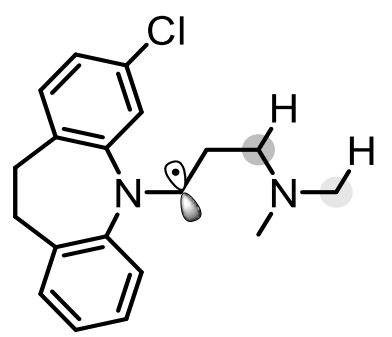

$E=-846.903205$

$\mathrm{H}_{\text {Corr. }}=0.39659$

C $\quad-1.07313900 \quad-2.59340400 \quad-1.46771600$

$\begin{array}{llll}\text { C } & -0.77795500 & -1.27988300 & -1.14024400\end{array}$

$\begin{array}{lllll}\mathrm{C} & 0.27618400 & -0.95737800 & -0.26897700\end{array}$

$\begin{array}{llll}\mathrm{C} & 1.06885700 & -1.98943200 & 0.27086600\end{array}$

C $\quad 0.75523100 \quad-3.30540500 \quad-0.09096200$

C $\quad-0.29837200-3.62350100 \quad-0.93733000$

$\mathrm{H} \quad-1.89613000 \quad-2.80928200 \quad-2.14538400$

H $\quad-1.35567700 \quad-0.46279000 \quad-1.56954300$

$\mathrm{H} \quad \begin{array}{llll}1.36450800 & -4.10647600 & 0.32858700\end{array}$

$\mathrm{H} \quad-0.51164000 \quad-4.66145400 \quad-1.18034000$ 


\begin{abstract}
$\begin{array}{lll}2.23941100 & -1.81984700 & 1.21413800\end{array}$
$\begin{array}{llll}3.15331300 & -2.14005600 & 0.68868700\end{array}$

$\begin{array}{llll}2.11016600 & -2.53351000 & 2.03999300\end{array}$

$\begin{array}{lll}2.48838700 & -0.43004300 & 1.79169200\end{array}$

$\begin{array}{lll}3.31922400 & -0.48923400 & 2.50545200\end{array}$

$\begin{array}{lll}1.60307500 & -0.09593800 & 2.35130100\end{array}$

$\begin{array}{lll}0.48960000 & 0.41726500 & 0.02955100\end{array}$

$\begin{array}{lll}1.79802800 & 0.95190400 & -0.16206900\end{array}$

$\begin{array}{llll}2.05297700 & 1.86407000 & -1.18554900\end{array}$

$\begin{array}{llll}2.81235500 & 0.56065100 & 0.71817200\end{array}$

$\begin{array}{lll}3.33178200 & 2.39047400 & -1.34227400\end{array}$

$\begin{array}{lll}1.24653400 & 2.14774600 & -1.85911700\end{array}$

$\begin{array}{lll}4.09205500 & 1.08539300 & 0.53661700\end{array}$

$\begin{array}{lll}4.35491200 & 1.99801200 & -0.48210000\end{array}$

$\begin{array}{llll}3.53005400 & 3.09904400 & -2.14292300\end{array}$

$\begin{array}{lll}4.88758100 & 0.78319200 & 1.21699100\end{array}$

$\begin{array}{llll}5.35631300 & 2.40420100 & -0.60287300\end{array}$

$\begin{array}{lll}-0.57953700 & 1.30990600 & 0.10734100\end{array}$

$\begin{array}{lll}-0.28709500 & 2.35720300 & 0.06821200\end{array}$

$\begin{array}{lll}-1.85739300 & 0.93542000 & 0.77828000\end{array}$

$\begin{array}{lll}-1.91456500 & 1.37933100 & 1.78791800\end{array}$

$\begin{array}{lll}-1.89359200 & -0.15121100 & 0.92227900\end{array}$

$\begin{array}{llll}-3.07329400 & 1.40954400 & -0.00976200\end{array}$

$\begin{array}{llll}-3.02274900 & 2.50406800 & -0.10650800\end{array}$

$\begin{array}{llll}-3.02492400 & 1.00471700 & -1.04718400\end{array}$

$\begin{array}{lll}-4.34326800 & 1.07098400 & 0.61443100\end{array}$

$\begin{array}{llll}-5.42802100 & 1.78933300 & -0.02751100\end{array}$

$\begin{array}{llll}-5.52669100 & 1.52770400 & -1.10194900\end{array}$

$\begin{array}{lll}-6.37796100 & 1.55436400 & 0.46593100\end{array}$

$\begin{array}{llll}-5.26174000 & 2.86992000 & 0.04656300\end{array}$

$\begin{array}{lll}-4.59913200 & -0.35727500 & 0.56066600\end{array}$

$\begin{array}{lll}-5.56809600 & -0.57764100 & 1.02198900\end{array}$

$\begin{array}{lll}-4.61919800 & -0.73476100 & -0.48418800\end{array}$

$\begin{array}{lll}-3.83504500 & -0.91810900 & 1.10856900\end{array}$
\end{abstract}

\section{Chlorpheniramine}<smiles>CN(C)CCC(c1ccc(Cl)cc1)c1ccccn1</smiles>

$\mathrm{E}=-1190.486931$

$\mathrm{H}_{\text {Corr. }}=0.335658$

$\mathrm{Cl} \quad 4.91669700 \quad 0.85026500 \quad 0.42593900$

$\begin{array}{lllll}\mathrm{N} & -2.25224400 & 1.39449100 & 0.94221500\end{array}$

$\mathrm{N} \quad \quad-1.50404100 \quad-3.69662900 \quad-0.08002600$

C $\quad-1.76395300 \quad 1.29426400 \quad-0.29883100$

$\begin{array}{lllll}\text { C } & -1.91381300 & 2.31489500 & -1.24269900\end{array}$

$\begin{array}{lllll}\text { C } & -2.59440800 & 3.47100600 & -0.88727400\end{array}$

$\begin{array}{llll}\text { C } & -3.10632800 & 3.57671000 & 0.40270400\end{array}$

$\begin{array}{llll}\mathrm{C} & -2.90580900 & 2.51211400 & 1.27368100\end{array}$

$\begin{array}{lllll}\text { C } & -0.98628000 & 0.04650800 & -0.66348800\end{array}$

$\begin{array}{lll}-1.52967500 & -1.21036600 & 0.01912700\end{array}$

$\begin{array}{llll}-0.82023900 & -2.46706600 & -0.45580300\end{array}$

$-0.84727100-4.83620200 \quad-0.69155000$

$\begin{array}{llll}-1.55525100 & -3.86763000 & 1.36061100\end{array}$

$\begin{array}{llll}0.49079400 & 0.25840600 & -0.39084700\end{array}$

$\begin{array}{llll}1.42084700 & 0.20544500 & -1.42937900\end{array}$

$\begin{array}{lll}2.78173300 & 0.38524400 & -1.19151200\end{array}$

$\begin{array}{lll}3.20752300 & 0.62289700 & 0.10817700\end{array}$

$\begin{array}{lll}2.30453200 & 0.68619600 & 1.16471000\end{array}$

$\begin{array}{lll}0.95092400 & 0.50456100 & 0.90675100\end{array}$

$\begin{array}{lll}-1.49502000 & 2.19361300 & -2.24037700\end{array}$

$\begin{array}{llll}-2.72599800 & 4.27702200 & -1.60521600\end{array}$

$\begin{array}{lll}-3.64820000 & 4.46006700 & 0.72834700\end{array}$

$\begin{array}{lll}-3.29124500 & 2.55734800 & 2.29239800\end{array}$

$\begin{array}{lll}-1.09320800 & -0.08871500 & -1.75171800\end{array}$

$-2.60370600 \quad-1.29985800 \quad-0.19954800$ $\begin{array}{lll}-1.44163300 & -1.09120900 & 1.10640300\end{array}$

$-0.75317600 \quad-2.45000500 \quad-1.55555800$

$\begin{array}{llll}0.22834700 & -2.47552300 & -0.07938200\end{array}$

$-1.38757200 \quad-5.75736800 \quad-0.44585800$

$\begin{array}{llll}0.20206300 & -4.95107000 & -0.34780900\end{array}$

$-0.83758400 \quad-4.72526600 \quad-1.78197900$

$\begin{array}{llll}-2.03222300 & -4.82440100 & 1.60002600\end{array}$

$\begin{array}{lll}-0.54240000 & -3.86365200 & 1.81632000\end{array}$

$\begin{array}{lll}-2.14593700 & -3.07458200 & 1.82985400\end{array}$

$\begin{array}{llll}1.07888400 & 0.01368400 & -2.44614300\end{array}$

$\begin{array}{llll}3.50186200 & 0.33951500 & -2.00408800\end{array}$

$\begin{array}{llll}2.65930600 & 0.87702400 & 2.17407900\end{array}$

$\begin{array}{llll}0.23763200 & 0.56194900 & 1.72768300\end{array}$

Chlorpheniramine $\mathbf{I}_{1}$<smiles>CN(C)CCC(c1ccc(Cl)cc1)c1ccccn1</smiles>

$E=-1189.829371$

$\mathrm{H}_{\text {Corr. }}=0.322277$

Cl $\quad 4.98877700 \quad 0.34063300 \quad 0.43063400$

$\begin{array}{lllll}\mathrm{N} & -2.10821900 & 1.59379300 & 0.93030900\end{array}$

$\begin{array}{llll}\mathrm{N} & -1.83386300 & -3.55643800 & 0.02011700\end{array}$

$\begin{array}{llll}-1.61289900 & 1.43148900 & -0.30096900\end{array}$

$\begin{array}{lll}-1.63621800 & 2.45352200 & -1.25470800\end{array}$

$\begin{array}{lll}-2.19422500 & 3.67900800 & -0.91912500\end{array}$

$\begin{array}{llll}-2.71291700 & 3.85043600 & 0.36106400\end{array}$

$\begin{array}{lll}-2.64313800 & 2.77811400 & 1.24283600\end{array}$

$\begin{array}{llll}-0.96606000 & 0.10483300 & -0.64318700\end{array}$

$\begin{array}{lll}-1.63350600 & -1.07615700 & 0.06414000\end{array}$

$\begin{array}{lll}-1.04179100 & -2.40233600 & -0.38014500\end{array}$

$\begin{array}{llll}-1.45085200 & -4.77836300 & -0.65719600\end{array}$

$\begin{array}{lll}-2.17493600 & -3.68578300 & 1.35418100\end{array}$

$\begin{array}{llll}0.52540900 & 0.17229300 & -0.37450600\end{array}$

$\begin{array}{llll}1.44338900 & 0.01728300 & -1.41352900\end{array}$

$\begin{array}{llll}2.81577600 & 0.06680000 & -1.17907400\end{array}$

$\begin{array}{llll}3.26527000 & 0.27642700 & 0.11746500\end{array}$

$\begin{array}{llll}2.37497000 & 0.43891300 & 1.17422500\end{array}$

$\begin{array}{llll}1.00966100 & 0.38716600 & 0.91984200\end{array}$

$\begin{array}{llll}-1.21619000 & 2.27907600 & -2.24398600\end{array}$

$\begin{array}{llll}-2.22651900 & 4.48815400 & -1.64482100\end{array}$

$\begin{array}{llll}-3.16168900 & 4.78983600 & 0.67100100\end{array}$

$\begin{array}{lll}-3.03867700 & 2.87310500 & 2.25414700\end{array}$

$\begin{array}{lll}-1.08848800 & -0.03881400 & -1.72875400\end{array}$

$\begin{array}{llll}-2.70991200 & -1.06443700 & -0.15941600\end{array}$

$\begin{array}{lll}-1.53744600 & -0.94443300 & 1.14875900\end{array}$

$-0.97624300 \quad-2.42164300 \quad-1.47912600$

$\begin{array}{lll}-0.00524700 & -2.50497500 & 0.00031600\end{array}$

$\begin{array}{llll}-2.15333100 & -5.57768800 & -0.40081400\end{array}$

$\begin{array}{llll}-0.43484100 & -5.10439900 & -0.37237900\end{array}$

$-1.47695500 \quad-4.62833400 \quad-1.74202800$

$\begin{array}{lll}-2.65953500 & -4.61487400 & 1.64094000\end{array}$

$\begin{array}{lll}-2.40188700 & -2.78336600 & 1.91191200\end{array}$

$\begin{array}{lll}1.08216400 & -0.15113400 & -2.42781100\end{array}$

$\begin{array}{llll}3.52641700 & -0.05740900 & -1.99176700\end{array}$

$\begin{array}{lll}2.74875800 & 0.60479500 & 2.18107600\end{array}$

$\begin{array}{lll}0.30738300 & 0.52242200 & 1.74110100\end{array}$

Chlorpheniramine $\mathbf{I}_{2}$ 
<smiles>CN(C)CCC(c1ccc(Cl)cc1)c1ccccn1</smiles>

$E=-1189.832756$

$\mathrm{H}_{\text {Corr. }}=0.322231$

$\mathrm{Cl} \quad 4.70144800 \quad 1.53217100 \quad 0.46742200$

$\begin{array}{llll}\mathrm{N} & -2.47415700 & 1.00438300 & 0.92223600\end{array}$

$\mathrm{N} \quad-0.74091000 \quad-3.86772600 \quad-0.13152900$

$\begin{array}{llll}\mathrm{C} & -1.94657500 & 1.00524200 & -0.30668600\end{array}$

$\begin{array}{llll}\text { C } & -2.19770500 & 2.03009400 & -1.22404400\end{array}$

$\begin{array}{llll}\mathrm{C} & -3.02320100 & 3.08268400 & -0.85433800\end{array}$

$\begin{array}{llll}\text { C } & -3.57579100 & 3.08309600 & 0.42319500\end{array}$

$\begin{array}{llll}\text { C } & -3.26803900 & 2.02235400 & 1.26754200\end{array}$

$\begin{array}{llll}\text { C } & -1.00979400 & -0.12315300 & -0.68425600\end{array}$

C $\quad-1.38503000 \quad-1.45218400 \quad-0.02780300$

C $\quad-0.52919000 \quad-2.55550400 \quad-0.53829800$

C $\quad 0.23257100 \quad-4.83273900 \quad-0.60186200$

$\begin{array}{llll}\mathrm{C} & 0.42369800 & 0.27664300 & -0.39262000\end{array}$

C $\quad 1.33844200 \quad 0.45217300 \quad-1.43131700$

$\begin{array}{llll}\mathrm{C} & 2.65395800 & 0.83537400 & -1.18039300\end{array}$

$\begin{array}{llll}\text { C } & 3.04911300 & 1.04763900 & 0.13316300\end{array}$

$\begin{array}{llll}\mathrm{C} & 2.15936900 & 0.88779400 & 1.19072300\end{array}$

$\begin{array}{llll}\mathrm{C} & 0.85114600 & 0.50540300 & 0.91925500\end{array}$

$\mathrm{H} \quad-1.74312800 \quad 1.99275600 \quad-2.21275200$

$\mathrm{H} \quad-3.23478900 \quad 3.88970600 \quad-1.55177900$

$\mathrm{H} \quad-4.22941600 \quad 3.88295300 \quad 0.75936800$

$\mathrm{H} \quad-3.68184200 \quad 1.98659600 \quad 2.27549600$

$\mathrm{H} \quad-1.08916500 \quad-0.25370100 \quad-1.77534700$

$\mathrm{H} \quad-2.46142800 \quad-1.64142000 \quad-0.21585400$

$\mathrm{H} \quad-1.30401800 \quad-1.34125900 \quad 1.06443400$

$\mathrm{H} \quad-0.08782300 \quad-2.46210600 \quad-1.53267800$

$\mathrm{H} \quad-0.16065900 \quad-5.84791400 \quad-0.48014300$

$\mathrm{H} \quad 1.18695600 \quad-4.75778900 \quad-0.05062600$

$\mathrm{H} \quad 0.43517100 \quad-4.66627200 \quad-1.66485500$

$\begin{array}{llll}\mathrm{H} & 1.02091000 & 0.28070100 & -2.45961900\end{array}$

$\mathrm{H} \quad 3.36302200 \quad 0.96581400 \quad-1.99352900$

$\begin{array}{llll}\mathrm{H} & 2.48873400 & 1.06368100 & 2.21135900\end{array}$

$\begin{array}{llll}\mathrm{H} & 0.14726900 & 0.39010000 & 1.74216200\end{array}$

C $\quad-1.15192600 \quad-4.09619500 \quad 1.23981100$

$\mathrm{H} \quad-0.34456900 \quad-3.85774400 \quad 1.95697800$

$\mathrm{H} \quad-2.02732700 \quad-3.49031900 \quad 1.48981500$

$\mathrm{H} \quad-1.42332300 \quad-5.14960900 \quad 1.36403300$

Chlorpheniramine $\mathbf{I}_{3}$<smiles>CN(C)C(CCC(=O)c1ccc(Cl)cc1)c1ccccn1</smiles>

$$
\mathrm{E}=-1189.848604
$$

$$
\mathrm{H}_{\text {Corr. }}=0.322582
$$

$\begin{array}{llcc}\mathrm{Cl} & 5.20392800 & 0.39919700 & -0.31077400 \\ \mathrm{~N} & -2.90167100 & 1.11695200 & -0.15088100 \\ \mathrm{~N} & -1.92368000 & -3.35645000 & -0.05488100 \\ \mathrm{C} & -1.62274900 & 1.38318800 & 0.21333600 \\ \mathrm{C} & -1.19641200 & 2.71131700 & 0.43159500 \\ \mathrm{C} & -2.08502300 & 3.75606300 & 0.23662700 \\ \mathrm{C} & -3.38484100 & 3.47358800 & -0.17516300\end{array}$

$\begin{array}{lll}-3.73428100 & 2.13351400 & -0.34327700\end{array}$

$\begin{array}{lll}-0.75843500 & 0.23883900 & 0.43289600\end{array}$

$\begin{array}{lll}-1.40089000 & -1.06892800 & 0.78537300\end{array}$

$-2.20294200 \quad-4.11041300 \quad-1.26325100$

$\begin{array}{llll}0.67657100 & 0.30341800 & 0.27123400\end{array}$

$\begin{array}{lll}1.29949100 & 1.25985100 & -0.56710100\end{array}$

$\begin{array}{llll}2.67237300 & 1.29263200 & -0.74880200\end{array}$

$\begin{array}{llll}3.47011900 & 0.35911700 & -0.09208300\end{array}$

$\begin{array}{llll}2.90204600 & -0.61560800 & 0.72345300\end{array}$

$\begin{array}{lll}1.52690000 & -0.64452000 & 0.89014500\end{array}$

$\begin{array}{lll}-0.19058800 & 2.90947200 & 0.79145100\end{array}$

$\begin{array}{llll}-1.76992100 & 4.78107400 & 0.41716700\end{array}$

$\begin{array}{llll}-4.11438800 & 4.26029100 & -0.34396900\end{array}$

$\begin{array}{llll}-4.74847700 & 1.87025500 & -0.64630900\end{array}$

$\begin{array}{lll}-2.44121000 & -0.90607300 & 1.08217500\end{array}$

$\begin{array}{lll}-0.88894300 & -1.51098200 & 1.65115700\end{array}$

$\begin{array}{llll}-2.63711700 & -5.08310600 & -1.00562400\end{array}$

$\begin{array}{llll}-1.28825600 & -4.29217300 & -1.86527600\end{array}$

$\begin{array}{lll}-2.92150200 & -3.56886900 & -1.88820100\end{array}$

$\begin{array}{llll}0.68768700 & 1.96558800 & -1.12282100\end{array}$

$\begin{array}{lll}3.12507400 & 2.02750700 & -1.40920100\end{array}$

$\begin{array}{llll}3.53480100 & -1.34418800 & 1.22325200\end{array}$

$\begin{array}{lll}1.10581700 & -1.41304100 & 1.53355500\end{array}$

$\begin{array}{lll}-1.01961600 & -4.10755700 & 0.79631700\end{array}$

$\begin{array}{llll}-0.03061600 & -4.26413300 & 0.31530500\end{array}$

$\begin{array}{lll}-0.85774600 & -3.59626600 & 1.75131600\end{array}$

$\begin{array}{lll}-1.44998600 & -5.09022900 & 1.01857100\end{array}$

$\begin{array}{lll}-1.38440600 & -2.04741700 & -0.39000500\end{array}$

$-0.35171500 \quad-2.14154800 \quad-0.79481100$

$\begin{array}{llll}-1.99968200 & -1.61595000 & -1.19199600\end{array}$

\section{Diltiazem}<smiles>CN=C(C)CN1C(=O)C(OC(C)=O)[C@@H](c2ccc(OC)cc2)Sc2ccccc21</smiles>

$\mathrm{E}=-1662.116187$

$\mathrm{H}_{\text {Corr. }}=0.477624$ 


$\begin{array}{lrrr}\mathrm{C} & 3.30788400 & -1.85612600 & -0.40578800 \\ \mathrm{H} & 2.74966300 & -2.14319300 & 0.50858000 \\ \mathrm{H} & 4.20667000 & -1.32041400 & -0.07058800 \\ \mathrm{C} & 4.90994400 & -3.63032900 & -0.56873000 \\ \mathrm{H} & 5.22307700 & -4.49457600 & -1.16542600 \\ \mathrm{H} & 5.73726000 & -2.91224100 & -0.54705900 \\ \mathrm{H} & 4.72496100 & -3.97995100 & 0.46756800 \\ \mathrm{C} & 2.66665300 & -4.00870200 & -1.25778400 \\ \mathrm{H} & 2.39659700 & -4.41805000 & -0.26331900 \\ \mathrm{H} & 1.76276900 & -3.57071900 & -1.69260900 \\ \mathrm{H} & 2.98450300 & -4.83815700 & -1.89921100 \\ \mathrm{C} & -3.57323900 & 1.19285500 & 0.73357600 \\ \mathrm{H} & -3.51927200 & 1.94083300 & 1.52373100 \\ \mathrm{C} & -4.78838800 & 0.59047400 & 0.45256800 \\ \mathrm{H} & -5.69012900 & 0.85257000 & 1.00028300 \\ \mathrm{C} & -3.72582000 & -0.72032900 & -1.27655200 \\ \mathrm{H} & -3.76158300 & -1.46384000 & -2.06682500 \\ \mathrm{C} & -2.51274600 & -0.10351700 & -0.97996200 \\ \mathrm{H} & -1.63113100 & -0.38581100 & -1.55134500 \\ \mathrm{C} & 3.54072400 & 1.66675900 & -0.18434700 \\ \mathrm{H} & 4.20628000 & 0.83383300 & 0.02874600 \\ \mathrm{C} & 4.03448500 & 2.96578900 & -0.19360600 \\ \mathrm{H} & 5.08952900 & 3.13900200 & 0.00184800 \\ \mathrm{C} & 3.17838300 & 4.03717900 & -0.43289400 \\ \mathrm{H} & 3.55688500 & 5.05588400 & -0.43219900 \\ \mathrm{C} & 1.82871300 & 3.79741000 & -0.66421200 \\ \mathrm{H} & 1.14542100 & 4.62353800 & -0.84741900\end{array}$

Diltiazem $\mathbf{I}_{1}$

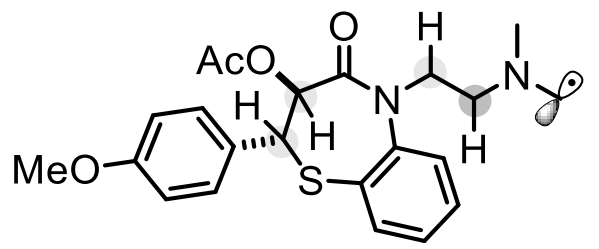

$E=-1661.458101$

$\mathrm{H}_{\text {Corr. }}=0.46394$

$\begin{array}{lrrr}\mathrm{S} & -0.36115200 & 2.17643700 & -1.20088400 \\ \mathrm{C} & 0.59260200 & -0.39221100 & 0.21870800 \\ \mathrm{~N} & 1.73598500 & 0.02293400 & -0.41438800 \\ \mathrm{C} & 1.33176000 & 2.43797700 & -0.70560100 \\ \mathrm{C} & 2.19487500 & 1.36501800 & -0.41149600 \\ \mathrm{C} & -2.42438900 & 0.83393700 & -0.01335700 \\ \mathrm{C} & -4.88487300 & -0.38997500 & -0.59763400 \\ \mathrm{O} & -6.11474600 & -0.92112900 & -0.80994200 \\ \mathrm{O} & -0.80753600 & -0.00484200 & 2.14714300 \\ \mathrm{C} & -0.03350600 & -0.63673700 & 3.05551800 \\ \mathrm{O} & 1.17658300 & -0.61092000 & 3.01426200 \\ \mathrm{O} & 0.17331400 & -1.53607400 & 0.12485400 \\ \mathrm{~N} & 3.80117800 & -3.05054400 & -0.99506600 \\ \mathrm{C} & -6.24195800 & -1.93585200 & -1.78599900 \\ \mathrm{H} & -7.29441200 & -2.22332800 & -1.79367400 \\ \mathrm{H} & -5.96029300 & -1.56978500 & -2.78219200 \\ \mathrm{H} & -5.62976600 & -2.81174200 & -1.53373200 \\ \mathrm{C} & -1.12874000 & 1.50942500 & 0.34780300 \\ \mathrm{H} & -1.35769100 & 2.35970300 & 1.00360300 \\ \mathrm{C} & -0.10752600 & 0.64535300 & 1.09468900 \\ \mathrm{H} & 0.65271100 & 1.30003100 & 1.53916600 \\ \mathrm{C} & -0.86535300 & -1.35614900 & 4.06305100 \\ \mathrm{H} & -0.24074600 & -1.67721000 & 4.89725300 \\ \mathrm{H} & -1.68367100 & -0.72265300 & 4.41528300 \\ \mathrm{H} & -1.31220000 & -2.23492700 & 3.58576800 \\ \mathrm{C} & 2.48434700 & -0.99568600 & -1.15832400 \\ \mathrm{H} & 3.13560100 & -0.48488700 & -1.87493200 \\ \mathrm{H} & 1.76375800 & -1.59427400 & -1.72730700 \\ \mathrm{C} & 3.29497500 & -1.91427700 & -0.24718600 \\ \mathrm{H} & 2.66618300 & -2.24540600 & 0.59576600 \\ \mathrm{H} & 4.14651100 & -1.37037700 & 0.18033300 \\ \mathrm{C} & 5.19337200 & -3.39903500 & -0.80120800 \\ \mathrm{H} & 5.49141600 & -4.13619500 & -1.55421900 \\ & & & \\ & & & \\ \mathrm{H} & & & \end{array}$ $\begin{array}{llll}5.82423500 & -2.51221300 & -0.92257900\end{array}$

$\begin{array}{llll}5.37927600 & -3.83271400 & 0.19596300\end{array}$

$2.91635400 \quad-4.08405700 \quad-1.23954400$

$\begin{array}{llll}1.85455400 & -3.86202300 & -1.19006300\end{array}$

$3.27419400 \quad-4.91819200 \quad-1.83561600$

$\begin{array}{llll}-3.59244700 & 1.20383600 & 0.66374800\end{array}$

$\begin{array}{lll}-3.54350500 & 1.97536600 & 1.43125200\end{array}$

$\begin{array}{lll}-4.80891400 & 0.60449900 & 0.38212400\end{array}$

$\begin{array}{lll}-5.71645800 & 0.89189400 & 0.90708700\end{array}$

$\begin{array}{llll}-3.73242000 & -0.76927100 & -1.28856600\end{array}$

$\begin{array}{llll}-3.76308900 & -1.53674900 & -2.05579900\end{array}$

$\begin{array}{llll}-2.51816700 & -0.15466800 & -0.99225600\end{array}$

$\begin{array}{llll}-1.63096500 & -0.46231200 & -1.54124800\end{array}$

$\begin{array}{llll}3.53946700 & 1.62752900 & -0.13961200\end{array}$

$\begin{array}{llll}4.20243800 & 0.80250300 & 0.10911900\end{array}$

$\begin{array}{llll}4.03224400 & 2.92647700 & -0.17458400\end{array}$

$\begin{array}{llll}5.08305500 & 3.10646800 & 0.03689100\end{array}$

$\begin{array}{llll}3.18007000 & 3.98961400 & -0.45997200\end{array}$

$\begin{array}{llll}3.55752600 & 5.00848700 & -0.47993000\end{array}$

$\begin{array}{llll}1.83547000 & 3.74146900 & -0.71124500\end{array}$

$\begin{array}{llll}1.15525200 & 4.56123900 & -0.93076500\end{array}$
${ }^{\text {Diltiazem }} \mathbf{I}_{2}$<smiles>CNCCN1C(=O)C(OC(C)=O)[C@@H](c2ccc(OC)cc2)Sc2ccccc21</smiles>

$E=-1661.460231$

$\mathrm{H}_{\text {Corr. }}=0.463992$

$\begin{array}{lrrr}\mathrm{S} & -0.39002000 & 2.37688100 & -0.91357500 \\ \mathrm{C} & 0.63867300 & -0.38440300 & 0.03212300 \\ \mathrm{~N} & 1.73947100 & 0.12868400 & -0.60646300 \\ \mathrm{C} & 1.33398000 & 2.55721300 & -0.49486600 \\ \mathrm{C} & 2.20788600 & 1.45376900 & -0.44073200 \\ \mathrm{C} & -2.38445200 & 0.85082400 & 0.16349800 \\ \mathrm{C} & -4.87860100 & -0.27602700 & -0.47185200 \\ \mathrm{O} & -6.12155700 & -0.76955200 & -0.70046300 \\ \mathrm{O} & -0.64420000 & -0.29641900 & 2.07458700 \\ \mathrm{C} & 0.18220000 & -1.04187300 & 2.83937600 \\ \mathrm{O} & 1.38822400 & -0.98775400 & 2.74632000 \\ \mathrm{O} & 0.21796300 & -1.50908700 & -0.19093700 \\ \mathrm{~N} & 3.73179300 & -2.98151800 & -1.05711100 \\ \mathrm{C} & -6.30177100 & -1.63298100 & -1.80495600 \\ \mathrm{H} & -7.35637700 & -1.91268500 & -1.80643300 \\ \mathrm{H} & -6.06061200 & -1.12864600 & -2.75014400 \\ \mathrm{H} & -5.68794200 & -2.53835500 & -1.70891200 \\ \mathrm{C} & -1.06916300 & 1.47072100 & 0.55157800 \\ \mathrm{H} & -1.25873900 & 2.20551500 & 1.34510100 \\ \mathrm{C} & -0.00716200 & 0.50977500 & 1.09166500 \\ \mathrm{H} & 0.78112300 & 1.09428700 & 1.58307500 \\ \mathrm{C} & -0.59072000 & -1.91891000 & 3.76622100 \\ \mathrm{H} & 0.07960200 & -2.35765400 & 4.50602300 \\ \mathrm{H} & -1.39030000 & -1.35704400 & 4.25626700 \\ \mathrm{H} & -1.06092400 & -2.71784200 & 3.18295500 \\ \mathrm{C} & 2.51197700 & -0.81050400 & -1.43784700 \\ \mathrm{H} & 3.13020200 & -0.18869500 & -2.10884000 \\ \mathrm{H} & 1.79664300 & -1.34300600 & -2.07735600 \\ \mathrm{C} & 3.28277900 & -1.75608000 & -0.58920300 \\ \mathrm{H} & 3.76299800 & -1.38800700 & 0.31639800 \\ \mathrm{C} & 4.24069600 & -3.87455700 & -0.03255900 \\ \mathrm{H} & 4.81303800 & -4.68454000 & -0.49679300 \\ \mathrm{H} & 4.90541600 & -3.32396800 & 0.64069600 \\ \mathrm{H} & 3.42534200 & -4.31438500 & 0.56810900 \\ \mathrm{C} & -3.51771400 & 1.12418800 & 0.93832100 \\ \mathrm{H} & -3.42810800 & 1.78168600 & 1.80222000 \\ \mathrm{C} & -4.75062300 & 0.57183000 & 0.63221300 \\ \mathrm{H} & -5.63128300 & 0.78495200 & 1.23293200\end{array}$ 


$\begin{array}{llcc}\mathrm{C} & -3.76126100 & -0.55808000 & -1.26018700 \\ \mathrm{H} & -3.83270100 & -1.21141200 & -2.12428600 \\ \mathrm{C} & -2.53000200 & 0.00736300 & -0.93751000 \\ \mathrm{H} & -1.67105800 & -0.22243400 & -1.56404800 \\ \mathrm{C} & 3.57458500 & 1.67774100 & -0.25071500 \\ \mathrm{H} & 4.25064900 & 0.82644600 & -0.20253800 \\ \mathrm{C} & 4.07244000 & 2.96829200 & -0.12095300 \\ \mathrm{H} & 5.13946900 & 3.11737300 & 0.02314800 \\ \mathrm{C} & 3.20895100 & 4.05996400 & -0.15905800 \\ \mathrm{H} & 3.59237800 & 5.07084500 & -0.04896000 \\ \mathrm{C} & 1.84683800 & 3.84782900 & -0.33614600 \\ \mathrm{H} & 1.15779500 & 4.68869900 & -0.37073300 \\ \mathrm{C} & 2.94473100 & -3.66140800 & -2.06924500 \\ \mathrm{H} & 1.94375100 & -3.94041500 & -1.69387900 \\ \mathrm{H} & 2.82283900 & -3.03468400 & -2.95795700 \\ \mathrm{H} & 3.46923100 & -4.57189700 & -2.37489500\end{array}$

Diltiazem $\mathbf{I}_{3}$

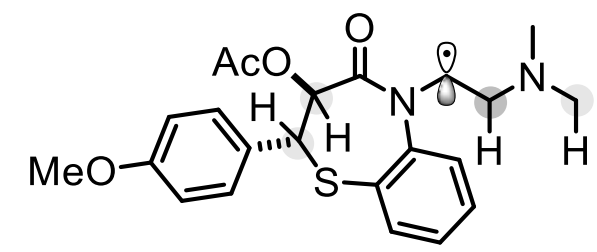

$\mathrm{E}=-1661.456198$

$$
\mathrm{H}_{\text {Corr. }}=0.463404
$$

S $\quad-0.21693100 \quad 2.11630300 \quad-1.28711400$

C $\quad 0.70361700 \quad-0.35960300 \quad 0.22279200$

$\begin{array}{llll}\mathrm{N} & 1.90639800 & 0.02557200 & -0.35514800\end{array}$

C $\quad 1.46516200 \quad 2.42430800 \quad-0.78253500$

$\begin{array}{llll}\text { C } & 2.33309300 & 1.38260800 & -0.40655800\end{array}$

C $\quad-2.31762800 \quad 0.92312100 \quad-0.01312700$

$\begin{array}{llll}\mathrm{C} & -4.83308900 & -0.22261800 & -0.51577400\end{array}$

$\begin{array}{llll}\mathrm{O} & -6.08620800 & -0.71096000 & -0.69197700\end{array}$

$\begin{array}{llll}\text { O } & -0.71013900 & 0.07272000 & 2.13285000\end{array}$

$\begin{array}{llll}\mathrm{C} & 0.04478400 & -0.58013300 & 3.04206300\end{array}$

$\begin{array}{llll}\text { O } & 1.25466500 & -0.60369200 & 2.99054500\end{array}$

$\begin{array}{lll}0.25302200 & -1.48496700 & 0.10156400\end{array}$

$\begin{array}{lll}3.45211900 & -3.24544800 & -1.14907600\end{array}$

$\begin{array}{llll}-6.26433700 & -1.76970700 & -1.61173000\end{array}$

$\begin{array}{llll}-7.32834100 & -2.01072000 & -1.59924900\end{array}$

$\begin{array}{lll}-5.97373300 & -1.46983800 & -2.62728900\end{array}$

$\begin{array}{lll}-5.68911000 & -2.65698600 & -1.31591200\end{array}$

$\begin{array}{lll}-0.99279100 & 1.56359300 & 0.30194000\end{array}$

$\begin{array}{lll}-1.18138100 & 2.45570100 & 0.91340400\end{array}$

$\begin{array}{lll}0.00872000 & 0.69902900 & 1.07728200\end{array}$

$\begin{array}{lll}0.77093900 & 1.35160200 & 1.52125900\end{array}$

$\begin{array}{lll}-0.80713700 & -1.25402300 & 4.06432200\end{array}$

$\begin{array}{llll}-0.18552000 & -1.61298200 & 4.88516000\end{array}$

$\begin{array}{lll}-1.57858900 & -0.57376800 & 4.43493300\end{array}$

$\begin{array}{lll}-1.31639800 & -2.10273700 & 3.59552000\end{array}$

$\begin{array}{lll}2.74526100 & -0.93382800 & -0.93818100\end{array}$

$\begin{array}{llll}3.31650400 & -0.59559300 & -1.80109500\end{array}$

$\begin{array}{llll}4.13834600 & -4.33165700 & -0.47699800\end{array}$

$\begin{array}{llll}4.37093800 & -5.12508100 & -1.19612300\end{array}$

$\begin{array}{llll}5.07983600 & -3.97515900 & -0.04339900\end{array}$

$\begin{array}{lll}3.52803600 & -4.77507400 & 0.33661300\end{array}$

$\begin{array}{lll}-3.46387100 & 1.38210800 & 0.64664500\end{array}$

$\begin{array}{lll}-3.37646600 & 2.19372100 & 1.36800200\end{array}$

$\begin{array}{lll}-4.70725700 & 0.82172000 & 0.40515100\end{array}$

$\begin{array}{llll}-5.59780700 & 1.17832000 & 0.91658300\end{array}$

$-3.70292300 \quad-0.69077200-1.18884000$

$\begin{array}{llll}-3.77239000 & -1.49838600 & -1.91108900\end{array}$

$-2.46069600 \quad-0.11472900 \quad-0.93358800$

$\begin{array}{llll}-1.59132300 & -0.49294100 & -1.46630600\end{array}$

$\begin{array}{lll}3.66508200 & 1.67048000 & -0.10010400\end{array}$

$\begin{array}{lll}4.31869700 & 0.85730500 & 0.20984900\end{array}$

$\begin{array}{llll}4.14419400 & 2.97160100 & -0.18208800\end{array}$

$\begin{array}{lll}5.18368100 & 3.17721600 & 0.05953600\end{array}$

$\begin{array}{llll}3.29090200 & 4.00661500 & -0.55806500\end{array}$ $\begin{array}{llll}3.65841800 & 5.02740500 & -0.62083300\end{array}$

$\begin{array}{llll}1.95831300 & 3.73044800 & -0.84318200\end{array}$

$\begin{array}{llll}1.27895800 & 4.52999000 & -1.12979100\end{array}$

$\begin{array}{llll}2.23283900 & -3.72775600 & -1.77631800\end{array}$

$\begin{array}{llll}1.53555400 & -4.17468000 & -1.03963800\end{array}$

$\begin{array}{lll}1.71142600 & -2.90026600 & -2.27068500\end{array}$

$2.48000700 \quad-4.48605500 \quad-2.52785900$

$3.16964600-2.16209000 \quad-0.21794300$

$\begin{array}{llll}2.42111900 & -2.45259800 & 0.54549300\end{array}$

$\begin{array}{lll}4.10610700 & -1.94719600 & 0.33147800\end{array}$

Diltiazem $\mathbf{I}_{4}$<smiles>COc1ccc(C2Sc3ccccc3N(CC(C)=O)C(=O)C2OC(C)=O)cc1</smiles>

$E=-1661.473032$

$\mathrm{H}_{\text {Corr. }}=0.464654$

S $\quad-0.68492400 \quad 2.23774000 \quad-0.70704200$

$\begin{array}{llll}\mathrm{C} & 0.81828300 & -0.52298000 & 0.08639800\end{array}$

$\mathrm{N} \quad \begin{array}{llll}\mathrm{N} & 1.78523700 & 0.23651400 & -0.52082700\end{array}$

$\begin{array}{llll}\mathrm{C} & 0.99148700 & 2.56159400 & -0.20012000\end{array}$

$\begin{array}{llll}\mathrm{C} & 2.02415200 & 1.60277400 & -0.21829900\end{array}$

$\begin{array}{llll}\text { C } & -2.49402500 & 0.31143900 & 0.00449800\end{array}$

C $\quad-5.08083100 \quad-0.70266400 \quad-0.56798900$

O $\quad-6.28431000 \quad-1.28285900 \quad-0.80148400$

$\begin{array}{llll}\text { O } & -0.60519700 & -0.66884700 & 2.03914600\end{array}$

C $\quad 0.32646900 \quad-1.20066200 \quad 2.86065300$

$\begin{array}{llll}\mathrm{O} & 1.49838500 & -0.90247800 & 2.79897300\end{array}$

$\begin{array}{lll}0.64950400 & -1.70563700 & -0.16537600\end{array}$

$\begin{array}{llll}4.30529100 & -2.28984100 & -1.68617900\end{array}$

$\begin{array}{lll}-7.30372800 & -0.48944800 & -1.37461600\end{array}$

$\begin{array}{lll}-8.17436600 & -1.13818300 & -1.48156000\end{array}$

$\begin{array}{llll}-7.56228900 & 0.35818600 & -0.72600700\end{array}$

$\begin{array}{lll}-7.00902600 & -0.11395800 & -2.36364200\end{array}$

$\begin{array}{lll}-1.19167400 & 0.84641800 & 0.25958000\end{array}$

$\begin{array}{lll}-0.09704000 & 0.22271400 & 1.06025500\end{array}$

$\begin{array}{llll}0.47763300 & 1.00191500 & 1.58038700\end{array}$

$\begin{array}{lll}-0.28865700 & -2.18067100 & 3.80163900\end{array}$

$\begin{array}{lll}0.44124400 & -2.48027700 & 4.55417600\end{array}$

$\begin{array}{lll}-1.17640700 & -1.75401200 & 4.27647600\end{array}$

$\begin{array}{lll}-0.61106600 & -3.06101300 & 3.23496600\end{array}$

$\begin{array}{llll}5.60520800 & -2.73960600 & -1.22262000\end{array}$

$\begin{array}{llll}6.03307800 & -3.44369200 & -1.94521000\end{array}$

$\begin{array}{lll}6.28986000 & -1.88939400 & -1.12763000\end{array}$

$\begin{array}{llll}5.54729500 & -3.25164500 & -0.24039700\end{array}$

$\begin{array}{llll}-2.83853700 & -1.04098400 & 0.27368800\end{array}$

$\begin{array}{llll}-2.09212800 & -1.71304400 & 0.68293300\end{array}$

$\begin{array}{llll}-4.09729800 & -1.52881900 & -0.00619700\end{array}$

$\begin{array}{lll}-4.34604700 & -2.56890900 & 0.19125000\end{array}$

$\begin{array}{llll}-4.77326000 & 0.63029000 & -0.85006000\end{array}$

$\begin{array}{llll}-5.51185300 & 1.30010900 & -1.28037100\end{array}$

$\begin{array}{llll}-3.50454300 & 1.11885300 & -0.56806300\end{array}$

$\begin{array}{llll}-3.30332100 & 2.16833100 & -0.77538000\end{array}$

$\begin{array}{lll}3.32987600 & 2.01921200 & 0.05292800\end{array}$

$\begin{array}{lll}4.12730000 & 1.28144900 & 0.06651500\end{array}$

$\begin{array}{lll}3.62137400 & 3.35244000 & 0.31390400\end{array}$

$\begin{array}{llll}4.64794400 & 3.64593000 & 0.51635700\end{array}$

$\begin{array}{lll}2.60007500 & 4.29698900 & 0.33829900\end{array}$

$\begin{array}{llll}2.81550400 & 5.33949600 & 0.55601500\end{array}$

$\begin{array}{llll}1.29266600 & 3.89492000 & 0.09647800\end{array}$

$\begin{array}{llll}0.48175100 & 4.61919400 & 0.12451000\end{array}$

$\begin{array}{llll}3.43037000 & -3.43463400 & -1.88487100\end{array}$

$\begin{array}{llll}3.29326900 & -4.01339500 & -0.94920300\end{array}$

$\begin{array}{llll}2.44005500 & -3.12011700 & -2.22823400\end{array}$

$\begin{array}{llll}3.85770600 & -4.09899100 & -2.64406000\end{array}$

$\begin{array}{llll}3.72383300 & -1.32888200 & -0.76161600\end{array}$

$\begin{array}{llll}3.27732100 & -1.82751400 & 0.12305500\end{array}$ 


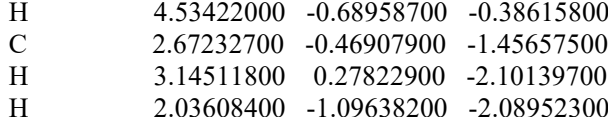

Diltiazem ${ }_{5}$

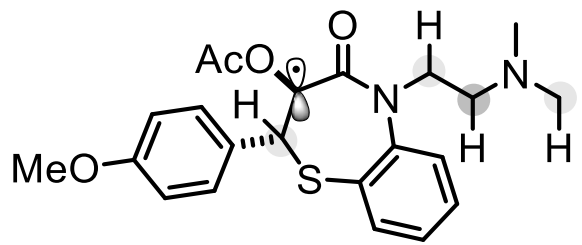

$$
\mathrm{E}=-1661.460941
$$

$\mathrm{H}_{\text {Corr. }}=0.464167$

\begin{tabular}{|c|c|c|c|}
\hline 5 & -0.65429100 & 1.96095700 & -1.20208700 \\
\hline $\mathrm{C}$ & 0.74340800 & -0.55110600 & 0.02577700 \\
\hline $\mathrm{N}$ & 1.75680000 & 0.14855100 & -0.56697900 \\
\hline $\mathrm{C}$ & 0.95192700 & 2.47897600 & -0.63059000 \\
\hline $\mathrm{C}$ & 1.96333900 & 1.53885200 & -0.36668000 \\
\hline $\mathrm{C}$ & -2.60974200 & 0.57442500 & 0.08833400 \\
\hline $\mathrm{C}$ & -5.14742900 & -0.46140000 & -0.49008000 \\
\hline $\mathrm{O}$ & -6.41560700 & -0.87449100 & -0.73568300 \\
\hline $\mathrm{O}$ & -0.59965000 & -0.39205500 & 2.03777900 \\
\hline $\mathrm{C}$ & 0.39887200 & -0.82864200 & 2.87019900 \\
\hline $\mathrm{O}$ & 1.56305900 & -0.59982400 & 2.65807000 \\
\hline $\mathrm{O}$ & 0.63720400 & -1.77218900 & -0.08272400 \\
\hline $\mathrm{N}$ & 4.39415800 & -2.41858000 & -1.31064300 \\
\hline $\mathrm{C}$ & -6.60441400 & -2.18280900 & -1.23707000 \\
\hline $\mathrm{H}$ & -7.68037900 & -2.30897800 & -1.36612800 \\
\hline $\mathrm{H}$ & -6.10614400 & -2.31488200 & -2.20657400 \\
\hline $\mathrm{H}$ & -6.23402100 & -2.93833000 & -0.53158100 \\
\hline $\mathrm{C}$ & -0.23901400 & 0.19641400 & 0.85350600 \\
\hline $\mathrm{C}$ & -0.17404000 & -1.59291700 & 4.01222500 \\
\hline $\mathrm{H}$ & 0.59505700 & -1.75786200 & 4.76719100 \\
\hline $\mathrm{H}$ & -1.02839200 & -1.06275800 & 4.44158300 \\
\hline $\mathrm{H}$ & -0.53790000 & -2.55923200 & 3.64666900 \\
\hline $\mathrm{C}$ & 5.66330500 & -2.79184700 & -0.71335300 \\
\hline $\mathrm{H}$ & 6.15426600 & -3.55313300 & -1.33016800 \\
\hline $\mathrm{H}$ & 6.32584500 & -1.92113900 & -0.65362900 \\
\hline $\mathrm{H}$ & 5.54128900 & -3.20689300 & 0.30816400 \\
\hline $\mathrm{C}$ & -3.75808000 & 1.34923000 & 0.28432500 \\
\hline $\mathrm{H}$ & -3.66085700 & 2.36571000 & 0.66480000 \\
\hline $\mathrm{C}$ & -5.01502100 & 0.84251200 & 0.00014400 \\
\hline $\mathrm{H}$ & -5.91298200 & 1.43612000 & 0.15221800 \\
\hline $\mathrm{C}$ & -4.01044400 & -1.24661500 & -0.69193100 \\
\hline $\mathrm{H}$ & -4.08738800 & -2.26233700 & -1.06753200 \\
\hline $\mathrm{C}$ & -2.75253900 & -0.72063200 & -0.40480600 \\
\hline $\mathrm{H}$ & -1.86987200 & -1.34264000 & -0.55713500 \\
\hline $\mathrm{C}$ & 3.21376500 & 1.98902400 & 0.06211900 \\
\hline $\mathrm{H}$ & 3.98794300 & 1.26315800 & 0.29650100 \\
\hline $\mathrm{C}$ & 3.46998000 & 3.34780000 & 0.20310900 \\
\hline $\mathrm{H}$ & 4.45145300 & 3.67565200 & 0.53585400 \\
\hline $\mathrm{C}$ & 2.47099800 & 4.27990000 & -0.06407700 \\
\hline $\mathrm{H}$ & 2.66476000 & 5.34318800 & 0.05010300 \\
\hline $\mathrm{C}$ & 1.21453700 & 3.84096400 & -0.46602400 \\
\hline $\mathrm{H}$ & 0.41808300 & 4.55403100 & -0.66688700 \\
\hline $\mathrm{C}$ & 3.55495000 & -3.59716800 & -1.45715200 \\
\hline $\mathrm{H}$ & 3.35411800 & -4.08212800 & -0.48038100 \\
\hline $\mathrm{H}$ & 2.58981700 & -3.34171200 & -1.90519000 \\
\hline $\mathrm{H}$ & 4.04999900 & -4.32459400 & -2.11009400 \\
\hline $\mathrm{C}$ & 3.73236700 & -1.38728600 & -0.52512600 \\
\hline $\mathrm{H}$ & 3.23662800 & -1.80932000 & 0.37241800 \\
\hline $\mathrm{H}$ & 4.50378700 & -0.69380300 & -0.16426300 \\
\hline $\mathrm{C}$ & 2.71423200 & -0.62310400 & -1.36701600 \\
\hline $\mathrm{H}$ & 3.21868900 & 0.06983600 & -2.04821400 \\
\hline $\mathrm{H}$ & 2.13339400 & -1.32323100 & -1.97692200 \\
\hline $\mathrm{C}$ & -1.25062300 & 1.16981800 & 0.36051600 \\
\hline $\mathrm{H}$ & -1.35729000 & 1.97228600 & 1.10831000 \\
\hline
\end{tabular}

\section{H radical}

db

$E=-0.499958$

$\mathrm{H}_{\text {Corr. }}=0.00236$

$\begin{array}{lll}0.00000000 & 0.00000000 & 0.00000000\end{array}$

\section{TMS}<smiles>C[Si](C)(C)C</smiles>

$\mathrm{C}_{\mathrm{NMR}}$ isotropic shielding tensor $(\mathrm{ppm})=$

176.72

$\begin{array}{cccc}\mathrm{Si} & -0.00000200 & -0.00004500 & 0.00002400 \\ \mathrm{C} & -0.39869400 & -1.83600700 & -0.10979800 \\ \mathrm{H} & 0.39806500 & -2.38209700 & -0.63007200 \\ \mathrm{H} & -0.51043800 & -2.27567600 & 0.88914700 \\ \mathrm{H} & -1.33433500 & -2.00419300 & -0.65752800 \\ \mathrm{C} & -1.39290500 & 0.89181200 & 0.89806400 \\ \mathrm{H} & -1.52144600 & 0.49968000 & 1.91464000 \\ \mathrm{H} & -1.18697700 & 1.96661500 & 0.97601500 \\ \mathrm{H} & -2.34561800 & 0.76977500 & 0.36780000 \\ \mathrm{C} & 1.60951700 & 0.23697800 & 0.94620200 \\ \mathrm{H} & 2.44067500 & -0.27403900 & 0.44443600 \\ \mathrm{H} & 1.86744200 & 1.30043800 & 1.02516200 \\ \mathrm{H} & 1.53204700 & -0.16646900 & 1.96351200 \\ \mathrm{C} & 0.18206600 & 0.70725800 & -1.73445200 \\ \mathrm{H} & -0.74337100 & 0.58243900 & -2.31039000 \\ \mathrm{H} & 0.41497000 & 1.77886400 & -1.70082500 \\ \mathrm{H} & 0.98911800 & 0.20504800 & -2.28232900\end{array}$

\section{1-Phenyl-1-pyridineethane}

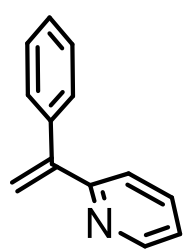

$$
E=-556.458702
$$

$\mathrm{G}_{\text {Corr. }}=0.16307$

$\begin{array}{lccc}\mathrm{C} & 0.04200400 & -2.20243800 & 1.02110200 \\ \mathrm{H} & 0.97033400 & -2.71621700 & 1.25982400 \\ \mathrm{H} & -0.88418400 & -2.71231900 & 1.27557800 \\ \mathrm{C} & 0.03128900 & -0.97497600 & 0.47869300 \\ \mathrm{C} & 1.31842200 & -0.30225700 & 0.15208500 \\ \mathrm{C} & 2.38642100 & -1.04602700 & -0.36897900 \\ \mathrm{C} & 3.58718200 & -0.40591700 & -0.64150000 \\ \mathrm{H} & 2.26307200 & -2.10532500 & -0.57740700 \\ \mathrm{C} & 2.57644500 & 1.61923700 & 0.10213700 \\ \mathrm{C} & 3.69425700 & 0.95920700 & -0.39701900 \\ \mathrm{H} & 4.42555200 & -0.96464900 & -1.05061500 \\ \mathrm{H} & 2.61495800 & 2.69112500 & 0.29733800 \\ \mathrm{H} & 4.61269300 & 1.50319300 & -0.59789800 \\ \mathrm{C} & -1.26460700 & -0.31399300 & 0.16956200 \\ \mathrm{C} & -2.22776900 & -1.02967900 & -0.55420500 \\ \mathrm{C} & -1.58010300 & 0.98131300 & 0.60074600 \\ \mathrm{C} & -3.47226100 & -0.47445400 & -0.83583500 \\ \mathrm{H} & -1.98667300 & -2.02923500 & -0.91211300 \\ \mathrm{C} & -2.82736600 & 1.53311200 & 0.32269500 \\ \mathrm{H} & -0.84178700 & 1.54881300 & 1.15817600 \\ \mathrm{C} & -3.77727100 & 0.81125000 & -0.39665100\end{array}$




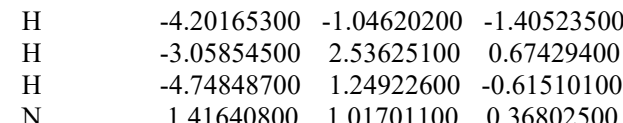

${ }^{30} \mathbf{I}_{1}$

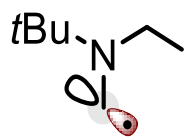

$$
E=-370.207851
$$

$$
\mathrm{G}_{\text {Corr. }}=0.209085
$$

N $\quad 0.14983000 \quad-0.27351500 \quad-0.41323000$

C $\quad 0.33899800 \quad-1.48800400 \quad-1.04803600$

$\mathrm{H} \quad-0.44776500 \quad-1.86292600 \quad-1.69231700$

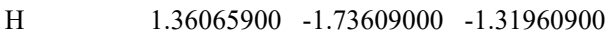

$\begin{array}{llll}\mathrm{C} & 1.32943500 & 0.32865400 & 0.22641900\end{array}$

$\begin{array}{llll}\text { C } & 2.21411800 & 1.00377900 & -0.81469600\end{array}$

$\begin{array}{llll}\mathrm{C} & 2.12835700 & -0.65041200 & 1.08364400\end{array}$

$\begin{array}{llll}\mathrm{H} & 0.95687500 & 1.10968900 & 0.89977100\end{array}$

$\begin{array}{llll}\mathrm{H} & 1.65330300 & 1.76681500 & -1.36696000\end{array}$

$\mathrm{H} \quad 3.07813300 \quad 1.48219900 \quad-0.33760500$

$\begin{array}{llll}\mathrm{H} & 2.59273600 & 0.27128800 & -1.53887900\end{array}$

$\mathrm{H} \quad \begin{array}{llll}\mathrm{H} & 1.48124900 & -1.18319400 & 1.78938400\end{array}$

$\mathrm{H} \quad 2.64801400 \quad-1.39738200 \quad 0.47228300$

$\begin{array}{llll}\mathrm{H} & 2.88864500 & -0.10453100 & 1.65497500\end{array}$

$\begin{array}{llll}\mathrm{C} & -1.18787700 & 0.12613500 & 0.08653100\end{array}$

$\begin{array}{llll}\text { C } & -1.36492000 & 1.63675900 & -0.08581400\end{array}$

$\begin{array}{llll}\text { C } & -1.34891700 & -0.27049100 & 1.55775100\end{array}$

$\mathrm{H} \quad-1.28111000 \quad 1.91059900 \quad-1.14491000$

$\mathrm{H} \quad-2.35823100 \quad 1.93635900 \quad 0.27042600$

$\begin{array}{llll}\mathrm{H} & -0.62979300 & 2.22218200 & 0.47729600\end{array}$

$\mathrm{H} \quad-1.19930300 \quad-1.35166200 \quad 1.67420500$

$\begin{array}{llll}\mathrm{H} & -0.63343500 & 0.25056300 & 2.20564200\end{array}$

$\begin{array}{llll}\mathrm{H} & -2.35733900 & -0.02173600 & 1.91253900\end{array}$

C $\quad-2.28656300 \quad-0.55658000 \quad-0.72266000$

$\mathrm{H} \quad-2.18158300 \quad-0.34875000 \quad-1.79455400$

$\mathrm{H} \quad-2.29817900 \quad-1.64151200 \quad-0.57104600$

$\mathrm{H} \quad-3.25747200 \quad-0.16635100 \quad-0.39687300$

${ }^{30} \mathbf{I}_{2}$

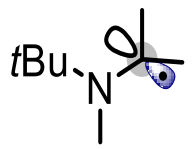

$$
\mathrm{E}=-370.206395
$$

$$
\mathrm{G}_{\text {Corr. }}=0.207465
$$

$\begin{array}{rrrr}\mathrm{N} & 0.12997800 & -0.33329100 & -0.54649800 \\ \mathrm{C} & 0.11704600 & -1.76417400 & -0.81489800 \\ \mathrm{H} & -0.66054300 & -2.01096300 & -1.54521300 \\ \mathrm{H} & 1.07508100 & -2.05156400 & -1.25860600 \\ \mathrm{H} & -0.04403700 & -2.39859300 & 0.07586200 \\ \mathrm{C} & 1.35799400 & 0.15116300 & -0.03171200 \\ \mathrm{C} & 2.24997800 & -0.71821100 & 0.79187200 \\ \mathrm{C} & 1.97268200 & 1.38078800 & -0.61488900 \\ \mathrm{H} & 2.95825800 & -1.30928600 & 0.18105000 \\ \mathrm{H} & 2.87006400 & -0.10052400 & 1.45679500 \\ \mathrm{H} & 1.69305600 & -1.42798200 & 1.41544200 \\ \mathrm{H} & 1.29437300 & 1.88602700 & -1.30788800 \\ \mathrm{H} & 2.28115000 & 2.10298200 & 0.15796400 \\ \mathrm{H} & 2.89146900 & 1.12957200 & -1.17628200 \\ \mathrm{C} & -1.10980200 & 0.19412600 & 0.10395100 \\ \mathrm{C} & -1.19149000 & -0.24366900 & 1.56954300 \\ \mathrm{C} & -2.33909600 & -0.30268500 & -0.65285200 \\ \mathrm{H} & -0.34042100 & 0.15646100 & 2.13673400 \\ \mathrm{H} & -2.11382300 & 0.13047600 & 2.03239000\end{array}$

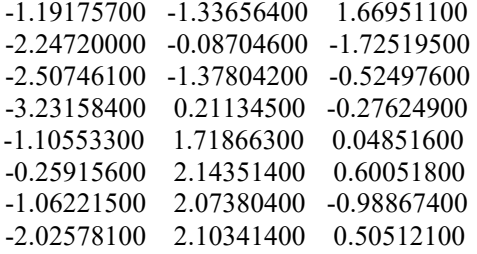

\section{${ }^{30}$ TS I- $\mathbf{I}_{1} \mathbf{I I}_{1}$}

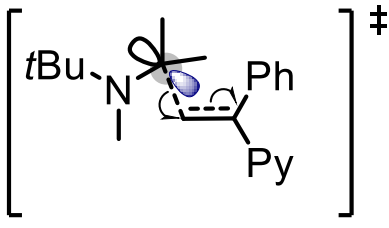

$\mathrm{E}=-926.672465$

$$
\mathrm{G}_{\text {Corr. }}=0.393274
$$

(1)

西

(

$\mathrm{H}$

$\begin{array}{llll}-3.16181100 & 0.78631300 & -2.01605900\end{array}$ $\begin{array}{llll}-3.06681300 & 0.75559600 & -0.48673000\end{array}$ $\begin{array}{lll}-3.86136100 & 0.02476400 & -2.38174200\end{array}$ $\begin{array}{llll}-3.51872500 & 1.76727300 & -2.35395400\end{array}$ $\begin{array}{llll}-2.18711700 & 0.60662700 & -2.48394600\end{array}$ $\begin{array}{llll}-2.10232400 & 1.84059500 & -0.00297100\end{array}$ $\begin{array}{lll}-2.06045100 & 1.85567000 & 1.09376700\end{array}$ $\begin{array}{lll}-1.08087800 & 1.70152000 & -0.37617100\end{array}$ $\begin{array}{llll}-2.44747800 & 2.82356900 & -0.34645300\end{array}$ $\begin{array}{llll}-2.57084700 & -0.57379900 & -0.02801400\end{array}$ $\begin{array}{lll}-3.47474000 & -1.62265400 & 0.47013300\end{array}$

$\begin{array}{lll}-2.81364200 & -2.45551300 & 1.56179200\end{array}$ $-4.00851700 \quad-2.50590200 \quad-0.65469700$ $\begin{array}{lll}-4.32210300 & -1.11429800 & 0.94020400\end{array}$ $\begin{array}{lll}-2.38912600 & -1.81352200 & 2.34190100\end{array}$ $\begin{array}{lll}-3.56072700 & -3.11206300 & 2.02199700\end{array}$ $\begin{array}{lll}-2.01352300 & -3.09717800 & 1.17156600\end{array}$ $-4.58465900 \quad-1.92530300 \quad-1.38449200$ $\begin{array}{llll}-3.18284400 & -2.99544000 & -1.18757600\end{array}$ $\begin{array}{llll}-4.66285400 & -3.28906000 & -0.25293900\end{array}$ $\begin{array}{lll}-1.30340000 & -0.95054100 & -0.38109400\end{array}$ $\begin{array}{llll}-1.09321900 & -2.01475500 & -0.45145200\end{array}$ $\begin{array}{lll}-0.74177600 & -0.30739000 & -1.05216700\end{array}$ $\begin{array}{llll}0.36836500 & -0.58234800 & 1.37615000\end{array}$ $\begin{array}{lll}-0.36586600 & 0.10203700 & 1.79263400\end{array}$ $\begin{array}{lll}0.29730300 & -1.61422000 & 1.71242000\end{array}$ $\begin{array}{lll}1.48326000 & -0.10754300 & 0.73916200\end{array}$ $\begin{array}{lll}-4.44198000 & 1.06600400 & 0.09507000\end{array}$ $\begin{array}{llll}-4.72519400 & 2.07884900 & -0.21291200\end{array}$ $\begin{array}{llll}-5.22198100 & 0.38717300 & -0.26998500\end{array}$ $\begin{array}{llll}-4.43379600 & 1.04384600 & 1.19191000\end{array}$ $\begin{array}{llll}1.58257200 & 1.34130200 & 0.46181400\end{array}$ $\begin{array}{lll}1.27882400 & 2.28274400 & 1.45409800\end{array}$ $\begin{array}{llll}1.89284600 & 1.81544800 & -0.82202400\end{array}$ $\begin{array}{lll}1.26635000 & 3.64611200 & 1.17373000\end{array}$ $\begin{array}{lll}1.05757800 & 1.93555700 & 2.46243500\end{array}$ $\begin{array}{llll}1.87507500 & 3.17618500 & -1.10658000\end{array}$ $\begin{array}{llll}2.13586500 & 1.10222100 & -1.60752600\end{array}$ $\begin{array}{llll}1.55915700 & 4.09994400 & -0.11033900\end{array}$ $\begin{array}{lll}1.03253800 & 4.35693800 & 1.96367700\end{array}$ $\begin{array}{llll}2.10704300 & 3.51860400 & -2.11294500\end{array}$ $\begin{array}{llll}1.54919100 & 5.16462300 & -0.33235400\end{array}$ $\begin{array}{llll}2.50846700 & -1.02056800 & 0.22446600\end{array}$ $2.24252200 \quad-2.38954300 \quad 0.02094900$ $\begin{array}{llll}4.68807800 & -1.32430000 & -0.46586300\end{array}$ $\begin{array}{llll}3.25452900 & -3.22490100 & -0.42200100\end{array}$ $\begin{array}{lll}1.24515400 & -2.78584500 & 0.19472700\end{array}$ $\begin{array}{llll}4.51700400 & -2.69059300 & -0.67133300\end{array}$ $\begin{array}{llll}5.65748100 & -0.86208100 & -0.65860100\end{array}$ $\begin{array}{lll}3.05821800 & -4.28267400 & -0.58346200\end{array}$ $\begin{array}{llll}5.33979900 & -3.30659600 & -1.02276200\end{array}$ $\begin{array}{lll}3.72950100 & -0.50675300 & -0.03510600\end{array}$ 
${ }^{30} \mathrm{TS} \mathrm{I}_{2}-\mathrm{II}_{2}$

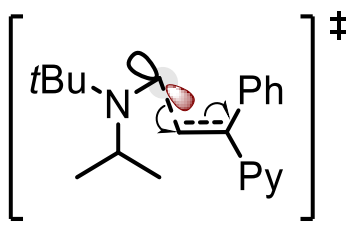

$\mathrm{E}=-926.669875$

$\mathrm{G}_{\text {Corr. }}=0.397047$

$\mathrm{N} \quad-2.64373400 \quad-0.28090900 \quad 0.61867800$

C $\quad-3.62222000 \quad-0.64023000 \quad-0.45972900$

$\begin{array}{lllll}\mathrm{C} & -3.68374600 & 0.47763500 & -1.51338200\end{array}$

$\begin{array}{llll}\text { C } & -4.99893600 & -0.82705000 & 0.18365700\end{array}$

$\begin{array}{lllll}\mathrm{H} & -2.70139300 & 0.65158600 & -1.96946100\end{array}$

$\mathrm{H} \quad-4.37711700 \quad 0.18275900 \quad-2.30984700$

$\mathrm{H} \quad-4.04258200 \quad 1.42952800 \quad-1.11076500$

$\mathrm{H} \quad-4.97142800 \quad-1.65275400 \quad 0.90527300$

$\mathrm{H} \quad \begin{array}{llll}-5.34262400 & 0.07167500 & 0.70794000\end{array}$

$\begin{array}{llll}\mathrm{H} & -5.74517400 & -1.06505500 & -0.58489000\end{array}$

$\begin{array}{llll}\mathrm{C} & -1.45031200 & -0.93861200 & 0.90543900\end{array}$

$\begin{array}{llll}\text { C } & -0.00741000 & -0.55873600 & -0.94852200\end{array}$

$\mathrm{H} \quad-0.00900800 \quad-1.59682100 \quad-1.27644000$

$\mathrm{H} \quad-0.81327900 \quad 0.07675700 \quad-1.29745200$

C $\quad 1.17949300 \quad 0.03800200 \quad-0.57979200$

$\begin{array}{llll}\mathrm{C} & -2.77440000 & 1.09309200 & 1.08834000\end{array}$

$\begin{array}{llll}\mathrm{H} & -2.13166600 & 1.79305500 & 0.52762200\end{array}$

$\begin{array}{llll}\mathrm{H} & -3.80894200 & 1.42508400 & 0.98729900\end{array}$

$\begin{array}{llll}\mathrm{H} & -2.52772600 & 1.16673200 & 2.14985000\end{array}$

$\begin{array}{lll}-1.45051000 & -2.44566600 & 1.02024100\end{array}$

$\begin{array}{lll}-1.33636700 & -2.98626200 & 0.07613500\end{array}$

$-0.62170900 \quad-2.75628500 \quad 1.66607600$

$\begin{array}{lll}-2.37976700 & -2.79945300 & 1.49221100\end{array}$

$\begin{array}{lll}-0.57717500 & -0.28614200 & 1.93860200\end{array}$

$\begin{array}{lll}-1.03059900 & -0.35703600 & 2.94260000\end{array}$

$\begin{array}{lll}0.38783000 & -0.80138300 & 1.98004200\end{array}$

$\begin{array}{lll}-0.36803000 & 0.76874600 & 1.73587200\end{array}$

$\begin{array}{lll}2.38525400 & -0.78178200 & -0.36314700\end{array}$

$\begin{array}{llll}3.66304600 & -0.32396800 & -0.73204600\end{array}$

$\begin{array}{lll}2.29210000 & -2.08876500 & 0.14523400\end{array}$

$\begin{array}{lll}4.78841500 & -1.12727400 & -0.58992400\end{array}$

$\begin{array}{llll}3.76939500 & 0.67000400 & -1.16309400\end{array}$

$\begin{array}{llll}3.41732400 & -2.89443000 & 0.28694300\end{array}$

$\begin{array}{lll}1.31867400 & -2.47623400 & 0.44094500\end{array}$

$\begin{array}{llll}4.67514900 & -2.41833400 & -0.07535000\end{array}$

$\begin{array}{lll}5.76082700 & -0.74528500 & -0.89383800\end{array}$

$\begin{array}{llll}3.30989900 & -3.89933800 & 0.69018900\end{array}$

$\begin{array}{llll}5.55595400 & -3.04590000 & 0.03751800\end{array}$

$\begin{array}{llll}1.19565700 & 1.48280600 & -0.32438200\end{array}$

$\begin{array}{lll}2.15201300 & 2.08350300 & 0.52091900\end{array}$

$\begin{array}{llll}0.13797700 & 3.52149600 & -0.58409900\end{array}$

$\begin{array}{lll}2.07436300 & 3.43840100 & 0.79640600\end{array}$

$\begin{array}{llll}2.92824800 & 1.47712000 & 0.98015500\end{array}$

$\begin{array}{lll}1.04710500 & 4.19202100 & 0.22925200\end{array}$

$\begin{array}{lll}-0.68810000 & 4.06904400 & -1.04228700\end{array}$

$\begin{array}{llll}2.80315700 & 3.90223400 & 1.45761300\end{array}$

$\begin{array}{llll}0.94716800 & 5.25770500 & 0.41416500\end{array}$

$\begin{array}{llll}0.19392600 & 2.22024600 & -0.85819200\end{array}$

$-3.28623900-1.92671100-1.21034700$

$-2.29114700 \quad-1.89842500-1.66566600$

$\begin{array}{lll}-3.37361500 & -2.81813100 & -0.58448500\end{array}$

$\begin{array}{lll}-4.01389400 & -2.03457000 & -2.02256600\end{array}$
${ }^{27}$ TS I $\mathbf{I}_{1}-\mathbf{I I}_{1}$

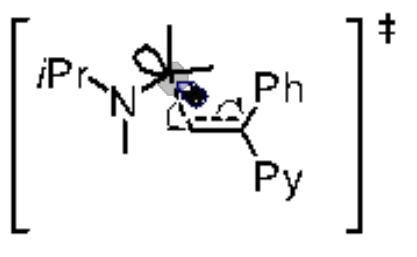

$E=-887.384683$

$\mathrm{G}_{\text {Corr. }}=0.36641$

C $\quad-3.16181100 \quad 0.78631300 \quad-2.01605900$

C $\quad-3.06681300 \quad 0.75559600 \quad-0.48673000$

$\mathrm{H} \quad-3.86136100 \quad 0.02476400 \quad-2.38174200$

$\mathrm{H} \quad-3.51872500 \quad 1.76727300 \quad-2.35395400$

H $\quad-2.18711700 \quad 0.60662700 \quad-2.48394600$

$\begin{array}{lllll}\text { C } & -2.10232400 & 1.84059500 & -0.00297100\end{array}$

$\begin{array}{lll}-2.06045100 & 1.85567000 & 1.09376700\end{array}$

$\begin{array}{lll}-1.08087800 & 1.70152000 & -0.37617100\end{array}$

$\begin{array}{lll}-2.44747800 & 2.82356900 & -0.34645300\end{array}$

$\begin{array}{lll}-2.57084700 & -0.57379900 & -0.02801400\end{array}$

$\begin{array}{llll}-3.47474000 & -1.62265400 & 0.47013300\end{array}$

$\begin{array}{lll}-2.81364200 & -2.45551300 & 1.56179200\end{array}$

$\begin{array}{llll}-4.00851700 & -2.50590200 & -0.65469700\end{array}$

$\begin{array}{lll}-4.32210300 & -1.11429800 & 0.94020400\end{array}$

$\begin{array}{lll}-2.38912600 & -1.81352200 & 2.34190100\end{array}$

$\begin{array}{lll}-3.56072700 & -3.11206300 & 2.02199700\end{array}$

$\begin{array}{lll}-2.01352300 & -3.09717800 & 1.17156600\end{array}$

$\begin{array}{lll}-4.58465900 & -1.92530300 & -1.38449200\end{array}$

$\begin{array}{llll}-3.18284400 & -2.99544000 & -1.18757600\end{array}$

$\begin{array}{llll}-4.66285400 & -3.28906000 & -0.25293900\end{array}$

$\begin{array}{llll}-1.30340000 & -0.95054100 & -0.38109400\end{array}$

$-1.09321900 \quad-2.01475500 \quad-0.45145200$

$\begin{array}{llll}-0.74177600 & -0.30739000 & -1.05216700\end{array}$

$\begin{array}{llll}0.36836500 & -0.58234800 & 1.37615000\end{array}$

$\begin{array}{lll}-0.36586600 & 0.10203700 & 1.79263400\end{array}$

$\begin{array}{lll}0.29730300 & -1.61422000 & 1.71242000\end{array}$

$\begin{array}{lll}1.48326000 & -0.10754300 & 0.73916200\end{array}$

$\begin{array}{lll}-4.44198000 & 1.06600400 & 0.09507000\end{array}$

$\begin{array}{lll}-4.72519400 & 2.07884900 & -0.21291200\end{array}$

$\begin{array}{llll}-5.22198100 & 0.38717300 & -0.26998500\end{array}$

$\begin{array}{lll}-4.43379600 & 1.04384600 & 1.19191000\end{array}$

$\begin{array}{lll}1.58257200 & 1.34130200 & 0.46181400\end{array}$

$\begin{array}{lll}1.27882400 & 2.28274400 & 1.45409800\end{array}$

$\begin{array}{lll}1.89284600 & 1.81544800 & -0.82202400\end{array}$

$\begin{array}{llll}1.26635000 & 3.64611200 & 1.17373000\end{array}$

$\begin{array}{lll}1.05757800 & 1.93555700 & 2.46243500\end{array}$

$\begin{array}{lll}1.87507500 & 3.17618500 & -1.10658000\end{array}$

$\begin{array}{llll}2.13586500 & 1.10222100 & -1.60752600\end{array}$

$\begin{array}{llll}1.55915700 & 4.09994400 & -0.11033900\end{array}$

$\begin{array}{llll}1.03253800 & 4.35693800 & 1.96367700\end{array}$

$\begin{array}{llll}2.10704300 & 3.51860400 & -2.11294500\end{array}$

$\begin{array}{lll}1.54919100 & 5.16462300 & -0.33235400\end{array}$

$\begin{array}{lll}2.50846700 & -1.02056800 & 0.22446600\end{array}$

$\begin{array}{llll}2.24252200 & -2.38954300 & 0.02094900\end{array}$

$\begin{array}{llll}4.68807800 & -1.32430000 & -0.46586300\end{array}$

$\begin{array}{llll}3.25452900 & -3.22490100 & -0.42200100\end{array}$

$\begin{array}{llll}1.24515400 & -2.78584500 & 0.19472700\end{array}$

$\begin{array}{llll}4.51700400 & -2.69059300 & -0.67133300\end{array}$

$\begin{array}{llll}5.65748100 & -0.86208100 & -0.65860100\end{array}$

$\begin{array}{llll}3.05821800 & -4.28267400 & -0.58346200\end{array}$

$\begin{array}{llll}5.33979900 & -3.30659600 & -1.02276200\end{array}$

$\begin{array}{llll}3.72950100 & -0.50675300 & -0.03510600\end{array}$

\section{${ }^{27}$ TS $\mathbf{I}_{2}-\mathbf{I I}_{2}$}




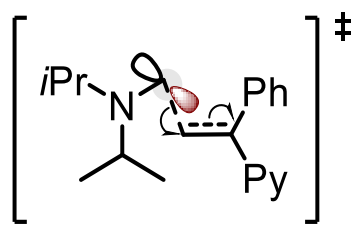

$E=-887.389996$

$\mathrm{G}_{\text {Corr. }}=0.368372$

N $\quad-2.64373400 \quad-0.28090900 \quad 0.61867800$

C $\quad-3.62222000 \quad-0.64023000 \quad-0.45972900$

$\begin{array}{llll}\mathrm{C} & -3.68374600 & 0.47763500 & -1.51338200\end{array}$

$\begin{array}{llll}\mathrm{C} & -4.99893600 & -0.82705000 & 0.18365700\end{array}$

$\mathrm{H} \quad-2.70139300 \quad 0.65158600 \quad-1.96946100$

$\begin{array}{lllll}\mathrm{H} & -4.37711700 & 0.18275900 & -2.30984700\end{array}$

$\mathrm{H} \quad-4.04258200 \quad 1.42952800 \quad-1.11076500$

$\begin{array}{llll}\mathrm{H} & -4.97142800 & -1.65275400 & 0.90527300\end{array}$

$\begin{array}{llll}\mathrm{H} & -5.34262400 & 0.07167500 & 0.70794000\end{array}$

$\mathrm{H} \quad-5.74517400 \quad-1.06505500 \quad-0.58489000$

$\begin{array}{llll}\mathrm{C} & -1.45031200 & -0.93861200 & 0.90543900\end{array}$

$\begin{array}{llll}\text { C } & -0.00741000 & -0.55873600 & -0.94852200\end{array}$

$\mathrm{H} \quad-0.00900800 \quad-1.59682100 \quad-1.27644000$

$\mathrm{H} \quad-0.81327900 \quad 0.07675700 \quad-1.29745200$

$\begin{array}{llll}\mathrm{C} & 1.17949300 & 0.03800200 & -0.57979200\end{array}$

$\begin{array}{llll}\mathrm{C} & -2.77440000 & 1.09309200 & 1.08834000\end{array}$

$\begin{array}{llll}\mathrm{H} & -2.13166600 & 1.79305500 & 0.52762200\end{array}$

$\begin{array}{llll}\mathrm{H} & -3.80894200 & 1.42508400 & 0.98729900\end{array}$

$\begin{array}{llll}\mathrm{H} & -2.52772600 & 1.16673200 & 2.14985000\end{array}$

$\begin{array}{llll}\mathrm{C} & -1.45051000 & -2.44566600 & 1.02024100\end{array}$

$\mathrm{H} \quad-1.33636700 \quad-2.98626200 \quad 0.07613500$

$\mathrm{H} \quad-0.62170900 \quad-2.75628500 \quad 1.66607600$

$\begin{array}{llll}\mathrm{H} & -2.37976700 & -2.79945300 & 1.49221100\end{array}$

$\begin{array}{llll}\mathrm{C} & -0.57717500 & -0.28614200 & 1.93860200\end{array}$

$\mathrm{H} \quad-1.03059900 \quad-0.35703600 \quad 2.94260000$

$\begin{array}{llll}\mathrm{H} & 0.38783000 & -0.80138300 & 1.98004200\end{array}$

$\begin{array}{llll}\mathrm{H} & -0.36803000 & 0.76874600 & 1.73587200\end{array}$

C $\quad 2.38525400 \quad-0.78178200 \quad-0.36314700$

$\begin{array}{llll}\text { C } & 3.66304600 & -0.32396800 & -0.73204600\end{array}$

$\begin{array}{llll}\text { C } & 2.29210000 & -2.08876500 & 0.14523400\end{array}$

C $\quad 4.78841500 \quad-1.12727400 \quad-0.58992400$

$\mathrm{H} \quad 3.76939500 \quad 0.67000400 \quad-1.16309400$

$\begin{array}{llll}\mathrm{C} & 3.41732400 & -2.89443000 & 0.28694300\end{array}$

$\mathrm{H} \quad 1.31867400 \quad-2.47623400 \quad 0.44094500$

C $\quad 4.67514900 \quad-2.41833400 \quad-0.07535000$

$\mathrm{H} \quad 5.76082700 \quad-0.74528500 \quad-0.89383800$

$\begin{array}{llll}\mathrm{H} & 3.30989900 & -3.89933800 & 0.69018900\end{array}$

$\begin{array}{llll}\mathrm{H} & 5.55595400 & -3.04590000 & 0.03751800\end{array}$

$\begin{array}{llll}\mathrm{C} & 1.19565700 & 1.48280600 & -0.32438200\end{array}$

$\begin{array}{llll}\text { C } & 2.15201300 & 2.08350300 & 0.52091900\end{array}$

C $\quad 0.13797700 \quad 3.52149600 \quad-0.58409900$

$\begin{array}{llll}\mathrm{C} & 2.07436300 & 3.43840100 & 0.79640600\end{array}$

$\begin{array}{llll}\mathrm{H} & 2.92824800 & 1.47712000 & 0.98015500\end{array}$

C $\quad 1.04710500 \quad 4.19202100 \quad 0.22925200$

$\mathrm{H} \quad-0.68810000 \quad 4.06904400 \quad-1.04228700$

$\begin{array}{llll}\mathrm{H} & 2.80315700 & 3.90223400 & 1.45761300\end{array}$

$\mathrm{H} \quad 0.94716800 \quad 5.25770500 \quad 0.41416500$

$\begin{array}{lll}0.19392600 & 2.22024600 & -0.85819200\end{array}$

$-3.28623900-1.92671100-1.21034700$

$-2.29114700 \quad-1.89842500 \quad-1.66566600$

$\begin{array}{lll}-3.37361500 & -2.81813100 & -0.58448500\end{array}$

$\begin{array}{lll}-4.01389400 & -2.03457000 & -2.02256600\end{array}$

${ }^{27} \mathbf{I I}_{1}$

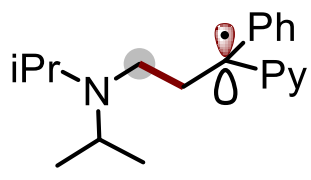

$\mathrm{E}=-887.4253372$

\begin{tabular}{|c|c|c|c|}
\hline $\mathrm{C}$ & -3.16181100 & 0.78631300 & -2.01605900 \\
\hline $\mathrm{C}$ & -3.06681300 & 0.75559600 & -0.48673000 \\
\hline $\mathrm{H}$ & -3.86136100 & 0.02476400 & -2.38174200 \\
\hline $\mathrm{H}$ & -3.51872500 & 1.76727300 & -2.35395400 \\
\hline $\mathrm{H}$ & -2.18711700 & 0.60662700 & -2.48394600 \\
\hline $\mathrm{C}$ & -2.10232400 & 1.84059500 & -0.00297100 \\
\hline $\mathrm{H}$ & -2.06045100 & 1.85567000 & 1.09376700 \\
\hline $\mathrm{H}$ & -1.08087800 & 1.70152000 & -0.37617100 \\
\hline $\mathrm{H}$ & -2.44747800 & 2.82356900 & -0.34645300 \\
\hline $\mathrm{N}$ & -2.57084700 & -0.57379900 & -0.02801400 \\
\hline $\mathrm{C}$ & -3.47474000 & -1.62265400 & 0.47013300 \\
\hline $\mathrm{C}$ & -2.81364200 & -2.45551300 & 1.56179200 \\
\hline $\mathrm{C}$ & -4.00851700 & -2.50590200 & -0.65469700 \\
\hline $\mathrm{H}$ & -4.32210300 & -1.11429800 & 0.94020400 \\
\hline $\mathrm{H}$ & -2.38912600 & -1.81352200 & 2.34190100 \\
\hline $\mathrm{H}$ & -3.56072700 & -3.11206300 & 2.02199700 \\
\hline $\mathrm{H}$ & -2.01352300 & -3.09717800 & 1.17156600 \\
\hline $\mathrm{H}$ & -4.58465900 & -1.92530300 & -1.38449200 \\
\hline $\mathrm{H}$ & -3.18284400 & -2.99544000 & -1.18757600 \\
\hline $\mathrm{H}$ & -4.66285400 & -3.28906000 & -0.25293900 \\
\hline $\mathrm{C}$ & -1.30340000 & -0.95054100 & -0.38109400 \\
\hline $\mathrm{H}$ & -1.09321900 & -2.01475500 & -0.45145200 \\
\hline $\mathrm{H}$ & -0.74177600 & -0.30739000 & -1.05216700 \\
\hline $\mathrm{C}$ & 0.36836500 & -0.58234800 & 1.37615000 \\
\hline $\mathrm{H}$ & -0.36586600 & 0.10203700 & 1.79263400 \\
\hline $\mathrm{H}$ & 0.29730300 & -1.61422000 & 1.71242000 \\
\hline $\mathrm{C}$ & 1.48326000 & -0.10754300 & 0.73916200 \\
\hline $\mathrm{C}$ & -4.44198000 & 1.06600400 & 0.09507000 \\
\hline $\mathrm{H}$ & -4.72519400 & 2.07884900 & -0.21291200 \\
\hline $\mathrm{H}$ & -5.22198100 & 0.38717300 & -0.26998500 \\
\hline $\mathrm{H}$ & -4.43379600 & 1.04384600 & 1.19191000 \\
\hline $\mathrm{C}$ & 1.58257200 & 1.34130200 & 0.46181400 \\
\hline $\mathrm{C}$ & 1.27882400 & 2.28274400 & 1.45409800 \\
\hline $\mathrm{C}$ & 1.89284600 & 1.81544800 & -0.82202400 \\
\hline $\mathrm{C}$ & 1.26635000 & 3.64611200 & 1.17373000 \\
\hline $\mathrm{H}$ & 1.05757800 & 1.93555700 & 2.46243500 \\
\hline $\mathrm{C}$ & 1.87507500 & 3.17618500 & -1.10658000 \\
\hline $\mathrm{H}$ & 2.13586500 & 1.10222100 & -1.60752600 \\
\hline $\mathrm{C}$ & 1.55915700 & 4.09994400 & -0.11033900 \\
\hline $\mathrm{H}$ & 1.03253800 & 4.35693800 & 1.96367700 \\
\hline $\mathrm{H}$ & 2.10704300 & 3.51860400 & -2.11294500 \\
\hline $\mathrm{H}$ & 1.54919100 & 5.16462300 & -0.33235400 \\
\hline $\mathrm{C}$ & 2.50846700 & -1.02056800 & 0.22446600 \\
\hline $\mathrm{C}$ & 2.24252200 & -2.38954300 & 0.02094900 \\
\hline $\mathrm{C}$ & 4.68807800 & -1.32430000 & -0.46586300 \\
\hline $\mathrm{C}$ & 3.25452900 & -3.22490100 & -0.42200100 \\
\hline $\mathrm{H}$ & 1.24515400 & -2.78584500 & 0.19472700 \\
\hline $\mathrm{C}$ & 4.51700400 & -2.69059300 & -0.67133300 \\
\hline $\mathrm{H}$ & 5.65748100 & -0.86208100 & -0.65860100 \\
\hline $\mathrm{H}$ & 3.05821800 & -4.28267400 & -0.58346200 \\
\hline $\mathrm{H}$ & 5.33979900 & -3.30659600 & -1.02276200 \\
\hline $\mathrm{N}$ & 3.72950100 & -0.50675300 & -0.03510600 \\
\hline
\end{tabular}

${ }^{27} \mathrm{II}_{2}$<smiles>CC(C)N(C)C(C)(C)CC(C)(P)P(=O)(O)c1ccccc1</smiles>

$\mathrm{E}=-887.4227282$

$\mathrm{G}_{\text {Corr. }}=0.374989$

$\begin{array}{llll}\mathrm{N} & -2.64373400 & -0.28090900 & 0.61867800\end{array}$

C $\quad-3.62222000 \quad-0.64023000 \quad-0.45972900$

$\begin{array}{llll}\text { C } & -3.68374600 & 0.47763500 & -1.51338200\end{array}$

$\begin{array}{llll}\text { C } & -4.99893600 & -0.82705000 & 0.18365700\end{array}$

$\mathrm{H} \quad-2.70139300 \quad 0.65158600 \quad-1.96946100$

$\begin{array}{llll}\mathrm{H} & -4.37711700 & 0.18275900 & -2.30984700\end{array}$

$\mathrm{H} \quad-4.04258200 \quad 1.42952800 \quad-1.11076500$

$\begin{array}{llll}\mathrm{H} & -4.97142800 & -1.65275400 & 0.90527300\end{array}$

$\mathrm{H} \quad-5.34262400 \quad 0.07167500 \quad 0.70794000$ 


$\begin{array}{lrrr}\mathrm{H} & -5.74517400 & -1.06505500 & -0.58489000 \\ \mathrm{C} & -1.45031200 & -0.93861200 & 0.90543900 \\ \mathrm{C} & -0.00741000 & -0.55873600 & -0.94852200 \\ \mathrm{H} & -0.00900800 & -1.59682100 & -1.27644000 \\ \mathrm{H} & -0.81327900 & 0.07675700 & -1.29745200 \\ \mathrm{C} & 1.17949300 & 0.03800200 & -0.57979200 \\ \mathrm{C} & -2.77440000 & 1.09309200 & 1.08834000 \\ \mathrm{H} & -2.13166600 & 1.79305500 & 0.52762200 \\ \mathrm{H} & -3.80894200 & 1.42508400 & 0.98729900 \\ \mathrm{H} & -2.52772600 & 1.16673200 & 2.14985000 \\ \mathrm{C} & -1.45051000 & -2.44566600 & 1.02024100 \\ \mathrm{H} & -1.33636700 & -2.98626200 & 0.07613500 \\ \mathrm{H} & -0.62170900 & -2.75628500 & 1.66607600 \\ \mathrm{H} & -2.37976700 & -2.79945300 & 1.49221100 \\ \mathrm{C} & -0.57717500 & -0.28614200 & 1.93860200 \\ \mathrm{H} & -1.03059900 & -0.35703600 & 2.94260000 \\ \mathrm{H} & 0.38783000 & -0.80138300 & 1.98004200 \\ \mathrm{H} & -0.36803000 & 0.76874600 & 1.73587200 \\ \mathrm{C} & 2.38525400 & -0.78178200 & -0.36314700 \\ \mathrm{C} & 3.66304600 & -0.32396800 & -0.73204600 \\ \mathrm{C} & 2.29210000 & -2.08876500 & 0.14523400 \\ \mathrm{C} & 4.78841500 & -1.12727400 & -0.58992400\end{array}$

$\begin{array}{rrr}3.76939500 & 0.67000400 & -1.16309400 \\ 3.41732400 & -2.89443000 & 0.28694300 \\ 1.31867400 & -2.47623400 & 0.44094500 \\ 4.67514900 & -2.41833400 & -0.07535000 \\ 5.76082700 & -0.74528500 & -0.89383800 \\ 3.30989900 & -3.89933800 & 0.69018900 \\ 5.55595400 & -3.04590000 & 0.03751800 \\ 1.19565700 & 1.48280600 & -0.32438200 \\ 2.15201300 & 2.08350300 & 0.52091900 \\ 0.13797700 & 3.52149600 & -0.58409900 \\ 2.07436300 & 3.43840100 & 0.79640600 \\ 2.92824800 & 1.47712000 & 0.98015500 \\ 1.04710500 & 4.19202100 & 0.22925200 \\ -0.68810000 & 4.06904400 & -1.04228700 \\ 2.80315700 & 3.90223400 & 1.45761300 \\ 0.94716800 & 5.25770500 & 0.41416500 \\ 0.19392600 & 2.22024600 & -0.85819200 \\ -3.28623900 & -1.92671100 & -1.21034700 \\ -2.29114700 & -1.89842500 & -1.66566600 \\ -3.37361500 & -2.81813100 & -0.58448500 \\ -4.01389400 & -2.03457000 & -2.02256600\end{array}$




\section{V. $\alpha-C\left(s^{3}\right)-H$ alkylation by dynamic radical trapping}

General Procedure A for Alkylation: A $8.0 \mathrm{~mL}$ disposable borosilicate glass tube with screw cap containing a stirring bar was charged with TPS-SH (10 mol\%, $5.9 \mathrm{mg}, 0.02 \mathrm{mmol})$, $\operatorname{Ir}(\text { ppy })_{2}(\mathrm{dtbbpy}) \mathrm{PF}_{6}(1 \mathrm{~mol} \%, 1.8 \mathrm{mg}, 0.002 \mathrm{mmol})$, trialkylamine $(0.6 \mathrm{mmol}$, added after solvent if volatile liquid) and olefin $(0.2 \mathrm{mmol}$, added after solvent if liquid). The tube was transferred to a nitrogen-filled glove-box where the dry Tol $(2.0 \mathrm{~mL}, 0.1 \mathrm{M})$ was added. Then the reaction was stirred for 1 minute and transferred outside, placing $\sim 10 \mathrm{~cm}$ away from a Kessil blue LED (34W maximum, 24 VDC) and rigorously stirred for the mentioned time (4 16 h) with cooling by fan. After completion of the reaction, the product was directly purified by flash column chromatography in silica gel with $\mathrm{CHCl}_{3} / \mathrm{MeOH}$.

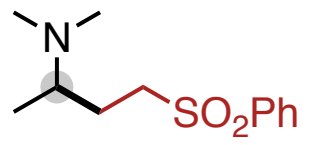

6

$\mathrm{N}, \mathrm{N}$-dimethyl-4-(phenylsulfonyl)butan-2-amine: Following the general procedure A, the tittle compound was obtained in $86 \%$ yield after $4 \mathrm{~h}$ as colorless oil. ${ }^{1} \mathrm{H}$ NMR $\left(400 \mathrm{MHz}, \mathrm{CDCl}_{3}\right)$ א 7.92-7.90 (m, 2H), 7.65-7.62 (m, 1H), 7.57-7.53 (m, 2H), 3.30-3.23 (m, 1H), 3.15-3.07 (m, 1H), 2.60-2.51 (m, 1H), $2.10(\mathrm{~s}, 6 \mathrm{H}), 1.81-1.72(\mathrm{~m}, 2 \mathrm{H}), 0.90(\mathrm{~d}, J=6.5 \mathrm{~Hz}, 3 \mathrm{H}) .{ }^{13} \mathrm{C}$ NMR $\left(101 \mathrm{MHz}, \mathrm{CDCl}_{3}\right) \delta 139.65,133.77,129.45,128.19,57.71,53.85,40.25,26.80,12.71 . \mathrm{IR}$ $\left(\mathrm{CDCl}_{3}, \mathrm{~cm}^{-1}\right): 3063,2960,2928,1447,1304,1286,1086,737$. HRMS calcd. for $\left(\mathrm{C}_{12} \mathrm{H}_{20} \mathrm{NO}_{2} \mathrm{~S}\right)$ $[\mathrm{M}+\mathrm{H}]^{+}: 242.1215$, found 242.1238 .

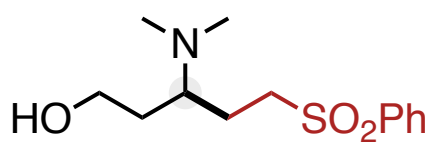

7

3-(dimethylamino)-5-(phenylsulfonyl)pentan-1-ol: Following the general procedure $\mathrm{A}$, the tittle compound was obtained in $53 \%$ yield after $8 \mathrm{~h}$ as colorless oil. ${ }^{1} \mathrm{H} \mathrm{NMR}\left(400 \mathrm{MHz}, \mathrm{CDCl}_{3}\right)$ $\delta$ 7.92-7.90 (m, 2H), 7.69-7.65 (m, 1H), 7.60-7.56 (m, 2H), 3.76-3.68 (m, 2H), 3.13-3.04 (m, 2H), 2.78-2.71 (m, 1H), $2.27(\mathrm{~s}, 6 \mathrm{H}), 2.08-1.99(\mathrm{~m}, 1 \mathrm{H}), 1.81-1.71(\mathrm{~m}, 1 \mathrm{H}), 1.71-1.60(\mathrm{~m}, 1 \mathrm{H})$, 1.38-1.32 (m, 1H), $1.04(\mathrm{~s}, 1 \mathrm{H}) .{ }^{13} \mathrm{C} \mathrm{NMR}\left(101 \mathrm{MHz}, \mathrm{CDCl}_{3}\right) \delta 139.34,133.99,129.53,128.06$, 63.74, 62.76, 54.31, 40.03, 30.78, 20.52. IR $\left(\mathrm{CDCl}_{3}, \mathrm{~cm}^{-1}\right): 3372,3061,2943,2781,1306,1083$, 739, 690.HRMS calcd. for $\left(\mathrm{C}_{13} \mathrm{H}_{22} \mathrm{NO}_{3} \mathrm{~S}\right)[\mathrm{M}+\mathrm{H}]^{+}: 272.1320$, found 272.1323.

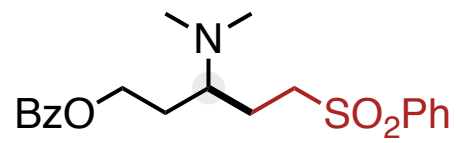


3-(dimethylamino)-5-(phenylsulfonyl)pentyl benzoate: Following the general procedure A, the tittle compound was obtained in $86 \%$ yield after $8 \mathrm{~h}$ as yellow oil. ${ }^{1} \mathrm{H}$ NMR $(500 \mathrm{MHz}$, $\left.\mathrm{CDCl}_{3}\right) \delta 8.06(\mathrm{~d}, J=7.4 \mathrm{~Hz}, 2 \mathrm{H}), 7.58(\mathrm{t}, J=7.3 \mathrm{~Hz}, 1 \mathrm{H}), 7.46(\mathrm{t}, J=7.5 \mathrm{~Hz}, 2 \mathrm{H}), 4.44-4.37$ (m, 2H), 2.62-2.26 (m, 9H), 2.00-1.93 (m, 1H), 1.85-1.60 (m, 3H), $1.46(\mathrm{~s}, 9 \mathrm{H}) .{ }^{13} \mathrm{C}$ NMR (126 $\left.\mathrm{MHz}, \mathrm{CDCl}_{3}\right) \delta 173.19,166.67,132.93,130.52,129.61,128.43,80.14,63.41,60.33,40.26$, 33.11, 28.20, 25.06. IR $\left(\mathrm{CDCl}_{3}, \mathrm{~cm}^{-1}\right): 3064,2966,2931,1718,1452,1270,1147,710$. IR $\left(\mathrm{CDCl}_{3}, \mathrm{~cm}^{-1}\right): 3060,3028,2934,1727,1436,1278,970,687$. HRMS calcd. for $\left(\mathrm{C}_{20} \mathrm{H}_{26} \mathrm{NO}_{4} \mathrm{~S}\right)$ $[\mathrm{M}+\mathrm{H}]^{+}: 376.1583$, found 376.1578 .

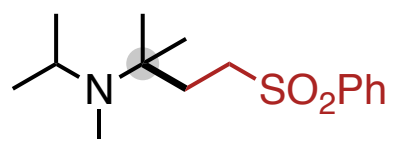

9

$N$-isopropyl- $N$,2-dimethyl-4-(phenylsulfonyl)butan-2-amine: Following the general procedure A, the tittle compound was obtained in $86 \%$ yield after $8 \mathrm{~h}$ as colorless oil. ${ }^{1} \mathrm{H}$ NMR $\left(400 \mathrm{MHz}, \mathrm{CDCl}_{3}\right) \delta 7.91(\mathrm{~d}, J=7.3 \mathrm{~Hz}, 2 \mathrm{H}), 7.64(\mathrm{t}, J=7.4 \mathrm{~Hz}, 1 \mathrm{H}), 7.56(\mathrm{t}, J=7.5 \mathrm{~Hz}, 2 \mathrm{H})$, 3.19-3.15 (m, 2H), $3.04(\mathrm{dt}, J=12.9,6.4 \mathrm{~Hz}, 1 \mathrm{H}), 1.97$ (s, 3H), 1.77-1.73 (m, 2H), 0.98 (s, 6H), $0.90(\mathrm{~d}, J=6.5 \mathrm{~Hz}, 1 \mathrm{H}) .{ }^{13} \mathrm{C} \mathrm{NMR}\left(101 \mathrm{MHz}, \mathrm{CDCl}_{3}\right) \delta 139.66,133.58,129.31,128.08,56.10$, 52.39, 45.79, 33.66, 26.63, 24.94, 21.06. IR $\left(\mathrm{CDCl}_{3}, \mathrm{~cm}^{-1}\right): 2970,2876,2788,1462,1292,1086$, 955, 742. HRMS calcd. for $\left(\mathrm{C}_{15} \mathrm{H}_{26} \mathrm{NO}_{2} \mathrm{~S}\right)[\mathrm{M}+\mathrm{H}]^{+}:$284.1684, found 284.1691.

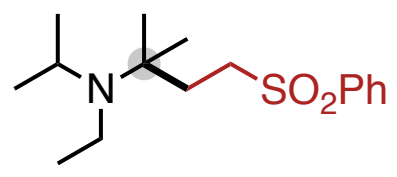

10

$\mathrm{N}$-ethyl- $\mathrm{N}$-isopropyl-2-methyl-4-(phenylsulfonyl)butan-2-amine: Following the general procedure A, the tittle compound was obtained in $38 \%$ yield after $8 \mathrm{~h}$ as yellow oil. ${ }^{1} \mathrm{H}$ NMR $\left(400 \mathrm{MHz}, \mathrm{CDCl}_{3}\right) \delta$ 7.93-7.91 (m, 2H), 7.67-7.63 (m, 1H), 7.59-7.55 (m, 3H), 3.24-3.19 (m, 2H), 3.00 (p, $J=6.6 \mathrm{~Hz}, 1 \mathrm{H}), 2.46$ (q, $J=7.0 \mathrm{~Hz}, 2 \mathrm{H}), 1.77-1.73(\mathrm{~m}, 2 \mathrm{H}), 1.01(\mathrm{~s}, 6 \mathrm{H}), 0.93$ (d, $J=6.6 \mathrm{~Hz}, 6 \mathrm{H}), 0.91(\mathrm{t}, J=7.1 \mathrm{~Hz}, 3 \mathrm{H}) .{ }^{13} \mathrm{C} \mathrm{NMR}\left(101 \mathrm{MHz}, \mathrm{CDCl}_{3}\right) \delta 139.72,133.61,129.35$, 128.15, 57.09, 52.63, 46.48, 35.34, 34.32, 26.56, 22.49, 20.27. IR $\left(\mathrm{CDCl}_{3}, \mathrm{~cm}^{-1}\right): 2970,2927$, 2872, 1446, 1303, 1087, 743, 689. HRMS calcd. for $\left(\mathrm{C}_{16} \mathrm{H}_{28} \mathrm{NO}_{2} \mathrm{~S}\right)[\mathrm{M}+\mathrm{H}]^{+}:$298.1841, found 298.1853.

Note: ratio was obtained by GC-MS, the other regioisomer decomposed during purification.

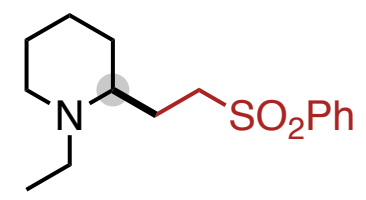


1-ethyl-2-(2-(phenylsulfonyl)ethyl)piperidine: Following the general procedure $\mathrm{A}$, the tittle compound was obtained in 53\% yield after $8 \mathrm{~h}$ as colorless oil. ${ }^{1} \mathrm{H}$ NMR $\left(400 \mathrm{MHz}, \mathrm{CDCl}_{3}\right) \delta$ $7.91(\mathrm{~d}, J=7.1 \mathrm{~Hz}, 2 \mathrm{H}), 7.65(\mathrm{t}, J=7.4 \mathrm{~Hz}, 1 \mathrm{H}), 7.56(\mathrm{t}, J=7.5 \mathrm{~Hz}, 2 \mathrm{H}), 3.24-3.06(\mathrm{~m}, 2 \mathrm{H})$, $2.82(\mathrm{~d}, J=11.6 \mathrm{~Hz}, 1 \mathrm{H}), 2.65-2.56(\mathrm{~m}, 1 \mathrm{H}), 2.37-2.26(\mathrm{~m}, 2 \mathrm{H}), 2.15(\mathrm{t}, J=10.7 \mathrm{~Hz}, 1 \mathrm{H}), 2.04-$ $1.96(\mathrm{~m}, 1 \mathrm{H}), 1.89-1.82(\mathrm{~m}, 1 \mathrm{H}), 1.65-1.37(\mathrm{~m}, 4 \mathrm{H}), 1.29-1.19(\mathrm{~m}, 2 \mathrm{H}), 0.94(\mathrm{t}, J=7.0 \mathrm{~Hz}, 1 \mathrm{H})$. ${ }^{13} \mathrm{C}$ NMR $\left(101 \mathrm{MHz}, \mathrm{CDCl}_{3}\right) \delta 139.47,133.74,129.38,128.13,57.77,52.32,51.02,46.65$, 30.06, 25.14, 24.40, 23.78, 10.60. IR $\left(\mathrm{CDCl}_{3}, \mathrm{~cm}^{-1}\right): 2930,2855,2796,1446,1304,1086,732$, 689. HRMS calcd. for $\left(\mathrm{C}_{15} \mathrm{H}_{24} \mathrm{NO}_{2} \mathrm{~S}\right)[\mathrm{M}+\mathrm{H}]^{+}: 282.1528$, found 282.1537 .

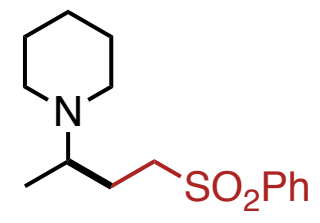

11

1-(4-(phenylsulfonyl)butan-2-yl)piperidine: Following the general procedure A, the tittle compound was obtained in $23 \%$ yield after $8 \mathrm{~h}$ as colorless oil. ${ }^{1} \mathrm{H}$ NMR $\left(400 \mathrm{MHz}, \mathrm{CDCl}_{3}\right) \delta$ $7.92(\mathrm{~d}, J=7.3 \mathrm{~Hz}, 2 \mathrm{H}), 7.65$ (t, $J=7.4 \mathrm{~Hz}, 1 \mathrm{H}), 7.57$ (t, $J=7.6 \mathrm{~Hz}, 2 \mathrm{H}), 3.39-3.32(\mathrm{~m}, 1 \mathrm{H})$, 3.18-3.10 (m, 1H), 2.57-2.16 (m, 5H), 1.85-1.64 (m, 2H), 1.49-1.38 (m, 6H), 0.90 (d, $J=6.0$ $\mathrm{Hz}, 3 \mathrm{H}) .{ }^{13} \mathrm{C} \mathrm{NMR}\left(101 \mathrm{MHz}, \mathrm{CDCl}_{3}\right) \delta 139.63,133.63,129.36,128.13,58.20,53.94,49.12$, 26.71, 26.53, 24.94, 13.45. IR ( $\left.\mathrm{CDCl}_{3}, \mathrm{~cm}^{-1}\right):$ 2932, 2851, 2798, 1446, 1303, 1144, 1086, 744. HRMS calcd. for $\left(\mathrm{C}_{15} \mathrm{H}_{24} \mathrm{NO}_{2} \mathrm{~S}\right)[\mathrm{M}+\mathrm{H}]^{+}: 282.1528$, found 282.1536 .

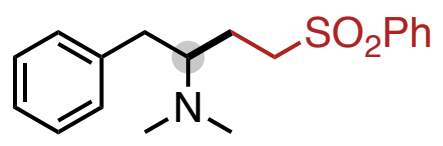

12

$N, N$-dimethyl-1-phenyl-4-(phenylsulfonyl)butan-2-amine: Following the general procedure A, the tittle compound was obtained in $70 \%$ yield after $8 \mathrm{~h}$ as colorless oil. ${ }^{1} \mathrm{H}$ NMR $(400 \mathrm{MHz}$, $\left.\mathrm{CDCl}_{3}\right) \delta 7.78(\mathrm{~d}, J=8.1 \mathrm{~Hz}, 2 \mathrm{H}), 7.60(\mathrm{t}, J=7.4 \mathrm{~Hz}, 1 \mathrm{H}), 7.49$ (t, $\left.J=7.6 \mathrm{~Hz}, 2 \mathrm{H}\right), 7.28-7.24$ (m, 2H), 7.21-7.17 (m, 1H), 7.08-7.07 (m, 2H), 3.31-3.24 (m, 1H), 3.01-2.94 (m, 2H), 2.64$2.58(\mathrm{~m}, 1 \mathrm{H}), 2.23(\mathrm{~s}, 6 \mathrm{H}), 2.23-2.18(\mathrm{~m}, 1 \mathrm{H}), 1.77-1.71(\mathrm{~m}, 2 \mathrm{H}) .{ }^{13} \mathrm{C} \mathrm{NMR}\left(101 \mathrm{MHz}, \mathrm{CDCl}_{3}\right)$ $\delta 139.79,139.47,133.51,129.25,129.21,128.68,128.08,126.23,64.74,53.67,40.11,33.53$, 23.73. IR $\left(\mathrm{CDCl}_{3}, \mathrm{~cm}^{-1}\right): 3099,3060,3028,2934,1506,1086,738,690$. HRMS calcd. for $\left(\mathrm{C}_{18} \mathrm{H}_{24} \mathrm{NO}_{2} \mathrm{~S}\right)[\mathrm{M}+\mathrm{H}]^{+}: 318.1528$, found 318.1546 .

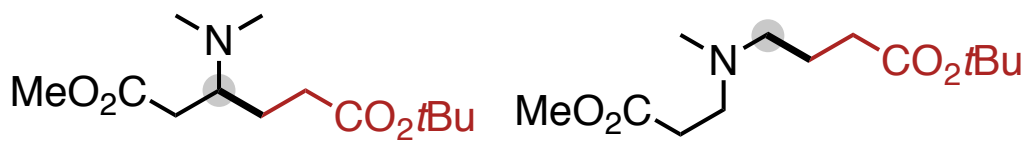


$13(5: 1)$

6-(tert-butyl) 1-methyl 3-(dimethylamino)hexanedioate (major) : Following the general procedure $\mathrm{A}$, the tittle compound was obtained in $89 \%$ yield after $8 \mathrm{~h}$ as a mixture of yellow oil. ${ }^{1} \mathrm{H}$ NMR (500 MHz, $\left.\mathrm{CDCl}_{3}\right) \delta 3.64$ (s, 3H), 2.93-2.18 (m, 11H), 1.72-1.56 (m, 2H), 1.41 (s, 9H). ${ }^{13} \mathrm{C} \mathrm{NMR}\left(126 \mathrm{MHz}, \mathrm{CDCl}_{3}\right) \delta 173.52,173.07,80.09,60.58,51.75,40.19,33.47,32.90$, 28.19, 26.59. IR $\left(\mathrm{CDCl}_{3}, \mathrm{~cm}^{-1}\right): 2932,2867,1728,1454,1366,1223,1148,1044$. IR $\left(\mathrm{CDCl}_{3}\right.$, $\left.\mathrm{cm}^{-1}\right): 2945,2781,1731,1723,1446,1254,1227,845$. HRMS calcd. for $\left(\mathrm{C}_{13} \mathrm{H}_{26} \mathrm{NO}_{4}\right)[\mathrm{M}+\mathrm{H}]^{+}$: 260.1862 , found 260.1873 .

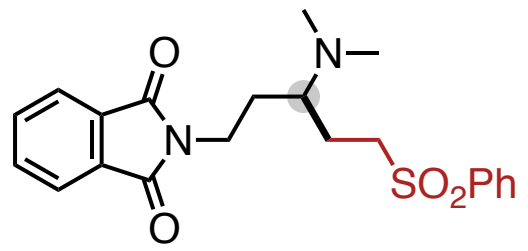

14

2-(3-(dimethylamino)-5-(phenylsulfonyl)pentyl)isoindoline-1,3-dione: Following the general procedure $\mathrm{A}$, the tittle compound was obtained in $82 \%$ yield after $8 \mathrm{~h}$ as colorless oil. ${ }^{1} \mathrm{H}$ NMR (400 MHz, $\left.\mathrm{CDCl}_{3}\right) \delta$ 7.93-7.91 (m, 2H), 7.83-7.80 (m, 2H), 7.73-7.69 (m, 2H), 7.66$7.62(\mathrm{~m}, 1 \mathrm{H}), 7.58-7.54(\mathrm{~m}, 2 \mathrm{H}), 3.68-3.65(\mathrm{~m}, 2 \mathrm{H}), 3.29-3.21(\mathrm{~m}, 1 \mathrm{H}), 3.16-3.08(\mathrm{~m}, 1 \mathrm{H}), 2.48$ (br, 1H), $2.14(\mathrm{~s}, 6 \mathrm{H}), 1.93-1.78(\mathrm{~m}, 3 \mathrm{H}), 1.51-1.42(\mathrm{~m}, 1 \mathrm{H}) .{ }^{13} \mathrm{C} \mathrm{NMR}\left(101 \mathrm{MHz}, \mathrm{CDCl}_{3}\right) \delta$ 168.39 , 139.52, 134.09, 133.74, 132.23, 129.41, 128.12, 123.35, 60.52, 53.98, 40.02, 36.07, 27.55, 22.89. IR ( $\left.\mathrm{CDCl}_{3}, \mathrm{~cm}^{-1}\right): 2937,2826,2780,1770,1707,1397,1146,720$. HRMS calcd. for $\left(\mathrm{C}_{21} \mathrm{H}_{25} \mathrm{~N}_{2} \mathrm{O}_{4} \mathrm{~S}\right)[\mathrm{M}+\mathrm{H}]^{+}:$401.1535, found 401.1539 .

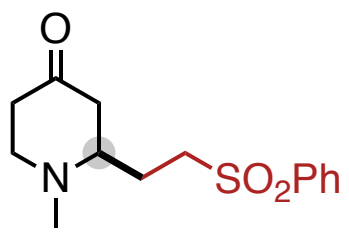

15

1-methyl-2-(2-(phenylsulfonyl)ethyl)piperidin-4-one: Following the general procedure A, the tittle compound was obtained in $72 \%$ yield after $8 \mathrm{~h}$ as colorless oil. ${ }^{1} \mathrm{H}$ NMR $(400 \mathrm{MHz}$, $\left.\mathrm{CDCl}_{3}\right) \delta$ 7.91-7.89 (m, 2H), 7.68-7.64 (m, 1H), 7.59-7.55 (m, 2H), 3.24-3.12 (m, 2H), 3.09$3.03(\mathrm{~m}, 1 \mathrm{H}), 2.69-2.57(\mathrm{~m}, 2 \mathrm{H}), 2.48-2.15(\mathrm{~m}, 8 \mathrm{H}), 2.01-1.92(\mathrm{~m}, 1 \mathrm{H}), 1.89-1.80(\mathrm{~m}, 1 \mathrm{H}) .{ }^{13} \mathrm{C}$ NMR (101 MHz, $\left.\mathrm{CDCl}_{3}\right) \delta 207.80,139.17,133.95,129.50,128.08,60.75,53.57,44.01,39.91$, 39.67, 25.50. IR $\left(\mathrm{CDCl}_{3}, \mathrm{~cm}^{-1}\right)$ : 2956, 2795, 1714, 1446, 1303, 1085, 915, 730. HRMS calcd. for $\left(\mathrm{C}_{14} \mathrm{H}_{20} \mathrm{NO}_{3} \mathrm{~S}\right)[\mathrm{M}+\mathrm{H}]^{+}:$282.1164, found 282.1171 . 


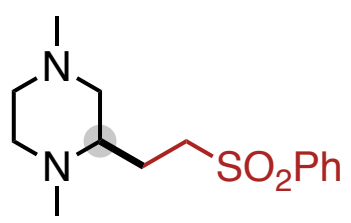

16

1,4-dimethyl-2-(2-(phenylsulfonyl)ethyl)piperazine: Following the general procedure A, the tittle compound was obtained in $73 \%$ yield after $8 \mathrm{~h}$ as colorless oil. ${ }^{1} \mathrm{H}$ NMR $\left(400 \mathrm{MHz}, \mathrm{CDCl}_{3}\right)$ $\delta 7.90(\mathrm{~d}, J=7.8 \mathrm{~Hz}, 2 \mathrm{H}), 7.65(\mathrm{t}, J=7.4 \mathrm{~Hz}, 1 \mathrm{H}), 7.56(\mathrm{t}, J=7.6 \mathrm{~Hz}, 2 \mathrm{H}), 3.27-3.19(\mathrm{~m}, 1 \mathrm{H})$, 3.09-3.01 (m, 1H), 2.72-2.63 (m, 2H), $2.29(\mathrm{td}, J=11.3,2.8 \mathrm{~Hz}, 1 \mathrm{H}), 2.21(\mathrm{~s}, 3 \mathrm{H}), 2.12(\mathrm{~s}, 3 \mathrm{H})$, 2.08-1.94 (m, 2H), 1.86-1.76 (m, 2H). ${ }^{13} \mathrm{C}$ NMR (126 MHz, $\left.\mathrm{CDCl}_{3}\right) \delta 139.18,133.84,129.43$, 128.15, 59.92, 59.07, 55.40, 54.92, 51.81, 46.07, 42.10, 23.36. IR $\left(\mathrm{CDCl}_{3}, \mathrm{~cm}^{-1}\right): 3062,2937$, 2795, 2795, 1446, 1300, 1143, 734. HRMS calcd. for $\left(\mathrm{C}_{14} \mathrm{H}_{23} \mathrm{~N}_{2} \mathrm{O}_{2} \mathrm{~S}\right)[\mathrm{M}+\mathrm{H}]^{+}: 283.1480$, found 283.1489 .

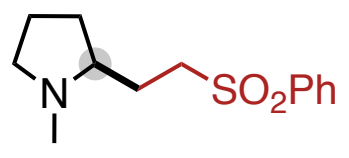

17

1-methyl-2-(2-(phenylsulfonyl)ethyl)pyrrolidine: Following the general procedure $A$, the tittle compound was obtained in $69 \%$ yield after $8 \mathrm{~h}$ as colorless oil. ${ }^{1} \mathrm{H}$ NMR $\left(400 \mathrm{MHz}, \mathrm{CDCl}_{3}\right)$ $\delta 7.91(\mathrm{~d}, J=8.0 \mathrm{~Hz}, 2 \mathrm{H}), 7.65(\mathrm{t}, J=7.9 \mathrm{~Hz}, 1 \mathrm{H}), 7.56(\mathrm{t}, J=7.7 \mathrm{~Hz}, 2 \mathrm{H}), 3.23-3.15(\mathrm{~m}, 1 \mathrm{H})$, 3.10-3.02 (m, 2H), 2.25-2.15 (m, 5H), 2.08-2.00 (m, 1H), 1.92-1.83 (m, 1H), 1.79-1.65 (m, 3H), 1.42-1.33 (m, 1H). $\left.{ }^{13} \mathrm{C} \mathrm{NMR} \mathrm{(126} \mathrm{MHz,} \mathrm{CDCl}_{3}\right) \delta 139.18,133.81,129.42,128.17,64.15,57.14$, 52.87, 40.31, 29.85, 25.66, 22.06. IR $\left(\mathrm{CDCl}_{3}, \mathrm{~cm}^{-1}\right): 3062,2945,2781,1446,1303,1086,739$, 689. HRMS calcd. for $\left(\mathrm{C}_{13} \mathrm{H}_{20} \mathrm{NO}_{2} \mathrm{~S}\right)[\mathrm{M}+\mathrm{H}]^{+}: 254.1215$, found 254.1219 .

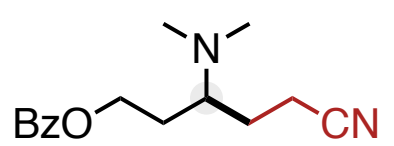

18

5-cyano-3-(dimethylamino)pentyl benzoate: Following the general procedure $A$, the tittle compound was obtained in $72 \%$ yield after $8 \mathrm{~h}$ as colorless oil. ${ }^{1} \mathrm{H}$ NMR $\left(500 \mathrm{MHz}, \mathrm{CDCl}_{3}\right) \delta$ 8.03-8.02 (m, 2H), 7.57-7.54 (m, 1H), 7.46-7.42 (m, 2H), 4.40-4.32 (m, 2H), 2.73-2.67 (m, 1H), $2.45(\mathrm{t}, J=7.3 \mathrm{~Hz}, 13 \mathrm{H}), 2.24(\mathrm{~s}, 6 \mathrm{H}), 2.09-2.02(\mathrm{~m}, 1 \mathrm{H}), 1.80-1.74(\mathrm{~m}, 2 \mathrm{H}), 1.59(\mathrm{~d}, J=34.9$ $\mathrm{Hz}, 1 \mathrm{H}) .{ }^{13} \mathrm{C} \mathrm{NMR}\left(126 \mathrm{MHz}, \mathrm{CDCl}_{3}\right) \delta 166.58,133.11,130.21,129.59,128.52,120.08,62.98$, 59.56, 40.03, 26.89, 26.83, 14.62. IR $\left(\mathrm{CDCl}_{3}, \mathrm{~cm}^{-1}\right): 2938,2826,2781,2240,1715,1452,1272$, 713. HRMS calcd. for $\left(\mathrm{C}_{15} \mathrm{H}_{21} \mathrm{~N}_{2} \mathrm{O}_{2}\right)[\mathrm{M}+\mathrm{H}]^{+}: 261.1603$, found 261.1610 . 


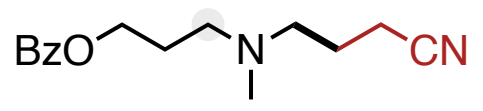

18 ,

3-((3-cyanopropyl)(methyl)amino)propyl benzoate: Following the general procedure A, the tittle compound was obtained in $14 \%$ yield after $8 \mathrm{~h}$ as colorless oil. ${ }^{1} \mathrm{H} \mathrm{NMR}\left(500 \mathrm{MHz}, \mathrm{CDCl}_{3}\right)$ $\delta$ 8.04-8.03 (m, 2H), 7.58-7.54 (m, 1H), 7.46-7.43 (m, 2H), $4.38(\mathrm{t}, J=6.5 \mathrm{~Hz}, 2 \mathrm{H}), 2.49(\mathrm{dt}, J$ $=15.0,6.8 \mathrm{~Hz}, 4 \mathrm{H}), 2.41(\mathrm{t}, J=7.1 \mathrm{~Hz}, 2 \mathrm{H}), 2.22(\mathrm{~s}, 3 \mathrm{H}), 1.93(\mathrm{p}, J=6.7 \mathrm{~Hz}, 2 \mathrm{H}), 1.80(\mathrm{p}, J=$ $6.9 \mathrm{~Hz}, 2 \mathrm{H}) .{ }^{13} \mathrm{C} \mathrm{NMR}\left(126 \mathrm{MHz}, \mathrm{CDCl}_{3}\right) \delta 166.72,133.05,130.45,129.65,128.52,119.95$, 63.22, 55.81, 54.36, 41.93, 26.81, 23.57, 14.85. IR $\left(\mathrm{CDCl}_{3}, \mathrm{~cm}^{-1}\right): 2955,2801,2246,1716$, 1452, 1274, 1115, 713. HRMS calcd. for $\left(\mathrm{C}_{15} \mathrm{H}_{21} \mathrm{~N}_{2} \mathrm{O}_{2}\right)[\mathrm{M}+\mathrm{H}]^{+}: 261.1603$, found 261.1600.

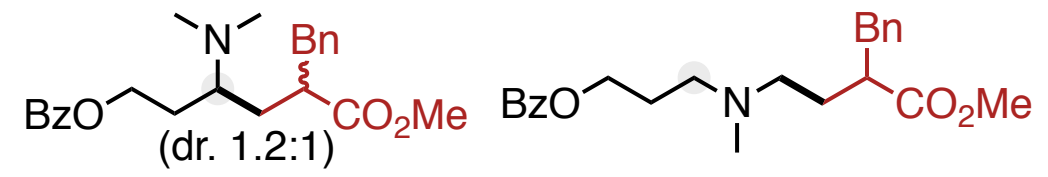

19

5-benzyl-3-(dimethylamino)-6-methoxy-6-oxohexyl benzoate (major): Following the general procedure $\mathrm{A}$, the tittle compound was obtained in $81 \%$ yield after $8 \mathrm{~h}$ as a mixture of yellow oil. Selected peaks for: ${ }^{1} \mathrm{H}$ NMR $\left(500 \mathrm{MHz}, \mathrm{CDCl}_{3}\right) \delta$ 8.05-8.08 (m, 2H), 7.57-7.60 (m, 1H), 7.45-7.48 (m, 2H), 7.28-7.31 (m, 2H), 7.17-7.23 (m, 3H), 4.30-4.41 (m, 2H). ${ }^{13} \mathrm{C}$ NMR $\left(126 \mathrm{MHz}, \mathrm{CDCl}_{3}\right) \delta 176.02,166.59,139.25,132.97,130.45,129.60,128.97,128.92,128.45$, 126.44, 63.19, 59.26, 51.32, 40.06. IR $\left(\mathrm{CDCl}_{3}, \mathrm{~cm}^{-1}\right): 2948,2840,1718,1602,1452,1272$, 1113, 712. HRMS calcd. for $\left(\mathrm{C}_{23} \mathrm{H}_{30} \mathrm{NO}_{4}\right)[\mathrm{M}+\mathrm{H}]^{+}:$384.2175, found 384.2167.

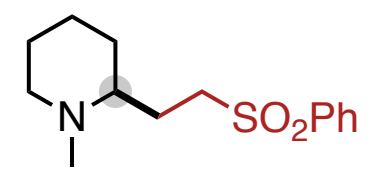

20

1-methyl-2-(2-(phenylsulfonyl)ethyl)piperidine: Following the general procedure A, the tittle compound was obtained in $86 \%$ yield after $4 \mathrm{~h}$ as colorless oil. ${ }^{1} \mathrm{H}$ NMR $\left(400 \mathrm{MHz}, \mathrm{CDCl}_{3}\right) \delta$ $7.91(\mathrm{~d}, J=7.9 \mathrm{~Hz}, 2 \mathrm{H}), 7.64(\mathrm{t}, J=7.4 \mathrm{~Hz}, 1 \mathrm{H}), 7.56(\mathrm{t}, J=7.7 \mathrm{~Hz}, 2 \mathrm{H}), 3.27-3.19(\mathrm{~m}, 1 \mathrm{H})$, 3.12-3.04 (m, 1H), $2.78(\mathrm{~d}, J=11.6 \mathrm{~Hz}, 1 \mathrm{H}), 2.11(\mathrm{~s}, 3 \mathrm{H}), 2.06-2.00(\mathrm{~m}, 3 \mathrm{H}), 1.87-1.80(\mathrm{~m}$, 1H), 1.69-1.64 (m, 1H), 1.57-1.40 (m, 3H), 1.27-1.16 (m, 2H). $\left.{ }^{13} \mathrm{C} \mathrm{NMR} \mathrm{(101} \mathrm{MHz,} \mathrm{CDCl}_{3}\right) \delta$ $139.44,133.75,129.38,128.14,61.59,57.05,51.81,42.74,30.36,25.63,25.46,24.33$. IR $\left(\mathrm{CDCl}_{3}, \mathrm{~cm}^{-1}\right): 3062,2928,2854,1446,1304,1284,1086,689$. HRMS calcd. for $\left(\mathrm{C}_{14} \mathrm{H}_{22} \mathrm{NO}_{2} \mathrm{~S}\right)$ $[\mathrm{M}+\mathrm{H}]^{+}:$268.1371, found 268.1376. 


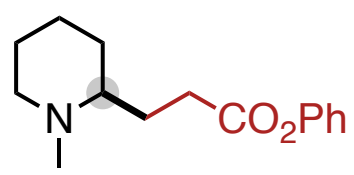

21

phenyl 3-(1-methylpiperidin-2-yl)propanoate: Following the general procedure A, the tittle compound was obtained in $76 \%$ yield after $8 \mathrm{~h}$ as colorless oil. ${ }^{1} \mathrm{H} \mathrm{NMR}\left(400 \mathrm{MHz}, \mathrm{CDCl}_{3}\right) \delta$ 7.39-7.35 (m, 2H), 7.24-7.20 (m, 1H), 7.09-7.06 (2H), 3.07 (d, J=10.2 Hz, 1H), 2.79-2.71 (m, $1 \mathrm{H}), 2.65-2.39(\mathrm{~m}, 6 \mathrm{H}), 2.19-2.12(\mathrm{~m}, 1 \mathrm{H}), 2.06-1.97(\mathrm{~m}, 1 \mathrm{H}), 1.87-1.68(\mathrm{~m}, 4 \mathrm{H}), 1.62-1.51$ (m, 1H), 1.41-1.35 (m, 1H). ${ }^{13} \mathrm{C}$ NMR (101 MHz, $\left.\mathrm{CDCl}_{3}\right) \delta 172.05,150.74,129.58,126.02$, 121.61, 63.16, 56.72, 41.95, 29.98, 29.16, 26.81, 24.47, 23.46. IR $\left(\mathrm{CDCl}_{3}, \mathrm{~cm}^{-1}\right): 2935,2855$, 1756, 1592, 1491, 1194, 1136, 752. HRMS calcd. for $\left(\mathrm{C}_{15} \mathrm{H}_{22} \mathrm{NO}_{2}\right)[\mathrm{M}+\mathrm{H}]^{+}: 248.1651$, found 248.1671 .

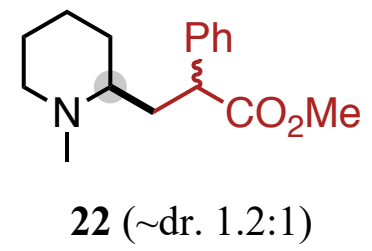

methyl 3-(1-methylpiperidin-2-yl)-2-phenylpropanoate: Following the general procedure A, the tittle compound was obtained in $69 \%$ yield $(\sim \mathrm{dr} .1 .2: 1)$ after $8 \mathrm{~h}$ as colorless oil. ${ }^{1} \mathrm{H}$ NMR $\left(400 \mathrm{MHz}, \mathrm{CDCl}_{3}\right) \delta$ 7.33-7.23 (m, 5H), 3.74-3.62 (m, 4H), 2.98-2.95 (m, 0.53H), 2.91-2.88 $(\mathrm{m}, 0.43 \mathrm{H}), 2.65-1.60(\mathrm{~m}, 11 \mathrm{H}), 1.47-1.20(\mathrm{~m}, 2 \mathrm{H}) .{ }^{13} \mathrm{C} \mathrm{NMR}\left(101 \mathrm{MHz}, \mathrm{CDCl}_{3}\right) \delta(174.21$, 174.15), (139.23, 138.25), (128.94, 128.90), 128.06, 127.79, (127.65, 127.54), (62.20, 61.43), (56.24, 55.96), (52.28, 52.25), (48.40, 47.90), (42.27, 41.92), (35.78, 34.65), (29.82, 29.34, (24.59, 24.39, (23.24, 23.03). IR ( $\left.\mathrm{CDCl}_{3}, \mathrm{~cm}^{-1}\right)$ : 3028, 2934, 2855, 1731, 1452, 1203, 733, 699. HRMS calcd. for $\left(\mathrm{C}_{16} \mathrm{H}_{24} \mathrm{NO}_{2}\right)[\mathrm{M}+\mathrm{H}]^{+}: 262.1807$, found 262.1825 .

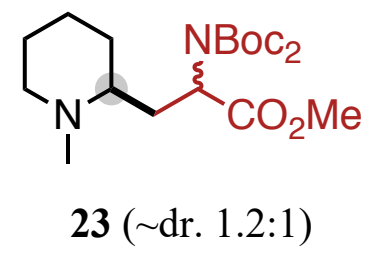

methyl 2-(bis(tert-butoxycarbonyl)amino)-3-(1-methylpiperidin-2-yl)propanoate: Following the general procedure A, the tittle compound was obtained in $81 \%$ yield ( $\sim$ dr. 1.2:1) after $8 \mathrm{~h}$ as colorless oil. ${ }^{1} \mathrm{H} \mathrm{NMR}\left(400 \mathrm{MHz}, \mathrm{CDCl}_{3}\right) \delta 4.96,4.88(\mathrm{~m}, 1 \mathrm{H}), 3.68$ (two single, $3 \mathrm{H}), 2.92-2.83(\mathrm{~m}, 1 \mathrm{H}), 2.72-2.03(\mathrm{~m}, 6 \mathrm{H}), 1.85-1.20(\mathrm{~m}, 25 \mathrm{H}) .{ }^{13} \mathrm{C} \mathrm{NMR}\left(101 \mathrm{MHz}, \mathrm{CDCl}_{3}\right)$ $\delta 171.53,(152.19,151.99),(83.35,83.34),(61.44,60.90),(56.32,56.25),(55.86,55.60),(52.39$, 52.32), (42.76, 42.37), (33.45, 31.83), (30.43, 30.31), 28.07, (25.22, 24.72), 23.37. IR ( $\mathrm{CDCl}_{3}$, 
$\left.\mathrm{cm}^{-1}\right): 2978,2933,1793,1743,1699,1365,994,852$. HRMS calcd. for $\left(\mathrm{C}_{20} \mathrm{H}_{37} \mathrm{~N}_{2} \mathrm{O}_{6}\right)[\mathrm{M}+\mathrm{H}]^{+}$: 401.2652, found 401.2665 .

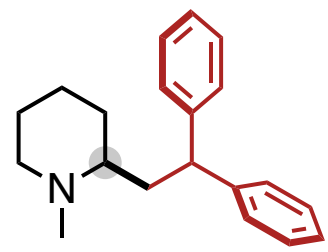

24

2-(2,2-diphenylethyl)-1-methylpiperidine: Following the general procedure B, $50 \mathrm{~mol} \%$ of 2tert-butyl-1,1,3,3-tetramethylguanidine (Barton's base) was used in Tol $(0.1 \mathrm{M})$, the tittle compound was obtained in $74 \%$ yield after $8 \mathrm{~h}$ as yellow oil. ${ }^{1} \mathrm{H}$ NMR $\left(400 \mathrm{MHz}, \mathrm{CDCl}_{3}\right) \delta$ 7.34-7.15 (m, 10H), 4.02 (dd, $J=10.9,5.1 \mathrm{~Hz}, 1 \mathrm{H}), 3.19$ (d, $J=11.9 \mathrm{~Hz}, 1 \mathrm{H}), 2.75-2.56$ (m, $3 \mathrm{H}), 2.60(\mathrm{~s}, 3 \mathrm{H}), 2.34-2.27(\mathrm{~m}, 1 \mathrm{H}), 2.01-1.90(\mathrm{~m}, 2 \mathrm{H}), 1.79-1.71(\mathrm{~m}, 3 \mathrm{H}), 1.36-1.25(\mathrm{~m}, 1 \mathrm{H})$. ${ }^{13} \mathrm{C} \mathrm{NMR}\left(101 \mathrm{MHz}, \mathrm{CDCl}_{3}\right) \delta 143.90,142.19,129.13,128.81,127.98,127.59,127.12,126.78$, 62.59, 55.29, 47.75, 40.25, 35.86, 27.49, 22.56, 21.78. IR $\left(\mathrm{CDCl}_{3}, \mathrm{~cm}^{-1}\right): 3057,3025,2935$, $1451,1439,841,751,702$. HRMS calcd. for $\left(\mathrm{C}_{20} \mathrm{H}_{26} \mathrm{~N}\right)[\mathrm{M}+\mathrm{H}]^{+}:$: 280.2065, found 280.2086.

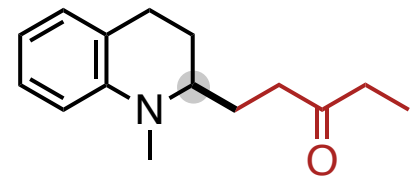

25

1-(1-methyl-1,2,3,4-tetrahydroquinolin-2-yl)pentan-3-one: Following the general procedure A, the tittle compound was obtained in $46 \%$ yield after $8 \mathrm{~h}$ as colorless oil. ${ }^{1} \mathrm{H}$ NMR $(400 \mathrm{MHz}$, $\left.\mathrm{CDCl}_{3}\right) \delta 7.09(\mathrm{t}, J=7.6 \mathrm{~Hz}, 1 \mathrm{H}), 6.97(\mathrm{~d}, J=7.2 \mathrm{~Hz}, 1 \mathrm{H}), 6.60(\mathrm{t}, J=7.3 \mathrm{~Hz}, 1 \mathrm{H}), 6.55(\mathrm{~d}, J$ $=8.1 \mathrm{~Hz}, 1 \mathrm{H}), 3.26(\mathrm{dq}, J=8.6,4.4 \mathrm{~Hz}, 1 \mathrm{H}), 2.95(\mathrm{~s}, 3 \mathrm{H}), 2.84-2.75(\mathrm{~m}, 1 \mathrm{H}), 2.71-2.64(\mathrm{~m}$, $1 \mathrm{H}), 2.44$ (q, $J=7.3 \mathrm{~Hz}, 3 \mathrm{H}), 1.99-1.80(\mathrm{~m}, 3 \mathrm{H}), 1.73-1.63(\mathrm{~m}, 1 \mathrm{H}), 1.07$ (t, $J=7.3 \mathrm{~Hz}, 2 \mathrm{H})$. ${ }^{13} \mathrm{C}$ NMR $\left(101 \mathrm{MHz}, \mathrm{CDCl}_{3}\right) \delta 211.18,145.41,128.77,127.28,121.96,115.74,111.02,58.18$, 38.67, 38.28, 36.09, 25.73, 24.64, 23.77, 7.99. IR $\left(\mathrm{CDCl}_{3}, \mathrm{~cm}^{-1}\right): 3019,2935,1712,1454,1500$, 1337, 1215, 745. HRMS calcd. for $\left(\mathrm{C}_{15} \mathrm{H}_{22} \mathrm{NO}\right)[\mathrm{M}+\mathrm{H}]^{+}: 232.1701$, found 232.1707.<smiles>COC(=O)C(C)C(CN(C(C)C)C(C)C)C(=O)OC</smiles>

26

dimethyl 2-(1-(diisopropylamino)propan-2-yl)malonate: Following the general procedure A, the tittle compound was obtained in $73 \%$ yield after $8 \mathrm{~h}$ as colorless oil. ${ }^{1} \mathrm{H}$ NMR $(400 \mathrm{MHz}$, $\left.\mathrm{CDCl}_{3}\right) \delta 4.19-4.13(\mathrm{~m}, 4 \mathrm{H}), 3.49(\mathrm{~d}, J=5.8 \mathrm{~Hz}, 1 \mathrm{H}), 2.96$ (hept, $\left.J=6.7 \mathrm{~Hz}, 2 \mathrm{H}\right), 2.43-2.36$ 
(m, 1H), 2.33-2.24 (m, 2H), $1.24(\mathrm{t}, J=7.1 \mathrm{~Hz}, 7 \mathrm{H}), 0.97(\mathrm{~d}, J=6.5 \mathrm{~Hz}, 3 \mathrm{H}), 0.94(\mathrm{~d}, J=6.7$ $\mathrm{Hz}, 15 \mathrm{H}) .{ }^{13} \mathrm{C} \mathrm{NMR}\left(101 \mathrm{MHz}, \mathrm{CDCl}_{3}\right) \delta 169.97,169.20,61.10,60.93,54.39,49.16,47.43$, 32.82, 21.02, 20.64, 15.14, 14.22. IR $\left(\mathrm{CDCl}_{3}, \mathrm{~cm}^{-1}\right): 2965,2934,1728,1462,1365,1228,1152$, 1030. HRMS calcd. for $\left(\mathrm{C}_{14} \mathrm{H}_{28} \mathrm{NO}_{4}\right)[\mathrm{M}+\mathrm{H}]^{+}:$274.2018, found 274.2022.

General Procedure B for amino alkylation of diary styrene: A $8.0 \mathrm{~mL}$ disposable borosilicate glass tube with screw cap containing a stirring bar was charged with TPS-SH (5 mol\%, $2.9 \mathrm{mg}$, $0.01 \mathrm{mmol}), \operatorname{Ir}(\mathrm{ppy})_{2}(\mathrm{dtbbpy}) \mathrm{PF}_{6}(1 \mathrm{~mol} \%, 1.8 \mathrm{mg}, 0.002 \mathrm{mmol})$, trialkylamine $(0.6 \mathrm{mmol}$, added after solvent if volatile liquid) and diary styrene ( $0.2 \mathrm{mmol}$, added after solvent if liquid). The tube was transferred to a nitrogen-filled glove-box where the dry 1,4-Dioxane $(2.0 \mathrm{~mL}, 0.1$ M) was added. Then the reaction was stirred for 1 minute and transferred outside, placing $~ 10$ $\mathrm{cm}$ away from a $34 \mathrm{~W}$ Blue-Leds and rigorously stirred for $8 \mathrm{~h}$ with cooling by fan. After completion of the reaction, the product was directly purified by flash column chromatography in silica gel with $\mathrm{CHCl}_{3} / \mathrm{MeOH}$.
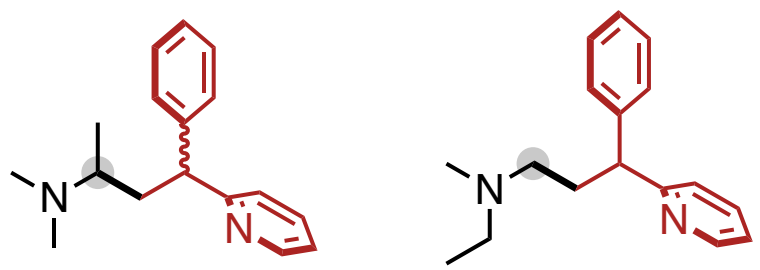

$27(2.6: 1)$

$\mathrm{N}, \mathrm{N}$-dimethyl-4-phenyl-4-(pyridin-2-yl)butan-2-amine (major): Following the general procedure B, the tittle compound was obtained in $91 \%$ as a mixture of yellow oil. ${ }^{1} \mathrm{H}$ NMR (400 $\left.\mathrm{MHz}, \mathrm{CDCl}_{3}\right) \delta$ 8.57-8.55 (m, 1H), 7.56-7.51 (m, 1H), 7.39-7.34 (m, 2H), 7.30-7.25 (m, 2H), 7.20-7.15 (m, 2H), 7.08-7.04 (m, 1H), 4.30-4.23 (m, 0.73H), 4.15-4.11 (m, 0.28H), 2.51-1.92 $(\mathrm{m}, 9 \mathrm{H}), 1.00-0.94(\mathrm{~m}, 3 \mathrm{H})$. Selected peaks: ${ }^{13} \mathrm{C}$ NMR $\left(101 \mathrm{MHz}, \mathrm{CDCl}_{3}\right) \delta 149.20,136.34$, $128.56,128.48,128.20,126.05,123.55,120.99,49.42,27.04,26.11,25.55,21.31,21.01$. IR $\left(\mathrm{CDCl}_{3}, \mathrm{~cm}^{-1}\right): 3060,2962,1587,1469,1431,1047,747,699$. HRMS calcd. for $\left(\mathrm{C}_{17} \mathrm{H}_{23} \mathrm{~N}_{2}\right)$ $[\mathrm{M}+\mathrm{H}]^{+}:$255.1861, found 255.1857.
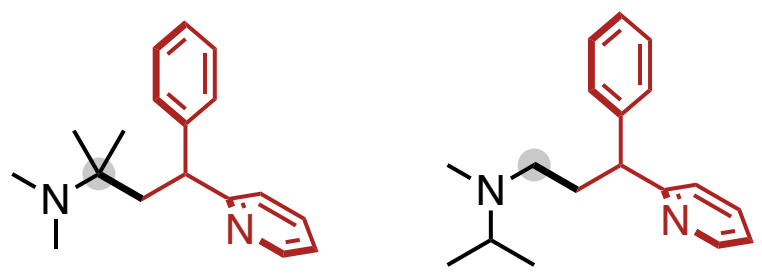

$28(1.3: 1)$

N,N,2-trimethyl-4-phenyl-4-(pyridin-2-yl)butan-2-amine (major): Following the general procedure $\mathrm{B}$, the tittle compound was obtained in $88 \%$ as a mixture of yellow oil. Selected peaks for ${ }^{1} \mathrm{H}$ NMR $\left(400 \mathrm{MHz}, \mathrm{CDCl}_{3}\right) \delta 8.5-8.53(\mathrm{~m}, 2.3 \mathrm{H}), 4.40(\mathrm{~m}, 1.3 \mathrm{H}), 4.16(\mathrm{t}, J=7.5 \mathrm{~Hz}$, 
$1 \mathrm{H}), 0.92(\mathrm{~d}, J=6.6 \mathrm{~Hz}, 6 \mathrm{H}), 0.87(\mathrm{~s}, 3.9 \mathrm{H}), 0.86(\mathrm{~s}, 3.9 \mathrm{H}) .{ }^{13} \mathrm{C} \mathrm{NMR}\left(101 \mathrm{MHz}, \mathrm{CDCl}_{3}\right) \delta$ $164.88,163.88,149.27,149.15,146.16,143.86,136.43,136.40,135.20,129.77,128.54$, $128.50,128.47,128.14,128.08,127.81,126.45,126.12,123.50,122.88,121.31,121.07,56.91$, $53.46,51.55,51.44,48.87,43.85,38.54,36.90,32.99,24.10,22.67,18.08,17.75 . \mathrm{IR}\left(\mathrm{CDCl}_{3}\right.$, $\left.\mathrm{cm}^{-1}\right): 3061,3025,2973,1469,1430,1047,747,700$. HRMS calcd. for $\left(\mathrm{C}_{18} \mathrm{H}_{25} \mathrm{~N}_{2}\right)[\mathrm{M}+\mathrm{H}]^{+}$: 269.2018, found 269.2029 .
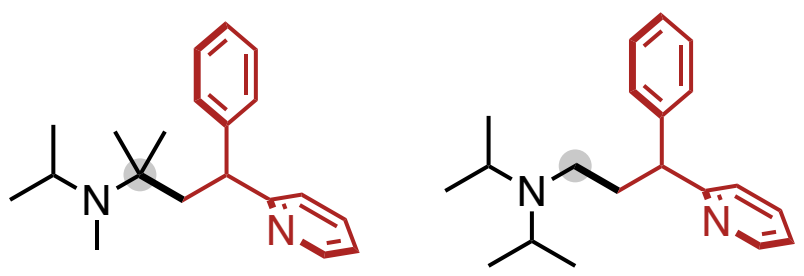

$29(2: 1)$

$N$-isopropyl- $N$,2-dimethyl-4-phenyl-4-(pyridin-2-yl)butan-2-amine (major): Following the general procedure $\mathrm{B}$, the tittle compound was obtained in $68 \%$ as a mixture of yellow oil. ${ }^{1} \mathrm{H}$ NMR (400 MHz, $\left.\mathrm{CDCl}_{3}\right) \delta$ 8.56-8.54 (m, 1.5H), 7.56-7.00 (m, 12H), 4.40-4.37 (m, 1H), 4.16$4.12(\mathrm{~m}, 0.5 \mathrm{H}), 3.27-3.21(\mathrm{~m}, 1 \mathrm{H}), 3.03-3.00(\mathrm{~m}, 1 \mathrm{H}), 2.75-2.70(\mathrm{~m}, 1 \mathrm{H}), 2.49-2.41(\mathrm{~m}, 1.5 \mathrm{H})$, 2.19-2.13 (m, 1.5H), $2.08(\mathrm{~s}, 3 \mathrm{H}), 1.01-0.88(\mathrm{~m}, 18 \mathrm{H})$. Selected peaks: ${ }^{13} \mathrm{C}$ NMR (101 MHz, $\left.\mathrm{CDCl}_{3}\right) \delta 149.20,136.34,128.56,128.48,128.20,126.05,123.55,120.99,49.42,27.04,26.11$, 25.55, 21.31, 21.01. IR $\left(\mathrm{CDCl}_{3}, \mathrm{~cm}^{-1}\right): 3024,2966,2872,1587,1469,1431,746,699$. HRMS calcd. for $\left(\mathrm{C}_{20} \mathrm{H}_{29} \mathrm{~N}_{2}\right)[\mathrm{M}+\mathrm{H}]^{+}:$297.2331, found 297.2333.

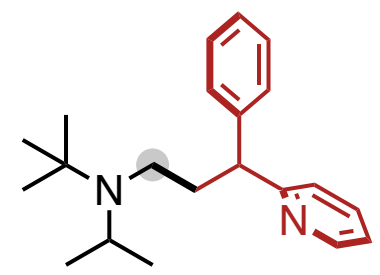

30

$N$-(tert-butyl)- $N$-isopropyl-3-phenyl-3-(pyridin-2-yl)propan-1-amine: $\quad$ Following the general procedure $\mathrm{B}$, the tittle compound was obtained in $95 \%$ as yellow oil. ${ }^{1} \mathrm{H}$ NMR (400 $\left.\mathrm{MHz}, \mathrm{CDCl}_{3}\right) \delta 8.55-8.53(\mathrm{~m}, 1 \mathrm{H}), 7.56-7.52(\mathrm{~m}, 1 \mathrm{H}), 7.38-7.35(\mathrm{~m}, 2 \mathrm{H}), 7.30-7.28(\mathrm{~m}, 2 \mathrm{H})$, 7.20-7.15 (m, 2H), 7.07-7.04 (m, 1H), 4.06 (t, $J=7.4 \mathrm{~Hz}, 1 \mathrm{H}), 3.34$ (br, 1H), 2.46 (br, 3H), $2.23(\mathrm{br}, 1 \mathrm{H}), 1.05(\mathrm{~s}, 9 \mathrm{H}), 0.95(\mathrm{~s}, 6 \mathrm{H})$. Selected peaks for ${ }^{13} \mathrm{C} \mathrm{NMR}\left(101 \mathrm{MHz}, \mathrm{CDCl}_{3}\right) \delta$ $149.21,136.43,128.50,128.09,126.39,122.57,121.27,52.45,47.15,41.20,40.14,28.93$, 22.44. IR $\left(\mathrm{CDCl}_{3}, \mathrm{~cm}^{-1}\right): 3024,2968,2870,1470,1387,1111,745,698$. HRMS calcd. for $\left(\mathrm{C}_{21} \mathrm{H}_{31} \mathrm{~N}_{2}\right)[\mathrm{M}+\mathrm{H}]^{+}:$311.2487, found 311.2488.

Note: rotamers were observed in NMR. 


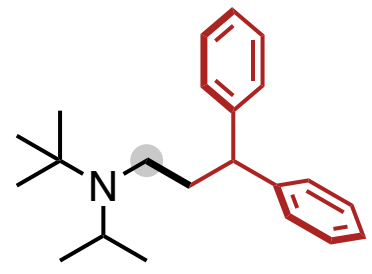

31

$N$-(tert-butyl)- $N$-isopropyl-3,3-diphenylpropan-1-amine: Following the general procedure B, the tittle compound was obtained in $86 \%$ as yellow oil. ${ }^{1} \mathrm{H}$ NMR $\left(400 \mathrm{MHz}, \mathrm{CDCl}_{3}\right) \delta 7.28$ 7.27 (m, 8H), 7.18-7.17 (m, 2H), 3.87 (t, $J=7.7 \mathrm{~Hz}, 1 \mathrm{H}), 3.35-3.29(\mathrm{~m}, 1 \mathrm{H}), 2.44-2.40(\mathrm{~m}, 2 \mathrm{H})$, 2.21-2.15 (2H), $1.03(\mathrm{~s}, 9 \mathrm{H}), 0.93(\mathrm{~d}, J=6.4 \mathrm{~Hz}, 6 \mathrm{H}) .{ }^{13} \mathrm{C} \mathrm{NMR}\left(101 \mathrm{MHz}, \mathrm{CDCl}_{3}\right) \delta$ 145.47, 128.49, 127.99, 126.12, 55.43, 50.16, 46.97, 41.34, 41.09, 29.13, 22.61. IR ( $\left.\mathrm{CDCl}_{3}, \mathrm{~cm}^{-1}\right)$ : 3060, $3025,2935,1450,1441,847,749,701$. HRMS calcd. for $\left(\mathrm{C}_{22} \mathrm{H}_{32} \mathrm{~N}\right)[\mathrm{M}+\mathrm{H}]^{+}: 310.2535$, found 310.2542 .
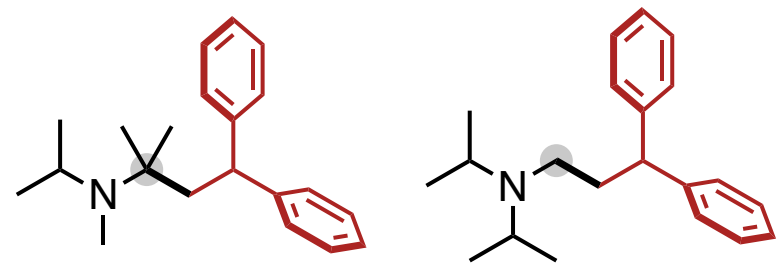

$32(1.8: 1)$

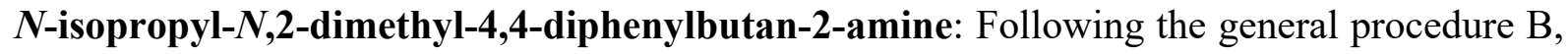
the tittle compound was obtained in $84 \%$ as a mixture of yellow oil. Selected peaks for ${ }^{1} \mathrm{H}$ NMR $\left(400 \mathrm{MHz}, \mathrm{CDCl}_{3}\right) \delta 4.20(\mathrm{t}, J=6.3 \mathrm{~Hz}, 1.78 \mathrm{H}), 3.92(\mathrm{t}, J=7.6 \mathrm{~Hz}, 1 \mathrm{H}) .{ }^{13} \mathrm{C} \mathrm{NMR}(101 \mathrm{MHz}$, $\left.\mathrm{CDCl}_{3}\right) \delta 128.74,128.67,127.84,126.64,126.17,49.38,47.40,27.64,24.96,20.58,18.86$. IR $\left(\mathrm{CDCl}_{3}, \mathrm{~cm}^{-1}\right): 3025,2964,2935,1491,1444,841,749,701$. HRMS calcd. for $\left(\mathrm{C}_{21} \mathrm{H}_{30} \mathrm{~N}\right)$ $[\mathrm{M}+\mathrm{H}]^{+}:$296.2378, found 296.2386.

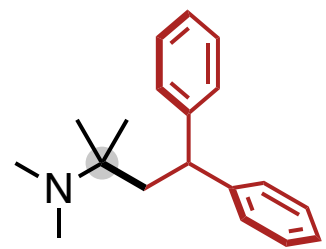

33

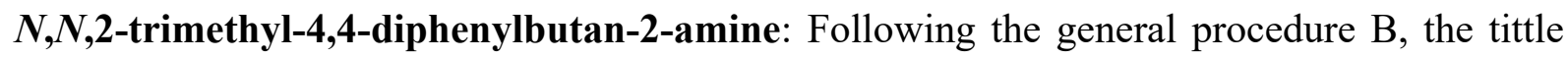
compound was obtained in $49 \%$ as a yellow oil. ${ }^{1} \mathrm{H}$ NMR $(400 \mathrm{MHz}, \mathrm{CDCl} 3) \delta$ 7.36-7.34 (m, 4H), 7.28-7.24 (m, 4H), 7.16-7.12 (m, 2H), 4.25 (t, $J=6.2 \mathrm{~Hz}, 1 \mathrm{H}), 2.33(\mathrm{~d}, J=6.2 \mathrm{~Hz}, 2 \mathrm{H})$, 2.27 (s, 6H), $0.93(\mathrm{~s}, 6 \mathrm{H}) .{ }^{13} \mathrm{C} \mathrm{NMR}\left(101 \mathrm{MHz}, \mathrm{CDCl}_{3}\right) \delta 146.88,128.59,127.91,126.01,47.05$, 44.30, 38.49, 23.62. IR $\left(\mathrm{CDCl}_{3}, \mathrm{~cm}^{-1}\right): 3026,2975,2940,1492,1261,1130,749,696$. HRMS calcd. for $\left(\mathrm{C}_{19} \mathrm{H}_{26} \mathrm{~N}\right)[\mathrm{M}+\mathrm{H}]^{+}:$268.2065, found 268.2070. 


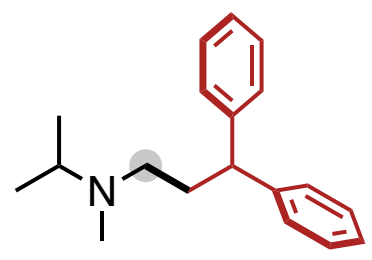

33'

$N$-isopropyl- $N$-methyl-3,3-diphenylpropan-1-amine: Following the general procedure B, the tittle compound was obtained in $18 \%$ as a yellow oil. ${ }^{1} \mathrm{H}$ NMR $\left(400 \mathrm{MHz}, \mathrm{CDCl}_{3}\right) \delta 7.29-7.25$ $(\mathrm{m}, 8 \mathrm{H}), 7.19-7.14(\mathrm{~m}, 2 \mathrm{H}), 4.01-3.97(\mathrm{~m}, 1 \mathrm{H}), 3.13-3.06(\mathrm{~m}, 1 \mathrm{H}), 2.58-2.54(\mathrm{~m}, 2 \mathrm{H}), 2.45-$ $2.42(\mathrm{~m}, 2 \mathrm{H}), 2.37(\mathrm{~s}, 3 \mathrm{H}), 1.08(\mathrm{~d}, J=6.6 \mathrm{~Hz}, 6 \mathrm{H}) .{ }^{13} \mathrm{C} \mathrm{NMR}\left(101 \mathrm{MHz}, \mathrm{CDCl}_{3}\right) \delta 144.04$, 128.70, 127.83, 126.53, 54.73, 51.97, 49.07, 36.18, 31.98, 17.26. IR $\left(\mathrm{CDCl}_{3}, \mathrm{~cm}^{-1}\right): 3026,2963$, 1492, 1451, 1154, 841, 752, 699. HRMS calcd. for $\left(\mathrm{C}_{19} \mathrm{H}_{26} \mathrm{~N}\right)[\mathrm{M}+\mathrm{H}]^{+}: 268.2065$, found 268.2065 .

General Procedure $\mathrm{C}$ for trialkylamine-containing pharmaceuticals: A $8.0 \mathrm{~mL}$ disposable borosilicate glass tube with screw cap containing a stirring bar was charged with TPS-SH (20 mol\%, $5.8 \mathrm{mg}, \quad 0.02 \mathrm{mmol}), \operatorname{Ir}(\text { ppy })_{2}(\mathrm{dtbbpy}) \mathrm{PF}_{6}(1 \mathrm{~mol} \%, 0.9 \mathrm{mg}, 0.001 \mathrm{mmol})$, pharmaceutical $(0.3 \mathrm{mmol})$ and olefin $(0.1 \mathrm{mmol}$, added after solvent if liquid). The tube was transferred to a nitrogen-filled glove-box where the dry Tol $(1.0 \mathrm{~mL}, 0.1 \mathrm{M})$ was added. Then the reaction was stirred for 1 minute and transferred outside, placing $\sim 10 \mathrm{~cm}$ away from a 34 W Blue-Leds and rigorously stirred for $8 \mathrm{~h}$ with cooling by fan. After completion of the reaction, the product was directly purified by flash column chromatography in silica gel with $\mathrm{CHCl}_{3} / \mathrm{MeOH}$.

Note: in case of solubility issue of the drug molecules in Tol, DMA or DMSO (1 mL) could be used as cosolvent.

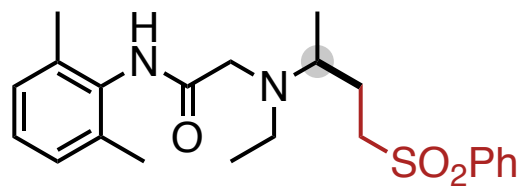

34

\section{$N$-(2,6-dimethylphenyl)-2-(ethyl(4-(phenylsulfonyl)butan-2-yl)amino)acetamide:}

Following the general procedure $\mathrm{C}$, the tittle compound was obtained in $65 \%$ yield after $8 \mathrm{~h}$ as colorless oil. ${ }^{1} \mathrm{H}$ NMR $\left(400 \mathrm{MHz}, \mathrm{CDCl}_{3}\right) \delta 8.55(\mathrm{~s}, 1 \mathrm{H}), 7.87(\mathrm{~d}, J=7.9 \mathrm{~Hz}, 2 \mathrm{H}), 7.66(\mathrm{t}, J=$ $7.4 \mathrm{~Hz}, 1 \mathrm{H}), 7.55(\mathrm{t}, J=7.7 \mathrm{~Hz}, 2 \mathrm{H}), 7.13-7.06(\mathrm{~m}, 3 \mathrm{H}), 3.24-3.10(\mathrm{~m}, 4 \mathrm{H}), 3.00-2.95(\mathrm{~m}, 1 \mathrm{H})$, 2.65-2.59 (m, 2H), $2.19(\mathrm{~s}, 6 \mathrm{H}), 2.04-1.94(\mathrm{~m}, 1 \mathrm{H}), 1.81-1.72(\mathrm{~m}, 1 \mathrm{H}), 1.14(\mathrm{t}, J=7.1 \mathrm{~Hz}, 1 \mathrm{H})$, $1.05(\mathrm{~d}, J=6.5 \mathrm{~Hz}, 1 \mathrm{H}) .{ }^{13} \mathrm{C} \mathrm{NMR}\left(101 \mathrm{MHz}, \mathrm{CDCl}_{3}\right) \delta 170.06,139.06,135.22,134.02,133.76$, $129.55,128.44,128.04,127.44,54.89,54.28,54.04,45.89,26.76,18.76,14.92,14.25$. IR 
$\left(\mathrm{CDCl}_{3}, \mathrm{~cm}^{-1}\right): 3300,3062,2967,1671,1491,1303,1086,731$. HRMS calcd. for $\left(\mathrm{C}_{22} \mathrm{H}_{31} \mathrm{~N}_{2} \mathrm{O}_{3} \mathrm{~S}\right)$ $[\mathrm{M}+\mathrm{H}]^{+}:$403.2055, found 403.2068.

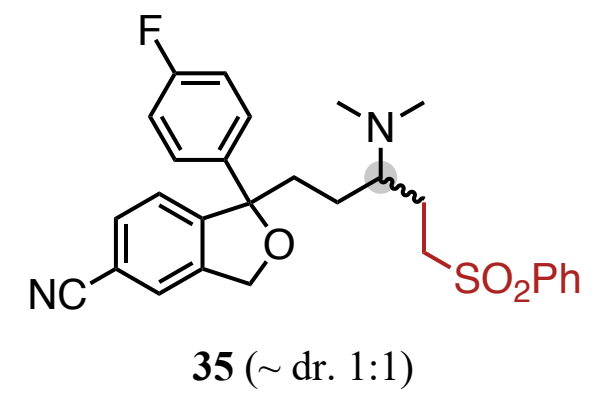

\section{1-(3-(dimethylamino)-5-(phenylsulfonyl)pentyl)-1-(4-fluorophenyl)-1,3-}

dihydroisobenzofuran-5-carbonitrile: Following the general procedure $\mathrm{C}$, the tittle compound was obtained in 55\% yield ( $\sim$ dr. 1:1) after $8 \mathrm{~h}$ as colorless oil. ${ }^{1} \mathrm{H}$ NMR $(400 \mathrm{MHz}$, $\left.\mathrm{CDCl}_{3}\right) \delta 7.89(\mathrm{~d}, J=7.7 \mathrm{~Hz}, 1 \mathrm{H}), 7.66-7.54(\mathrm{~m}, 4 \mathrm{H}), 7.50(\mathrm{~s}, 1 \mathrm{H}), 7.41-7.36(\mathrm{~m}, 3 \mathrm{H}), 7.01(\mathrm{t}$, $J=8.6 \mathrm{~Hz}, 1 \mathrm{H}), 5.19-5.10$ (m, 2H), 3.20-2.99 (m, 2H), 2.35 (br, 1H), 2.22-2.05 (m, 2H), 2.05 (s, 6H), 1.81-1.71 (m, 2H), 1.52-1.33 (m, 1H), 1.18-1.00 (m, 1H). $\left.{ }^{13} \mathrm{C} \mathrm{NMR} \mathrm{(101} \mathrm{MHz,} \mathrm{CDCl}_{3}\right)$ $\delta 163.45,161.00,149.50,149.46,140.40,140.26,139.69,139.67,139.33,133.76,132.10$, $132.05,129.41,128.04,128.03,126.87,126.84,126.79,126.77,125.42,122.80,122.77$, $118.69,115.69,115.48,112.04,91.21,91.21,71.36,71.33,62.76,62.70,54.05,40.13,40.11$, 38.60, 22.79, 22.71, 22.65. ${ }^{19} \mathrm{~F}$ NMR $\left(376 \mathrm{MHz}, \mathrm{CDCl}_{3}\right) \delta$-115.12. IR $\left(\mathrm{CDCl}_{3}, \mathrm{~cm}^{-1}\right): 3064$, 2937, 2826, 2228, 1506, 1303, 1032, 732. HRMS calcd. for $\left(\mathrm{C}_{28} \mathrm{H}_{30} \mathrm{~N}_{2} \mathrm{O}_{3} \mathrm{FS}\right)[\mathrm{M}+\mathrm{H}]^{+}: 493.1961$, found 493.1969.

Note: less than 10\% of minor isomer (impure) was observed.

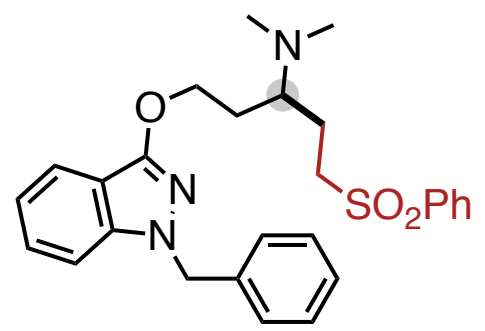

36

1-((1-benzyl-1H-indazol-3-yl)oxy)- $N, N$-dimethyl-5-(phenylsulfonyl)pentan-3-amine:

Following the general procedure $\mathrm{C}$, the tittle compound was obtained in $63 \%$ yield after $8 \mathrm{~h}$ as colorless oil. ${ }^{1} \mathrm{H}$ NMR (400 MHz, $\left.\mathrm{CDCl}_{3}\right) \delta$ 7.90-7.88 (m, 2H), 7.61-7.56 (m, 2H), 7.50-7.46 $(\mathrm{m}, 2 \mathrm{H}), 7.33-7.23(\mathrm{~m}, 4 \mathrm{H}), 7.19-7.16(\mathrm{~m}, 3 \mathrm{H}), 7.03(\mathrm{t}, J=7.8 \mathrm{~Hz}, 1 \mathrm{H}), 5.38(\mathrm{~s}, 2 \mathrm{H}), 4.37$ (tt, $J=10.4,5.3 \mathrm{~Hz}, 2 \mathrm{H}), 3.39-3.32$ (m, 1H), 3.19-3.12 (m, 1H), 2.68 (br, 1H), 2.17 (s, 6H), 2.15$2.08(\mathrm{~m}, 1 \mathrm{H}), 1.98-1.86(\mathrm{~m}, 2 \mathrm{H}), 1.62(\mathrm{dq}, J=14.1,6.4 \mathrm{~Hz}, 1 \mathrm{H}) .{ }^{13} \mathrm{C} \mathrm{NMR}\left(101 \mathrm{MHz}, \mathrm{CDCl}_{3}\right)$ $\delta 155.82,141.71,139.59,137.57,133.61,129.32,128.71,128.09,127.64,127.54,127.16$, 
$120.10,119.32,113.14,108.99,66.95,59.79,53.98,52.48,40.05,27.54,24.13$. IR $\left(\mathrm{CDCl}_{3}\right.$, $\left.\mathrm{cm}^{-1}\right): 3061,2937,2780,1528,1444,1303,1086,742$. HRMS calcd. for $\left(\mathrm{C}_{27} \mathrm{H}_{32} \mathrm{~N}_{3} \mathrm{O}_{3} \mathrm{~S}\right)$ $[\mathrm{M}+\mathrm{H}]^{+}:$478.2164, found 478.2166 .

Note: less than 10\% of minor isomer (impure) was observed.

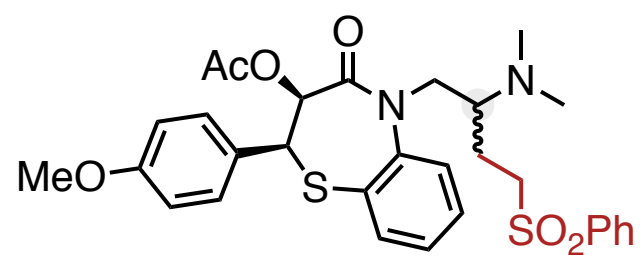

$37(\sim$ dr. 1.2:1)

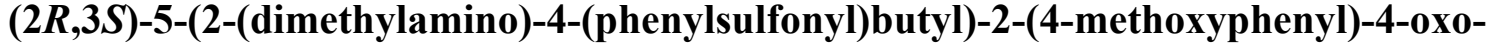

2,3,4,5-tetrahydrobenzo $[b][1,4]$ thiazepin-3-yl acetate: Following the general procedure $C$, the tittle compound was obtained in $76 \%$ yield $(\sim \mathrm{dr} .1 .2: 1)$ after $8 \mathrm{~h}$ as colorless oil. ${ }^{1} \mathrm{H}$ NMR $\left(400 \mathrm{MHz}, \mathrm{CDCl}_{3}\right) \delta 7.88(\mathrm{~d}, J=7.8 \mathrm{~Hz}, 1 \mathrm{H}), 7.75(\mathrm{~d}, J=7.6 \mathrm{~Hz}, 1 \mathrm{H}), 7.71-7.56(\mathrm{~m}, 2 \mathrm{H}), 7.56-$ $7.24(\mathrm{~m}, 7 \mathrm{H}), 6.94(\mathrm{~d}, J=8.6 \mathrm{~Hz}, 1 \mathrm{H}), 6.87(\mathrm{~d}, J=8.6 \mathrm{~Hz}, 1 \mathrm{H}),[5.13(\mathrm{~d}, J=7.7 \mathrm{~Hz}, 0.55 \mathrm{H})$, $5.08(\mathrm{~d}, J=7.5 \mathrm{~Hz}, 0.45 \mathrm{H})],[4.99(\mathrm{~d}, J=7.8 \mathrm{~Hz}, 0.55 \mathrm{H}), 4.96(\mathrm{~d}, J=7.5 \mathrm{~Hz}, 0.45 \mathrm{H})], 4.47-$ $4.41(\mathrm{~m}, 1 \mathrm{H}),[3.83(\mathrm{~s}, 1.58 \mathrm{H}), 3.81(\mathrm{~s}, 1.29 \mathrm{H})], 3.60-2.96(\mathrm{~m}, 4 \mathrm{H}), 2.52-2.19(\mathrm{~m}, 4 \mathrm{H}), 2.06-$ $2.04(\mathrm{~m}, 4 \mathrm{H}), 1.88-1.87(\mathrm{~m}, 3 \mathrm{H}), 1.75-1.43(\mathrm{~m}, 1 \mathrm{H})$. Major isomer: ${ }^{13} \mathrm{C} \mathrm{NMR}\left(126 \mathrm{MHz}, \mathrm{CDCl}_{3}\right)$ $\delta 170.03,159.96,139.21,135.95,133.58,131.43,130.91,129.37,128.98,128.31,128.13$, $127.46,126.45,124.85,123.68,114.07,71.32$, 60.89, 55.41, 54.63, 53.74, 46.18, 39.98, 22.37, 20.60. IR $\left(\mathrm{CDCl}_{3}, \mathrm{~cm}^{-1}\right): 3063,2937,2833,1744,1676,1511,1147,731$. HRMS calcd. for $\left(\mathrm{C}_{30} \mathrm{H}_{35} \mathrm{~N}_{2} \mathrm{O}_{6} \mathrm{~S}_{2}\right)[\mathrm{M}+\mathrm{H}]^{+}:$583.1937, found 583.1946.

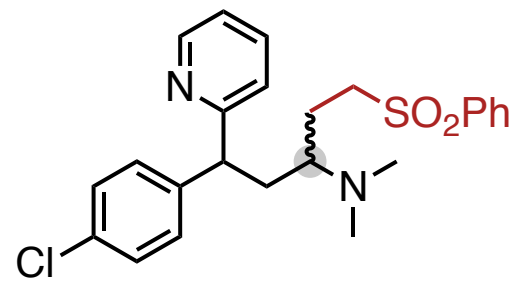

$38(\sim$ dr. $1: 1)$

\section{1-(4-chlorophenyl)- $N, N$-dimethyl-5-(phenylsulfonyl)-1-(pyridin-2-yl)pentan-3-}

amine :Following the general procedure $\mathrm{C}$, the tittle compound was obtained in $59 \%$ yield $(\sim \mathrm{dr}$. 1:1) after $8 \mathrm{~h}$ as colorless oil. ${ }^{1} \mathrm{H}$ NMR $\left(400 \mathrm{MHz}, \mathrm{CDCl}_{3}\right) \delta 8.58-8.54(\mathrm{~m}, 1 \mathrm{H}), 7.90-7.87(\mathrm{~m}$, 2H), $7.66(\mathrm{t}, J=7.4 \mathrm{~Hz}, 1 \mathrm{H}), 7.56(\mathrm{t}, J=7.7 \mathrm{~Hz}, 3 \mathrm{H}), 7.28-7.23(\mathrm{~m}, 4 \mathrm{H}), 7.13-7.09(\mathrm{~m}, 2 \mathrm{H})$, 4.16-4.09 (m, 1H), 3.23-3.02 (m, 2H), 2.51-2.18 (m, 2H), 2.12 (s, 3H), 2.10 (s, 3H), 1.93-1.76 (m, 3H). ${ }^{13} \mathrm{C}$ NMR $\left(101 \mathrm{MHz}, \mathrm{CDCl}_{3}\right) \delta(162.79,162.64),(149.53,149.49),(142.42,141.88)$, (139.64, 139.57), 136.62, (133.68, 133.63), (132.49, 132.40), (129.48, 129.45), (129.35, 129.33), (128.81, 128.75), 128.06, 123.42, 121.69, (60.12, 59.95), (54.14, 54.02), (49.66, 
49.46), 39.88, (34.49, 33.74), (22.57, 22.39). IR ( $\left.\mathrm{CDCl}_{3}, \mathrm{~cm}^{-1}\right): 3063,2934,2824,1587,1470$, 1302, 1086, 731. HRMS calcd. for $\left(\mathrm{C}_{24} \mathrm{H}_{28} \mathrm{~N}_{2} \mathrm{O}_{2} \mathrm{SCl}\right)[\mathrm{M}+\mathrm{H}]^{+}$: 443.1560, found 443.1560 .

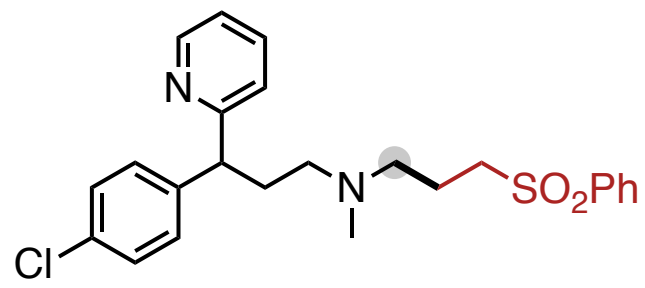

38 '

\section{3-(4-chlorophenyl)- $N$-methyl- $N$-(3-(phenylsulfonyl)propyl)-3-(pyridin-2-yl)propan-1-}

amine: Following the general procedure $\mathrm{C}$, the tittle compound was obtained in $16 \%$ yield after $8 \mathrm{~h}$ as colorless oil. ${ }^{1} \mathrm{H}$ NMR $\left(400 \mathrm{MHz}, \mathrm{CDCl}_{3}\right) \delta 8.57(\mathrm{~d}, J=4.3 \mathrm{~Hz}, 1 \mathrm{H}), 7.92(\mathrm{~d}, J=7.6 \mathrm{~Hz}$, 2H), $7.67(\mathrm{t}, J=7.4 \mathrm{~Hz}, 1 \mathrm{H}), 7.60-7.55(\mathrm{~m}, 3 \mathrm{H}), 7.28-7.24(\mathrm{~m}, 4 \mathrm{H}), 7.13-7.10(\mathrm{~m}, 2 \mathrm{H}), 4.08$ (t, $J=7.5 \mathrm{~Hz}, 1 \mathrm{H}) .3 .16-3.12(\mathrm{~m}, 2 \mathrm{H}), 2.45-2.36(\mathrm{~m}, 3 \mathrm{H}), 2.29-2.21(\mathrm{~m}, 2 \mathrm{H}), 2.17-2.09(\mathrm{~m}, 4 \mathrm{H})$, 1.88-1.79 (m, 2H). ${ }^{13} \mathrm{C}$ NMR (101 MHz, $\left.\mathrm{CDCl}_{3}\right) \delta 163.01,149.55,142.20,139.43,136.67$, $133.76,132.40,129.49,129.41,128.78,128.16,123.01,121.67,55.61,54.26,50.54,41.64$, 32.44, 20.70. IR $\left(\mathrm{CDCl}_{3}, \mathrm{~cm}^{-1}\right): 3062,2949,2797,1587,1305,1087,822,750$. HRMS calcd. for $\left(\mathrm{C}_{24} \mathrm{H}_{28} \mathrm{~N}_{2} \mathrm{O}_{2} \mathrm{SCl}\right)[\mathrm{M}+\mathrm{H}]^{+}$: 443.1560, found 443.1562 .
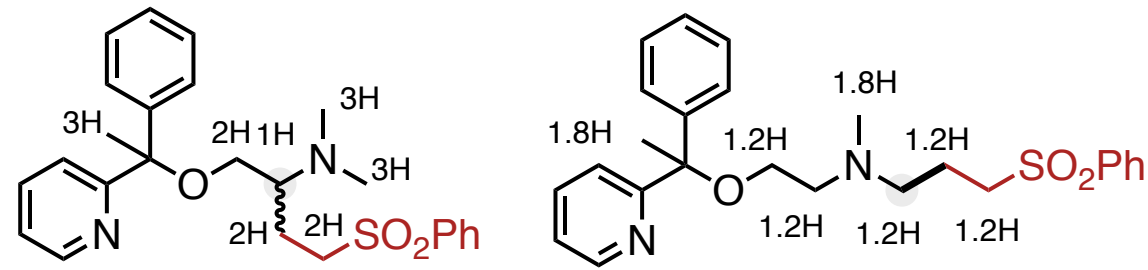

$39(\sim$ dr. $1: 1, \sim$ rr. 1.7:1)

Following the general procedure $\mathrm{C}$, the tittle compound was obtained in $73 \%$ yield as a yellow oil mixture after 8h. ${ }^{1} \mathrm{H}$ NMR $\left(400 \mathrm{MHz}, \mathrm{CDCl}_{3}\right) \delta 8.52-7.08(\mathrm{~m}, 22.4 \mathrm{H}), 3.41-3.16(\mathrm{~m}, 6.4 \mathrm{H})$, 2.69-2.64 (m, 1H), $2.60(\mathrm{t}, J=5.9 \mathrm{~Hz}, 1.2 \mathrm{H}), 2.48(\mathrm{t}, J=6.6 \mathrm{~Hz}, 1.2 \mathrm{H}), 2.23(\mathrm{~s}, 3 \mathrm{H}), 2.22(3 \mathrm{H})$, $2.18(\mathrm{~s}, 1.8 \mathrm{H}), 2.01-1.85(\mathrm{~m}, 8 \mathrm{H})$. Selected peaks: ${ }^{13} \mathrm{C}$ NMR $\left(101 \mathrm{MHz}, \mathrm{CDCl}_{3}\right) \delta 165.05$, $148.54,136.52,133.62,129.32,128.16,126.45,121.86,121.02,82.41,62.28,57.39,54.22$, 41.22, 24.06, 20.85. IR $\left(\mathrm{CDCl}_{3}, \mathrm{~cm}^{-1}\right): 3058,2934,2783,1446,1303,1085,789,748$. HRMS calcd. for $\left(\mathrm{C}_{25} \mathrm{H}_{31} \mathrm{~N}_{2} \mathrm{O}_{3} \mathrm{~S}\right)[\mathrm{M}+\mathrm{H}]^{+}:$439.2055, found 439.2057 .<smiles>CCCN(CCC)S(=O)(=O)c1ccc(C(=O)N2CCN(C)C(CCS(=O)(=O)Oc3ccccc3)C2)cc1</smiles> 


\section{4-(4-methyl-3-(2-(phenylsulfonyl)ethyl)piperazine-1-carbonyl)- $N, N$ -}

dipropylbenzenesulfonamide: Following the general procedure $\mathrm{C}$, the tittle compound was obtained in $77 \%$ yield after $8 \mathrm{~h}$ as colorless oil. ${ }^{1} \mathrm{H}$ NMR $\left(400 \mathrm{MHz}, \mathrm{CDCl}_{3}\right) \delta$ 7.95-7.78 (m, 4H), 7.68-7.64 (m, 1H), 7.59-7.55 (m, 2H), 7.50-7.42 (br, 2H), 4.36-4.11 (br, 1H), 3.42-2.61 (m, $10 \mathrm{H}), 2.40-2.15$ (m, $5 \mathrm{H}), 2.05-1.73$ (br, 2H), 1.55 (h, $J=7.4 \mathrm{~Hz}, 4 \mathrm{H}), 0.86$ (t, $J=7.4 \mathrm{~Hz}$, $6 \mathrm{H}) .{ }^{13} \mathrm{C} \mathrm{NMR}\left(101 \mathrm{MHz}, \mathrm{CDCl}_{3}\right) \delta 168.77,141.67,139.18,139.08,134.00,129.55,128.08$, 127.73, 127.49, 59.48, 54.22, 51.69, 50.24, 47.16, 44.91, 41.99, 22.19, 21.80, 11.26. IR $\left(\mathrm{CDCl}_{3}\right.$, $\left.\mathrm{cm}^{-1}\right): 3064,2965,1630,1443,1147,1086,913,726$. HRMS calcd. for $\left(\mathrm{C}_{26} \mathrm{H}_{38} \mathrm{~N}_{3} \mathrm{O}_{5} \mathrm{~S}_{2}\right)$ $[\mathrm{M}+\mathrm{H}]^{+}:$536.2253, found 536.2262.

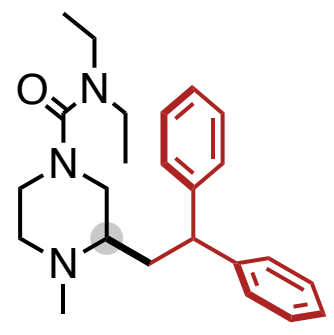

41

3-(2,2-diphenylethyl)- $N, N$-diethyl-4-methylpiperazine-1-carboxamide: Following the general procedure C, $5 \mathrm{~mol} \%$ of TPS-SH and $50 \mathrm{~mol} \%$ of 2-tert-butyl-1,1,3,3tetramethylguanidine (Barton's base) was used instead of $10 \mathrm{~mol} \%$ of TPS-SH, the tittle compound was obtained in $45 \%$ yield after $8 \mathrm{~h}$ as colorless oil. ${ }^{1} \mathrm{H}$ NMR $\left(400 \mathrm{MHz}, \mathrm{CDCl}_{3}\right) \delta$ 7.31-7.14(m, 10H), 4.02 (dd, $J=10.0,5.7 \mathrm{~Hz}, 1 \mathrm{H}), 3.44-3.35$ (m, 2H), 3.14-3.00 (m, 5H), 2.82$2.73(\mathrm{~m}, 2 \mathrm{H}), 2.52(\mathrm{ddd}, J=13.5,10.1,2.9 \mathrm{~Hz}, 1 \mathrm{H}), 2.30$ (s, 3H), $2.24(\mathrm{td}, J=11.5,3.1 \mathrm{~Hz}$, 1H), 2.07-2.03 (m, 1H), $1.95(\mathrm{ddd}, J=14.1,8.4,5.7 \mathrm{~Hz}, 1 \mathrm{H}), 1.05(\mathrm{t}, J=7.1 \mathrm{~Hz}, 6 \mathrm{H}) .{ }^{13} \mathrm{C} \mathrm{NMR}$ $\left(101 \mathrm{MHz}, \mathrm{CDCl}_{3}\right) \delta 164.40,(145.26,143.86),(128.76,128.68),(128.11,127.78),(126.61$, 126.41), 60.24, 54.44, 51.32, 48.45, 46.40, 42.52, 41.86, 35.55, 13.35. IR $\left(\mathrm{CDCl}_{3}, \mathrm{~cm}^{-1}\right): 3065$, 2968, 2794, 1640, 1451, 1416, 1261, 701. HRMS calcd. for $\left(\mathrm{C}_{24} \mathrm{H}_{34} \mathrm{~N}_{3} \mathrm{O}\right)[\mathrm{M}+\mathrm{H}]^{+}$:, 380.2702 , found 380.2710 .<smiles>CCN(CC)C(=O)N1CCN(C)C(CCS(=O)(=O)Oc2ccccc2)C1</smiles>

42

$\mathrm{N}, \mathrm{N}$-diethyl-4-methyl-3-(2-(phenylsulfonyl)ethyl)piperazine-1-carboxamide: Following the general procedure $\mathrm{C}$, the tittle compound was obtained in $82 \%$ yield after $8 \mathrm{~h}$ as colorless 
oil. ${ }^{1} \mathrm{H}$ NMR $\left(400 \mathrm{MHz}, \mathrm{CDCl}_{3}\right) \delta 7.90(\mathrm{~d}, J=8.0 \mathrm{~Hz}, 2 \mathrm{H}), 7.65(\mathrm{t}, J=6.9 \mathrm{~Hz}, 1 \mathrm{H}), 7.56(\mathrm{t}, J$ $=7.7 \mathrm{~Hz}, 2 \mathrm{H}), 3.37-3.23(\mathrm{~m}, 3 \mathrm{H}), 3.16(\mathrm{q}, J=7.1 \mathrm{~Hz}, 4 \mathrm{H}), 3.11-3.02(\mathrm{~m}, 1 \mathrm{H}), 2.93(\mathrm{t}, J=11.6$ $\mathrm{Hz}, 1 \mathrm{H}), 2.71(\mathrm{~d}, J=11.7 \mathrm{~Hz}, 1 \mathrm{H}), 2.61$ (t, $J=11.3 \mathrm{~Hz}, 1 \mathrm{H}), 2.39-2.24(\mathrm{~m}, 2 \mathrm{H}), 2.19$ (s, 3H), 2.05-1.87 (m, 2H), $1.08(\mathrm{t}, J=7.1 \mathrm{~Hz}, 6 \mathrm{H}) .{ }^{13} \mathrm{C} \mathrm{NMR}\left(101 \mathrm{MHz}, \mathrm{CDCl}_{3}\right) \delta 164.31,139.21$, $133.89,129.46,128.12,59.71,54.73,51.79,49.86,47.22,42.23,41.87,22.63,13.29$. IR $\left(\mathrm{CDCl}_{3}, \mathrm{~cm}^{-1}\right): 3062,2969,2797,1634,1419,1262,1086,735$. HRMS calcd. for $\left(\mathrm{C}_{18} \mathrm{H}_{30} \mathrm{~N}_{3} \mathrm{O}_{3} \mathrm{~S}\right)$ $[\mathrm{M}+\mathrm{H}]^{+}:$368.2008, found 368.2030 .

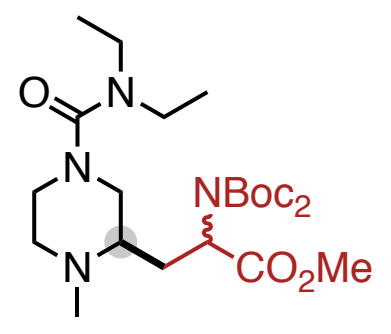

43

methyl 2-(bis(tert-butoxycarbonyl)amino)-3-(4-(diethylcarbamoyl)-1-methylpiperazin-2yl)propanoate: Following the general procedure $\mathrm{C}$, the tittle compound was obtained in $73 \%$ yield ( dr. 1.4:1) after $8 \mathrm{~h}$ as colorless oil. ${ }^{1} \mathrm{H}$ NMR (400 MHz, $\left.\mathrm{CDCl}_{3}\right) \delta 5.00-4.96(\mathrm{~m}, 1 \mathrm{H})$, $3.70(\mathrm{~s}, 3 \mathrm{H}), 3.45-2.57(\mathrm{~m}, 10 \mathrm{H}), 2.34-2.15(\mathrm{~m}, 6 \mathrm{H}), 1.48(\mathrm{~s}, 18 \mathrm{H}), 1.10(\mathrm{t}, J=7.1 \mathrm{~Hz}, 3 \mathrm{H})$, $1.09(\mathrm{t}, J=7.1 \mathrm{~Hz}, 3 \mathrm{H}) .{ }^{13} \mathrm{C} \mathrm{NMR}\left(101 \mathrm{MHz}, \mathrm{CDCl}_{3}\right) \delta(171.46,171.35), 164.49,(152.24$, 152.11), (83.42, 83.35), (59.78, 59.36), (55.87, 55.80), (54.74, 53.97), 52.42, (51.42, 51.07), (46.55, 46.27), (42.67, 42.42), 41.91, (31.95, 29.19), 28.13, 13.31. IR $\left(\mathrm{CDCl}_{3}, \mathrm{~cm}^{-1}\right)$ : 2977, 2934, 1793, 1745, 1699, 1645, 1366, 777. HRMS calcd. for $\left(\mathrm{C}_{24} \mathrm{H}_{45} \mathrm{~N}_{4} \mathrm{O}_{7}\right)[\mathrm{M}+\mathrm{H}]^{+}: 501.3288$, found 501.3291 .

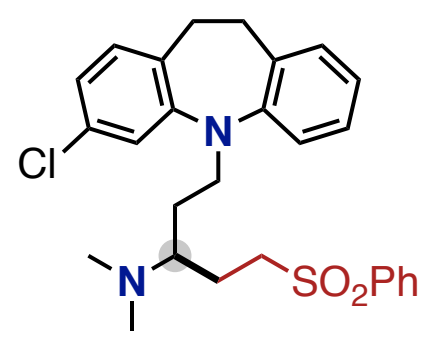

44

\section{1-(3-chloro-10,11-dihydro-5H-dibenzo $[b, f]$ azepin-5-yl)- $N, N$-dimethyl-5-}

(phenylsulfonyl)pentan-3-amine: Following the general procedure $\mathrm{C}$ with ethyl acetate and hexane as column eluent, the tittle compound was obtained in $62 \%$ yield after $8 \mathrm{~h}$ as colorless oil. ${ }^{1} \mathrm{H}$ NMR (400 MHz, $\left.\mathrm{CDCl}_{3}\right) \delta 7.85(\mathrm{~d}, J=8.0 \mathrm{~Hz}, 2 \mathrm{H}), 7.62(\mathrm{t}, J=7.4 \mathrm{~Hz}, 1 \mathrm{H}), 7.52(\mathrm{t}, J$ $=7.7 \mathrm{~Hz}, 2 \mathrm{H}), 7.17-7.09(\mathrm{~m}, 2 \mathrm{H}), 7.03-6.94(\mathrm{~m}, 4 \mathrm{H}), 6.87-6.85(\mathrm{~m}, 1 \mathrm{H}), 3.75-3.72(\mathrm{~m}, 1 \mathrm{H})$, $3.59(\mathrm{dt}, J=13.5,7.0 \mathrm{~Hz}, 1 \mathrm{H}), 3.20-2.99(\mathrm{~m}, 6 \mathrm{H}), 2.51(\mathrm{br}, 1 \mathrm{H}), 2.03(\mathrm{~s}, 6 \mathrm{H}), 1.82-1.71$ (m, 
3H), 1.30-1.24 (m, 1H). ${ }^{13} \mathrm{C} \mathrm{NMR}\left(101 \mathrm{MHz}, \mathrm{CDCl}_{3}\right) \delta 148.96,147.61,139.33,135.28,133.73$, 131.68, 131.56, 131.46, 129.68, 129.36, 128.07, 126.74, 123.66, 122.33, 120.52, 119.76, 60.19, 53.78, 48.04, 39.94, 32.21, 31.54, 26.20, 23.11. IR $\left(\mathrm{CDCl}_{3}, \mathrm{~cm}^{-1}\right): 3062,2929,2826,1585$, 1483, 1303, 1086, 731. HRMS calcd. for $\left(\mathrm{C}_{27} \mathrm{H}_{32} \mathrm{~N}_{2} \mathrm{O}_{2} \mathrm{SCl}\right)[\mathrm{M}+\mathrm{H}]^{+}:$: 483.1873, found 483.1879 .

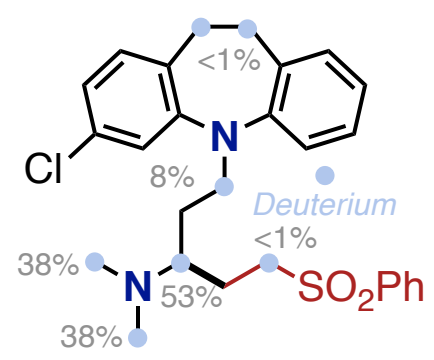

44-d

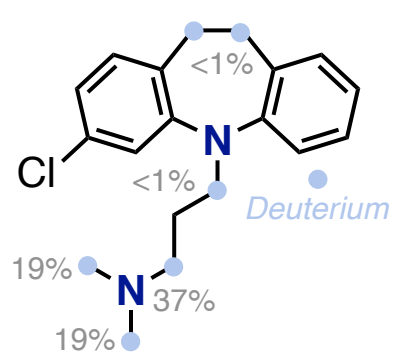

44-SM-d

Following the general procedure $\mathrm{C}$ in presence of $\mathrm{D}_{2} \mathrm{O}$ (50 eq.), compound 33- $\boldsymbol{d}$ was obtained in $60 \%$ yield after $8 \mathrm{~h}$ as colorless oil, the remaining unreactive starting material 33-SM-d (2.2 eq.) was recovered.

Note: see below H-NMR spectrums for details.

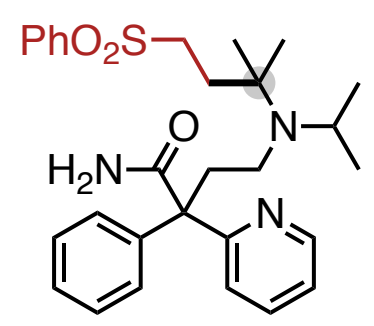

45

\section{4-(isopropyl(2-methyl-4-(phenylsulfonyl)butan-2-yl)amino)-2-phenyl-2-(pyridin-2-}

yl)butanamide: Following the general procedure $\mathrm{C}$, the tittle compound was obtained in $71 \%$ yield after $8 \mathrm{~h}$ as yellow oil. ${ }^{1} \mathrm{H}$ NMR $\left(400 \mathrm{MHz}, \mathrm{CDCl}_{3}\right) \delta 8.69(\mathrm{br}, 1 \mathrm{H}), 8.55-8.53(\mathrm{~m}, 1 \mathrm{H})$, 7.92-7.90 (m, 2H), 7.66-7.60 (m, 2H), 7.58-7.54 (m, 2H), 7.31-7.16 (m, 7H), $5.80(\mathrm{br}, 1 \mathrm{H})$, 3.24-3.20 (m, 2H), 2.90 (p, $J=6.5 \mathrm{~Hz}, 1 \mathrm{H}), 2.68-2.62(\mathrm{~m}, 1 \mathrm{H}), 2.42-2.30$ (m, 2H), 2.25-2.19 (m, 1H), $1.70(\mathrm{dd}, J=11.6,4.7 \mathrm{~Hz}, 1 \mathrm{H}), 0.92(\mathrm{~s}, 3 \mathrm{H}), 0.88(\mathrm{~s}, 3 \mathrm{H}), 0.78(\mathrm{~d}, J=6.5 \mathrm{~Hz}, 3 \mathrm{H})$, $0.74(\mathrm{~d}, J=6.5 \mathrm{~Hz}, 3 \mathrm{H}) .{ }^{13} \mathrm{C} \mathrm{NMR}\left(101 \mathrm{MHz}, \mathrm{CDCl}_{3}\right) \delta 175.64,163.44,147.26,144.44,139.71$, $136.95,133.61,129.35,128.37,128.22$, 128.10, 124.50, 121.82, 60.70, 57.36, 52.61, 46.76, 42.66, 37.91, 34.01, 26.60, 26.40, 22.23. IR $\left(\mathrm{CDCl}_{3}, \mathrm{~cm}^{-1}\right): 3274,3060,2970,1675,1586,1290$, 1145, 730. HRMS calcd. for $\left(\mathrm{C}_{29} \mathrm{H}_{38} \mathrm{~N}_{3} \mathrm{O}_{3} \mathrm{~S}\right)[\mathrm{M}+\mathrm{H}]^{+}$: 508.2634, found 508.2652.

Note: single isomer was obtained due to the $\beta$-cleavage of secondary a-amino radical. The byproduct diaryl acetamide was isolated as an evidence. 


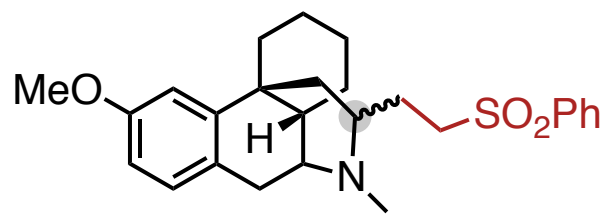

$46(\sim$ dr. 5.5:1)

(4bS,8aS)-3-methoxy-11-methyl-12-(2-(phenylsulfonyl)ethyl)-6,7,8,8a,9,10-hexahydro5H-9,4b-(epiminoethano)phenanthrene: Following the general procedure $\mathrm{C}$, the tittle compound was obtained in $45 \%$ yield ( dr. 5.5:1) after $8 \mathrm{~h}$ as colorless oil. ${ }^{1} \mathrm{H}$ NMR $(400 \mathrm{MHz}$, $\left.\mathrm{CDCl}_{3}\right) \delta$ 7.93-7.86 (m, 2H), 7.65-7.61 (m, 1H), 7.57-7.52 (m, 2H), $6.99(\mathrm{~d}, J=8.3 \mathrm{~Hz}, 1 \mathrm{H})$, $6.74(\mathrm{~d}, J=2.6 \mathrm{~Hz}, 1 \mathrm{H}), 6.69(\mathrm{dd}, J=8.3,2.5 \mathrm{~Hz}, 1 \mathrm{H}), 3.77$ (s, 3H), 3.26-2.16 (m, $10 \mathrm{H}), 1.85-$ $1.03(\mathrm{~m}, 12 \mathrm{H})$. Major isomer: ${ }^{13} \mathrm{C} \mathrm{NMR}\left(101 \mathrm{MHz}, \mathrm{CDCl}_{3}\right) \delta 158.34,141.66,139.29,133.59$, 129.28, 129.23, 128.74, 128.01, 111.09, 110.93, 60.22, 55.20, 52.81, 51.38, 46.84, 44.76, 39.37, 38.04, 36.59, 26.46, 26.36, 24.68, 21.98. IR $\left(\mathrm{CDCl}_{3}, \mathrm{~cm}^{-1}\right): 3061,2926,2854,1497,1303,1238$, 1145, 730. HRMS calcd. For $\left(\mathrm{C}_{26} \mathrm{H}_{34} \mathrm{NO}_{3} \mathrm{~S}\right)[\mathrm{M}+\mathrm{H}]^{+}:$440.2260, found 440.2250. 


\section{References}

1. Cismesia, M. A.; Yoon, T. P. Characterizing chain processes in visible light photoredox catalysis. Chem. Sci., 2015, 6, 5426-5434

2. Zhou, R., Goh, Y. Y., Liu, H., Tao, H., Li, L., Wu, J. Visible-light-mediated metal-free hydrosilylation of alkenes through selective hydrogen atom transfer for $\mathrm{Si}-\mathrm{H}$ activation. Angew. Chem. Int. Ed. 56, 16621-16625 (2017).

3. Gaussian 16, Revision A.03, Frisch, M. J., Trucks, G. W., Schlegel, H. B., Scuseria, G. E., Robb, M. A., Cheeseman, J. R., Scalmani, G., Barone, V., Petersson, G. A., Nakatsuji, H., Li, X., Caricato, M., Marenich, A. V., Bloino, J., Janesko, B. G., Gomperts, R., Mennucci, B., Hratchian, H. P., Ortiz, J. V., Izmaylov, A. F., Sonnenberg, J. L., Williams-Young, D., Ding, F., Lipparini, F., Egidi, F., Goings, J., Peng, B., Petrone, A., Henderson, T., Ranasinghe, D., Zakrzewski, V. G., Gao, J., Rega, N., Zheng, G., Liang, W., Hada, M., Ehara, M., Toyota, K., Fukuda, R., Hasegawa, J., Ishida, M., Nakajima, T., Honda, Y., Kitao, O., Nakai, H., Vreven, T., Throssell, K., Montgomery Jr. J. A., Peralta, J. E., Ogliaro, F., Bearpark, M. J., Heyd, J. J., Brothers, E. N., Kudin, K. N., Staroverov, V. N., Keith, T. A., Kobayashi, R., Normand, J., Raghavachari, K., Rendell, A. P., Burant, J. C., Iyengar, S. S., Tomasi, J., Cossi, M., Millam, J. M., Klene, M., Adamo, C., Cammi, R., Ochterski, J. W., Martin, R. L., Morokuma, K., Farkas, O., Foresman, J. B., Fox, D. J. Gaussian, Inc., Wallingford CT, 2016.

4. Legault, C. Y. CYLview, Version 1.0b. Université de Sherbrooke. (2009); http://www.cylview.org. 
VII. ${ }^{1} \mathrm{H},{ }^{13} \mathrm{C}$ and ${ }^{19} \mathrm{~F}$ NMR spectra
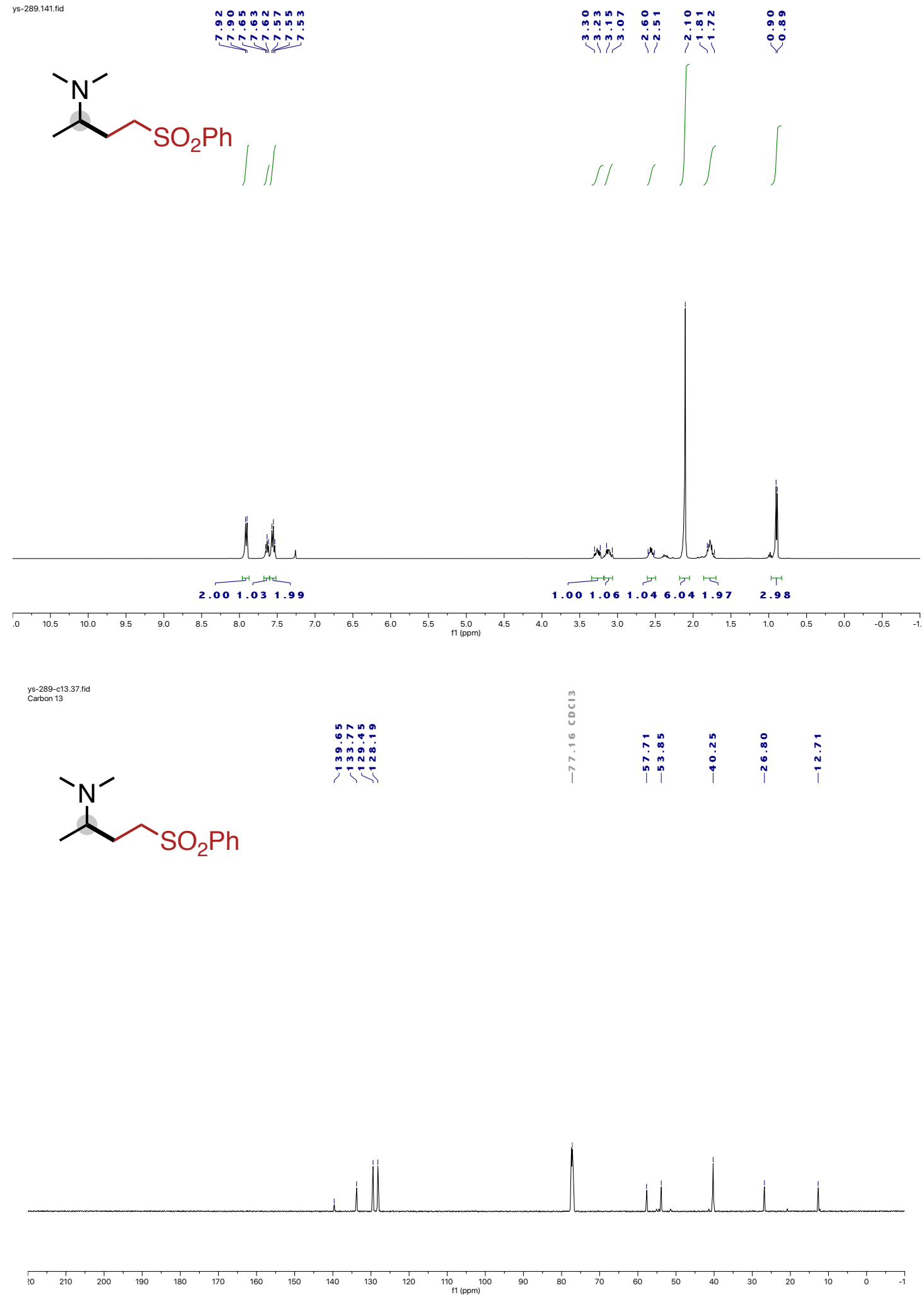


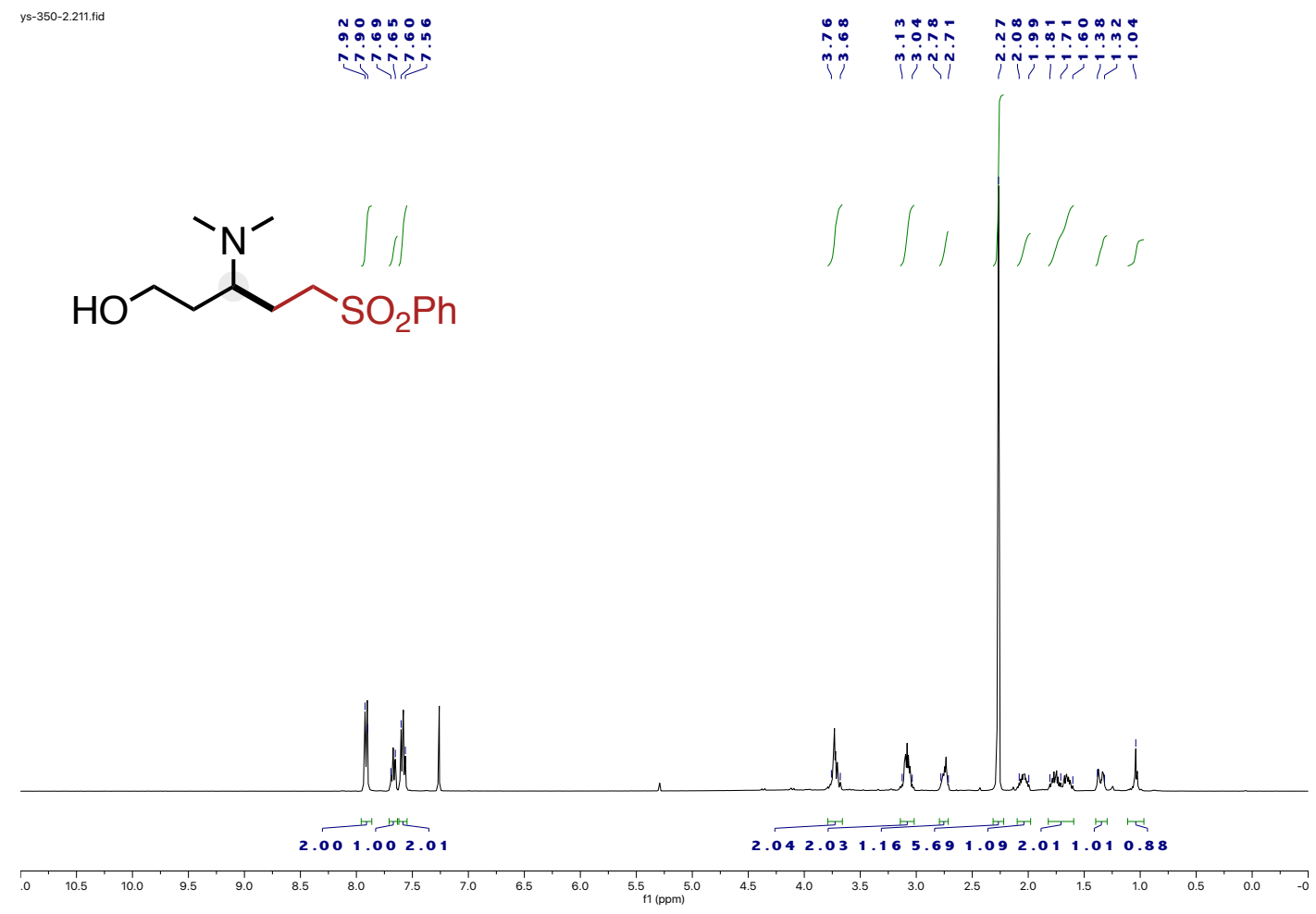

ys-350-2-c13.57.fid
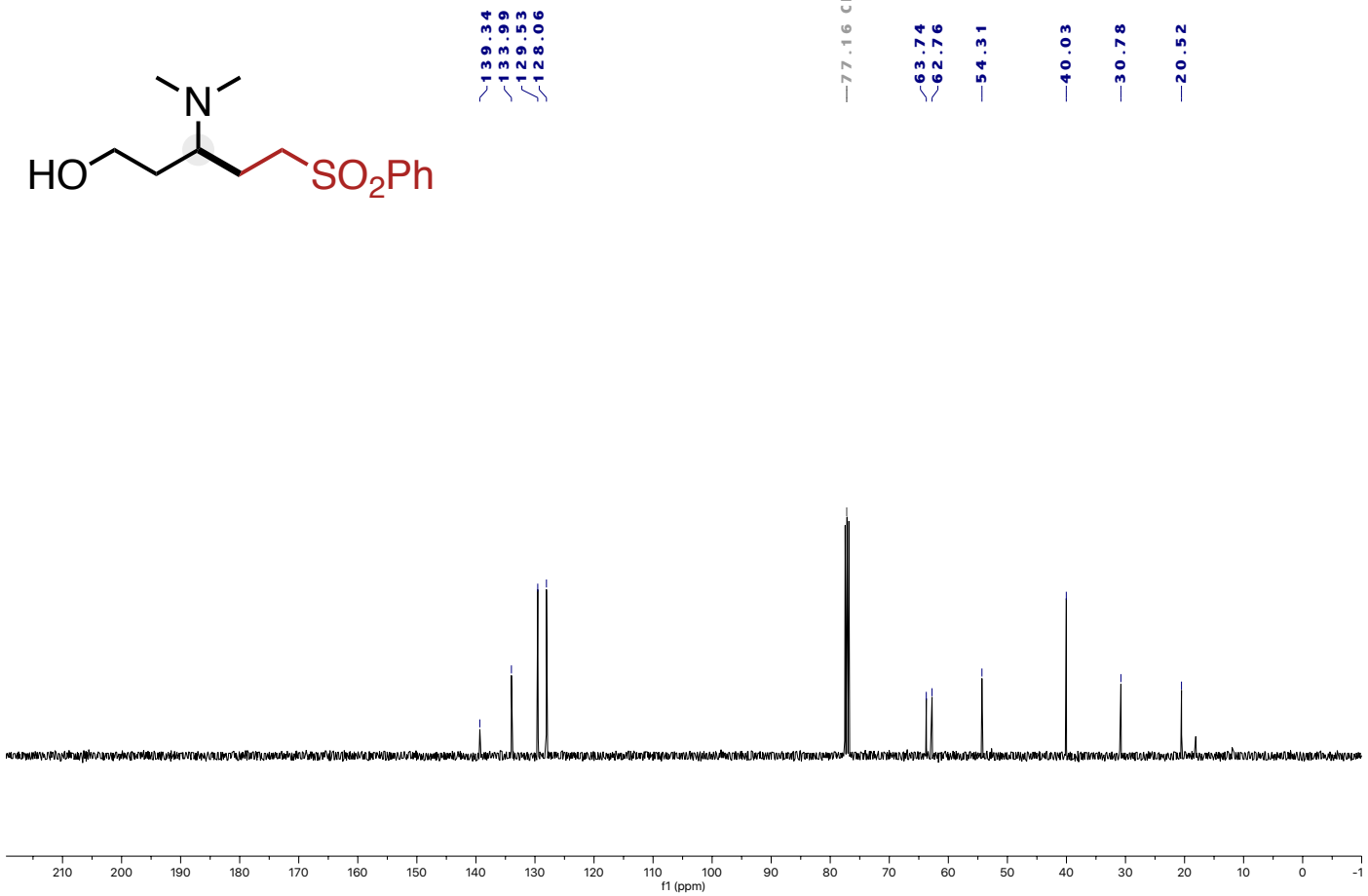


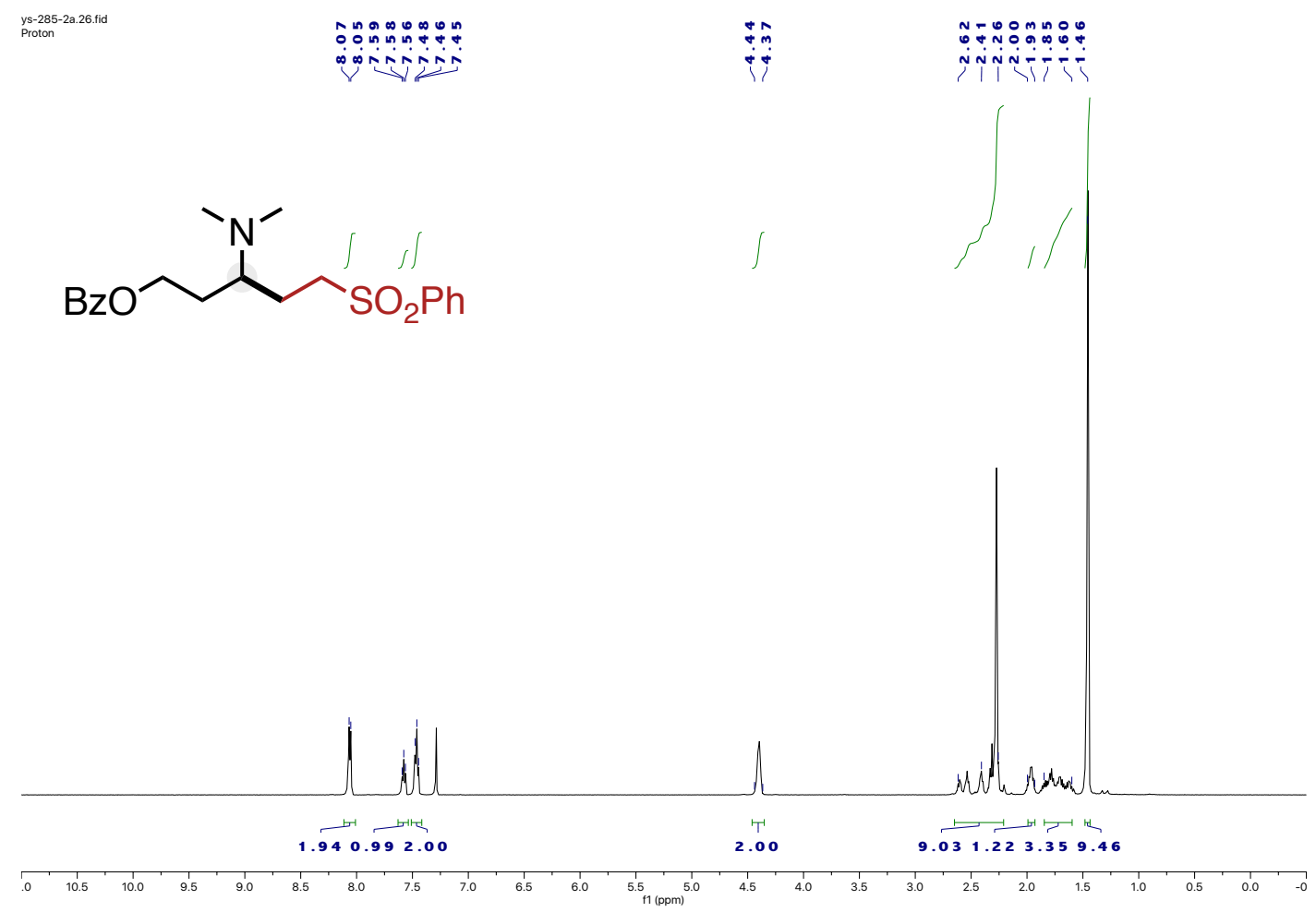

ys-285-1-1-c13.28.fid
Carbon 13
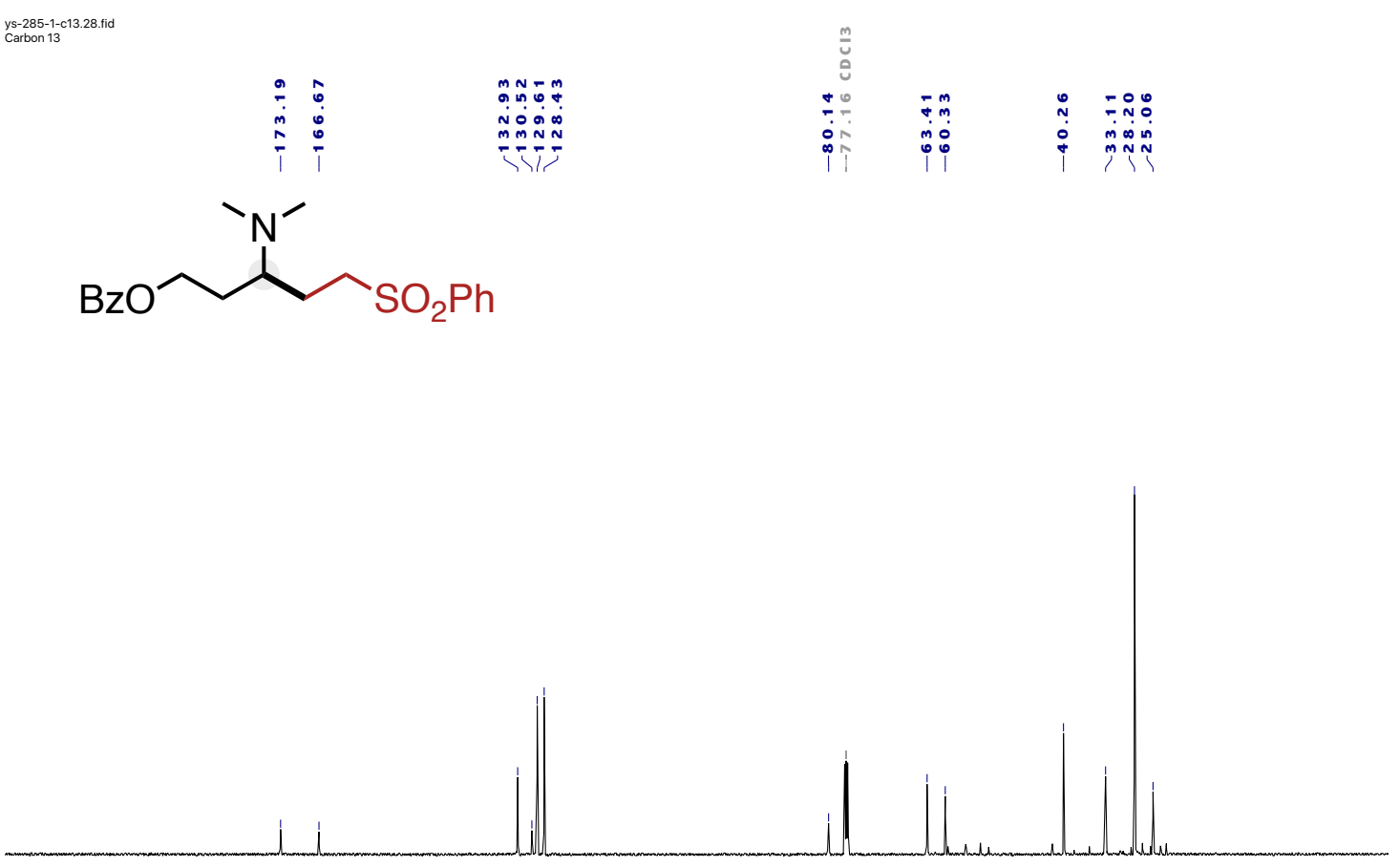

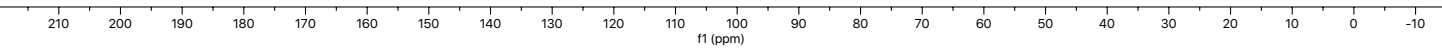




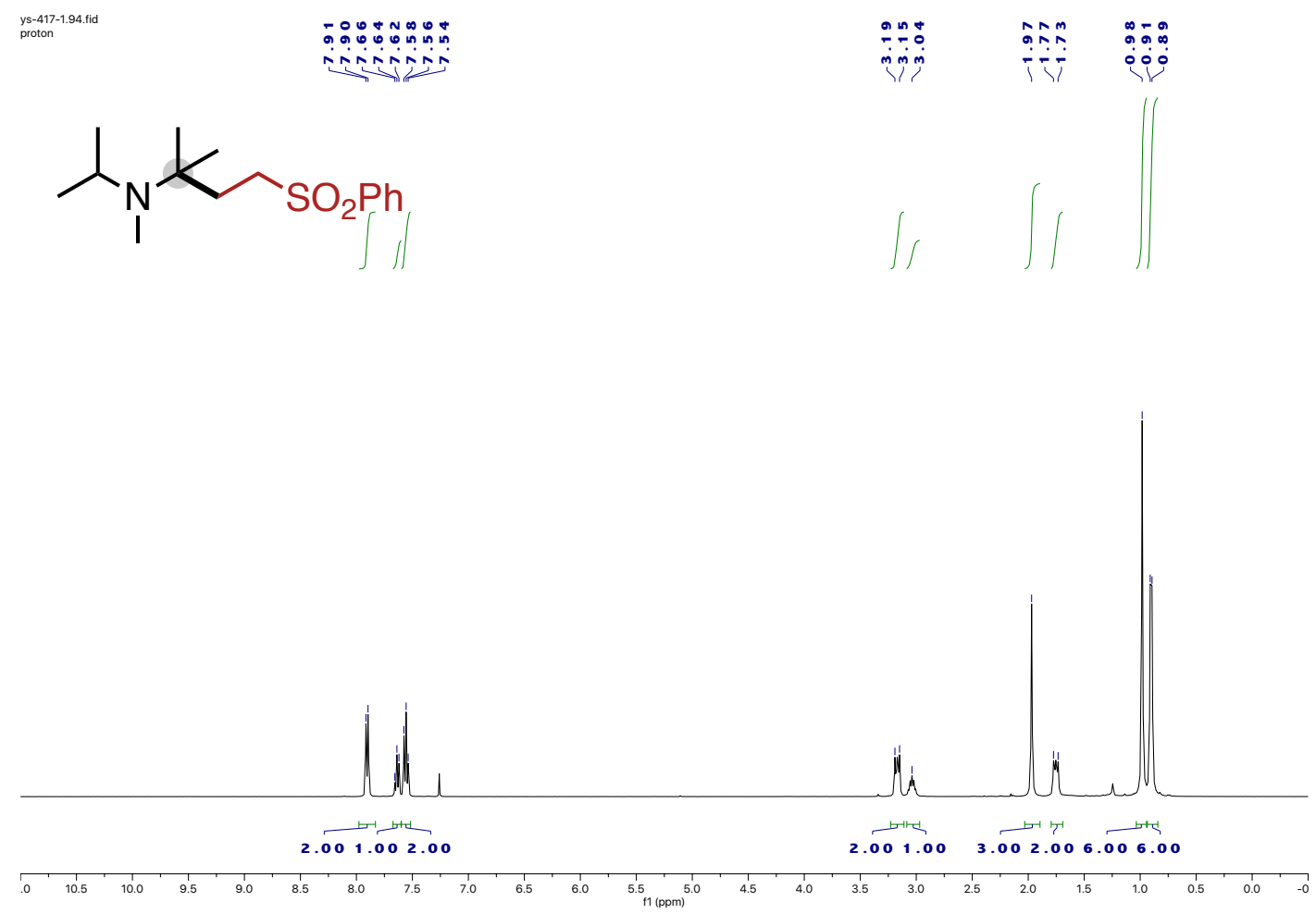

ys-417-1-c13.100.fid

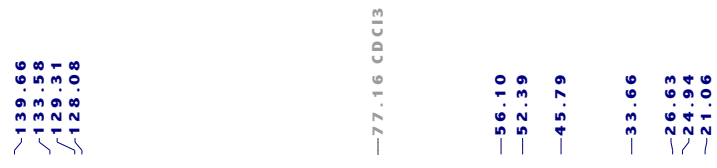

$\lambda_{\mathrm{I}}<\mathrm{SO}_{\mathrm{SO}_{2} \mathrm{Ph}}$
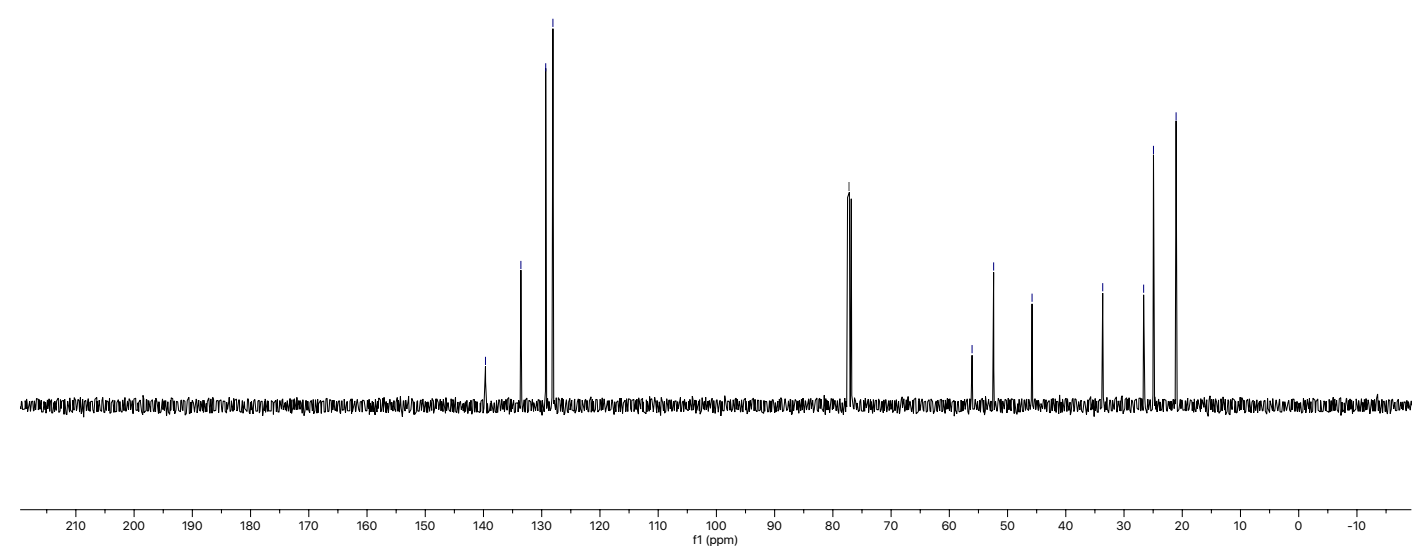
$\mathrm{J}_{\mathrm{SO}^{2}} \mathrm{Ph}_{\mathrm{H}}$

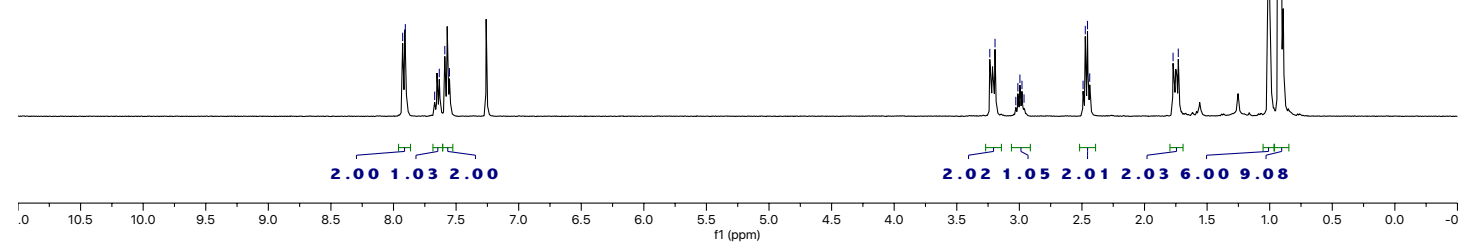<smiles>CCN(C(C)C)C(C)(C)CCS(=O)(=O)c1ccccc1</smiles>
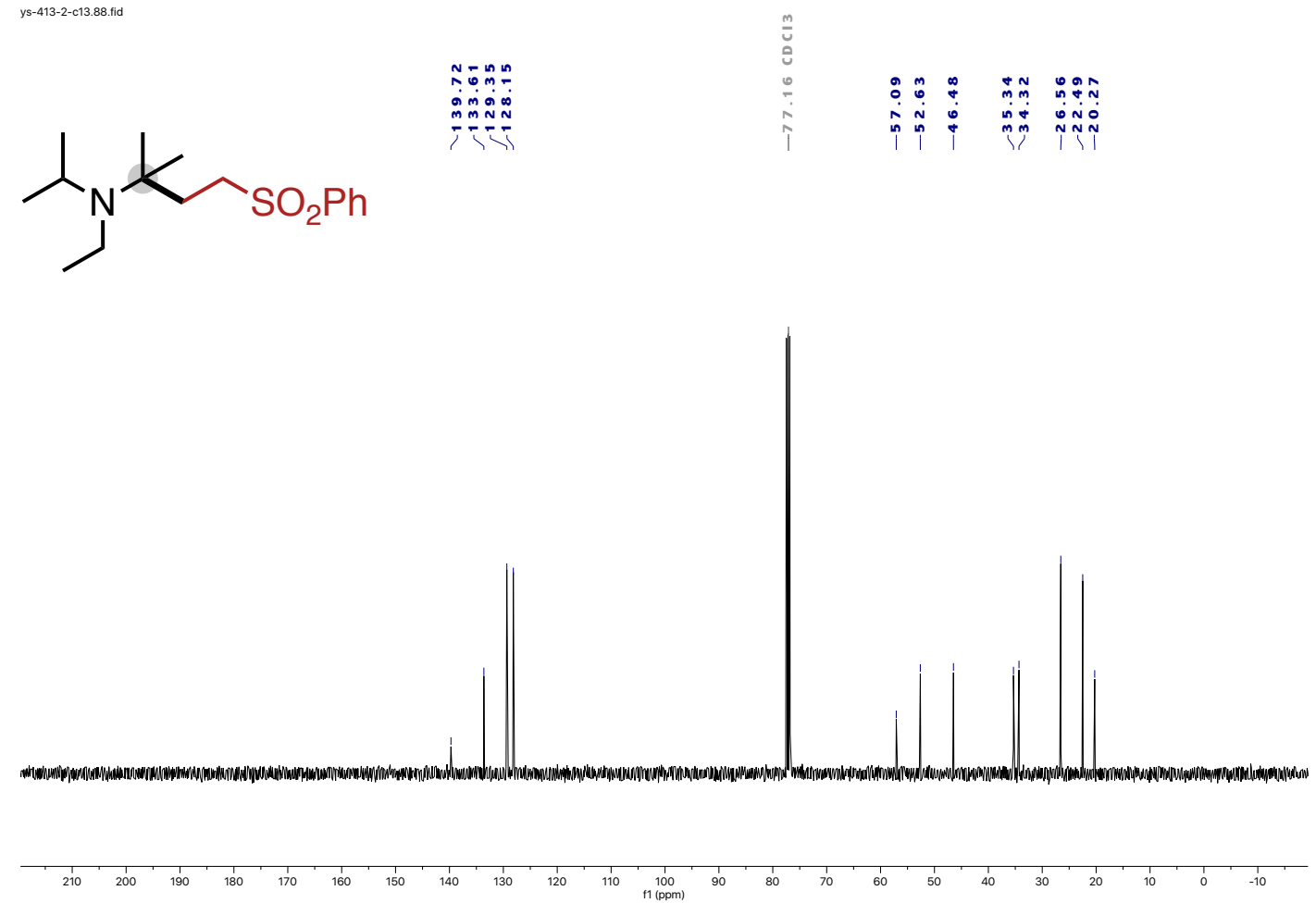

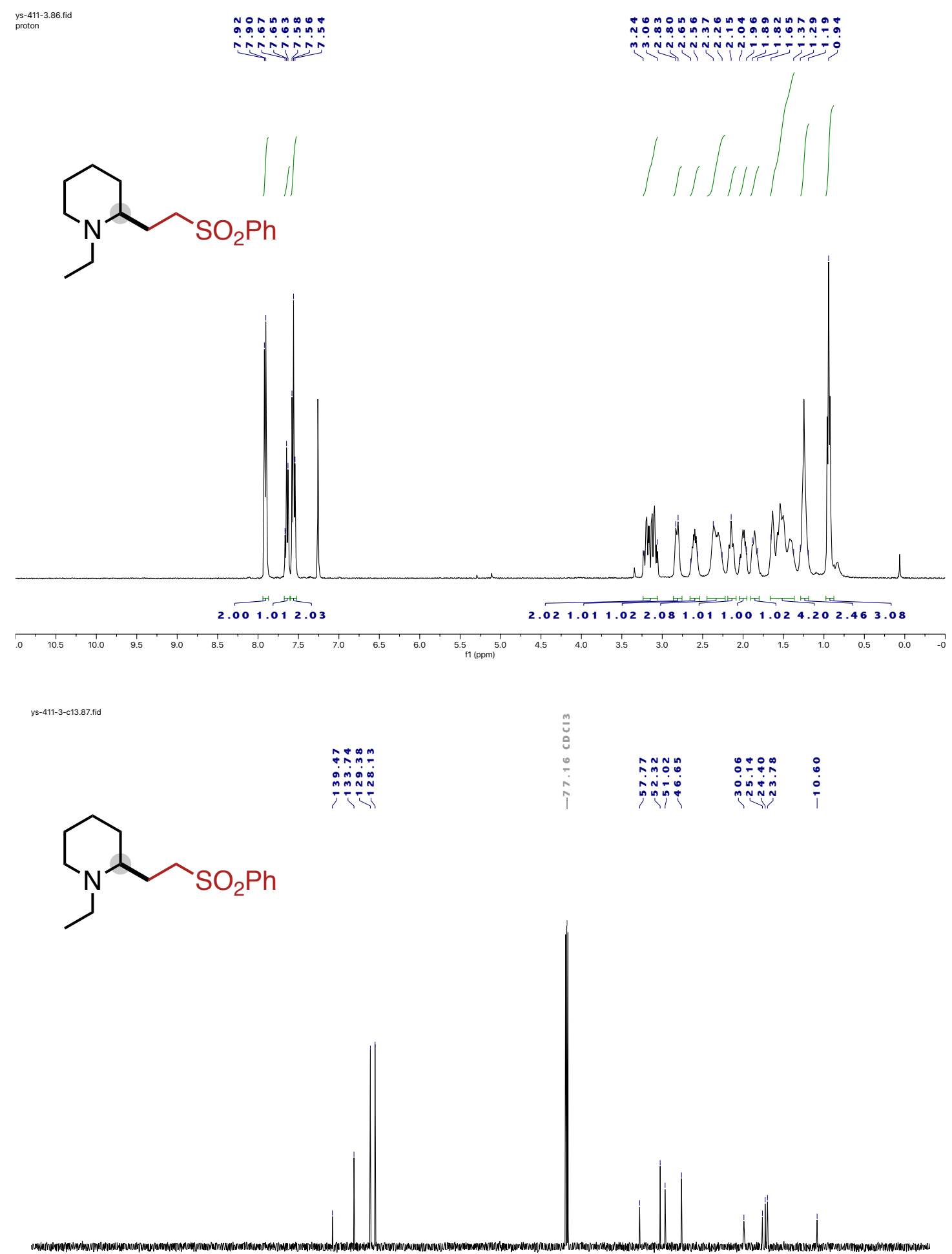
$\underset{\substack{\text { ys-411-2.25. fid } \\ \text { proton }}}{\text { id }}$

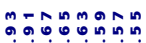

inisinis

$\overbrace{\mathrm{SO}_{2} \mathrm{Ph}}$

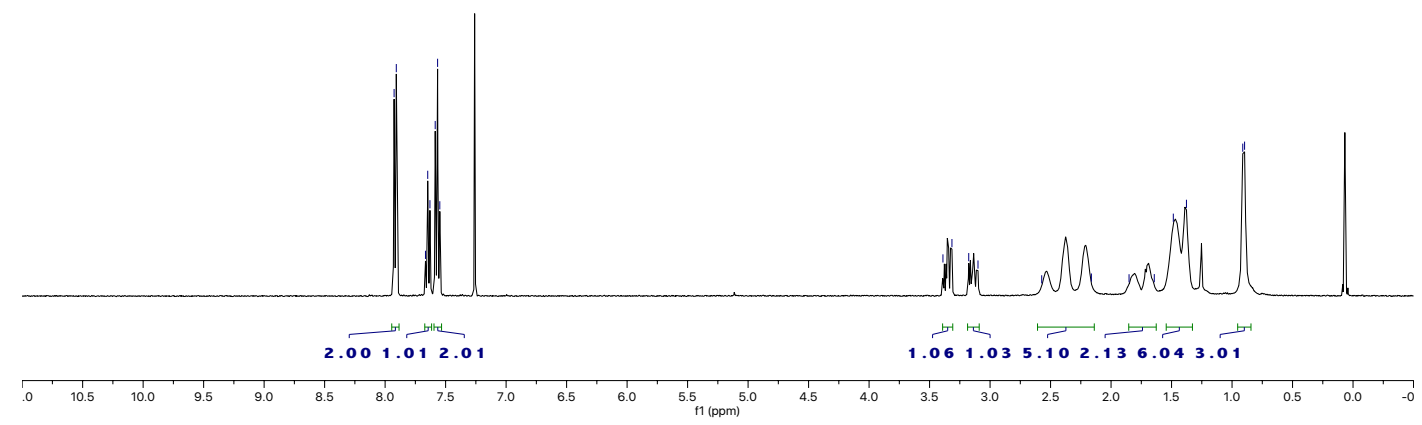

ys-411-2-c13.89.fid

imin

$\overbrace{\mathrm{SO}_{2} \mathrm{Ph}}$
भกำ

योग्यें

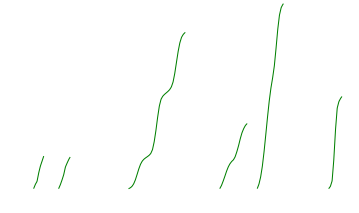

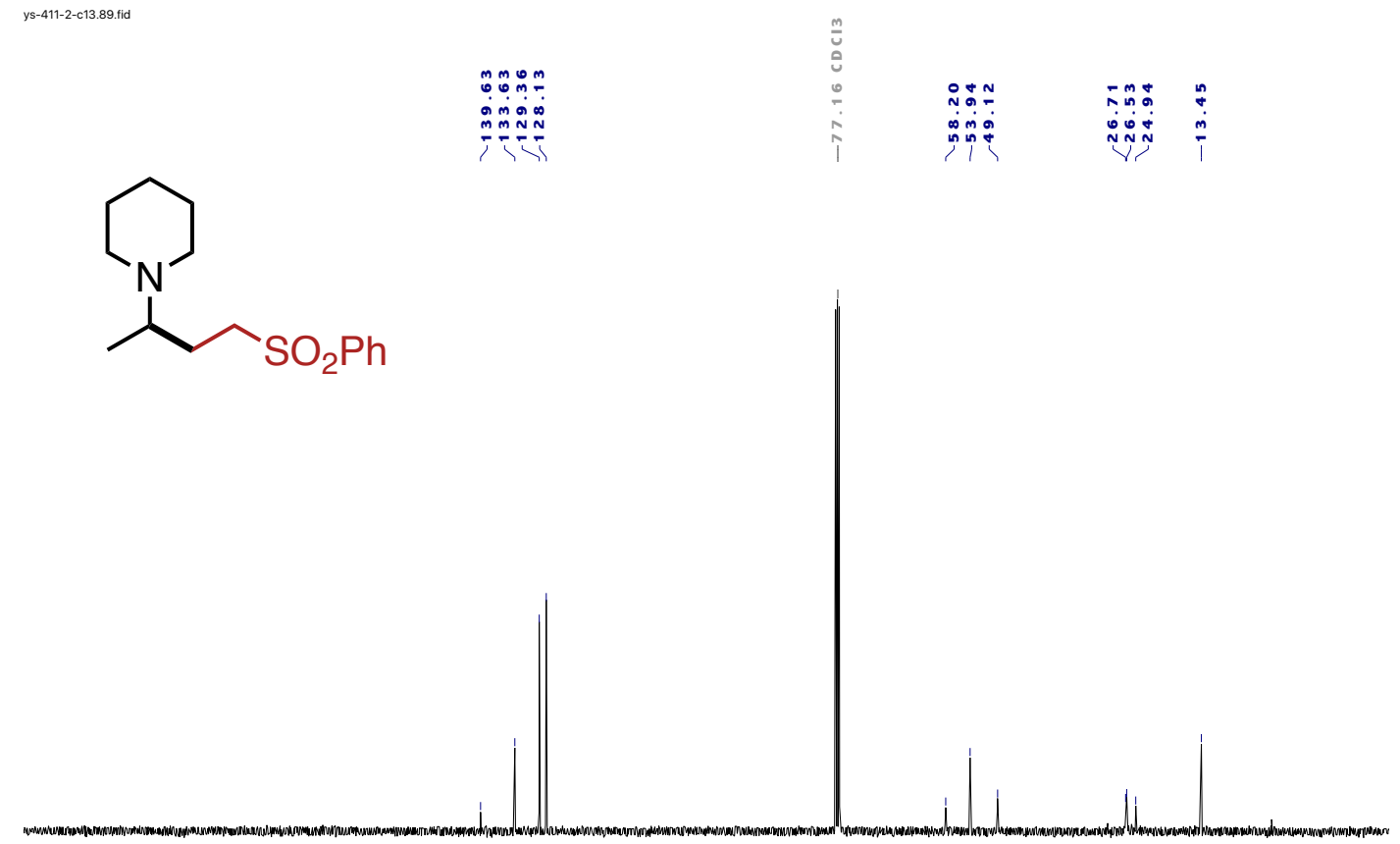

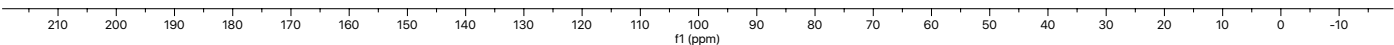



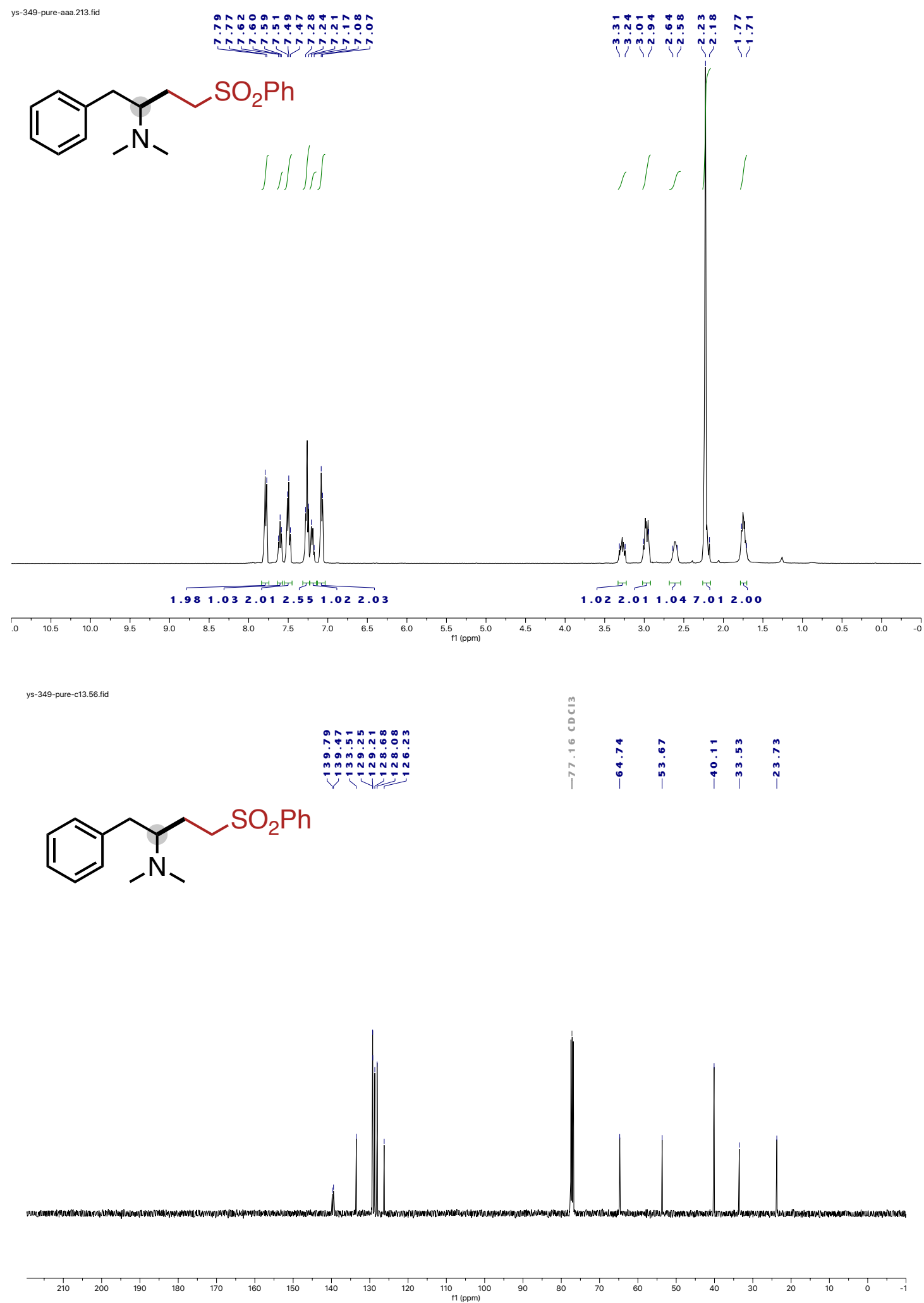


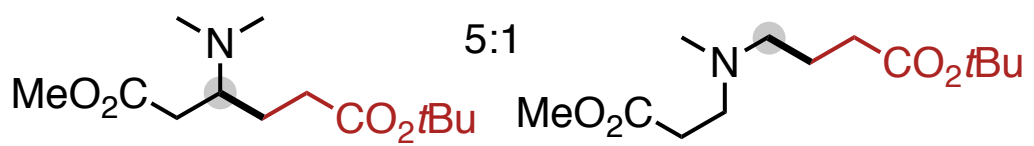

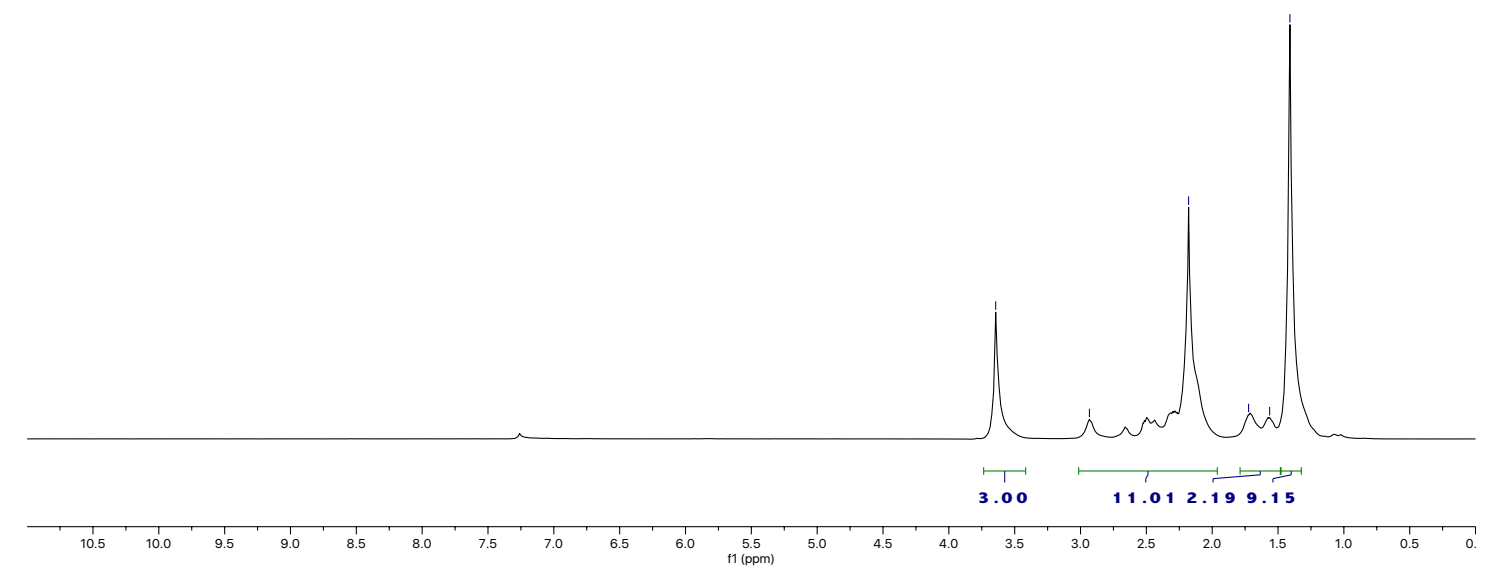

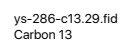

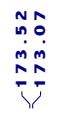

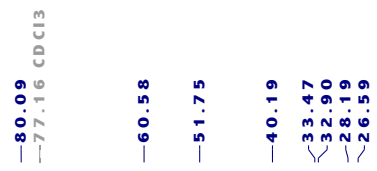

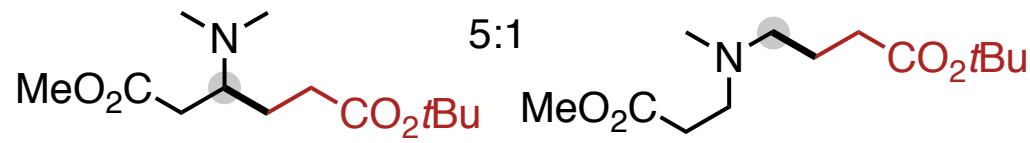

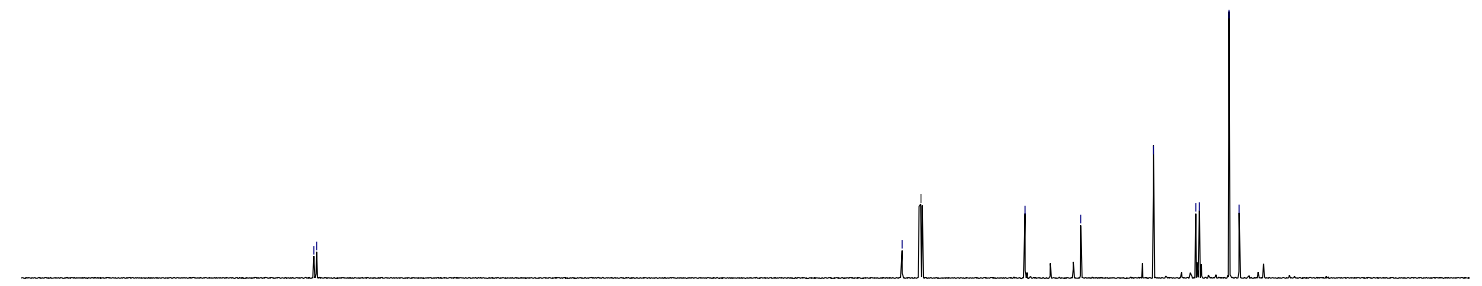

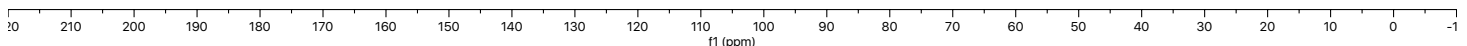


<smiles>C#CN(C)C(CCN1C(=O)c2ccccc2C1=O)CCS(=O)O[Na]</smiles>
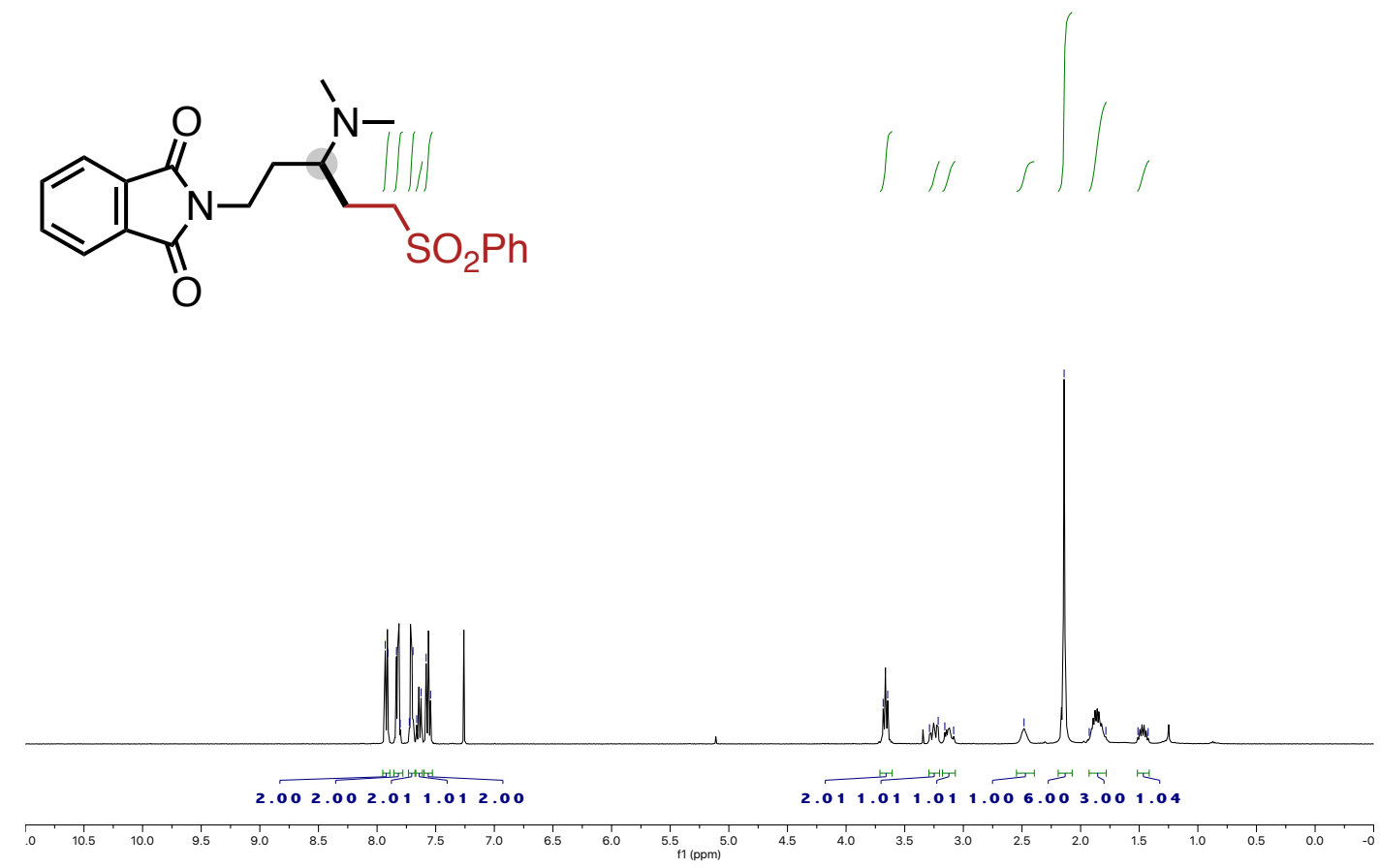

ys-421-1-c13.101.fid<smiles>C[Al]I(N)C(CCSOc1ccccc1)CCN1C(=O)c2ccccc2C1=O</smiles>
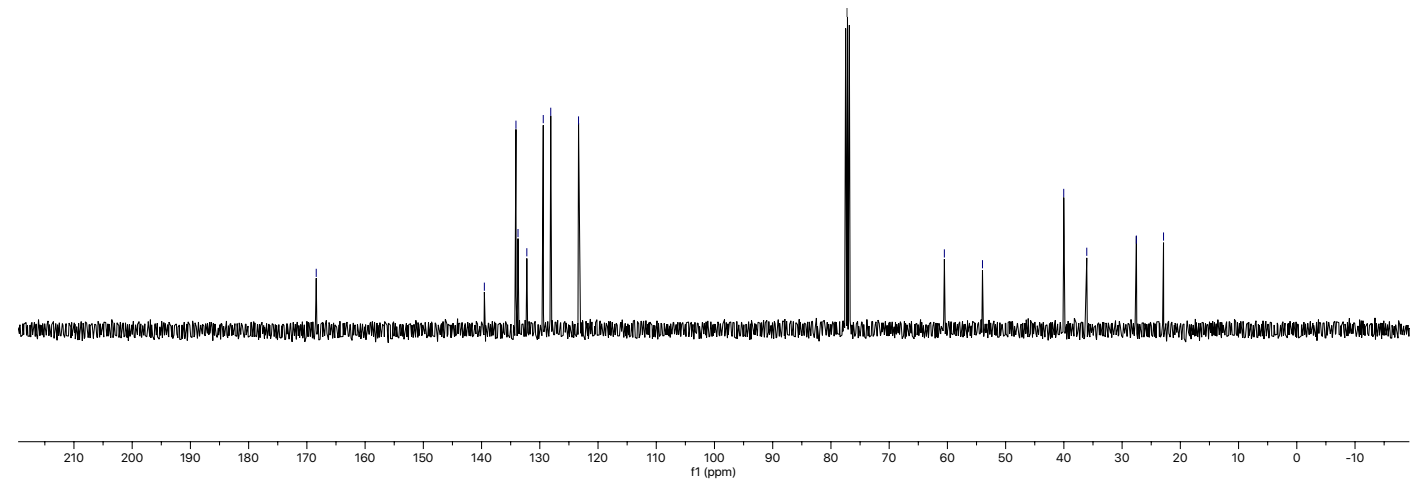
O<smiles>CN1CCC(=O)CC1CCS(=O)(=O)Oc1ccccc1</smiles>

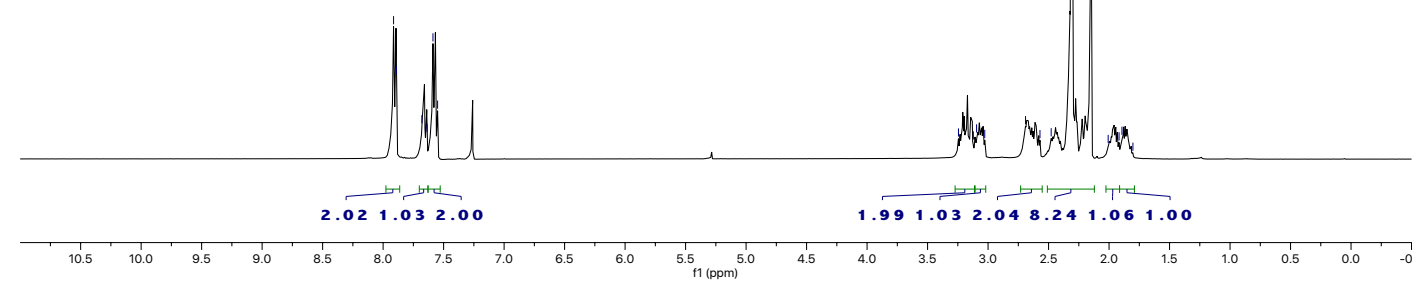

ys-362-2-c13-a.54.fid

ڤ̊

วกำ

มंm

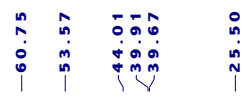<smiles>CN1CCC(=O)CC1CCS(=O)(=O)c1ccccc1</smiles>
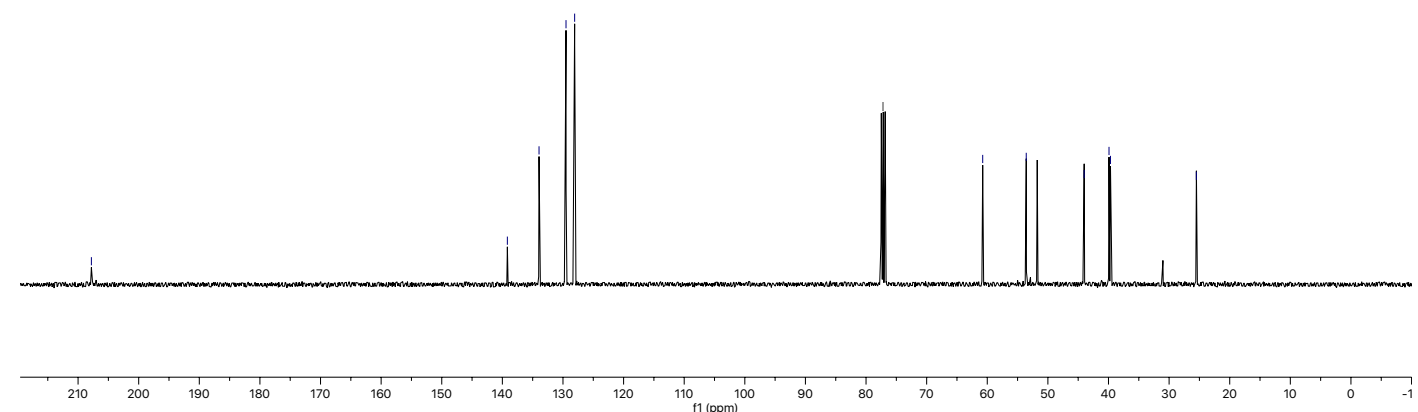


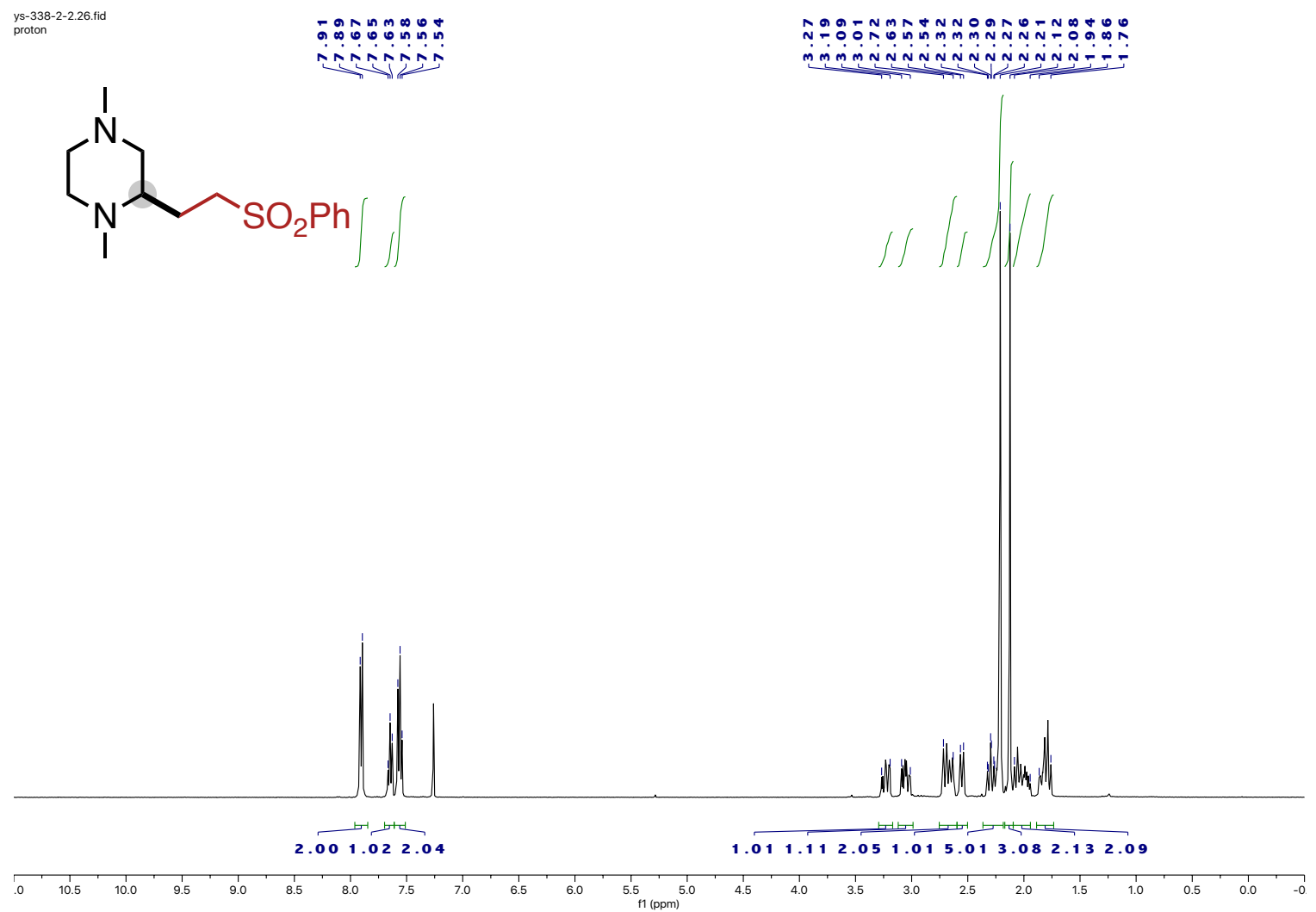

ys-338-2-2-c13.39.fid
Carbon 13

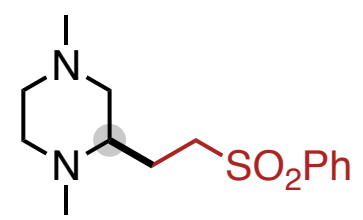

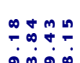

ํำ

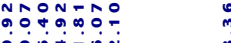

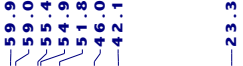

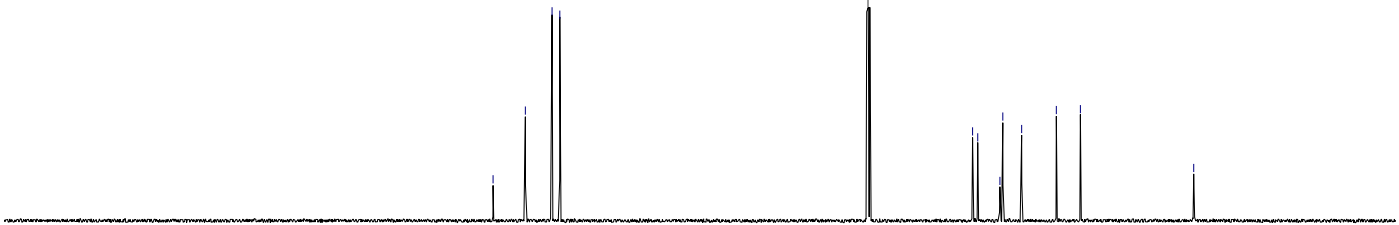

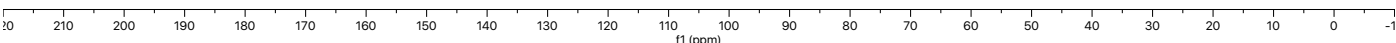




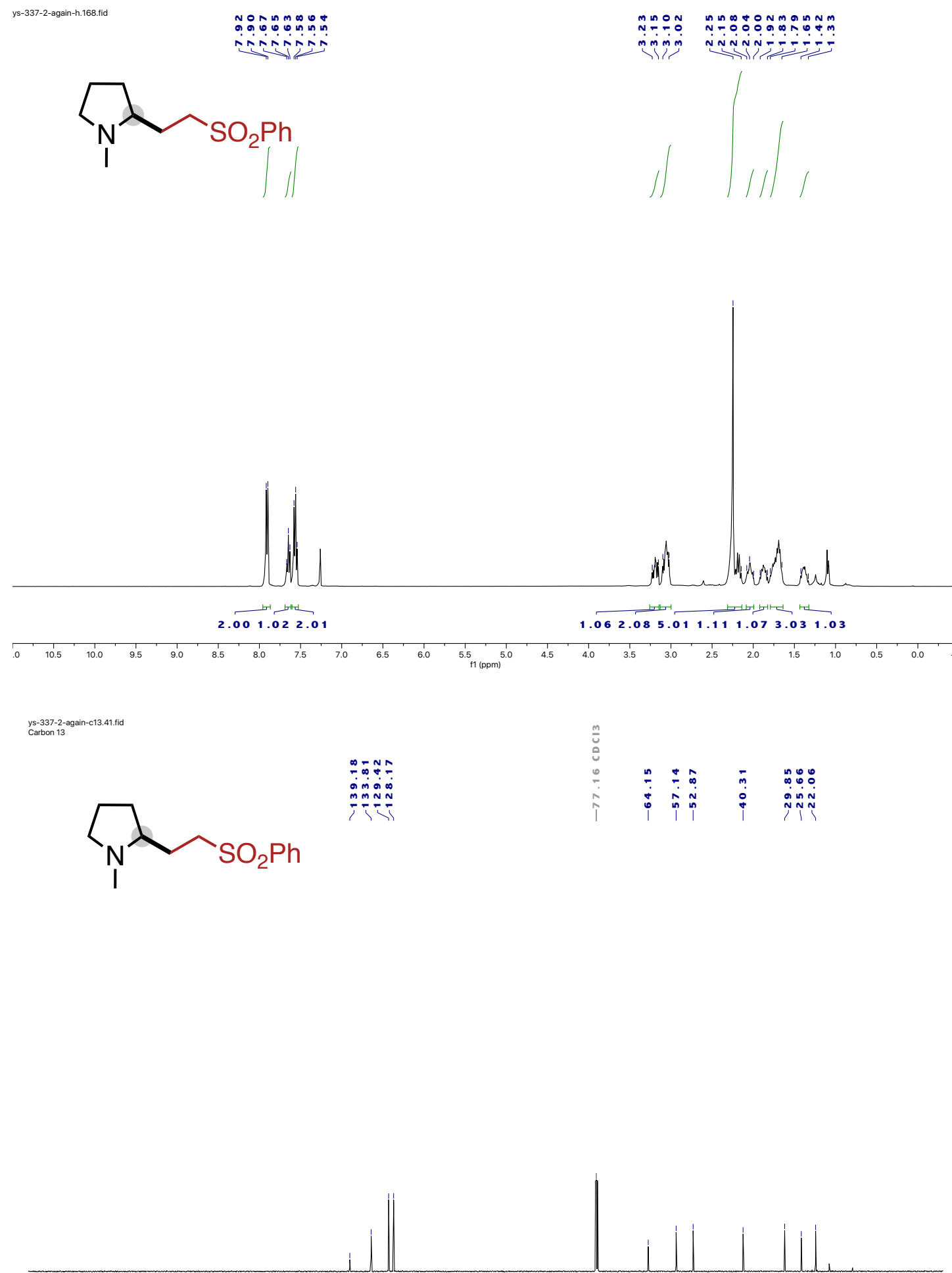

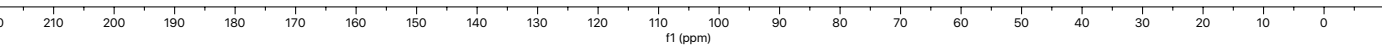




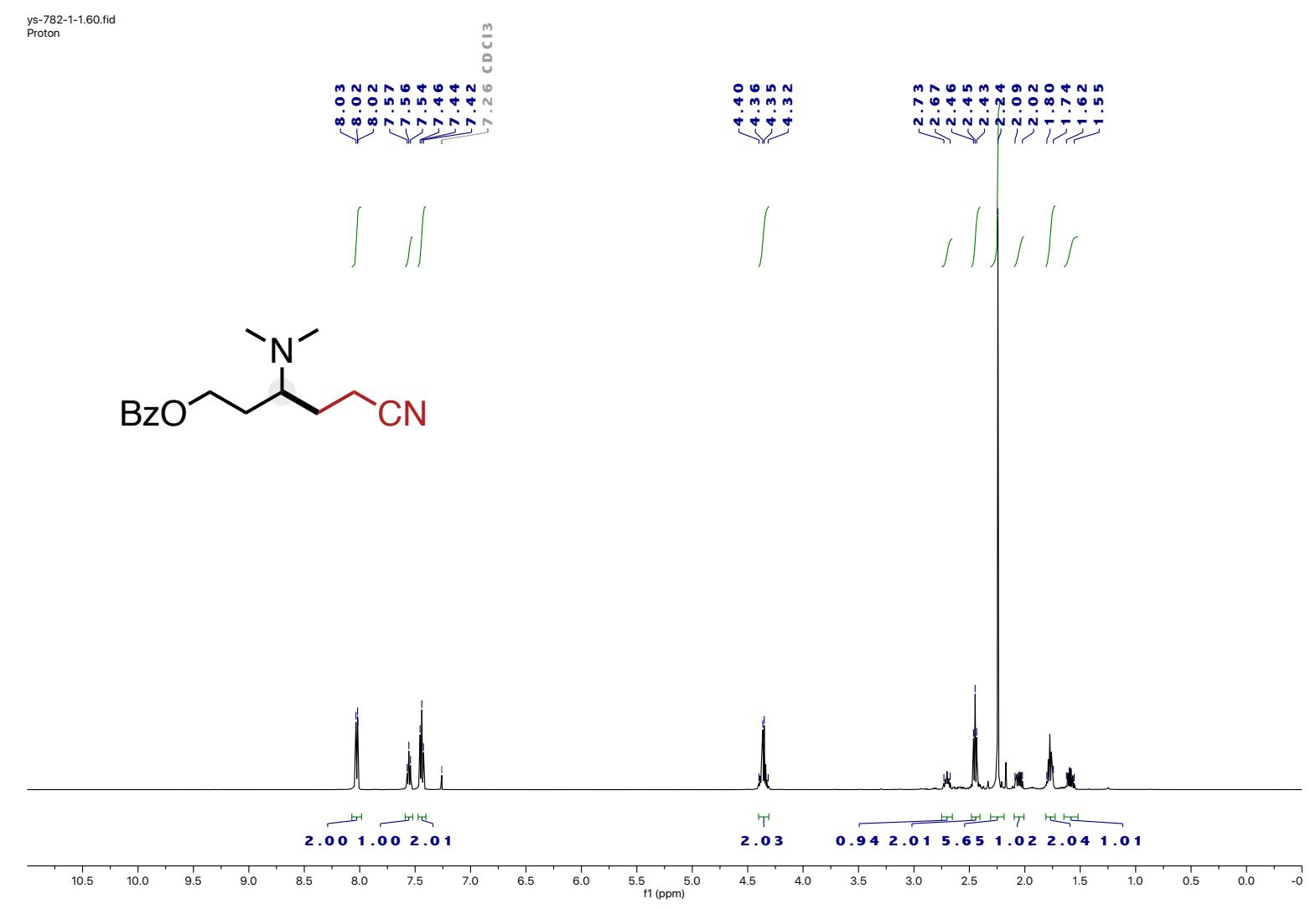

ys-782-1-1-500c.61.fid
Carbon 13
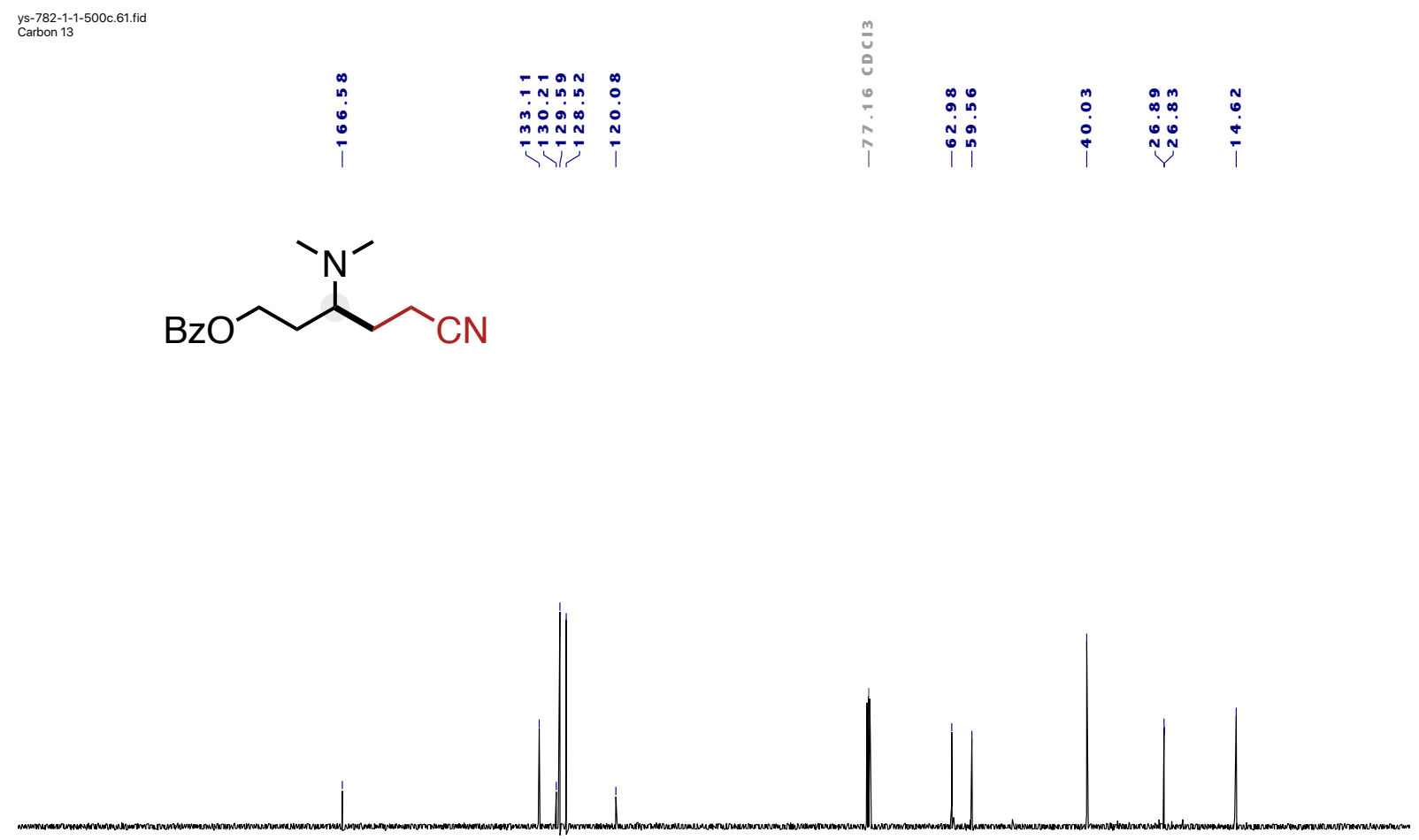

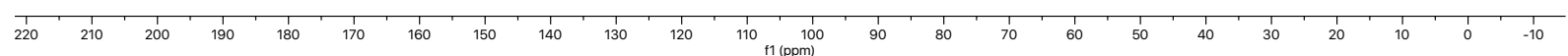




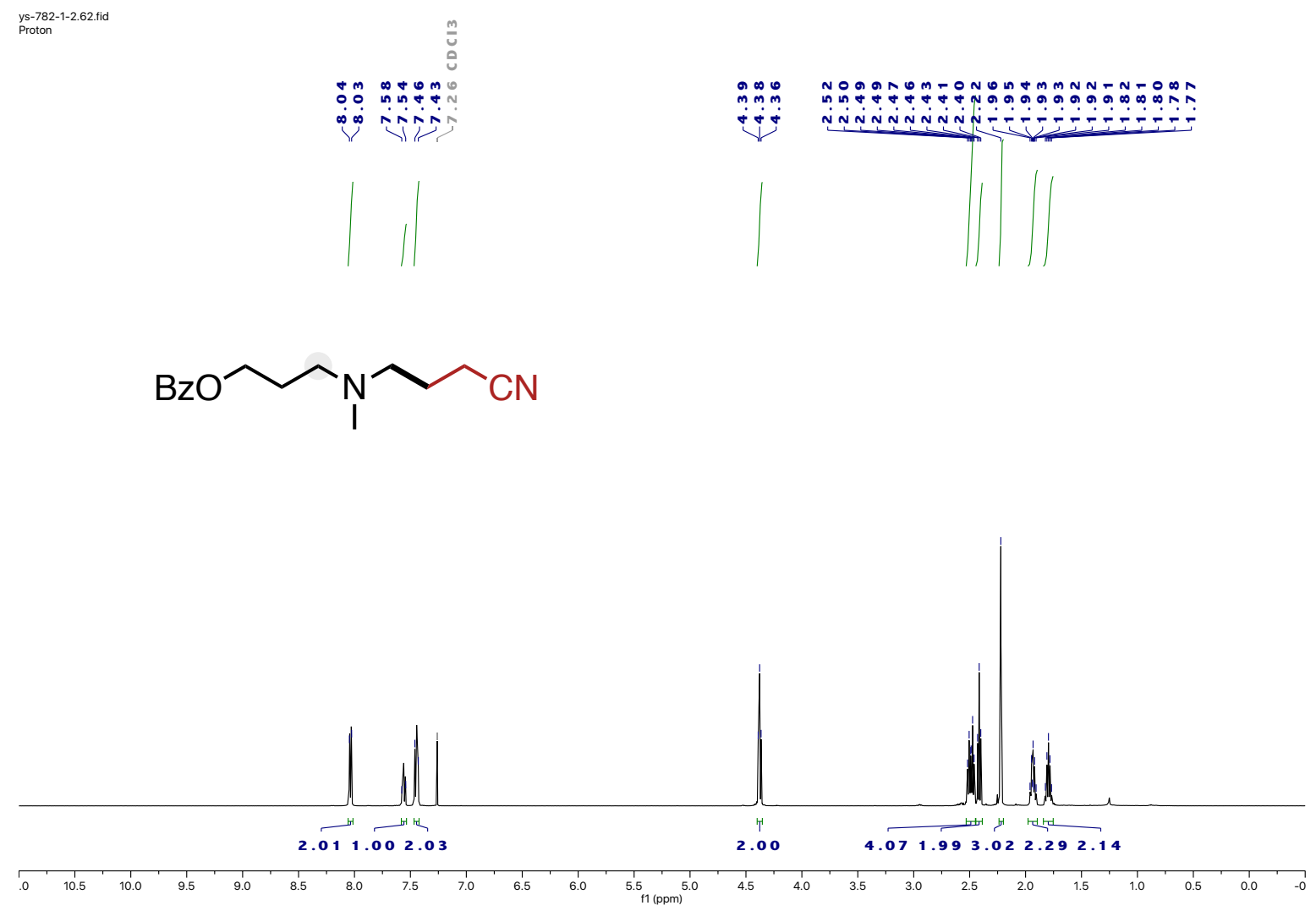

ys-782-1-2-500c.63.fid
Carbon 13

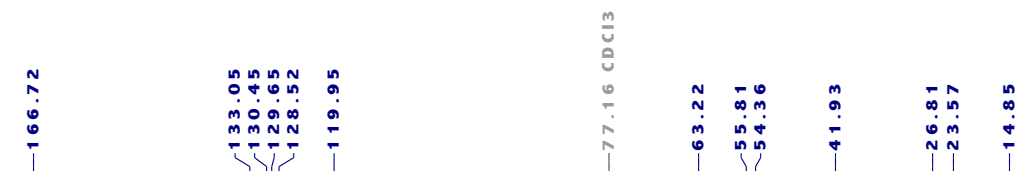

$\mathrm{BzO}_{\mathrm{N}}^{\mathrm{N}} \overbrace{\mathrm{CN}}$

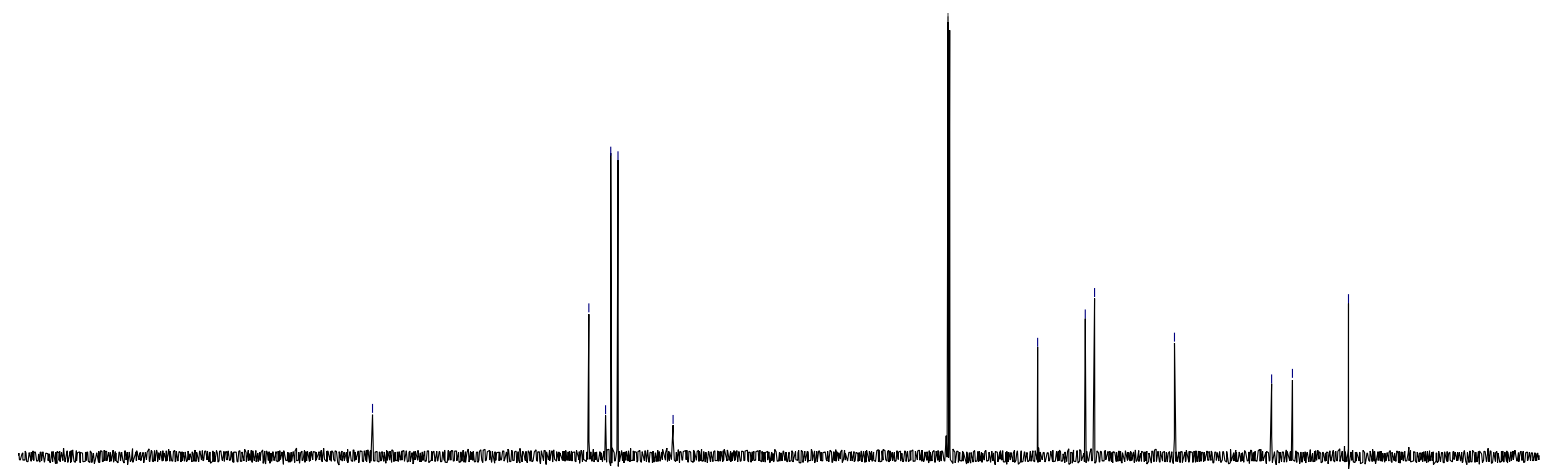

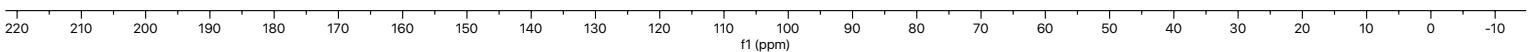




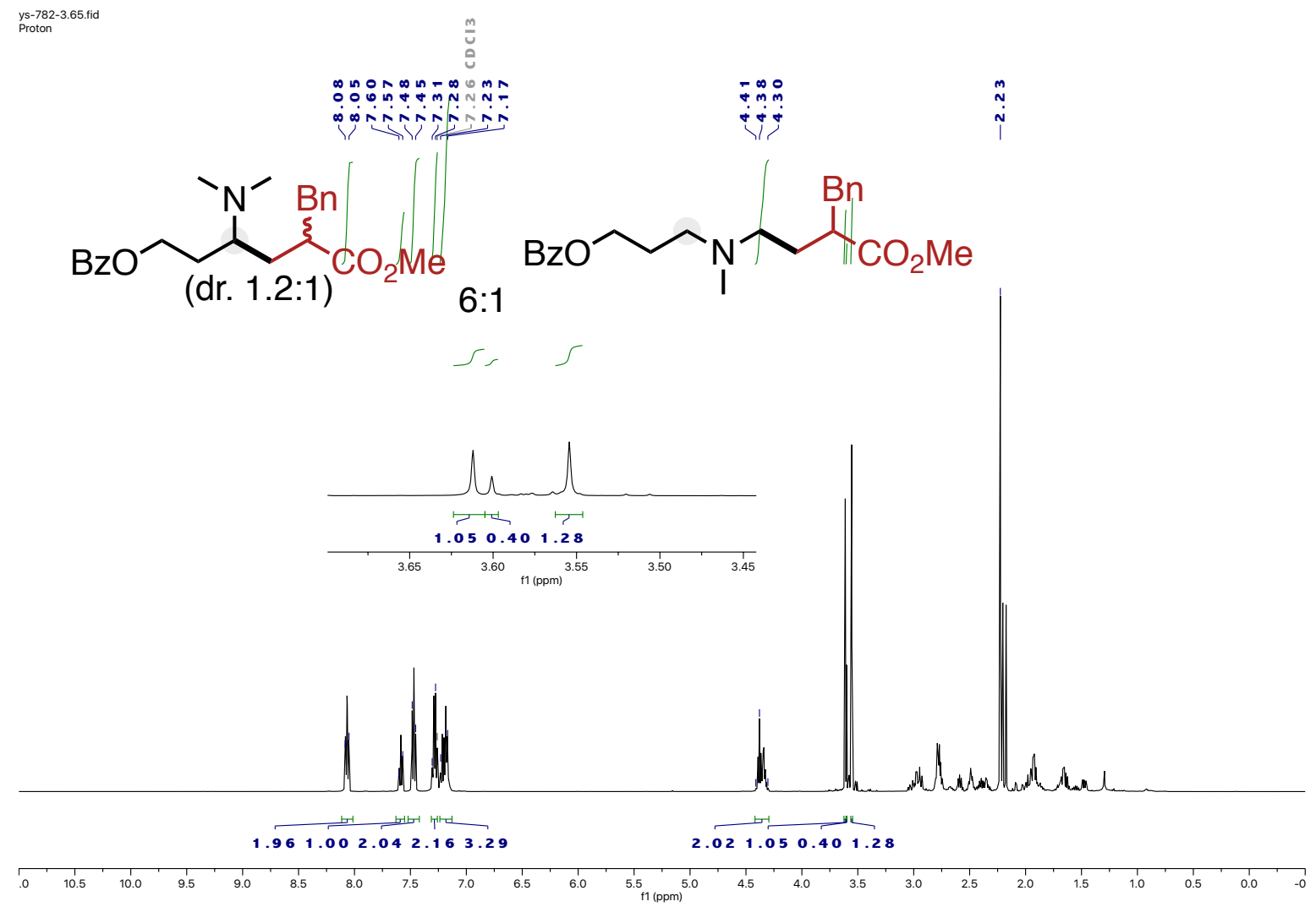

ys-782-3-500c.66.fid
Carbon 13
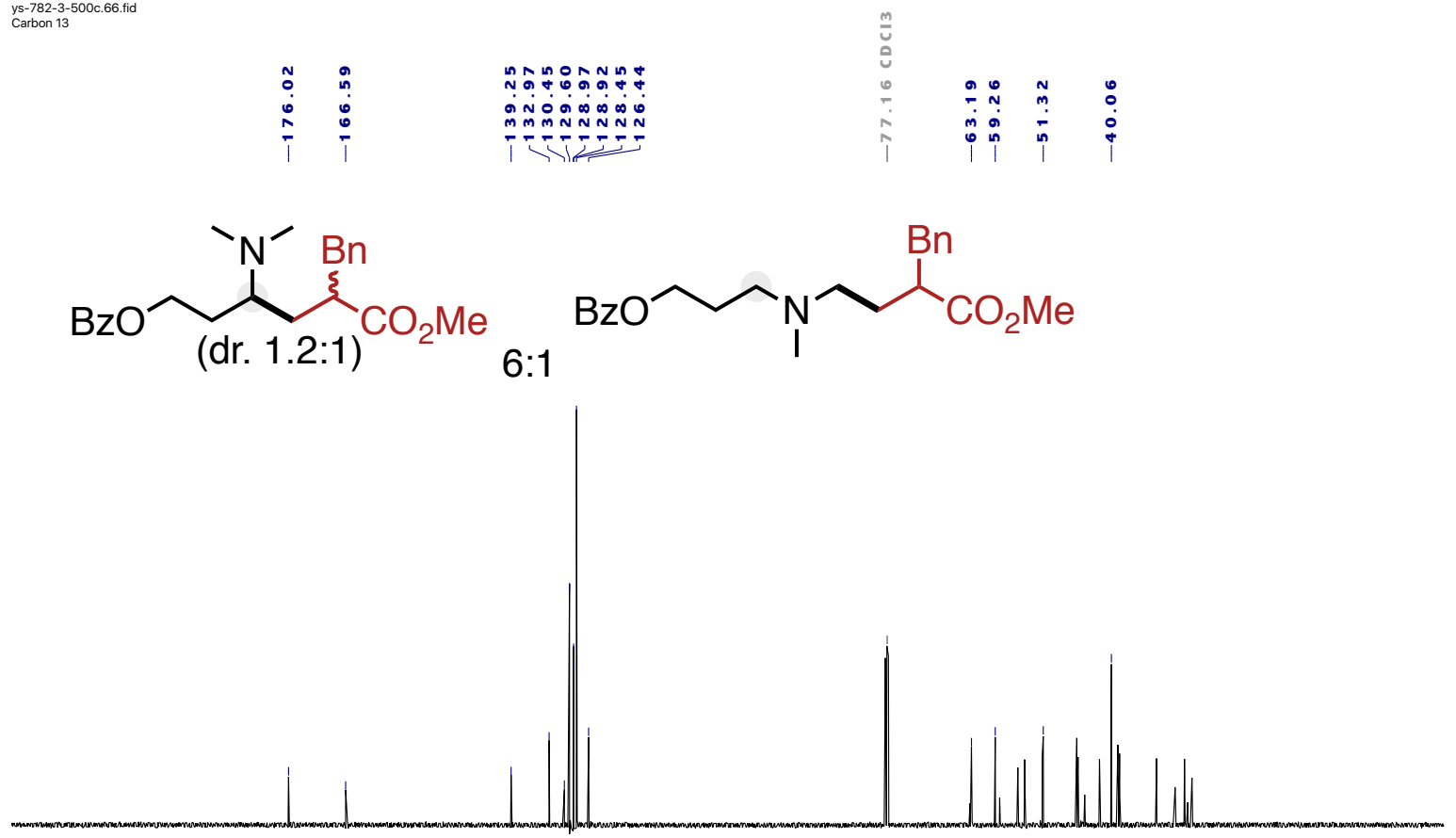

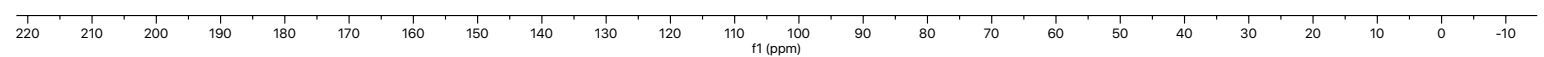



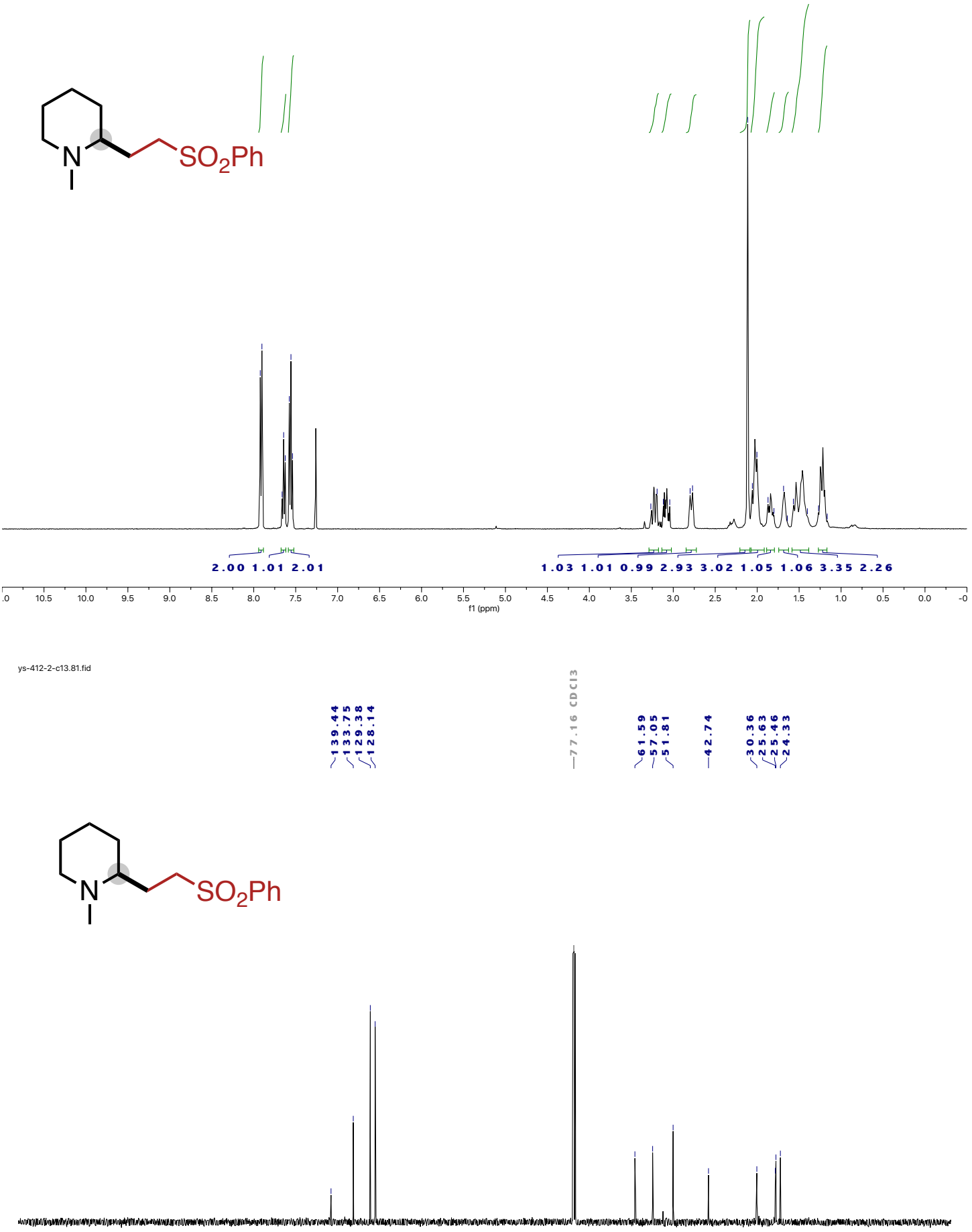
minn

$\longrightarrow \mathrm{CO}_{2} \mathrm{Ph}$
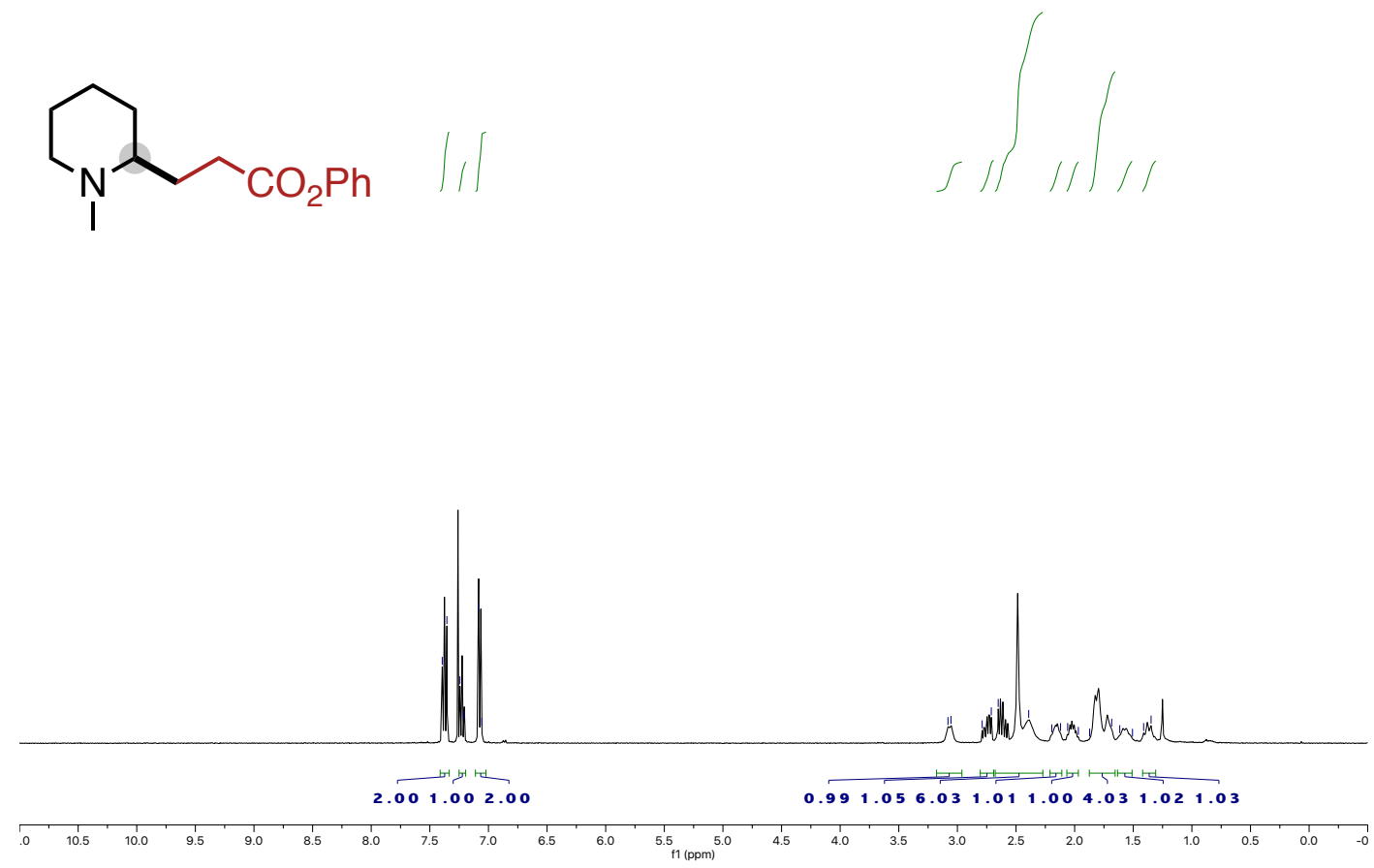

ys-503-1-c13-aaa.173.fid
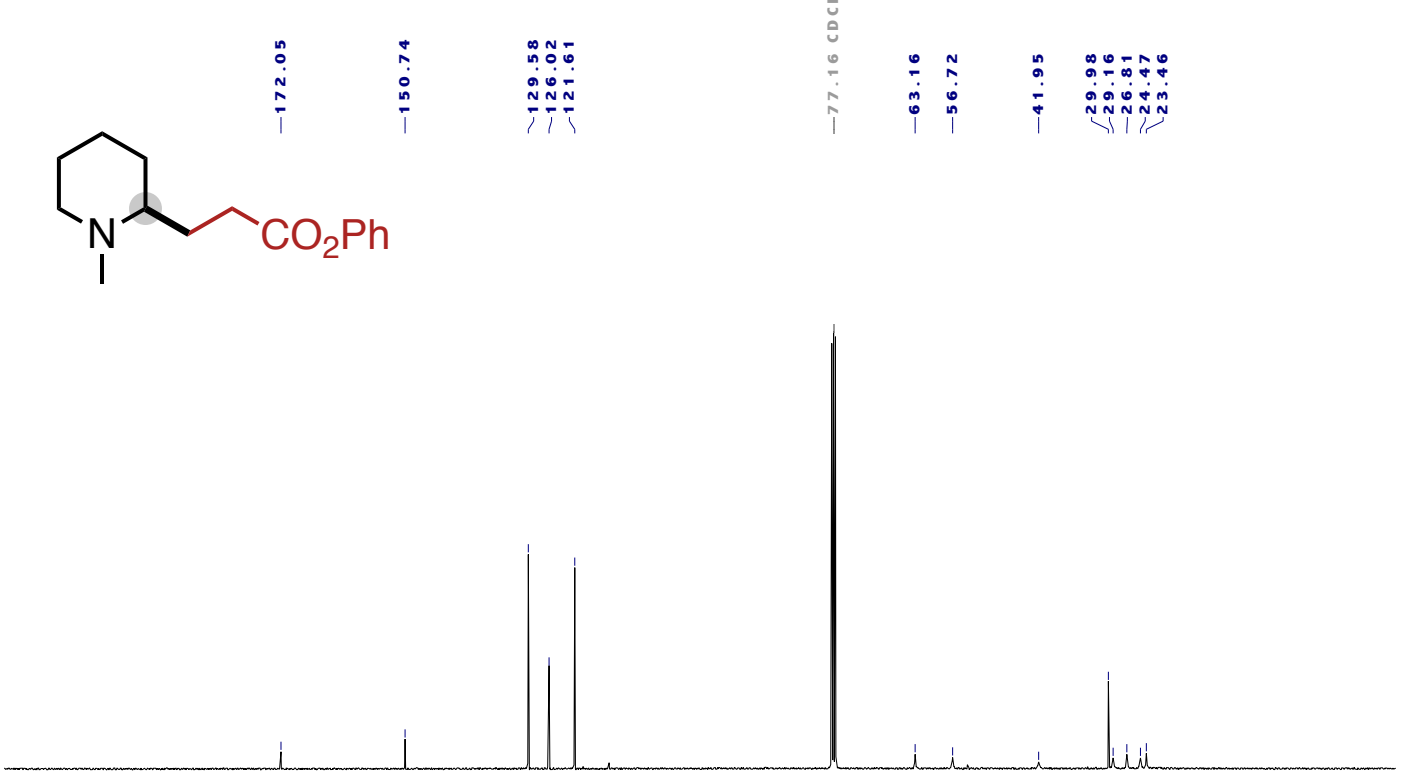

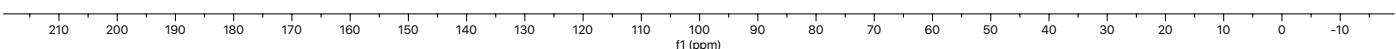


<smiles>COC(=O)C(C[C@@H]1CCCCN1C)c1ccccc1</smiles>
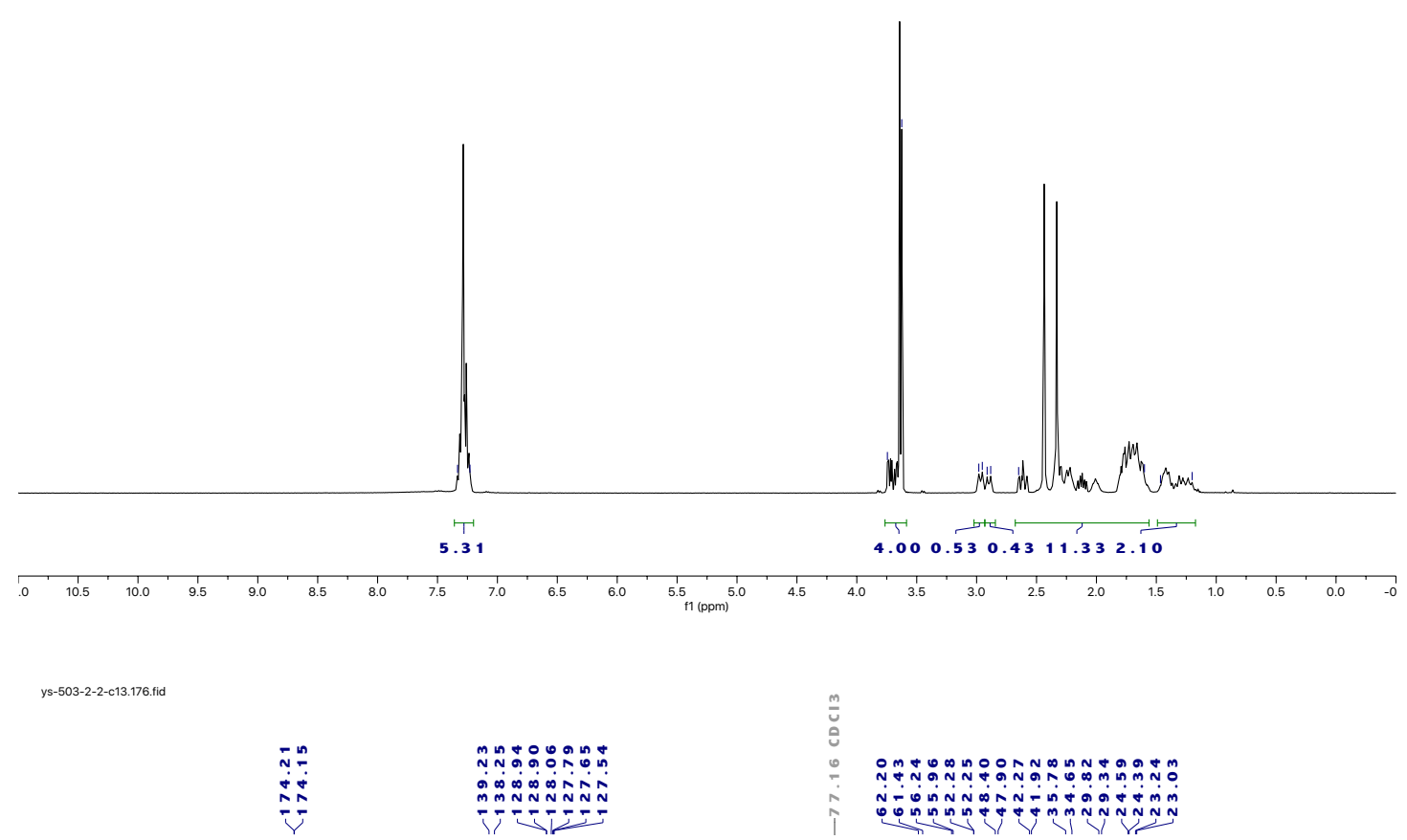<smiles>COC(=O)C(CC1CCCCN1C)c1ccccc1</smiles>
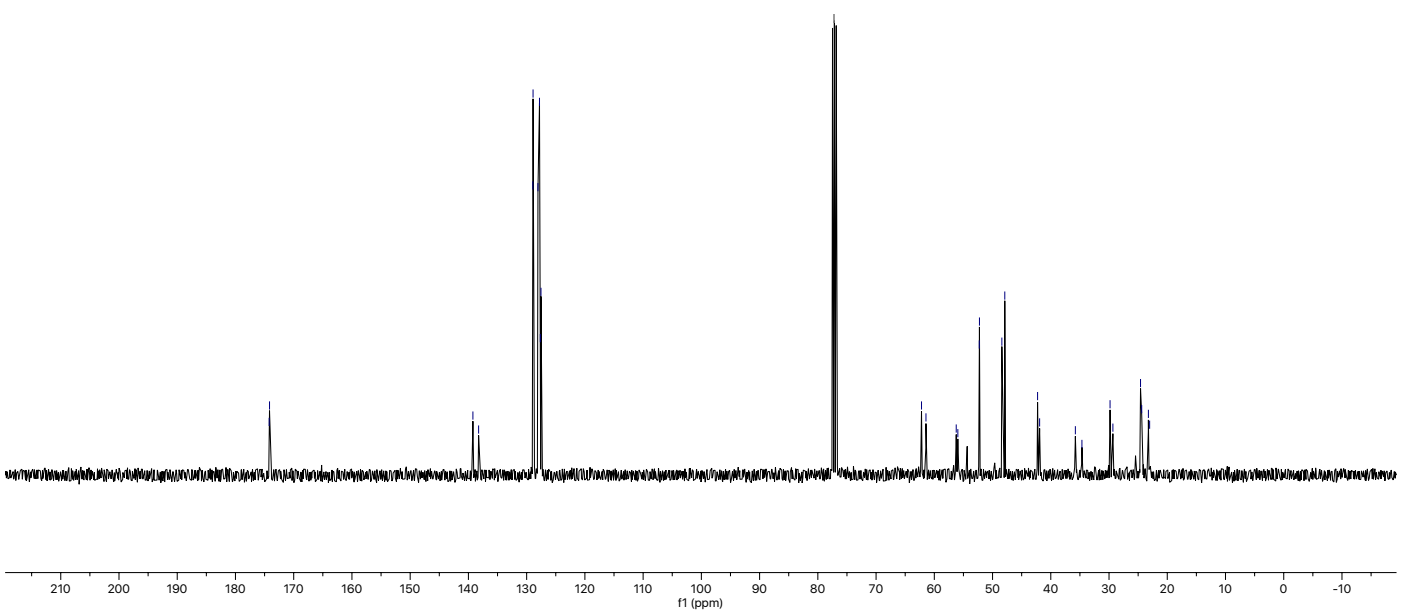


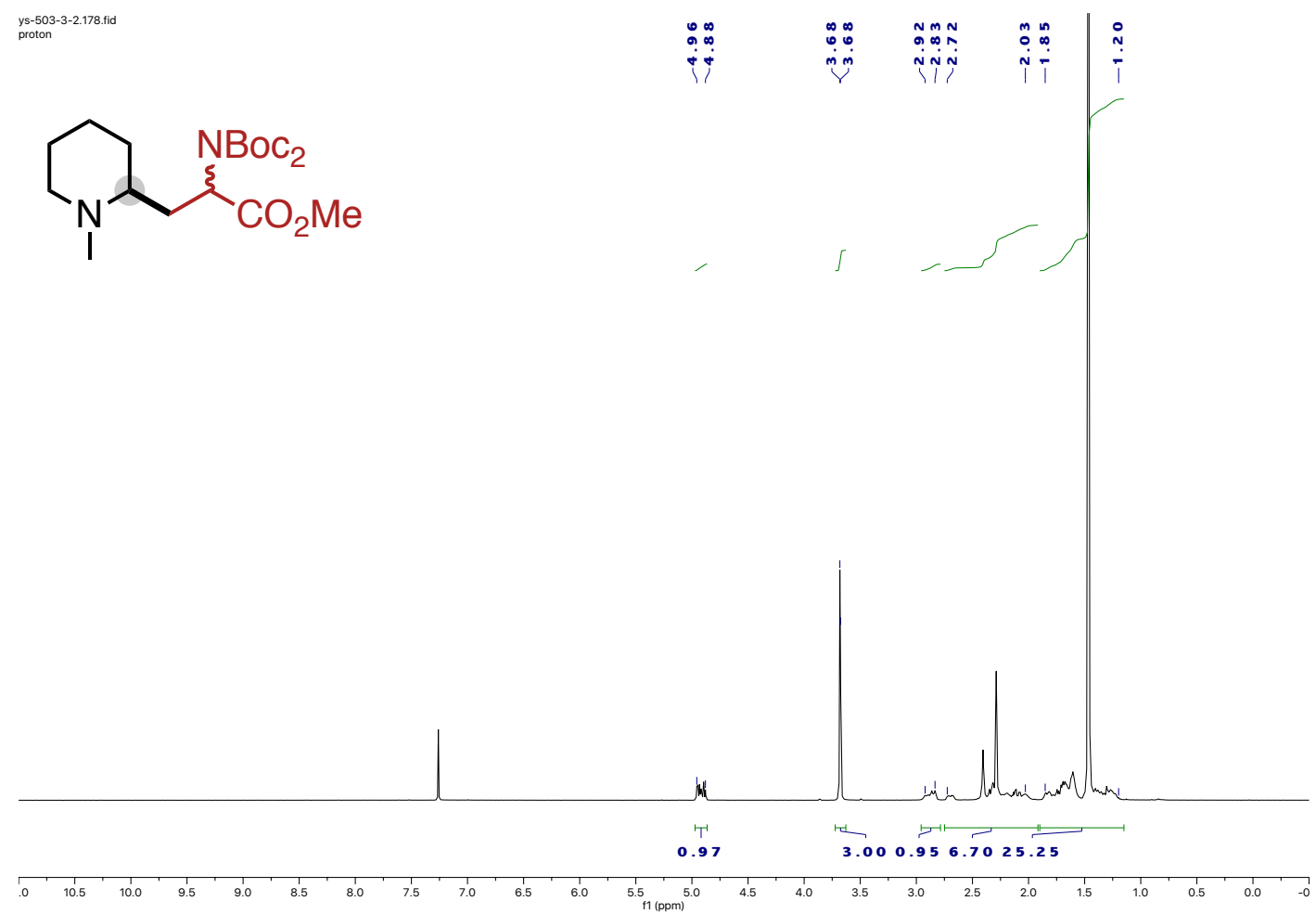

ys-503-3-2-c13.179.fid

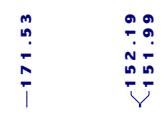

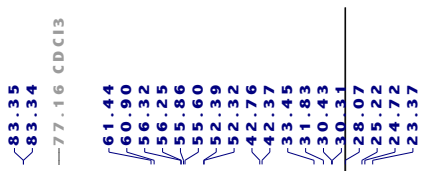

$\overbrace{\mathrm{I}}^{\mathrm{NBoc}} \mathrm{CO}_{2} \mathrm{Me}$
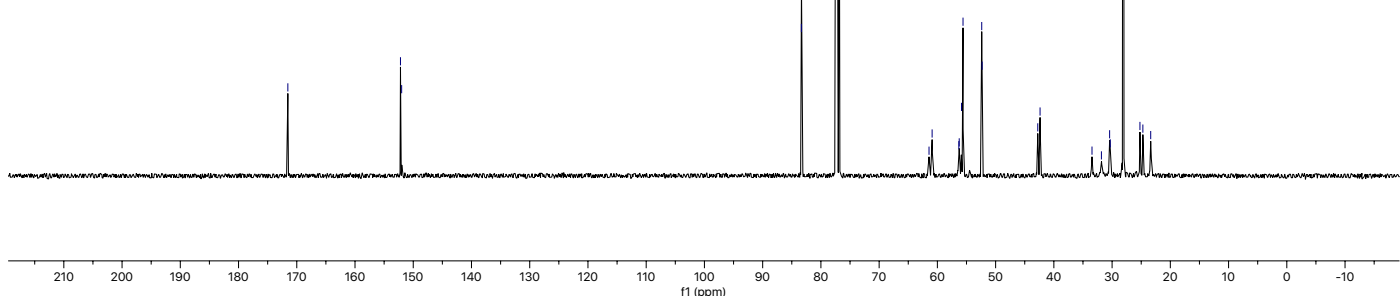

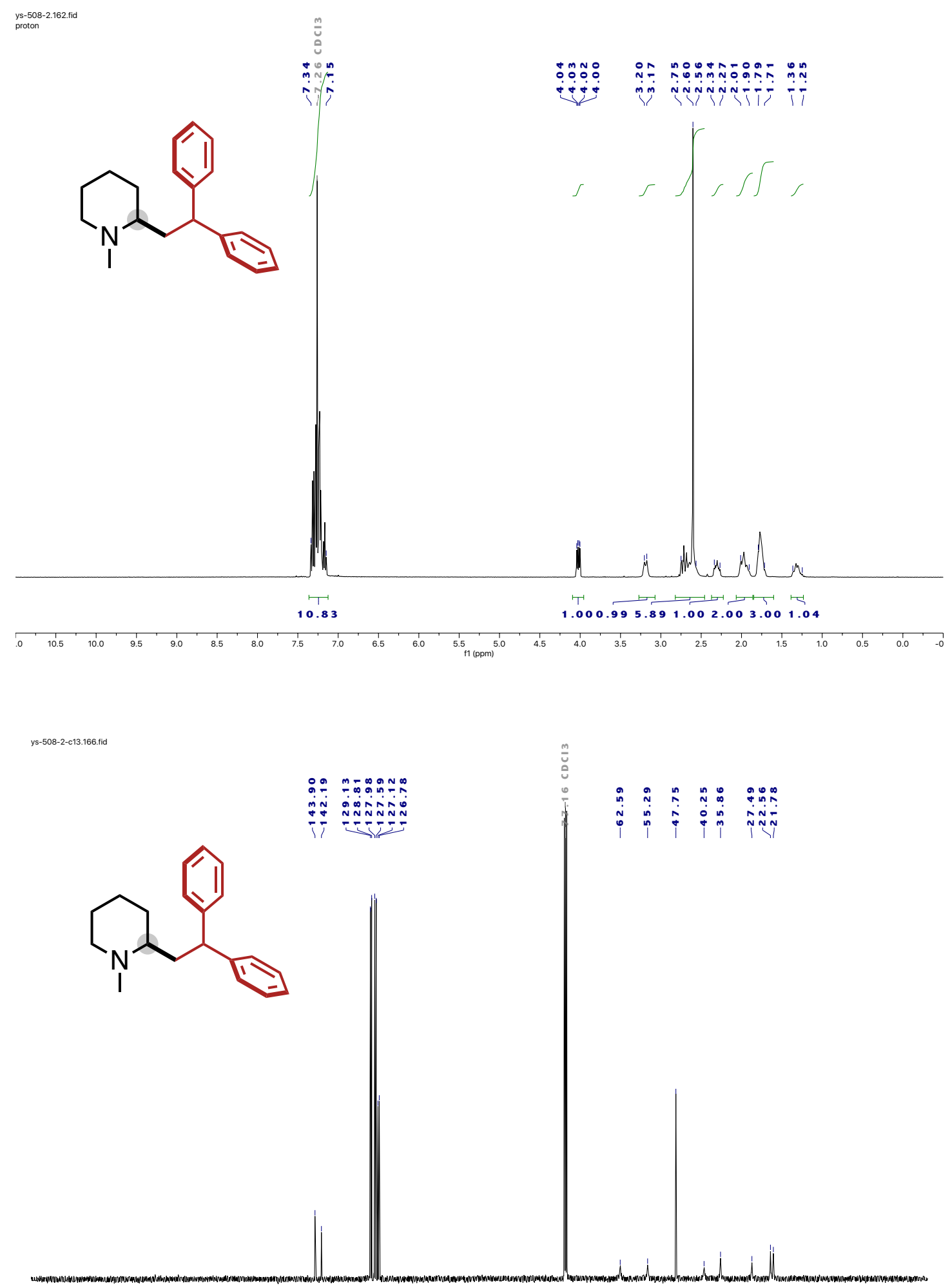
$\underbrace{}_{\mathrm{O}}$
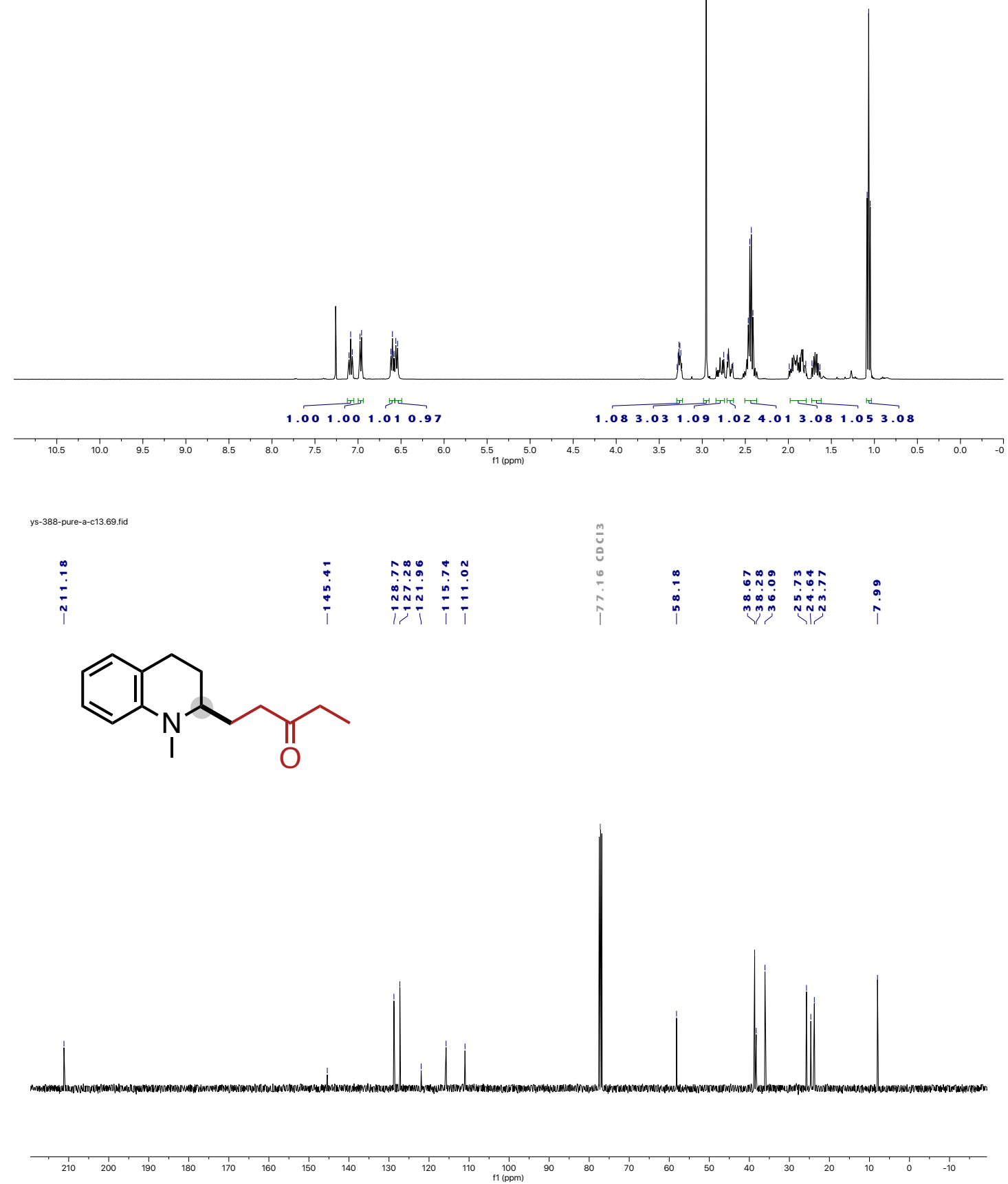


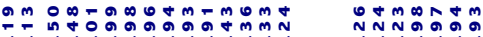

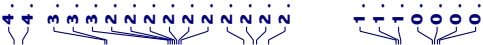

$\overbrace{\mathrm{CO}_{2} \mathrm{Me}}^{\mathrm{CO}_{2} \mathrm{Me}}$

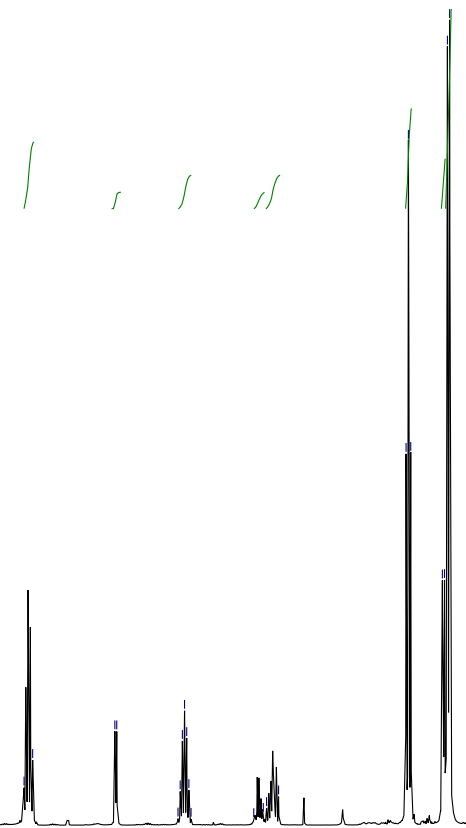

4.031 .002 .021002026 .043 .021202

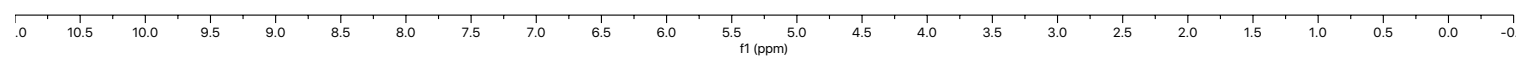

ys-665-1-c13.336.fid
carbon 13

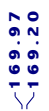

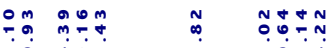

in<smiles>COC(=O)C(C)C(C)CN(C(C)C)C(C)C</smiles>

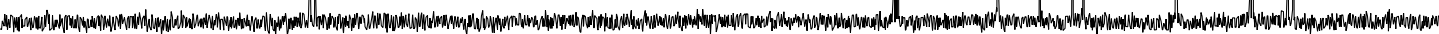

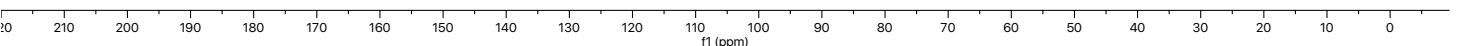




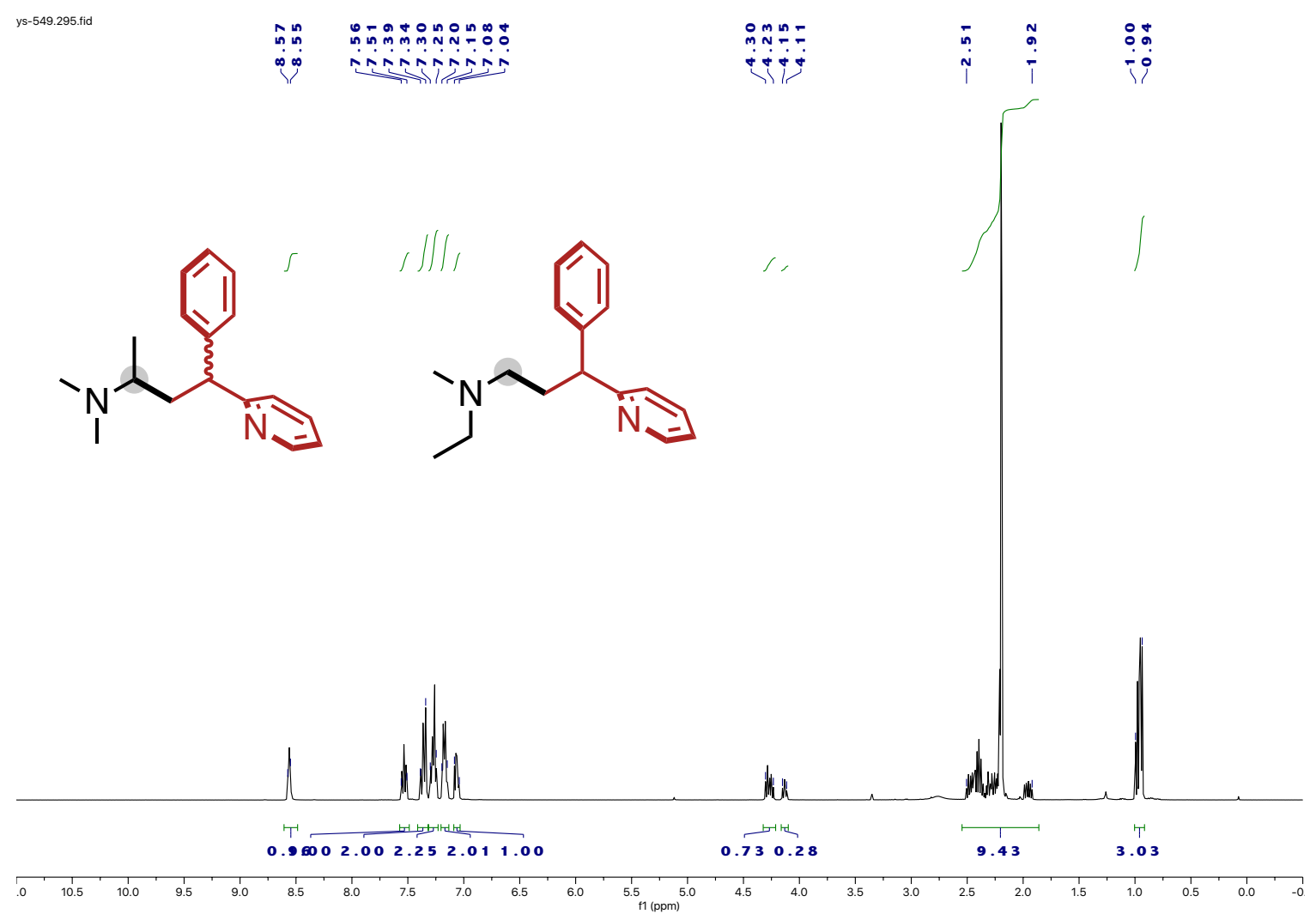

ys-551-3--13.303. fid
carbon 13

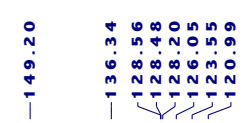

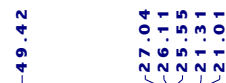<smiles>CC(C[C@@H](c1ccccc1)c1ccccn1)N(C)C</smiles><smiles>CCN(C)CCC(c1ccccc1)c1ccccn1</smiles>

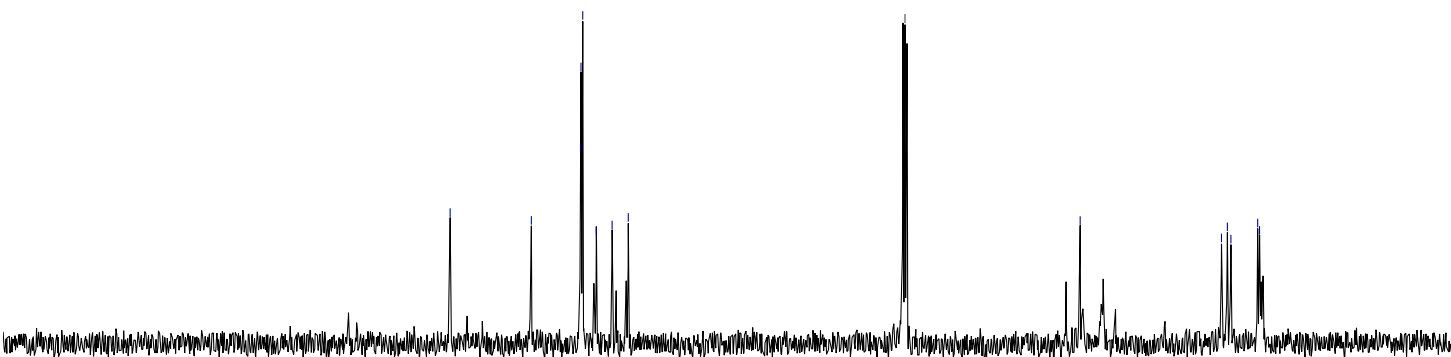

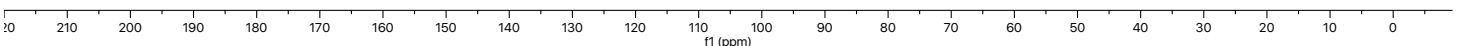




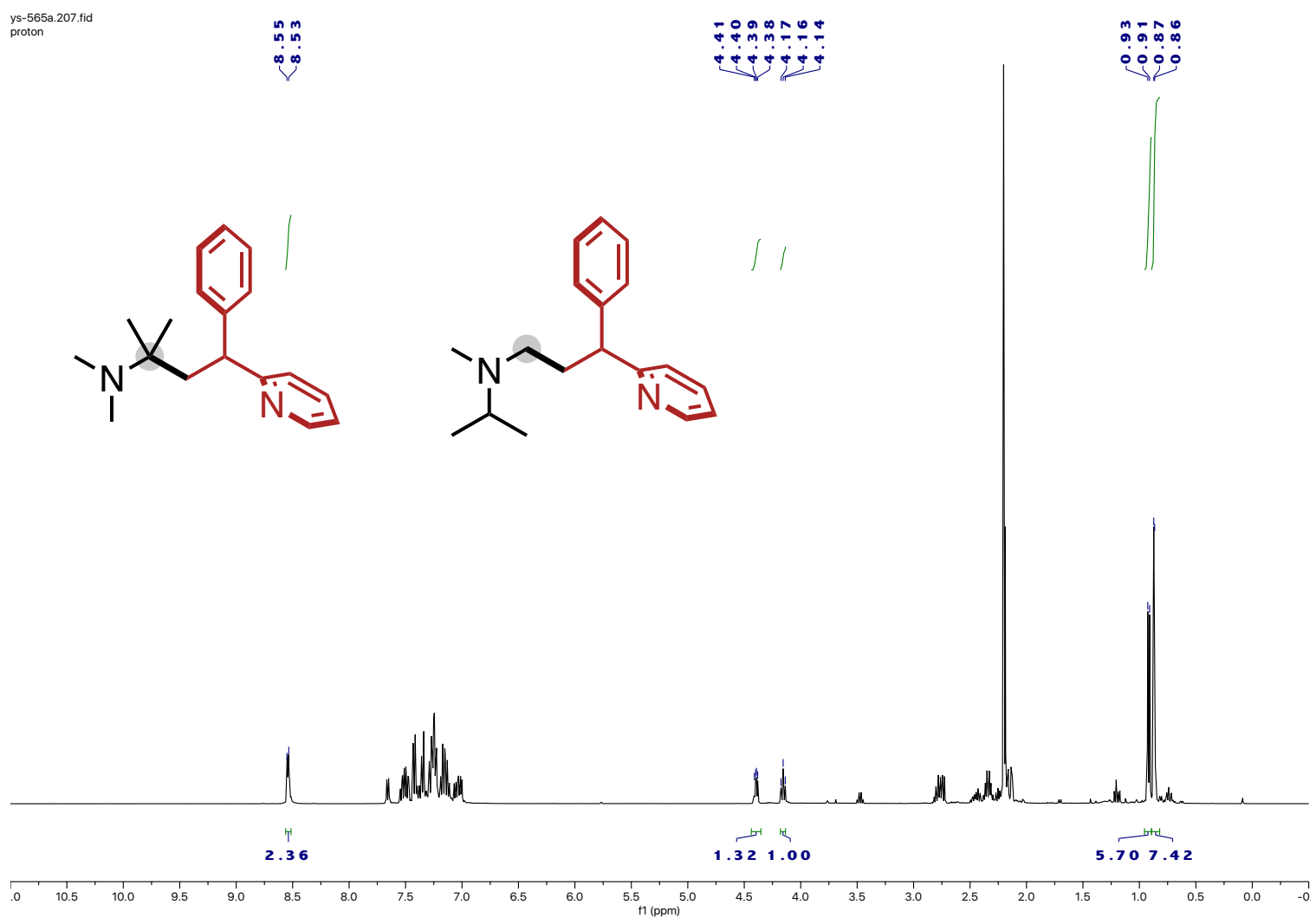

ys-565a-c13.210.fid
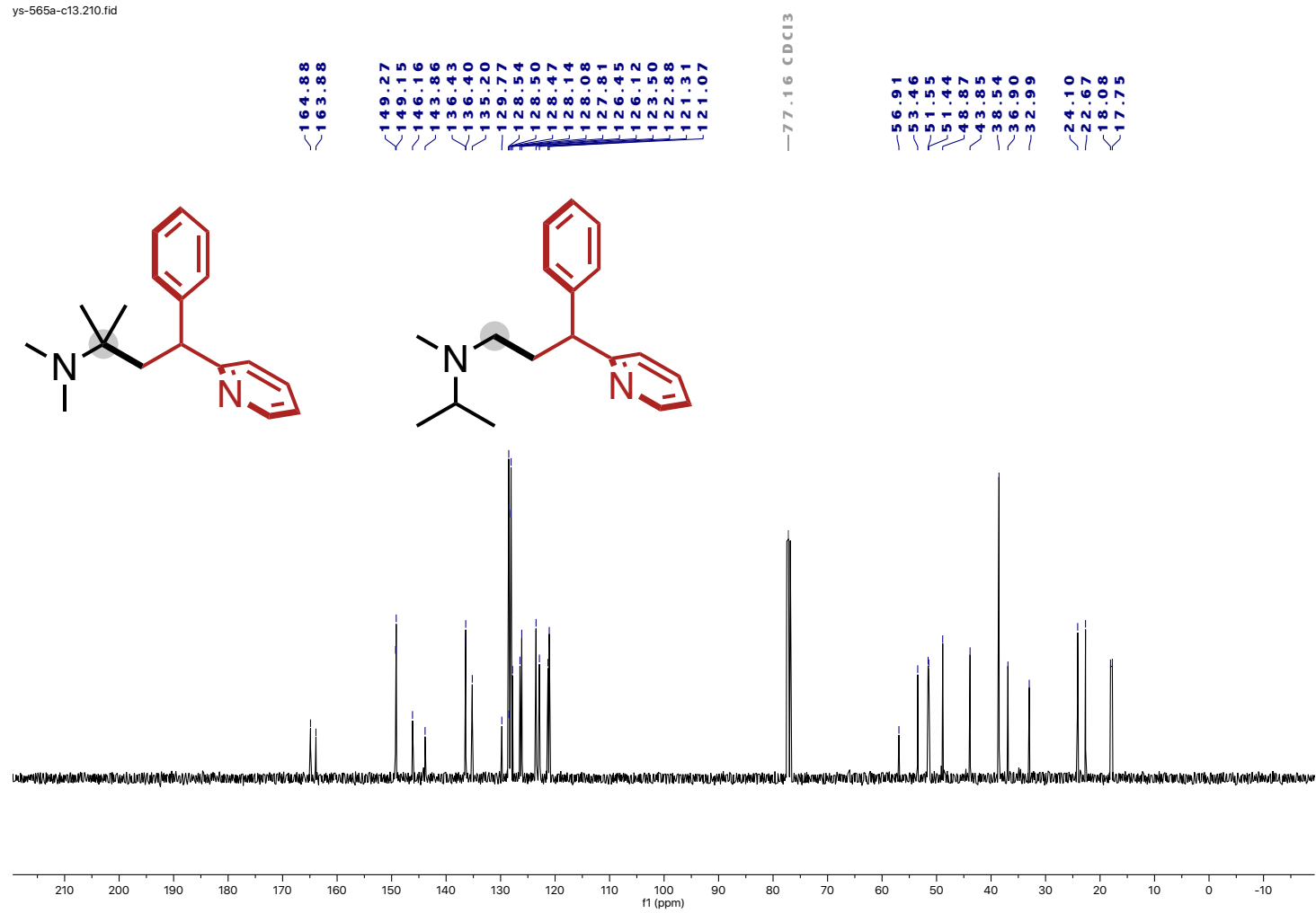

108 


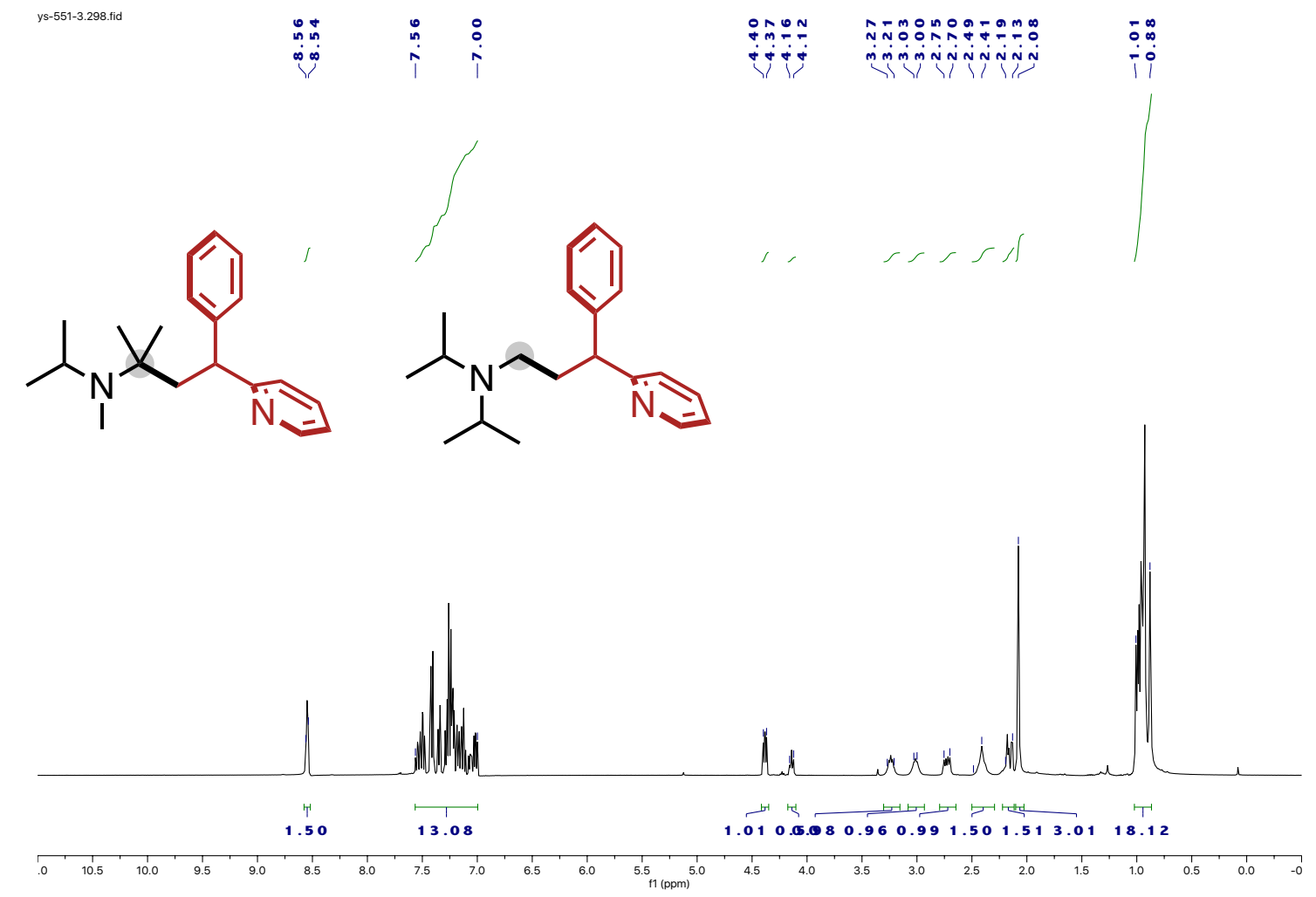

ys-531-3-c13.303.fid
carbon 13
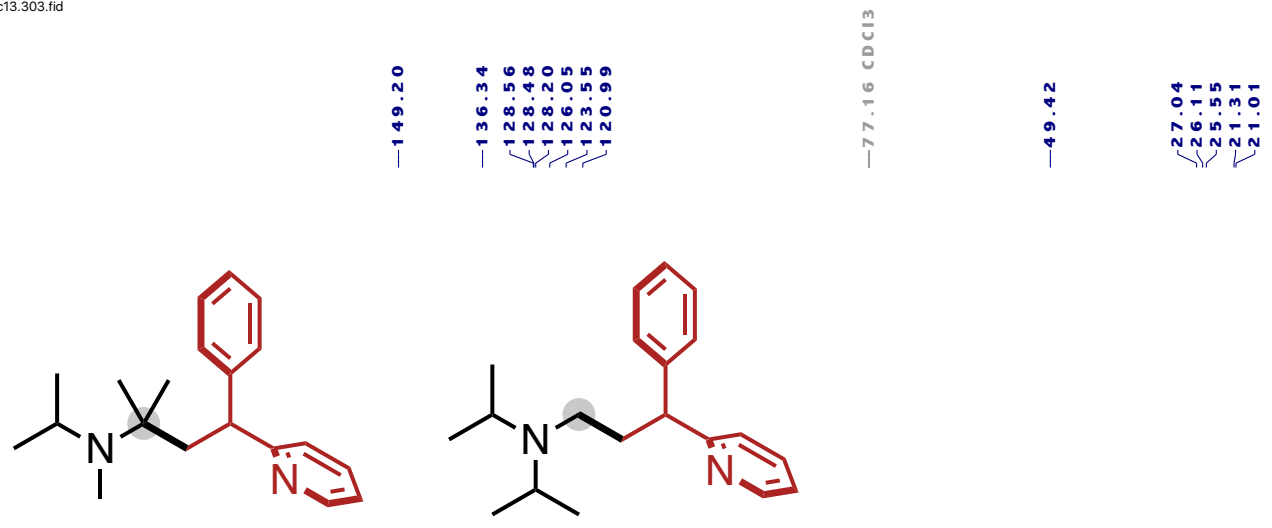

.

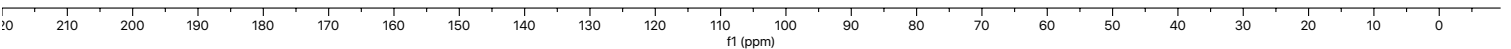




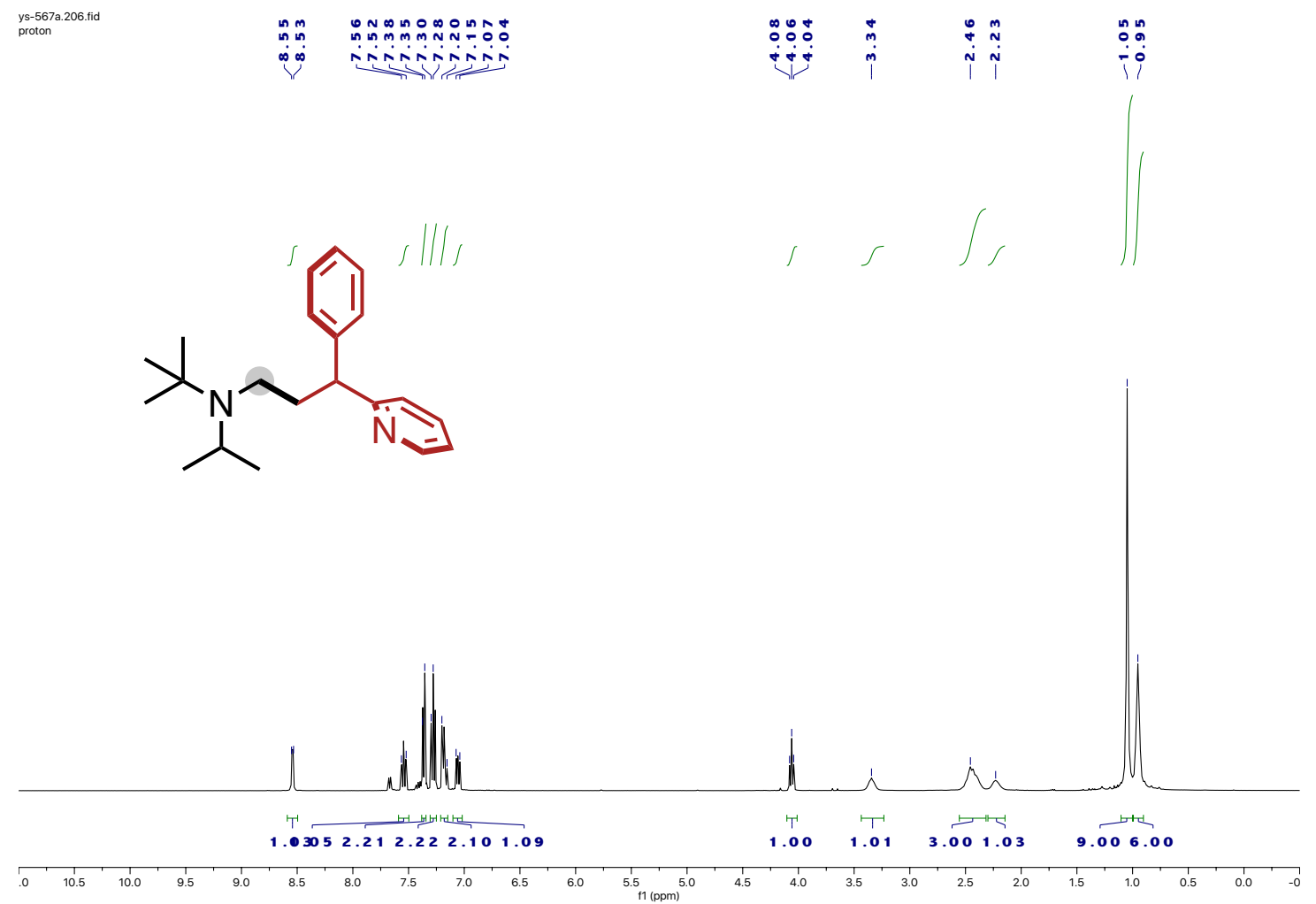

ys-567a-c13.209.fid
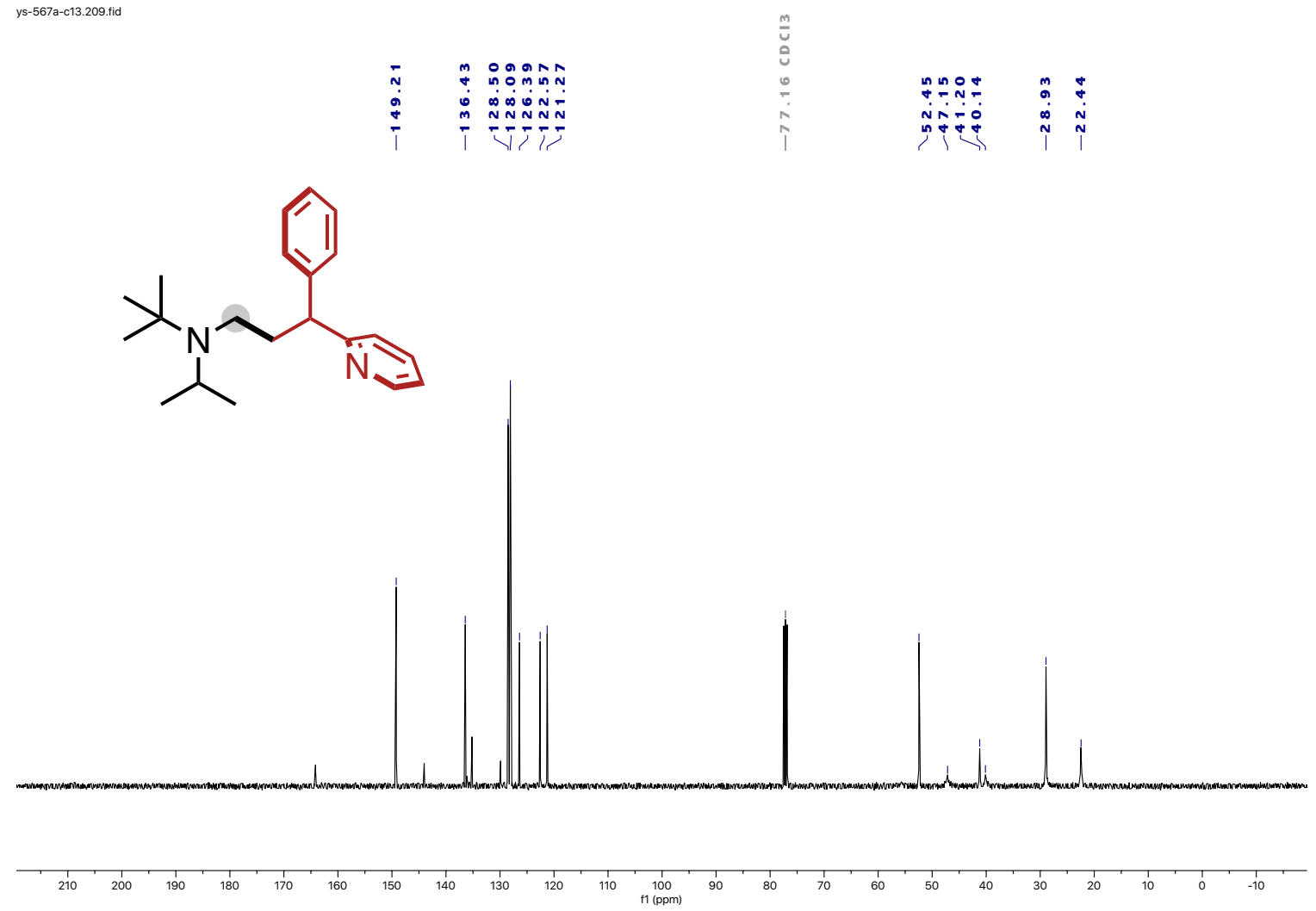


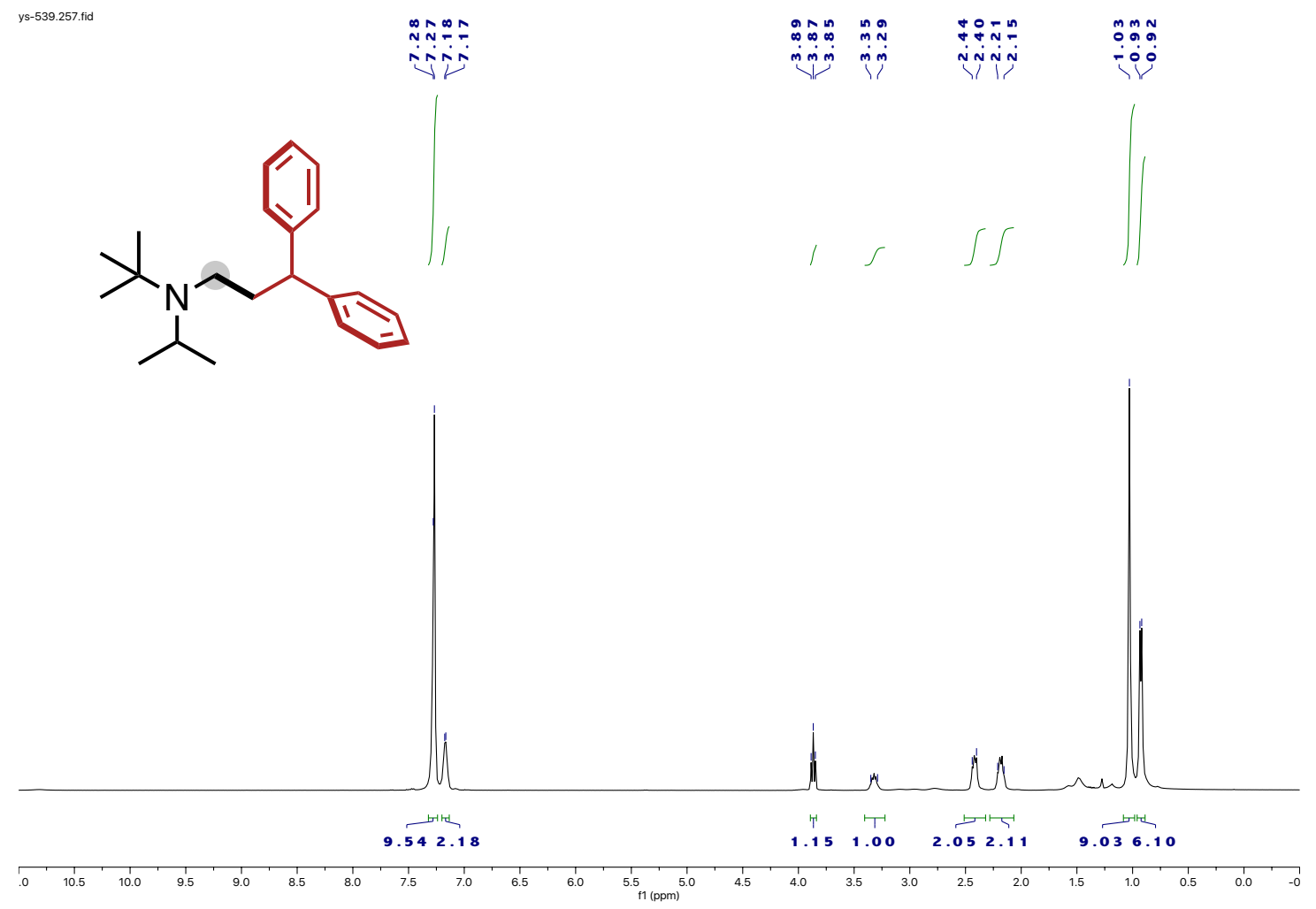

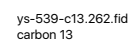
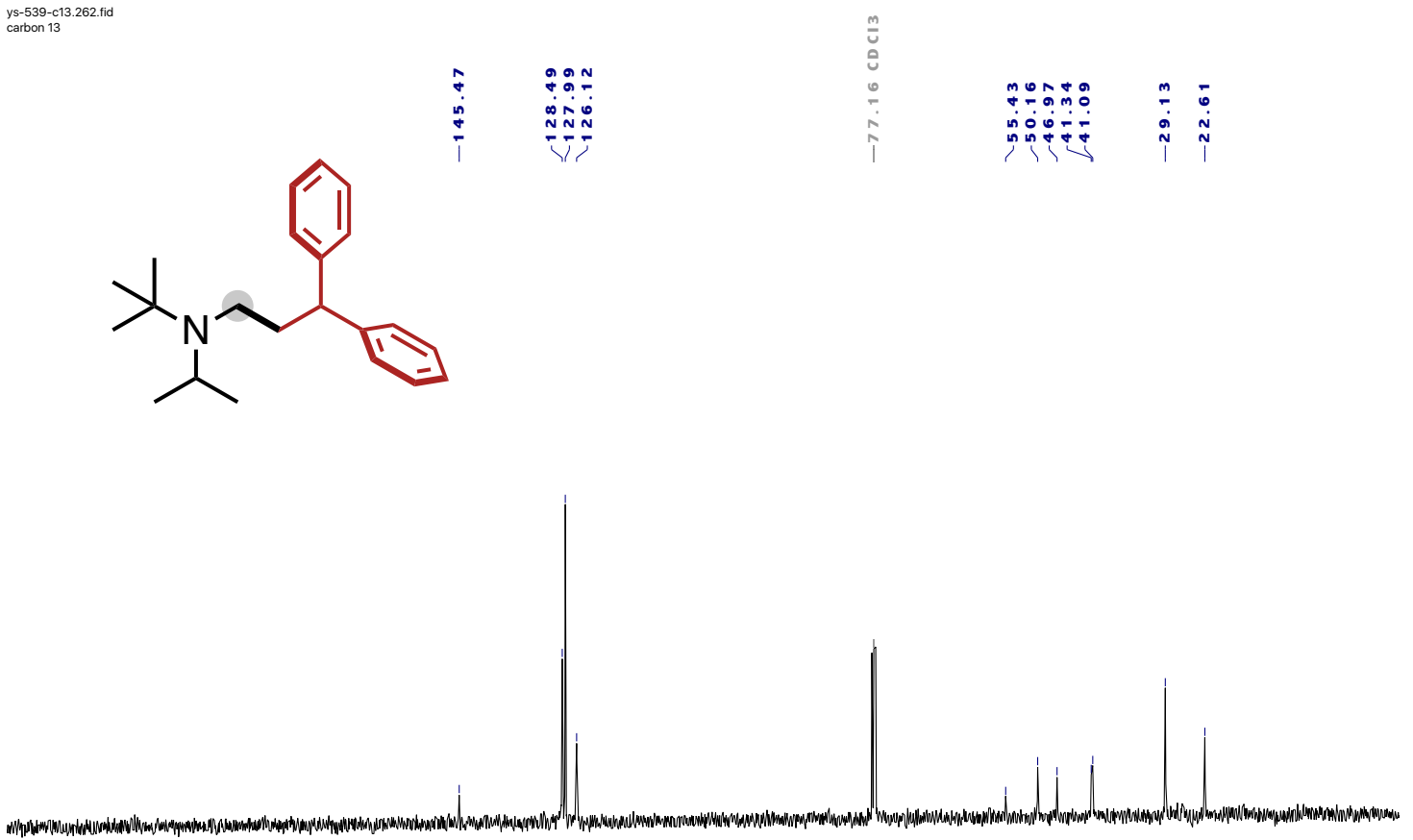

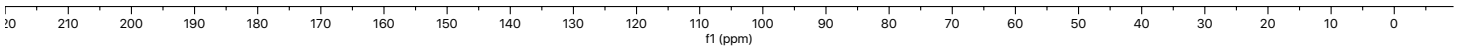




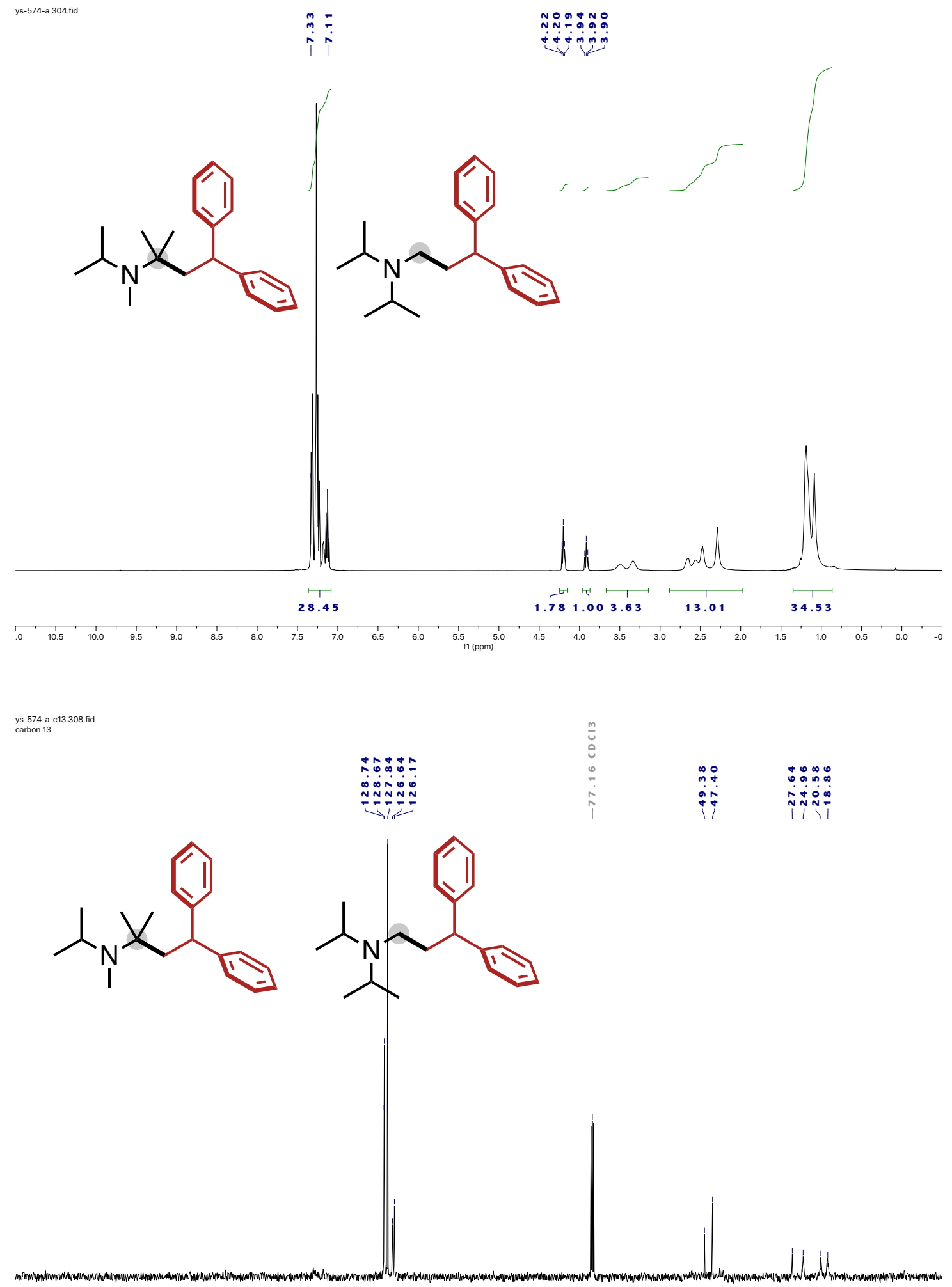

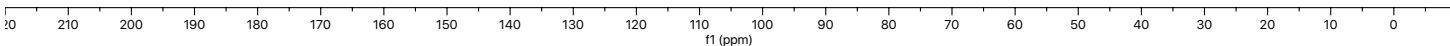




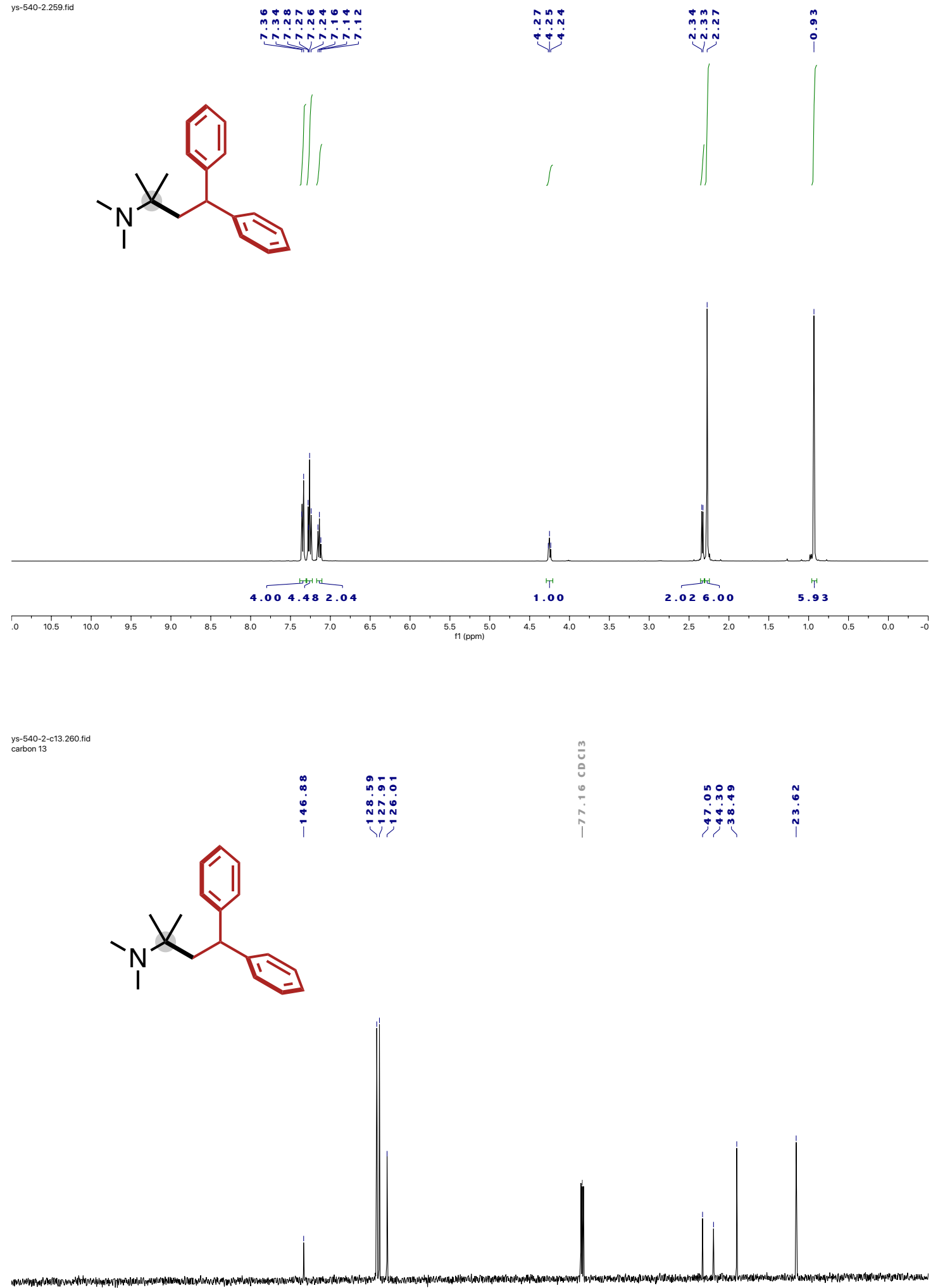

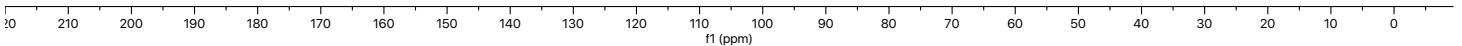




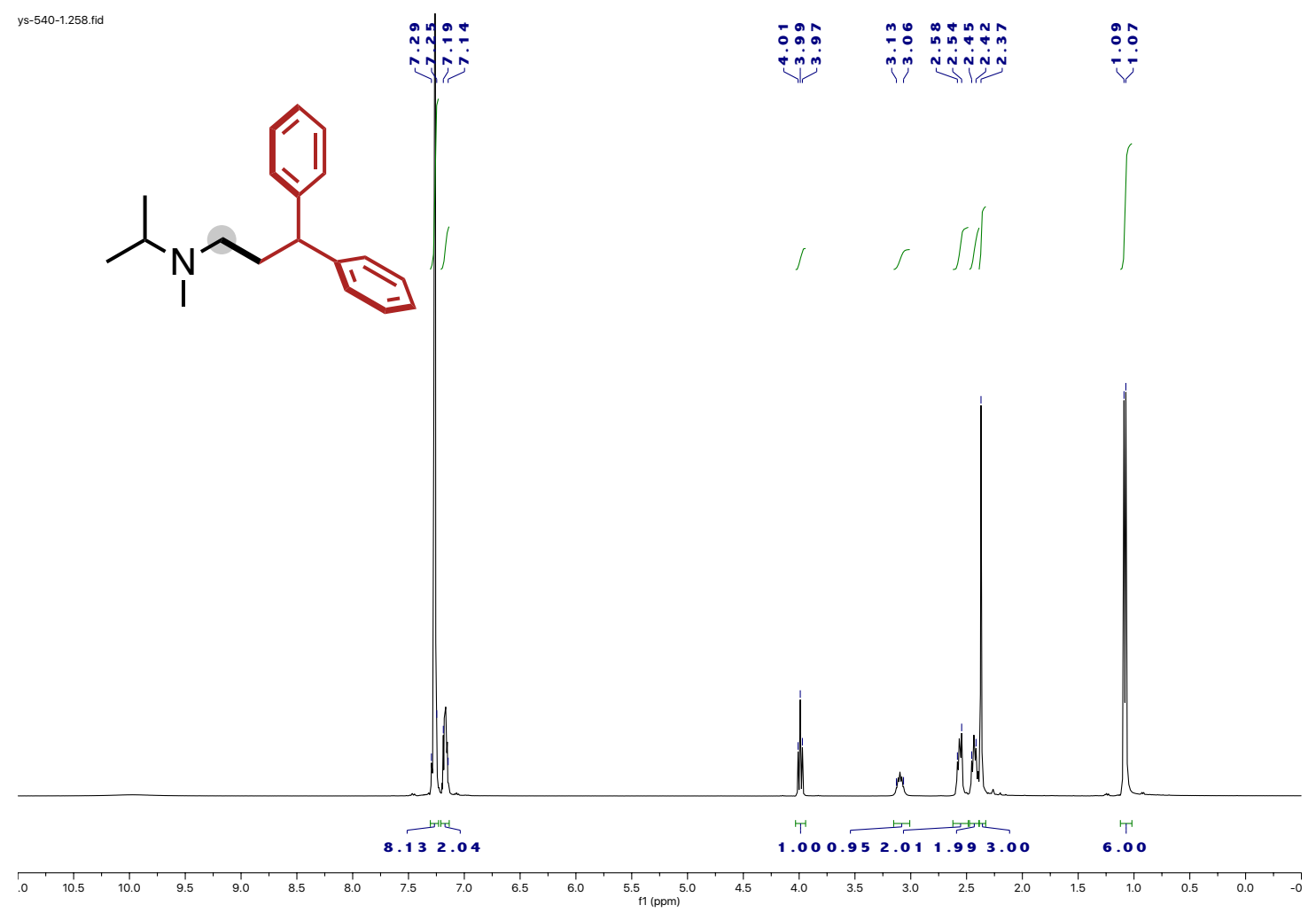

ys-540-1-c13.261.fid
carbon 13
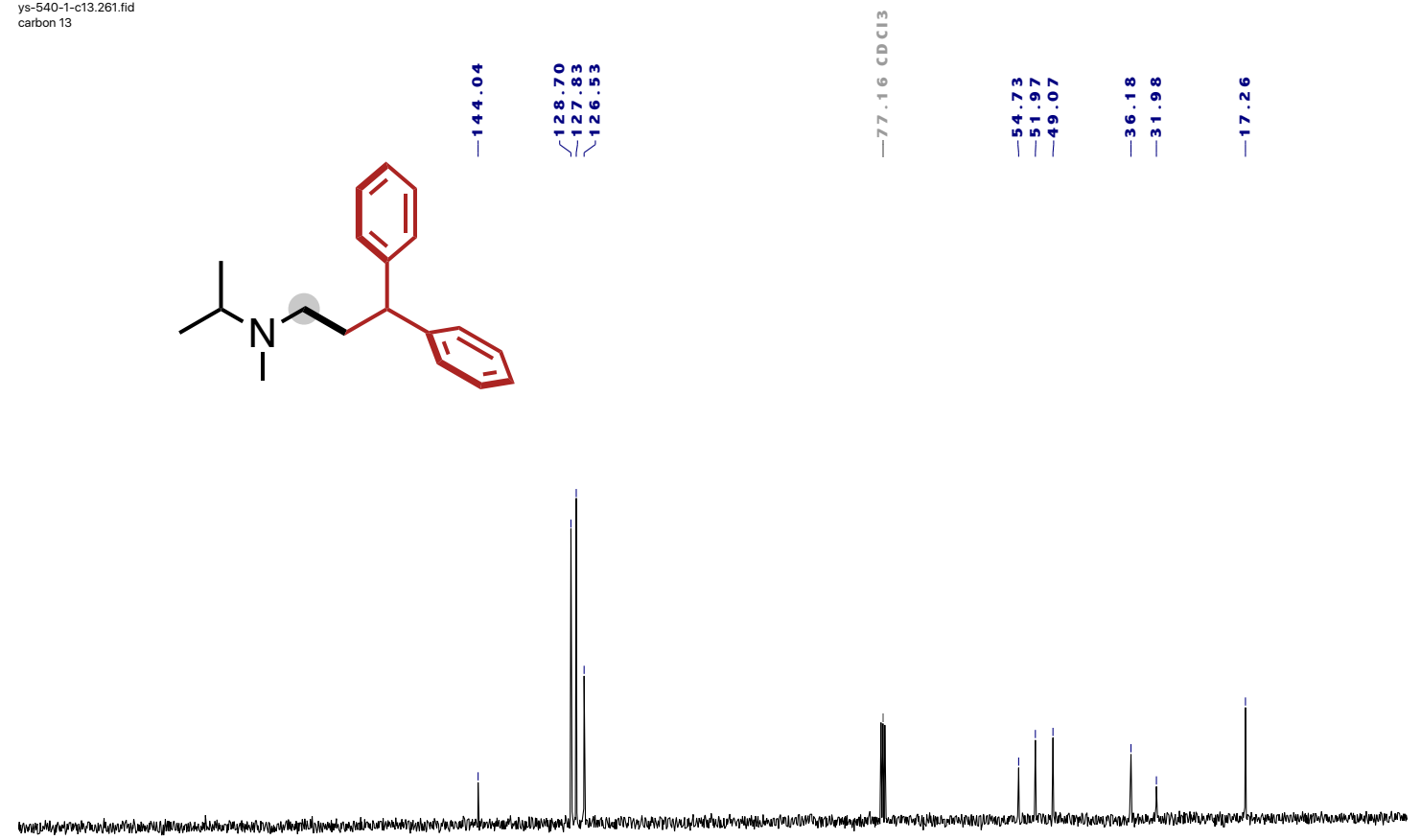

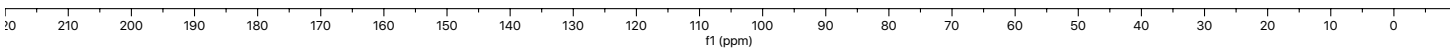


$\mathrm{C}_{\mathrm{O}}^{\mathrm{N}} \mathrm{SO}_{\mathrm{SO}_{2} \mathrm{Ph}}^{\mathrm{N}}$

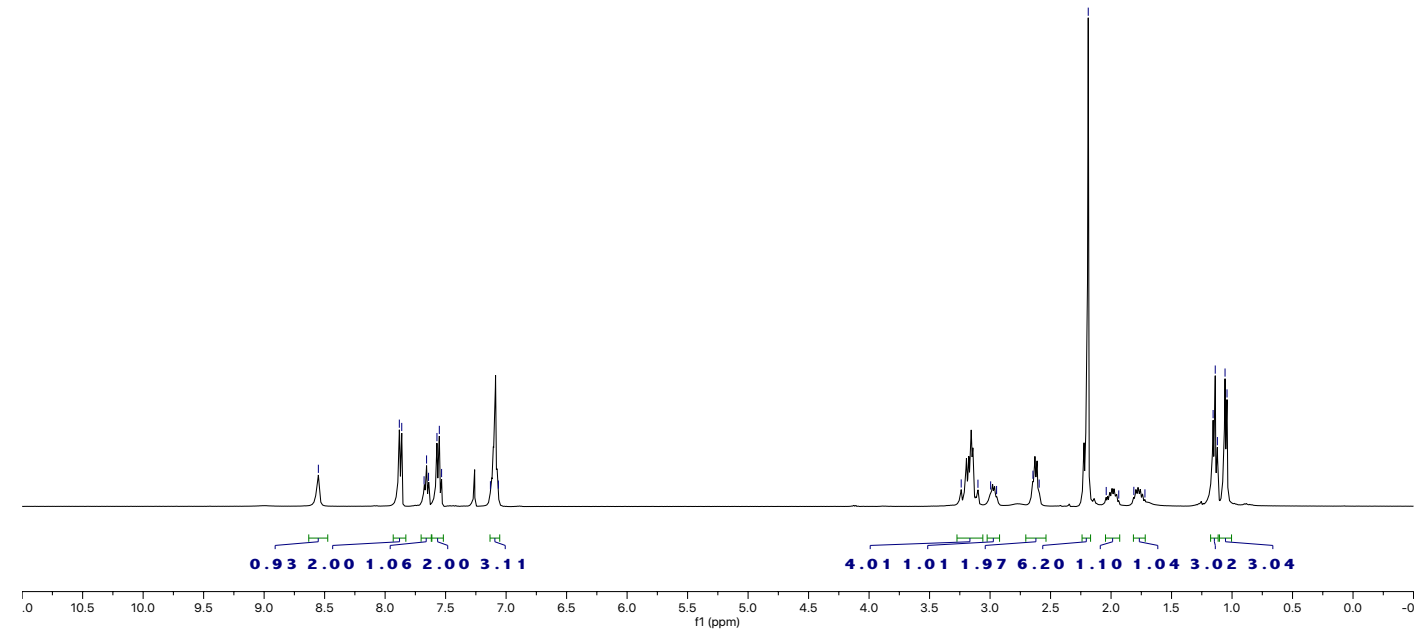

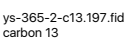

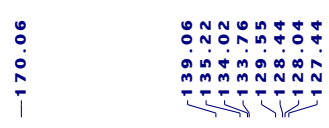

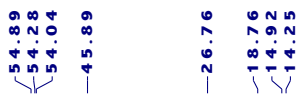<smiles>CCN(CC(=O)Nc1c(C)cccc1C)C(C)CCS(=O)(=O)c1ccccc1</smiles>
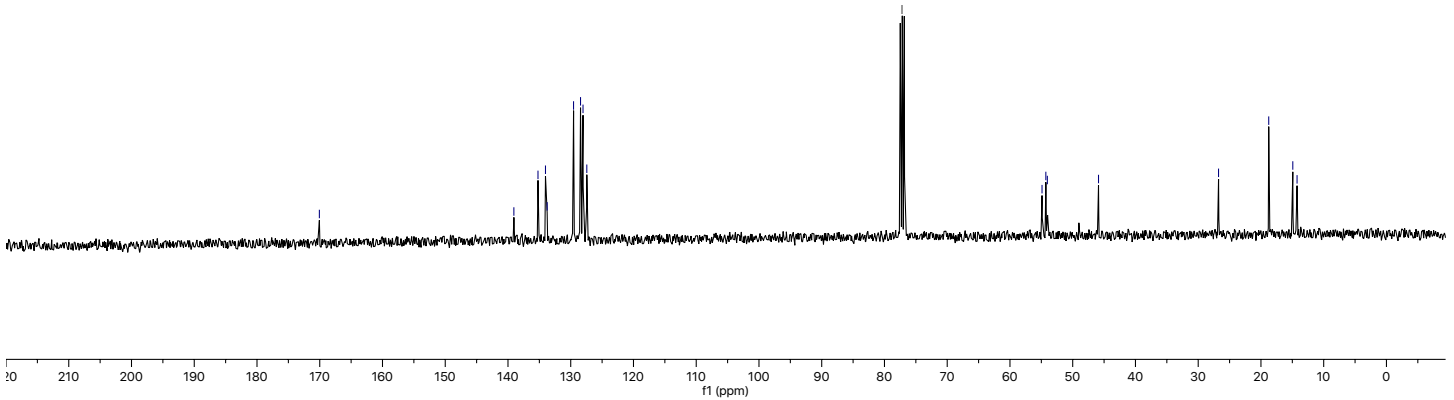
<smiles>CC(CSOc1ccccc1)[C@@H](CCC1(c2ccc(F)cc2)OCc2cc(C#N)ccc21)N(C)C</smiles>

$\mathrm{NC}$
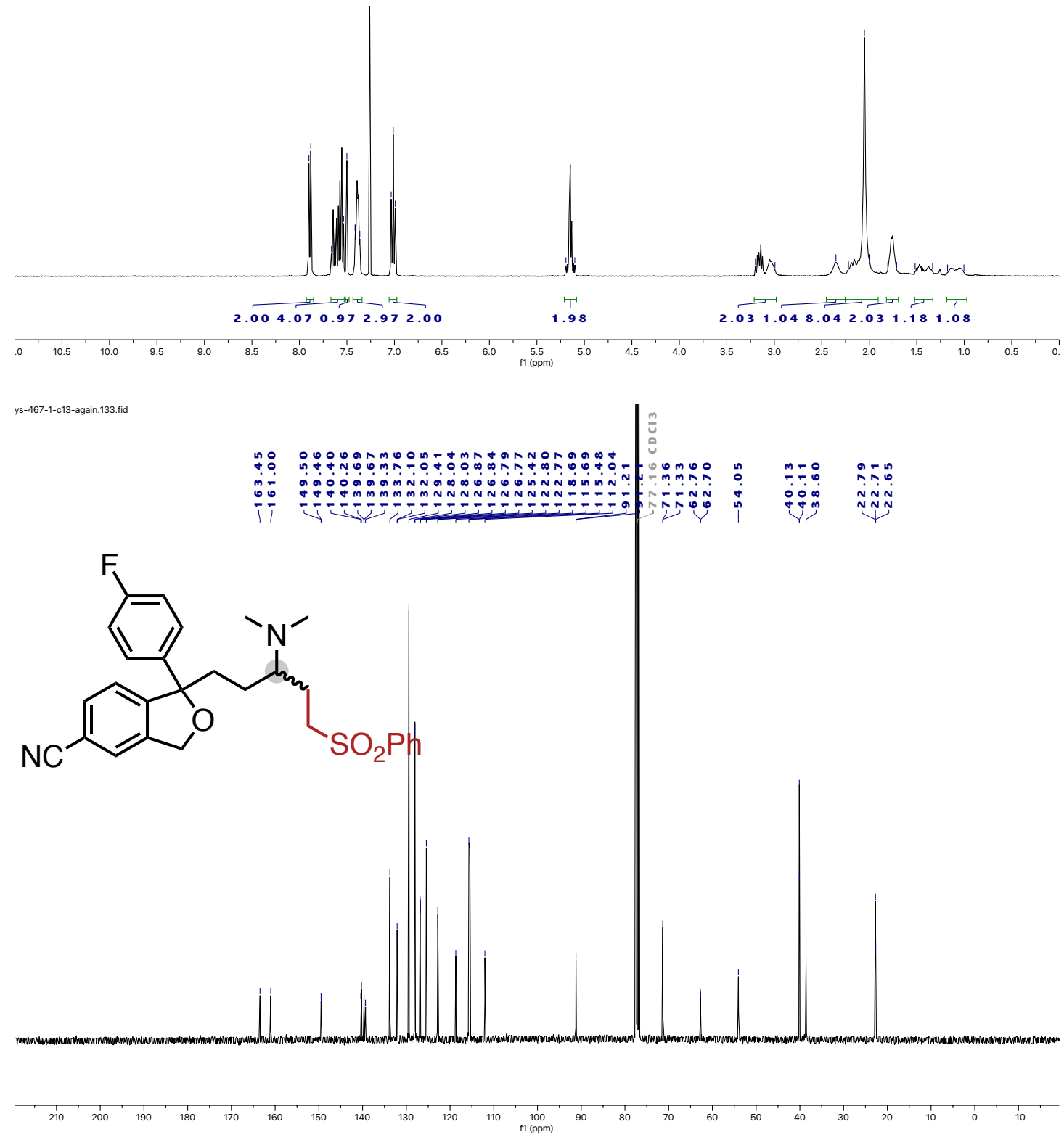
<smiles>CN(C)[C@@H](CCC1(c2ccc(F)cc2)OCc2cc(C#N)ccc21)CC[SH](=O)(O)Oc1ccccc1</smiles>
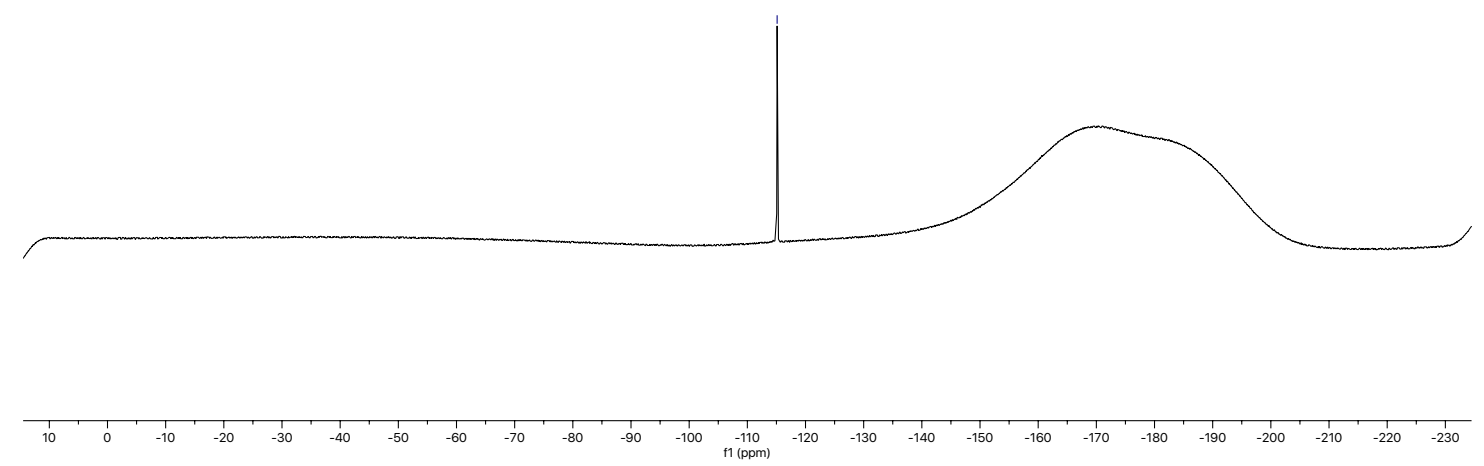<smiles>C#CCC[C@H](CCOc1ccccc1)N(C)C</smiles>
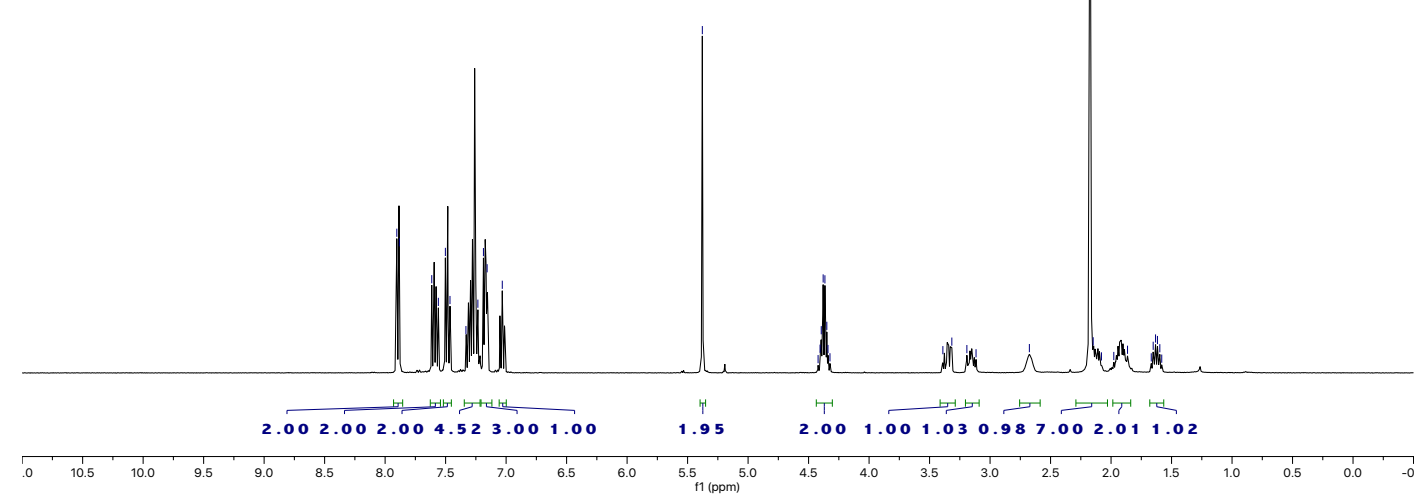

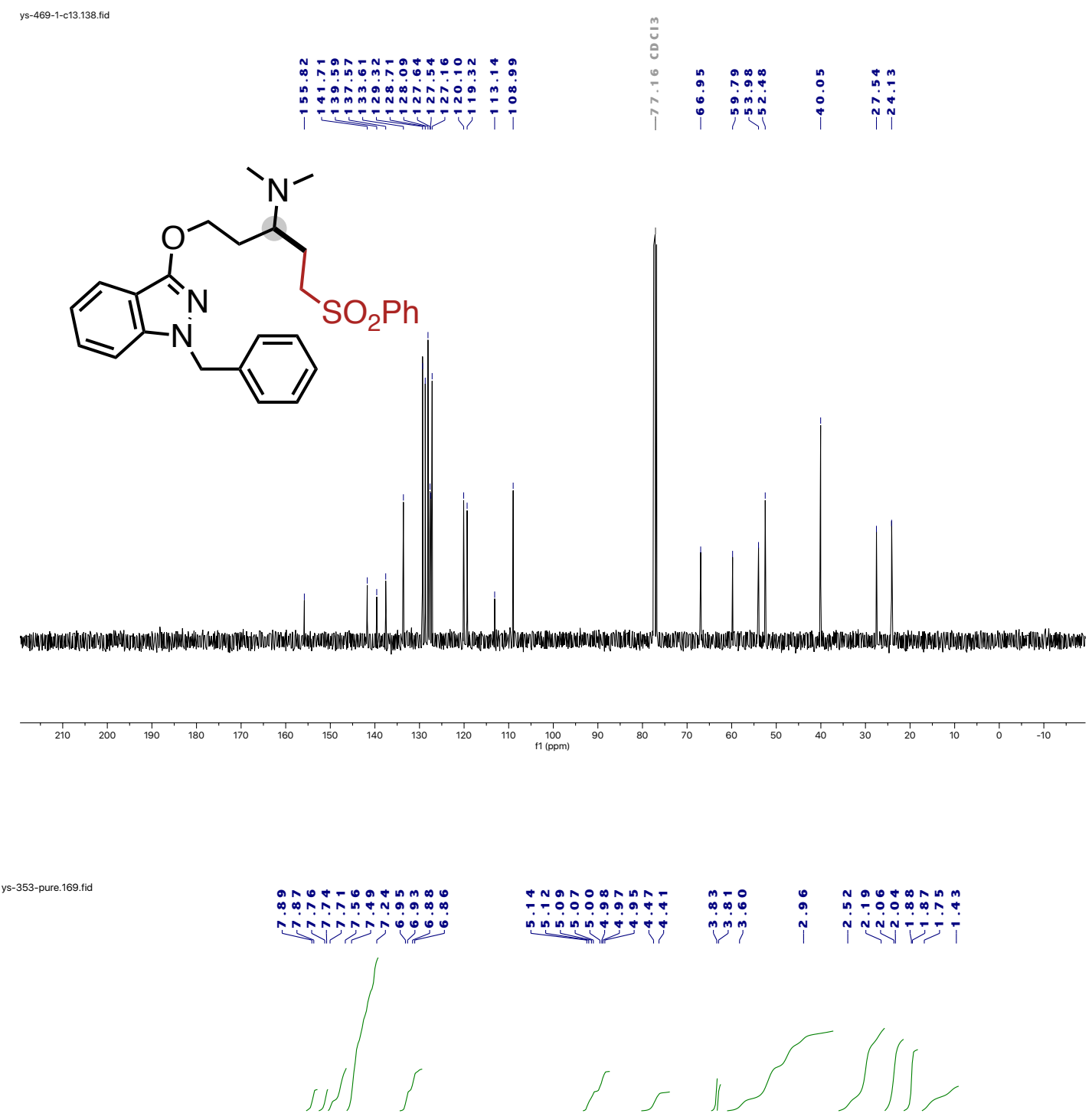<smiles>COc1ccc(C2Sc3ccccc3N(C[C@H](CCS(=O)(=O)c3ccccc3)N(C)C)C(=O)C2OC(C)C)cc1</smiles>

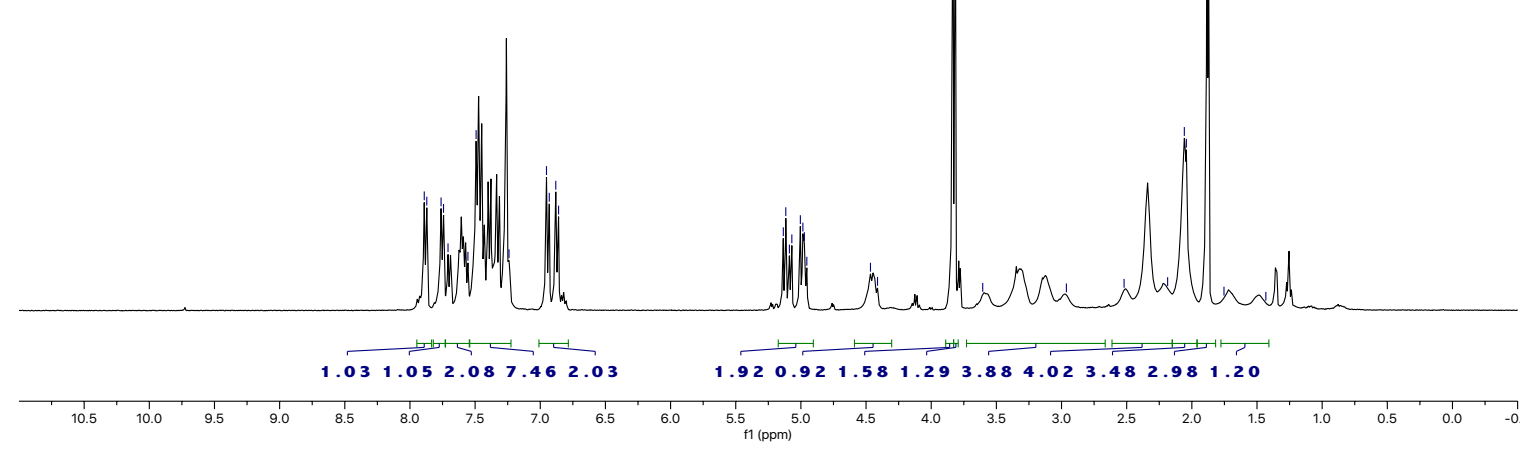



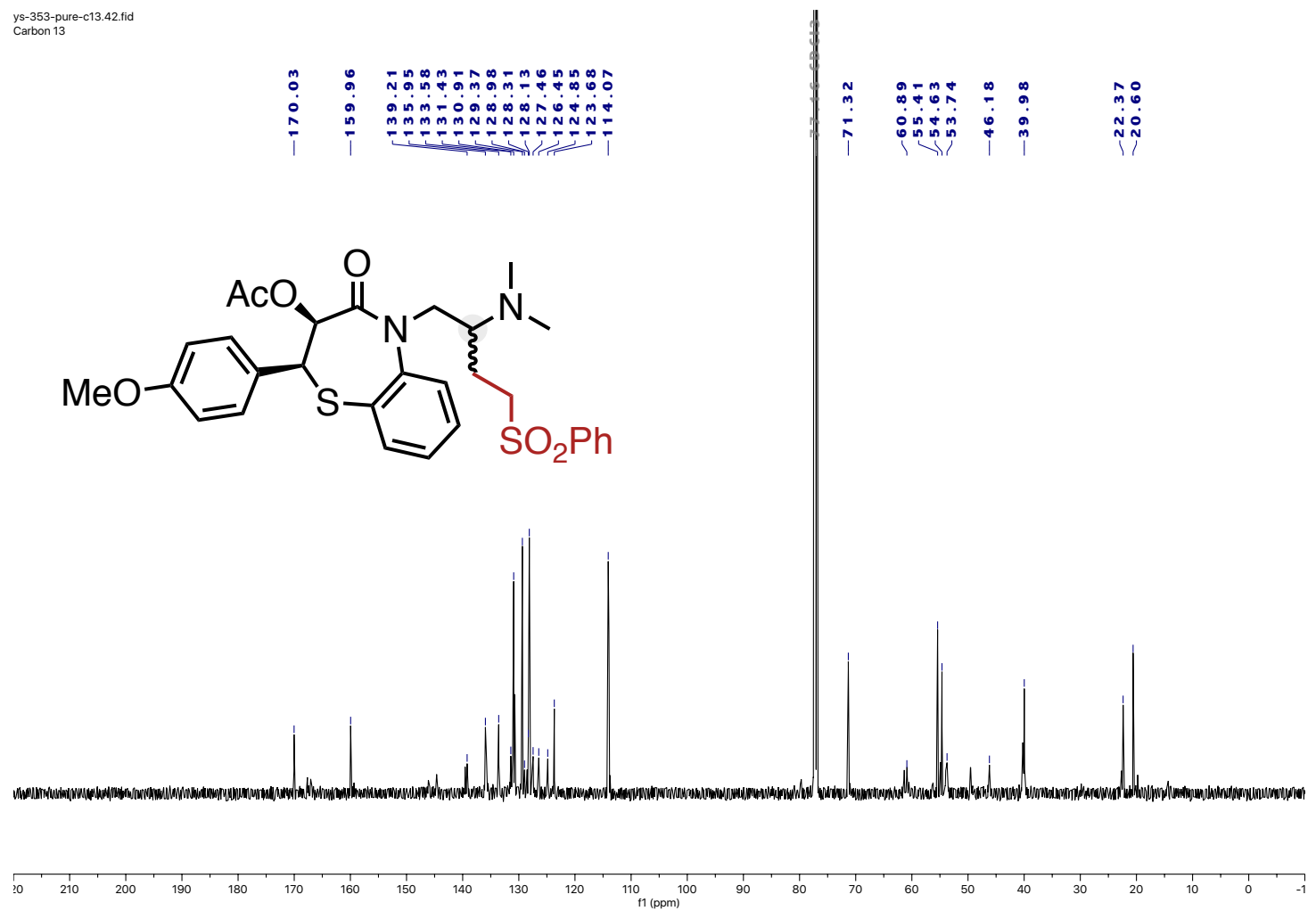

ys-477-1.150.fid
proton

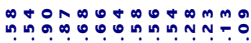

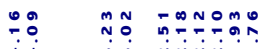

(c)

म) $m$ IINNT;

$\int_{\infty}$
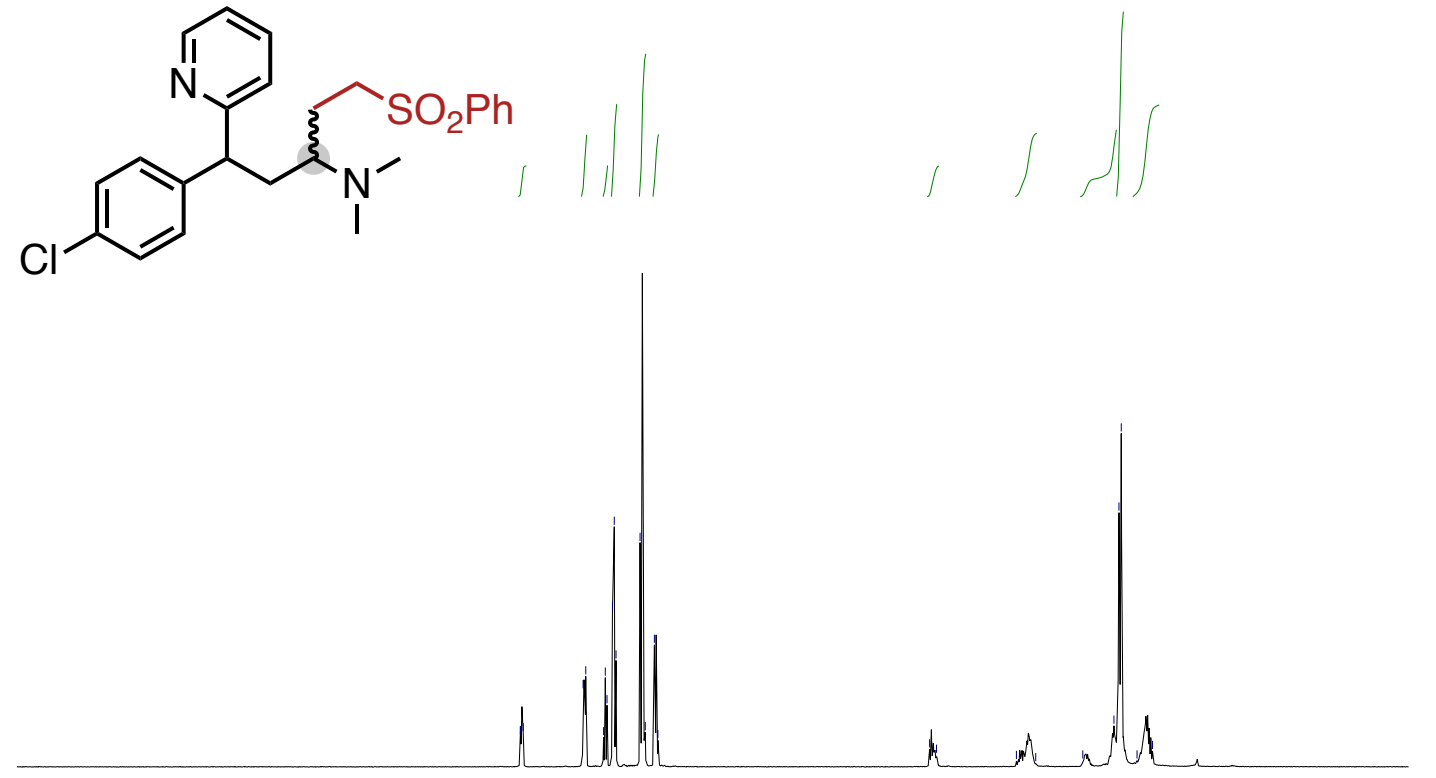

1.002 .001 .013 .004 .642 .02

1.002 .062 .176 .012 .98

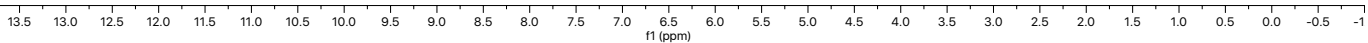



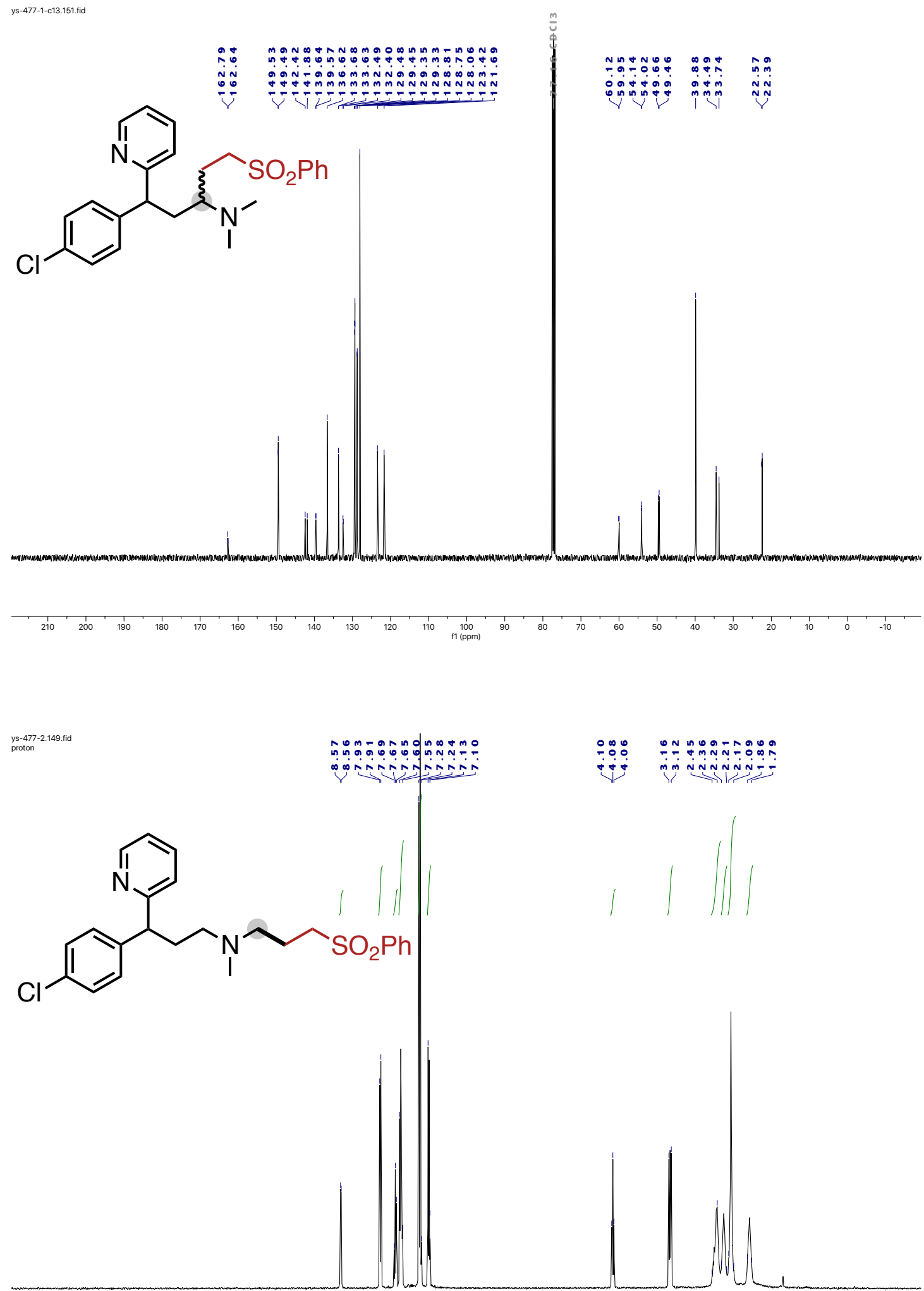

$1.002 .001 .063 .024 .902 .01 \quad 1.052 .013 .012 .004 .002 .03$

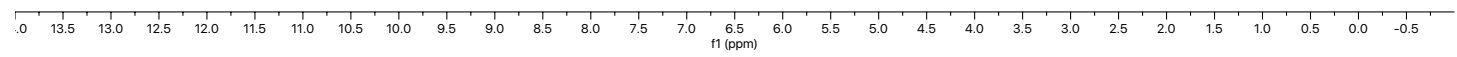



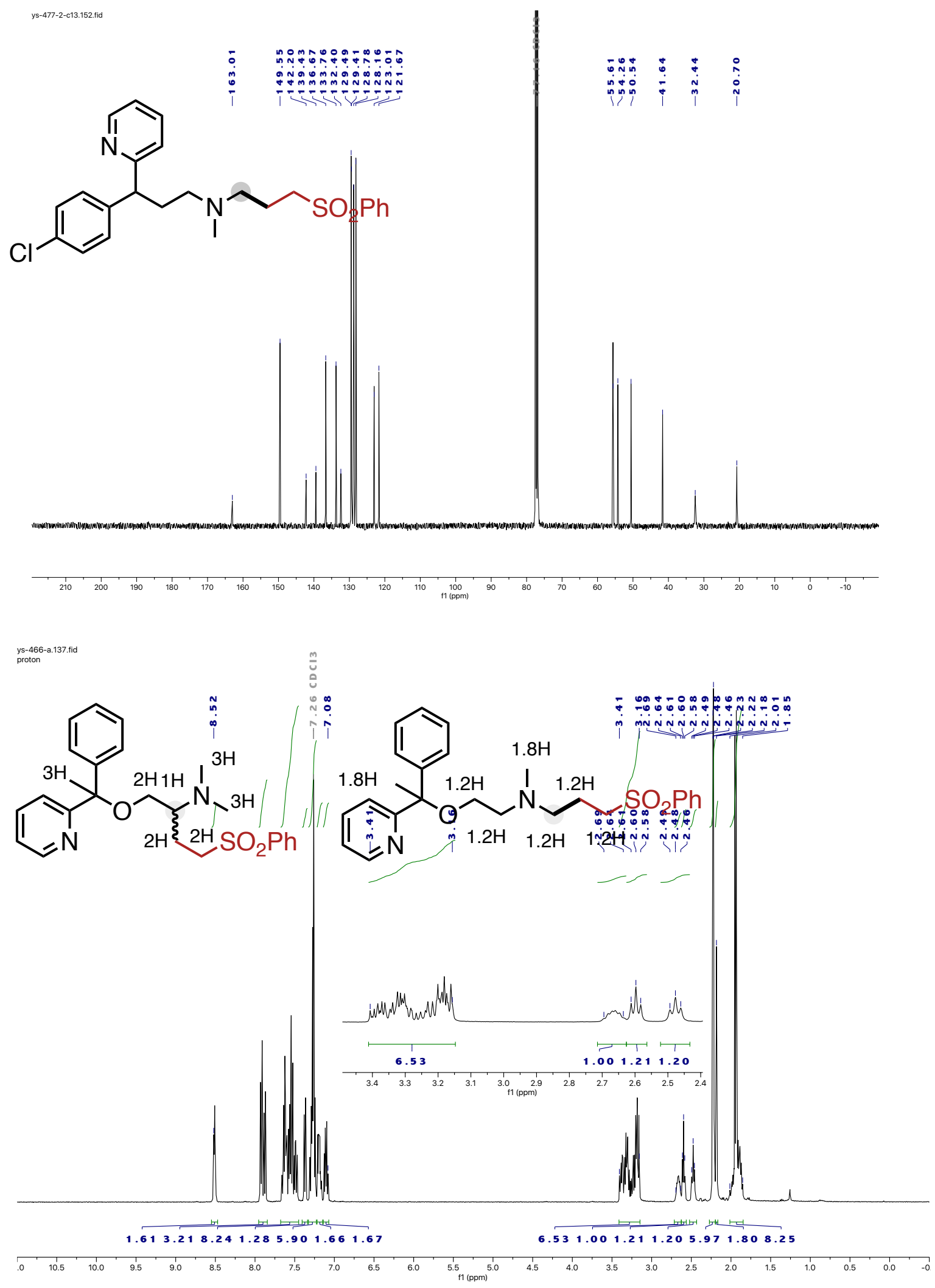

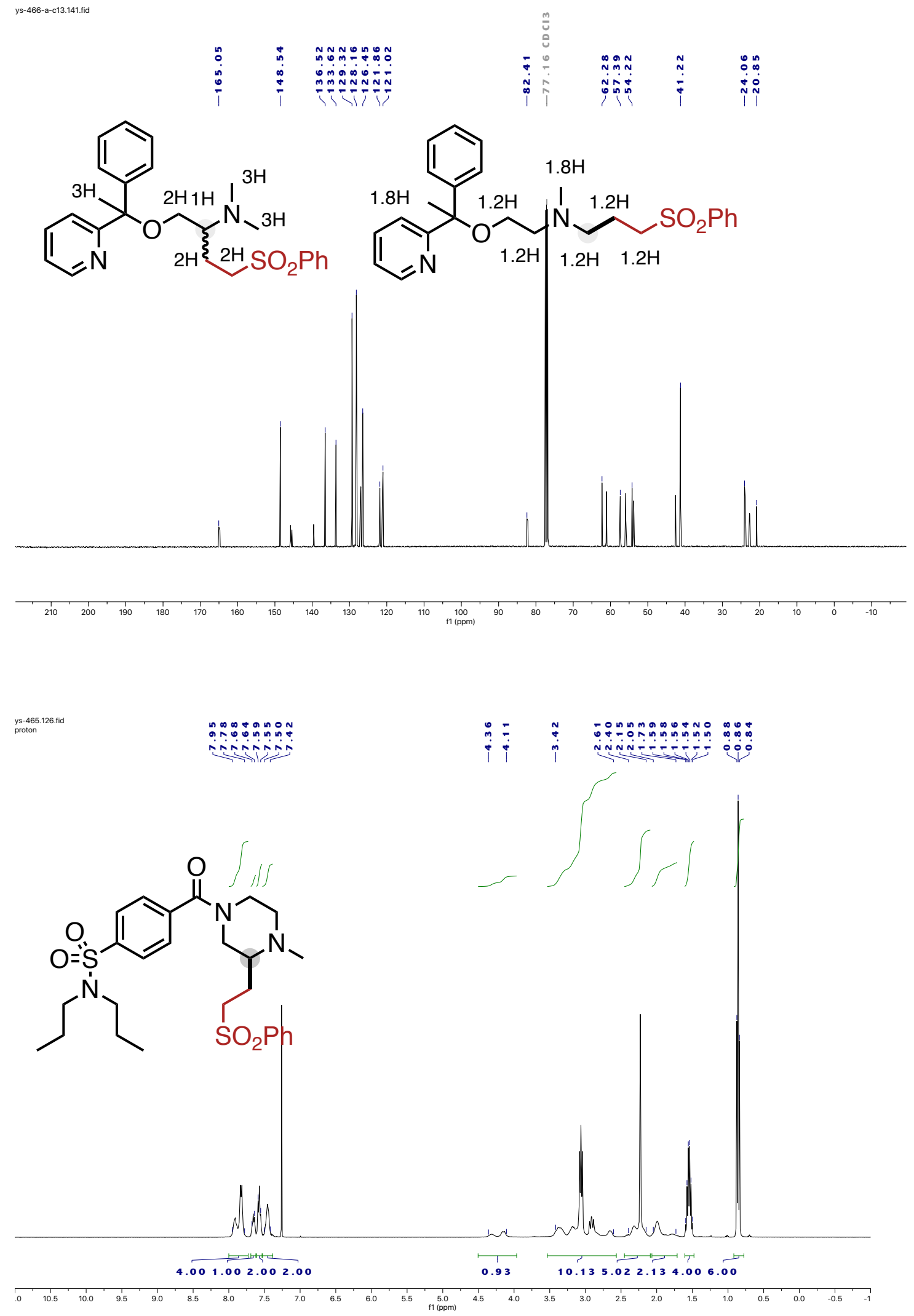

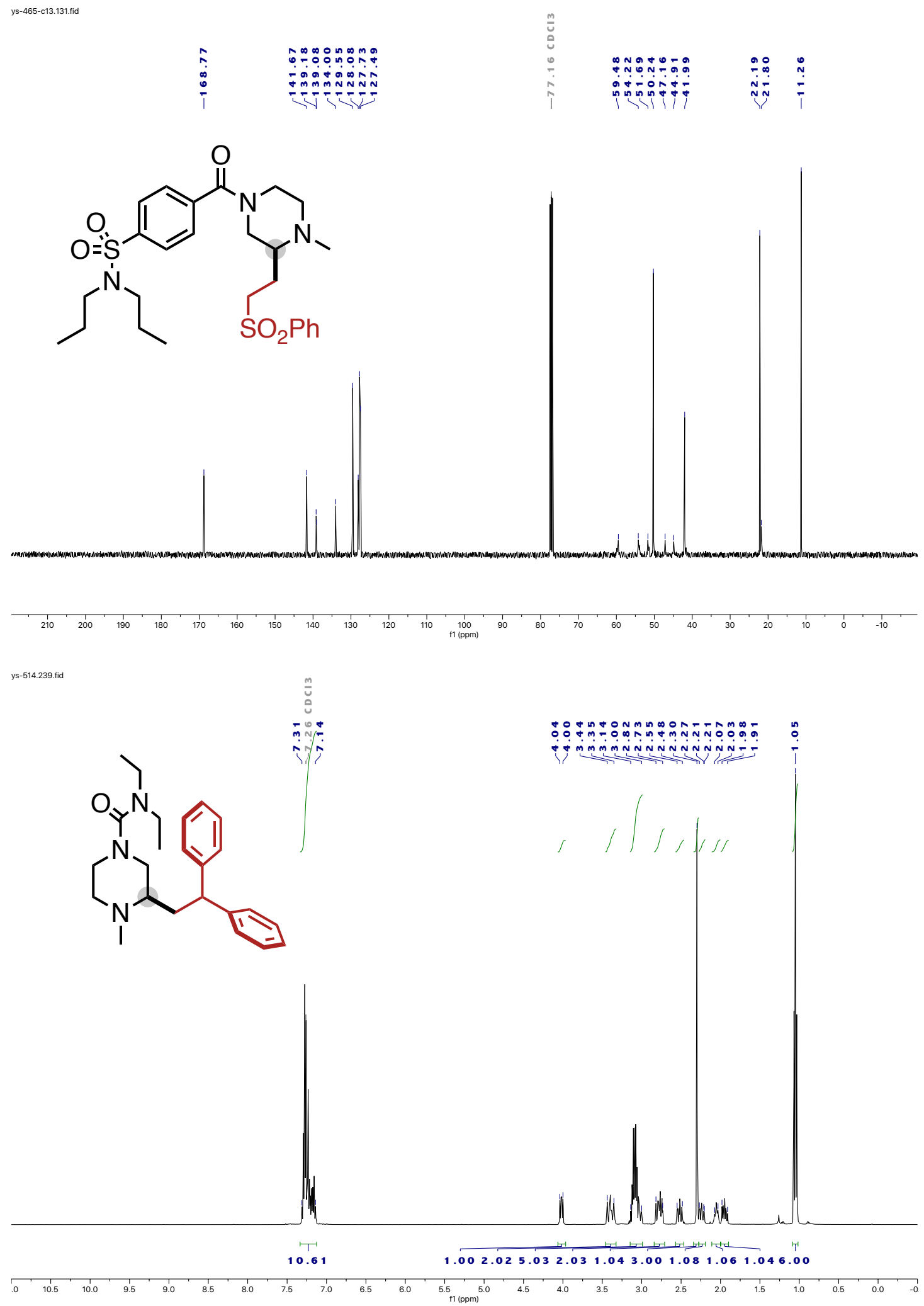

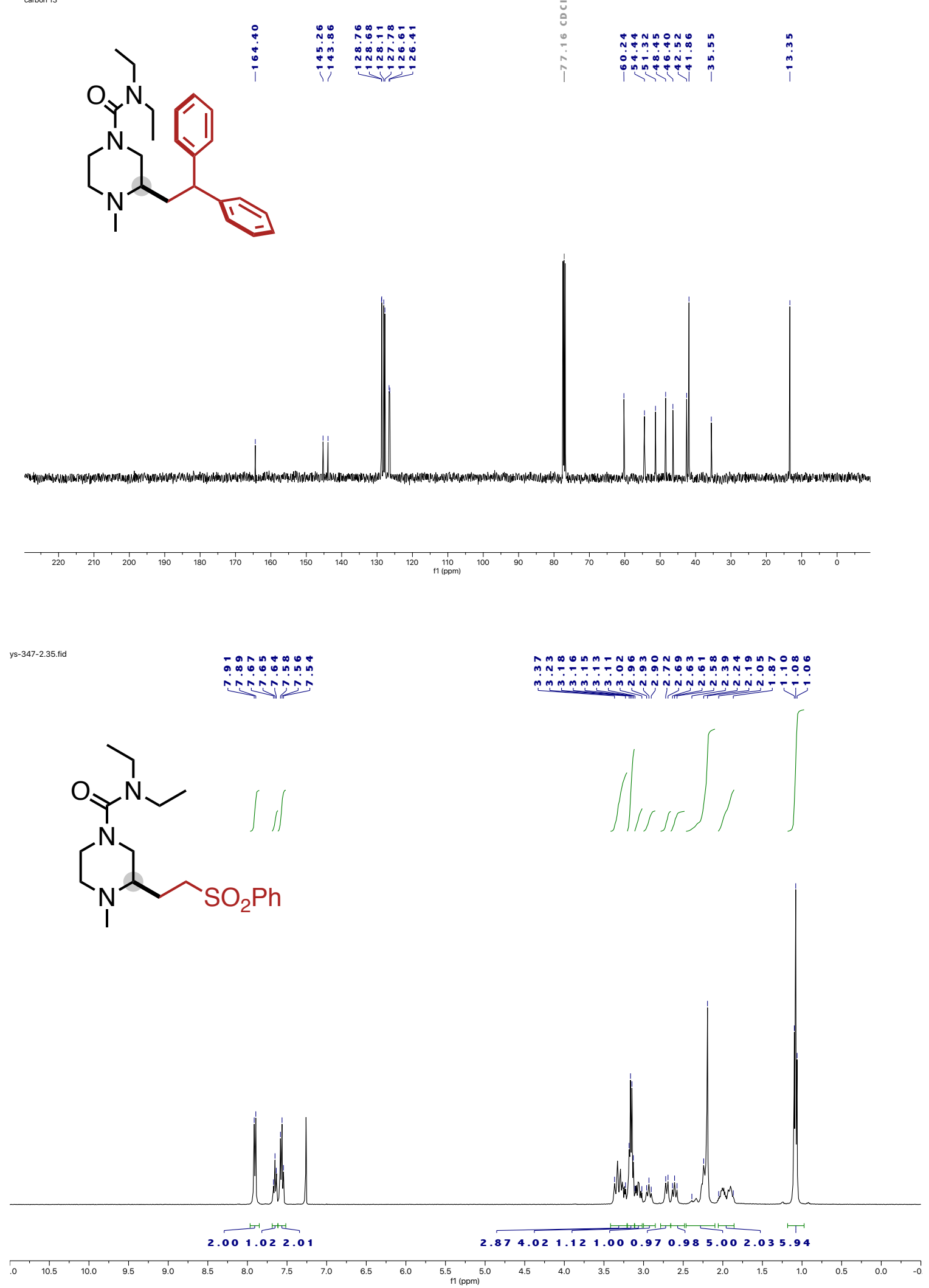


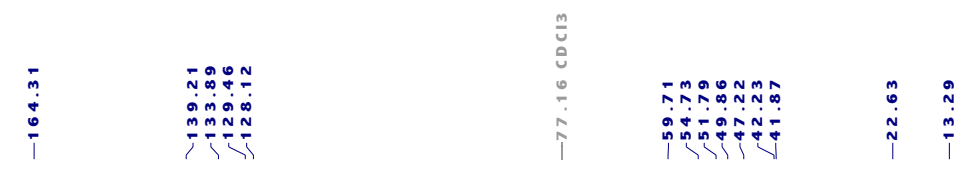<smiles>CCN(CC)C(=O)N1CCN(C)C(CCS(=O)(=O)Oc2ccccc2)C1</smiles>
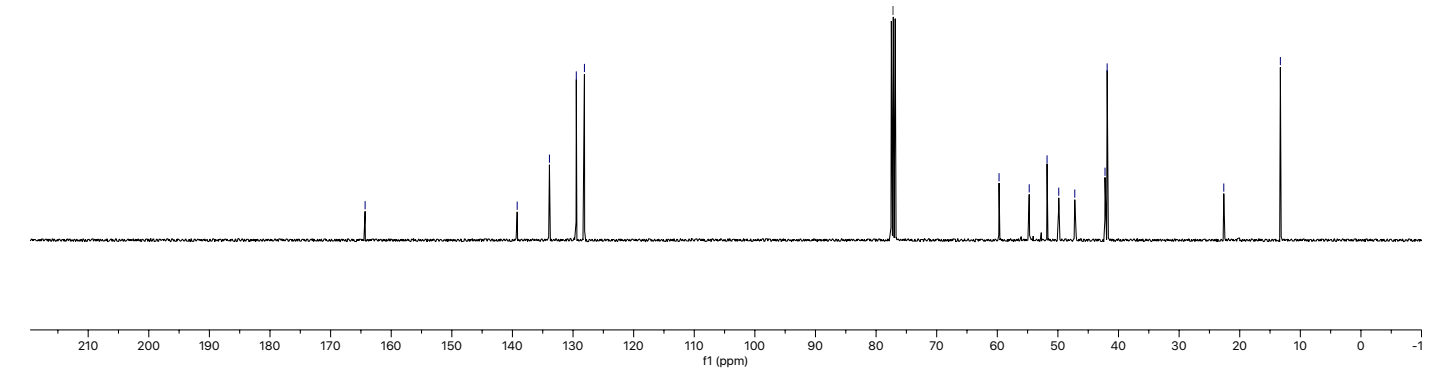

ys-510.240.fid
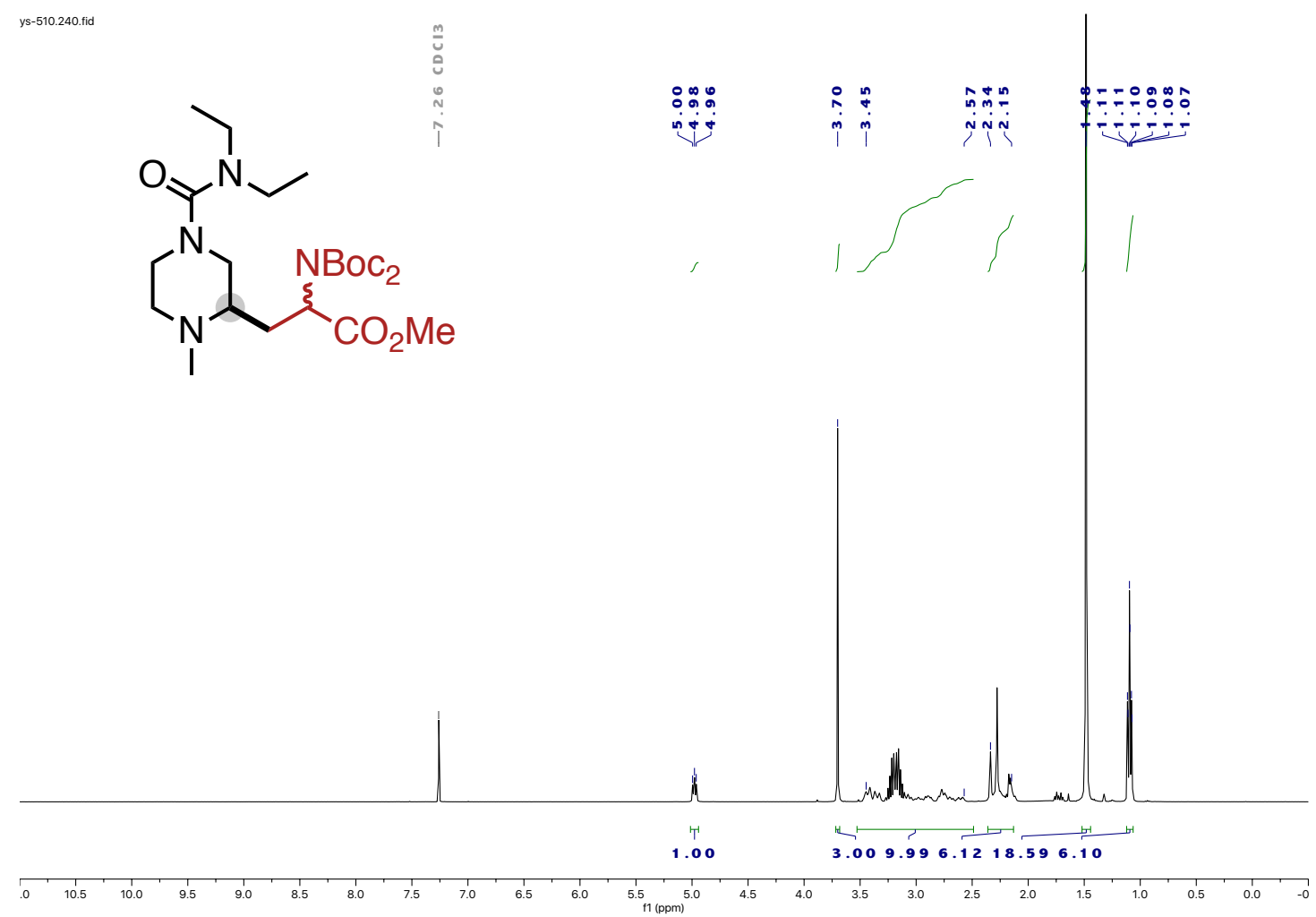

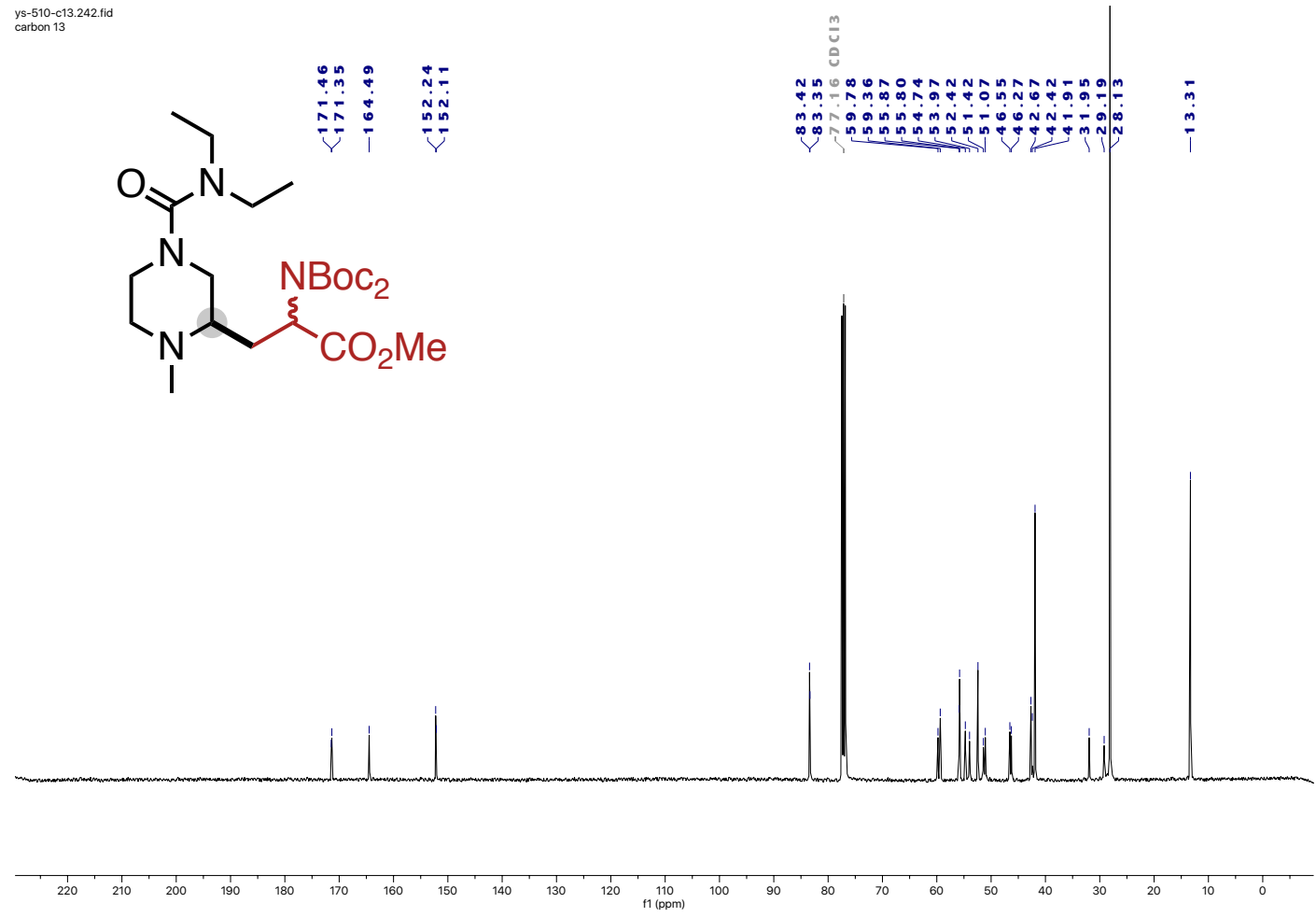

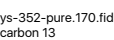

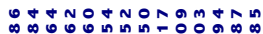
incinisisiono<smiles>CC#CCCC(CCS(=O)(=O)Oc1ccccc1)N(C)C</smiles>

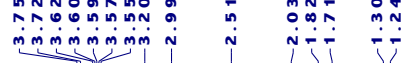

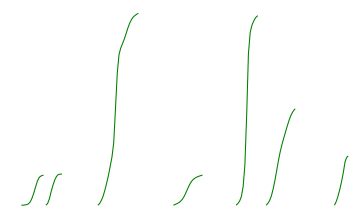

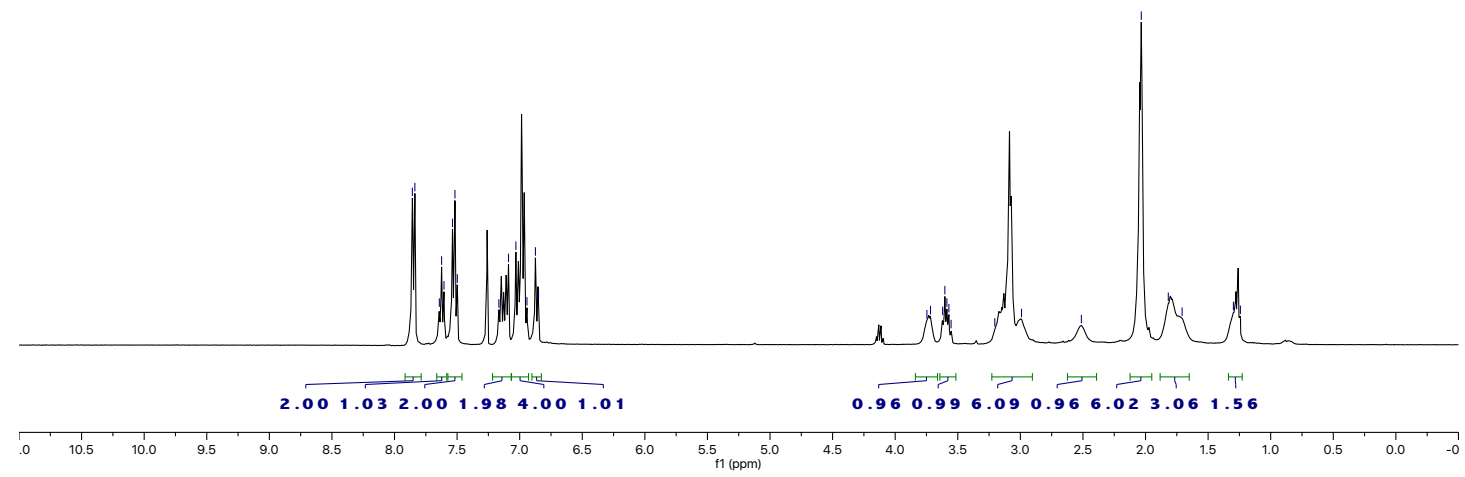



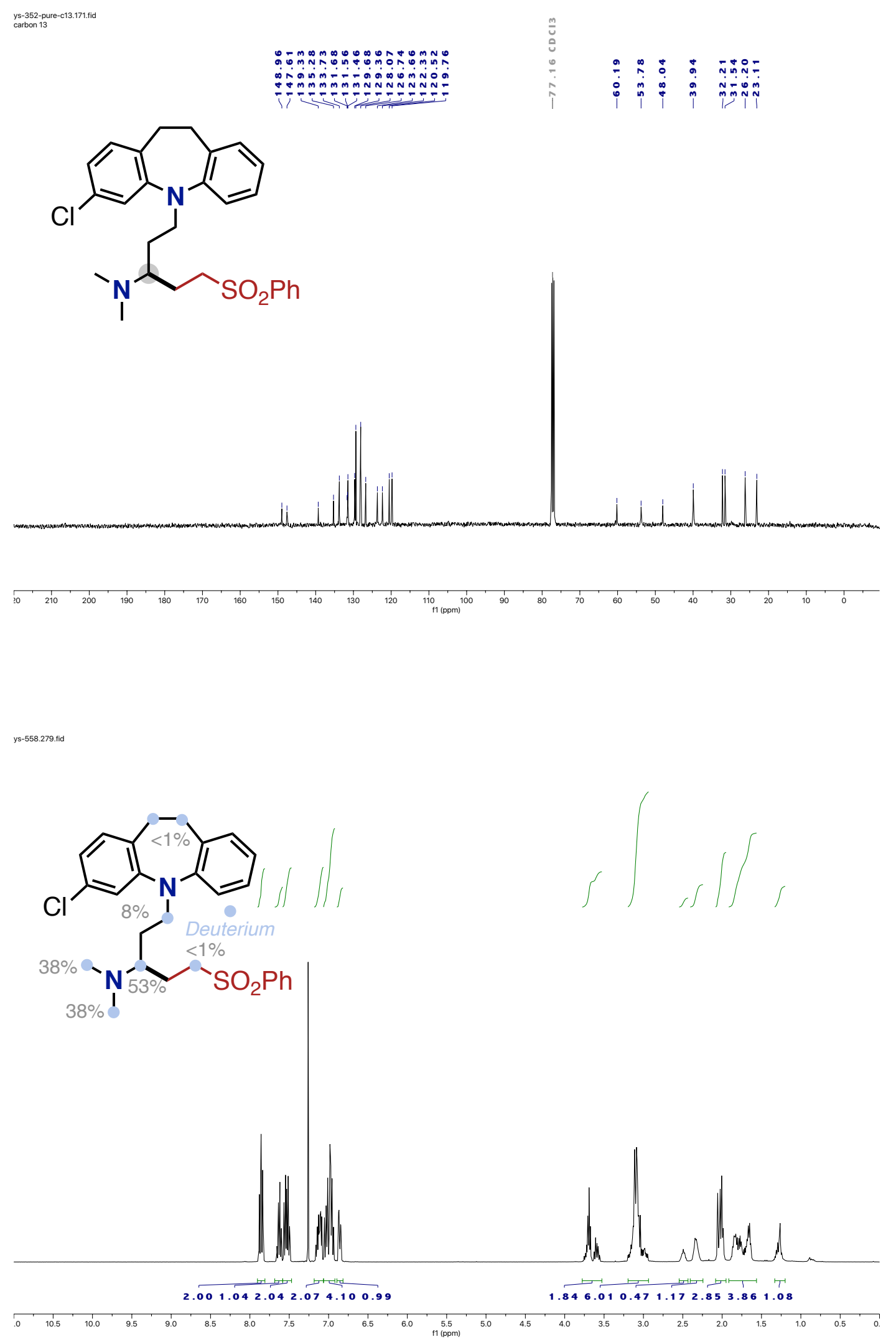

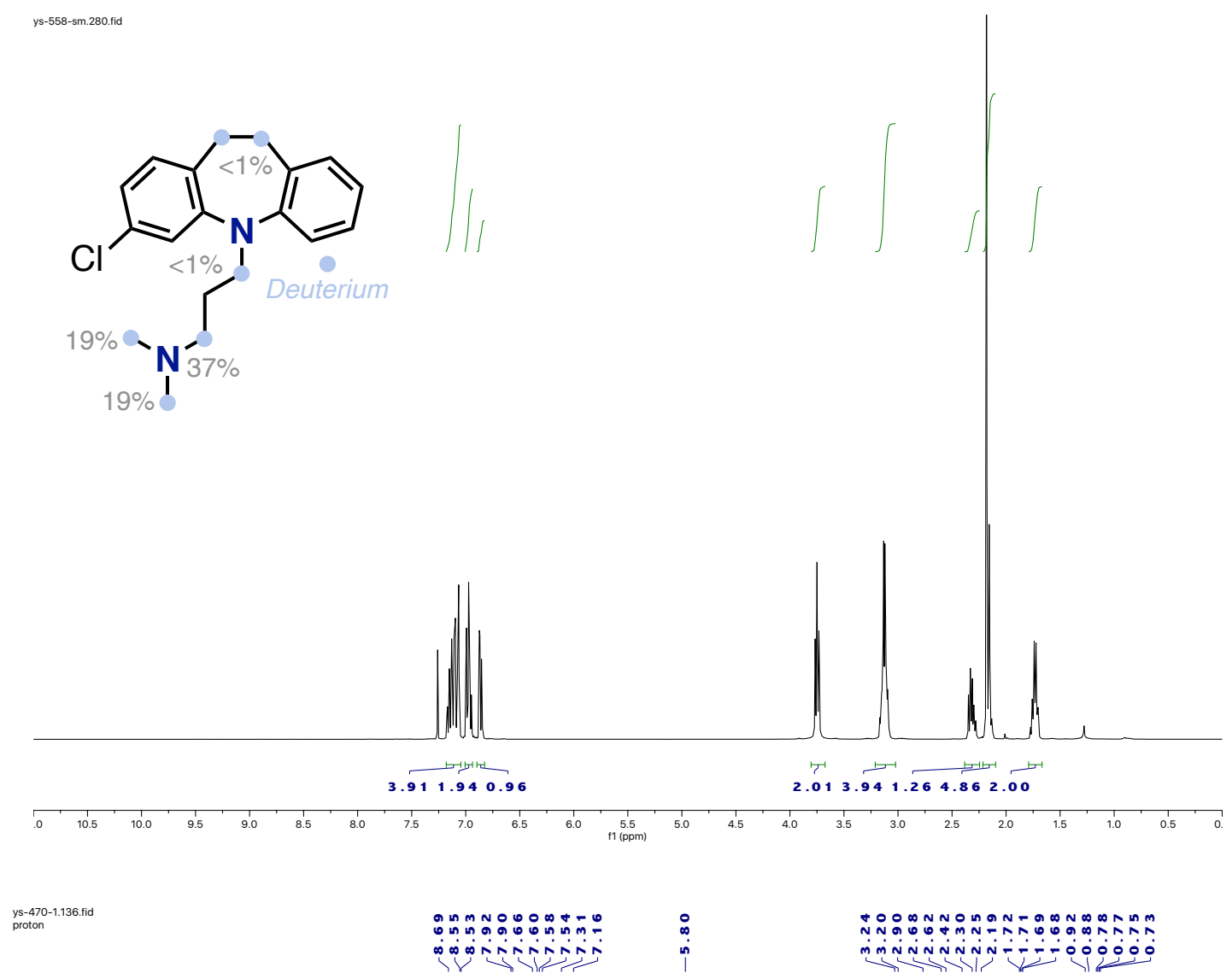

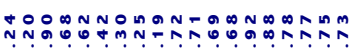

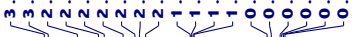

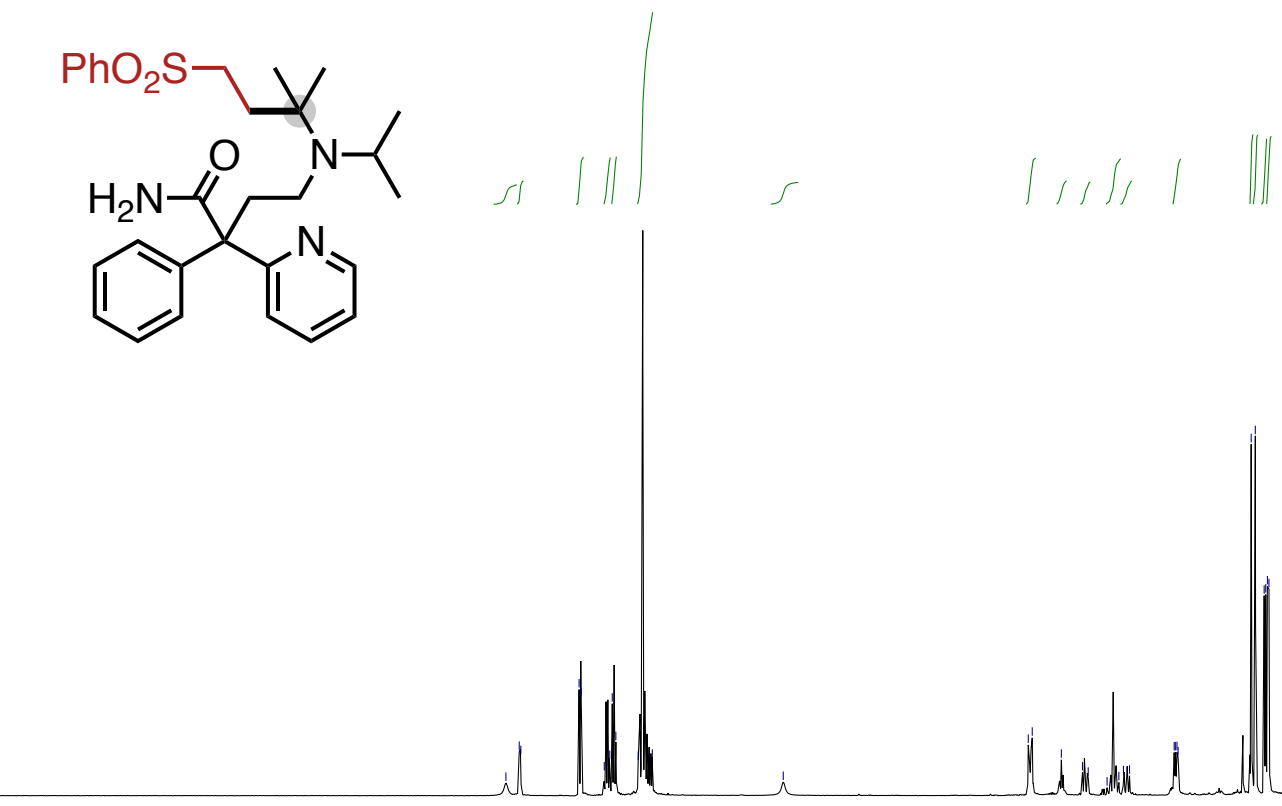

0.851 .002 .032 .022 .068 .310 .942 .001 .010 .971 .971 .001 .953 .023 .002 .842 .95

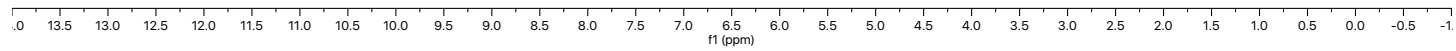




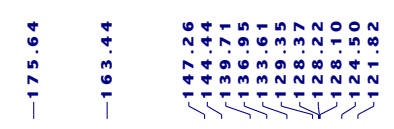

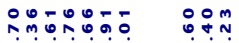

Oinnion
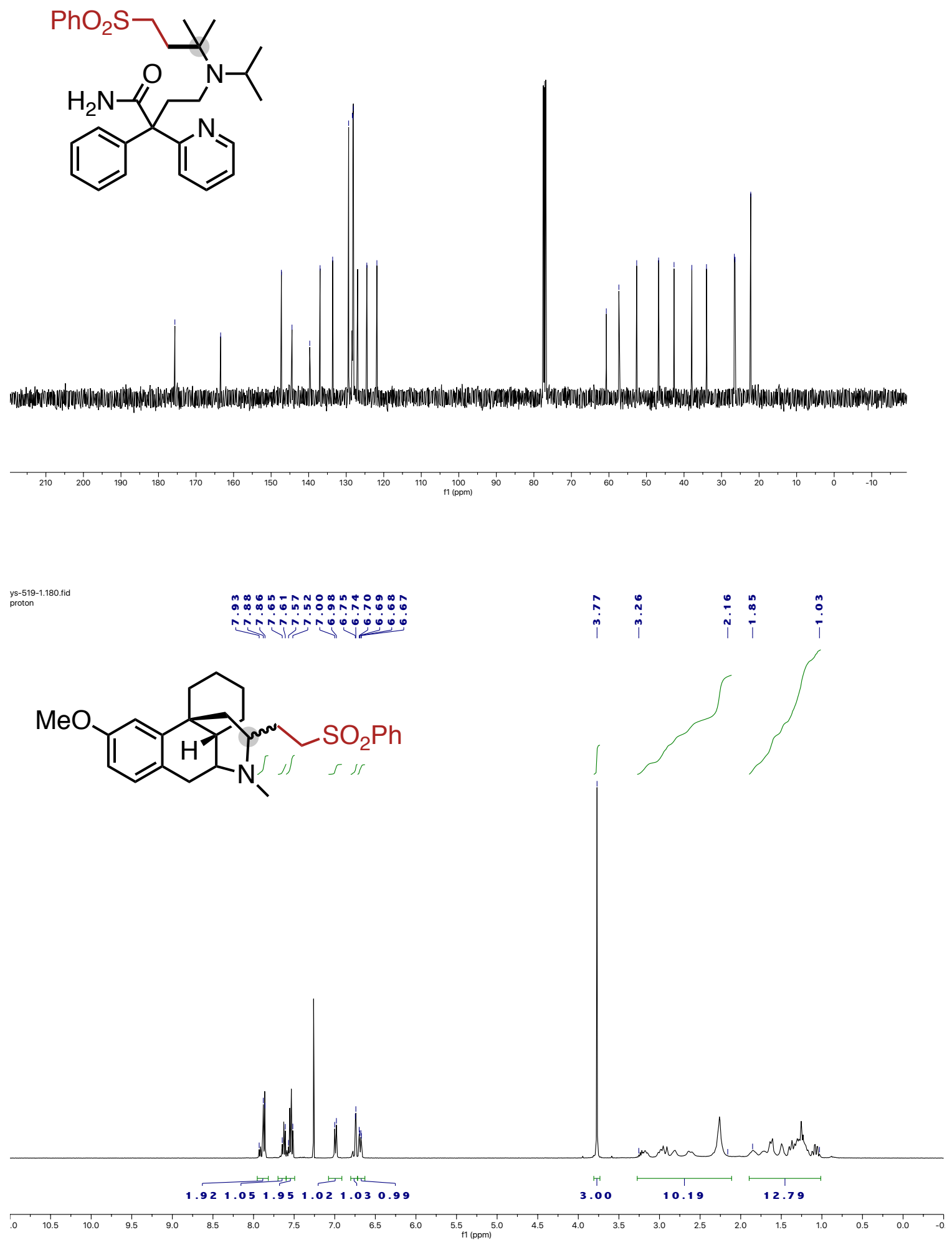

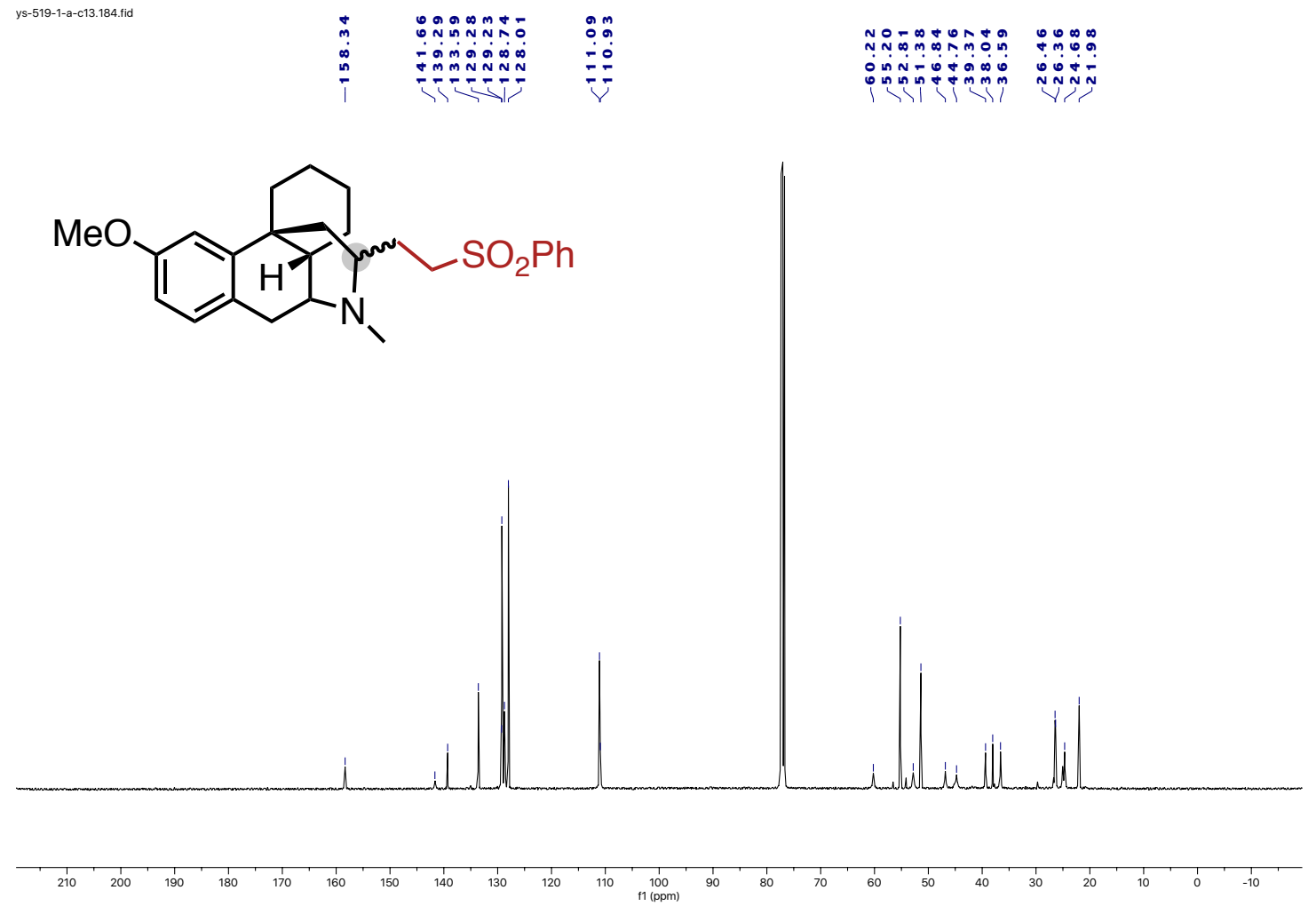
